\title{
Synthese und biologische Evaluierung von
}

\section{fluoreszenzmarkierten Duocarmycin-Analoga}

\author{
Dissertation \\ zur Erlangung des Doktorgrades \\ „Doctor rerum naturalium"
}

der Mathematisch-Naturwissenschaftlichen Fakultäten

der Georg-August-Universität zu Göttingen

vorgelegt von

Frank Behrendt

aus Paderborn

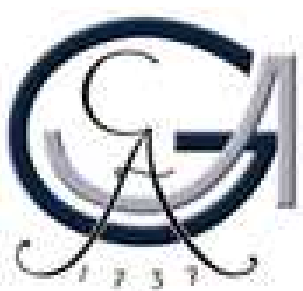

Göttingen 2011 

D7

Referent:

Prof. Dr. Dr. h.c. Lutz F. Tietze

Korreferent:

Prof. Dr. Hartmut Laatsch

Tag der mündlichen Prüfung: 25. November 2011 

Die vorliegende Arbeit wurde in der Zeit von September 2008 bis September 2011 am Institut für Organische und Biomolekulare Chemie der Georg-August-Universität Göttingen unter der Leitung von Prof. Dr. Dr. h.c. Lutz F. Tietze angefertigt

Mein besonderer Dank gilt Herrn Prof. Dr. Dr. h.c. Lutz F. Tietze für die interessante Themenstellung, sein stetes Interesse am Fortgang dieser Arbeit sowie für die zahlreichen Diskussionen und Anregungen. 

MeIner FAMILIE 

„Auch aus Steinen, die einem in den Weg gelegt werden, kann man Schönes bauen." 



\section{INHALTSVERZEICHNIS}

\section{INHALTSVERZEICHNIS.}

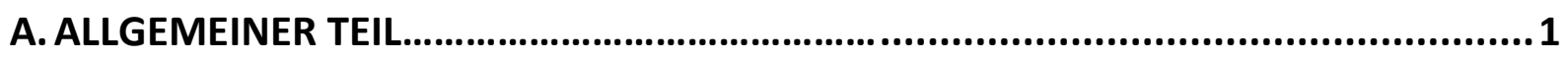

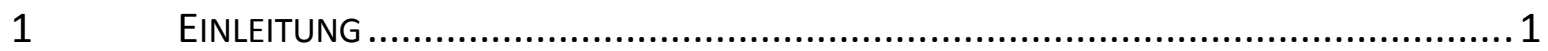

2 CANCEROGENESE - VON DER KREBSZELLE ZUM TUMOR ...................................... 4

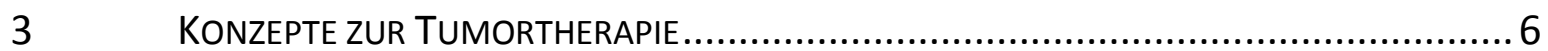

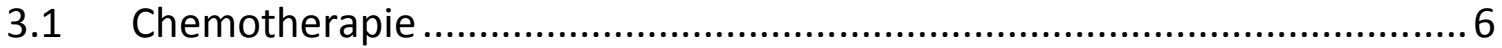

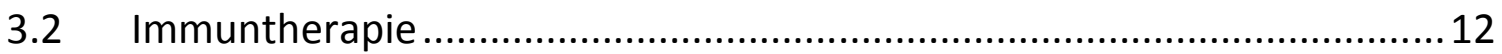

3.3 Selektive Krebstherapie mittels reversibel detoxifizierter Prodrugs ......... 15

3.3.1 Antibody-Directed Enzyme Prodrug Therapy (ADEPT) ....................17

3.3.2 Prodrug-Monotherapie (PMT) …............................................. 21

$4 \quad$ CC-1065, DuOCARMYCIN SA UND YATAKEMYCIN ......................................... 25

4.1 Analoga von (+)-CC-1065 und (+)-Duocarmycin SA.............................. 28

4.2 Seco-Verbindungen und Prodrugs einiger Duocarmycin-Analoga .............. 32

4.2.1 Forschungsergebnisse anderer Arbeitsgruppen ............................33

4.2.2 Forschungsergebnisse aus dem Arbeitskreis Tietze ......................... 34

5 CONFOCAL LASER SCANNING MicROSCOPY (CLSM) \& OPTICAL IMAGING ................ 39

5.1 Confocal Laser Scanning Microscopy (CLSM) ....................................... 39

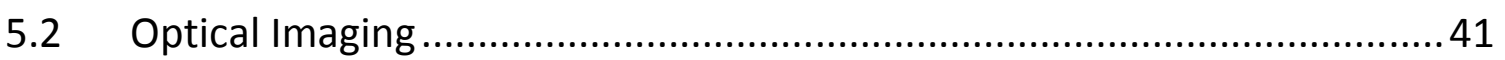

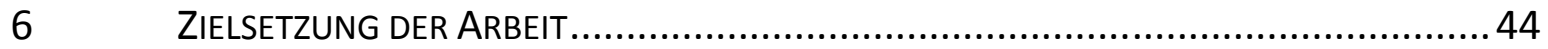

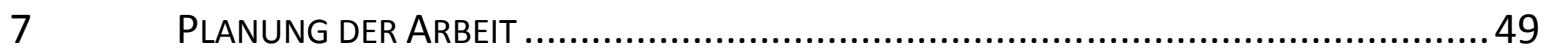

7.1 Synthese des fluoreszenzmarkierten CBI-DMAI-Prodrugs (1S)-72 ........... 49

7.2 Verkürtzte Synthese zur Darstellung des fluoreszenzmarkierten

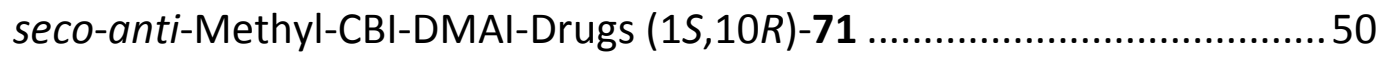

7.3 Synthese neuartiger fluoreszenzmarkierter Duocarmycin-Analoga ..........53

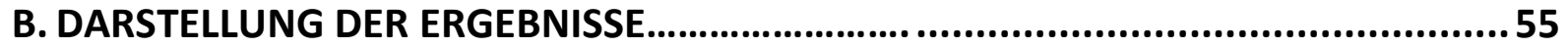

1 SYNTHESE DES FLUORESZENZMARKIERTEN ANTI-METHYL-SECO-CBI-DRUGS

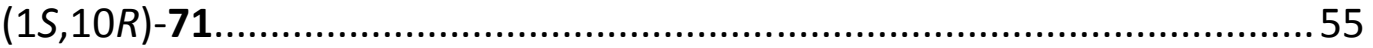

1.1 Synthese des anti-Methyl-seco-CCBI-Grundgerüstes $(1 S, 10 R)-82 \ldots \ldots \ldots \ldots 5$ 
1.2 Synthese des anti-Methyl-seco-CCBI-DMAI-Derivates $(1 S, 10 R)-88$. 59

1.3 Synthese des anti-Methyl-seco-CBI-DMAI-Derivates mit Spacer-Einheit $(1 S, 10 R)-106$ 59

1.4 Synthese des anti-Methyl-seco-CBI-DMAI-Derivates ohne Spacereinheit $(1 S, 10 R)-87$

1.5 Synthese des fluroreszenzmarkierten anti-Methyl-seco-CBI-DMAI-Drugs $(1 S, 10 R)-71$

1.5.1 Diskussion ausgewählter spektroskopischer Daten des

fluoreszenzmarkierten anti-Methyl-seco-Drugs $(1 R, 10 S)-71$ 66

CHARAKTERISIERUNG UND UNTERSUCHUNG DER ZELLULÄREN AUFNAHME VON FLUORESZENZMARKIERTEN DAPOXYL-ANTI-METHYL-SECO-CBI-DMAI-DERIVATEN ....69

2.1 Fluorimetrische Bestimmung der Absorptions- und Emissionsmaxima ....69

2.2 Untersuchung der zellulären Aufnahme fluroreszenzmarkierter antiMethyl-seco-CBI-DMAI-Derivate in lebende Zellen durch CLSM .............. 71

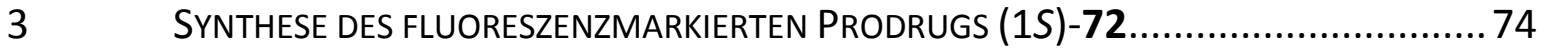

3.1 Synthese des seco-CCBI-Grundgerüstes (1S)-115 ................................ 74

3.2 Synthese des CBI-DMAI-Prodrugs (1S)-118 ......................................... 75

3.3 Synthese des fluoreszenzmarkierten CBI-DMAI-Prodrugs (1S)-72 ...........77

3.4 In-vitro-Zytotoxizitätsuntersuchungen des fluoreszenzmarkierten

(1S)-CBI-DMAI-Galaktosides (1S)-72 sowie des Galaktosides (1S)-118 .... 78

3.4.1 Der HTCFA-Test zur Bestimmung der Zytotoxizität ......................... 78

3.4.2 Ergebnisse zur Untersuchung der In-vitro-Zytotoxizität an humanen Bronchialkarzinomzellen (A549) für das fluoreszenzmarkierte (1S)-CBI-DMAI-Galaktosid (1S)-72 sowie das Galaktosid (1S)-118 .....80

4 SYNTHESE DES SECO-CBI-GRUNDGERÜSTES (1S)-128 82

5 SYNTHESE NEUARTIGER FLUORESZENZMARKIERTER CBI-CUMARIN-DUOCARMYCINANALOGA .85

5.1 Synthese der DNA-Binder Indol-Cumarin-2-Carbonsäure (130) sowie DMACA (62) 86

5.2 Synthese der seco-CBI-Cumarin-Drugs (1S)-92, (1S)-137 und (1S)-138 .... 87 
5.2.1 Diskussion ausgewählter spektroskopischer Daten des seco-CBI-

DMACA-Drugs (1S)-137 88

5.3 Synthese der (1S)-CBI-Cumarin-Galaktoside (1S)-69, (1S)-139 und (1S)-140

5.4 Ergebnisse zur Untersuchung der In-vitro-Zytotoxizität an humanen Bronchialkarzinomzellen (A549) für die (1S)-CBI-Cumarin-Derivate 92

6 CHARAKTERISIERUNG UND UNTERSUCHUNG DER ZELLULÄREN AUfNAHME VON FLUORESZENZMARKIERTEN (1S)-CBI-CUMARIN-DERIVATEN 94

6.1 Fluorimetrische Bestimmung der Absorptions- und Emissionsmaxima ....94

6.1.1 Bestimmung der Fluoreszenz- und Emissionsmaxima für die dargestellten Cumarin-3-Carbonsäure-Derivate (1S)-92, (1S)-69 sowie 131 . 94

6.1.2 Bestimmung der Fluoreszenz- und Emissionsmaxima für die dargestellten DMACA-Derivate (1S)-137, (1S)-139 sowie 62 96

6.1.3 Bestimmung der Fluoreszenz- und Emissionsmaxima für die dargestellten Indol-Cumarin-Derivate (1S)-138, (1S)-140 sowie $\mathbf{1 3 0 . 9 7}$

6.2 Untersuchung der zellulären Aufnahme fluroreszenzmarkierter (1S)-CBI-

Cumarin-Derivate in lebende Zellen durch CLSM 99

6.2.1 Untersuchung der zellulären Aufnahme fluroreszenzmarkierter (1S)CBI-Cumarin-3-Carbonsäure-Derivate (1S)-92, (1S)-69 sowie 131 in lebende Zellen 100

6.2.2 Untersuchung der zellulären Aufnahme fluroreszenzmarkierter (1S)CBI-DMACA-Derivate (1S)-137, (1S)-139 sowie 62 102

6.2.3 Untersuchung der zellulären Aufnahme fluroreszenzmarkierter (1S)-CBI-Indol-Cumarin-Derivate (1S)-138, (1S)-140 sowie 130 ....... 104 SYNTHESE NEUARTIGER FLUORESZENZMARKIERTER (1S)-CBI-BENZOTHIAZOLDUOCARMYCIN-ANALOGA 107

7.1 Synthese der seco-CBI-Benzothiazol-Drugs (1S)-141 und (1S)-145 ......... 108

7.1.1 Diskussion ausgewählter spektroskopischer Daten des seco-CBI-BODrugs (1S)-141 108 
7.2 Synthese der (1S)-CBI-Benzothiazol-Galaktoside (1S)-70 und (1S)-147... 112

7.2.1 Ergebnisse zur Untersuchung der In-vitro-Zytotoxizität an humanen Bronchialkarzinomzellen (A549) für die seco-CBI-BenzothiazolDerivate (1S)-141 und (1S)-145 sowie den Galaktosiden (1S)-147 und (1S)-70 114

7.3 Synthese der Benzothiazol-Methylester 143 und 144 115

8 ChARAKTERISIERUNG UND UNTERSUCHUNG DER ZELLULÄREN AUFNAHME VON FLUORESZENZMARKIERTEN (1S)-CBI-BENZOTHIAZOL-DERIVATEN....

8.1 Fluorimetrische Bestimmung der Absorptions- und Emissionsmaxima .. 117

8.1.1 Bestimmung der Fluoreszenz- und Emissionsmaxima für die dargestellten BO-Derivate (1S)-141, (1S)-70 sowie 144

8.1.2 Bestimmung der Fluoreszenz- und Emissionsmaxima für die dargestellten TO3-Derivate (1S)-145, (1S)-147 sowie 143

8.2 Untersuchung der zellulären Aufnahme fluroreszenzmarkierter (1S)-CBI-

Benzothiazol-Derivate in lebende Zellen durch CLSM 121

8.2.1 Untersuchung der zellulären Aufnahme fluroreszenzmarkierter (1S)-CBI-BO-Derivate (1S)-141, (1S)-70 und 144 in lebende Zellen .. 122

8.2.2 Untersuchung der zellulären Aufnahme fluroreszenzmarkierter (1S)-CBI-TO3-Derivate (1S)-145, (1S)-147 sowie 143. 124

C. ZUSAMMENFASSUNG. 127

D. EXPERIMENTELLER TEIL 145

1 ALLGEMEINE ARBEITSVORSCHRIFTEN 145

1.1 Verwendete Geräte ...................................................................... 145

1.2 Chromatographische Methoden ...................................................... 147

1.3 Materialien für die In-vitro-Zytotoxizitätsuntersuchungen ................... 149

2 SYNTHESE DER FLUORESZENZAKTIVEN DNA-BINDER ...................................150

2.1 1-(5-Methoxycarbonyl-pentyl)-4-(3-Methyl-3H-benzothiazol-2-

ylidenmethyl)-pyridinium-chlorid (144) 150

2.2 1-(5-Methoxycarbonyl-pentyl)-4-[3-(3-Methyl-3H-benzothiazol-2-yliden)propenyl]-quinolinium-chlorid (143) 
2.3 (7-Dimethylamino-2-oxo-2H-chromn-4-yl)-essigsäure (62)

2.4 7-Oxo-3,7-dihydro-pyrano[3,2-e]indol-2-carbonsäure (130)... 153 SYNTHESE DES SECO-CCBI-GRUNDGERÜSTES (1S)-115

3.1 2-Amino-4-benzyloxy-1-iod-N-(tert-butyloxycarbonyl)-7-cyano-naphthalin (75) 154

3.2 (E/Z)-2-Amino-4-benzyloxy-N-(tert-butyloxycarbonyl)- $N$-(3-chlor-prop-2enyl)-7-cyano-1-iod-naphthalin (104) 155

3.3 rac-\{(1S)-5-Benzyloxy-3-(tert-butyloxycarbonyl)-1-chlormethyl-8-cyano1,2-dihydro-3H-benz[e]indol (rac-(1S)-74) 156

3.4 Trennung der Enantiomere von rac-\{(1S)-5-Benzyloxy-3-(tert-butyloxycarbonyl)-1-chlormethyl-8-cyano-1,2-dihydro-3H-benz[e]indol (rac-(1S)-74) 158

3.5 (-)-\{(1S)-5-Benzyloxy-3-(tert-butyloxycarbonyl)-1-chlormethyl-8-cyano1,2-dihydro-3H-benz[e]indol ((-)-(1S)-115). 159

$4.1(+)-\left\{\left[(1 S)-1-c h l o r m e t h y l-8-c y a n o-3-\left[\left(5-\left(2-\left(N, N^{\prime}-\operatorname{dimethyl-amino}\right)\right.\right.\right.\right.\right.$-ethoxy)indol-2-yl)carbonyl]-1,2-dihydro-3H-benz[e]indol-5-yl]-2,3,4,6-tetra-Oacetyl- $\beta$-D-Galaktopyranosid\} ((+)-(1S)-73) 161

$4.2(+)-\left\{\left[(1 S)-8-A m i n o m e t h y l-1-c h l o r m e t h y l-3-\left[\left(5-\left(2-\left(N, N^{\prime}-\right.\right.\right.\right.\right.\right.$ dimethylamino $)-$ ethoxy)-indol-2-yl)carbonyl]-1,2-dihydro-3H-benz[e]indol-5-yl]-2,3,4,6tetra-O-acetyl- $\beta$-D-Galaktopyranosid\} ((+)-(1S)-117) 163

4.3 [(1S)-8-Aminomethyl-1-chlormethyl-3-[(5-(2-( $N, N^{\prime}-$ dimethylamino)ethoxy)-indol-2-yl)carbonyl]-1,2-dihydro-3H-benz[e]indol-5-yl]-O- $\beta$-DGalaktopyranosid-ditrifluoracetat \} ((1S)-118) 164

4.4 6-(Fluorescein-5-carboxamido)-Hexansäure-[(1S)-1-chlormethyl-3-[(5-(2$\left(N, N^{\prime}\right.$-dimethylamino)-ethoxy)-indol-2-yl)carbonyl]-5-O- $\beta$-D-Galaktopyranosyl-1,2-dihydro-3H-benz[e]indol-8-ylmethyl]-amid-trifluoracetat ((1S)-72) 167

5 SYNTHESE DES ANTI-METHYL-SECO-CCBI-GRUNDGERÜSTES $(1 S, 10 R)-82$ 170

5.1 Ethyl-1-benzyloxy-7-brom-3-naphthalincarboxylat (98). 170 
5.2 Ethyl-1-benzyloxy-7-cyano-3-naphthalincarboxylat (99)

5.3 1-Benzyloxy-7-cyano-3-naphthalincarbonsäure (100)

5.4 3-Amino-1-benzyloxy- $N$-(tert-butyloxycarbonyl)-7-cyano-naphthalin (79)

5.5 2-Amino-4-benzyloxy-1-brom- $N$-(tert-butyloxycarbonyl)-6-cyanonaphthalin (101)

$5.6(+)-\left\{\left(2^{\prime} R, 3^{\prime} R\right)-2-A m i n o-4-b e n z y l o x y-N-(2,3-e p o x y b u t y l)-1-b r o m-N\right.$-(tertbutyloxycarbonyl)-6-cyano-naphthalin\} $\left((+)-\left(2^{\prime} R, 3^{\prime} R\right)-80\right)$.

5.7 (+)-\{(1S,10R)-5-Benzyloxy-3-(tert-butyloxycarbonyl-1-(10-Hydroxyethyl)-7cyano-1,2-dihydro-3H-benz[e]indol ((+)-(1S,10R)-81)

$5.8(+)-\{(1 S, 10 R)-5-B e n z y l o x y-3-($ tert-butyloxycarbonyl-1-(10-chlorethyl)-7cyano-1,2-dihydro-3H-benz[e]indol ((+)-(1S,10R)-82) 177

5.9 (E/Z)-2-Amino-4-benzyloxy-1-brom-N-(tert-butyloxycarbonyl)-N-(3-chlor2-butenyl)-6-cyano-naphthalin (104) 178

5.10 rac-\{(1S,10R)-5-Benzyloxy-3-(tert-butyloxycarbonyl)-1-(10-chlor-ethyl)-7cyano-1,2-dihydro-3H-benz[e]indol\} $(\operatorname{rac}-(1 S, 10 R)-82)$ und $\operatorname{rac}-\{(1 S, 10 S)-5-$ Benzyloxy-3-(tert-butyloxycarbonyl)-1-(10-chlor-ethyl)-7-cyano-1,2dihydro-3H-benz[e]indol\} (rac-(1S,10S)-82) 179

5.11 Trennung der Enantiomere von rac-\{(1S,10R)-5-Benzyloxy-3-(tertbutyloxycarbonyl)-1-(10-chlor-ethyl)-7-cyano-1,2-dihydro-3Hbenz $[e]$ indol $\}(\operatorname{rac}-(1 S, 10 R)-82)$ 180

$5.12(+)-\{(1 S, 10 R)-3-($ tert-Butyloxycarbonyl)-1-(10-chlor-ethyl)-7-cyano-

5-hydroxy-1,2-dihydro-3H-benz[e]indol\} $((+)-(1 S, 10 R)-149)$ 181 SYNTHESE DER ANTI-METHYL-CBI-DERIVATE MIT DMAI-SEITENKETTE..... 182

6.1 rac-\{(1S,10S)-5-Benzyloxy-1-(10-chlor-ethyl)-7-cyano-3-[(5-(2-(N,N'dimethylamino)-ethoxy)-indol-2-yl)carbonyl]-1,2-dihydro-3H-benz[e]indol\} (rac- $(1 S, 10 S)-88)$ 182

$6.2(+)-\left\{(1 S, 10 R)-5-B e n z y l o x y-1-(10-c h l o r-e t h y l)-7-c y a n o-3-\left[\left(5-\left(2-\left(N, N^{\prime}-\right.\right.\right.\right.\right.$ dimethylamino)-ethoxy)-indol-2-yl)carbonyl]-1,2-dihydro-3H-benz[e]indol\} $((+)-(1 S, 10 R)-88)$ 183 
6.3 (-)-\{(1R,10S)-5-Benzyloxy-1-(10-chlor-ethyl)-7-cyano-3-[(5-(2-(N,N'dimethylamino)-ethoxy)-indol-2-yl)carbonyl]-1,2-dihydro-3H-benz[e]indol\} $((-)-(1 R, 10 S)-88)$ 185

6.4 rac-\{(1S,10S)-7-Aminomethyl-5-benzyloxy-1-(10-chlor-ethyl)-3-[(5-(2$\left(\mathrm{N}, \mathrm{N}^{\prime}\right.$-dimethylamino)-ethoxy)-indol-2-yl)carbonyl]-1,2-dihydro-3Hbenz $[e]$ indol\} $($ rac- $(1 S, 10 S)-81)$ 186

$6.5\left\{(1 S, 10 R)-7-A m i n o m e t h y l-5-b e n z y l o x y-1-(10-c h l o r-e t h y l)-3-\left[\left(5-\left(2-\left(N, N^{\prime}-\right.\right.\right.\right.\right.$ dimethylamino)-ethoxy)-indol-2-yl)carbonyl]-1,2-dihydro-3H-benz[e]indol\} $((1 S, 10 R)-81)$

$6.6\left\{(1 R, 10 S)-7-A m i n o m e t h y l-5-b e n z y l o x y-1-(10-c h l o r-e t h y l)-3-\left[\left(5-\left(2-\left(N, N^{\prime}-\right.\right.\right.\right.\right.$ dimethylamino)-ethoxy)-indol-2-yl)carbonyl]-1,2-dihydro-3H-benz[e]indol\} $((1 R, 10 S)-81)$

6.7 \{(1S,10R)-2-(\{5-Benzyloxy-1-(10-chlor-ethyl)-3-[(5-(2-N,N'-dimethylamino)ethoxy)-1H-indol-2-yl)carbonyl]-1,2-dihydro-3H-benz[e]indol-7-ylmethyl\}carbamoyl)-ethyl]-carbaminsäure-benzylester ((1S,10R)-90). 190

$6.8\{(1 S, 10 R)-3-A m i n o-N-2-(\{1-(10-$ chlor-ethyl)-3-[(5-(2-N,N'-dimethylamino)ethoxy)-1H-indol-2-yl)carbonyl]-5-hydroxy-1,2-dihydro-3H-benz[e]indol-7ylmethyl\}-propionamid ((1S,10R)-106)

$6.9\{(1 R, 10 S)$-[7-Aminomethyl-1-(1-chlor-ethyl)-5-hydroxy-1,2-dihydrobenz[e]indol-3-yl]-[5-(2-dimethylamino-ethoxy)-1H-indol-2-yl]-methanon $((1 R, 10 S)-87)$ 193

$6.10\{(1 S, 10 R)$-[7-Aminomethyl-1-(1-chlor-ethyl)-5-hydroxy-1,2-dihydrobenz[e]indol-3-yl]-[5-(2-dimethylamino-ethoxy)-1H-indol-2-yl]-methanon $(1 S, 10 R)-87$ 196 SYNTHESE DER FLUORESZENZMARKIERTEN ANTI-METHYL-SECO-CBI-DMAI-DERIVATE (1S,10R)-71 UND (1R,10S)-71 199

7.1 3-[4-(5-(4-Dimethylaminophenyl)oxazol-2-yl)benzolsulfonylamino]propionsäure-[(1S,10R)-1-(10-chlor-ethyl)-3-[(5-(2-(N,N'dimethylamino)ethoxy)indol-2-yl)carbonyl]-5-hydroxy-1,2-dihydro-3Hbenz[e]indol-7-ylmethyl]amid $((1 S, 10 R)-71)$ 
7.2 3-[4-(5-(4-Dimethylaminophenyl)oxazol-2-yl)benzolsulfonylamino]propionsäure-[(1S,10R)-1-(10-chlor-ethyl)-3-[(5-(2-(N,N'-

dimethylamino)ethoxy)indol-2-yl)carbonyl]-5-Hydroxy-1,2-dihydro-3Hbenz[e]indol-7-ylmethyl]amid ((1R,10S)-71)

7.3 3-[4-(5-(4-Dimethylaminophenyl)oxazol-2-yl)benzolsulfonylamino]propionsäure-[(1S,10R)-1-(10-chlor-ethyl)-3-[(5-(2-(N,N'-

dimethylamino)ethoxy)indol-2-yl)carbonyl]-5-hydroxy-1,2-dihydro-3Hbenz[e]indol-7-ylmethyl]amid $((1 S, 10 R)-71)$ 203

8 SYNTHESE DES SECO-CBI-GRUNDGERÜSTES (1S)-128 205

8.1 tert-Butyl-(E)-3-(ethoxycarbonyl)-4-phenyl-3-butenoat (121)................ 205

8.2 (E)-3-Ethoxycarbonyl-4-phenyl-3-butencarbonsäure (96) ......................206

8.3 Ethyl-1-acetoxy-3-naphthalincarboxylat (122)....................................207

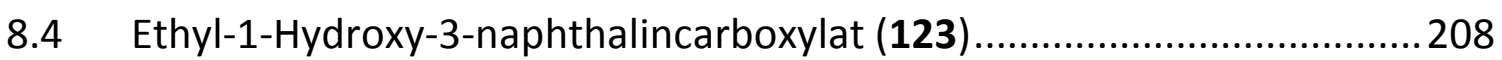

8.5 Ethyl-1-benzyloxy-3-naphthalincarboxylat (124) ................................209

8.6 1-Benzyloxy-3-naphthalincarbonsäure (125) .....................................210

8.7 3-Amino-1-benzyloxy- $N$-(tert-butyloxycarbonyl)-naphthalin (126)........ 211

8.8 2-Amino-4-benzyloxy-N-(tert-butyloxycarbonyl)-1-iod-naphthalin (95) . 212

8.9 (+)-\{(2'R)-2-Amino-4-benzyloxy-N-(tert-butyloxycarbonyl)-N-(2,3epoxypropyl)-1-iod-naphthalin\} ((+)-(2'R)-94)

$8.10(+)-\{(1 S)-5-B e n z y l o x y-3-($ tert-butyloxycarbonyl)-1-hydroxymethyl-1,2-

dihydro-3H-benz[e]indol\} $((+)-(1 S)-127)$

8.11 (-)-\{(1S)-5-Benzyloxy-3-(tert-Butyloxycarbonyl)-1-chlormethyl-1,2-

dihydro-3H-benz[e]indol\} ((-)-(1S)-93) 216

8.12 (-)-\{(1S)-3-(tert-Butyloxycarbonyl)-1-chlormethyl-5-hydroxy-1,2-dihydro$3 H$-benz[e]indol\} $((-)-(1 S)-128)$ 217

8.13 Anreichung von $\{(1 S)$-3-(tert-butyloxycarbonyl)-1-(chlormethyl)-1,2di-hydro-5-hydroxy-3H-benz[e]indol\} ((1S)-128) durch präp. HPLC 217

9 SYNTHESE NEUARTIGER FLUORESZENZMARKIERTER CBI-PRODRUGS, SOWIE DER ENTSPRECHENDEN SECO-CBI-DERIVATE 
9.1 (-)-(1S)-1-[6-(1-Chlormethyl-5-hydroxy-1,2-dihydro-benz[e]indol-3-yl)-6oxo-hexyl]-4-(3-Methyl-3H-benzothiazol-2-ylidenmethyl)-pyridiniumchlorid ((-)-(1S)-141) 219

9.2 (-)-\{(1S)-[1-[6-(1-Chlormethyl-1,2-dihydro-benz[e]indol-3-yl)-6-oxo-hexyl]4-(3-Methyl-3H-benzothiazol-2-ylidenmethyl)-pyridinium-chlorid-5-yl]2,3,4,6-tetra-O-acetyl- $\beta$-D-Galaktopyranosid\} ((-)-(1S)-146)

9.3 (-)-\{(1S)-[1-[6-(1-Chlormethyl-1,2-dihydro-benz[e]indol-3-yl)-6-oxo-hexyl]4-(3-Methyl-3H-benzothiazol-2-ylidenmethyl)-pyridinium-chlorid-5-yl]-O$\beta$-D-Galaktopyranosid\} ((-)-(1S)-70)

9.4 (1S)-1-[6-(1-Chlormethyl-5-hydroxy-1,2-dihydro-benz[e]indol-3-yl)-6oxo-hexyl]-4-[3-(3-Methyl-3H-benzothiazol-2-yliden)-propenyl]quinolinium-chlorid ((1S)-145) 226

9.5 \{(1S)-[1-[6-(1-Chlormethyl-5-hydroxy-1,2-dihydro-benz[e]indol-3-yl)-6oxo-hexyl]-4-[3-(3-Methyl-3H-benzothiazol-2-yliden)-propenyl]quinolinium-chlorid-5-yl]-2,3,4,6-tetra-O-acetyl- $\beta$-D-galatopyranosid\} ((1S)-150)

9.6 \{(1S)-[1-[6-(1-Chlormethyl-5-hydroxy-1,2-dihydro-benz[e]indol-3-yl)-6oxo-hexyl]-4-[3-(3-Methyl-3H-benzothiazol-2-yliden)-propenyl]quinolinium-chlorid-5-yl]-O- $\beta$-D-galatopyranosid\} ((1S)-147) 232

9.7 (-)-(1S)-3-(1-Chlormethyl-5-hydroxy-1,2-dihydro-benz[e]indol-3-carbonyl)chromn-2-on ((-)-(1S)-92) 234

$9.8\{(1 S)$-[3-(1-Chlormethyl-5-hydroxy-1,2-dihydro-benz[e]indol-3-carbonyl)chromn-2-on]-2,3,4,6-tetra-O-acetyl- $\beta$-D-Galaktopyranosid\} ((1S)-151) 236

$9.9\{(1 S)$-[3-(1-Chlormethyl-5-hydroxy-1,2-dihydro-benz[e]indol-3-carbonyl)chromn-2-on]-O- $\beta$-D-Galaktopyranosid\} ((1S)-69)

$9.10\{(-)-(1 S)-[4-[2-(1-C h l o r m e t h y l-5-h y d r o x y-1,2-d i h y d r o-b e n z[e]$ indol-3-yl)-2oxo-ethyl]-7-dimethylamino-chromn-2-on]\} ((-)-(1S)-137). 239

$9.11\{[(1 S)-[4-[2-(1-C h l o r m e t h y l-5-h y d r o x y-1,2-d i h y d r o-b e n z[e]$ indol-3-yl)-2oxo-ethyl]-7-dimethylamino-chromn-2-on]-5-yl]-2,3,4,6-tetra-O-acetyl- $\beta$ D-Galaktopyranosid\} ((1S)-151) 
$9.12\{[(-)-(1 S)-[4-[2-(1-C h l o r m e t h y l-5-h y d r o x y-1,2-d i h y d r o-b e n z[e] i n d o l-3-y l)-2-$ oxo-ethyl]-7-dimethylamino-chromn-2-on]-5-yl]-O- $\beta$-D-Galaktopyranosid\} $((-)-(1 S)-139)$

9.13 (1S)-2-(1-Chlormethyl-5-hydroxy-1,2-dihydro-benz[e]indol-3-carbonyl)-3Hpyrano[3,2-e]indol-7-on ((1S)-138) 245

$9.14\{(1 S)$-[2-(1-Chlormethyl-5-hydroxy-1,2-dihydro-benz[e]indol-3-carbonyl)$3 H$-pyrano[3,2-e]indol-7-on-5-yl]-2,3,4,6-tetra-O-acetyl- $\beta$-D-

Galaktopyranosid\} ((1S)-152) 248

$9.15\{(1 S)$-[2-(1-Chlormethyl-5-hydroxy-1,2-dihydro-benz[e]indol-3-carbonyl)3H-pyrano[3,2-e]indol-7-on-5-yl]-O- $\beta$-D-Galaktopyranosid\} ((1S)-140) . . 250 UNTERSUCHUNG DER FLUORESZENZEIGENSCHAFTEN UND DER ZELLULÄREN AUFNAHME DER DARGESTELLTEN CBI-DERIVATE. 253

10.1 Charakterisierung der Fluoreszenzeigenschaften der synthetisierten CBIDerivate. 253

10.1.1 AAV 10.1.1. Allgemeine Arbeitsvorschrift zur Bestimmung der Fluoreszenzintensität bei festgelegter Wellenlänge in Abhängigkeit von der Anregungswellenlänge (Absorption-Scan) . 253

10.1.2 AAV 10.1.2 Allgemeine Arbeitsvorschrift zur Messung des Fluoreszenzemissionsspektrums bei festgelegter Anregungswellenlänge (Emission-Scan) 253

10.2 Fluoreszenzmikroskopische Untersuchungen zur Aufnahme und intrazellulären Verteilung der synthetisierten fluoreszenzmarkierten CBIDerivate. 254

10.2.1 AAV 10.2.1. Allgemeine Arbeitsvorschrift zur Vorbereitung der A549Zellen zur Untersuchung der zellulären Aufnahme fluoreszenzmarkierter CBI-Derivate am konfokalen Laserscanning-Mikroskop. 254

10.2.2 AAV 10.2.2. Allgemeine Arbeitsvorschrift zur Vorbereitung der HeLa SS6-Zellen zur Untersuchung der zellulären Aufnahme fluoreszenzmarkierter CBI-Derivate am konfokalen LaserscanningMikroskop. 
10.2.3 AAV 10.2.2. Allgemeine Arbeitsvorschrift zur Untersuchung der zellulären Aufnahme fluoreszenzmarkierter $\mathrm{CBI}$ - Derivate inklusive des Waschens der Zellen nach Inkubation 255

10.2.4 AAV 10.2.4. Allgemeine Arbeitsvorschrift zur Anfärbung mit Hoechst 33342 (64), Mitochondrienfarbstoff MitoTracker Deep Red FM (65) oder dem Mitochondrienfarbstoff MitoTracker Grenn FM (148) 255

E. ANHANG. 257

1 IN-VITRO-ZYTOTOXIZITÄTSTESTS. 257

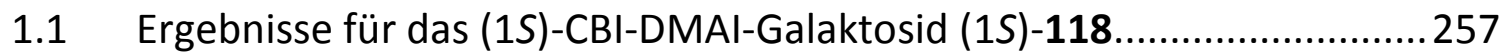

1.2 Ergebnisse für das fluoreszenten (1S)-CBI-DMAI-Galaktosid (1S)-72 ....258

1.3 Ergebnisse für das (1S)-CBI-BO-Galaktosid (1S)-70 ...............................258

1.4 Ergebnisse für das (1S)-seco-CBI-BO-Drug (1S)-141 .................................259

1.5 Ergebnisse für das (1S)-CBI-TO3-Galaktosid (1S)-147 ............................259

1.6 Ergebnisse für das (1S)-seco-CBI-TO3-Drug (1S)-145 ..............................260

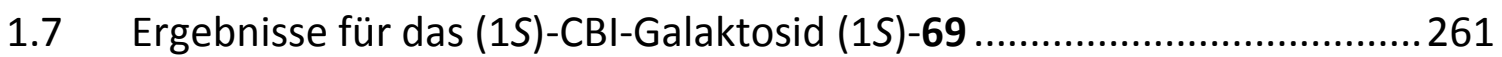

1.8 Ergebnisse für das (1S)-seco-CBI-Drug (1S)-92 .....................................261

1.9 Ergebnisse für das (1S)-CBI-DMACA-Galaktosid (1S)-139 ........................262

1.10 Ergebnisse für das (1S)-seco-CBI-DMACA-Drug (1S)-137 ........................262

1.11 Ergebnisse für das (1S)-CBI-Cumarinindol-Galaktosid (1S)-140 ...............263

1.12 Ergebnisse für das (1S)-seco-CBI-Cumarinindol-Drug (1S)-138_...............263

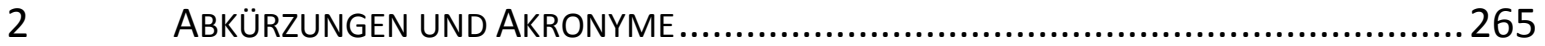

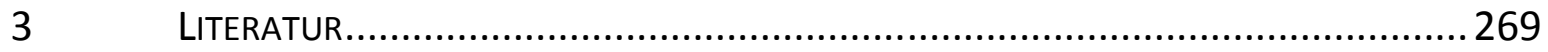

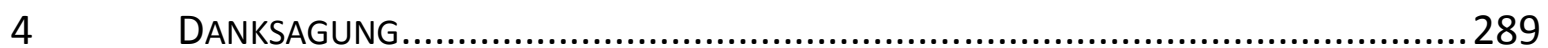

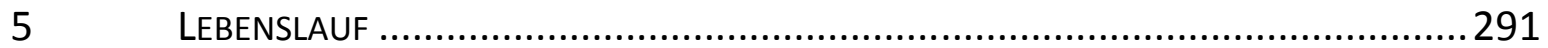




\section{A. Allgemeiner TeIL}

\section{$1 \quad$ Einleitung}

Betrachtet man die Entwicklung der Bevölkerung nach Altersgruppen in der Bundesrepublik Deutschland fällt auf, dass der Anteil der über 65-jährigen in den Jahren 2005-2009 von 15.9 Mio auf 16.9 Mio zugenommen hat, während die Gesamtbevölkerungszahl von 82.4 Mio auf 81.8 Mio gesunken ist. ${ }^{1}$ Betrachtet man weiterhin den prozentualen Anteil der über 60-jährigen in Deutschland wird der demographische Wandel noch deutlicher. So veränderte sich der Anteil der über 60-jährigen an der Gesamtbevölkerung im Zeitraum von 1950-2009 von 14.6\% auf 25.9\%, während der Anteil der unter 40-jährigen von 56.8\% auf 43.1\% sank.

Nimmt man nun die Vohersagen des Bundesministeriums für Wirtschaft und Forschung München hinzu, wird deutlich, dass sich dieser Trend in den nächsten Jahrzehnten auf Grund geringer Geburtenraten und steigender Lebenserwartungen weiter fortsetzt (Abbildung 1). ${ }^{2}$

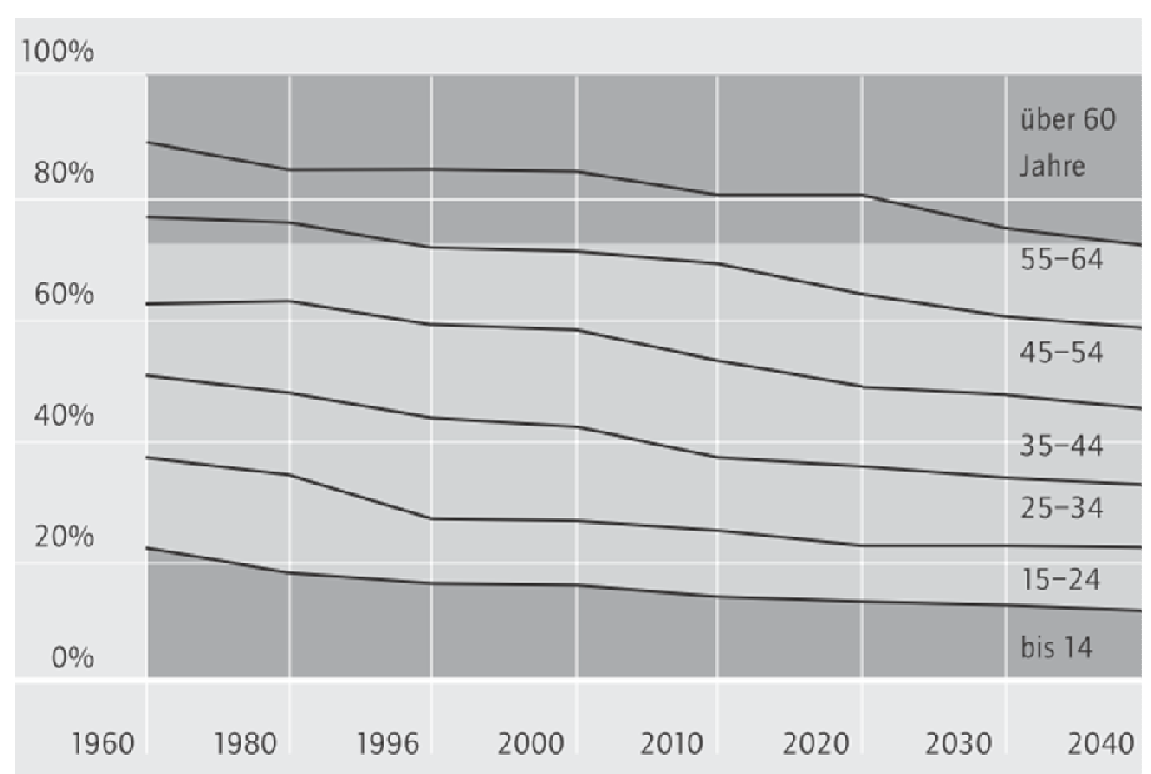

Abbildung 1. Entwicklung der Bevölkerung nach Altersgruppen. 
Ebenso zeigen die aktuellen Daten des Deutschen Krebsforschungszentrum, ${ }^{3}$ dass die Auswirkungen des Demographischen Wandels im Bezug auf Krebs nicht zu verkennen sind. Auf Grund der veränderten Altersstruktur hat die Zahl der neu aufgetretenen Krebserkrankungen bei Männern seit 1980 um 80\%, bei Frauen um 35\% zugenommen. Betrachtet man die Neuerkrankungen bei Männern seit 1990 fällt auf, dass bei den 55-80-jährigen eine steigende Zahl der Neukrankungen zu beobachten ist, während diese bei den unter 55-jährigen sinkt (Abbildung 2).

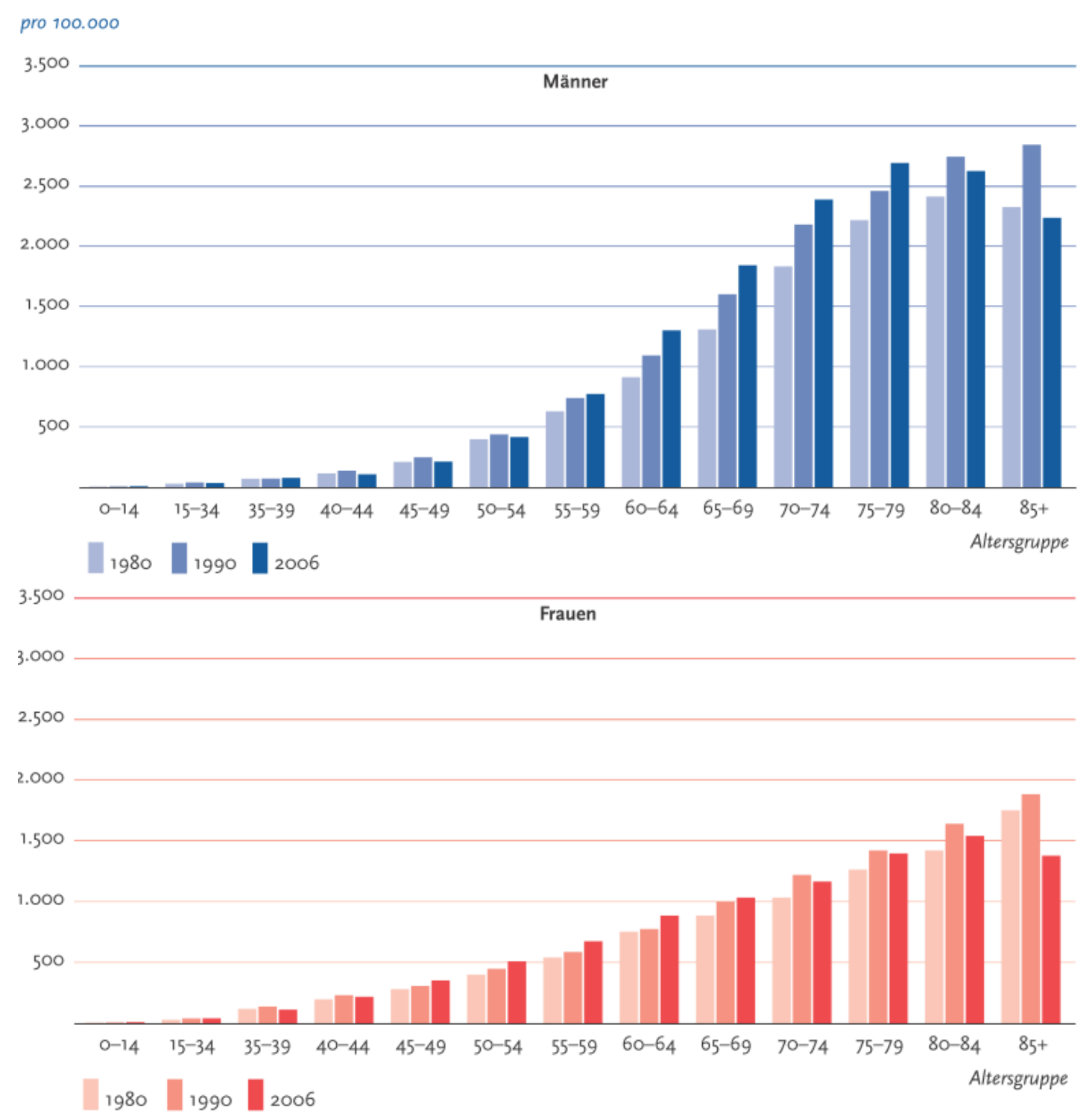

Abbildung 2. Altersspezifische Erkrankungsraten in Deutschland nach Geschlecht für 1980, 1990 und 2006. 
Die aus Abbildung 2 abgeleiteten Aussagen unterstützen die aus Abbildung 1 gemachten Beobachtungen in Hinblick auf den Demographsichen Wandel und die damit verbundene Wichtigkeit der Intensivierung der Krebsforschung.

Hierbei steht die Weiterentwicklung der Chemotherapie und damit einhergehend die Entwicklung neuer effizienter Wirkstoffe im Mittelpunkt. So ist es von entscheidender Bedeutung Wirkstoffe darzustellen, die in ihrer Anwendung eine möglichst vollständige Unterscheidung zwischen gesunden und malignen Zellen.

Einen Ansatz zur Verringerung der starken Nebenwirkungen der zur Zeit zur Verfügung stehenden Zytostatika beschreibt das Konzept der Antibody-DirectedEnyzme-Prodrug-Therapy. Hierbei wird eine Identifizierung der Krebszellen Antikörper-Enzym-Konjugate geschaffen. Der Antikörper bindet selektiv an Immunglobuline der Krebszellen und das an den Antikörper gebundene Enzym kann dort eine weitestgehend untoxische Wirkstoffvorstufe (Prodrug) enzymatisch zum Wirkstoff (Drug) umwandeln. Erste erfolgreiche Analoga des zytotoxischen Naturstoffs Duocarmycin konnten in der Arbeitsgruppe von Tietze entwickelt werden. ${ }^{4}$

Zur weiteren Aufklärung des Wirkmechanismus' und des Wirkortes werden in dieser Arbeit neuartige fluoreszenzmarkierte Analoga Duocarmycin synthetisiert und in Live Cell Imaging Experimenten untersucht. 


\section{Cancerogenese - Von der Krebszelle zum Tumor ${ }^{5}$}

Ein Tumor entsteht durch eine Veränderung der normalen Regulationsmechanismen zur Proliferation (Vermehrung) von Körperzellen. ${ }^{6}$ Maligne (bösartige) Tumore, die zu unkontrolliertem Wachstum neigen, können aus verschiedenen Gründen entstehen. ${ }^{7}$ Wenn die Signale der Proliferation dauerhaft aktiv sind, der Übergang in einen teilungsfähigen Zellzustand verhindert wird oder die Apoptose (programmierter, nicht nekrotischer Zelltod) nicht eingeleitet werden kann, kommt es zur Ausbildung von Tumoren. ${ }^{8}$

Eine Störung der Regulationsmechanismen zur Proliferation wird durch eine Veränderung der für die Regulation verantwortlichen Gene hervorgerufen. ${ }^{9}$ Dies kann ebenfalls verschiedene Ursachen haben. Zum Einen Mutationen, die spontan, vererbt oder durch chemische Substanzen (z.B. polyzyklische aromatische Kohlenwasserstoffe, Nitrosamine, halogenierte Kohlenwasserstoffe, Asbest und aromatische Amine) ${ }^{10,11}$ initiiert werden, zum Anderen Mutationen, die durch physikalische Einflüsse, wie UV-Licht oder radioaktive Strahlung entstehen. ${ }^{12}$ Des Weiteren können der Einbau viraler Gene ${ }^{13,14}$ in das menschliche Genom und chronische Entzündungen ${ }^{15}$ für das Entstehen von Tumoren verantwortlich sein.

Die betroffenen, meist regulatorischen, Gene sind die Protoonkogene, die das Zellwachstums fördern sowie die Tumorsuppressorgene, deren Aufgaben die Hemmung des Zellwachstums in gesunden Zellen, die Verhinderung der Zellteilung bei DNA-Schäden sowie die Einleitung der Apoptose sind. ${ }^{16}$

Ein Tumor entsteht jedoch erst, wenn mehrere Mutationen eingetreten sind, da der Zellstoffwechsel über viele Kontroll- und Steuerungsmechanismen verfügt, die im Stande sind eine einzelne Mutationen zu reparieren.

Werden Protoonkogene, wie im Fall des Ras-Onkogens, durch Mutationen in Onkogene umgewandelt, führt dies zu einem erhöhten Zellwachstum. ${ }^{13,17}$ Für die Entstehung eines Tumors müssen jedoch zusätzlich, wie oben beschrieben, Tumorsuppressorgene durch Mutationen deaktiviert werden, wie im Beispiel des 
p53-Gens. ${ }^{13,18}$ Dieses in mehr als der Hälfte aller menschlichen Tumore gefundene Gen, sorgt für den Eintritt von gesunden Zellen in die S-Phase des Zellzyklus' und ermöglicht hierdurch, z.B. bei DNA-Schädigungen, dessen Reparatur. ${ }^{19}$ Ist dieses Gen jedoch deaktiviert, kann die DNA-Schädigung nicht repariert werden und es kommt zum programmierten Zelltod (Apoptose).

Die Entstehung eines Tumors kann in vier Phasen eingeteilt werden: Initiierungsphase, Promotionsphase, Progressionsphase und Implantationsphase. In der Initiierungs-phase (Pathogenese) treten erste Mutationen am Erbgut auf, die an die Tochterzellen weitergegeben werden. Die Promotionsphase tritt nach einer Latenzzeit von bis zu mehreren Jahren ein und wird durch eine ansteigende Zellproliferation sowie das Sichtbarwerden von Kern- und Zellatypien beschrieben. In der folgenden Progessionsphase kann zwischen benigner oder maligner Geschwulst unter-schieden werden. In der vierten Phase, der Implantationsphase, verteilen sich maligne Zellen im gesamten Organismus, nachdem sie Blut- und Lymphgefäße infiltriert haben. Auf diese Weise können sie sich an verschiedenen Organen (Lymphknoten, Leber, Lunge, Knochenmark) festsetzen und Metastasen bilden. ${ }^{20}$ Durch Ausbildung von Blutgefäßen (Angiogenese) und der damit einhergehenden Versorgung der Metastasen wird die Tumorentstehung abgeschlossen. 


\section{$3 \quad$ Konzepte zur Tumortherapie}

Auf Grund ihrer vielfältigen Erscheinungsformen und der sich daraus ergebenden notwendigen Einzelbetrachtung erfordern Krebserkrankungen individuell angepasste Therapiekonzepte. ${ }^{21}$ Bei den meisten klinschen Anwendungen handelt es sich um die Kombination aus verschiedenen Methoden. Hierzu zählen: Chemotherapie, Strahlenbehandlung und die chirurgische Entfernung des Tumorgewebes. Bei soliden, gut zugänglichen und klar abgegrenzten Tumoren, kann eine chirurgische Entfernung vorgenommen werden. Hierbei handelt es sich um die Methode mit den geringsten Nebenwirkungen und den besten Heilungschancen. Ist eine derartige Abgrenzung gegenüber gesundem Gewebe nicht gegeben oder betrifft der Tumor lebenswichtige Strukturen, ist eine Strahlenbehandlung (z.B. Radiotherapie mit $\gamma$-Strahlung oder radioaktiven Isotopen) unumgänglich. Ist es hingegen bereits zur Bildung von Metastasen gekommen wird die Behandlung der Patienten durch die Anwendung einer Chemotherapie durchgeführt. Neue Therapieansätze, wie zum Beispiel die Behandlung mit Angiogenesehemmern ${ }^{22}$ und Kinaseinhibitoren ${ }^{23}$ oder auch die Behandlung durch Immun- ${ }^{24}$ und Hormontherapie ${ }^{25}$ konnten bereits erfolgreich eingesetzt werden.

\subsection{Chemotherapie}

Wie oben bereits erläutert, wird im Fall einer bereits eingetretenen oder aufkommenden Metastasierung die klassische antineoplastische Chemotherapie gewählt, wenngleich diese meist durch schwere Nebenwirkungen gekennzeichnet ist. Zu diesen, teils drastischen Nebenwirkungen werden Störungen der Hämatopoese sowie des Gastrointestinaltraktes (Übelkeit, Erbrechen), Mucositis (Schleimhautentzündung), Alopezie (Haarausfall), Fieber, Immunschwäche, Infertilität und Teratogenität gezählt. Wird eine klassische Chemotherapie angewendet, werden Medikamente verabreicht, die sich über den Blutkreislauf im gesamten Körper 
verteilen. Die Wirkung der Chemotherapeutika auf menschliche Zellen ist entweder zytostatisch, im Falle einer Hemmung des Zellwachstum, oder sie ist zytotoxisch, wenn sie zum Tod der Zelle führt.

Chemotherapeutika schädigen auf Grund ihres Wirkmechanismus' vorwiegend schnell-proliferierende Zellen. Durch ihren erhöhten Stoffwechsel nehmen diese Zellen die verabreichten Wirkstoffe schneller auf. Das größte Problem dieser Anwendung besteht in der Tatsache, dass auch gesunde Zellen, wie beispielsweise die des Knochenmarks und der Haarwurzel- oder Schleimhautzellen zu den schnell proliferierenden Zellen gehören. Eine Unterscheidung durch die Chemotherapeutika ist hier nicht gegeben und es kommt folglich zu einer Schädigung von gesunden Organismen und damit einhergehend zum Auftreten der oben genannten Nebenwirkungen. Bei der Vermehrung von Zellen, kommt es zu verschiedenen Phasen, die während eines Zellzyklus' durchlaufen werden (Abbildung 3).

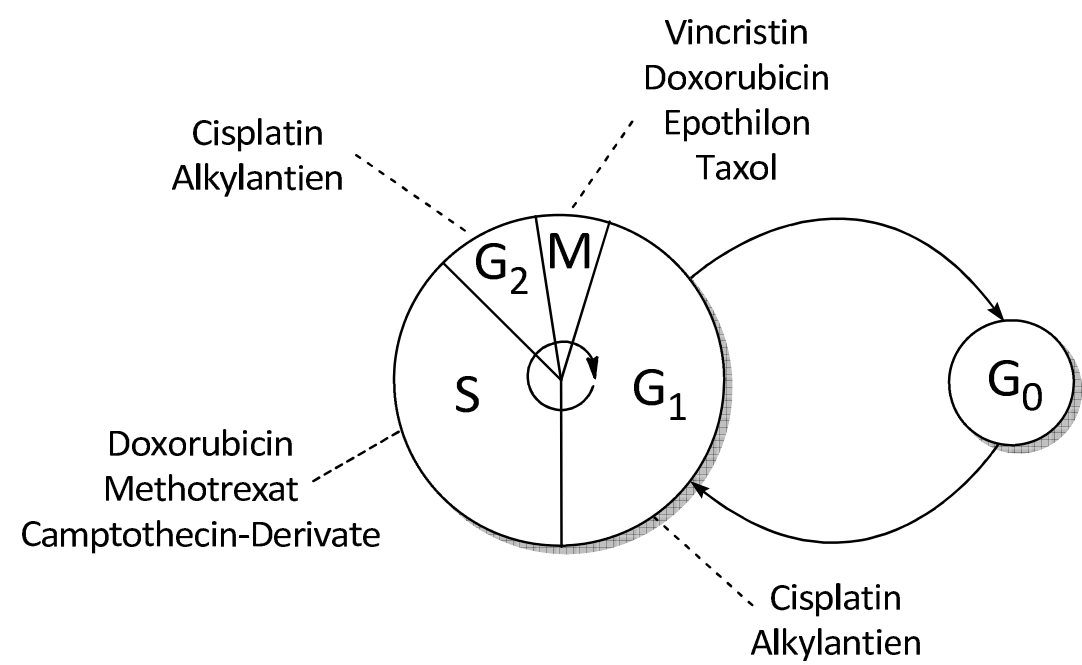

Abbildung 3. Phasen des Zellzyklus' und Angriffspunkte verschiedener Chemotherapeutika.

In der Wachstumsphase ( $\mathrm{G}_{1}$-Phase) der Zelle werden die RNA sowie erste Proteine synthetisiert. Von der $G_{1}$-Phase können die Zellen in die Ruhephase $\left(G_{0}\right.$-Phase) übergehen. Die meisten gesunden Zellen befinden sich, im Gegensatz zu den Tumorzellen (nur ca. 10\%) in der Ruhephase, in der sie für die meisten Zytostatika nicht zugänglich sind. In der Synthesephase (S-Phase) findet die DNA-Replikation 
statt. Eine Überprüfung der Replikation wird in der kurzen Postsynthesephase $\left(\mathrm{G}_{2}{ }^{-}\right.$ Phase) vorgenommen, um in der anschließenden Mitosephase (M-Phase) den doppelten Chromosomensatz zu trennen und an jede der zwei Tochterzellen zu übergeben. Eine Überprüfung der durchlaufenen Phasen erfolgt an Kontrollpunkten zwischen Wachstumsphase und Synthesephase sowie zwischen Synthesephase und Postsynthesephase. An diesen Kontrollpunkten wird im positiven Fall eine Stimulierung der Wachstumsfaktoren oder im negativen Fall eine Reparatur der DNASchäden, ein Zellzyklusstop oder ein programmierter Zelltod (Apoptose) eingeleitet. ${ }^{26}$ Eine Differenzierung der derzeit eingesetzen Chemotherapeutika kann auf Grund ihrer Wirkmechanismen und ihrer Angriffspunkte im Zellzyklus vorgenommen werden.

Hierbei wird zwischen Alkylantien, Antimetaboliten, Mitosehemmstoffen, Topoisomerase-Hemmstoffen und zytostatischen Antibiotika unterschieden.

Die Klasse der Alkylantien wirkt hauptsächlich phasenunspezifisch. Das für die Alkylierung häufig bevozugte Carbokation des Chemotherapeutikums wird erst im Körper dargestellt, bevor es mit $\mathrm{N}$-, $\mathrm{O}$ - oder $\mathrm{S}$-Nucleophilen der Proteine oder Nukleinsäuren reagieren kann und kovalente Bindungen ausbildet. Daraus resultieren Quervernetzungen der DNA-Stränge (cross links), abnorme Basenpaarungen oder Strangbrüche, die eine Replikation und somit eine Zellteilung verhindern, wodurch es letzlich zum Zelltod kommt. Wichtige Vertreter dieser Substanzklasse sind StickstoffLost-Derivate, wie etwa das untoxische Cyclophosphamid (1), ${ }^{27}$ das erst durch Biotransformationen im Körper in den toxischen Wirkstoff 2 überführt wird (Giftung) (Abbildung 4).

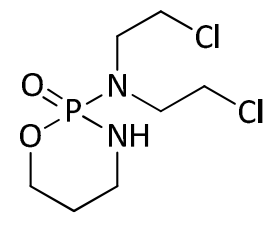

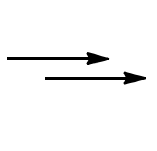

Cyclophosphamid (1)

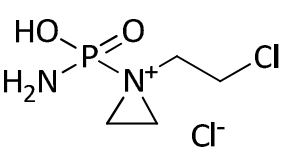

2

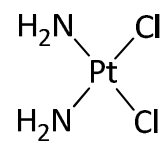

Cisplatin (3)

Abbildung 4. Cyclophosphamid (1) als ein Beispiel für die Gruppe der Alkylantien. 
Zu der Klasse der Alkylantien gehören vor allem auch die Platinkomplexe des Cisplatin

(3). Der Wirkmechanismus dieser Verbindungen wird mit Intra- oder InterstrangQuervernetzungen der DNA beschrieben. ${ }^{28}$ Darüber hinaus zählten das in dieser Arbeit als Leitstruktur verwendete natürliche Antibiotikum CC-1065 sowie die Duocarmycine, Yatakemycin und deren Derivate (Kapitel 4) zur Klasse der Alkylantien.

Antimetabolite sind, wie aus der Namensgebung zu erkennen ist, Strukturanaloga körpereigener Stoffwechselbausteine, die als Antagonisten die eigentlichen Metabolite ersetzen. Im Gegensatz zu den phasenunspezifischen Alkylantien wirken Antimetabolite bevorzugt in der Synthesephase. Hier hemmen sie wichtige Enzyme oder führen zur Entstehung funktionsuntüchtiger Makromoleküle., ${ }^{8,26}$ Der Folsäureantagonist Methotrexat (4) ist ein wichtiges Beispiel für die Klasse der Antimetabolite. Der Wirkmechanismus beruht auf einer Inhibition der DihydrofolatReduktase und einer damit einhergehenden Verhinderung der Bildung von Tetrahydrofolsäure (Abbildung 5). Tetrahydrofolsäure ist ein wichtiger Bestandteil im Aminosäure- und Purinstoffwechsel und damit essentiell für die Zellproliferation. ${ }^{29}$<smiles>[R1]c1nc(N)nc2ncc(CN([R])c3ccc(C(=O)NC(CCC(=O)O)C(=O)O)cc3)nc12</smiles>

Methotrexat (4): $\quad \mathrm{R}^{1}=\mathrm{NH}_{2}, \mathrm{R}^{2}=\mathrm{CH}_{3}$ Folsäure (5): $\quad \mathrm{R}^{1}=\mathrm{OH}, \mathrm{R}^{2}=\mathrm{H}$

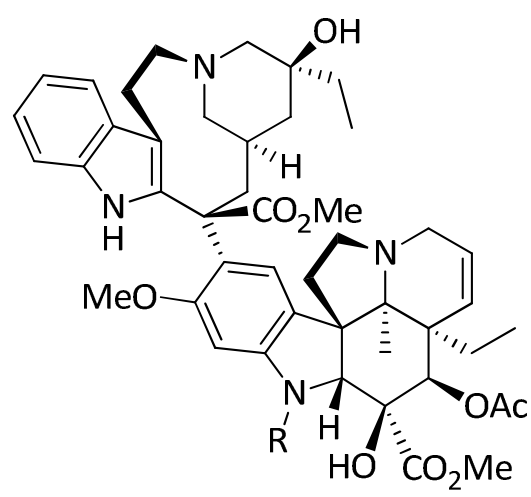

Vincristin (6): $\quad \mathrm{R}=\mathrm{CHO}$ Vinblastin (7): $\quad \mathrm{R}=\mathrm{CH}_{3}$

Abbildung 5. Methotrexat (4) als Beispiel für die Wirkstoffklasse der Antimetabolite sowie Vincristin (6) und Vinblastin (7) als Beispiele für die Gruppe der Mitosehemmstoffe.

Vincristin (6) und Vinblastin (7) greifen als Mitosehemmstoffe (Spindelgifte) in die Mitosephase des Zellzyklus' ein, indem sie an die $\beta$-Einheit des Tubulindimers binden und so den Aufbau der Kernspindel ${ }^{30}$ hemmen oder im Fall von Taxol und Epothilon 
dessen Abbau verhindern. ${ }^{31}$ Auf Grund der Blockierung des Auf- und Abbaus des Spindelapparates kann eine Kern- und Zellteilung nicht mehr stattfinden.

Die zytotoxische Wirkung der Topoisomerase-Hemmstoffe, wie Etoposid, Irinotecan und Derivate des Alkaloids Camptothecin (8) beruht auf der Störung der Topoisomerasen I und II. Ihre Aufgaben sind die Entwindung der verdrillten Stränge während der DNA-Replikation, deren Unterbrechung und der anschließende Zusammenschluss der Stränge. Eine Hemmung dieser Enzyme durch die beschriebenen Wirkstoffe, führt zu einem Verlust der Fähigkeit von der DNA zu dissoziieren. Hierdurch werden Strangbrüche hervorgerufen, die schließlich den Zelltod auslösen (Abbildung 6).<smiles>C[C@H](O)[C@@]1(O)C(=O)OCc2c1cc1n(c2=O)Cc2cc3ccccc3nc2-1</smiles>

Camptothecin (8)

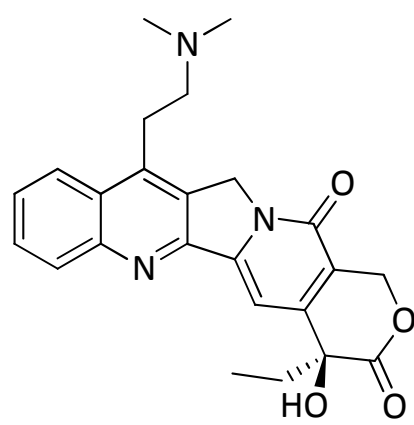

Belotecan (9)<smiles>CCc1c(C)nc2ccc(OC(=O)N3CCC(N4CCCCC4)CC3)cc2c1C</smiles>

Irinocetan (10)

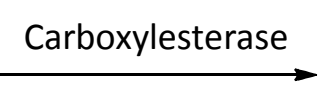

SN-38 (11)

Abbildung 6. Beispiele für die Gruppe der Topoisomerasehemmstoffe (8,9 und 11)

Zu den zytostatischen Antibiotika zählen in erster Linie die aus Streptomyces-Arten isolierten Anthracycline Daunorubicin (12) und Doxorubicin (13) (Abbildung 6), die bevorzugt in der S-Phase des Zellzyklus' wirken. Sie interkalieren in die DNA und stören dadurch die DNA- und RNA-Synthese. Des Weiteren können sie durch Radikalbildung und Hemmung der Topoisomerase II Strangbrüche induzieren. ${ }^{32}$ 
<smiles>[R]CC(=O)[C@]1(O)Cc2c(O)c3c(c(O)c2[C@@H](OC2CC(N)(O)C(C)(C)O2)C1)C(=O)c1cccc(OC)c1C3=O</smiles>

Daunorubicin (12): $\mathrm{R}=\mathrm{H}$

Doxorubicin (13): $\quad \mathrm{R}=\mathrm{OH}$<smiles>COc1cccc2c1C(=O)c1c(O)c3c(c(O)c1C2=O)C[C@@](O)(C(=O)CO)C[C@@H]3OC1CC2(N)CC2(O)C1(N)O</smiles>

Epirubicin (14)<smiles>CC(=O)[C@]1(O)Cc2c(O)c3c(c(O)c2[C@@H](OC2C[C@](N)(O)C(C)(C)O2)C3)C(=O)c2ccccc2C1=O</smiles>

Idarubicin (15)

Abbildung 7. Beispiele für die Wirkstoffklasse der zytostatischen Antibiotika: Daunorubicin (12), Doxorubicin (13), Epirubicin (14) und Idarubicin (15).

Der Vorteil der Chemotherapeutika gegenüber der Strahlentherapie und der chirurgischen Entfernung von Tumoren bei der Bekämpfung von Krebszellen ist insbesondere in Fällen chirurgisch schwer zugänglicher Tumoren oder bei Metastasenbildung von Bedeutung. Allerdings treten, wie bereits beschrieben, schwere Nebenwirkungen auf, die unter Umständen eine weitere Behandlung des Patienten nicht zulassen. Außerdem bringt eine Chemotherapie häufig Spätfolgen mit sich, wozu die Induktion von Sekundärtumoren, Schädigungen des Knochenmarks, Lungenfibrosen oder Immundefekte zählen. Ebenso stellt die Resistenzentwicklung von Tumoren gegen einzelne angewendete Zytostatika eine Limitation in der Behandlung dar. ${ }^{33}$

Trotz der beschriebenen schweren Sekundäreffekte und Resistenzen konnte sich die Chemotherapie als unentbehrliche Behandlungsmethode durchsetzen. Auf Grund dieser Notwendigkeit ist eine stetige Weiterentwicklung der vorhandenen Therapieansätze und eine Verbesserung der Wirkstoffe unumgänglich. 


\subsection{Immuntherapie}

Neben den bereits erwähnten Therapiemaßnahmen, hat sich die Immuntherapie in den letzten Jahren stetig weiter entwickelt. Bei der Anwendung der Immuntherapie werden zum Beispiel Cytokine oder Antikörper verwendet. Diese haben die Eigenschaft immunmodulierend oder direkt antiproliferierend $\mathrm{zu}$ wirken. ${ }^{26}$ Von entscheidender Bedeutung sind hierbei die charakteristischen Zelloberflächen, die sich im Falle von gesunden Zellen und Tumorzellen stark unterscheiden.

Jede Zellmembran weist auf ihrer extrazellulären Seite die Glykokalyx auf, die aus Glykolipiden, Glykoproteinen und Glykosaminoglykanen besteht und unter anderem der Zellerkennung, der Kommunikation und der Signalaufnahme dient. ${ }^{\mathrm{X}}$ Eine Unterscheidung zwischen gesunden Zellen und Krebszellen und damit einhergehend der Einsatz der Immuntherapie kann auf Grundlage der Andersartigkeit der Antigene erfolgen. ${ }^{34}$ Bestimmte tumorassoziierte Antigene sind spezifisch für Tumorzellen oder sie sind im Vergleich zu gesunden Zellen überexprimiert. ${ }^{35}$

Eine Bindung dieser tumorspezifischen Antigene mit monoklonalen Antikörpern und damit eine selektive Markierung der Krebszellen ermöglicht eine selektive Zerstörung des Gewebes. ${ }^{36}$ Köhler und Milstein enwickelten 1975 die ersten Immunoglobuline, die heute durch die Hybridomtechnik darstellbar sind. ${ }^{37}$

$\mathrm{Zu}$ den bekanntesten Vertretern der Antikörper gehören Trastuzumab ${ }^{38}$ gegen HER2/neu-positive Mammakarzinome ${ }^{39}$ sowie Bevacizumab ${ }^{40}$ als Angiogeneseinhibitor. So kann das Immunglobulin mit Zytokinen (z.B. Interleukin-2, IL-2) gekuppelt werden, wobei das resultierende Immunzytokin die körpereigene Immunabwehr am Tumor auslöst (A). ${ }^{41,42}$ Außerdem können Antikörper mit T-Lymphozyten gekuppelt werden, was eine direkte Zytolyse der Tumorzelle hervorruft (B). ${ }^{43} \mathrm{Um}$ die körpereigene Immunabwehr zur Bekämpfung von Tumoren einzusetzen, wird mit der Verschmelzung von Krebszellen und Antigen-präsentierenden Zellen ein weiterer Ansatz verfolgt. Die auf diese Weise erhaltenen Hybride aktivieren durch ihre Überexprimierung an tumorassoziierten Antigenen zytotoxische Lymphozyten (CTL), 
die wiederum Krebszellen mit identischen Antigenen zerstören. ${ }^{44}$ Eine Beladung dendritischer Zellen (DZ) mit Tumorproteinen, Tumorpeptiden oder Tumor-DNA, kann einen vergleichbaren Effekt hervorrufen (C). ${ }^{45}$

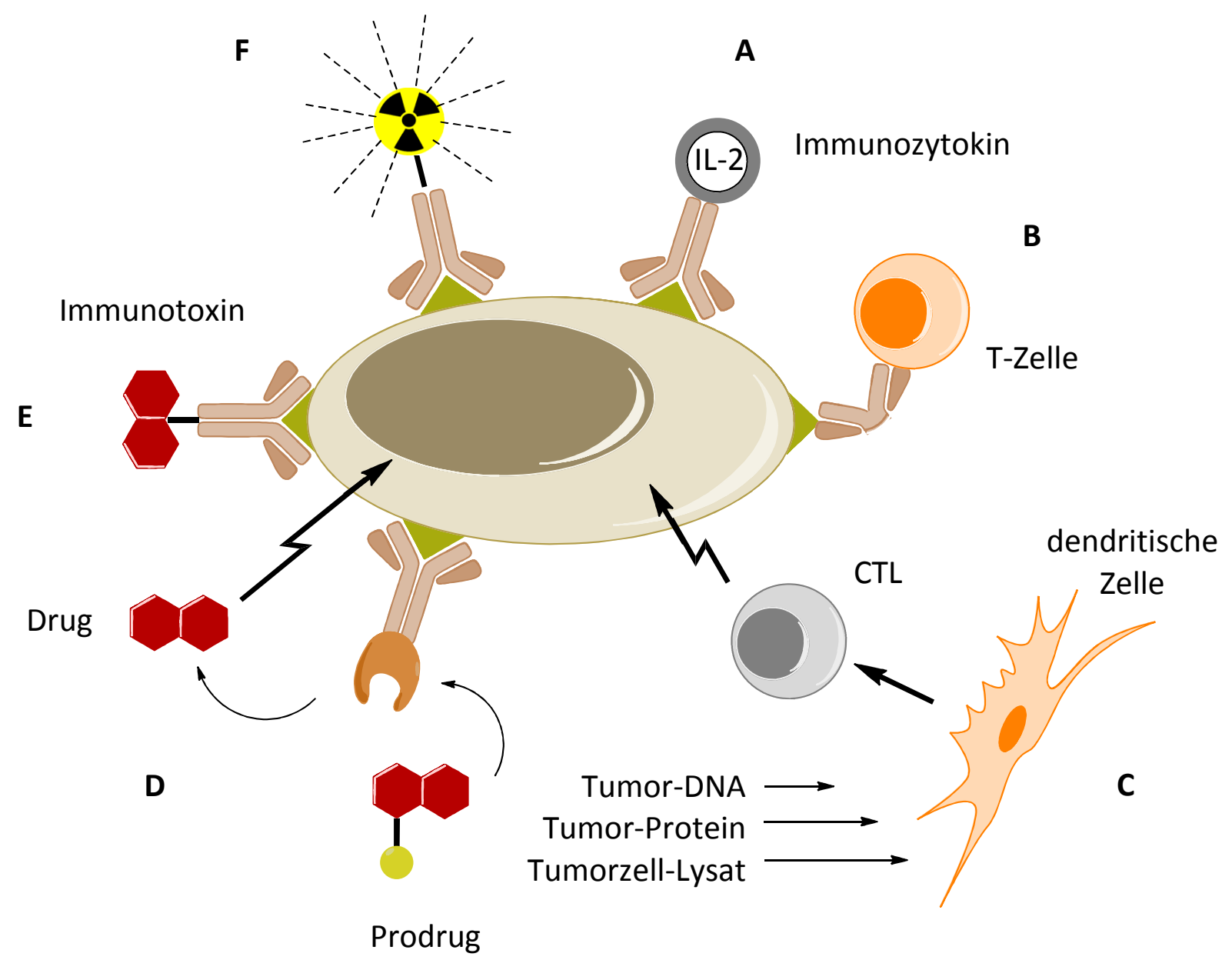

Abbildung 8. Beispiele für die Bekämpfung maligner Tumore durch Anwendung der Immuntherapie. A: Immunzytokine, B: Antikörper-vermittelte Tumorzytolyse, C: Aktivierung von zytotoxischen Lymphozyten (CTL) durch dendritische Zellen, D: Enzym-vermittelte Umwandlung von Prodrugs in Drugs, E: Immunotoxin, F: Antikörper-Radioisotopen-Konjugat. ${ }^{46}$

Über die bereits erwähnten Therapiemöglichkeiten hinaus ist eine Verwendung von Toxin-Immunokonjugaten möglich (E). ${ }^{47}$ Hierbei wird die Antikörperspezifität der Tumorzellen ausgenutzt um Toxine direkt zum Tumor zu leiten, während gesunde Zellen nicht beschädigt werden. Der erste Vertreter dieser Immunotoxine war Gemtuzumab-Ozogamicin (Mylotarg ${ }^{\circledR}$, Konjugat aus Antikörper gegen CD-33 und einem Calicheamicin-Derivat ${ }^{48}$. Dessen Marktzulassung für die USA wurde allerdings im Herbst 2010 wegen schwerwiegender Nebenwirkungen wie Myelosuppression 
aufgehoben. Neuere Präparate wie T-DM1 (Konjugat aus Trastuzumab und einem Maytansinoid-Derivat) ${ }^{49}$ sowie CMD-193 (Konjugat aus G193-Antikörper und einem Calicheamicin-Derivat ${ }^{50}$ befinden sich derzeit in klinischen Phasen.

Ein neues Konzept verwendet die tumorassoziierten Antigene um selektiv AntikörperEnzym-Konjugate an Krebszellen zu kupplen. Das ADEPT-Konzept (Antibody-Directed Enzyme Prodrug Therapy) ermöglicht die Umwandlung eines detoxifizierten Wirkstoffes (Prodrugs) ${ }^{51}$ ausschließlich an der Krebszelle in ein zytotoxisches Drug (D). ${ }^{52}$ Neben den erwähnten Methoden verwendet die Radioimmuntherapie Antikörper, die mit radioaktiven Isotopen $\left({ }^{131} \mathrm{I},{ }^{90} \mathrm{Y}\right)$ gekuppelt wurden $(\mathrm{F})$. Neben der Verwendung dieses Therapieansatzes in der Tumorbekämpfung wird er ebenso in der Diagnostik angewandt, wo er unter anderem die Lokalisation von Metastasen ermöglicht. $^{53}$

Mit Ausnahme von Ansatz C nutzen alle Ansätze die Möglichkeit der Identifikation von Krebszellen durch Antikörper, die an tumorassoziierte Antigene binden. Auch dieser vielversprechende Ansatz der Immunotherapie weist zwei Probleme auf, die als Immunogenität beschrieben werden. Behandelt man die Patienten mit Antikörpern murinen Ursprungs, wird eine Immunantwort auf körperfremde Immunglobuline ausgelöst. ${ }^{36}$ Werden humane Antigene verwendet, verbessert man die Verträglichkeit dieser, mindert aber die Selektivität. ${ }^{52 b}$ Ähnlich problematisch ist auch hier die große Individualität der Zellen in Bezug auf verschiedene Krebsarten und die damit verbundene Andersartigkeit der Antigene. Eine optimale Therapie wäre nur durch die individuelle Anpassung der Antikörper an die Antigene des Patienten möglich. 


\subsection{Selektive Krebstherapie mittels reversibel detoxifizierter Prodrugs}

Auf Grund der starken Nebenwirkungen vieler Chemotherapeutika ist eine selektive Krebsbehandlung von entscheidender Bedeutung. Ein vielversprechender Ansatz nutzt die Möglichkeit untoxische Prodrugs gezielt am Tumor in das entsprechende toxische Drug zu überführen (Abbildung 9). ${ }^{54} \mathrm{Um}$ eine bessere Abgrenzung zwischen gesunden Zellen und Krebszellen zu erreichen, lassen sie sich anhand von genotypischen und phänotypischen Eigenschaften unterscheiden.
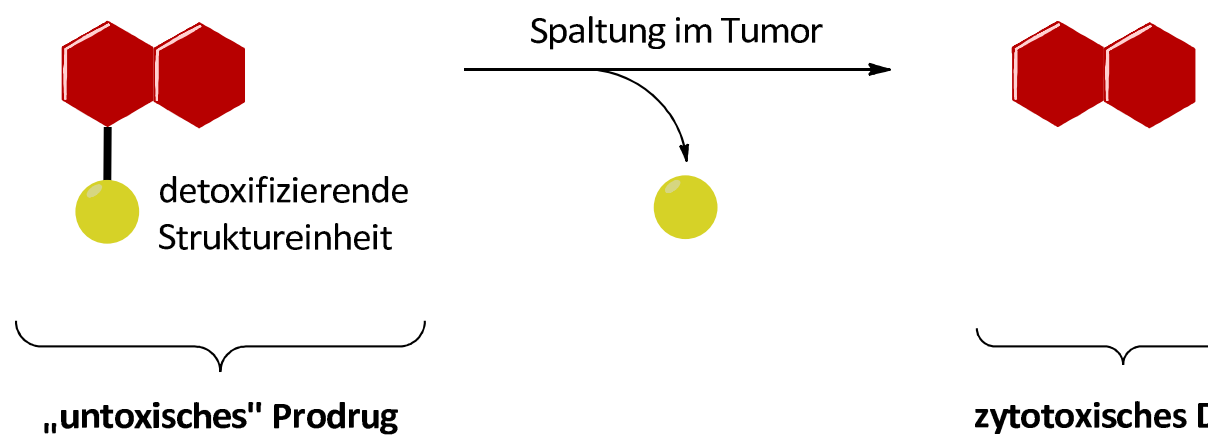
Abbildung 9. Überführung des „untoxischen“ Prodrugs in das zytotoxische Drug durch Spaltung
am Tumor.

So unterscheiden sich gesunde Zellen von Tumorzellen zum Beispiel durch eine Abweichung des pH-Wertes im extrazellulären Raum. Dieser ist bei Tumoren, hervorgerufen durch eine erhöhte anaerobe Glykolyserate, signifikant niedriger als bei gesundem Gewebe. ${ }^{55}$ Dieser Unterschied geht einher mit der gesteigerten anabolischen und mitotischen Aktivität der Tumorzelle ${ }^{56}$ und führt durch die nicht vorhandene Möglichkeit zum Abtransport der gebildeten Milchsäure zum Absinken des $\mathrm{pH}$-Wertes im Tumorgewebe. ${ }^{57}$ Des Weiteren ist die Glykolyserate der Krebszellen von der extrazellulären Glucosekonzentration abhängig, da diese nicht in der Lage sind, die Kontrollmechanismen zur Glucosezufuhr zu steuern. ${ }^{58}$ Eine exogene Glucosezufuhr führt aus diesen Gründen zu einer Absenkung des pH-Wertes um 1.2 Einheiten. ${ }^{59}$ Dieser Unterschied kann für therapeutische Zwecke genutzt werden und 
ist für eine Dauer von $24 \mathrm{~h}$ stabil. Angelehnt an diese Untersuchungen entwickelte L.F. Tietze 1981 das Prinzip der säurelabilen acetalischen Prodrugs. Eine Überführung des Prodrugs durch eine säurekatalysierte Hydrolyse in das deutlich toxischere Drug geschieht erst im Tumorgewebe, wodurch das gesunde Gewebe nicht geschädigt wird. ${ }^{4 d, 60}$ Versuche hierzu wurden unter anderem mit Acetalglykosiden von Ketonen durchgeführt. $^{61}$ Das Ketophosphamid BE-1 (16) wird unter säurekatalysierter Hydrolyse in das Keton 18 umgewandelt und spaltet dabei D-Glucose (17) und Methanol ab. Im Anschluss kann 18 wie auch das Cyclophosphamid (1) über die Friedmannsäure 19 zum Aziridiniumion 2 als aktives Drug umgewandelt werden. Bei Untersuchungen von BE-1 (16) an Mammakarzinomzellen der Marshallrate (M1R) unter physiologischen Bedingungen und bei pH 6.2 konnte gezeigt werden, dass $\mathrm{BE}-1$ (16) unter physiologischen Bedinungen 17-fach weniger toxisch ist.

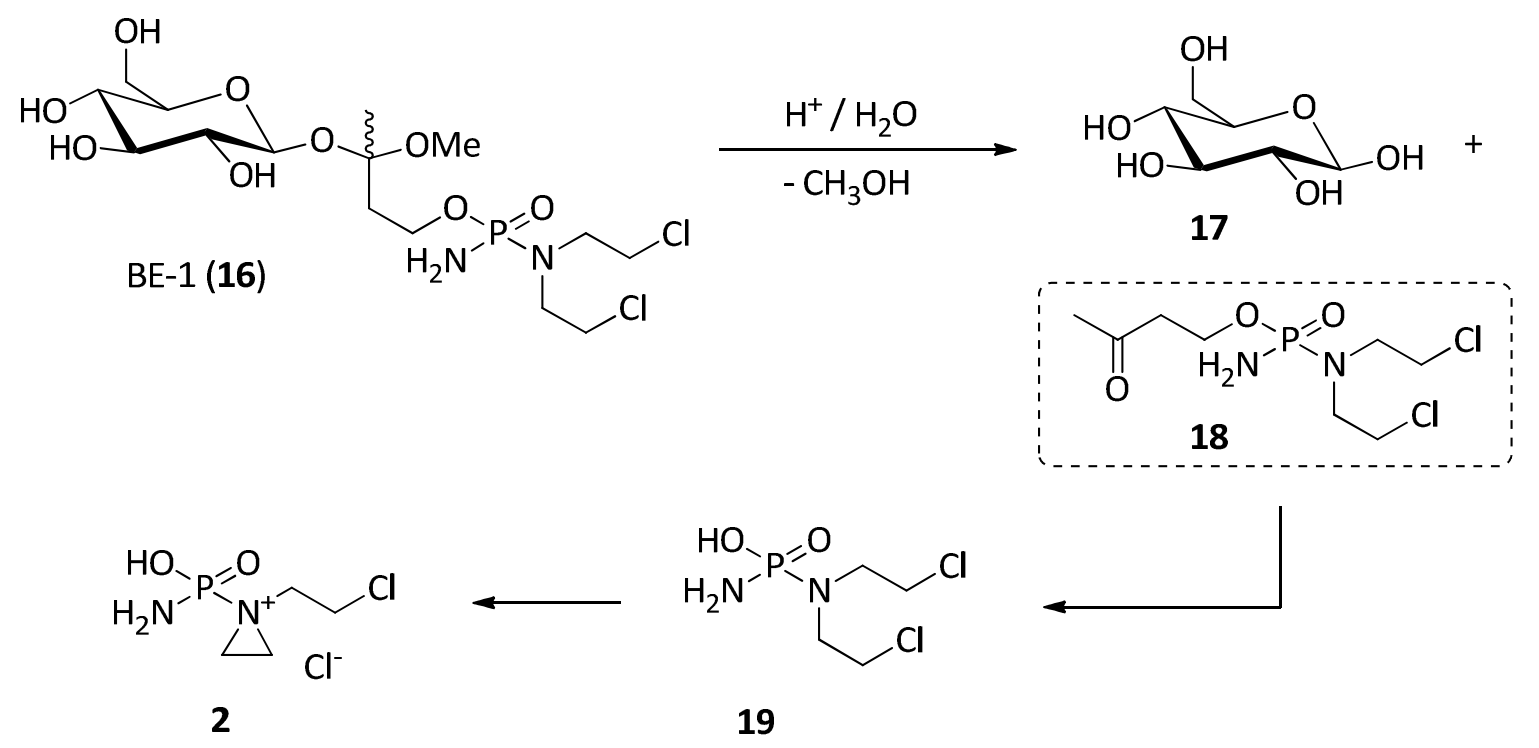

Abbildung 10. Darstellung von Drug 2 durch säurekatalysierte Aktivierung des acetalischen Prodrugs BE-1 (11). 


\subsubsection{Antibody-Directed Enzyme Prodrug Therapy (ADEPT)}

Wie in Kapitel 3.2 erwähnt, kann die Tumorzelloberfläche als Ziel für Marker genutzt werden. Ein binäres Therapiekonzept beschreibt Bagshawe 1987 als AntibodyDirected Enzyme Prodrug Therapy. Hierbei bindet ein systemisch verabreichtes Antikörper-Enzym-Konjugat (AEC) selektiv an tumorassoziierte Antigene. Dieses AEC ist nun in der Lage, in direkter Nähe zum Tumor, das ebenfalls systemische untoxische Prodrug durch das entsprechende Enzym in das toxische Drug zu überführen. ${ }^{52,62}$ Ein wichtiger Bestandteil der Therapie ist die Clearance-Zeit. ${ }^{63,64}$ Das verabreichte AEC muss vor der Gabe des Prodrugs aus dem Blutkreislauf verschwunden sein, um eine unselektive Freisetzung des Drugs zu verhindern. Eine Umsetzung des Prodrugs in das entsprechende Drug ist hierdurch auf das Tumorgewebe beschränkt und die gewünschte Differenzierung zwischen gesundem Gewebe und Tumorgewebe ist erfolgreich. Das Drug penetriert die Zellmembran und entfaltet seine toxische Wirkung innerhalb der Zelle. Das AEC ist weiterhin in der Lage, die außerhalb der Zelle befindlichen Prodrugs in die Drugs zu überführen und einen Zelleintritt zu ermöglichen.

Auf Grund der Unterscheidung zwischen untoxischem Prodrug und toxischem Drug kann eine weitaus höhere Wirkstoffdosis verabreicht und somit eine effizientere Behandlung ermöglicht werden. Ein weiterer Vorteil des ADEPT-Konzeptes besteht in der Möglichkeit der Drugs, durch solide Tumoren zu diffundieren und Krebszellen zu töten, die nicht über die vom AEC benötigten Antigene verfügen (Bystander-Effekt). ${ }^{65}$ Zur Abschätzung einer möglichen Therapie im Rahmen von ADEPT durch ein Prodrug wurden folgende Kriterien erarbeitet: ${ }^{66}$ „Das Prodrug sollte ein adäquates Substrat für das Enzym im AEC, jedoch nicht für andere Enzyme im Organismus sein. Außerdem sollte es eine möglichst geringe Zytotoxizität aufweisen und nicht zellgängig sein. Darüber hinaus ist eine große therapeutische Breite des Prodrugs anzustreben, die in vitro durch den Quotienten der $\mathrm{IC}_{50}$-Werte in $\mathrm{Ab}$ - und Anwesenheit des spaltenden Enzyms widergespiegelt wird $\left(Q I C_{50}=I C_{50}\{\right.$ Prodrug\} $/ I C_{50}$ \{Prodrug in Anwesenheit des Enzyms\}). Als Richtlinie gilt, dass der 
$\mathrm{QIC}_{50}>1000$ sein sollte und das zytotoxische Drug einen $\mathrm{IC}_{50}<10 \mathrm{nM}$ besitzen sollte. ${ }^{4 d, 67}$ Das Drug sollte ferner die Zellmembran sehr leicht penetrieren können und eine Halbwertszeit aufweisen, die lang genug ist, um einen Bystander-Effekt zu bewirken, und ausreichend kurz, um eine Rückdiffusion des Drugs vom Tumor in das umliegende Gewebe zu verhindern." Das Enzym sollte eine hohe katalytische Aktivität haben und entweder nicht-humanen oder humanen Ursprungs sein. Im letzten Fall darf das Enzym allerdings gar nicht oder nur marginal in Normalgewebe und Körperflüssigkeiten exprimiert sein, um eine unselektive Prodrug-Aktivierung und damit systemische Toxizität zu vermeiden.

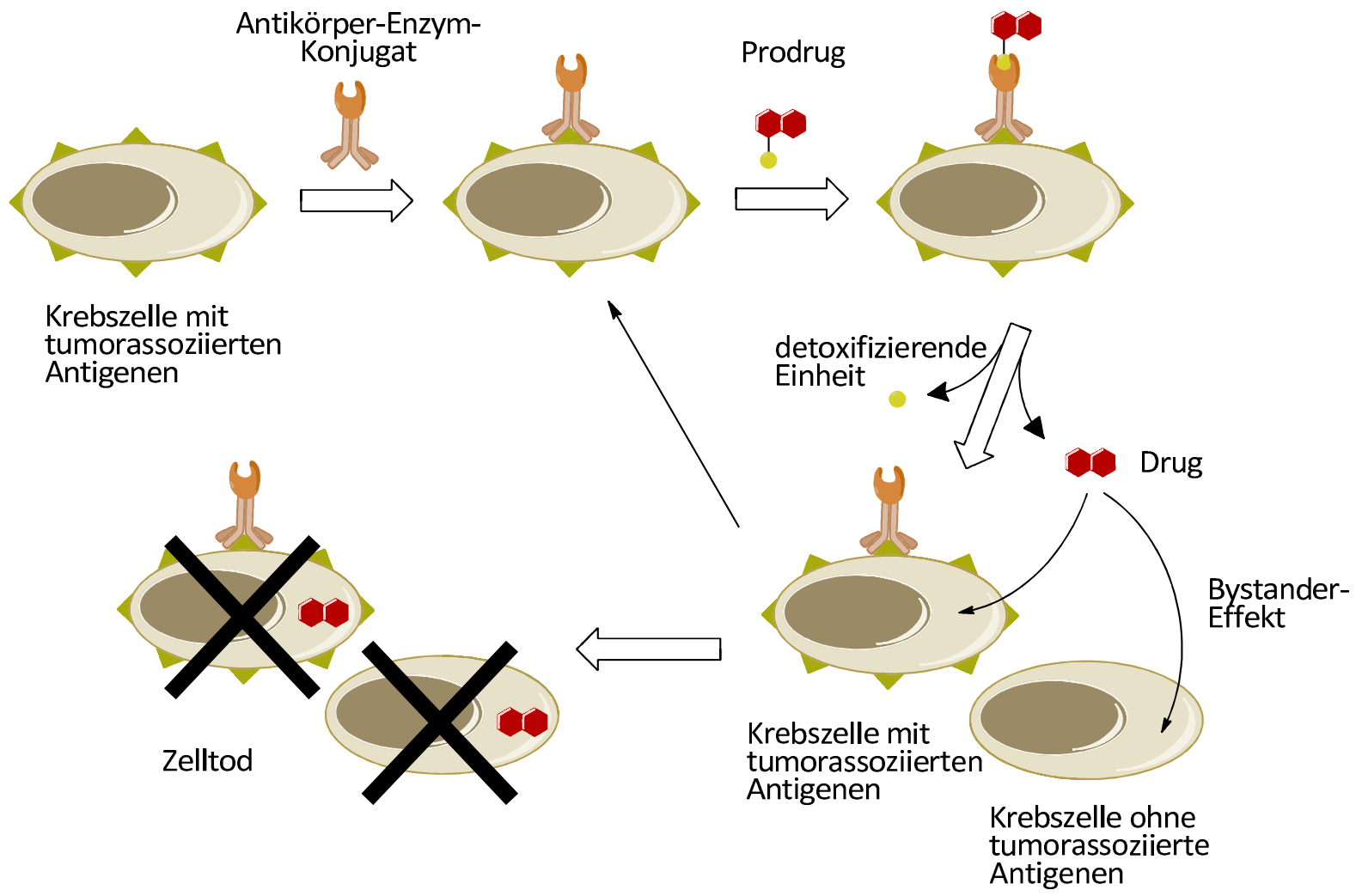

Abbildung 11. Vereinfachte Darstellung des ADEPT-Konzeptes.

Wie im Fall der bereits vorgestellten Antikörper und einer damit einhergehenden Immunogenität tritt diese Problematik auch beim ADEPT-Konzept auf. Um sie zu vermeiden, wurde das ADAPT-Konzept (Antibody-Directed Abzyme Prodrug Therapy) entwickelt, das humanisierte, katalytisch wirksame Antikörper (Abzyme) nutzt. ${ }^{68}$ 
Seit den 1980er Jahren konnten zahlreiche Prodrugs für eine Anwendung im ADEPTKonzept entwickelt werden. Besonders erwähnenswert sind hier „untoxische” Derivate der Wirkstoffe Lost, $^{69}$ Methotrexat, $^{70}$ Doxorubicin und Daunorubicin, $^{71}$ 5-Fluoruracil, ${ }^{72}$ Etoposid, $^{73}$ Vinblastin, $^{74}$ Paclitaxel $^{75}$ und Camptothecin. ${ }^{76}$ Eine Aktivierung der Prodrugs erfolgt durch Antikörper-Enzym-Konjugate oder Fusionsproteine aus Single-chain-Antikörpern. Eine weitere Möglichkeit liegt in der Aktivierung durch die Enzyme Carboxypeptidase G2 und A (CPG2, CPA), ${ }^{77} \beta$-Lactamase $^{78}$ Nitroreduktase, ${ }^{79}$ Cytosin-Deaminase ${ }^{80}$ oder $\beta$-D-Galaktosidase ${ }^{81}$. Allerdings konnten auch hier die typischen Nachteile der bisher bekannten Prodrugs in zu kleinen therapeutischen Fenstern sowie zu geringen Zytotoxizitäten des gebildeten Drugs gefunden werden.

Erste klinische Studien im Rahmen des ADEPT-Konzeptes folgten 1995 durch Bagshawe und Sharma. ${ }^{52 \mathrm{~b} 52 \mathrm{~d}}$ Ein geeignetes Prodrug wurde in CMDA (20) gefunden und wurde durch Umsetzung mit dem A5B7-CPG2-Konjugat in das toxische N-LostDrug 21 überführt.<smiles>COS(=O)(=O)CCN(CCCl)c1ccc(C(=O)N[C@@H](CCC(=O)O)C(=O)O)cc1</smiles>

CMDA (20)<smiles>O=C(O)CC[C@H](NC(=O)Oc1ccc(N(CCI)CCI)cc1)C(=O)O</smiles>

ZD2767P (22)

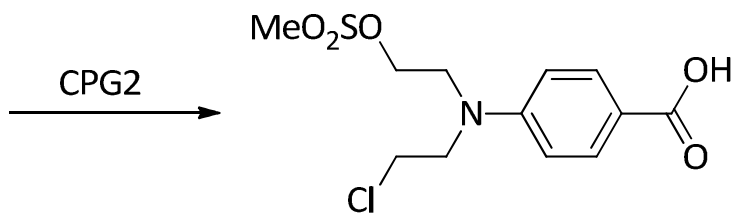

21

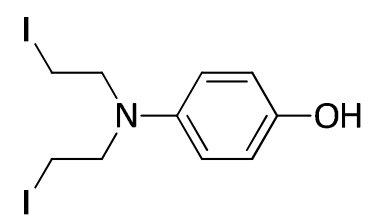

ZD2767 (23)

Abbildung 12. Aktivierung der Prodrugs CMDA (20) und ZD2767P (22) zu den entsprechenden Drugs 21 und 23 durch Carboxypeptidase G2 (CPG2).

Mit einer therapeutischen Breite von $\mathrm{QIC}_{50}=100$ und einer relativ langen Plasmahalbwertszeit für $\mathbf{2 1}$ von $t_{1 / 2}=30 \mathrm{~min}$ sowie der Eigenschaft, aus dem 
Tumorgewebe zu diffundieren und eine Myelosuppression hervorzurufen, konnte kein erfolgreiches Ergebnis erzielt werden.

2002 folgte eine weitere Studie, in der das Glutamat-Prodrug ZD2767P ${ }^{82}$ (22) eingesetzt wurde, das im Gegensatz zu CMDA (20) über eine geringere Plasmahalbwertszeit verfügt. Wie bei 20 erfolgt eine Umsetzung durch das Enzym CPG2 zu dem entsprechenden Drug ZD2767 (23). Glutamat-Prodrug ZD2767P ${ }^{83}$ (22) zeigte mit einer therapeutischen Breite von $\mathrm{QIC}_{50}=100-200$ einen akzeptablen Wert. Im Falle von 22 wurde im Gegensatz zur Anwendung von 20 kein AEC verwendet, sondern auf ein mannosyliertes Fusionsprotein aus Single-Chain-Antikörper und Enzym zurückgegriffen. ${ }^{64}$ Der Vorteil dieses Proteins liegt in der deutlich geringeren Clearence-Zeit, da es über Mannose-Rezeptoren in die Leber aufgenommen wird. ${ }^{84}$ Bei der Behandlung von Patienten mit fortgeschrittenem kolorektalem Karzinom konnte nach 2-3 Therapiezyklen eine signifikante Anti-Tumor-Response festgestellt werden. $^{52 \mathrm{c}}$

Neben den beschriebenen klinischen Studien konnte auch ein von Tietze et al. synthetisiertes Galaktosysl-Prodrug ${ }^{85}$ in einer präklinischen Studie in einem Mammakarzinommodell (MDA-MB-231) untersucht werden. ${ }^{86}$ Auch hier konnte nach 2 Therapiezyklen eine signifikante Anti-Tumor-Response festgestellt werden. Im direkten Vergleich mit Kontrolltieren wurde ein um den Faktor 1.7 geringeres Tumorwachstum festgestellt. Untersuchungen mit dem Lymphommodell (A20) zeigte sogar ein um den Faktor 3.6 kleineres Tumorwachstum. ${ }^{134 b}$

Neben den bereits beschrieben Konzepten ADEPT und ADAPT haben sich weitere Therapiekonzepte herauskristallisiert. Unter anderem wurden die Konzepte GDEPT (gene-directed), ${ }^{87}$ PDEPT (polymer-directed), PELT (polymer-enzyme liposome therapy), ${ }^{88}$ CDEPT (clostridial-directed), ${ }^{89}$ BDEPT (bacteria-directed), ${ }^{90}$ FDEPT (folatedirected) ${ }^{91}$ und LEAPT (lectin-directed enzyme activated prodrug therapy) ${ }^{92}$ weiterentwickelt und zeigen teils vielversprechende Ansätze.

Weitere Studien untersuchen selbstauflösende Dendrimer-Systeme, die terminal mit Wirkstoffen wie Doxorubicin, Camptothecin, Paclitaxel oder Etoposid beladen sind. 
Unter Verwendung des ADAPT-Konzeptes können die Zytostatika mit Hilfe eines Abzyms kaskadenartig und somit hocheffizient freigesetzt werden. ${ }^{54 a, 71 a, 93}$

\subsubsection{Prodrug-Monotherapie (PMT)}

Neben den bereits beschriebenen Konzepten der Immuntherapie und der AntibodyDirected Enzyme Prodrug Therapy wurde mit der Prodrug-Monotherapie (PMT) ${ }^{94}$ ein weiteres vielversprechendes Konzept entwickelt. Im Gegensatz zum ADEPT-Konzept, bei dem zusätzliche AEC verabreicht werden müssen, um das Prodrug in das Drug umzuwandeln, werden im Fall der PMT die Abnormitäten des Tumorgewebes genutzt. Auf diese Weise können sowohl metabolische als auch physiologische Eigenschaften des Tumorgewebes genutzt werden, um gezielt Prodrugs in toxische Drugs umzuwandeln. Hierbei stellen zum Beispiel der Unterschied im pH-Wert, Hypoxie oder tumorassoziierte Rezeptoren und Enzyme mögliche Angriffspunkte dar. $^{95}$

Besonders das Enzym $\beta$-D-Glucuronidase, das in Zellen bei akuten und chronischen Entzündungsprozessen in den Extrazellularbereich von nekrotischen Tumoren freigesetzt wird, eignet sich für eine Anwendung im Rahmen der Prodrug-MonoTherapie. ${ }^{96}$ Des Weiteren handelt es sich bei $\beta$-D-Glucuronidase um ein humanes Enzym und ruft somit keine Immunantwort hervor und es besitzt bei niedrigem $\mathrm{pH}$ Wert, wie es im Tumorgewebe der Fall ist, eine höherer Aktivität. ${ }^{97}$ Das AcetalGlucoronid $\mathbf{2 4}$ konnte im Arbeitskreis von Tietze et al. entwickelt werden und wird in Anwesenheit von $\beta$-D-Glucuronidase nach erfolgreicher Esterase zu Butyraldehyd (26) umgesetzt, wobei D-Glucuronsäure (27) und Methanol abgespalten werden (Abbildung 13). ${ }^{98}$ Leider konnten weitere In-vivo-Studien nicht die gewünschten Ergebnisse liefern, was auf die geringe Toxizität des Butyraldehydes zurückzuführen ist. 

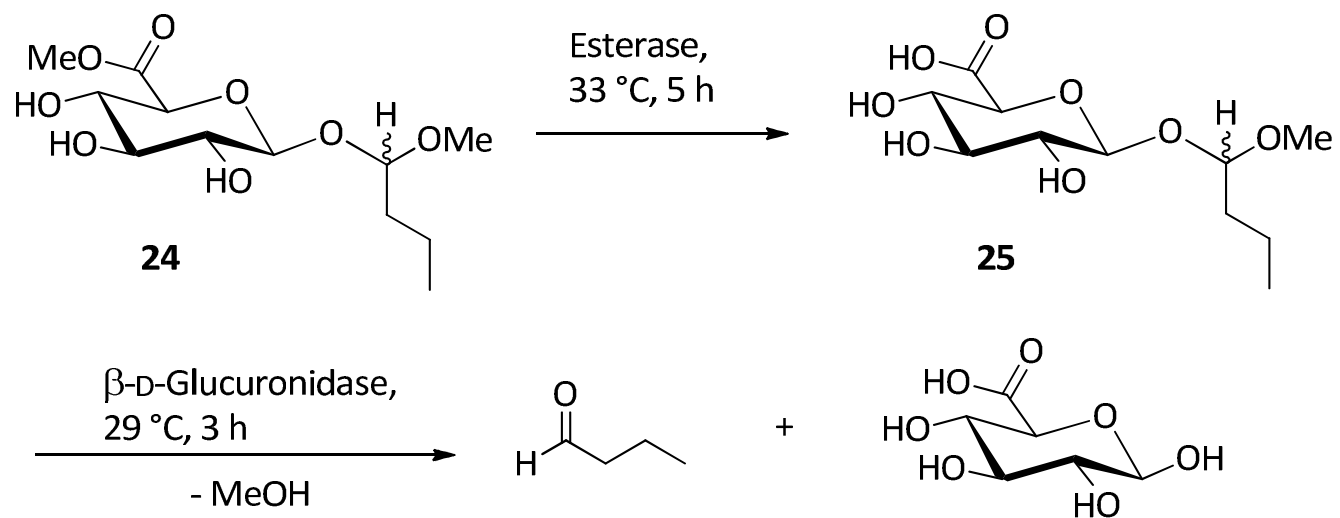

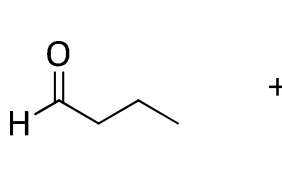

26

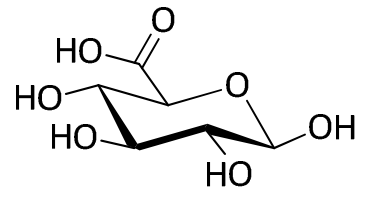

27

Abbildung 13. Aktivierung des Acetal-Glucuronids 24 durch Esterase und $\beta$-D-Glucuronidase.

Zahlreiche Glucuronsäure-Prodrugs folgten auf die Arbeiten von Tietze et al. ${ }^{99}$ Bei der Verwendung einer selbstauflösenden Spacer-Einheit zeigte sich an dem Glucuronsäurerest eine Erhöhung der Enzymaktivität. Mit steigender Entfernung des Pharmakophors von der Glucuronsäure konnte der sterische Anspruch verringert und die Aktivität des Enzyms verbessert werden. Häufig wurden Pharmakophoren der Typen Doxorubicin, Paclitaxel, Docetaxel und Camptothecin verwendet. Zunächst wurden Glucuronsäure-Prodrugs für die Prodrug-Mono-Therapie entwickelt. Eine Anwendung in Bezug auf GDEPT und ADEPT ist aber ebenso denkbar. Hierzu könnten Gen-kontrollierte Erhöhungen der Glucuronidase-Konzentration im Tumorgewebe (GDEPT) oder Antikörper-Glucuronidase-Konjugate (ADEPT) verwendet werden. ${ }^{100}$

Ein prominentes Beispiel für die Anwendung der PMT in Zusammenhang mit einer Spacer-Einheit wurde von Boven et al. Entwickelt. Der Wirkstoff DOX-GA3 (29) zeigte eine verbesserte Wachstumshemmung in Ovarialkarzinom-Modellen an Nacktmäusen. Auch dessen entsprechendes Derivat, der Methylester 28, zeigte in vitro eine ebenso große Zytotoxizität wie Doxorubicin, was auf eine vollständige Umsetzung des Prodrugs schließen lässt. DOX-GA3 (29) ist über einen p-Aminobenzyloxycarbonyl-Spacer an die Glucuronsäureeinheit gekuppelt und kann durch $\beta$-DGlucuronidase und anschließende 1,6-Eliminierung des Spacers in Doxorubicin (13), p-Aminobenzylalkohol (30) und $\mathrm{CO}_{2}$ überführt werden (Abbildung 14). ${ }^{101}$ 

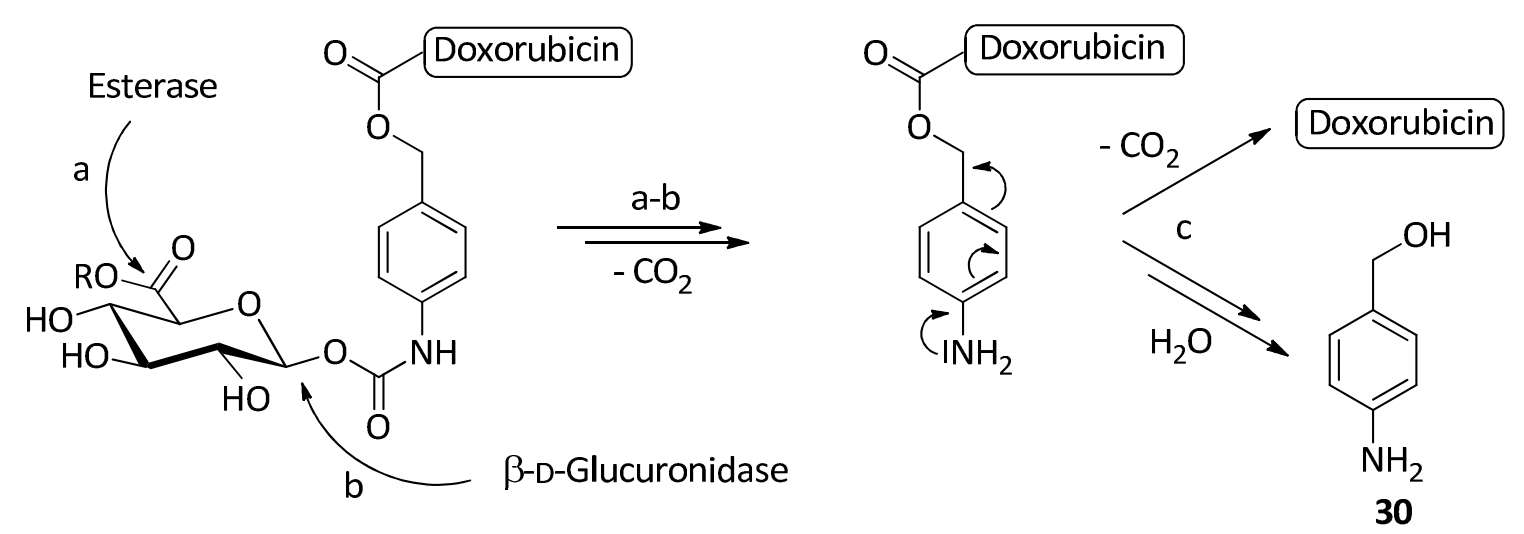

a

28: $R=M e(D O X-m G A 3)$

29: $\quad R=H \quad(D O X-G A 3)$

Abbildung 14. Aktivierung des Prodrugs DOX-GA3 (29) durch Hydrolyse des Methylglucuronats DOX-mGA3 (28) durch Serumesterase (a) sowie anschließende Spaltung der Zuckereinheit durch $\beta$-D-Glucuronidase unter Freisetzung von $\mathrm{CO}_{2}(\mathrm{~b})$ und spontane 1,6-Eliminierung des Spacers unter Freisetzung von Doxorubicin (13), p-Aminobenzylalkohol (30) und $\mathrm{CO}_{2}$ (c).

Weitere Untersuchungen wurden von Monneret et al. zu Glucuronidase-Prodrugs angestellt. Sie synthetisierten ein Paclitaxel-Prodrug mit einem kurzen Spacer und erzielten in vitro einen $\mathrm{QIC}_{50}$-Wert von 700. Dieses Ergebnis konnte in vivo jedoch nicht wiederholt werden, was auf eine unvollständige Umsetzung des Prodrugs zum Drug schließen lässt. ${ }^{102}$ Weitere Untersuchungen mit einem doppelten Spacersystem, das zu einer quantitativen enzymatischen Spaltung, jedoch auch zum Absinken des QIC $_{50}$-Wertes führte, konnten nicht die gewünschten Ergebnisse liefern. ${ }^{103,104}$ Natürlich wurden auch die Spacer-Einheiten auf ihre Toxizität untersucht, konnten aber als vernachlässigbar angesehen werden, da die Toxizität des Drugs die des Spacers bei Weitem übersteigt.

Außer den bereits erwähnten Untersuchungen zu den $\beta$-D-Glucuronidase-Prodrugs folgten weitere Untersuchungen zu Enzymen, die in Tumoren überexprimiert sind. Hierzu zählen Enzyme wie Plasmin, Prolidase, Tyrosinase uvm., die aber in der Forschung auf weniger Interesse stoßen. ${ }^{54 a, 105}$

Neben der erwähnten Überexprimierung von Enzymen und den physiologischen Bedingungen im Tumorgewebe kann auch die Hypoxie als wichtiges Merkmal von Tumorzellen angesehen werden und stellt einen wichtigen Ansatzpunkt in der 
Prodrug-Mono-Therapie dar. Wie bereits beschrieben, neigen Tumorzellen zu einer abnormen und ausgeprägten Vaskularisierung, was eine schlechte Nährstoff- und Sauerstoffzufuhr der betroffenen Zellen zur Folge hat. Des Weiteren können hieraus Resistenzen gegenüber Chemotherapeutika und Radiotherapie enstehen. Im Rahmen einer selektiven Krebstherapie wird das Vorliegen hypoxischer Areale ausgenutzt, um gezielt Prodrugs zu toxifizieren. ${ }^{106}$ 


\section{CC-1065, Duocarmycin SA und Yatakemycin}

1978 wurde das natürliche Antibiotikum (+)-CC-1065 (31) erstmals von Mitarbeitern der Upjohn Company aus Kulturen von Streptomyces zelensis isoliert. ${ }^{107}$ (+)-CC-1065 (31) zeigte sowohl in vitro als auch in vivo eine sehr hohe Zytotoxizität sowie ein breites Spektrum der antibiotischen Wirksamkeit und eine starke Antitumoraktivität. $^{108}$

31 ist aus drei substituierten Pyrrolo[2,3-e]-indol-Einheiten aufgebaut, die jeweils durch eine Amidbindung miteinander verknüpft sind (Abbildung 15). Eine wichtige Rolle übernehmen die 1,2-Dihydro-3H-pyrrolo-[3,2-e]-indol-Systeme (PDE I und PDE II), da sie auf Grund ihrer hohen Affinität zu Doppelstrang-DNA, als DNAbindende Einheiten fungieren. Die mit CPI (Cyclopropapyrroloindol) gekennzeichnete Einheit ist verantwortlich für die Alkylierung der DNA und damit ein entscheidendes Strukturmerkmal für (+)-CC-1065 (31), Duocarmycin SA (32) und Yatakemycin (33).

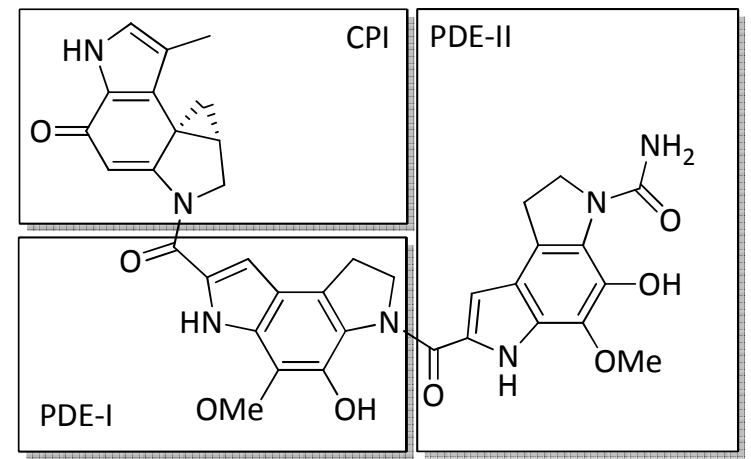

(+)-CC-1065 (31)

$\mathrm{IC}_{50}: 20 \mathrm{pM}(\mathrm{L} 1210)$

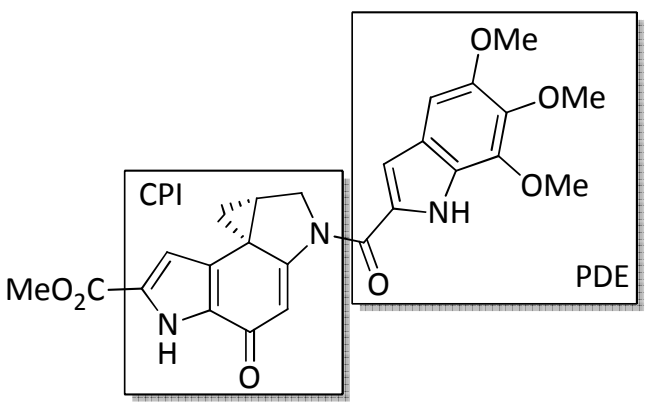

(+)-Duocarmycin SA (32) $\mathrm{IC}_{50}: 10 \mathrm{pM}(\mathrm{L} 1210)$

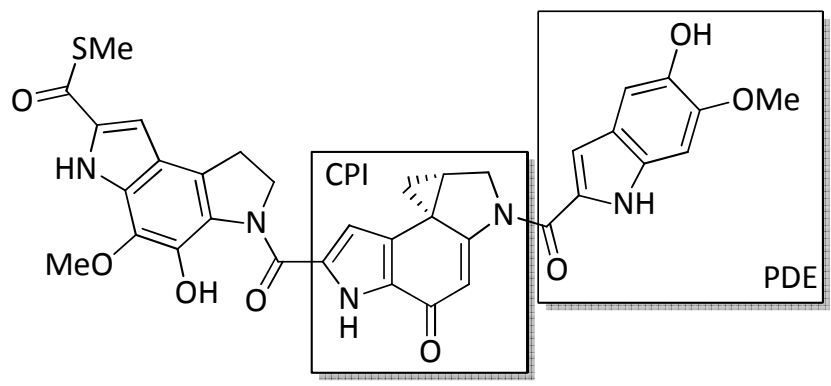

(+)-Yatakemycin (33)

$\mathrm{IC}_{50}:$ 3-5 pM (L1210)

Abbildung 15. (+)-CC-1065 (25), (+)-Duocarmycin SA (26) und (+)-Yatakemycin (27) als Beispiele für zytotoxische Antibiotika. 
Verwandte Verbindungen von $\mathbf{3 1}$ konnten aus anderen Streptomycetenstämmen isoliert werden. Hierzu gehören insbesondere (+)-Duocarmycin SA (32) (aus Streptomyces DO-113) ${ }^{109}$ sowie Yatakemycin (33) (aus Streptomyces TP-A0356), ${ }^{110}$ das mit einem $\mathrm{IC}_{50}$-Wert von 3 pM eines der potentesten Zytostatika darstellt.

(+)-CC-1065 (31) entfaltet seine zytotoxische Wirkung auf Grund einer sequenzspezifischen Alkylierung der DNA, bei der ein nucleophiler Angriff des AdeninN-3 aus der kleinen Furche AT-reicher Regionen von doppelsträngiger DNA auf das niedrigst substituierte Kohlenstoffatom der Spirocyclopropyleinheit (CPI) erfolgt (Abbildung 16). Die lipophile Innenseite der gekrümmten Struktur des CC-1065 und seiner Analoga begünstigt dabei eine Wechselwirkung mit den Nucleobasen, während die hydrophile Außenseite die Einlagerung in die kleine Furche durch Wechselwirkungen mit den polaren Funktionalitäten des DNA-Rückgrates stabilisiert.

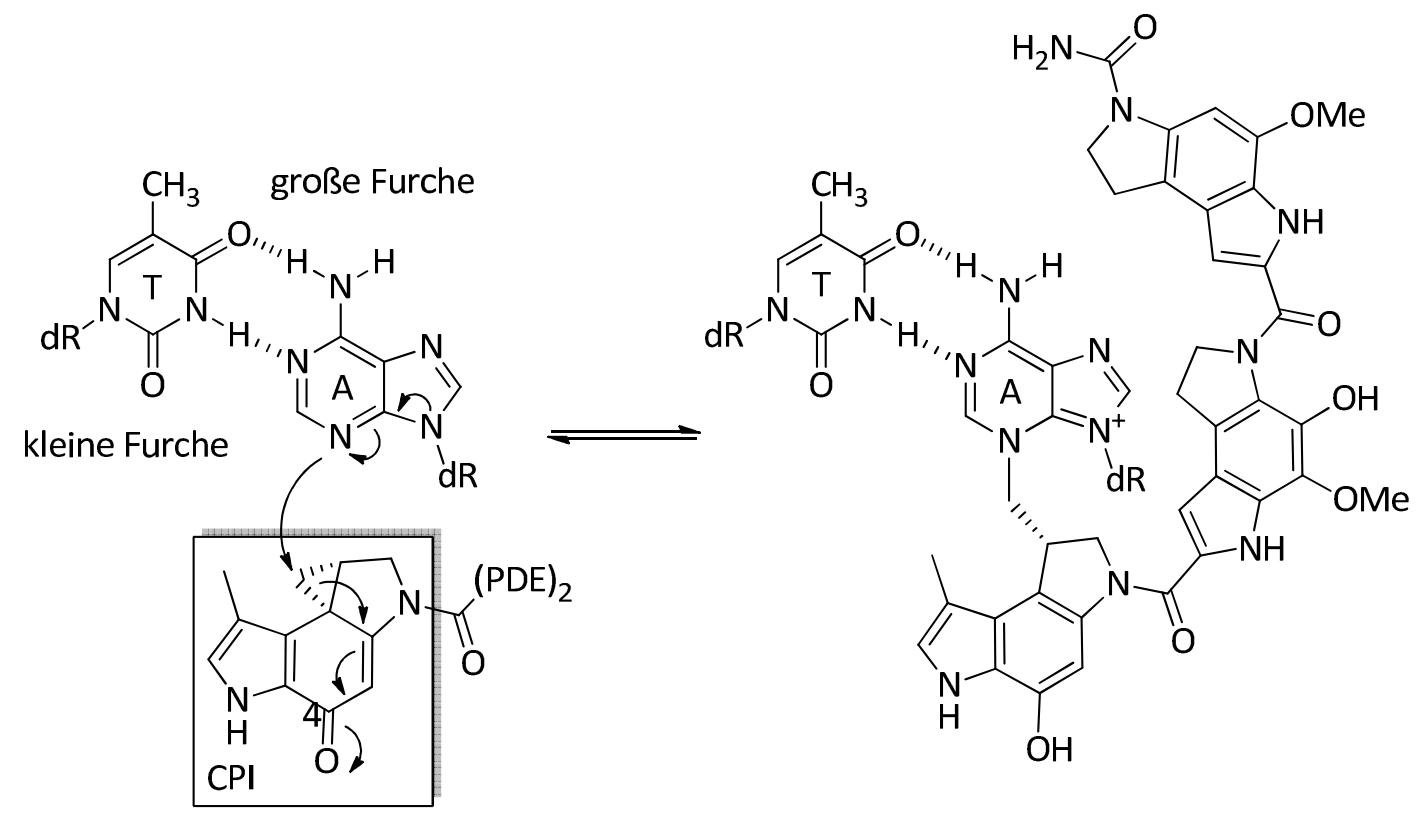

Abbildung 16. DNA-Alkylierung CC-1065 (31) durch Adenin-N-3 in der kleinen Furche.

Zu der beobachteten Alkylierungsselektivität wurden verschiedenste Hpothesen aufgestellt. Hurley vertritt das Modell einer Protonierung der Carbonylfunktion an C-4, die aus einer günstig angeordneten Phosphatgruppe im flexiblen DNA-Rückgrat resultiert, wodurch sich die Konformation der DNA ändert. Hieraus resultiert ein günstigerer Abstand des Adenin-N-3-Atoms zu dem Brückenkopfatom der CPI- 
Einheit. ${ }^{111}$ Auf Grund der Tatsache, dass Strukturanaloga auch ohne die von Hurleys Modell benötigte Carbonylfunktion an C-4 alkylierende Eigenschaften aufweisen, reicht dieses Modell zur Beschreibung des Wirkmechanismus' nicht aus. Weitere Studien haben zusätzlich gezeigt, dass die von Hurley postulierte Änderung der DNAKonformation bei der Bindung des Drugs nicht signifikant eintritt. ${ }^{112}$

Einen weitereren Ansatz beschreibt Boger als das Modell der konformativen Aktivierung. Eine besondere Bedeutung kommt hierbei der DNA-bindenden Einheit (PDE) zu, die über eine formspezifische Erkennung tief in die kleine Furche der DNA eindringt (shape-selective recognition) und dort Wasserstoffbrückenbindungen mit AT-reichen Sequenzen ausbildet. ${ }^{113}$ Eine daraus resultierende Verdrillung der Amidbindung führt zu einer Aufhebung der vinylogen Amidstabilisierung des Enons (shape-dependent catalysis) und einer Erhöhung der Reaktivität der CPI-Einheit in Hinblick auf einen Angriff des Adenin-N-3-Atoms (target-based activation). Diese von Boger postulierte These konnte durch die Untersuchung von 34 und $\mathbf{3 5}$ gestärkt werden (Abbildung 17). ${ }^{114} 34$ zeigt auf Grund des Fehlens der Amidbindung und dem damit einhergehenden Verlust der vinylogen Konjugation keinerlei DNA-alkylierende Eingenschaften. Das Indol $\mathbf{3 5}$ wurde über eine alternative Spirozyklisierung, die die vinyloge Stabilisierung ausschaltet, synthetisiert und ist 420 -fach weniger stabil sowie 500-fach weniger potent als Duocarmycin SA (32).

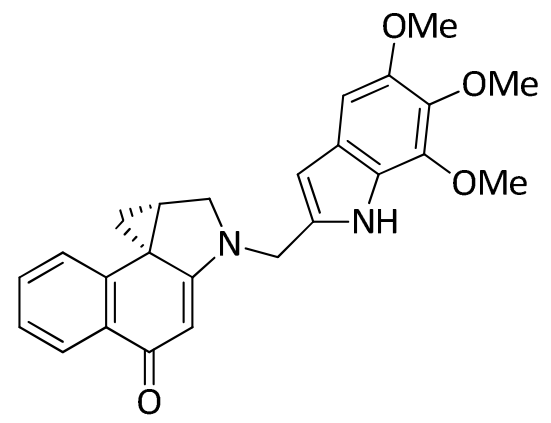

34

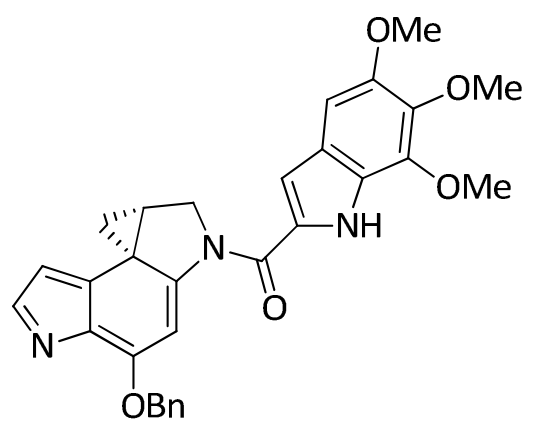

35

Abbildung 17. Verbindungen $\mathbf{3 4}$ ohne Amidfunktion und $\mathbf{3 5}$ aus einer alternativen Spirozyklisierung.

Wie neueste DFT-Berechnungen zeigen konnten, ist eine Aufhebung der vinylogen Amidstabilisierung nicht allein entscheidend für die Alkylierungsselektivität der 
beschriebenen Verbindungen. ${ }^{115}$ Untersuchungen zu Verbindungen mit unterschiedlichsten Reaktivitäten gegenüber Duplex-DNA haben eine ähnliche Verdrillung der Amidbindung gezeigt. Das Modell des Proximitätseffektes geht davon aus, dass nicht kovalente Bindungen mit den funktionellen Gruppen in der kleinen Furche der DNA für die Selektivität der Wirkstoffe verantwortlich sind. Hierdurch wird die Aktivierungsenergie im Vergleich zu einer nucleophilen Reaktion in Lösung vermindert und die Reaktivität somit erhöht. ${ }^{116}$

\subsection{Analoga von (+)-CC-1065 und (+)-Duocarmycin SA}

Obwohl sich (+)-CC-1065 (31) (IC $50=20$ pM) und (+)-Duocarmycin SA (32) $\left(\mathrm{IC}_{50}=10 \mathrm{pM}\right)$ durch enorm hohe Zytotoxizitäten auszeichnen, sind sie für eine klinische Anwendung ungeeignet. Verantwortlich hierfür sind die starken Nebenwirkungen, die sich im Fall von (+)-CC-1065 (31) bei Tierversuchen durch eine verzögerte letale Hepatoxizität gezeigt haben. ${ }^{117}$ Gleiches gilt für die Duocarmycine, die in therapeutisch wirksamen Dosen zu einer hohen Myelotoxizität geführt haben. Auf Grund dieser unerwünschten starken Nebenwirkungen wurden natürliche und synthetische Analoga der Duocarmycine untersucht, die eine vergleichbare Zytotoxizität zeigten. Bei den angestellten Untersuchungen konnten Nebenwirkungen wie die Myelo-suppression nachgewiesen werden, während das Phänomen der Hepatoxizität ausgeschlossen werden konnte. ${ }^{118}$ Auf Grund dieser Untersuchungen lässt sich festhalten, dass die beschriebene Hepatoxizität ein spezifisches Charakteristikum des Wirkstoffs (+)-CC-1065 (31), jedoch vom Wirkmechanismus unabhängig ist. ${ }^{119}$

Wie bereits erwähnt kommt, der Spirocyclopropylcyclohexadienon Struktur (Cl) eine besondere Bedeutung zu, da sie in allen natürlich vorkommenden (CPI, DA, DSA) und den synthetisierten (z.B. Cl, CBI, CBIQ, CPyI, MCBI und MCCPI) Analoga des (+)-CC1065 (31) als pharmakophore Einheit vorliegt (Abbildung 18). ${ }^{120}$ 


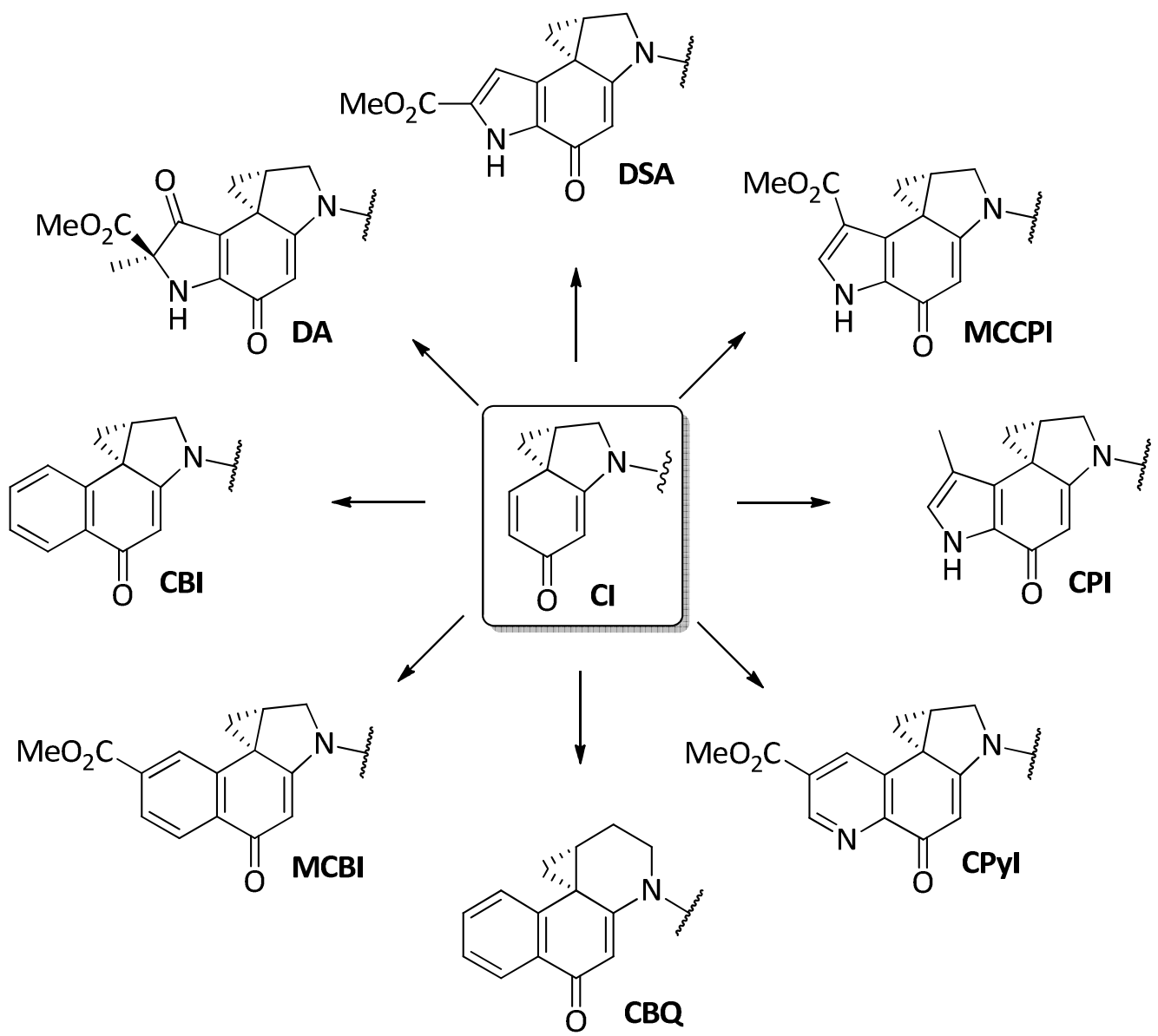

Abbildung 18. Analoga der aus Duocarmycin bekannten pharmakophoren CyclopropylindolEinheit.

Untersuchungen zur Struktur-Wirkbeziehung haben eine parabolische Beziehung zwischen der Zytotoxizität und der Solvolysestabilität der pharmakophoren Einheit gezeigt. $^{114,121}$ So sind chemisch stabile Verbindungen zytotoxischer, wobei gesagt werden muss, das eine zu hohe Stabilität gegenüber einem nucleophilen Angriff wiederum zum Abfall der Zytotoxizität führt. Eine Ausgewogenheit zwischen der beschriebenen Solvolysestabilität sowie der DNA-Alkylierung ist hierbei von entscheidender Bedeutung.

Neben der beschriebenen Variation des Pharmakophors wurde eine Variation der DNA-bindenden Einheit untersucht. ${ }^{119,122}$ Tabelle 1 zeigt die untersuchten DNAbindenden Einheiten (TMI, MMI, Ind, $(\mathrm{PDE})_{2}$, (Ind) $)_{2}$ und Boc) in Bezug auf ihre Zytotoxizität bei gleichzeitiger Variation des Pharmakophors (DSA, CPI und CBI). 


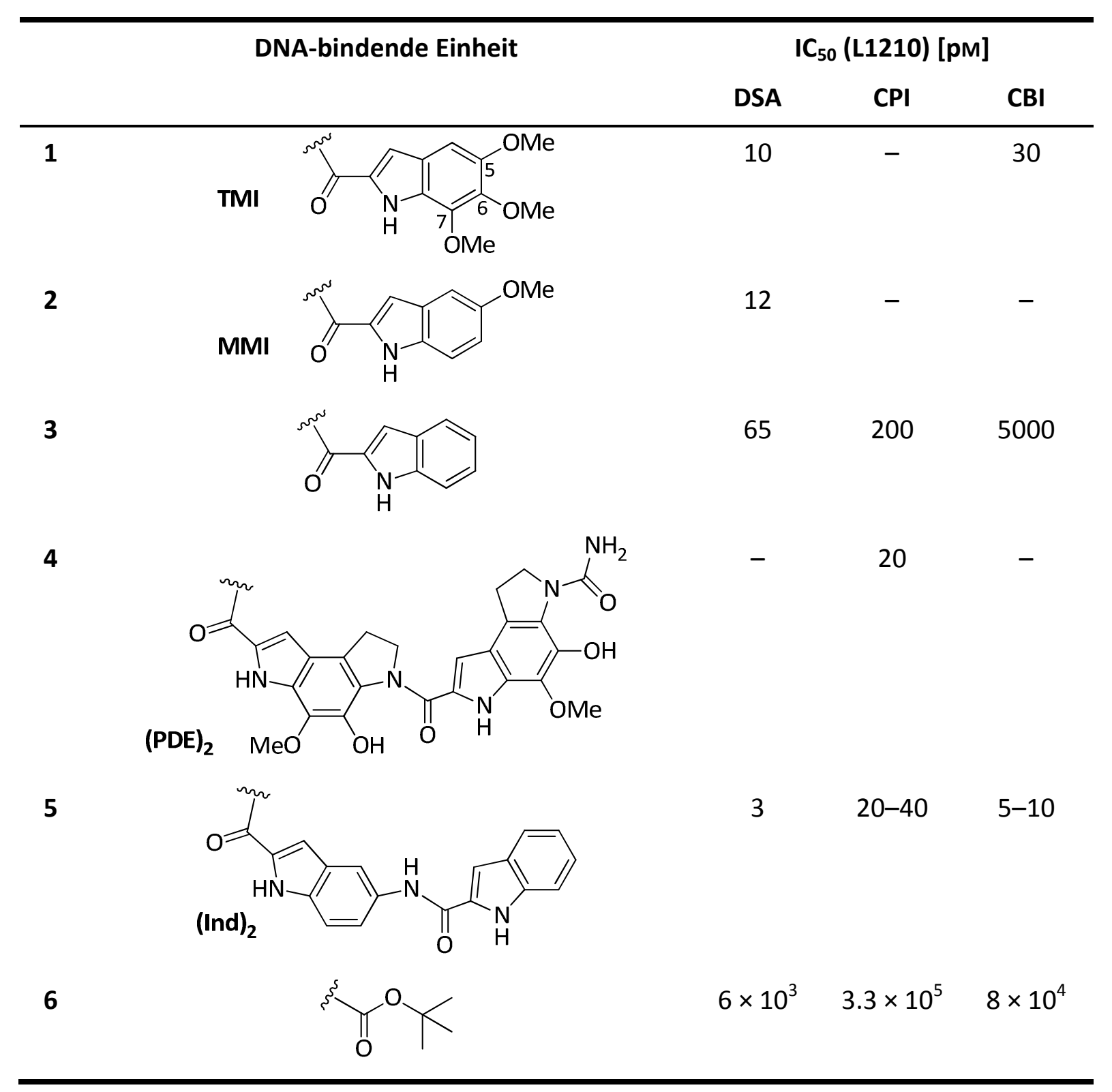

Tabelle 1. Einfluss der DNA-Binder auf die Zytotoxizität von DSA-, CPI- und CBI-Analoga.

Eine genaue Beobachtung der Ergebnisse aus Tabelle 1 zeigt, dass die Einführung eines Methoxysubstituenten in C-6- und C-7-Position des TMI nur zu einer sehr geringen Steigerung der Zytotoxizität führt, wohingegen eine Methoxygruppe an C-5 für eine hohe Zytotoxizität von entscheidender Bedeutung ist (Einträge 1-3).

Außerdem kann festgehalten werden, dass Bisindolderivate (Einträg 4 und 5) eine um den Faktor drei bis sechs höhere Toxizität aufweisen, während Verbindungen ohne DNA-bindende Einheit (Eintrag 6) 1000- bis 10000-fach weniger zytotoxisch sind. 
Sowohl der Austausch der DNA-bindenden Einheit als auch der Austausch des Pharmakophors hat einen Einfluss auf die Aktivität des Wirkstoffes. So zeigt der Austausch von CPI durch CBI eine Erhöhung der Zytotoxizität, was durch die geringere Ringspannung und die damit einhergehende höhere Stabilität erklärt werden kann (Eintrag 5). ${ }^{119 b, 123}$

Ein weiteres wichtiges Merkmal für die Einstufung der Zytotoxizität ist im Vergleich der Konfigurationen verschiedener Wirkstoffe zu finden. ${ }^{124}$ Dementsprechend zeigen die natürlichen Konfigurationen höhere (z.B. Duocarmycin A Faktor 110) oder ähnliche (z.B. CC-1065) Aktivitäten, als die entsprechenden nicht natürlichen Enantiomere (Abbildung 19).

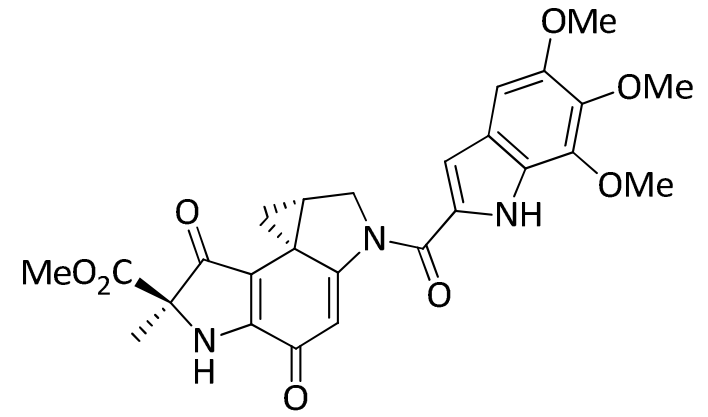

(+)-Duocarmycin A ((+)-36)

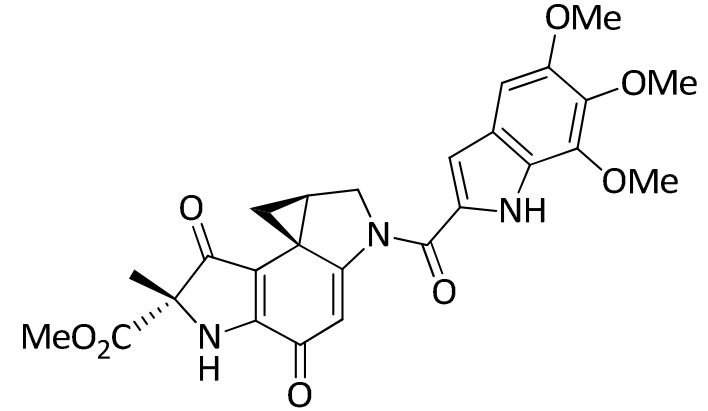

$(-)$-Duocarmycin A ((-)-36)

Abbildung 19. (+)-Duocarmycin A ((+)-36) und (-)-Duocarmycin A ((-)-36).

Während die natürlichen vorkommenden Enantiomere die Duplex-DNA durch das Adenin-N-3 am 3'-Ende einer AT-reichen Region alkyliert werden, zeigen die entsprechenden unnatürlichen Enantiomere eine Alkylierung am 3'-Ende des komplementären Strangs, da es auf Grund der andersartigen Konfiguration des Wirkstoffes zu einer entgegengesetzten Orientierung des Drugs in der AT-reichen Region kommt. ${ }^{119}$

Zu den bekanntesten Vertretern der synthetischen Duocarmycin-Analoga gehören auf Grund ihrer ausgezeichneten In-vitro-Ergebnisse Adozelesin (37), ${ }^{125}$ Carzelesin $(38),{ }^{126}$ Bizelesin $(39)^{127}$ und KW-2189 (40) ${ }^{128}$ (Abbildung 20). Obwohl bei allen Wirkstoffen eine signifikante Anti-Tumor-Response beobachtet werden konnte, mussten die Studien für alle Kandidaten mit Ausnahme von Bizelesin (39) eingestellt 
werden, da die Wirkstoffe bei Applikation therapeutischer Dosen eine ausgeprägte Myelotoxizität zeigten. ${ }^{125-128}$

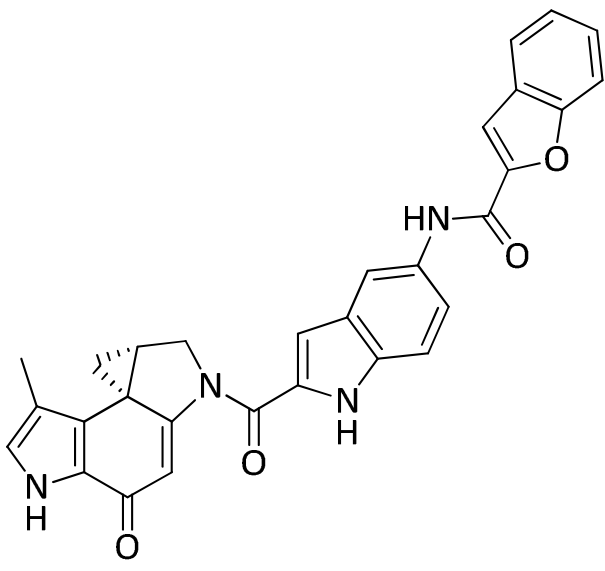

Adozelesin (37)

$\mathrm{IC}_{50}: 60 \mathrm{pM}(\mathrm{L} 1210)$
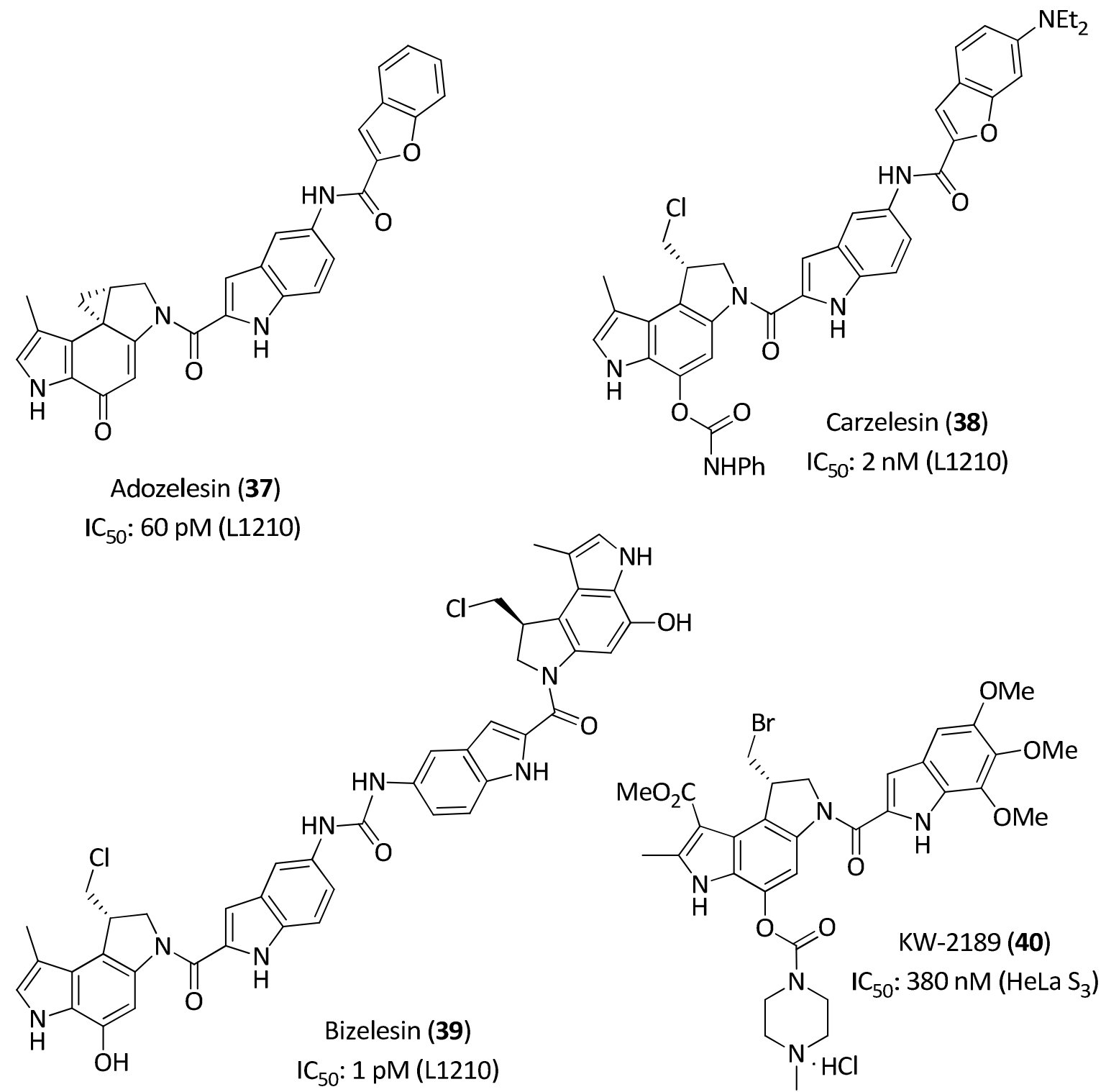

Abbildung 20. Duocarmycin-Analoga aus klinischen Phasen: Adozelesin (37), Carzelesin (38), Bizelesin (39) und KW-2189 (40).

\subsection{Seco-Verbindungen und Prodrugs einiger Duocarmycin-Analoga}

Obwohl die strukturverwandten Verbindungen 41 und $\mathbf{4 3}$ keine Spirocyclopropyleinheit aufweisen, haben sie dennoch eine ähnliche Zytotoxizität gezeigt wie CC-1065 (31) und die Duocarmycine (32 und 36). ${ }^{4 d, 119}$ Auf Grund dieser Egebnisse kann darauf geschlossen werden, dass der für die Aktivität verantwortliche Cyclopropanring in situ 
aus den seco-Verbindungen über eine Winstein-Aryl-3'-Spirozyklisierung gebildet wird (Abbildung 21). ${ }^{129}$ Im Anschluss daran erfolgt die wirkstofftypische Alkylierung der DNA durch das CBI-Drug 42. Von entscheidender Bedeutung ist die phenolische Hydroxyfunktion, da sie für die Zyklisierung unabdinglich ist und eine Blockade dieser Funktion zu einem Verlust der Toxizität führen sollte. Diese Blockade führt zur Ausbildung sogenannter detoxifizierter Prodrugs. ${ }^{130}$

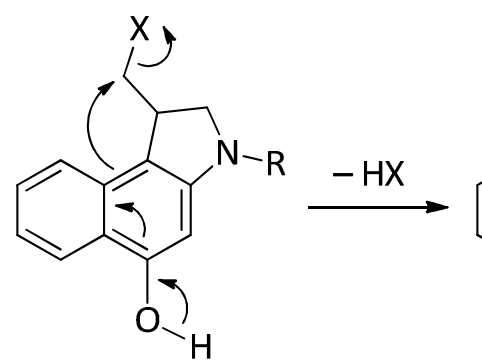

41

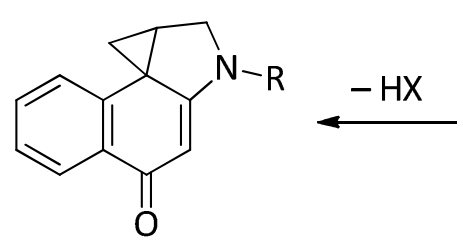

42

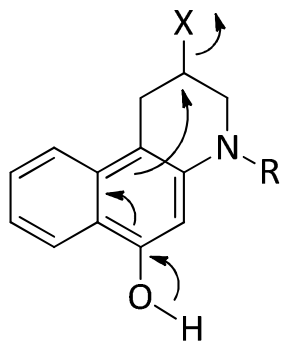

43

Abbildung 21. Winstein-Aryl-3'-Spirozyklisierung der seco-CBI-Derivate 41 und 43 unter Abspaltung von $\mathrm{HX}$.

\subsubsection{Forschungsergebnisse anderer Arbeitsgruppen}

Denny et al. konnten Analoga der Duocarmycine darstellen, die im Rahmen der GDEPTherapie angewendet wurden. Sie nutzten eine genetische Veränderung der Krebszellen durch eine Transfizierung mit einem Vektor, der dazu führt, dass das Enzym Nitroreduktase überexprimiert wurde. So können die applizierten Prodrugs selektiv im Krebsgewebe zu den enstprechenden seco-Drugs umgesetzt werden. ${ }^{131}$

Bei dem von Denny et al. verfolgten Ansatz wird ein durch Carbamat blockiertes Amino-seco-CBI-Derivat 44 in Kombination mit TMI als DNA-bindende Einheit verwendet. Nach Abspaltung der detoxifizierenden Einheit durch Nitroreduktase zu 45 und anschließender Hydrolyse wird das seco-Drug 46 gebildet, welches im Anschluss eine zum $\mathrm{CBI}$ analoge Winstein-Spirocyclisierung eingehen kann (Abbildung 22). 


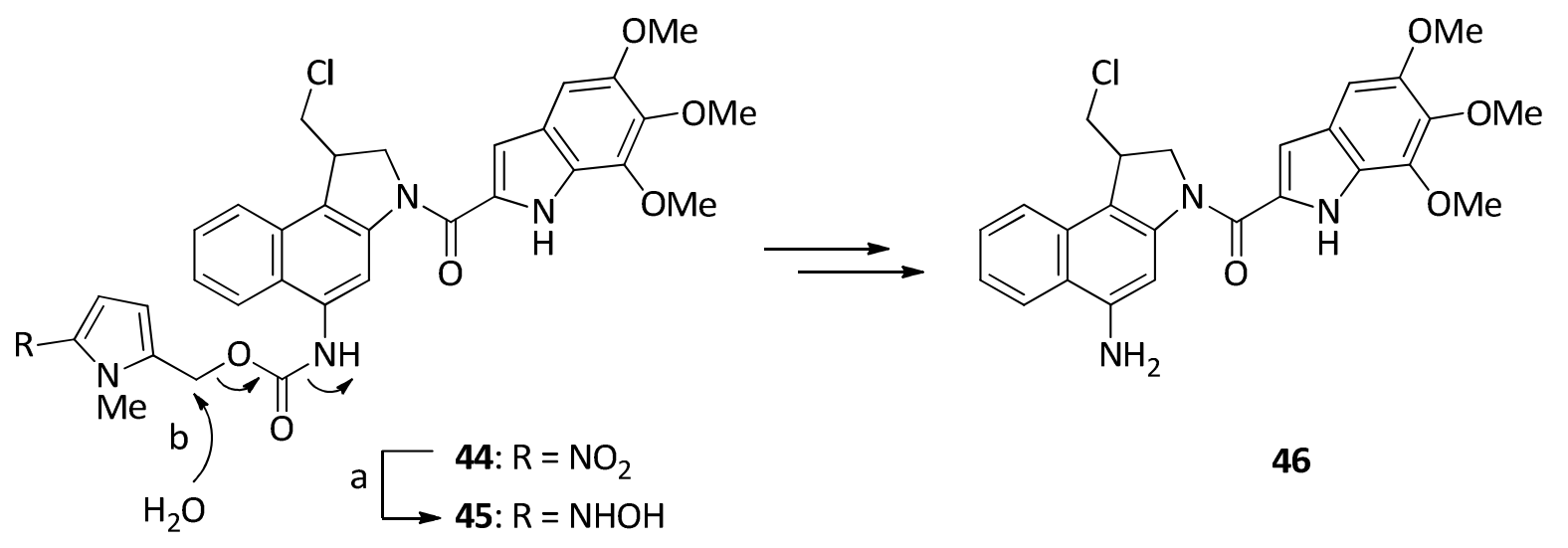

Abbildung 22. Reduktion des Prodrugs $\mathbf{4 4}$ durch Nitroreduktase (a) zu $\mathbf{4 5}$ und folgender Hydrolyse zum seco-Drug 46 (b).

Leider lieferte dieser vielversprechende Ansatz nicht die gewünschten Ergebnisse. Das Prodrug 44 zeigt bei Nitroreduktase-transfizierten Ovarialkarzinomzellen (SKOV3) nur eine etwa 20-fach höhere Zytotoxizität als bei entsprechenden nichttransfizierten Zellen. Man kann daher von einer unvollständigen Aktivierung des Prodrugs durch Nitroreduktase ausgehen, woraufhin Denny et al. neue 4-NitrobenzylCarbamat-Prodrugs entwickelten, die jedoch nur eine therapeutische Breite von bis zu 87 zeigten. $^{132}$

Wie Denny et al. synthetisierten auch Boger et al. CBI-Prodrugs, die durch eine Carbamat-Funktion blockiert sind. Im Gegensatz zu Denny nutzte Boger jedoch die phenolische $\mathrm{OH}-G r u p p e$ statt der Aminfunktion. Eine Freisetzung und damit Aktivierung der OH-Funktion sollte durch reduzierende Nucleophile erfolgen, die die schwache $\mathrm{N}$-O-Bindung spalten. ${ }^{133}$ Eine nicht ausreichende Differenzierung zwischen Prodrug und seco-Drug zeigte sich in vitro durch eine nur 37-fach stärkere zytotoxische Wirkung des entsprechenden seco-Drugs.

\subsubsection{Forschungsergebnisse aus dem Arbeitskreis Tietze}

In den letzten Jahren konnten verschiedene Analoga des Duocarmycins und CC-1065 synthetisiert und auf ihre biologische Aktivität hin untersucht werden., ${ }^{434,135}$ Dabei haben sich eine Anwendung in Bereichen des ADEPT- sowie des PMT-Konzeptes 
herauskristallisiert. Im Gegensatz zu vielen anderen Ansätzen werden in diesen Fällen Glykoside als detoxifizierende Einheit genutzt. Prodrugs wie 47a-g und 48-50 können im Rahmen von ADEPT durch Antikörper-Glycohydrolase-Konjugate sowie im Rahmen von PMT zum Beispiel durch tumorassoziierte $\beta$-D-Glucuronidase zu den entsprechenden seco-Drugs 51-54 umgesetzt werden, die im Anschluss in situ die bereits beschriebene Winstein-Zyklisierung zu 55-58 eingehen (Abbildung 23).

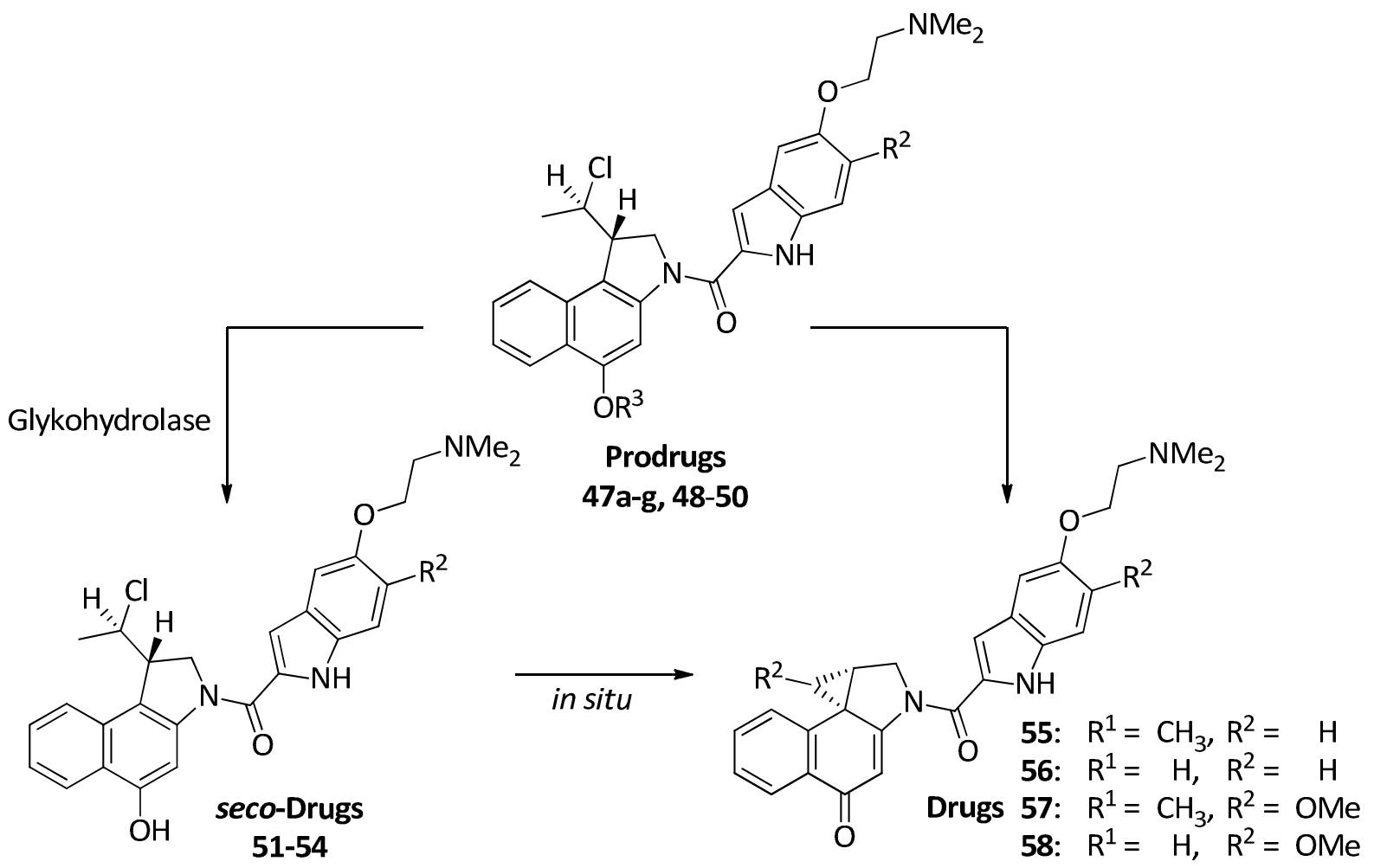

Abbildung 23. Glykosidische Prodrugs als Analoga des zytotoxischen Duocarmycin (32) aus dem Arbeitskreis Tietze.

Im Mittelpunkt der Forschung der Arbeitsgruppe Tietze steht eine hohe therapeutische Breite mit $\mathrm{QIC}_{50}>1000$ sowie eine hohe biologische Aktivität der entsprechenden seco-Drugs $\left(\mathrm{IC}_{50}<10 \mathrm{nM}\right)$. Neueste Untersuchungen erfolgten an CBI- und anti-Methyl-CBI als Pharmakophor sowie verschiedenen DNA-bindenden Einheiten in Kombination mit einer Variation von verschiedenen Glykosiden (Abbildung 23).

Unterschieden werden kann hier vor allem zwischen $\mathrm{CBI}$ - und anti-methy-CBIDerivaten, da die Einführung einer Methylgruppe die sterische Hinderung am 
Spirocyclopropylsystem erhöht und damit eine geringere Zytotoxizität der entsprechenden Verbindungen begründet.

Tabelle 2 zeigt die ermittelten In-vitro-Zytotoxizitäten ausgewählter Prodrugs und seco-Drugs an der humanen Bronchialkarzinomzellinie A549.

\begin{tabular}{|c|c|c|c|c|c|c|}
\hline & $\mathbf{R}^{1}$ & $\mathbf{R}^{2}$ & $\mathbf{R}^{3}$ & $I C_{50}$ O.E. $[\mathrm{nM}]^{[\mathrm{a}]}$ & $\begin{array}{c}\mathrm{IC}_{50} \mathrm{~m} . \mathrm{E} . \\
{[\mathrm{nM}]^{[\mathrm{a}]}}\end{array}$ & $\mathrm{QIC}_{50}{ }^{[\mathrm{b}]}$ \\
\hline $47 a$ & $\mathrm{CH}_{3}$ & $\mathrm{H}$ & $\beta$-D-Galaktosyl & 3600 & 0.75 & 4800 \\
\hline $47 b$ & $\mathrm{CH}_{3}$ & $\mathrm{H}$ & $\alpha$-D-Mannosyl & 2700 & 0.60 & 4500 \\
\hline $47 c$ & $\mathrm{CH}_{3}$ & $\mathrm{H}$ & $\beta$-D-Glucosyl & 2000 & 1.90 & 1050 \\
\hline $47 d$ & $\mathrm{CH}_{3}$ & $\mathrm{H}$ & $\beta$-D-Cellobiosyl & 2400 & 2.60 & 920 \\
\hline $47 e$ & $\mathrm{CH}_{3}$ & $\mathrm{H}$ & $\beta$-D-Lactosyl & 2700 & 2.10 & 1290 \\
\hline $47 f$ & $\mathrm{CH}_{3}$ & $\mathrm{H}$ & $\beta$-D-Glucuronyl & 610 & 0.90 & 700 \\
\hline $47 g$ & $\mathrm{CH}_{3}$ & $\mathrm{H}$ & $\begin{array}{l}\beta \text {-D-Methyl- } \\
\text { glucuronyl }\end{array}$ & 3300 & 2.10 & 1600 \\
\hline 48 & $\mathrm{H}$ & $\mathrm{H}$ & $\beta$-D-Galaktosyl & 56.0 & 0.016 & 3500 \\
\hline 49 & $\mathrm{CH}_{3}$ & OMe & $\beta$-D-Galaktosyl & 940 & 0.22 & 4300 \\
\hline 50 & $\mathrm{H}$ & OMe & $\beta$-D-Galaktosyl & 9.5 & 0.005 & 2100 \\
\hline \multicolumn{7}{|c|}{ SECO-DRUGS } \\
\hline 51 & $\mathrm{CH}_{3}$ & $\mathrm{H}$ & $\mathrm{H}$ & - & 0.75 & - \\
\hline 52 & $\mathrm{H}$ & $\mathrm{H}$ & $\mathrm{H}$ & - & 0.026 & - \\
\hline 53 & $\mathrm{CH}_{3}$ & OMe & $\mathrm{H}$ & - & 0.20 & - \\
\hline 54 & $\mathrm{H}$ & OMe & $\mathrm{H}$ & - & 0.009 & - \\
\hline
\end{tabular}

Tabelle 2.

Ergebnisse der In-vitro-Zytotoxizitätstests der glykosidischen Prodrugs (47a-g und 48-50) und seco-Drugs (51-54). [a] Bestimmung der $\mathrm{IC}_{50}$-Werte mittels Klonogenitätsassays an der Lungenkrebszellinie A549; [b] QIC $\mathrm{C}_{50}=\mathrm{IC}_{50}$ des Prodrugs $/ \mathrm{IC}_{50}$ des Prodrugs in Anwesenheit des spaltenden Enzyms. o.E. $=$ ohne Enzym, m.E. $=$ mit Enzym.

Auffällig sind hierbei vor allem die direkten Einflüsse der DNA-bindenden Einheit sowie des Glykosides auf die Zytotoxizität der seco-Drugs und die therapeutische Breite der Prodrugs. Bei der Betrachtung der DNA-bindenden Einheit fällt auf, dass die besten Ergebnisse mit $\operatorname{DMAl}\left(R^{2}=H\right)$ sowie DMMI $\left(R^{2}=O M e\right)$ erzielt werden 
konnte. Ein entscheidender Vorteil der genannten DNA-Binder liegt in der Möglichkeit durch Anwesenheit eines tertiären Amins eine Salzbildung zu formulieren, die die Wasserlöslichkeit der Verbindung erhöht.

Vergleicht man das anti-Methyl-seco-CBI-DMAI- $\beta$-D-Galaktosid 47a und das seco-CBIDMAI- $\beta$-D-Galaktosid 48, fällt auf, dass beide einen hervorragenden $\mathrm{QIC}_{50}$-Wert liefern (47a: 4800, 48: 3500), wobei das zu 48 entsprechende seco-Drug 52 mit einem $\mathrm{IC}_{50}$-Wert von $26 \mathrm{pM}$ den des anti-Methyl-seco-Drugs 51 um den Faktor 29 übersteigt. $^{136}$

Des Weiteren wurden Derivate synthetisiert, bei denen die Variation des Glykosides im Vordergrund stand. Hierdurch konnten sehr gute Resultate mit dem $\alpha$-D-Mannosid 47b ( $\mathrm{QIC}_{50}$ von 4500) erzielt werden, während Disaccharid-Prodrugs (47d und 47e) auf Grund einer unvollständigen enzymatischen Aktivierung nur moderate bis gute $\mathrm{QIC}_{50}$-Werte lieferten. Für den Einsatz in einer Prodrug-Mono-Therapie (PMT) wurden das Glucuronid 47f $\left(\mathrm{QIC}_{50}=700\right)$ und dessen Methylester 47g $\left(\mathrm{QIC}_{50}=1600\right)$ entwickelt. $^{137}$

Zur Aufklärung des Wirkmechanismus' der im Arbeitskreis Tietze synthetisierten Analoga des Duocarmycin wurden ESI-FTICR-MS-Experimente (electrospray ionization fourier transform ion cyclotron resonance mass spectrometry), CD-Spektroskopie sowie HPLC-Untersuchen durchgeführt. ${ }^{138}$

Hierbei wurde bestätigt, dass die seco-Drugs 51 und 52 als Analoga der Duocarmycine sowie die daraus resultierenden Drugs 55 und 56 AT-reiche Regionen in synthetischen Doppelstrang-DNA-Oligonucleotiden selektiv alkylieren. Ebenfalls konnte gezeigt werden, dass Glutathion (Modellpeptid), RNA-Oligonucleotide sowie Einzelstrang-DNA kein Ziel für die Duocarmycine darstellt.

Auffällig war vor allem der Unterschied in der Alkylierungseffizienz von Duplex-DNA durch Methyl-CBI 55 und CBI 56. So weist das bioaktivere 56 eine deutlich geringere Alkylierungseffizienz auf als 55, was darauf schließen lässt, dass Zytotoxizität und DNA-Alkylierungseffizienz nicht in direktem Zusammenhang stehen.

Es konnte gezeigt werden, dass das akitve CBI-Drug 56 langsamer aus 52 gebildet wird als das entsprechende Methyl-CBI-Drug 55 aus 51. Begründet wird dies mit einer 
Stabilisierung des seco-CBI-Drugs 52 durch nicht kovalente Wechselwirkungen mit der DNA, was eine verzögerte Zyklisierung und eine damit einhergehende geringere Alkylierungseffizienz zur Folge hat. Somit kann eine direkte Korrelation zwischen der Zytotoxizität und der Stabilisierung des seco-Drugs bestätigt werden.

Die höhere Zytotoxizität kann durch eine „Überstabilisierung“ des Doppelstranges und einer daraus resultierenden Verhinderung von Reparaturmechanismen, Replikation und Transkription erklärt werden.

Die beobachtete Selektivität der synthetisierten Analoga der Duocarmycine ist in erster Linie durch Proximitätseffekte zu erklären. So können die dargestellten seco-Drugs besonders gut in AT-reiche Regionen der kleinen Furche einlagern, wobei die Alkylierung der DNA lediglich einer irreversiblen Fixierung dient. ${ }^{138 e}$

Neueste Untersuchungen zu bifunktionalen Prodrugs vom Typ 59 zeigen extrem hohe QIC $_{50}$-Werte von bis zu einer Million und eine sehr hohe Zytotoxizität der seco-Drugs bis in den femtomolaren Bereich (Abbildung 24). ${ }^{139}$ Diese extrem hohen Zytotoxizitäten wurden zunächst mit DNA-Inter- oder Intrastrangvernetzung begründet. ${ }^{140}$ Untersuchungen der seco-Drugs durch ESI-FTICR-MS- und CD-Spektroskopie konnten allerdings keine Alkylierung der DNA nachweisen. ABPP-Studien (activity based protein profiling) konnten den Wirkmechanismus aufklären und zeigten das in Krebszellen überexprimierte Protein ADH-A1 als spezifisches Target.

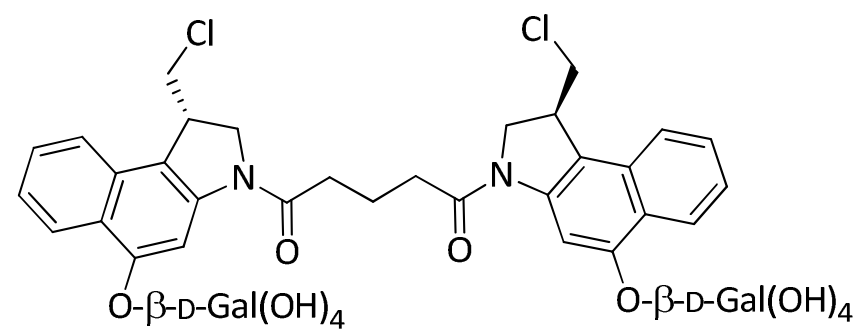

59

$$
\begin{aligned}
\mathrm{IC}_{50} \text { o.E. } & =146 \mathrm{nM} \quad(\mathrm{A} 549) \\
\mathrm{IC}_{50} \text { m.E. } & =0.15 \mathrm{pM}(\mathrm{A} 549) \\
\mathrm{QIC}_{50} & =970000
\end{aligned}
$$

Abbildung 24. Bifunktionales Galaktosyl-Prodrug 59 mit einem QIC50-Wert von 970000. 


\section{Confocal Laser Scanning Microscopy (CLSM) \& Optical Imaging}

\subsection{Confocal Laser Scanning Microscopy (CLSM)}

Das seit mehreren hundert Jahren bekannte Phänomen der Fluoreszenz konnte vor allem in den letzten Jahrzehnten auf Grund der Weiterentwicklung von Lasern und hochsensitiven Sensoren zur Aufklärung von Wirkmechanismen beitragen. Neben der konventionellen Epifluoreszenzmikroskopie erfreut sich die Confocal Laser Scanning Microscopy (CLSM) steigender Beliebtheit. Fluoreszenzmikroskopie im Allgemeinen nutzt die Eigenschaft organischer Moleküle (Fluorochrom), mit Licht bestimmter Wellenlänge in einen angeregten Zustand angehoben zu werden. ${ }^{141}$ Beim Relaxieren aus eben diesem angeregtem Zustand wird unter Abgabe eines Lichtquants der Grundzustand erreicht.

So können von Natur aus fluoreszierende (autofluoreszierende) Verbindungen (z.B. GFP und Chlorophylle ${ }^{142}$ ), synthetisch hergestellte Konjugate (z.B. FITC-Derivate 60, Dapoxyl-Derivate 61, Cumarin-Derivate 62 und Benzothiazol-Derivate 63) oder durch Gentransfektion mit einem Fluorochrom markierte Verbindungen ${ }^{143}$ in Zellen sichtbar gemacht werden, um die Aufnahme der Substanz in vitro an lebenden Zellen zu untersuchen.

Eine genaue Aussage der Zuordnung bestimmter Zellregionen kann erst nach einer erfolgten Colokalisation erfolgen. Hierbei wird die bereits mit der fluoreszierenden Substanz behandelte Zelle zusätzlich mit sogenannten Markern behandelt. Auf Grund der bereits gemachten Untersuchungen kann eine Auswahl an zelltypischen Markern erstellt werden. Häufig werden Zellkernmarker (z.B. Hoechst 33342 64) ${ }^{144}$ und Mitochondrienmarker (z.B. MitoTracker Deep Red 633 FM 65) ${ }^{145,146}$ verwendet (Abbildung 25).

Die Wahl des verwendeten Fluorochroms gestaltet sich hierbei allerdings deutlich schwieriger. Hierbei müssen zum Einen die Eigenschaften der Fluoreszenz (Anregungs- und Emissionswellenlänge sowie Quantenausbeute) und zum Anderen 
die chemi-schen Eigenschaften im Hinblick auf Ladung und Hydrophilität beachtet werden. Ebenso wichtig ist eine Anpassung des Markers an den verwendeten Fluorochrom. Hierbei muss eine Überschneidung der Anregungs- bzw. Emissionswellenlängen vermieden werden, da sonst keine klare Aussage über die Aufnahme der fluoreszenzmarkierten Substanz gemacht werden kann. Auf Grund der Vielzahl der heute erhältlichen Fluorochrom ist eine derartige Unterscheidung häufig zu realisieren. ${ }^{147}$<smiles>O=C(O)c1cc(N=C=S)ccc1-c1c2ccc(=O)cc-2oc2cc(O)ccc12</smiles>

FITC (60)

$$
\lambda_{\text {max, Abs. }}=490 \mathrm{~nm}
$$$$
\lambda_{\text {max }, \mathrm{Em} .}=520 \mathrm{~nm}
$$<smiles>CN(C)c1ccc2oc(=O)cc(CC(=O)O)c2c1</smiles>

DMACA (62)

$\lambda_{\text {max }, \text { Abs. }}=346 \mathrm{~nm}$

$\lambda_{\text {max, } \mathrm{Em} .}=460 \mathrm{~nm}$

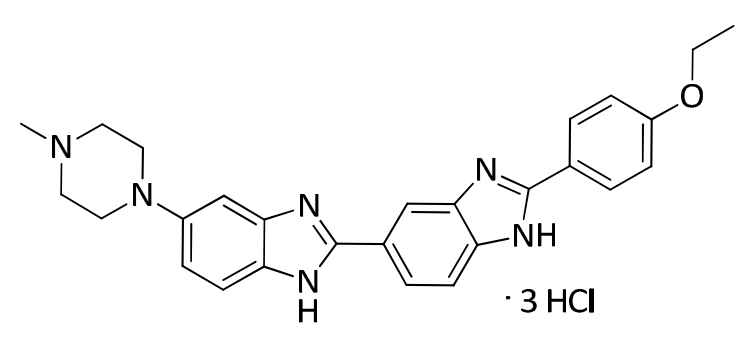

Hoechst 33342 (64)

$\lambda_{\text {max }, \text { Abs. }}=346 \mathrm{~nm}$

$\lambda_{\text {max }, \mathrm{Em} .}=460 \mathrm{~nm}$<smiles>CN(C)c1ccc(-c2cnc(-c3ccc(S(=O)(=O)NCCC(=O)ON4C(=O)CCC4=O)cc3)o2)cc1</smiles>

D10162 (61)

$$
\begin{aligned}
& \lambda_{\text {max Abs. }}=373 \mathrm{~nm} \\
& \lambda_{\text {max, Em. }}=574 \mathrm{~nm}
\end{aligned}
$$<smiles>CN1/C(=C\c2cc[n+](C(F)(F)CC(C)(C)O)cc2)Sc2ccccc21</smiles>

$$
\begin{gathered}
\mathrm{BO}(63) \\
\lambda_{\text {max }, \mathrm{Abs} .}=346 \mathrm{~nm} \\
\lambda_{\text {max, Em. }}=460 \mathrm{~nm}
\end{gathered}
$$<smiles>C[N+]1=C(C=CC=C2N(Cc3ccc(CCl)cc3)c3ccccc3C2(C)C)C(C)(C)c2ccccc21</smiles>

MitoTracker Deep Red 633 FM (65)

$$
\begin{aligned}
& \lambda_{\text {max }, \text { Abs. }}=644 \mathrm{~nm} \\
& \lambda_{\text {max }, \text { Em. }}=665 \mathrm{~nm}
\end{aligned}
$$

Abbildung 25. Die Fluoreszenzfarbstoffe FITC (60), D10162 (61),DMACA (62) und BO (63) sowie die Colokalisationsfarbstoffe Hoechst 33342 (64) zur Zellkernfärbung und der Mitochondrienfarbstoff MitoTracker ${ }^{\circledR}$ Deep Red 633 FM (63). 
Untersuchungen zur Bindungskinetik von fluoreszenzmarkierten Antikörper- $\beta$-DGalaktosidase-Konjugaten konnten im Arbeitskreis von F. Alves erfolgreich durchgeführt werden. So konnte das FITC-markierte anti-uPAR- $\beta$-D-GalaktosidaseKonjugat die Theorie des ADEPT-Konzeptes in vitro bestätigen. ${ }^{148}$

Das applizierte Konjugat bindet nach $0.5 \mathrm{~h}$ spezifisch an das in Mammakarzinomzellen (MDA-MB-231) überexprimierte Antigen UPAR und erreicht nach 65 Stunden eine stabile Bindung (Abbildung 26). Da eine Internalisierung des Konjugates nicht beobachtet wurde und die behandelten Zellen bis zu einer Inkubationszeit von $65 \mathrm{~h}$ vital blieben, konnte bestätigt werden, das sich das anti-uPAR- $\beta$-D-GalaktosidaseKonjugat für einen Einsatz im Rahmen des ADEPT-Konzeptes für In-vitroUntersuchungen sehr gut eignet.

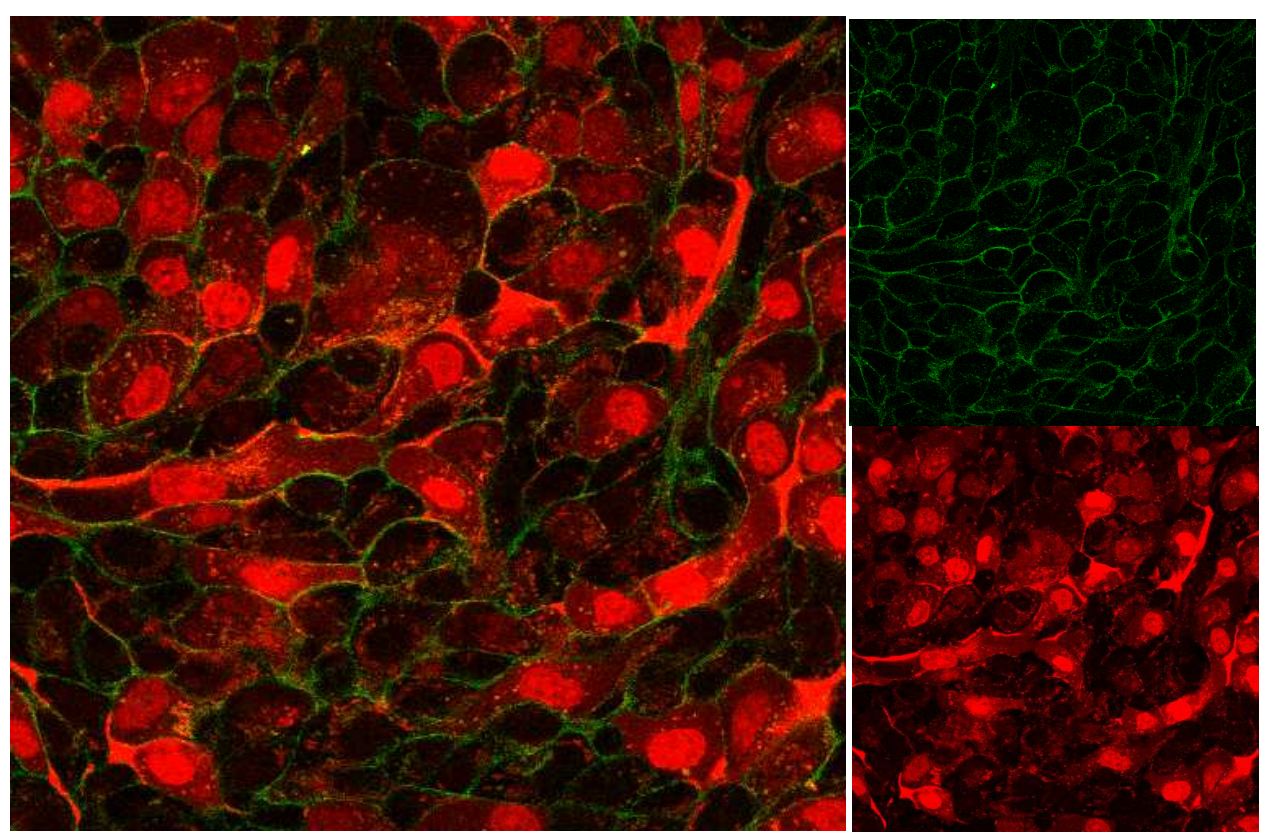

Abbildung 26. Fluoreszenzmikroskopische In-vitro-Untersuchung zur Bindung des FITC-markierten anti-uPAR- $\beta$-D-Galaktosidase-Konjugates (grün) an die Zellmembranen von MDA-MB-231-Zellen. Die DNA der Zellkerne und die RNA im Zytosol wurden mit Syto 61 (rot) angefärbt.

\subsection{Optical Imaging}

Um die in vitro durch CLSM gemachten Untersuchungen auch in vivo zu bestätigen, kann das Verfahren des Optical Imaging eingesetzt werden, um die applizierte 
fluoreszenzmarkierte Verbindung so zu untersuchen, dass neue Einblicke über den Wirkort und -mechanismus in lebenden Zellen, Geweben und Organismen erhalten werden. ${ }^{149,150,151}$

Eine Auswahl des Fluorochroms ist auch hier von entscheidender Bedeutung, da das Gewebe des Tieres ein besonderes Hindernis darstellt. Bis zu einer Absorptionswellenlänge von $\lambda_{\text {max,Abs. }}=650 \mathrm{~nm}$ kommt es zu einer starken Absorption des Laserstrahls durch das Gewebe und zu einer unerwünschten Autofluoreszenz. ${ }^{152}$ Die angesprochenen Limitierungen der Fluorochrome können durch die richtige Wahl der Absorptions- und Emissionswellenlängen im roten bzw. infraroten Bereich (650$900 \mathrm{~nm}$ ) ausgeschlossen werden. Häufig eingesetzte Fluorochrome sind Derivate von Cy5.5 (66), das mit $\lambda_{\text {max,Abs. }}=675 \mathrm{~nm}$ und $\lambda_{\text {max,Em. }}=694 \mathrm{~nm}$ die notwendigen Eigenschaften mitbringt (Abbildung 27).

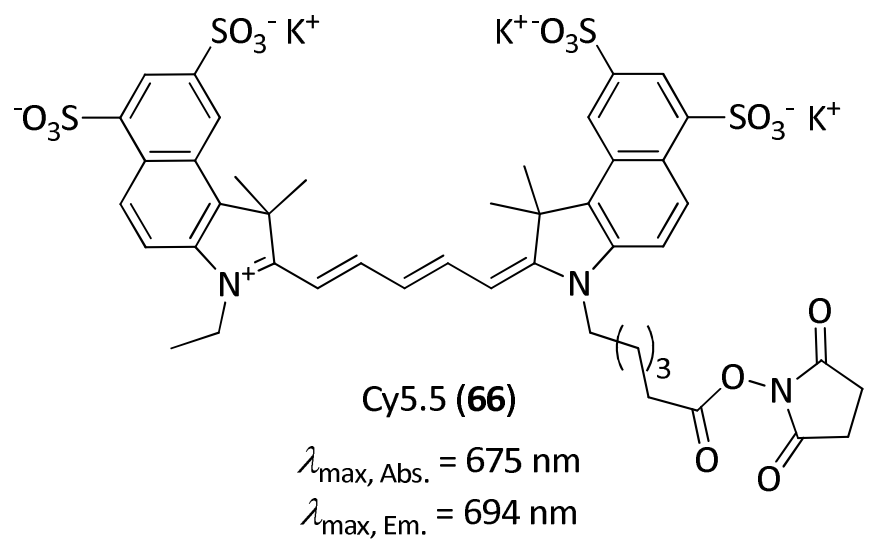

Abbildung 27. Der Fluoreszenzfarbstoff Cy5.5 (66) für Anwendungen im Optical Imaging.

Neben der angesprochenen Möglichkeit, Fluoreszenz als bildgebende Eigenschaft der zu untersuchenden Substanzen zu nutzen, kann auch das Phänomen der Lumineszenz genutzt werden. Beim Bioluminescence Imaging wird ein spezielles Luziferin-Substrat enzymatisch durch das lichtgenerierende Enzym Luziferase aktiviert. Als Vorteil dieser Messmethode gilt die hohe Spezifität (schwaches Hintergrundsignal), während die geringe Intensität und die damit verbundene lange Messzeit als Nachteil angesehen werden können. Nach der erfolgten Transfizierung eines Gens des Luziferase-Enzyms in das Tumorgewebe kann eine Konjugation der Farbstoffe mit kleinen Molekülen, 
Antikörpern oder AEC genutzt werden, um neue Erkenntnisse in Bezug die Wirkweise von Antikörper-Enzym-Konjugaten und Prodrugs des ADEPT-Konzeptes zu erhalten.

Durch Anwendung dieser Optical-Imaging-Methode konnten Alves et al. Cy5.5markierte Antikörper-Enzym-Konjugate in vivo untersuchen. ${ }^{148}$

Wie in Abbildung $28 \mathrm{zu}$ sehen ist, konnten nach der Applikation des AEC starke Fluoreszenzsignale im Tumorbereich (rechte Brustdrüse) gemessen werden (B-J), während die gemessen Fluoreszenze im Darmbereich (A) als Hintergrundfluoreszenz betrachtet werden kann. Da die Messungen gezeigt haben, dass eine Bindung des AEC nach 3-7 Tagen maximal ist und bis zum 11. Tag wieder abnimmt, wird eine Gabe des Prodrugs 7 Tage nach der Applikation des AEC empfohlen.
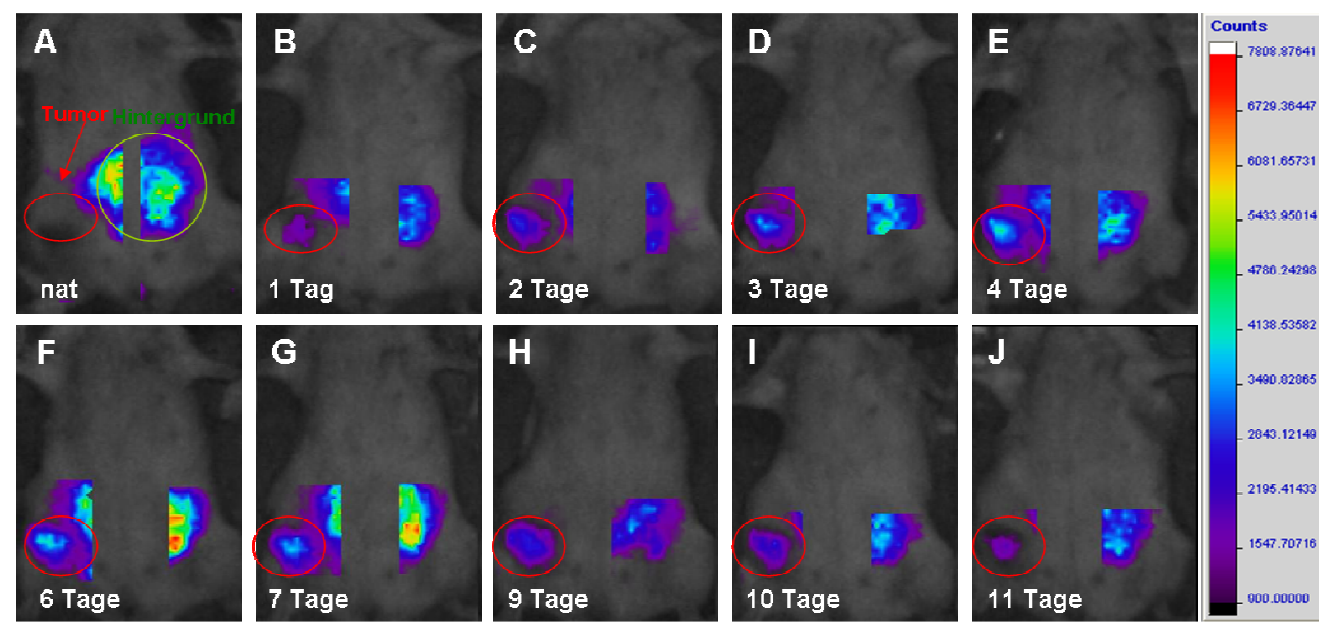

Abbildung 28. Zeitabhängige Bindung des Cy5.5-markierten anti-uPAR- $\beta$-D-GalaktosidaseKonjugates an MDA-MB-231-Tumorzellen in SCID-Mäusen. A: nativer Scan vor der Verabreichung des Cy5.5-markierten anti-uPAR- $\beta$-D-Galaktosidase-Konjugates, B-J: Scans zu den angegebenen Zeitpunkten nach Applikation des anti-uPAR- $\beta$-DGalaktosidase-Konjugats 


\section{Zielsetzung der Arbeit}

Wie in den vorangegangenen Kapiteln bereits erläutert, stellt eine selektive Behandlung maligner Zellen mit nur geringer Beeinträchtigung gesunder Zellen eine der größten Herausforderungen für die moderne Wirkstoffforschung dar. So werden Wirkstoffe, die auf das Merkmal der schnelleren Proliferation von Tumorzellen zurückgreifen, häufig nicht mehr weiter entwickelt, sondern eine Anwendung dieser Zytostatika z.B. im Rahmen der Konzepte ADEPT und PMT vorangetrieben. Hierdurch wird vor allem versucht, die starken Nebenwirkungen zu unterbinden, die häufig einen Abbruch der Therapie erforderlich machen.

Im Rahmen von ADEPT (Antibody-Directed Prodrug Therapy) und PMT (Prodrug Mono Therapy) können relativ untoxische Substanzen (sogenannte Prodrugs) selektiv im Krebsgewebe in den eigentlichen Wirkstoff und damit das toxische Drug überführt werden. In diesem Zusammenhang ist eine Erwähnung der therapeutischen Breite von besonderer Bedeutung. So kann zwischen der detoxifizierten Variante des Drugs und dem daraus resultierenden toxischen Drug unterschieden werden. Der hierfür gebräuchliche Index wird als $\mathrm{QIC}_{50}$ beschrieben und ist der Quotient aus dem $I C_{50}$ in Abwesenheit des spaltenden Enzyms und dem $\mathrm{IC}_{50}$ in Anwesenheit des Enzyms definiert. Auf Grund dieser Möglichkeit zur Klassifizierung wird ein Wirkstoff mit einem $\mathrm{QIC}_{50}>1000$ für das Prodrug (therapeutische Breite) und einem $\mathrm{IC}_{50}<10 \mathrm{nM}$ des resultierenden Drugs (systemische Toxizität) als möglicher Kandidat für eine Anwendung im Rahmen der Konzepte ADEPT und PMT betrachtet. ${ }^{4,52,95}$ Während bei der Anwendung des ADEPT-Konzeptes Antikörper-Enzym-Konjugate (AEC) genutzt werden, um die „detoxifizierten“ Prodrugs selektiv im Tumorgewebe zu den entsprechenden Drugs umzusetzen, werden im Rahmen der Prodrug-Mono-Therapie tumorspezifische Eigenschaften, wie z.B. ein erniedrigter pH-Wert oder überexprimierte Enzyme wie $\beta$-D-Glucuronidase genutzt, um das Prodrug in das Drug zu überführen. 
Im Arbeitskreis Tietze konnten in den letzten Jahren hervorragende Ergebnisse mit glykosidierten seco-CBI- und anti-Methyl-seco-CBI-Analoga (1S)-48 und (1S,10R)-47a (Abbildung 29) des zytotoxischen Duocarmycins (32) erreicht werden.<smiles>[R][R1](Cl)C1(C([R])Cl)CN(C(=O)c2cc3cc(OCCN(C)C)ccc3[nH]2)c2cc(O[R6]([H])([H])[H])c3cc([R])c([R])cc3c21</smiles>

$$
\begin{array}{llll}
\mathrm{R}_{1}=\mathrm{CH}_{3} & \mathrm{R}_{2}=\mathrm{H} & \mathrm{R}_{3}=\mathrm{H} & \mathbf{4 7 a} \\
\mathrm{R}_{1}=\mathrm{H} & \mathrm{R}_{2}=\mathrm{H} & \mathrm{R}_{3}=\mathrm{H} & \mathbf{4 8} \\
\mathrm{R}_{1}=\mathrm{CH}_{3} & \mathrm{R}_{2}=\mathrm{CN} & \mathrm{R}_{3}=\mathrm{H} & \mathbf{6 7} \\
\mathrm{R}_{1}=\mathrm{H} & \mathrm{R}_{2}=\mathrm{H} & \mathrm{R}_{3}=\mathrm{CN} & \mathbf{6 8}
\end{array}
$$

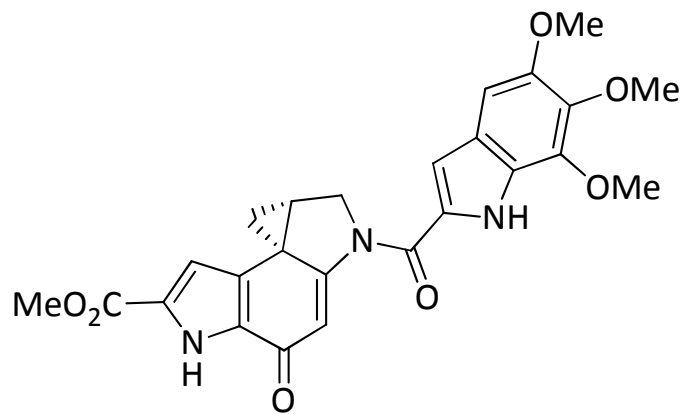

(+)-Duocarmycin SA (32) $\mathrm{IC}_{50}: 10 \mathrm{pM}(\mathrm{L} 1210)$

Abbildung 29. Im Arbeitskreis Tietze entwickelte seco-CBI-Analoga 48 und $\mathbf{6 8}$ sowie die antiMethyl-seco-CBI-Analoga $47 a$ und 67 des zytotoxischen Antibiotikums (+)-Duocarmycin SA (32).

Ziel der Arbeit war es, neue fluoreszenzmarkierte seco-CBI- sowie anti-Methyl-secoCBI-Analoga für Untersuchungen im Rahmen von Live Cell Imaging darzustellen, um weitere Aufschlüsse über den Wirkort der Duocarmycin-Analoga innerhalb des ADEPT-Konzeptes zu erhalten.

In Anlehnung an Arbeiten von F. Major ${ }^{5 \mathrm{C}}$ und B. Krewer ${ }^{5 \mathrm{a}}$ sollten seco-CCBI- und antiMethyl-seco-CCBI-Analoga 68 und $\mathbf{6 7}$ als Vorläufer für eine Fluoreszenzmarkierung durch FITC (60) und Dapoxyl-Derivat 61 an der eingeführten Aminofunktion synthetisiert werden.

Ebenso sollten neuartige fluoreszenzmarkierte seco-CBI-Analoga des (+)-Duocarmycins SA (32) synthetisiert werden, die als Substrate für das Enzym $\beta$-DGalaktosidase dienen können. Bei diesem neuen Ansatz solllte der bis dahin verwendete DNA-Binder DMAI (104) durch einen fluoreszenzaktiven DNA-Binder aus der Klasse der Cumarine, wie DMACA (62), und der Klasse der Benzothiazole, wie BO (Benzothiazole Orange) (63), ersetzt werden. 
<smiles>O=C(c1cc2ccccc2oc1=O)N1C[C@H](CCl)c2c1cc(Oc1ccccc1)c1ccccc21</smiles>

(1S)-69<smiles>CC(C)Oc1cc2c(c3ccccc13)[C@@H](CCl)CN2C(=O)C(C)(C)[n+]1ccc(/C=C2/Sc3ccccc3N2C)cc1</smiles>

(1S)-70

Abbildung 30. Neuartige fluoreszenzmarkierte seco-CBI-Galaktoside (1S)-69 und (1S)-70 als Analoga des zytotoxischen Antibiotikums Duocarmycin (32) durch Verwendung von Cumarin-3-Carbonsäure (131) und BO (63) als DNA-Binder.

Die Farbstoffe sollten eine langwellige Fluoreszenz-Absorption besitzen, um ein Optical Imaging im Tier zu ermöglichen. Eine weitere Herausforderung bestand in der richtigen Auswahl der möglichen Fluoreszenzmarker in Hinblick auf ihre DNAbindenden Eigenschaften in AT-reichen Regionen. Ein Ziel war die Synthese der Verbindungen (1S)-69 und (1S)-70 (Abbildung 30).

Neben der Synthese der fluoreszenzmarkierten Prodrugs, wie (1S)-69 und (1S)-70 sowie der entsprechenden fluoreszenzmarkierten seco-Drugs sollte eine verkürtzte Synthese des fluoreszenzmarkierten anti-Methyl-seco-CBI-DMAI-Drugs (1S,10R)-71 aus der Arbeit von B. Krewer ${ }^{5 a}$ durchgeführt werden.

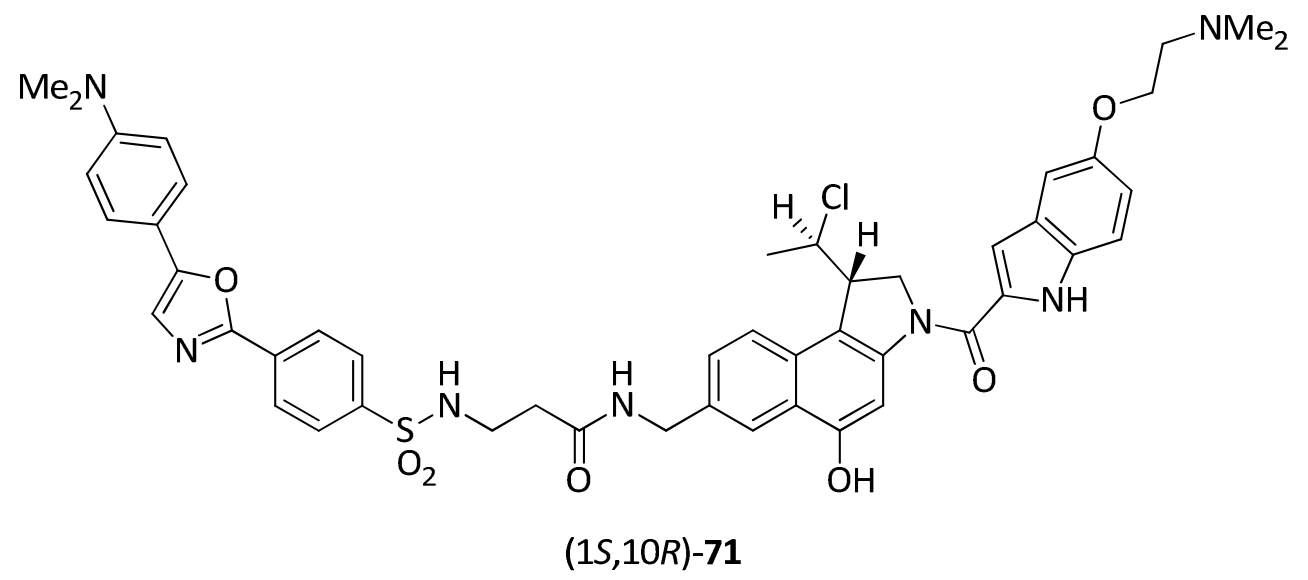

Abbildung 31. Dapoxylmarkiertes anti-Methyl-seco-CBI-Drug $(1 S, 10 R)-71$. 
Ebenso sollte das FITC-markierte Prodrug (1S)-72 (Abbildung 32) synthetisiert werden, um dessen Aufnahme in lebende Zellen durch Live Cell Imaging zu untersuchen.

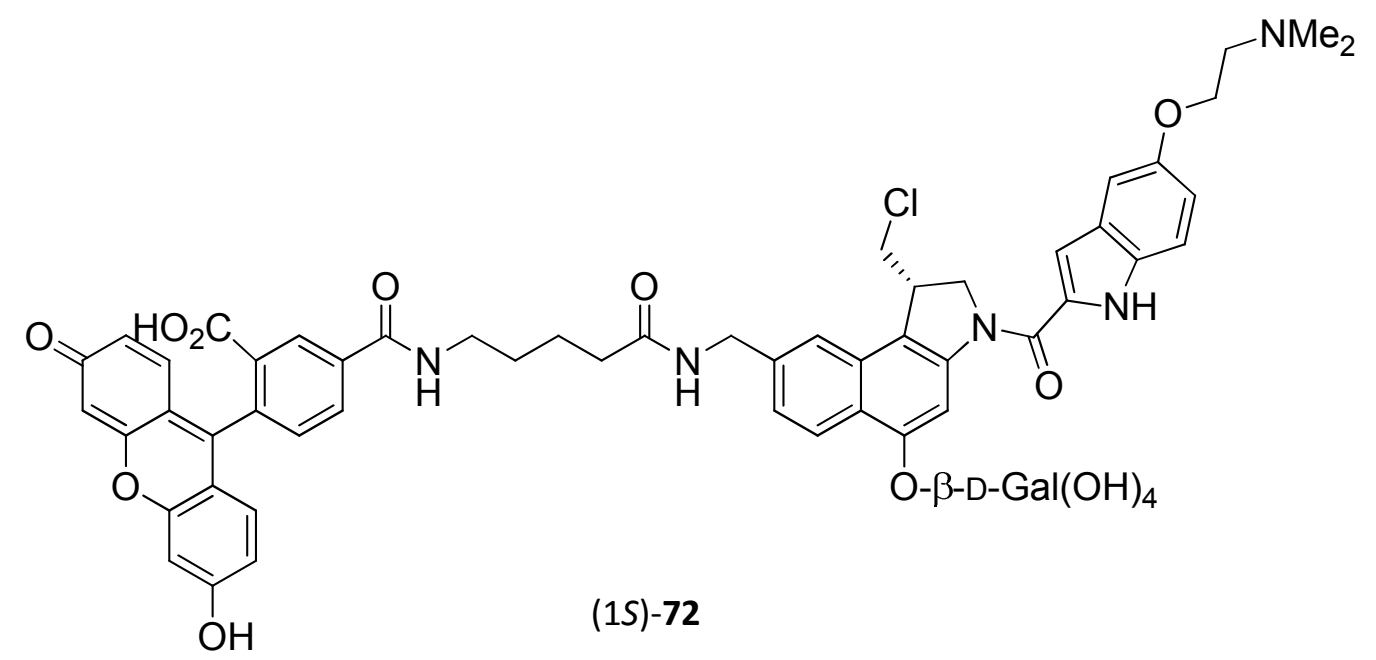

Abbildung 32. Struktur des FITC-markierten Galaktosides (1S)-72.

Alle synthetisierten Prodrugs, seco-Drugs und die dargestellten fluoreszenzmarkierten DNA-Binder waren in Kooperation mit Dr. Mišo Mitkovski am Max-PlanckInstitut für experimentelle Medizin in Göttingen auf ihre zelluläre Aufnahme zu untersuchen. Hierzu sollten zunächst die Absorptions- und Emissionsspektren der dargestellten Verbindungen am Fluorimeter bestimmt werden, um die anschließenden Live Cell Imaging Experimente durchzuführen.

Ein weiteres Ziel war die Bestimmung der therapeutischen Breite $\left(\mathrm{QIC}_{50}\right)$ der dargestellten Prodrugs und der Zelltoxizität $\left(\mathrm{IC}_{50}\right)$ der synthetisierten seco-Drugs in Kollaboration mit Dr. Ingrid Schuberth durch In-vitro-Assays.

Hieraus ergaben sich zusammenfassend folgende Aufgaben:

- $\quad$ Synthese des enantiomerenreinen FITC-markierten CBI-DMAI-Prodrugs (1S)-72

- Verkürzte Synthese des enantiomerenreinen Dapoxyl-markierten anti-Methylseco-CBI-DMAI-Drugs $(1 S, 10 R)-71$ 
- Synthese der enantiomerenreinen CBI-Prodrugs (1S)-69 und (1S)-70 sowie der entsprechenden seco-CBI-Drugs

- In-vitro-Untersuchungen zur Toxizität der neuen Verbindungen an Krebszellen der Linie A549

- Messung der Fluoreszenzspektren der dargestellten Verbindungen sowohl in Lösung als auch in Puffer

- In-vitro-Untersuchungen der dargestellten Verbindungen durch Confocal Laser Scanning Microscopy (Live Cell Imaging) 


\section{Planung der Arbeit}

\subsection{Synthese des fluoreszenzmarkierten CBI-DMAI-Prodrugs (1S)-72}

In Anlehnung an Arbeiten von F. Major ${ }^{5 \mathrm{c}}$ sollte das fluoreszenzmarkierte seco-CBIDMAI Prodrug (1S)-72 ausgehend vom iodierten Naphthalinderivat 75 dargestellt werden (Abbildung 33).

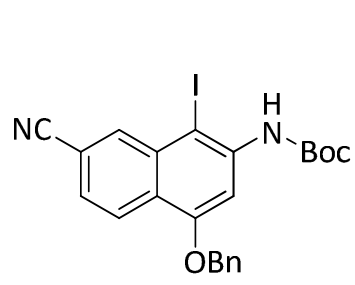

75

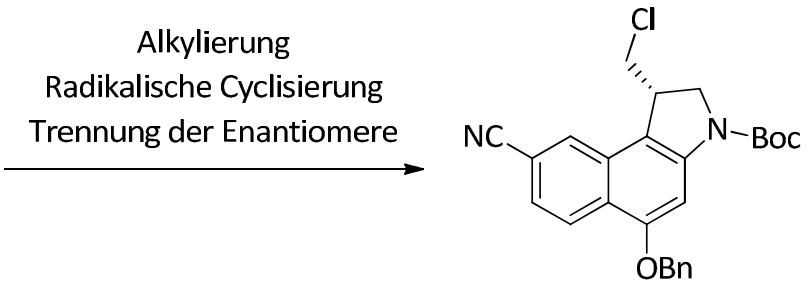

(1S)-74

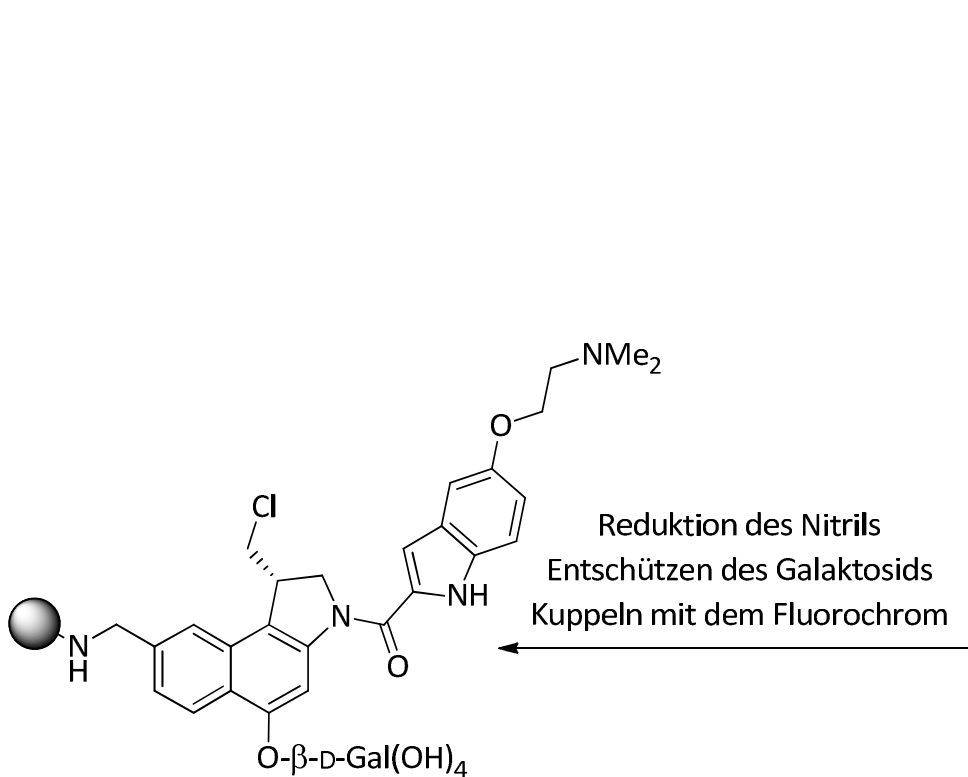

(1S)-72

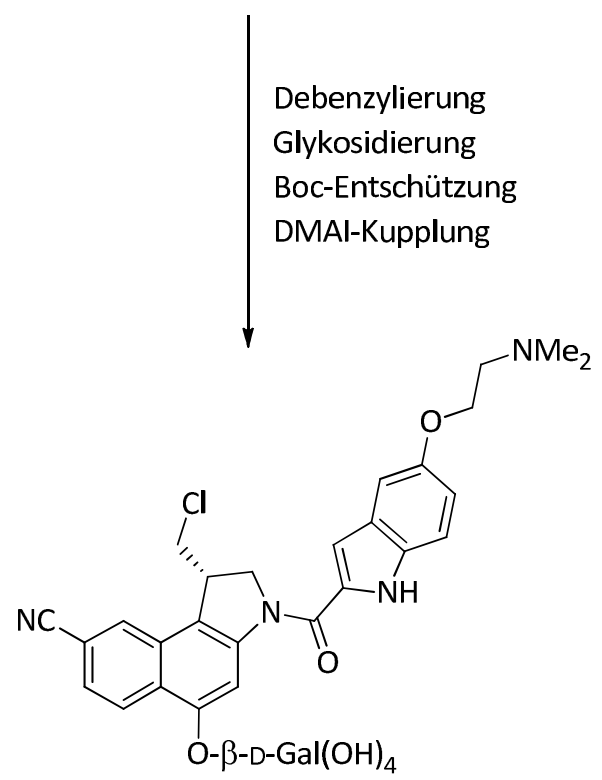

(1S)-73

Abbildung 33. Synthese des fluoreszenzmarkierten seco-CBI-DMAI-Prodrugs (1S)-72 ausgehend von Naphthalinderivat 75.

Hierzu war das lodnaphthalinderivat $\mathbf{7 5}$, das in der Abteilung Tietze verfügbar war ${ }^{5 e}$ erhältlich war, mit (E/Z)-1,3-Dichlorpropen zu alkylieren, um nach anschließender radikalischer Cyclisierung und Trennung der entstandenen Enantiomere das seco-CBI- 
Derivat (1S)-74 zu erhalten. Dieses sollte daraufhin debenzyliert und mit tetraacetyliertem Galaktose-Trichloracetimidat (116) gekuppelt werden. Anschliessende $N$-Boc-Entschützung und Bindungsknüpfung mit $D M A I-\mathrm{CO}_{2} \mathrm{H}$ (104) liefert den Vorläufer (1S)-73. Abschließend sollte die Nitrilfunktion reduziert, das Galaktosid entschützt und mit dem FITC-Derivat 119 gekuppelt werden, um das fluoreszenzmarkierte CBI-DMAI-Prodrug (1S)-72 zu erhalten.

\subsection{Verkürzte Synthese zur Darstellung des fluoreszenzmarkierten seco- anti-Methyl-CBI-DMAI-Drugs (1S,10R)-71}

In Anlehnung an Arbeiten von B. Krewer ${ }^{5 a}$ sollte das fluoreszenzmarkierte seco-antiMethyl-CBI-DMAI-Drug $(1 S, 10 R)-71$ in einer um fünf Stufen verkürzten Synthese dargestellt werden, bei der auf eine Glykosidierung des Phenols verzichtet wurde, um die Bedingungen der Zuckerabspaltung zu umgehen (Abbildung 34).

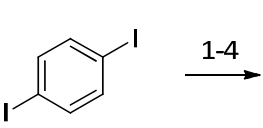

76

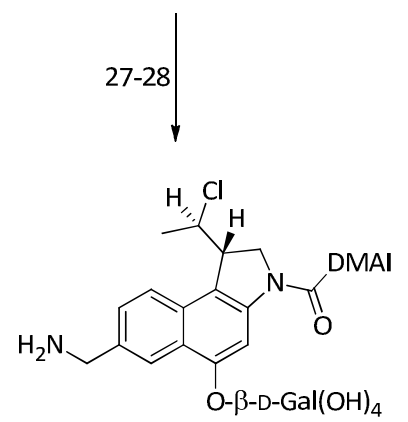

$(1 S, 10 R)-84$<smiles>CCOC(=O)CC(=Cc1ccc(I)cc1)C(=O)OCC</smiles>

77<smiles>CCOC(=O)c1cc(OCC)cc2ccc(I)cc12</smiles>

78<smiles>CC(C)(C)OC(=O)Nc1cc(O)c2cc(C#N)ccc2c1</smiles>

79 $\downarrow 12-15$<smiles></smiles>

$(15,10 R)-83$

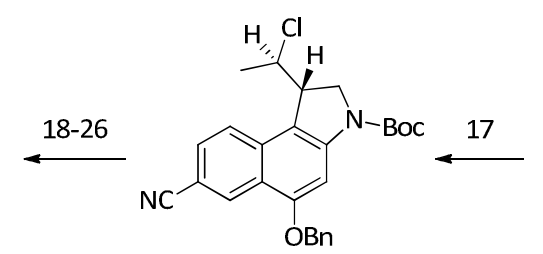

$(1 S, 10 R)-82$<smiles>CCOC(CN(C(C)=O)c1cc(O)c2cc(C#N)ccc2c1Br)C(=O)O</smiles>

(1S,10R)-81

80

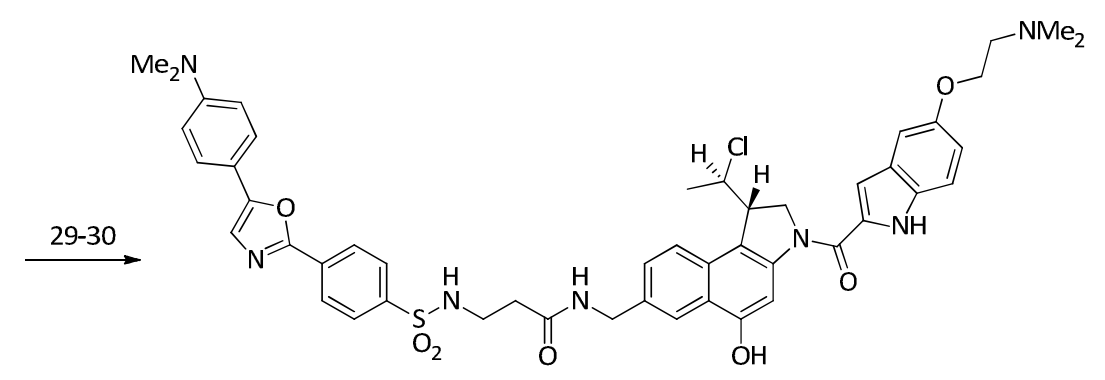

$(1 S, 10 R)-71$

Abbildung 34. Syntheseschema zur Darstellung von $(1 S, 10 R)-71$ nach dem von B. Krewer verwendeten Verfahren über eine Gesamtzahl von 30 Stufen. 
Unter Bezug auf Vorarbeiten von J. M. von $\operatorname{Hof}^{5 \mathrm{~b}}$ war das Bromnaphthalin 86, nach Halogen-Nitril-Austausch, Verseifung, Curtius-Umlagerung und Bromierung zu 85 umzusetzen. Nachfolgend sollte eine Alyklierung mit enantiomerenreinem Epoxynosylat 102, Zyklisierung zu enantiomerenreinem Alkohol (1S,10R)-81 und Appel-Reaktion zum seco-CBI-Grundgerüst $(1 S, 10 R)-82$ folgen. Alternativ sollte eine Alkylierung von 85 mit (E/Z)-1,3-Dichlor-2-buten (103) und anschließende radikalische Zyklisierung erfolgen. Trennung der gebildeten Diastereomere und chromatographische Racematspaltung sollte zu den Enantiomeren (+)-(1S,10R)-82 und (-)-(1R,10S)-82 führen (Abbildung 35).<smiles>C#CC(=O)N1C[C@H](C(C)Cl)c2c1cc(OCc1ccccc1)c1cc(C#N)ccc21</smiles>

$(1 S, 10 R)-82$<smiles>CC(C)(C)OC(=O)Nc1cc(OCc2ccccc2)c2cc(C#N)ccc2c1Br</smiles>

85<smiles>C=CC=CC</smiles>

86

Abbildung 35. Retrosynthetische Analyse zur Darstellung von (1S,10R)-82 ausgehend von Bromnaphthalin 85.

Im Gegensatz zu der Synthese von B. Krewer ${ }^{5 a}$ soll im Anschluss keine Debenzylierungs-Glykosidierungs-Kaskade erfolgen, sondern das Nitril nach erfolgter N-Boc-Entschützung und DMAI-Kupplung durch Verwendung von Palladium oder Platin sowie Wasserstoffgas reduziert werden.

Anschließende Kupplung des primären Amins (1S,10R)-87 mit dem Dapoxyl-Derivat 61 sollte das gewünschte fluoreszenzmarkierte anti-Methyl-seco-CBI-DMAI-Drug (1S,10R)-71 liefern (Abbildung 36).

Auf Grund der enormen Kosten von $5265 €$ / 25 mg für das Dapoxyl-Derivat 61 sollte ein analoges Verfahren zur Darstellung von $(1 S, 10 R)-71$ durch das DapoyxlSulfonylchlorid 89 entwickelt werden. 
<smiles>CC(Cl)C1(C(C)(Cl)Cl)CN(C(=O)c2cc3cc(OCCN(C)C)ccc3[nH]2)c2cc(O)c3cc(CNC(=O)CCNS(=O)c4ccc(-c5ncc(-c6ccc(N(C)C)cc6)o5)cc4)ccc3c21</smiles>

Abbildung 36. Retrosynthetische Analyse zur Darstellung von $(1 S, 10 R)-71$ ausgehend von $(1 S, 10 R)-88$ durch Kupplung mit dem Dapoxylderivat 61.<smiles>CNc1ccc(-c2cnc(-c3ccc(S(=O)(=O)NCCC(=O)ON4C(=O)CCC4=O)cc3)o2)cc1</smiles>

61<smiles>CN(C)c1ccc(-c2cnc(-c3ccc(S(=O)(=O)Cl)cc3)o2)cc1</smiles>

89

Abbildung 37. Strukturen der verwendeten Dapoxyl-Derivate $\mathbf{6 1}$ und $\mathbf{8 9}$. 
Hierzu sollte das primäre Amin des benzylgeschützten Naphthalinderivates (1S,10R)-91 mit Cbz-geschütztem $\beta$-Alanin (105) alkyliert werden. Anschließende simultane Entschützung der Benzyl- und Cbz-Schutzgruppe und Kupplung mit Dapoxyl-Sulfonylchlorid (89) soll das gewünschte fluoreszenzmarkierte anti-Methylseco-CBI-DMAI-Drug (1S,10R)-71 liefern (Abbildung 38).
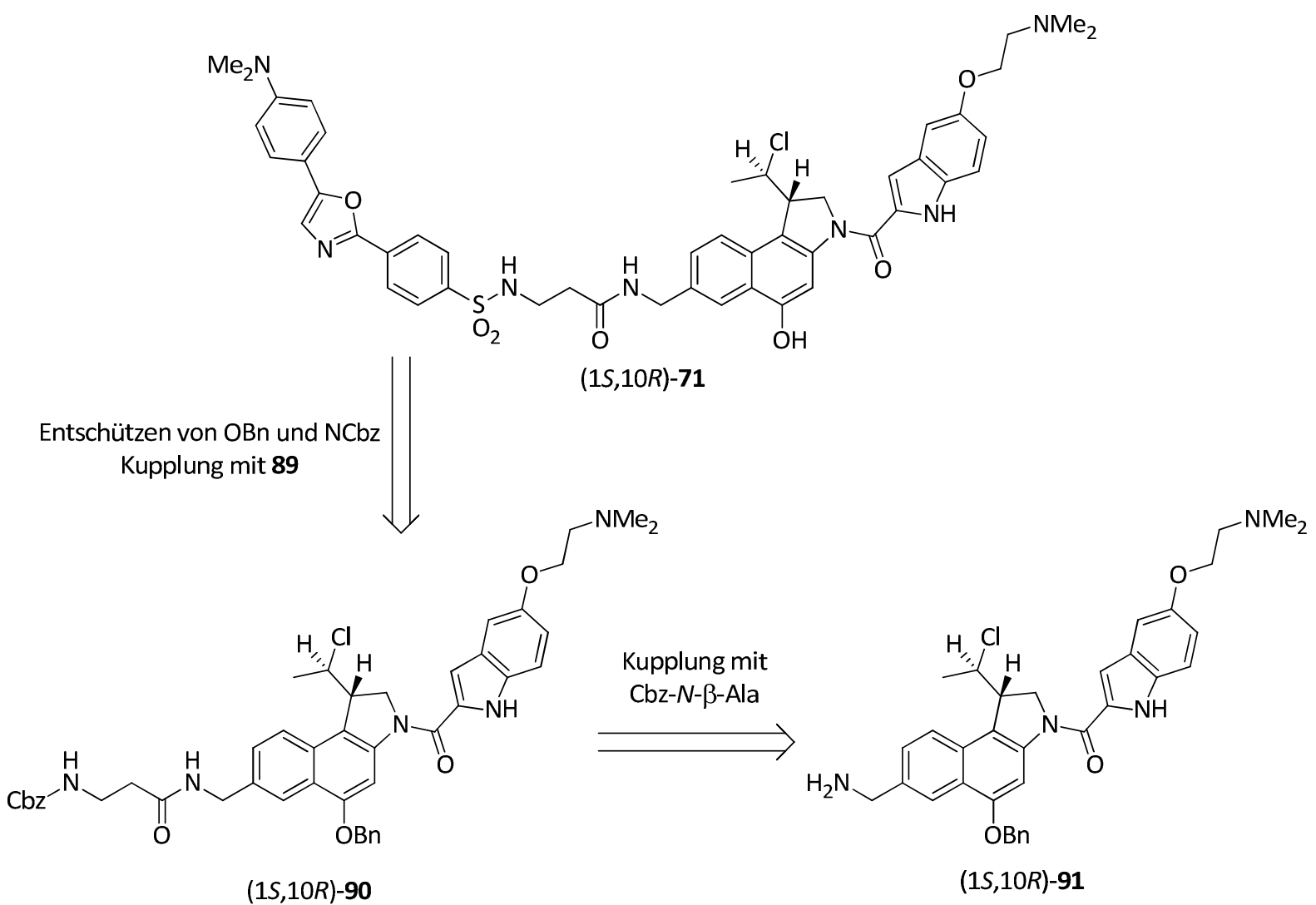

Abbildung 38 Retrosynthetische Analyse zur Darstellung von (1S,10R)-71 unter Verwendung des Dapoxyl-Sulfonylchlorides 89.

\subsection{Synthese neuartiger fluoreszenzmarkierter Duocarmycin-Analoga}

Um eine bessere Vergleichbarkeit der fluoreszenzmarkierten Wirkstoffe zu den bereits synthetisierten Verbindungen, wie (1S,10R)--47a und (1S)-48 zu haben, sollten sowohl seco-Drugs als auch Prodrugs entwickelt werden, die in ihrer räumlichen und chemischen Struktur den CBI-DMAI-Analoga nahe kommen. Daher sollte der bisher genutzte DNA-Binder DMAI durch einen fluoreszenzmarkierten DNA-Binder 
ausgetauscht werden. Hierbei sollten vor allem Cumarine und Benzothiazole genutzt werden, da bekannt war, dass letztere über DNA-bindende Eigenschaften verfügen. Ausgehend von Benzaldehyd (97) sollte in Anlehnung an Arbeiten von F. Major und J. M. von Hof der Grundkörper (1S)-93 in einer 16-stufigen Synthese enantiomerenrein dargestellt werden (Abbildung 39). Anschließend sollte nach dem bekannten Protokoll zur Glykosidierung und DNA-Binder-Kupplung, sowie folgender Entschüzung des Galaktosides fluoreszenzmarkierte (1S)-CBI-Galaktoside wie (1S)-69 erhalten werden. Analog sollte zur Darstellung der seco-CBI-Drugs wie (1S)-92 verfahren werden.

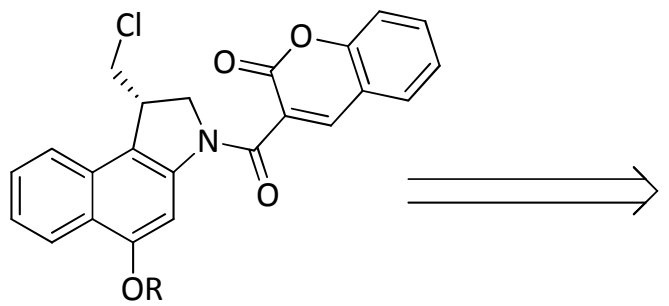

$\mathrm{R}=\mathrm{H}$

(1S)-92

$R=\beta$-D-Gal $(\mathrm{OH})_{4}(1 S)-69$<smiles>O=Cc1ccccc1</smiles>

97<smiles>CC(C)(C)OC(=O)N1C[C@@H](CCl)c2c1cc(OCc1ccccc1)c1ccccc21</smiles>

(1S)-93<smiles>CC(C)(C)OC(=O)c1ccccc1Cc1ccccc1OCc1ccccc1</smiles>

94<smiles>C1CCCCC1</smiles><smiles>CC(C)(C)Nc1cc(OCc2ccccc2)c2ccccc2c1I</smiles>

95

Abbildung 39. Retrosynthetische Analyse zur Darstellung neuartiger fluoreszenzmarkierter(1S)CBI-Galaktoside und seco-CBI-Drugs ausgehend von Benzaldeyhd (97) am Beispiel von (1S)-69 und (1S)-92, als Vertreter der Cumarine. 


\section{B. DARSTELLUNG DER ERGEBNISSE}

\section{Synthese des fluoreszenzmarkierten anti-Methyl-seco-CBI- Drugs $(1 S, 10 R)-71$}

\subsection{Synthese des anti-Methyl-seco-CCBI-Grundgerüstes $(1 S, 10 R)-82$}

In Anlehnung an die von Tietze und Boger entwickelten Verfahren konnte das antiMethyl-seco-CCBI-Grundgerüst $(1 S, 10 R)-82$ über 8 Stufen ausgehend von der Naphthalincarbonsäure 85 dargestellt werden. ${ }^{153}$

Durch Veresterung der Carbonsäure 85 via Bildung des Säurechlorides und anschließender Umsetzung mit Ethanol unter Rückfluss für $19 \mathrm{~h}$ konnte der Ethylester 98 erhalten werden. Der Ethylester 98 konnte nun zunächst in DMF unter Rückfluss für $20 \mathrm{~h}$ bei Verwendung von Kupfercyanid einem Halogen-Nitril-Austausch zu 99 unterzogen und anschließend in einem Lösungsmittelgemisch von THF/MeOH/ $\mathrm{H}_{2} \mathrm{O}$ (3:1:1) unter Zugabe von $\mathrm{LiOH} \cdot \mathrm{H}_{2} \mathrm{O}$ bei Raumtemperatur und Rühren für $20 \mathrm{~h}$ zur Carbonsäure 100 verseift werden (Abbildung 40).<smiles>O=C(O)c1cc(OCc2ccccc2)c2cc(Br)ccc2c1</smiles>

85

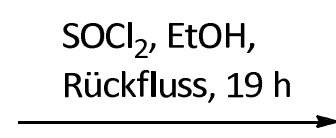<smiles>CCOC(=O)c1cc(OCc2ccccc2)c2cc(Br)ccc2c1</smiles>

98

CuCN, DMF, Rückfluss, $20 \mathrm{~h}$<smiles>N#Cc1ccc2cc(C(=O)O)cc(OCc3ccccc3)c2c1</smiles>

100
$\mathrm{LiOH} \cdot \mathrm{H}_{2} \mathrm{O}, \mathrm{THF} / \mathrm{MeOH} / \mathrm{H}_{2} \mathrm{O}$

(3:1:1), RT, $20 \mathrm{~h}$

Abbildung 40. Synthese der Carbonsäure $\mathbf{1 0 0}$ ausgehend von Bromnaphthalin 85 über 3 Stufen. 
Die Synthese des N-Boc-geschützten Bromnaphthailnderivates 101 erfolgte durch eine Curtius-Umlagerung unter Verwendung von Diphenylphosphorylazid (DPPA) und Triethylamin in tert-Buthanol und Erhitzen auf Rückfluss für $22 \mathrm{~h}$, gefolgt von einer säurekatalysierten Bromierung des Aromaten 79 durch N-Bromsuccinimid in THF bei $-78^{\circ} \mathrm{C}$ für $4 \mathrm{~h}$ sowie $1 \mathrm{~h}$ bei Raumtemperatur lieferte die gewünschte Verbindung 101 (Abbildung 41).<smiles>N#Cc1ccc2cc(C(=O)O)cc(Br)c2c1</smiles>

100

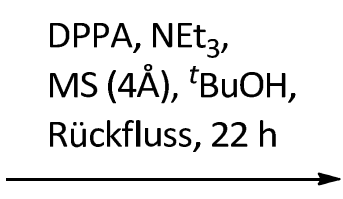<smiles>CC(C)(C)OC(=O)Nc1cc(OCc2ccccc2)c2cc(C#N)ccc2c1</smiles>

79<smiles>CC(=O)OCc1cc(OCc2ccccc2)c2cc(C#N)ccc2c1Br</smiles>

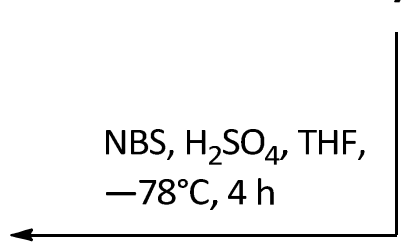

101

Abbildung 41. Syntheseschema für die Darstellung des N-Boc-geschützten Bromnaphthalins 101 ausgehend von 100 über Curtius-Umlagerung und Bromierung.

Um das gewünschte anti-Methyl-seco-CCBI Grundgerüst $(1 S, 10 R)-82$ darzustellen, gibt es zwei mögliche Reaktionswege. Zum Einen die Alkylierung von 101 durch das enantiomerenreine Epoxynosylat 102 sowie anschließende enantioselektive Zinkatvermittelte Zyklisierung zum gewünschten Produkt (1S,10R)-81 (Abbildung 42)..$^{154}$ Zum Anderen eine Alkylierung von 101 durch (E/Z)-1,3-Dichlor-2-buten (103) und anschließende AIBN-vermittelte radikalische Zyklisierung zu den Diastereomeren rac$(1 S, 10 R)-82$ und rac-(1S,10S)-82 (Abbildung 43). Auf Grund der besseren Gesamtausbeute bei der Anwendung der radikalischen Methode und der damit verbundenen größeren Menge an Substanz für folgende Untersuchungen wurde diese zunächst gewählt. Anschließend wurde auch die enantioselektive Variante verwendet, da diese keine Reinigung durch HPLC an chiralier stationärer Phase erfordert. 
Nach der Alkylierung des Bromnaphthalins 101 durch das Epoxynosylat 102 unter Verwendung von Natriumhydrid als Base und DMF als Lösungsmittel konnte das Derivat $\mathbf{8 0}$ mit einer hervorragenden Ausbeute von 95\% isoliert werden. Die Umsetzung von $\mathbf{8 0}$ mit dem Zinkat $\mathrm{Li}_{2} \mathrm{Zn}(\mathrm{SCN}) \mathrm{Me}_{3}$, welches aus Zinkchlorid $\left(\mathrm{ZnCl}_{2}\right)$, Methyllithium (MeLi) und Trimethylsilylisothiocyanat (TMS-SCN) in situ hergestellt wurde, liefert den gewünschten Alkohol (1S,10R)-81 mit einer sehr guten Ausbeute von 69\%. Anschließende Chlorierung mit Thionylchlorid und Pyridin bei Raumtemperatur in Dichlormethan für $4 \mathrm{~h}$ liefert das anti-Methyl-seco-CCBIGrundgerüst $(1 S, 10 R)-82$ in einer moderaten Ausbeute von 34\%. Leider führte die Anwendung der Appel Reaktion, wie sie bei der Darstellung von (1S)-93 benutzt wird, nicht zum gewünschten Produkt.<smiles>CC(C)(C)OC(=O)Nc1cc(OCc2ccccc2)c2cc(C#N)ccc2c1Br</smiles>
101

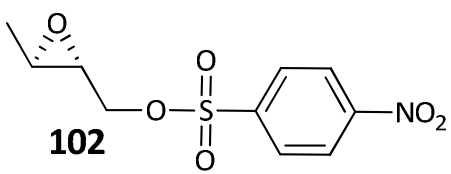

1. $\mathrm{NaH}, \mathrm{DMF}, 60^{\circ} \mathrm{C}, 40 \mathrm{~min}$

2. 102, RT, $24 \mathrm{~h}$

$95 \%$<smiles>CC(=O)OC(=O)N(CC1OC1C(=O)OCc1ccccc1)c1cc(OCc2ccccc2)c2cc(C#N)ccc2c1Br</smiles>

80

$$
\begin{gathered}
\mathrm{Li}_{2} \mathrm{Zn}(\mathrm{SCN}) \mathrm{Me}_{3}, \mathrm{THF}, \\
-78^{\circ} \mathrm{C} \rightarrow \mathrm{RT}, 3.5 \mathrm{~h} \\
69 \%
\end{gathered}
$$<smiles>CC(Cl)[C@@H]1CN(C(=O)OC(C)(C)C)c2cc(OCc3ccccc3)c3cc(C#N)ccc3c21</smiles>

(1S,10R)-82

$$
\frac{\begin{array}{l}
\mathrm{SOCl}_{2}, \text { Pyridin, } \\
\mathrm{CH}_{2} \mathrm{Cl}_{2}, \mathrm{RT}, 4 \mathrm{~h}
\end{array}}{34 \%}
$$<smiles>CC(O)[C@@H]1CN(C(=O)OC(C)(C)C)c2cc(OCc3ccccc3)c3cc(C#N)ccc3c21</smiles>

$(1 S, 10 R)-81$

Abbildung 42. Syntheseschema zur enantioselektiven Darstellung des anti-Methyl-seco-CCBIGrundgerüstes (1S,10R)-82 ausgehend vom Bromnaphthalin 101. 
Als Alternative zur enantioselektiven Synthese von $(1 S, 10 R)-82$ wurde auch eine radikalische racemische Syntheseroute auf Grund der bereits beschriebenen Vorteile verfolgt. Hierbei wurde das Bromnaphtalin 101 durch (E/Z)-1,3-Dichlor-2-buten (103) alkyliert und anschließend unter Verwendung von Tris-Tri-Methyl-Silyl-Silan (TTMSS) und Azoisobutyronitril (AIBN) in Toluol zyklisiert. (1S,10R)-82 konnte in einer hervorragenden Ausbeute von $90 \%$ isoliert werden, wobei das gewünschte antiDiastereomer rac-(1S,10R)-82 mit einer Ausbeute von 46\% dargestellt werden konnte. Nach Trennung der Diastereomere rac-(1S,10R)-82 und rac-(1S,10S)-82 durch Säulenchromatographie an Kieselgel konnte eine Trennung der Enantiomere von rac-(1S,10R)-82 an chiraler stationärer Phase (Chrialpak $I A)$ erfolgen und das reine Enantiomer (+)-(1S,10R)-82 erhalten werden (Abbildung 43).<smiles>CC(C)(C)Nc1cc(OCc2ccccc2)c2cc(C#N)ccc2c1Br</smiles>

101

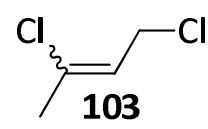

1. $\mathrm{NaH}, \mathrm{DMF}, \mathrm{RT}, 2.5 \mathrm{~h}$

$\frac{\text { 2. 103, RT, } 4 \mathrm{~h}}{66 \%}$
NC<smiles>CC(=O)Oc1cc(N(CC=C(C)Cl)C(=O)OCc2ccccc2)c(Br)c2ccc(C#N)cc12</smiles>

104
TTMSS, AIBN, Toluol, $80^{\circ} \mathrm{C}, 4 \mathrm{~h}$<smiles>CC(C)(C)OC(=O)N1C[C@@H](C(C)(C)C)c2c1cc(OCc1ccccc1)c1cc(C#N)ccc21</smiles>

rac- $(1 S, 10 R)-82$

$46 \%$<smiles>CC(C)(C)OC(=O)N1C[C@@H](C(C)(C)C)c2c1cc(OCc1ccccc1)c1cc(C#N)ccc21</smiles>

rac-(1S,10S)-82

$44 \%$

Abbildung 43. Dastellung des anti-Methyl-seco-CCBI-Grundgerüstes (1S,10R)-82 unter Verwendung der radikalischen Syntheseroute. 


\subsection{Synthese des anti-Methyl-seco-CCBI-DMAI-Derivates (1S,10R)-88}

Zur Darstellung des fluoreszenzmarkierten anti-Methyl-seco-CBI-Drugs (1S,10R)-71 unter Verwendung des Dapoxylsulfonylchlorides (61) wurde zunächst das antiMethyl-seco-CCBI-Grundgerüst $(+)-(1 S, 10 R)-82$ in Anlehnung an Arbeiten von $F$. Major $^{5 \mathrm{C}}$ zu anti-Methyl-seco-CCBI-DMAI (1S,10R)-88 umgesetzt. Hierzu wurde zunächst die säurelabile $N$-Boc-Schutzgruppe aus (1S,10R)-82 durch Anwendung einer $4 \mathrm{M} \mathrm{HCl}$ /Essigester Lösung bei Raumtemperatur entfernt, um das entstandene sekundäre Amin mit dem DNA-Binder DMAI- $\mathrm{CO}_{2} \mathrm{H}$ (104) zu kuppeln. Hierbei wurde EDAC. $\mathrm{HCl}$, das als Kupplungsreagenz von Aminen mit Carbonsäure bekannt ist, eingesetzt. $(1 S, 10 R)-88$ und $(1 R, 10 S)-88$ sowie rac-(1S,10S)-88 konnten in sehr guten Ausbeuten dargestellt werden (Abbildung 44).<smiles>CC(C)(C)OC(=O)N1CC(C(C)(Cl)Cl)c2c1cc(OCc1ccccc1)c1cc(C#N)ccc21</smiles>

82

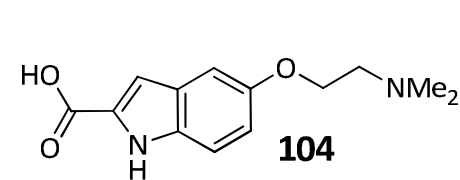

1. $4 \mathrm{M} \mathrm{HCl} / \mathrm{EE}, \mathrm{RT}, 4 \mathrm{~h}$

2. 104, EDAC.HCl, RT, $18 \mathrm{~h}$

$66 \%$ rac- $(1 S, 10 S)-88$

$62 \%(1 S, 10 R)-88$

$47 \%(1 R, 10 S)-88$

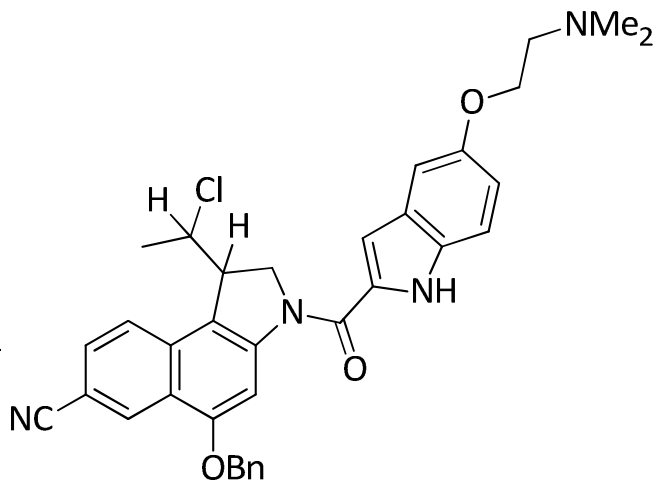

88

Abbildung 44. Syntheseschema zur Darstellung der seco-CCBI-DMAI-Derivate.

\subsection{Synthese des anti-Methyl-seco-CBI-DMAI-Derivates mit Spacer- Einheit $(1 S, 10 R)-106$}

Zur verkürtzten Darstellung des fluoreszenzmarkierten anti-Methyl-seco-CBI-Drugs (1S,10R)-71 sollte eine $\beta$-Alanin-Einheit eingeführt werden, um den kostengünstigeren Dapoxylfarbstoff als Sulfonylchlorid (89) nutzen zu können. Hierzu wurde zunächst versucht, die Nitrilfunktion in $(1 S, 10 R)-88$ unter Verwendung 
verschiedener $\mathrm{Pd}$ - und $\mathrm{Pt}$-Verbindungen zum primären Amin zu reduzieren (Abbildung 45 und Tabelle 3).

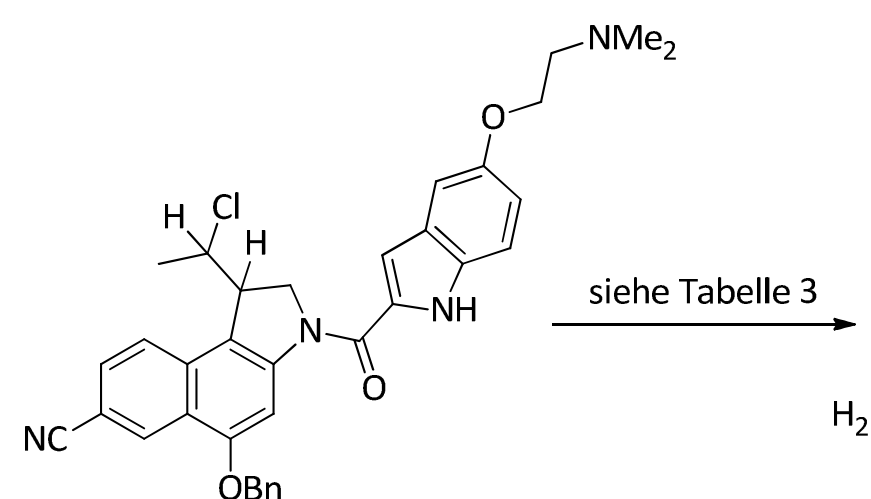

88

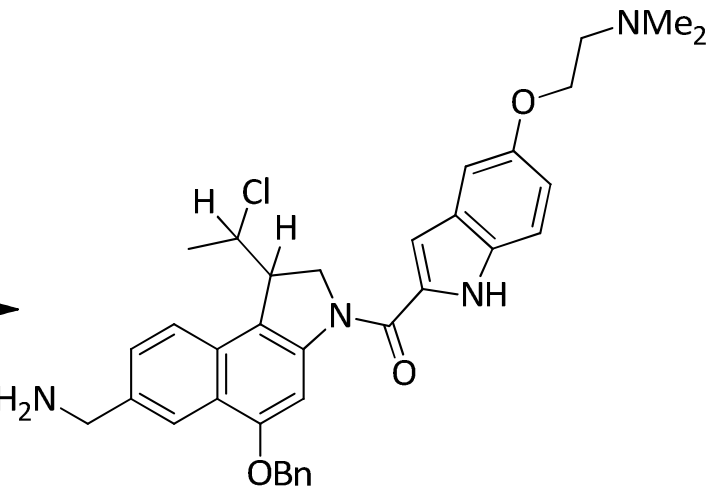

81

Abbildung 45. Versuche zur Darstellung des primären Amins $\mathbf{8 1}$ durch Reduktion der Nitrilfunktion in 88.

\begin{tabular}{|c|c|}
\hline Bedingungen & Ausbeute (Verwendetes Substrat) \\
\hline $\begin{array}{l}\mathrm{PtO}_{2} \cdot \mathrm{H}_{2} \mathrm{O}, \mathrm{H}_{2}(1 \mathrm{~atm}), \mathrm{EtOH} / \mathrm{H}_{2} \mathrm{O}(96: 4), 1.25 \mathrm{M} \\
\mathrm{HCl} / \mathrm{EtOH}(2 \text { Äq.), RT, } 48 \mathrm{~h}\end{array}$ & Edukt (rac-(1S,10S)-88) \\
\hline $\begin{array}{l}\mathrm{PtO}_{2} \cdot \mathrm{H}_{2} \mathrm{O}, \mathrm{H}_{2}(1 \mathrm{~atm}), \mathrm{MeOH} / \mathrm{H}_{2} \mathrm{O}(96: 4), 1.25 \mathrm{M} \\
\mathrm{HCl} / \mathrm{EtOH}(2 \text { Äq.), RT, } 48 \mathrm{~h}\end{array}$ & Edukt (rac-(1S,10S)-88) \\
\hline $\mathrm{LiAlH}_{4}$ (2.0 Äq.), THF, RT, $15 \mathrm{~min}$ & Zersetzung (rac-(1S,10S)-88) \\
\hline $4 \mathrm{M} \mathrm{HCl} / \mathrm{EE}, \mathrm{RT}, 1.5 \mathrm{~h}$ & \\
\hline $\begin{array}{l}\text { 2. } \mathrm{Pd} / \mathrm{C}, \mathrm{H}_{2}(1 \mathrm{~atm}), \mathrm{THF} / \mathrm{MeOH}(2: 1), 40^{\circ} \mathrm{C} \text {, } \\
\mathrm{NH}_{4} \mathrm{CO}_{2}\end{array}$ & Debenzylierung (rac-(1S,10S)-88) \\
\hline $\begin{array}{l}\mathrm{PtO}_{2} \cdot \mathrm{H}_{2} \mathrm{O}, \mathrm{H}_{2}(1 \mathrm{~atm}), \mathrm{THF} / \mathrm{EtOH} / \mathrm{H}_{2} \mathrm{O}(2: 4: 1), \\
1.25 \mathrm{M} \mathrm{HCl} / \mathrm{EtOH}(2, \mathrm{q} .), \mathrm{RT}, 8 \mathrm{~h}\end{array}$ & $\begin{array}{c}54 \%(\text { rac }-(1 S, 10 S)-81 \\
58 \%(1 S, 10 R)-81 \\
53 \%(1 R, 10 S)-81\end{array}$ \\
\hline
\end{tabular}

Tabelle 3. Versuche zur Reduktion des Nitrils in $\mathbf{8 8 .}$

Erste Versuche erfolgten in Anlehnung an Arbeiten von F. Major, ${ }^{5 \mathrm{c}}$ in denen statt der Benzylschutzgruppe an C-4 tetraacetyliete Galaktose gebunden war. Hierzu wurde das Edukt in $\mathrm{EtOH} / \mathrm{H}_{2} \mathrm{O}(96: 4)$ oder $\mathrm{MeOH} / \mathrm{H}_{2} \mathrm{O}$ (96:4) suspendiert und mit $1.25 \mathrm{M} \mathrm{HCl}$ in $\mathrm{EtOH}$ versetzt, wobei sich das Edukt löste. Anschließende Zugabe von $\mathrm{PtO}_{2} \cdot \mathrm{H}_{2} \mathrm{O}$ und 
Rühren unter $\mathrm{H}_{2}$-Atmosphäre lieferte jedoch keine Umsetzung des Eduktes (Eintrag 1 und 2). Versuche mit $\mathrm{LiAlH}_{4}{ }^{155}$ als Hydridquelle in THF bei Raumtemperatur führten im Fall von rac-(1S,10S)-88 zur Zersetzung des Eduktes (Eintrag 3). Überführung von rac-(1S,10S)-88 in dessen Hydrochlorid und anschließende Versetzung mit Pd auf Aktivkohle, sowie Ammoniumformiat als $\mathrm{H}_{2}$-Quelle ${ }^{4 c}$ führte, wie erwartet zur Debenzylierung des Eduktes (Eintrag 4). Die erfolgreich Nitrilreduktion konnte in einem Lösungsmittelgemisch aus THF/EtOH/ $\mathrm{H}_{2} \mathrm{O}(2: 4: 1)$ beobachtet werden. Das gebildete Hydrochlorid von $\mathbf{8 8}$ ist gut wasserlöslich und konnte durch die Wahl des genannten Lösungsmittels über die gesamte Reaktionsdauer in Lösung gehalten werden. Eine Reduktion des Nitrils konnte somit in guten Ausbeuten ermöglicht werden (Eintrag 5).

Nach der erfolgreichen Reduktion des Nitrils $(1 S, 10 R)-88$ zum primären Amin $(1 S, 10 R)-81$ konnte nun das $N$-Cbz geschützte $\beta$-Alanin (105) unter Verwendung von PyBOP als Kupplungsreagenz und Hünigbase in DMF nach einem Standardprotokoll in einer sehr guten Ausbeute von 82\% zu (1S,10R)-90 umgesetzt werden (Abbildung 46). ${ }^{156}$

$(1 S, 10 R)-81$<smiles>CON1C[C@H](C(C)Cl)c2c1cc(Cc1ccccc1)c1cc(CN)ccc21</smiles>

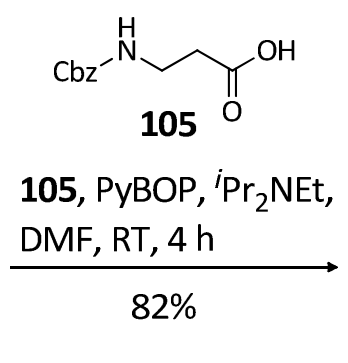

\begin{tabular}{l} 
105, PyBOP, ${ }^{i} \mathrm{Pr}_{2} \mathrm{NEt}$, \\
DMF, RT, $4 \mathrm{~h}$ \\
\hline $82 \%$
\end{tabular}

Abbildung 46. Einführung der Spacer-Einheit in $(1 S, 10 R)-81$ durch Verwendung von Cbzgeschütztem $\beta$-Alanin (105).

Anschließende simultane Entfernung der Cbz- und Benzylschutzgruppe in (1S,10R)-90 unter Verwendung eines Lösungsmittelgemisches aus $\mathrm{MeOH} / \mathrm{AcOH} / \mathrm{H}_{2} \mathrm{O}$ (3:1:1) und Pd auf Aktivkohle in einer $\mathrm{H}_{2}$-Atmosphäre lieferte den Vorläufer (1S,10R)-106 des 
fluoreszenzmarkierten anti-Methyl-seco-CBI-DMAI-Drugs $(1 S, 10 R)-106$ in einer guten Ausbeute von 57\% (Abbildung 47).<smiles></smiles>

$(1 S, 10 R)-90$<smiles></smiles>

$(1 S, 10 R)-106$

Abbildung 47. Synthese des freien Phenols (1S,10R)-106 durch simultane hydrogenolytische Spaltung der Cbz- und Benzylschutzgruppe in (1S,10R)-90.

\subsection{Synthese des anti-Methyl-seco-CBI-DMAI-Derivates ohne Spacereinheit (1S,10R)-87}

Neben der Synthese des anti-Methyl-seco-CBI-DMAI-Derivates (1S,10R)-106 mit der Spacereinheit zur Darstellung des fluoreszenzmarkierten anti-Methyl-seco-CBI-Drugs (1S,10R)-71 durch Dapoxylsulfonylchlorid (89) konnte ebenso eine Synthese zur Darstellung von $(1 S, 10 R)$-71 über das anti-Methyl-seco-CBI-DMAI-Derivat $(1 S, 10 R)-87$ ohne Spacereinheit unter Verwendung des Dapoxyl-derivates 61 durchgeführt werden. Hierzu konnte in Analogie zur Synthese von (1S,10R)-106 das Phenol $(1 S, 10 R)-87$ in guten Ausbeuten von $51 \%$ für $(1 R, 10 S)-87$ bis $67 \%$ für $(1 S, 10 R)-87$ dargestellt werden (Abbildung 48).

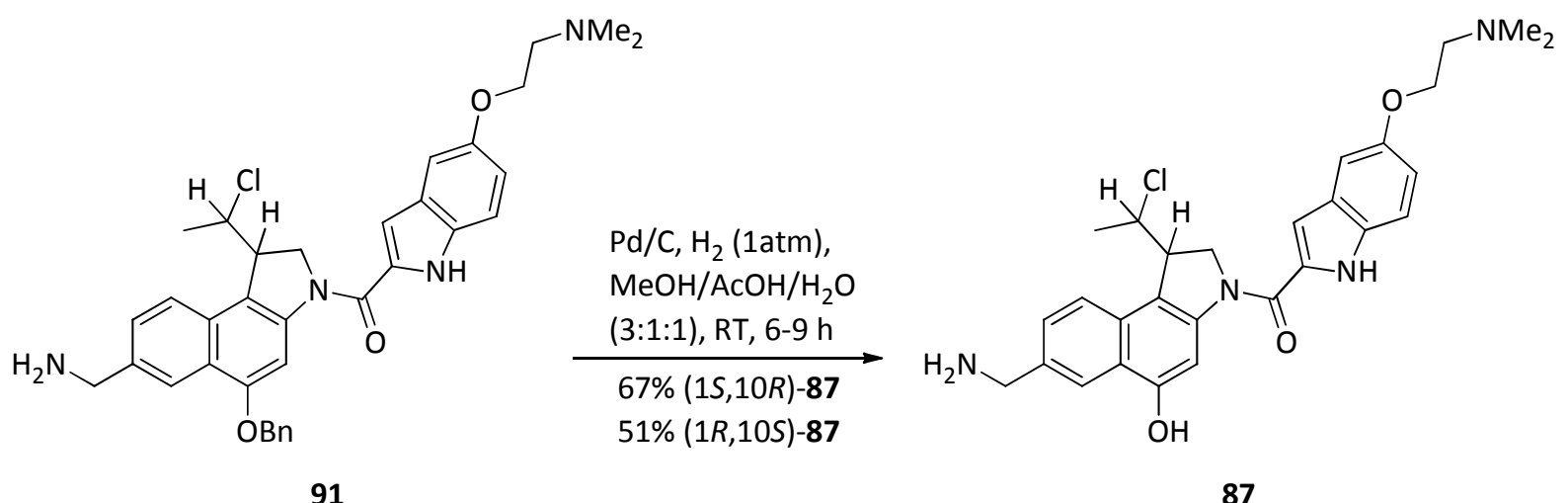

Abbildung 48. Synthese des freien Phenols $\mathbf{8 7}$ durch hydrogenolytische Debenzylierung von $\mathbf{8 1 .}$ 


\subsection{Synthese des fluroreszenzmarkierten anti-Methyl-seco-CBI-DMAI- Drugs $(1 S, 10 R)-71$}

Ausgehend von den primären Aminen $(1 S, 10 R)-87$ und $(1 S, 10 R)-106$ konnte das gewünschte Produkt $(1 S, 10 R)-71$ durch die Verwendung der Dapoxylderivate 61 und 89 dargestellt werden. In Anlehnung an Arbeiten von B. Krewer ${ }^{5 \mathrm{a}}$ wurde das Amin mit Hünig-Base deprotoniert, um es im Anschluss daran mit dem entsprechenden Dapoxylderivat zu kuppeln. Auf Grund der freien Phenolfunktion und der damit verbundenen Winstein-Zyklisierung unter basischen Bedingungen wurden neben dem gewünschten Produkt auch dessen Substitutionsprodukte erhalten (Abbildung 49).

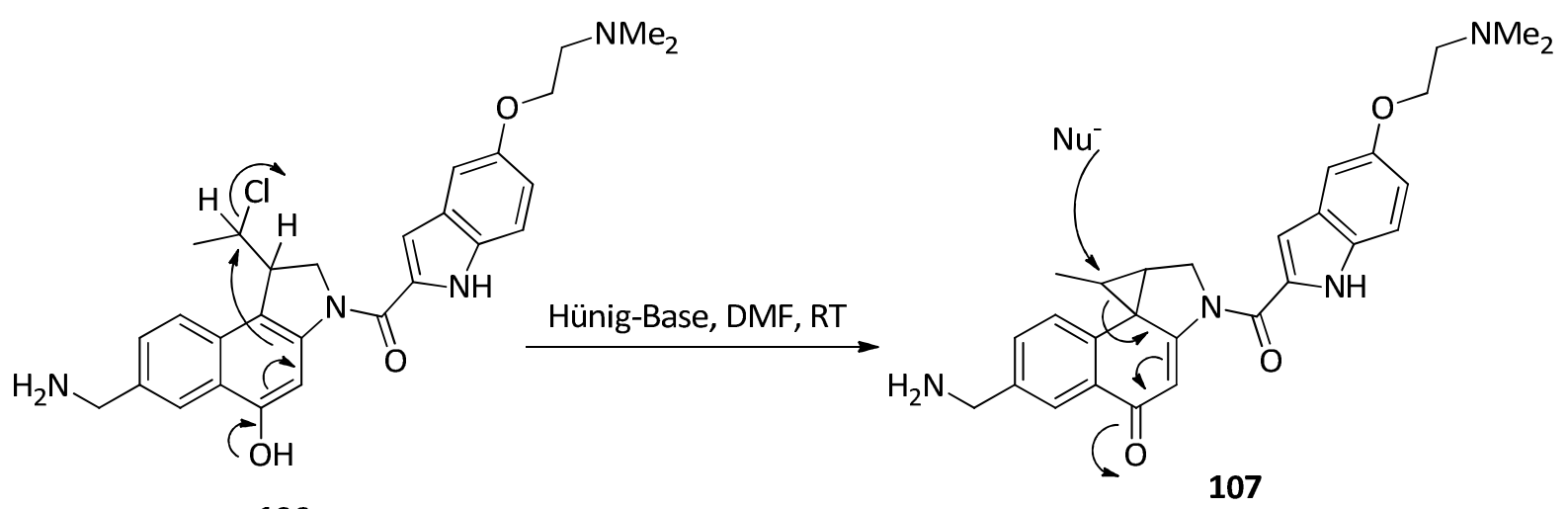

106

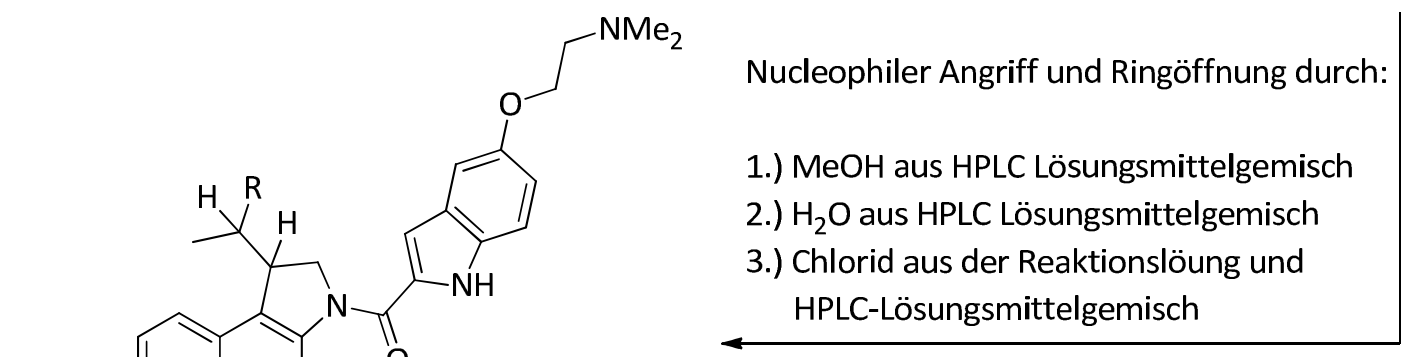

Abbildung 49. Durch Hünig-Base vermittelte Winstein-Zyklisierung zu 107 und anschließende Ringöffnung bei Versetzen mit Lösungsmittelgemisch für präparative $\mathrm{HPLC}\left(\mathrm{H}_{2} \mathrm{O}\right.$ $(0.06 \% \mathrm{HCl})$ und $\mathrm{MeOH})$ zu 108, 109 und 106. 
Eine mögliche Debenzylierung im Produkt unter Verwendung eines O-benzylierten Derivates und einer damit einhergehenden Verhinderung der Winstein-Zyklisierung wurde nicht durchgeführt, da es bei der Debenzylierung zu einer Dechlorierung kommt und eine Abtrennung des dechlorierten Nebenproduktes vom gewünschten Produkt nicht möglich ist. Auf Grund der beschriebenen Problematik wurde nach ersten Versuchen mit der Hünigbase und einer damit einhergehenden WinsteinZyklisierung erfolgreich versucht, eben diese durch den Einsatz einer schwächeren Base (Pyridin) zu umgehen. Auf diese Weise konnte das gewünschte Produkt $(1 S, 10 R)-71$ sowie dessen Enantiomer $(1 R, 10 S)$-71 in mäßigen bis guten Ausbeuten erhalten werden (Abbildung 50). 


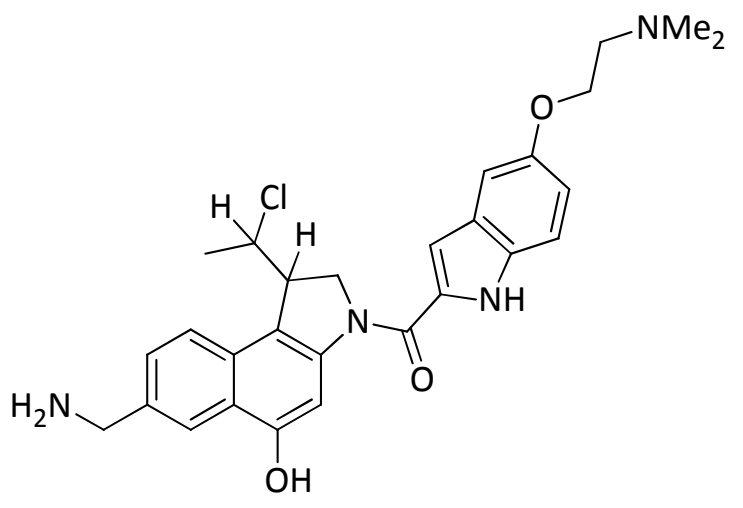

87

für (1S,10R)-87 und Verwendung von $i \operatorname{Pr}_{2} \mathrm{NEt}$

29\% $(1 S, 10 R)-71,13 \%(1 S, 10 R)-110,26 \%(1 S, 10 R)-111$

für $(1 R, 10 S)-87$ und Verwendung von Pyridin

$47 \%(1 R, 10 S)-71$<smiles>CSc1ccc(-c2ncc(-c3ccc(N(C)C)cc3)o2)cc1</smiles>

61, Base, DMF, RT, 23 h, prep. HPLC $38 \%(1 S, 10 R)-7120 \%(1 S, 10 R)-111$

89, Base, DMF, RT, 23 h, prep. HPLC

$$
\begin{array}{ll}
\mathrm{R}=\mathrm{Cl} & \mathbf{7 1} \\
\mathrm{R}=\mathrm{OMe} & 110 \\
\mathrm{R}=\mathrm{OH} & 111
\end{array}
$$

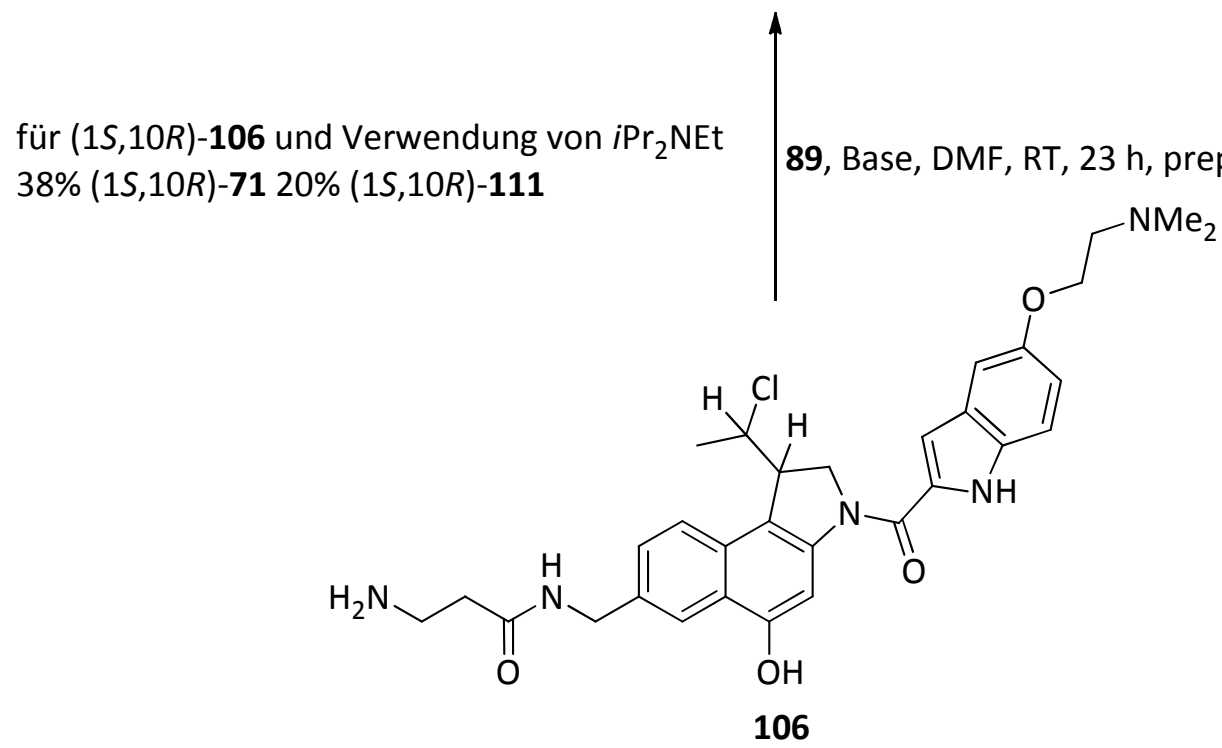

Abbildung 50. Synthese von 71 durch Verwendung von 87 und Dapoxylderivat 61 sowie 106 und Dapoxylderivat 89. 


\subsubsection{Diskussion ausgewählter spektroskopischer Daten des}

fluoreszenzmarkierten anti-Methyl-seco-Drugs $(1 R, 10 S)-71$

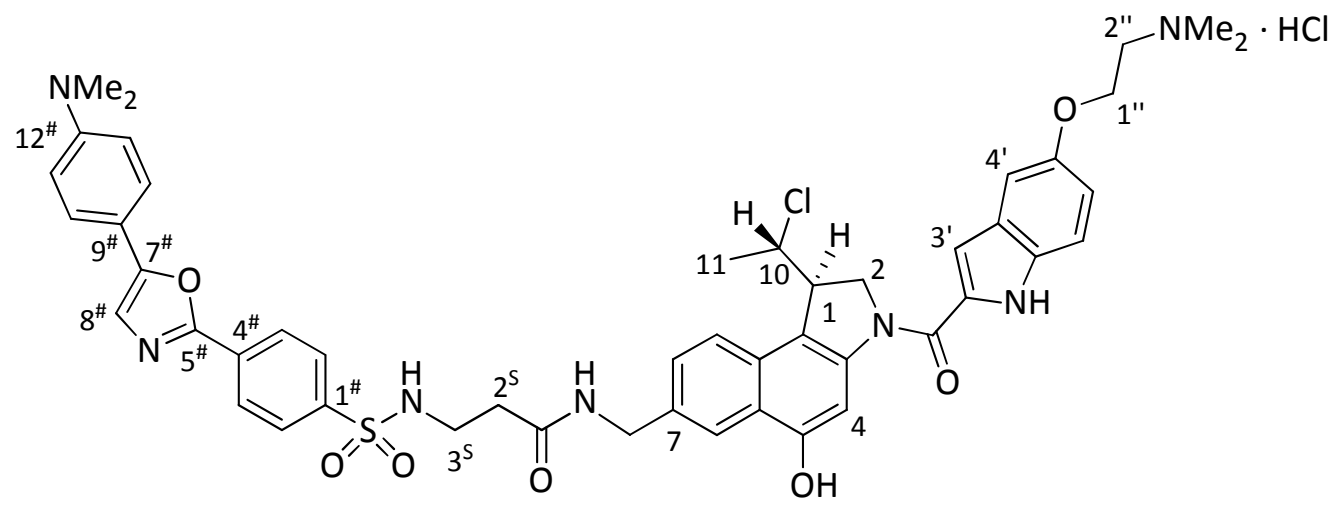

$(1 R, 10 S)-71$

Abbildung 51. Struktur des fluoreszenzmarkierten (1R,10S)-anti-Methyl-seco-Drugs $(1 R, 10 S)-\mathbf{7 1}$.

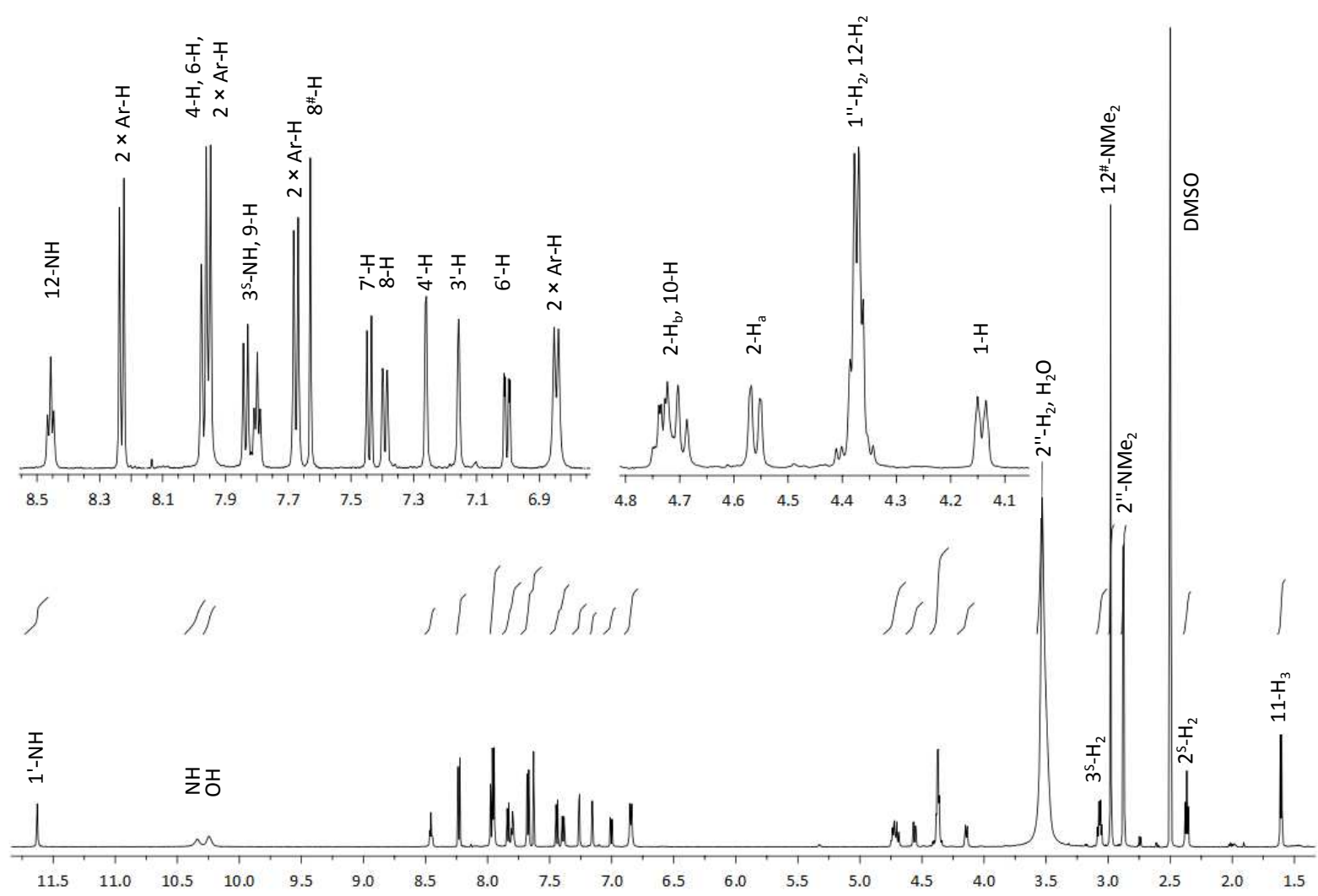

Abbildung 52. ${ }^{1} \mathrm{H}-\mathrm{NMR}$-Spektrum $\left(600 \mathrm{MHz}\right.$, DMSO- $\left.\mathrm{d}_{6}\right)$ des fluoreszenzmarkiertern $(1 R, 10 S)$-antiMethyl-seco-Drugs $(1 R, 10 S)-71$.

Im ${ }^{1} \mathrm{H}$-NMR-Spektrum von $(1 R, 10 S)-71$ findet man bei $\delta=1.61 \mathrm{ppm}$ ein Dublett mit einer Kopplungskonstante von $J=6.6 \mathrm{~Hz}$, das charakteristisch für die Methylgruppe 
der anti-Methyl-Verbindungen ist. Bei $\delta=2.37 \mathrm{ppm}$ resonieren als Triplett mit einer Kopplungskonstante von $J=7.4 \mathrm{~Hz}$ die Methylenprotonen an $\mathrm{C}-2^{\mathrm{S}}$. Bei $\delta=2.88 \mathrm{ppm}$ ist das Signal für die sechs Protonen der $\mathrm{NMe}_{2}$-Funktionalität des DNA-Binders DMAI als Dublett zu beobachten, während das Resonanzsignal für die Protonen der $\mathrm{NMe}_{2}{ }^{-}$ Gruppe des Fluorochroms bei $\delta=2.97 \mathrm{ppm}$ zu sehen ist. Es folgt das Signal für die Methylenprotonen an $\mathrm{C}-3^{\mathrm{S}}$ bei $\delta=3.07 \mathrm{ppm}$ als Doppeldublett sowie ein Multiplett für die Protonen der Methyleneinheit an C-2" bei $\delta=3.43-3.59$. Die weiteren Signale für die $\mathrm{CBI}$-Einheit findet man bei $\delta=4.14 \mathrm{ppm}$ resoniert ein Dublett für $1-\mathrm{H}$ mit einer Kopplungs-konstante von $J=9.2 \mathrm{~Hz}$, was durch eine Kopplung mit dem Proton an C10 erklärt werden kann, dessen Resonanz im Multiplett bei $\delta=4.67-4.77$ ppm zu sehen ist. Bei $\delta=4.32-4.42 \mathrm{ppm}$ ist ein Multiplett zu beobachten, dass den Protonen an C-1" sowie den beiden Methylenprotonen an C-12 zugewiesen werden kann. Weiter im Tieffeld folgt die beschriebene Resonanz für das Proton an C-1, sowie die Signale für die diastereotopen Protonen an C-2. Zunächst findet man bei $\delta=4.56 \mathrm{ppm}$ ein Dublett mit einer Kopplungskonstante von $J=9.2 \mathrm{~Hz}$ für die geminale Kopplung von $2-\mathrm{H}_{\mathrm{a}} \mathrm{zu} 1-\mathrm{H}$ sowie bei $\delta=4.67-4.77 \mathrm{ppm}$ die Resonanz für 2$\mathrm{H}_{\mathrm{b}}$ zusammen mit 10-H als Mutiplett. Im typischen Bereich der Aromaten findet man bei $\delta=6.85 \mathrm{ppm}$ ein Dublett mit einer Kopplungskonstante von $J=8.4 \mathrm{~Hz}$ und einer Intensität von 2 Protonen, was darauf schließen lässt, dass es sich hierbei im zwei chemisch identische Protonen mit einer ortho-Kopplung handelt. Hierbei muss es sich also um zwei aromatische Protonen des Fluorochroms handeln. Es folgt bei $\delta=7.00$ ppm ein Dublett vom Dublett mit $J=8.9$ und $2.3 \mathrm{~Hz}$, das auf Grund seiner chemischen Verschiebung, Kopplungskonstanten und der Kopplungspartner aus dem Korrelationsspektrum COSY eindeutig dem 6'-H zugewiesen werden kann. Bei $\delta=7.16 \mathrm{ppm}$ folgt ein Singulett, das dem Proton an C-3' des DNA-Binders DMAI zugeordnet werden kann. Bei $\delta=7.26 \mathrm{ppm}$ etwas weiter im Tieffeld folgt das Resonanzsignal für 4'-H als Dublett mit $J=2.0 \mathrm{~Hz}$, das durch eine meta-Kopplung mit 6 '-H hervorgerufen wird. Mit einer chemischen Verschiebung von $\delta=7.39 \mathrm{ppm}$ ist das Signal für das 8-H Proton des CBI-Grundkörpers als Dublett mit $J=8.8 \mathrm{~Hz} z u$ 
beobachten. Bei $\delta=7.44 \mathrm{ppm}$ ist das Resonanzsignal für 7'- $\mathrm{H}$ zu beobachten, welches durch ortho-Kopplung mit $6^{\prime}-\mathrm{H}$ als Dublett mit $J=8.9 \mathrm{~Hz}$ entsteht. Es folgen die Signale für die Protonen des Fluorochroms bei $\delta=7.63 \mathrm{ppm}$ für $8^{\#}-\mathrm{H}$ als Singulett sowie als Dublett mit $J=8.7 \mathrm{~Hz}$ bei $\delta=7.68 \mathrm{ppm}$ für zwei weitere aromatische Protonen. Ein Multiplett bei $\delta=7.77-7.85 \mathrm{ppm}$ mit einem Integral für zwei Protonen steht für das Proton an C-9 sowie dem Amin an C-3 ${ }^{5}$. Ein weiteres Multiplett bei $\delta=7.93-7-99$ ppm mit einem Integral für vier Protonen steht für die Protonen an C-4 und C-6 sowie zwei weiteren Arylprotonen des Fluorochroms, gefolgt von zwei aromatische Protonen des Fluorochroms bei $\delta=8.23 \mathrm{ppm}$ mit einer typischen Kopplungskonstante für ortho-Kopplungen mit $J=8.4 \mathrm{~Hz}$. Es folgt bei $\delta=8.46 \mathrm{ppm}$ ein Triplett mit $J=5.8 \mathrm{~Hz}$ als Signal für die Aminprotonen an C-12. Bei $\delta=10.25,10.34$ und $11.63 \mathrm{ppm}$ folgen die Resonanzen für die phenolische Hydroxyfunktion, das Proton an der $\mathrm{NMe}_{2}$-Gruppierung des DNA-Binders DMAl sowie für das IndolAminproton. 


\section{Charakterisierung und Untersuchung der zellulären Aufnahme von fluoreszenzmarkierten Dapoxyl-anti-Methyl-seco-CBI- DMAI-Derivaten}

\subsection{Fluorimetrische Bestimmung der Absorptions- und Emissionsmaxima}

Zunächst wurden die Absorptions- und Emissionsspektren der zu untersuchenden Verbindungen $(1 S, 10 R)-71,(1 R, 10 S)-71,(1 S, 10 R)-110$ sowie $(1 S, 10 R)-111$ (Abbildung 53) sowohl in Methanol als auch in PBS gemessen.

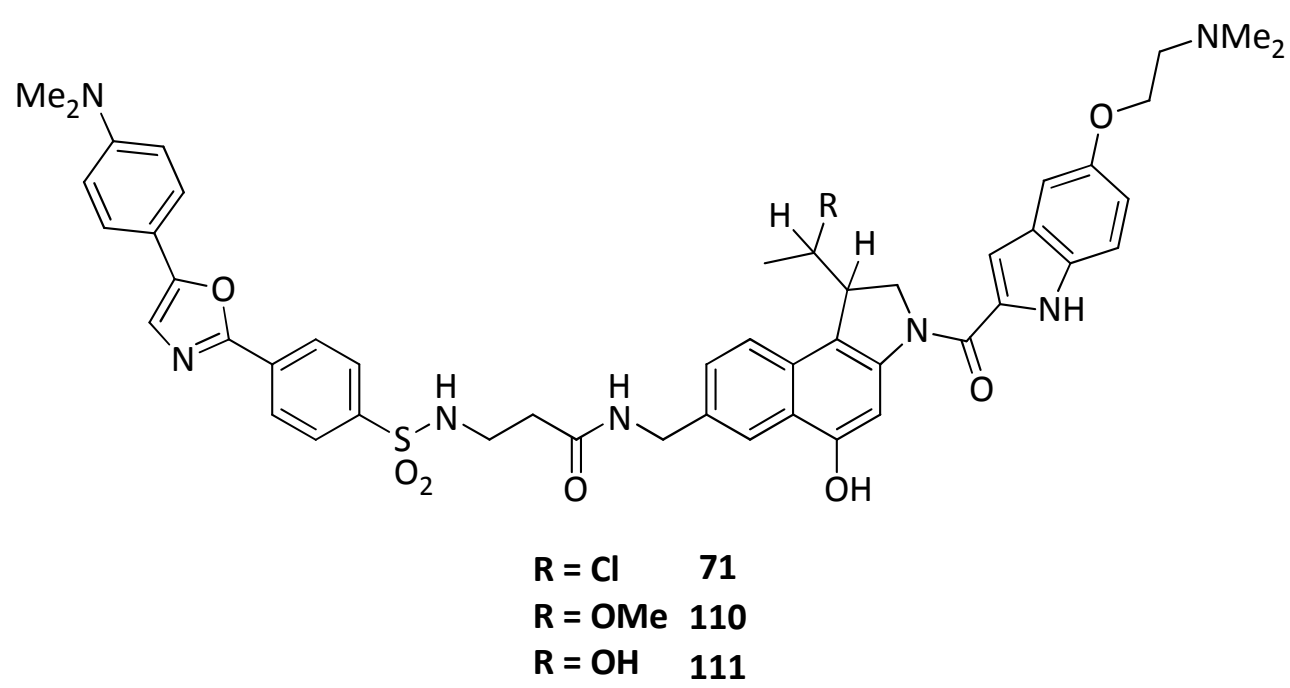

Abbildung 53. Struktur der untersuchten fluoreszenzmarkierten Derivate.

Hierbei konnte gezeigt werden, dass sich die synthetisierten Verbindungen in ihren Absorptions- und Emissionsmaxima kaum unterscheiden. In Abbildung 54 sind stellvertretend die Absorptions- und Emssionsspektren für die Verbindung (1S,10R)-71 zu sehen. In Methanol findet man das Absorptionsmaximum bei $\lambda_{\text {max,Abs }}=376 \mathrm{~nm}$, während es in wässrigem Puffer bei $\lambda_{\text {max,Abs }}=366 \mathrm{~nm}$ zu finden ist. Außerdem fällt auf, dass das Emissionsmaximum in Methanol mit $\lambda_{\text {max }, \mathrm{Em}}=564 \mathrm{~nm}$ gegenüber dem in wässrigem Bildgebungspuffer mit $\lambda_{\max , \mathrm{Em}}=534 \mathrm{~nm}$ um $32 \mathrm{~nm}$ verschoben ist, was gleichzeitig zu einer Verringerung des Stokes-Shifts um $50 \mathrm{~nm}$ auf $138 \mathrm{~nm}$ führt. 

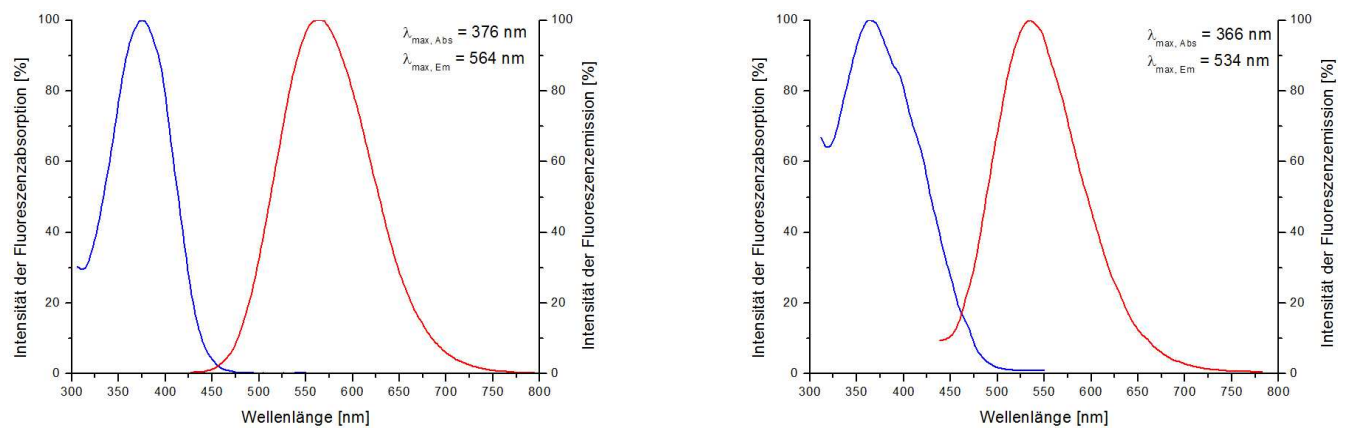

Abbildung 54. Fluoreszenzspektren von (1S,10R)-71 in MeOH (links) und wässrigem Bildgebungspuffer (rechts).

Betrachtet man eine Überlagung der Spektren, der synthetisierten Verbindungen $(1 S, 10 R)-71, \quad(1 R, 10 S)-71, \quad(1 S, 10 R)-110,(1 S, 10 R)-111$ sowie der von $B$. Krewer synthetisierten Verbindung $(1 S, 10 R)-71$, fällt auf, dass die Spektren sehr gut übereinstimmen. Eine Ausnahme bildet hier jedoch die Probe aus (1S,10R)-71 und einem unbekannten Nebenprodukt von B. Krewer, die über ein anderes Verfahren synthetisiert wurde (s. Kapitel 7.2) und neben den normalen Maxima ein weiteres Absorptionsmaximum bei $\lambda_{\max , A b s}=525 \mathrm{~nm}$ (Abbildung 55) besitzt.

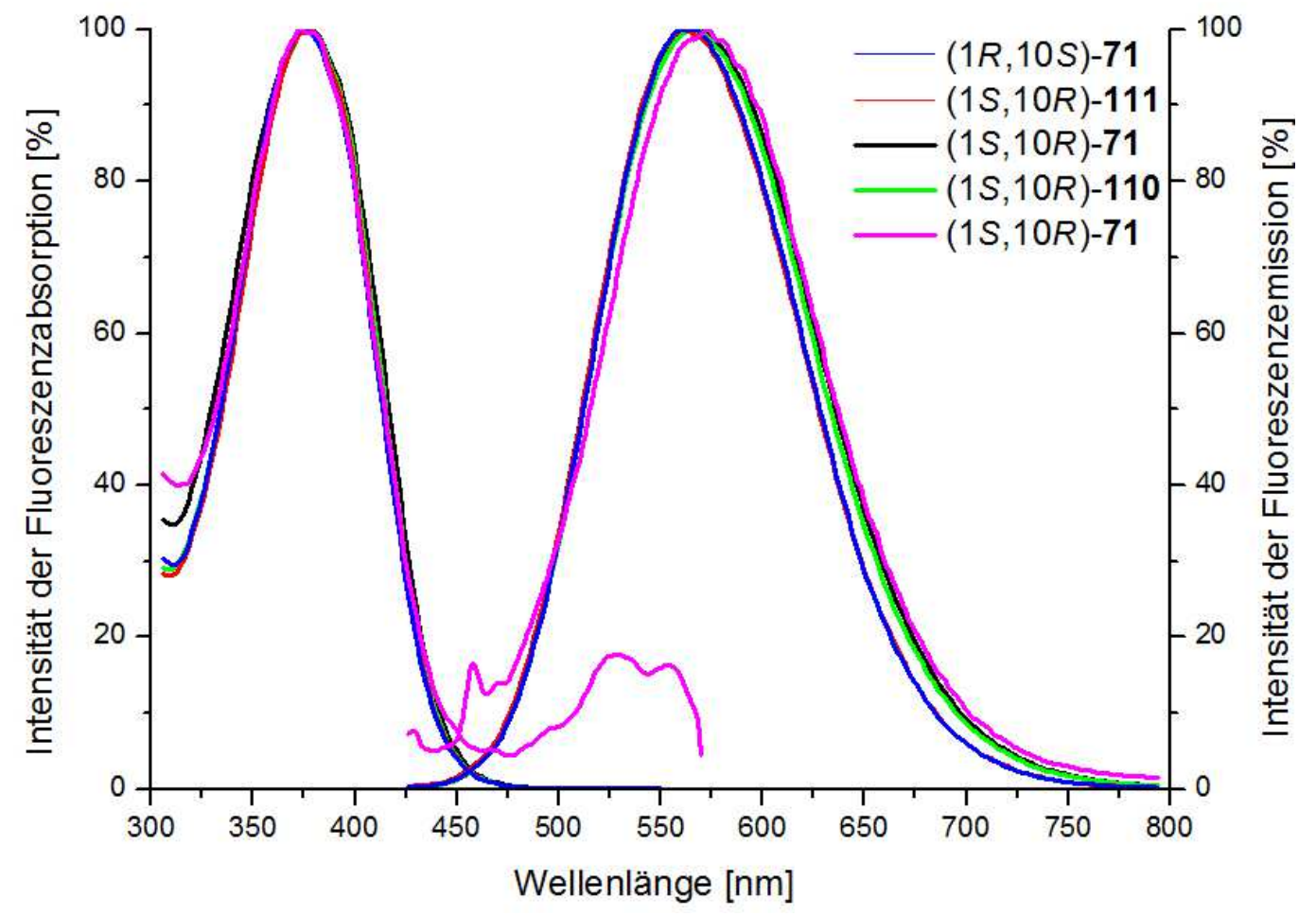

Abbildung 55. Flurorimetrische Bestimmung der Absorptions- und Emissionsspektren in $\mathrm{MeOH}$ für die in dieser Arbeit synthetisierten Verbindungen (blaue, rote, schwarze und grüne Linie) sowie der von B. Krewer synthetisierten Verbindung (magentafarbene Linie). 
Auf Grund der fluorimetrischen sowie der LC-MS-spektroskopischen Untersuchungen der Proben lässt sich festhalten, dass es bei der Synthese von $(1 S, 10 R)-71$ über das Galaktosid und dessen Abspaltung unter harschen Bedingungen $(\mathrm{MeOH}$, konzentrierte $\mathrm{HCl}$ für 6 Tage) zur Bildung eines unerwünschten Nebenproduktes kommt, dem die zusätzliche Absorptionsbande zugeschrieben werden muss.

\subsection{Untersuchung der zellulären Aufnahme fluroreszenzmarkierter anti- Methyl-seco-CBI-DMAI-Derivate in lebende Zellen durch CLSM}

B. Krewer konnte mit der von ihr entwickelten Synthese zur Darstellung von fluoreszenzmarkiertem anti-Methyl-seco-CBI-DMAI-Derivat $\mathbf{7 1}$ eine bis dahin unbekannte Zweifachfärbung untersuchen. Die verunreinigte Probe zeigte zum Einen Fluoreszenz bei $\lambda_{\text {max,Abs }}=405 \mathrm{~nm}$ und $\lambda_{\text {max }, \mathrm{Em}}=500 \mathrm{~nm}$ sowie zum Anderen bei $\lambda_{\text {max }, \mathrm{Abs}}=514 \mathrm{~nm}$ und $\lambda_{\max , \mathrm{Em}}=560 \mathrm{~nm}$. Während die erste erwähnte Fluoreszenz in Zellregionen wie Endosomen und Lysosomen beobachtet werden konnte, wurde die zweite Fluoreszenz in Mitochondrien beobachtet (Abbildung 56).
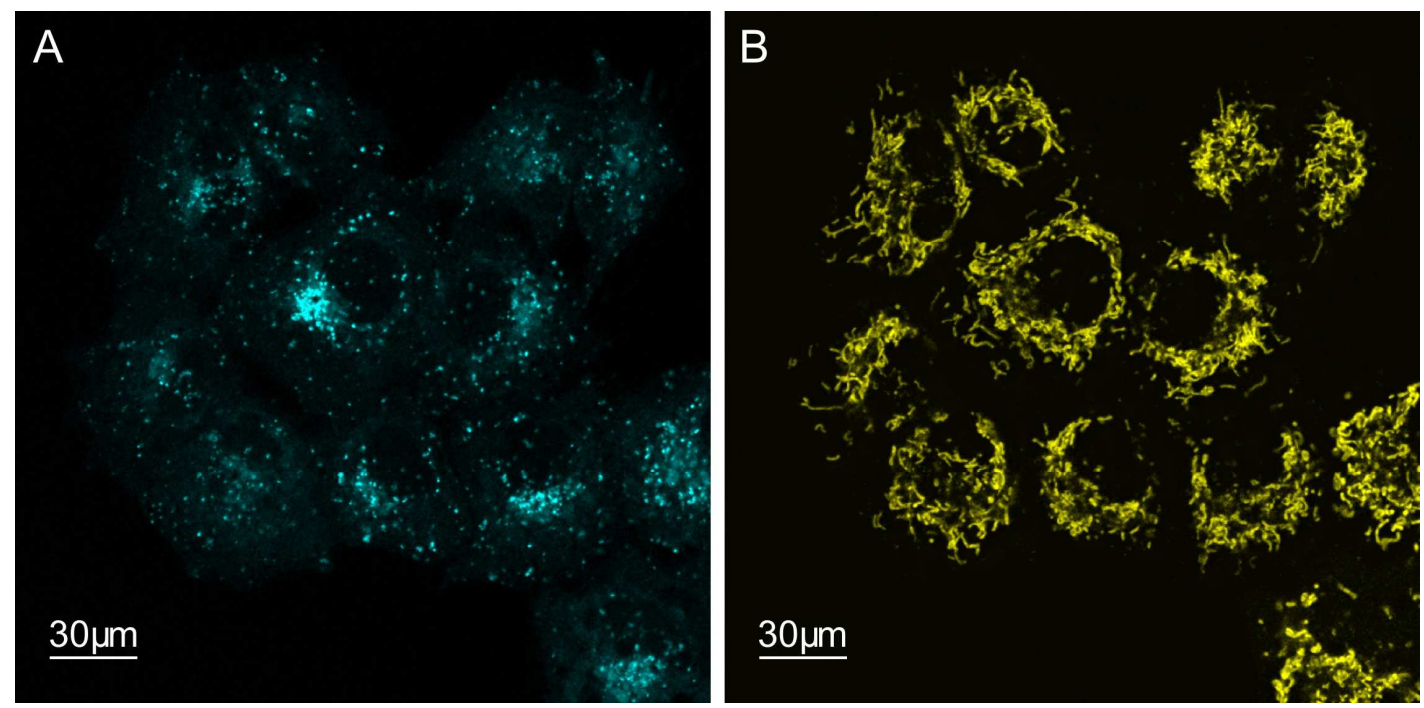

Abbildung 56. Intrazelluläre Verteilung des Gemisches aus Zwei-Farb-Fluoreszenzmarkers $(1 S, 10 R)-71$ und eines nicht identifizierbaren Nebenproduktes in A549 Zellen. Endosomale Vesikel werden sichtbar bei $\lambda_{\mathrm{abs}}=405 \mathrm{~nm}, \lambda_{\mathrm{em}}=500 \mathrm{~nm}$ (A) während bei $\lambda_{\text {abs }}=514 \mathrm{~nm}$ und $\lambda_{\mathrm{em}}=560 \mathrm{~nm}$ Mitochondrien sichtbar werden (B). 
Die durch das neue Synthesekonzept hergestellten Derivate $(1 S, 10 R)-\mathbf{7 1},(1 R, 10 S)-\mathbf{7 1}$, $(1 S, 10 R)-110$ und $(1 S, 10 R)-111$, sowie die von $B$. Krewer synthetisierte verunreinigte Probe von $(1 S, 10 R)-71$ und einem Nebenprodukt wurden mit einem KonfokalMikroskop untersucht. Obwohl intrazelluläre Vesikel bei den, in dieser Arbeit synthetisierten Substanzen bei $\lambda_{\mathrm{abs}}=405 \mathrm{~nm}, \lambda_{\mathrm{em}}=500 \mathrm{~nm}$ sichtbar werden (linke Spalte), sind, mit Ausnahme von Autofluoreszenz keine fluoreszierenden Spezies bei $\lambda_{\mathrm{abs}}=514 \mathrm{~nm}, \lambda_{\mathrm{em}}=560 \mathrm{~nm}$ vorhanden (rechte Spalte Abbildungen 57 und 58). Auf Grund der in dieser Arbeit durchgeführten synthetischen, wie auch fluoreszenzmikroskopischen Untersuchungen konnte festgestellt werden, dass es sich bei $(1 S, 10 R)$-71 nicht um einen Zweifarb-Fluoreszenzmarker handelt, da die zweite Zellfärbung durch ein nicht indentifizierbares Nebenprodukt hervorgerufen wird.

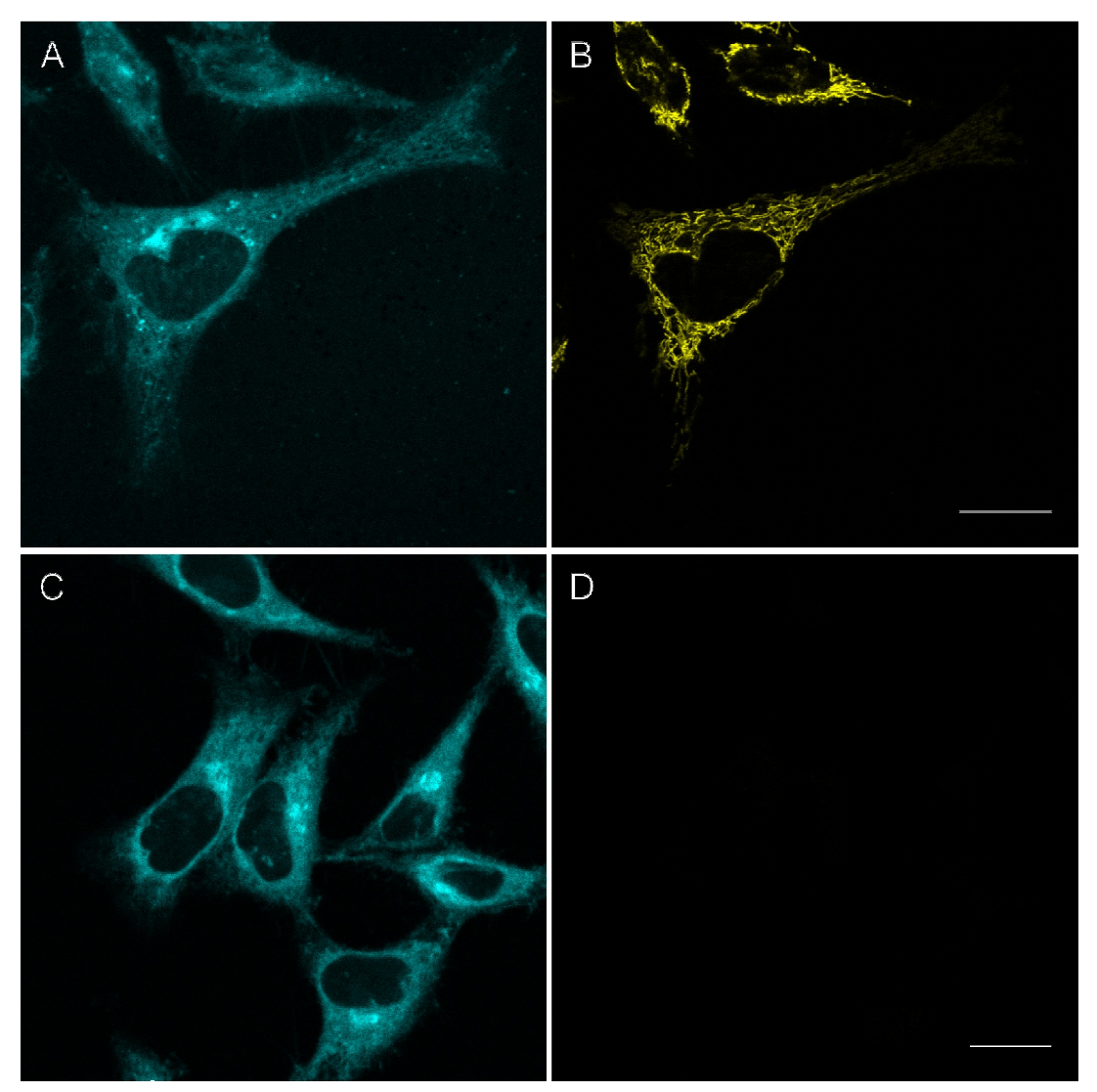

Abbildung 57. Untersuchung der zellulären Aufnahme von einem Gemisch aus (1S,10R)-71 und nicht identifizierbarem Nebenprodukt ( $A$ und $B$ ) sowie von (1S,10R)-71 (C und D). Maßstab $20 \mu \mathrm{m}$. 

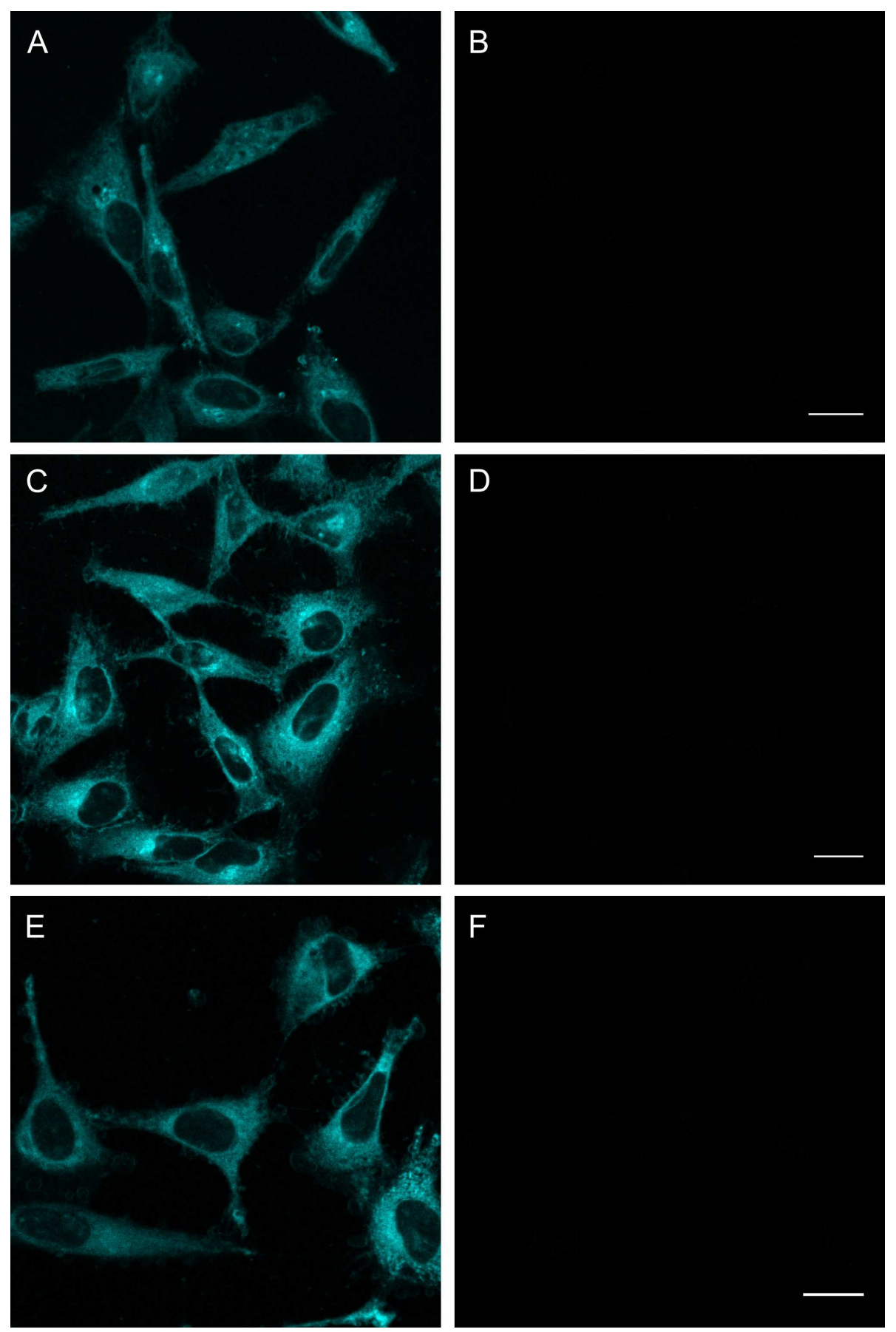

Abbildung 58. Untersuchung der zellulären Aufnahme der synthetisierten Substanzen $(1 S, 10 R)-110$ (A und $B),(1 S, 10 R)-111$ ( $C$ und $D)$ sowie $(1 R, 10 S)-71$ (E und $F$ ). Maßstab $20 \mu \mathrm{m}$. 


\section{Synthese des fluoreszenzmarkierten Prodrugs (1S)-72}

\subsection{Synthese des seco-CCBI-Grundgerüstes (1S)-115}

Ausgehend vom N-Boc geschützten Naphthalin-Derivat 112 konnte das seco-CCBIGrundgerüst (1S)-115 in Anlehnung an Arbeiten aus den Arbeitskreisen Tietze und Boger dargestellt werden. ${ }^{134 f, 153}$

Hierzu musste 112 zunächst regioselektiv an C-1 iodiert werden. Lösen des Naphthalins 112 in einem 1:1 Gemisch aus $\mathrm{MeOH}$ und THF, sowie Versetzen bei Raumtemperatur mit $N$-lod-Succinimid und katalytischen Mengen Toluolsulfonsäure lieferte das Produkt 75 (Abbildung 59). Da es sich hierbei um eine literaturbekannte Stufe handelte, wurde auf Grund der großen Ansatzmenge auf eine Aufreinigung durch Säulenchromatograhpie verzichtet.<smiles>N#Cc1ccc2c(Cc3ccccc3)cc(NC(=O)OCc3ccccc3)cc2c1</smiles>

112
$\mathrm{NIS}, \mathrm{TsOH} \cdot \mathrm{H}_{2} \mathrm{O}$,

$\mathrm{MeOH} / \mathrm{THF}(1: 1)$,

$50^{\circ} \mathrm{C}, 1.5 \mathrm{~h}$<smiles>CC(C)(C)Oc1cc(NC(=O)OCc2ccccc2)c(I)c2cc(C#N)ccc12</smiles>

Abbildung 59. lodierung des N-Boc geschützten Naphthalins 112.

Da anschließende Versuche zur enantiomerenreinen Darstellung fehlschlugen ${ }^{4 e}$, musste auf die Kupplung mit (E/Z)-1,3-Dichlorpropen (113) und anschließende radikalische Zyklisierung zum gewünschten seco-CCBI-Grundgerüst (1S)-74 zurückgegriffen werden (Abbildung 60).

Hierzu wurde der lodaromat $\mathbf{7 5}$ bei Raumtemperatur in DMF mit $\mathrm{NaH}$ versetzt und anschließend mit (E/Z)-1,3-Dichlorpropen (103) alkyliert. Das resultierende Alken 104 konnte unter radikalischen Bedinungen mit AIBN (Azobisisobutyronitril) und TTMSS (Tetra-Tri-Methyl-Silyl-Silan) in Toluol bei $80^{\circ} \mathrm{C}$ zum gewünschten rac-seco-CBI- 
Grundgerüst rac-(1S)-74 zyklisiert werden. Anschließende Trennung der Enantiomere durch HPLC an chiraler stationärer Phase (Chiralpak IA) und Debenzylierung durch hydrogenolytische Spaltung durch $\mathrm{H}_{2}$ und Palladium auf Aktivkohle bei Raumtemperatur in Essigester lieferte das gewünschte Produkt (1S)-115.

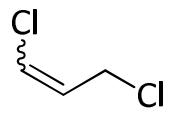

113<smiles>CC(C)(C)OC(=O)Nc1cc(OCc2ccccc2)c2ccc(C#N)cc2c1I</smiles>

75

1. $\mathrm{NaH}, \mathrm{DMF}, \mathrm{RT}, 1.5 \mathrm{~h}$

2. $113, \mathrm{RT}, 14 \mathrm{~h}$

$77 \%$<smiles>CC(C)(C)OC(=O)N(C/C=C/Cl)c1cc(OCc2ccccc2)c2ccc(C#N)cc2c1I</smiles>

114<smiles>CC(C)(C)OC(=O)N1C[C@H](CCl)c2c1cc(O)c1ccc(C#N)cc21</smiles>

(1S)-115
1. Trennung der Enantiomere an chiralerer stationärer Phase EtOAc, RT, $5 \mathrm{~h}$ $90 \%$

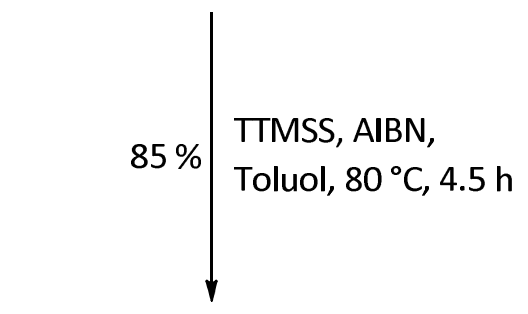<smiles>CC(C)(C)OC(=O)N1CC(CCl)c2c1cc(OCc1ccccc1)c1ccc(C#N)cc21</smiles>

74

Abbildung 60. Syntheseschema zur Darstellung des enantiomerenreinen seco-CCBI-Grundgerüstes (1S)-115.

\subsection{Synthese des CBI-DMAI-Prodrugs (1S)-118}

Das freie Phenol (1S)-115 wurde durch $\mathrm{BF}_{3} \cdot \mathrm{OEt}_{2}$ vermittelte Abspaltung der $\mathrm{N}$-BocSchutzgruppe in dessen sekundäres Amin überführt, das im Anschluss mit DMAI- $\mathrm{CO}_{2} \mathrm{H}$ (104) und EDAC. $\mathrm{HCl}$ als Kupplungsreagenzien sowie Glykosidierung nach der Trichloracetimidatmethode nach Schmidt ${ }^{157}$ mit 116 das tetraacetylierte Galaktosid (1S)-73 in einer Ausbeute von 53\% lieferte (Abbildung 61). 
<smiles>CC(C)(C)OC(=O)N1C[C@@H](CCl)c2c1cc(O)c1ccc(C#N)cc21</smiles>

(1S)-115

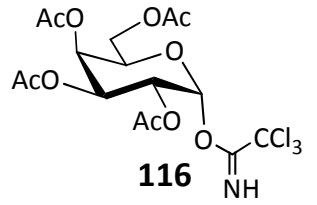

1. 116, $\mathrm{BF}_{3} \cdot \mathrm{OEt}_{2}, \mathrm{CH}_{2} \mathrm{Cl}_{2}$, $\operatorname{MS}(4 \AA),-10{ }^{\circ} \mathrm{C}, 3.5 \mathrm{~h}$ dann $\mathrm{BF}_{3} \cdot \mathrm{OEt}_{2}, \mathrm{RT}, 6 \mathrm{~h}$

2. 104, EDAC. $\mathrm{HCl}, \mathrm{DMF}, \mathrm{RT}$, $18 \mathrm{~h}$

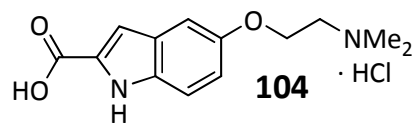

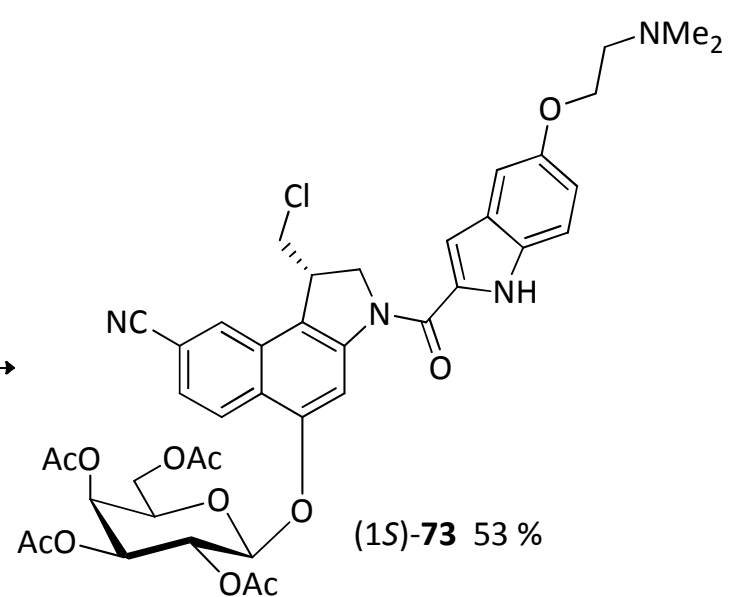

Abbildung 61. Syntheseschema zur Darstellung des tetraacetylierten Galaktosids (1S)-73 ausgehend vom Phenol (1S)-115.

Anschließende Reduktion des Nitrils in (1S)-73 unter Verwendung von $\mathrm{PtO}_{2} \cdot \mathrm{H}_{2} \mathrm{O}$ und $\mathrm{H}_{2}$ in Ethanol für $48 \mathrm{~h}$ bei Raumtemperatur sowie basische Spaltung der Acetylschutzgruppen in (1S)-117 durch Natriummethanolat in Methanol lieferte das Galaktosid (1S)-118 mit einer Ausbeute von 84\% (Abbildung 62). Hierdurch konnte die primäre Aminofunktion dargestellt werden, die zur Kupplung mit dem gewählten Fluorochrom 119 essentiell ist.
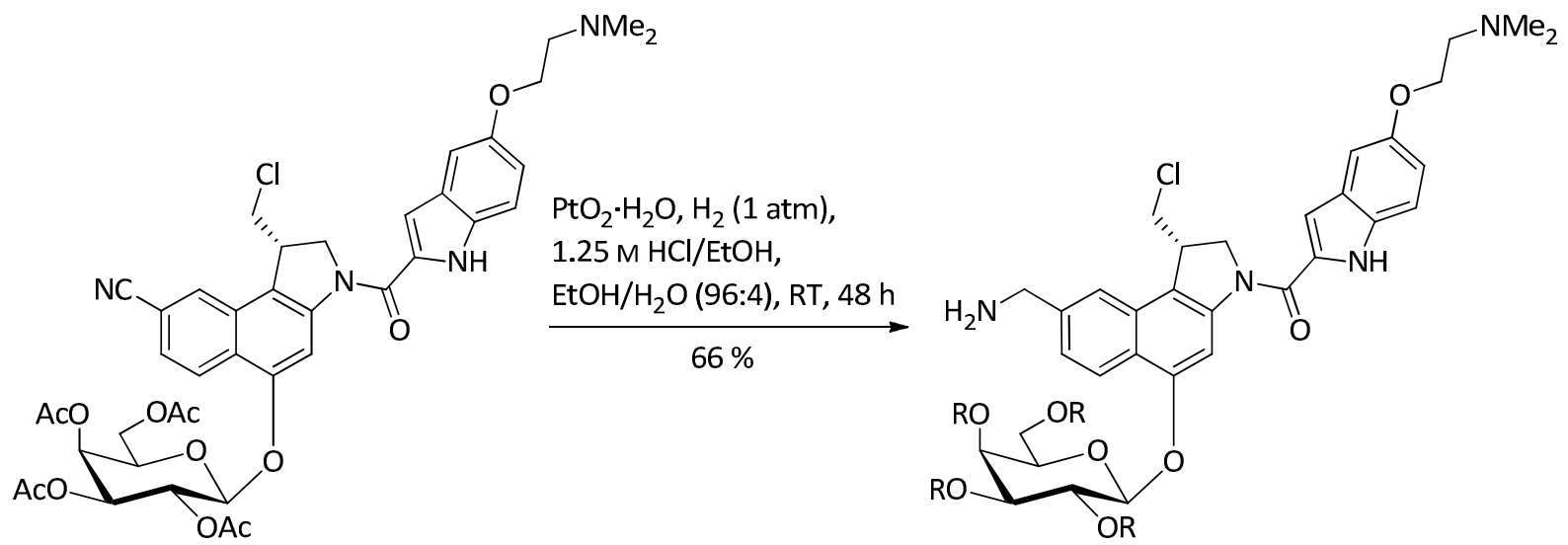

$(15)-73$

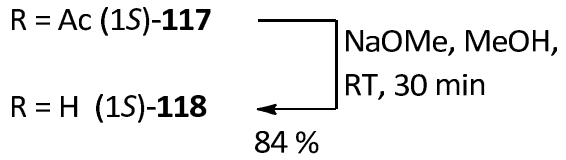

Abbildung 62. Syntheseschema zur Darstellung des Galaktosides (1S)-118. 


\subsection{Synthese des fluoreszenzmarkierten CBI-DMAI-Prodrugs (1S)-72}

Zur Darstellung des gewünschten fluoreszenzmarkierten (1S)-CBI-DMAI-Galaktosides (1S)-72 wurde das Amin (1S)-118 mit dem Fluorescein-Derivat 119 umgesetzt. Deprotonierung des Amins mit Hünig-Base in DMF bei Raumtemperatur und Versetzen mit einer Lösung des $\mathrm{N}$-Hydroxy-Succinimid-Esters 119 in DMF für $18 \mathrm{~h}$ lieferte das gewünschte Galaktosid (1S)-72 in einer Ausbeute von 46\% nach präparativer HPLC.

(1S)-118

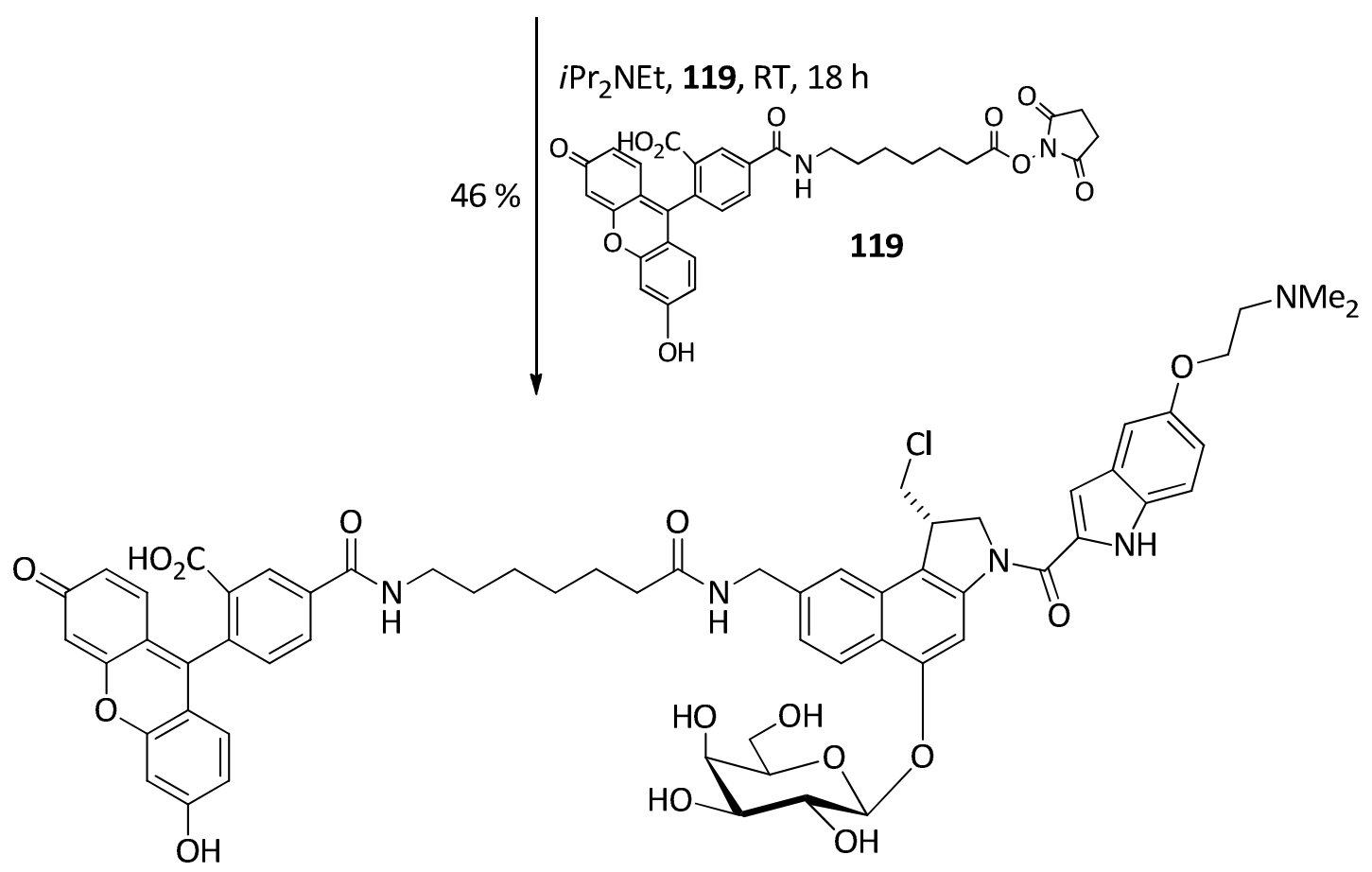

(1S)-72

Abbildung 63. Synthese des fluoreszenzmarkierten(1S)-CBI-DMAI-Galakosides (1S)-72. 


\subsection{In-vitro-Zytotoxizitätsuntersuchungen des fluoreszenzmarkierten (1S)- CBI-DMAI-Galaktosides (1S)-72 sowie des Galaktosides (1S)-118}

Die In-vitro-Zytotoxizität der dargestellten Verbindungen wurde von Dr. Ingrid Schuberth, Angela Rübeling und Anja Herdlitschke in der Abteilung für Zellbiologie am Institut für Organische und Biomolekulare Chemie unter Verwendung eines

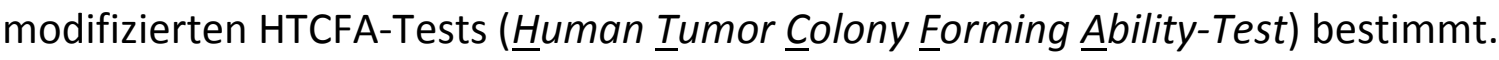

\subsubsection{Der HTCFA-Test zur Bestimmung der Zytotoxizität}

Für den Klonogenitätstest, der sich an den HTCFA-Test anlehnt und die Proliferationsfähigkeit von einzelnen Zellen widergibt (Abbildung 64) wurde das adhärent wachsende humane Bronchialkarzinom der Linie A549 verwendet. Die Aussaat der Tumorzellen erfolgte aus einer Stammkultur, in der sie in einem Kulturmedium (DMEM mit Zusatz von 10\% fötalem Kälberserum, $44 \mathrm{mM} \mathrm{NaHCO}$ und $4 \mathrm{mM}$ Glutamin) bei $37^{\circ} \mathrm{C}$ und $7.5 \% \mathrm{CO}_{2}$-Begasung in Luft permanent gehalten wurden, in Konzentrationen von $10^{2}$ bis $10^{4}$ Zellen in 6-well Multischalen. Nach 24 Stunden wurde das Kulturmedium entfernt, die Zellen einmal mit dem serumfreien Inkubationsmedium UltraCulture gewaschen und die Zellen anschließend in diesem Medium 24 Stunden mit zehn bis zwölf verschiedenen Konzentrationen (drei dekadische Größenordnungen) der zu testenden Substanz inkubiert. Diese wurde vorher frisch in einer DMSO-Stammlösung angesetzt und mit dem Inkubationsmedium verdünnt, so dass in den Näpfen letztlich eine DMSO-Konzentration von $1 \%$ vorlag. Nach Entfernen der Testsubstanz und einem weiteren Waschgang mit dem Kulturmedium wurden die verbliebenen Zellen 12 Tage in normalem Kulturmedium kultiviert. Anschließend wurde dieses entfernt, die gebildeten Klone wurden getrocknet, mit Löffler's Methylenblau angefärbt und ab einer Klongröße von ca. $500 \mu \mathrm{m}$ makroskopisch ausgezählt. Die Bestimmung der relativen Klonbildungsraten erfolgte anhand der in Abbildung 64 angegebenen Formel, wobei sich die Klonzahl 
des Kontrollversuches aus unbehandelten Zellen ergab und gleich $100 \%$ gesetzt wurde. Die Freisetzung der zytotoxischen Verbindungen aus den Prodrugs erfolgte durch Zugabe von $4.0 \mathrm{U} / \mathrm{mL} \beta$-D-Galaktosidase. Jede Versuchsreihe wurde in der Regel mindestens dreimal als Duplikat durchgeführt, um reproduzierbare Testergebnisse zu erhalten.

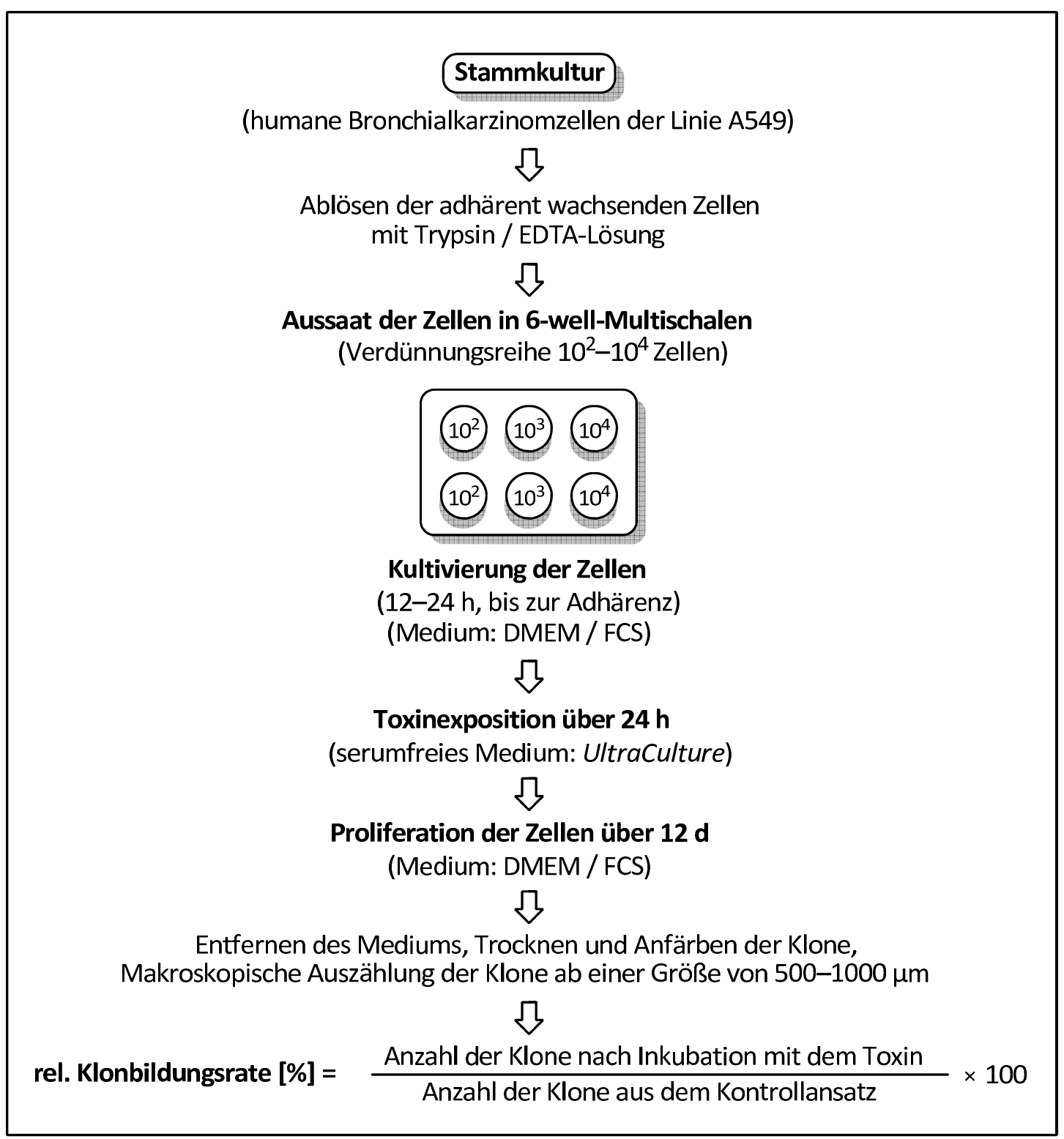

Abbildung 64. Schematische Darstellung des nach Dr. Ingrid Schuberth modifizierten HTCFA-Tests. 
3.4.2 Ergebnisse zur Untersuchung der In-vitro-Zytotoxizität an humanen Bronchialkarzinomzellen (A549) für das fluoreszenzmarkierte (1S)-CBI-DMAI-Galaktosid (1S)-72 sowie das Galaktosid (1S)-118

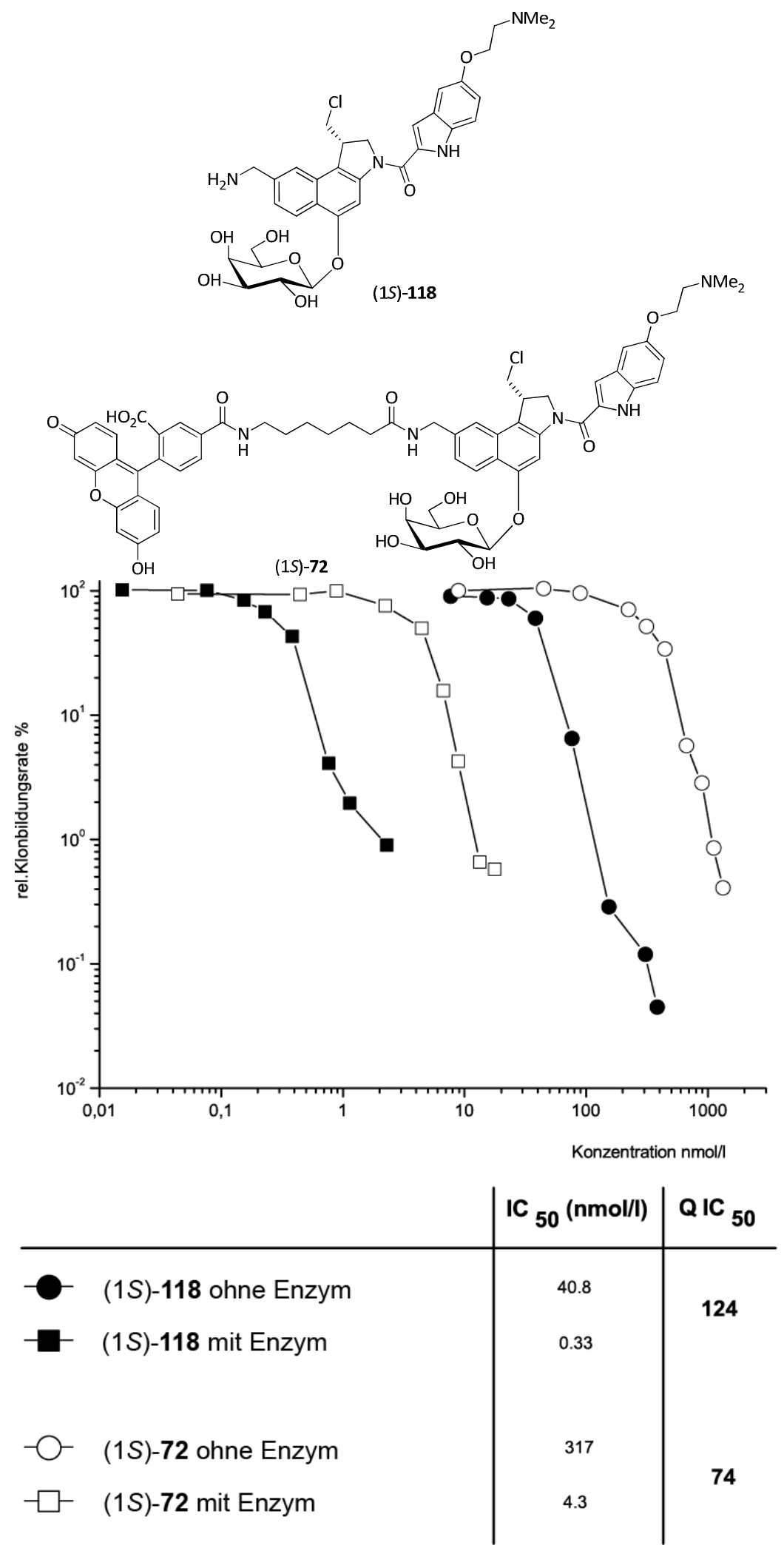

Abbildung 65. In-vitro-Zytotoxizitäten (A549) des fluoreszenzmarkierten (1S)-CBI-DMAI-Galaktosides (1S)-72 sowie des nicht markierten Galaktosides (1S)-118. 
Abbildung 65 zeigt die Ergebnisse der In vitro-Untersuchungen an humanen Bronchialkarzinomzellen (A549) für das fluoreszenzmarkierte (1S)-CBI-DMAIGalaktosid (1S)-72 sowie das nicht markierte Galaktosid (1S)-118.

Betrachtet man die IC $\mathrm{C}_{50}$-Werte für das Aminomethyl-Galaktosid (1S)-118, so fällt auf, dass es in Abwesenheit des Enzyms $\beta$-D-Galaktosidase, wie erwartet mit $\mathrm{IC}_{50}=40.8 \mathrm{nM}$ um einem Faktor 7.8 toxischer ist, als das fluoreszenzmarkierte Galaktosid (1S)-72 (IC $50=317 \mathrm{nM})$. Die Galaktoside (1S)-118 und (1S)-72 zeigen in Anwesenheit des Enzyms IC50-Werte von IC $50=0.33 \mathrm{nM}((1 S)-118)$ bzw. IC $C_{50}=4.3 \mathrm{nM}$ ((1S)-72) und damit QIC $_{50}$-Werte von QIC $_{50}=124$ für das (1S)-118 Galaktosid sowie $\mathrm{QIC}_{50}=74$ für das fluoreszenzmarkierte Galaktosid (1S)-72. Auf Grund der Kriterien für Wirkstoffe, die im Rahmen von ADEPT eingesetzt werden können $\left(\right.$ QIC $_{50}>1000$, $\left.\mathrm{IC}_{50}<10 \mathrm{nM}\right)$ kann festgestellt werden, dass sich die synthetisierten Verbindungen auf Grund der nur geringen $\mathrm{QIC}_{50}$-Werte nicht für eine Anwendung eignen. 


\section{Synthese des seco-CBI-Grundgerüstes (1S)-128}

Ausgehend von Benzaldehyd (97) sollte das seco-CBI-Grundgerüst (1S)-128 in Anlehnung an Arbeiten von Tietze und Boger dargestellt werden. ${ }^{134 f, 153}$ Auf Grund der großen Anzahl von Stufen bis zur Darstellung neuartiger fluoreszenzmarkierter CBIDuocarmycin-Analoga wurde die Synthese mit einer Ansatzgröße von $20 \mathrm{~g}$ Benzaldehyd (97) begonnen. Da es sich hierbei zum größten Teil um literaturbekannte Stufen handelte, wurde auf eine Aufreinigung durch Säulenchromatographie zur Einsparung von Zeit und Kosten verzichtet, wenn möglich aber eine Umkristallisation vorgenommen.

Das Alken 121 konnte in einer Wittig-Horner-Reaktion mit Benzaldehyd (97) E-selektiv dargestellt werden. Hierzu wurde das Phosphonat 120 mit Natriumhydrid in THF deprotoniert und Benzaldehyd (97) zugegeben. Anschließende Hydrolyse des tertButylesters 121 in einem Lösungsmittelgemisch aus Trifluoressigsäure und Wasser (9:1) für $20 \mathrm{~h}$ bei Raumtemperatur lieferte die Carbonsäure 96, die nach einer intramolekularen Friedel-Crafts-Acylierung durch Verwendung von Kaliumacetat in Essigsäureanhydrid das acetylgeschützte Naphthol 122 lieferte. Die Schutzgruppe in 122 wurde durch Kaliumcarbonat in $\mathrm{EtOH}$ und Rühren für $1 \mathrm{~h}$ unter Rückfluss entfernt und das erhaltene freie Phenol 123 mit Benzylbromid, katalytischen Mengen Tetrabutylammoniumiodid (TBAI) und Kaliumcarbonat in DMF zum benzylgeschütztem Naphthol 124 umgesetzt. Anschließende Verseifung des Ethylesters 124 durch $\mathrm{LiOH} \cdot \mathrm{H}_{2} \mathrm{O}$ in einem Lösungsmittelgemisch aus THF/MeOH/ $\mathrm{H}_{2} \mathrm{O}$ (3:1:1) lieferte nach 2 Tagen die Carbonsäure 125, welche nach Umkristallisation aus Essigester und $n$-Hexan mit einer Ausbeute von 53\% über 6 Stufen erhalten wurde (Abbildung 66). 


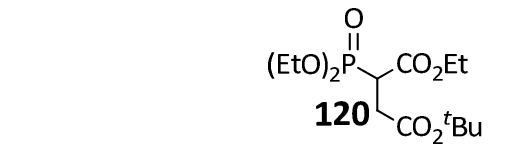

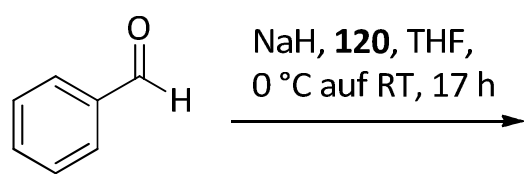

97<smiles>CCOC(=O)CC(=Cc1ccccc1)C(=O)OCC</smiles>

121

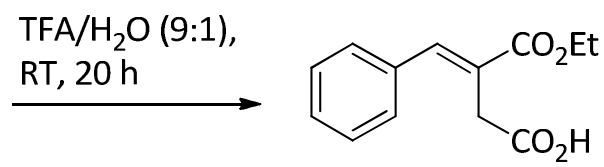

96 KOAc, $\mathrm{Ac}_{2} \mathrm{O}$, Rückfluss, $2.5 \mathrm{~h}$<smiles>CCCOC(=O)c1cc(OCc2ccccc2)c2ccccc2c1</smiles>

124<smiles>CCOC(=O)c1ccc2c(O)cc(C(=O)OCC)cc2c1</smiles>

123

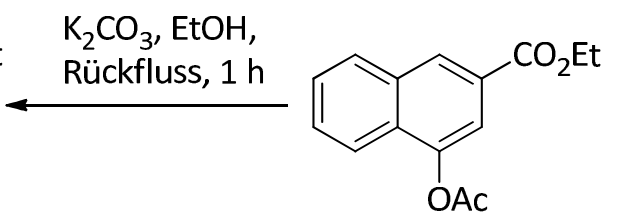

122<smiles>O=C(O)c1cc(OCc2ccccc2)c2ccccc2c1</smiles>

125

Abbildung 66. Syntheseschema zur Darstellung der Carbonsäure $\mathbf{1 2 5}$ ausgehend von Benzaldehyd (97) über 6 Stufen.

Die Überführung der Carbonsäure 125 in das N-Boc geschützte Naphthalin 126 wurde in einer Curtius-Umlagerung ermöglicht. Hierzu wurde Diphenylphosphorylazid (DPPA) als Azidquelle genutzt und mit der Carbonsäure 125, tert-Butanol sowie Molsieb (4 ̊̊) und Triethylamin für 3 Tage unter Rückfluss gerührt. Das Produkt 126 wurde nach Königstein ${ }^{158}$ durch Einsatz von lod und lodsäure in einem Lösungsmittelgemisch aus $\mathrm{MeOH}$ und $\mathrm{H}_{2} \mathrm{O}$ (4:1) regioselektiv an C-4 zu 95 iodiert (Abbildung 67). 


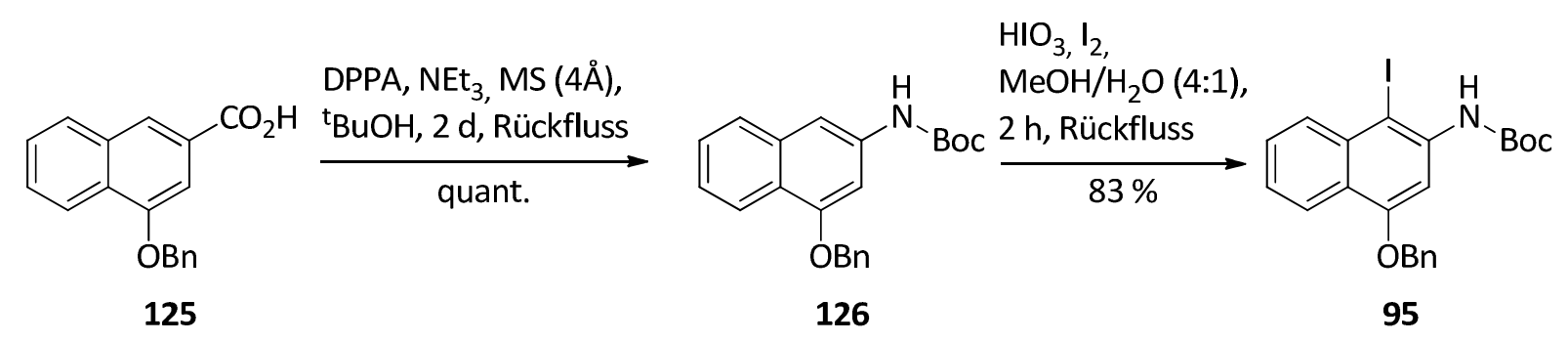

Abbildung 67. Synthese des Aryliodids 95 durch Curtius-Umlagerung und anschließende lodierung nach Königstein.

Um den Zyklisierungsvorläufer 94 darzustellen, wurde das $N$-Boc geschütze Naphthalinderivat 95 durch Natriumhydrid in DMF deprotoniert und mit enantiomerenreinem Epoxynosylat 126 alkyliert. Anschließende Zinkat-vermittelte Zyklisierung zu (1S)-127 sowie Chlorierung durch Verwendung einer Appel-Reaktion zu (1S)-93 und abschließende Debenzylierung durch Palladium auf Aktivkohle und $\mathrm{H}_{2}$-Atmosphäre der naphtholischen Schutzgruppe lieferte das seco-CBI-Grundgerüst (-)-(1S)-128 (Abbildung 68). Anreichung des enantiomeren Überschusses sowie Abtrennung des dechlorierten Produktes wurde durch HPLC an chiraler stationärer Phase erreicht.

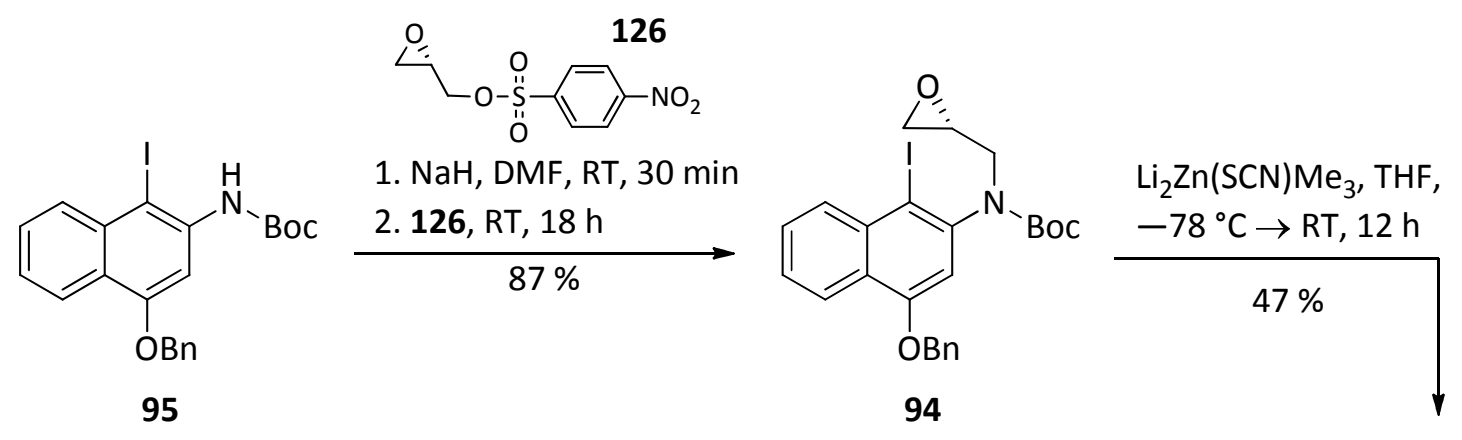<smiles>CC(C)(C)OC(=O)N1C[C@@H](CCl)c2c1cc(O)c1ccccc21</smiles>

(1S)-128<smiles>CC(C)(C)OC(=O)N1C[C@H](CCl)c2c1cc(OCc1ccccc1)c1ccccc21</smiles>

(1S)-93<smiles>CC(C)(C)OC(=O)N1C[C@H](CO)c2c1cc(OCc1ccccc1)c1ccccc21</smiles>

(1S)-127

Abbildung 68. Syntheseschema zur Darstellung des seco-CBI-Grundgerüstes (1S)-128 ausgehend von Aryliodid 95. 


\section{Synthese neuartiger fluoreszenzmarkierter CBI-Cumarin- Duocarmycin-Analoga}

Zur Untersuchung der Zellaufnahme von synthetischen Analoga der Duocarmycine, wurde der DNA-Binder DMAI- $\mathrm{CO}_{2} \mathrm{H}$ durch einen fluoreszenzativen DNA-Binder aus der Klasse der Cumarine ersetzt (Abbildung 69). Hierzu wurden drei verschiedene Cumarine ausgewählt. Zum einen die käuflich erwerbliche Cumarin-3-Carbonsäure (131) auf Grund des einfach Zugangs, das Dimethylaminocumarinsäure-Derivat DMACA (62), dass in vielen CLSM zur Aufklärung der zellulären Aufnahme von Naturstoffen ${ }^{159}$ sowie in Multiphotonenapplikationen ${ }^{160}$ eingesetzt wurde und auf Grund der Aminofunktion zu einer erhöhten Wasserlöslichkeit beitragen sollte. Außerdem wurde die in Abbildung 69 gezeigte Indol-Cumarin-2-Carbonsäure (130), die in seiner chemischen Struktur nur sehr gering von der, des DNA-Binders DMAI abweicht, eingesetzt, um die entsprechenden seco-Drugs sowie Galaktoside zu synthetisieren.
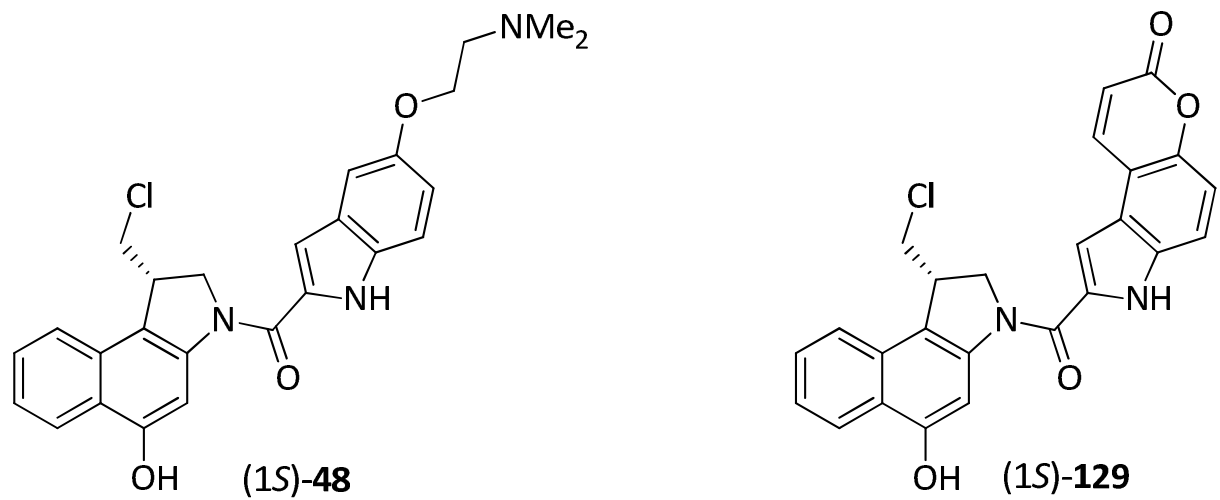<smiles>O=C(O)c1cc2c(ccc3oc(=O)ccc32)[nH]1</smiles>

130<smiles>O=C(O)c1cc2ccccc2oc1=O</smiles>

131<smiles>CN(C)c1ccc2c(CC(=O)O)cc(=O)oc2c1</smiles>

62

Abbildung 69. Strukturvergleich der seco-CBI-Analoga mit DMAI (1S)-48 oder Cumarin (1S)-129 als DNA-bindende Einheit sowie die Darstellung der verwendeten Cumarine: IndolCumarin-2-Carbonsäure (130), Cumarin-3-Carbonsäure (131) und DMACA (DimethylaminoCumarinicacid) (62). 


\subsection{Synthese der DNA-Binder Indol-Cumarin-2-Carbonsäure (130) sowie DMACA (62)}

Zur Darstellung des Cumarin-Derivates DMACA (62) wurde N,N-Dimethylaminophenol (132) in einer Pechmann-Kondensation mit 1,3-Aceton-Dicarboxylate (133) sowie $\mathrm{ZnCl}_{2}$ für $18 \mathrm{~h}$ unter Rückfluss gerührt und der entstandene Ethylester $134 \mathrm{im}$ Anschluss durch $\mathrm{LiOH} \cdot \mathrm{H}_{2} \mathrm{O}$ in einem Lösungsmittelgemisch aus THF/ $\mathrm{H}_{2} \mathrm{O}$ (3:1) zur Carbonsäure DMACA (62) mit einer Ausbeute von 12\% über 2 Stufen verseift (Abbildung 70). ${ }^{159 a}$

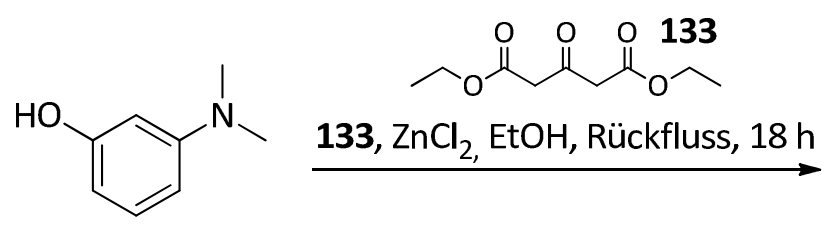

132<smiles>CN(C)c1ccc2c(CC(=O)O)cc(=O)oc2c1</smiles>

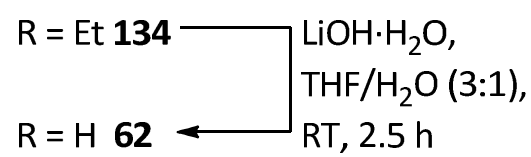

Abbildung 70. Synthese von DMACA (62) ausgehend von $m-N, N$-Dimethylaminophenol (132) über 2 Stufen mit einer Ausbeute von 12\%.

Indol-Cumarin-2-Carbonsäure (130) konnte in Schwefelsäure aus 5-Hydroxy-Indol-2Carbonsäure (135) und Äpfelsäure (136) in einer Mikrowellenapparatur dargestellt werden (Abbildung 71). ${ }^{161}$

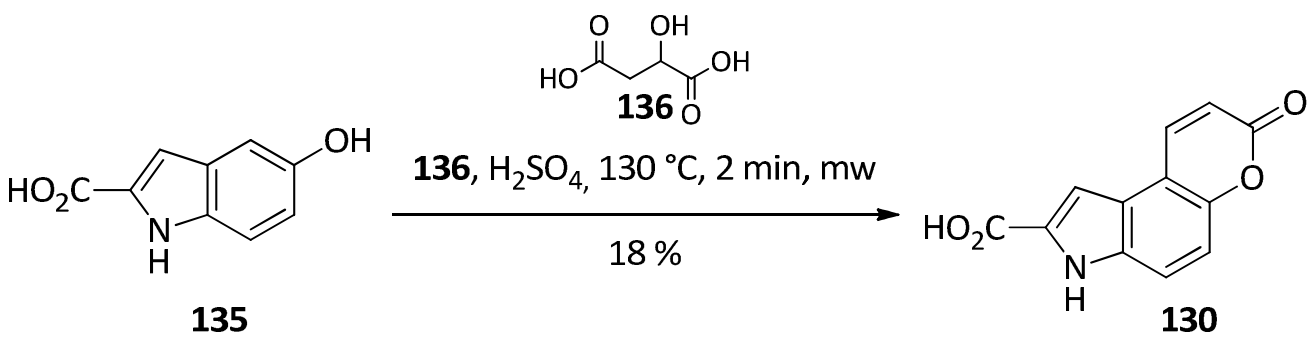

Abbildung 71. Synthese von IndolCumarin-2-Carbonsäure (130) ausgehend von 135 unter Verwendung einer Mikrowellenapparatur. 


\subsection{Synthese der seco-CBI-Cumarin-Drugs (1S)-92, (1S)-137 und (1S)-138}

Ausgehend vom Phenol (1S)-128 konnten die seco-CBI-Cumarin-Drugs (1S)-92, (1S)137 und (1S)-138 über 2 Stufen hergestellt werden. So wurde zunächst die N-BocSchutzgruppe durch Suspendieren des Phenols (1S)-128 in $4 \mathrm{M} \mathrm{HCl}$ /Essigester-Lösung entfernt, um das freie sekundäre Amin im Anschluss unter Verwendung von EDAC. $\mathrm{HCl}$ als Kupplungsreagenz mit der Carbonsäure des Cumarins (131, 130 oder 62) zu kuppeln. Abschließend wurden alle erhaltenen seco-CBI-Drugs durch präparative HPLC (Kromasil 100 C-18) gereinigt (Abbildung 72).

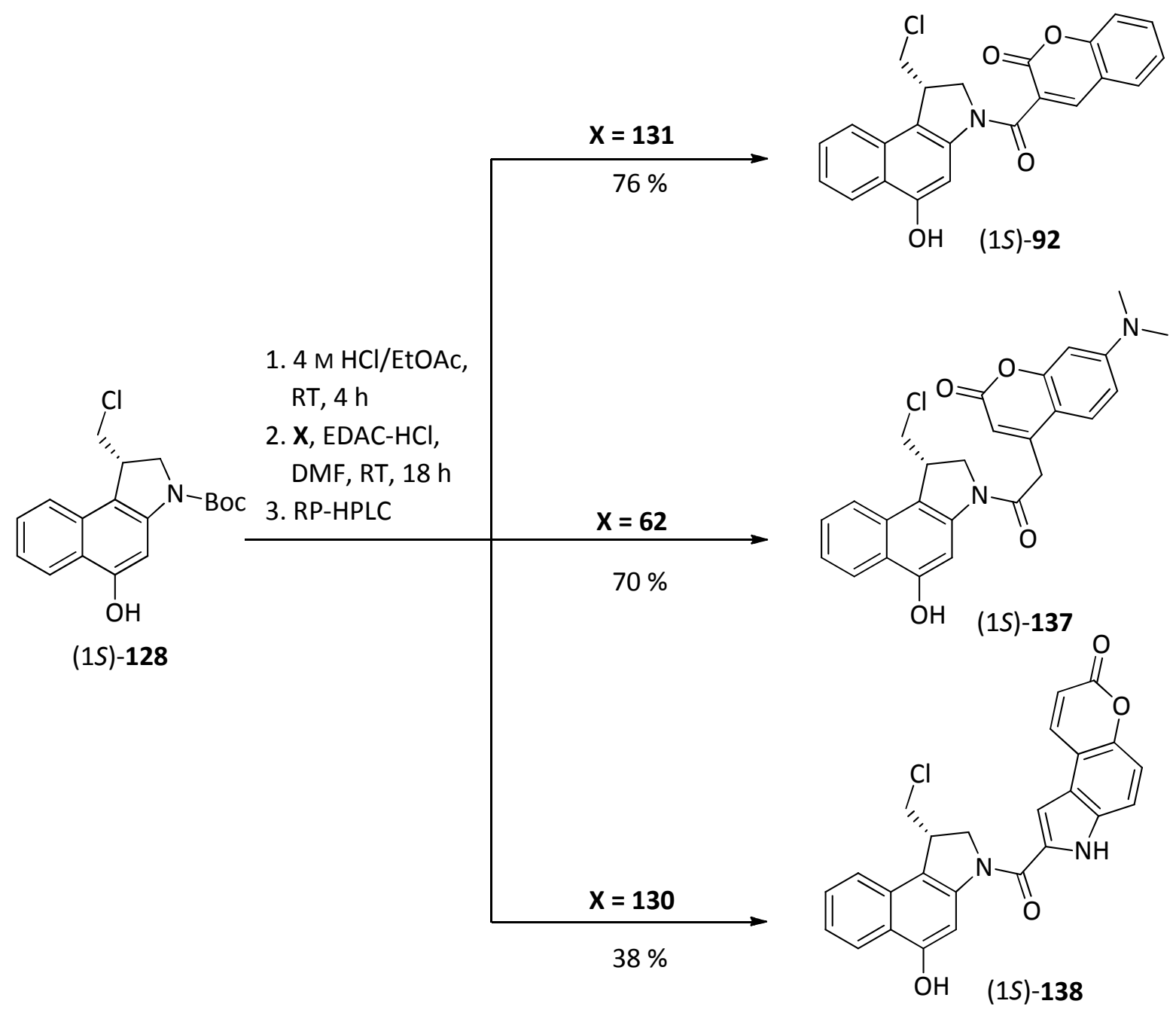

Abbildung 72. Synthese neuartiger fluoreszenzmarkierter seco-CBI-Cumarin-Drugs (1S)-92, (1S)137 und (1S)-138 ausgehend von Phenol (1S)-128. 
5.2.1 Diskussion ausgewählter spektroskopischer Daten des seco-CBIDMACA-Drugs (1S)-137<smiles>CCC(=O)N1C[C@H](C(Cl)Cl)c2c1cc(O)c1ccccc21</smiles>

(1S)-137

Abbildung 73. Struktur des seco-CBI-DMACA-Drugs (1S)-137.
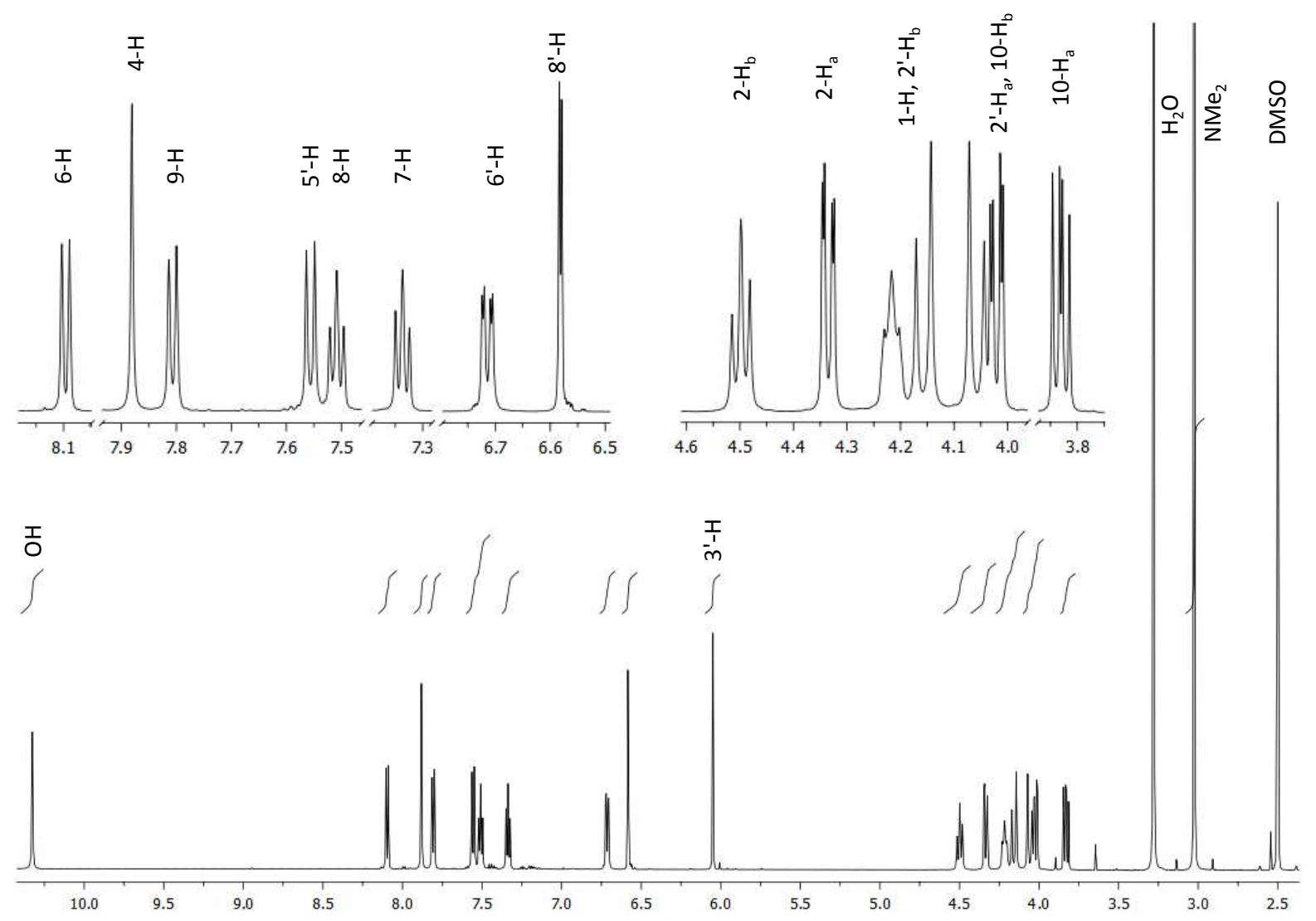

Abbildung 74. $\quad{ }^{1} \mathrm{H}-\mathrm{NMR}$ Spektrum (600 Mhz, DMSO-d $\mathrm{d}_{6}$ ) des seco-CBI-DMACA-Drugs (1S)-137.

Abbildung 74 zeigt das ${ }^{1} \mathrm{H}-\mathrm{NMR}$ Spektrum sowie die Zuordnung der Signale des secoCBI-DMACA-Drugs (1S)-137, das in DMSO- $\mathrm{d}_{6}$ bei $600 \mathrm{MHz}$ aufgenommen wurde. 
Das Resonanzsignal für die Protonen der Dimethylaminfunktionalität des Cumarins findet man als Singulett bei $\delta=3.02 \mathrm{ppm}$. Es folgt ein Mulitplett bei $\delta=3.76-$ $3.88 \mathrm{ppm}$ für das $10-\mathrm{H}_{\mathrm{a}}$-Proton sowie ein Multiplett bei $\delta=3.97-4.00 \mathrm{ppm}$ für das $10-\mathrm{H}_{\mathrm{b}}$-Proton und eines der beiden Methylenprotonen an C-2'. Das zweite Proton von C-2' folgt im Multiplett bei $\delta=4.12-4.27 \mathrm{ppm}$. In diesem Multiplett befindet sich auch das Resonanzsignal für das Proton an C-1. Die Signale für die diastereotopen Protonen an C-2 resonieren zum einen bei $\delta=4.34 \mathrm{ppm}$ als Dublett vom Dublett mit $J=10.9 \mathrm{~Hz}$ für die geminale und $J=2.4 \mathrm{~Hz}$ für die vicinale Kopplung zum Anderen bei $\delta=4.50 \mathrm{ppm}$ als Triplett mit einer Kopplungskonstante von $J=10.1 \mathrm{~Hz}$. Bei $\delta=6.05 \mathrm{ppm}$ findet man das Signal für das 3"-H Proton als Singulett sowie bei $\delta=6.58 \mathrm{ppm}$ ein Dublett mit einer Kopplungskonstante von $J=2.5 \mathrm{~Hz}$ für $8^{\prime \prime}-\mathrm{H}$, welches aus einer meta-Kopplung mit 6"-H resultiert. Das entsprechende Signal für 6"-H findet man bei $\delta=6.71 \mathrm{ppm}$ als Dublett vom Dublett mit $J=9.1 \mathrm{~Hz}$ für die orthoKopplung zu 5"-H und $J=2.5 \mathrm{~Hz}$ für die meta-Kopplung zu 8"-H. Bei $\delta=7.34$ ppm und $\delta=7.51$ ppm folgen die Resonanzsignale für die Protonen des CBI-Grundgerüsts an C7 und C-8. Hierbei zeigt 7-H ein Triplett mit $J=7.3 \mathrm{~Hz}$, das aus zwei ortho-Kopplungen, nämlich zu 6-H und $8-\mathrm{H}$, resultiert. Bei $\delta=7.51 \mathrm{ppm}$ findet man ein Triplett mit $J=7.6 \mathrm{~Hz}$, dass durch Kopplungen des 8- $\mathrm{H}$ mit 9-H und 7- $\mathrm{H}$ entsteht. Es folgt bei $\delta=7.56 \mathrm{ppm}$ ein Dublett mit einer typischen Kopplungskonstante für orthoKopplungen im Aromaten von $J=9.0 \mathrm{~Hz}$. Diese Resonanz kann 5"-H zugeordnet werden und resultiert aus einer Kopplung mit 6"-H. Bei $\delta=7.81 \mathrm{ppm}$ beobachtet man das Resonanzsignal für 9-H, das mit einer Kopplungskonstante von $J=8.4 \mathrm{~Hz}$ für eine ortho-Kopplung zu 8-H steht. Bei $\delta=7.88$ ppm findet man das Signal für das $4-\mathrm{H}$ als Singulett sowie bei $\delta=8.10 \mathrm{ppm}$ das Signal für $6-\mathrm{H}$ als Dublett mit $J=8.3 \mathrm{~Hz}$. Abschließend findet man bei $\delta=10.32 \mathrm{ppm}$ das Resonanzsignal für die phenolische Hydroxyfunktion als breites Singulett. 

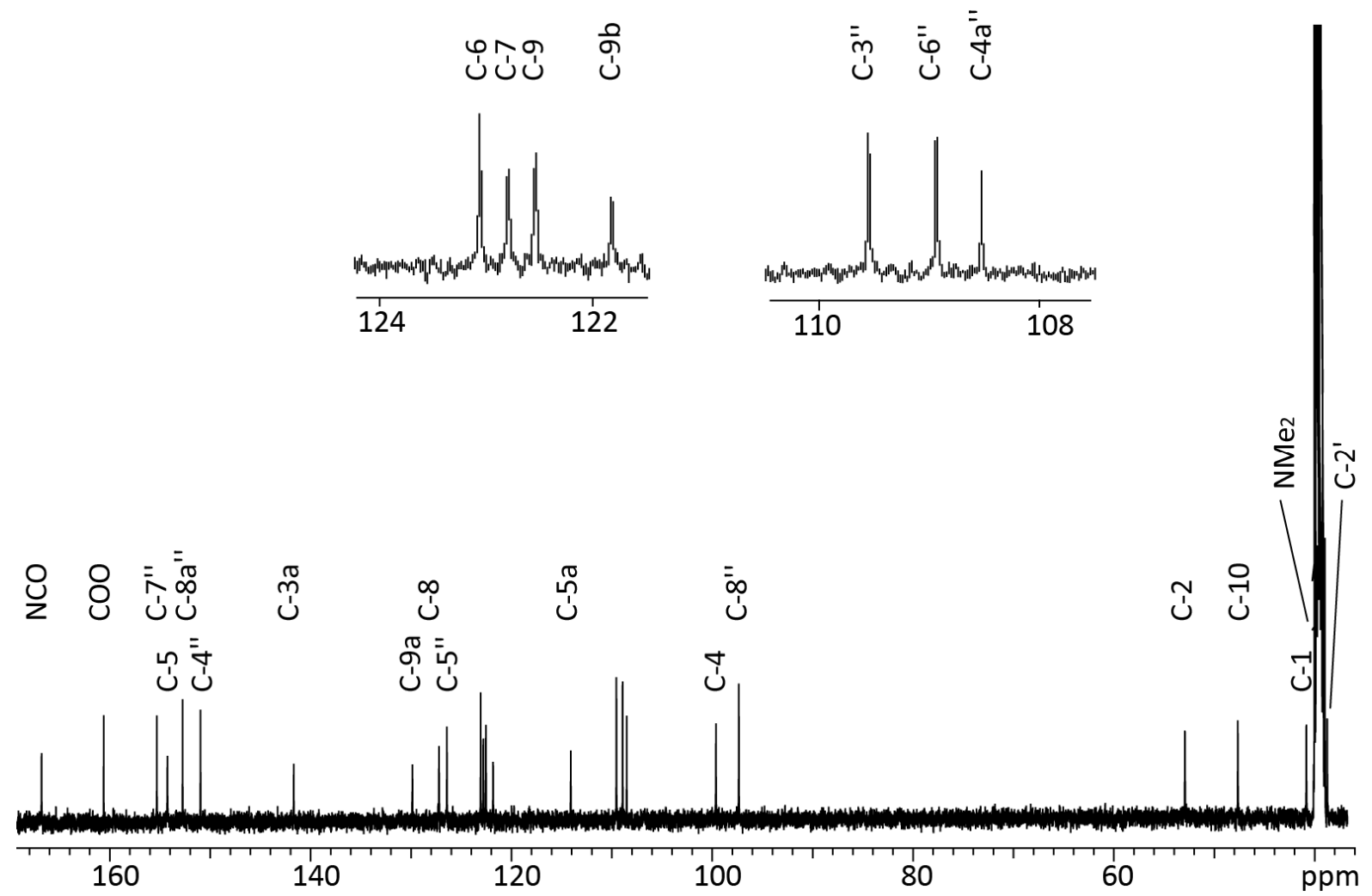

Abbildung 75. $\quad{ }^{13} \mathrm{C}-\mathrm{NMR}$ Spektrum (125 Mhz, DMSO- $\mathrm{d}_{6}$ ) des seco-CBI-DMACA-Drugs (1S)-137.

Abbildung 75 zeigt das ${ }^{13} \mathrm{C}$-NMR Spektrum (125 MHz, DMSO-d $\mathrm{d}_{6}$ ) sowie die Zuordnung der Signale für (1S)-137.

Bei $\delta=38.7 \mathrm{ppm}$ resoniert die Methylengruppe des Cumarins, gefolgt von dem Signal für die Kohlenstoffatome der Dimethylaminofunktionalität bei $\delta=39.6 \mathrm{ppm}$. Es folgen die Signale für die aliphatischen Kohlenstoffatome des CBI-Grundgerüstes bei $\delta=40.8$ (C-1), 47.6 (C-10) und $52.9 \mathrm{ppm}$ (C-2). Es folgen die Resonanzen für die aromatischen Kohlen-stoffatome. Zunächst die Signale für C-8" und C-4 bei $\delta=97.3$ und $99.6 \mathrm{ppm}$. Es folgt das Brückenkopfatom C-4a" bei $\delta=108.5 \mathrm{ppm}$. Die Signale für den aromatischen Cumarinkern findet man bei $\delta=108.9$ (C-6"), 109.5 (C-3"), 126.4 (C-5"), 151.0 (C-4"), 152.7 (C-8a") und 155.3 ppm (C-7"). Die Signale für die aromatischen Kohlenstoffatome des CBI-Grundgerüstes werden bei $\delta=114.1$ (C-5a), 121.8 (C-9b), 122.5 (C-9), 122.8 (C-7), 123.0 (C-6), 127.2 (C-8), 129.8 (C-9a), 141.7 (C-3a), 154.2 (C-5) ppm detektiert. Abschließend findet man die Signale für die Carbonylkohlenstoffatome bei $\delta=160.6 \mathrm{ppm}$ für das Cumarin-Carbonyl sowie bei $\delta=166.8$ ppm für das Amidcarbonyl. 


\subsection{Synthese der (1S)-CBI-Cumarin-Galaktoside (1S)-69, (1S)-139 und (1S)-140}

Ausgehend vom Phenol (1S)-128 konnten die CBI-Cumarin-Galaktoside (1S)-69, (1S)-139 und (1S)-140 über 4 Stufen hergestellt werden. Zunächst wurde das freie Phenol (1S)-128 nach der Trichloracetimidatmethode nach Schmidt ${ }^{157}$ unter $\mathrm{BF}_{3} \cdot \mathrm{OEt}_{2^{-}}$ Katalyse mit tetraacetyliertem D-Galaktose-Trichloracetimidat 116 gekuppelt und anschließend die säurelabile $\mathrm{N}$-Boc-Schutzgruppe $\mathrm{BF}_{3} \cdot \mathrm{OEt}_{2}$-vermittelt abgespalten (Abbildung 76).

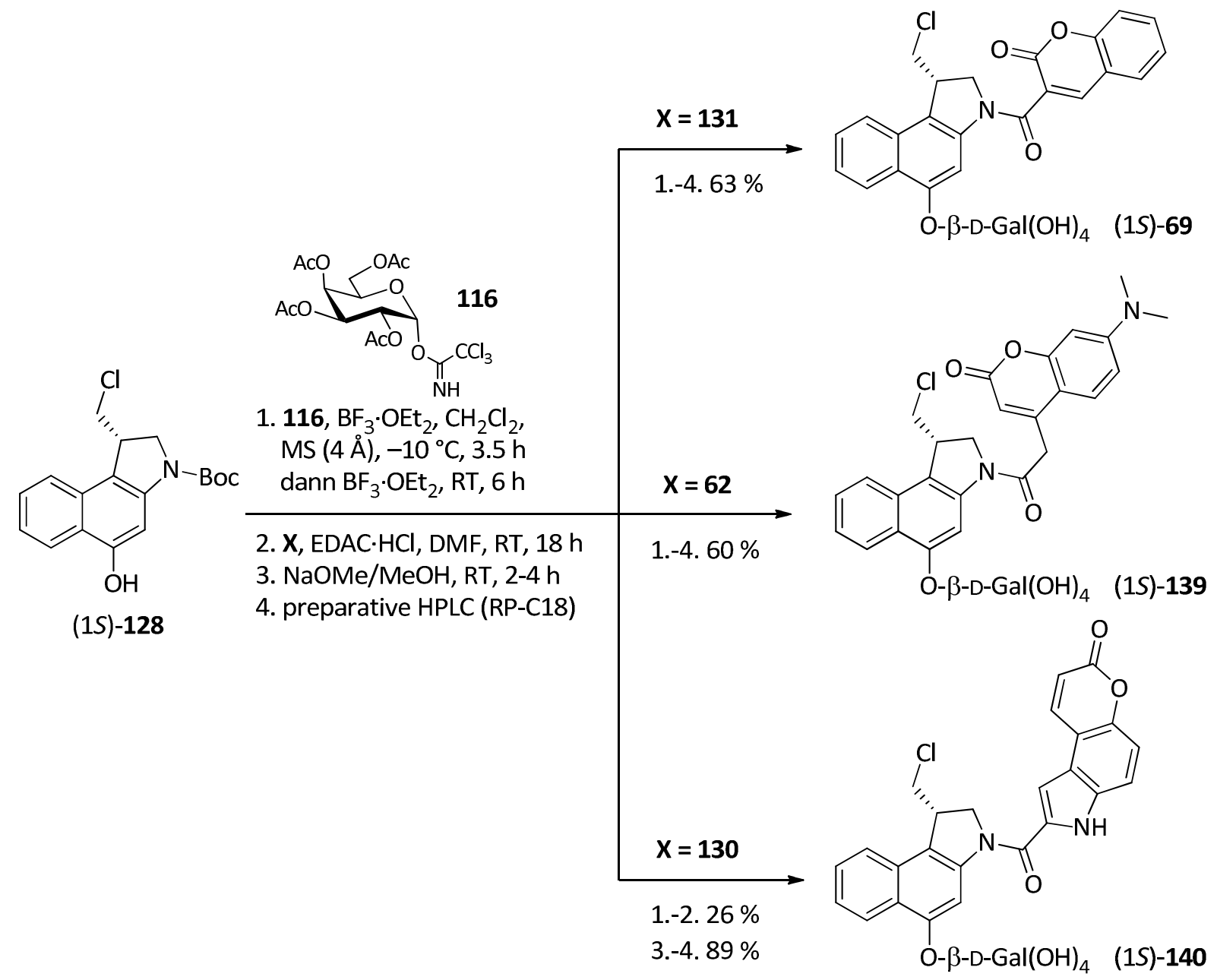

Abbildung 76. Synthese der (1S)-CBI-Cumarin-Galaktoside ausgehend von Phenol (1S)-128.

Das freie sekundäre Amin wurde dann mit EDAC $\cdot \mathrm{HCl}$ als Kupplungsreagenz in DMF mit der Carbonsäure des entsprechenden Cumarins (62, 130 oder 131) gekuppelt um 
die tetraacetylierten CBI-Cumarin-Galaktoside zu erhalten. Anschließende Deacetylierung nach Zemplén durch Natriummethanolat in Methanol bei Raumtemperatur lieferte die entsprechenden (1S)-CBI-Cumarin-Galaktoside (1S)-69, (1S)-139 und (1S)140 in guten bis sehr guten Ausbeuten. Abschließend wurden alle erhaltenen Prodrugs durch präparative HPLC (Kromasil 100 C-18) gereinigt.

\subsection{Ergebnisse zur Untersuchung der In-vitro-Zytotoxizität an humanen Bronchialkarzinomzellen (A549) für die (1S)-CBI-Cumarin-Derivate}<smiles>[R]Oc1cc2c(c3ccccc13)[C@@H](CCl)CN2C(=O)c1cc2ccccc2oc1=O</smiles>

$$
\begin{array}{ll}
\mathrm{R}=\mathrm{H} & (1 \mathrm{~S})-92 \\
\mathrm{R}=\beta-\mathrm{D}-\mathrm{Gal}(\mathrm{OH})_{4} & (1 \mathrm{~S})-69
\end{array}
$$

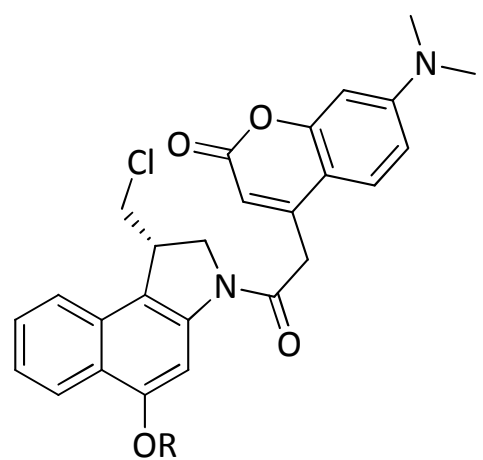

$$
\begin{array}{ll}
R=H & (1 S)-137 \\
R=\beta-D-G a l(O H)_{4} & (1 S)-139
\end{array}
$$<smiles>O=C(c1cc2c(ccc3oc(=O)ccc32)[nH]1)N1C[C@H](CCl)c2c1cc(O)c1ccccc21</smiles>

$$
\begin{array}{ll}
R=H & (1 S)-138 \\
R=\beta-D-G a l(O H)_{4} & (1 S)-140
\end{array}
$$

Abbildung 77. Untersuchte (1S)-CBI-Cumarine: Galaktoside (1S)-69, (1S)-139 und (1S)-140 sowie seco-CBI-Cumarin-Drugs (1S)-92, (1S)-137 und (1S)-138.

Abbildung 78 zeigt die Ergebnisse der Untersuchungen zur In-vitro-Zytotoxizität der in dieser Arbeit synthetisierten (1S)-CBI-Cumarin-Derivate. Hierbei ist vor allem auffällig, dass das (1S)-CBI-Cumarinindol-Derivat (1S)-138 deutlich zytotoxischer ist als die (1S)CBI-Cumarin-Derivate (1S)-92 und (1S)-137. So erreicht das (1S)-CBI-CumarinindolDrug (1S)-138 mit IC $\mathrm{C}_{50}=0.014 \mathrm{nM}$ einen hervorragenden Wert, der den des CumarinDrugs $(1 S)-137\left(\mathrm{IC}_{50}=0.38 \mu \mathrm{M}\right)$ um den Faktor 27143 übersteigt. Allerdings haben die Prodrugs aller Cumarin-Derivate nur eine geringe therapeutische Breite mit $\mathrm{QIC}_{50}{ }^{-}$ Werten von 2-14. Auffällig ist außerdem, dass die ermittelte In-vitro-Zytotoxizität von (1S)-138 $\left(I C_{50}=0.014 \mathrm{nM}\right)$ annähernd identisch ist mit der des Strukturanalogen 
(1S)-CBI-DMAI-Drugs (1S)-52 $\left(\mathrm{IC}_{50}=0.026 \mathrm{nM}\right)$. Eine Erklärung für die vom Strukturanalogon abweichende hohe Zytotoxizität des Galaktosides (1S)-140 könnte auf eine schnelle Aufnahme in Mitochondrien und damit einer Einleitung der Apoptose zurückzuführen sein.

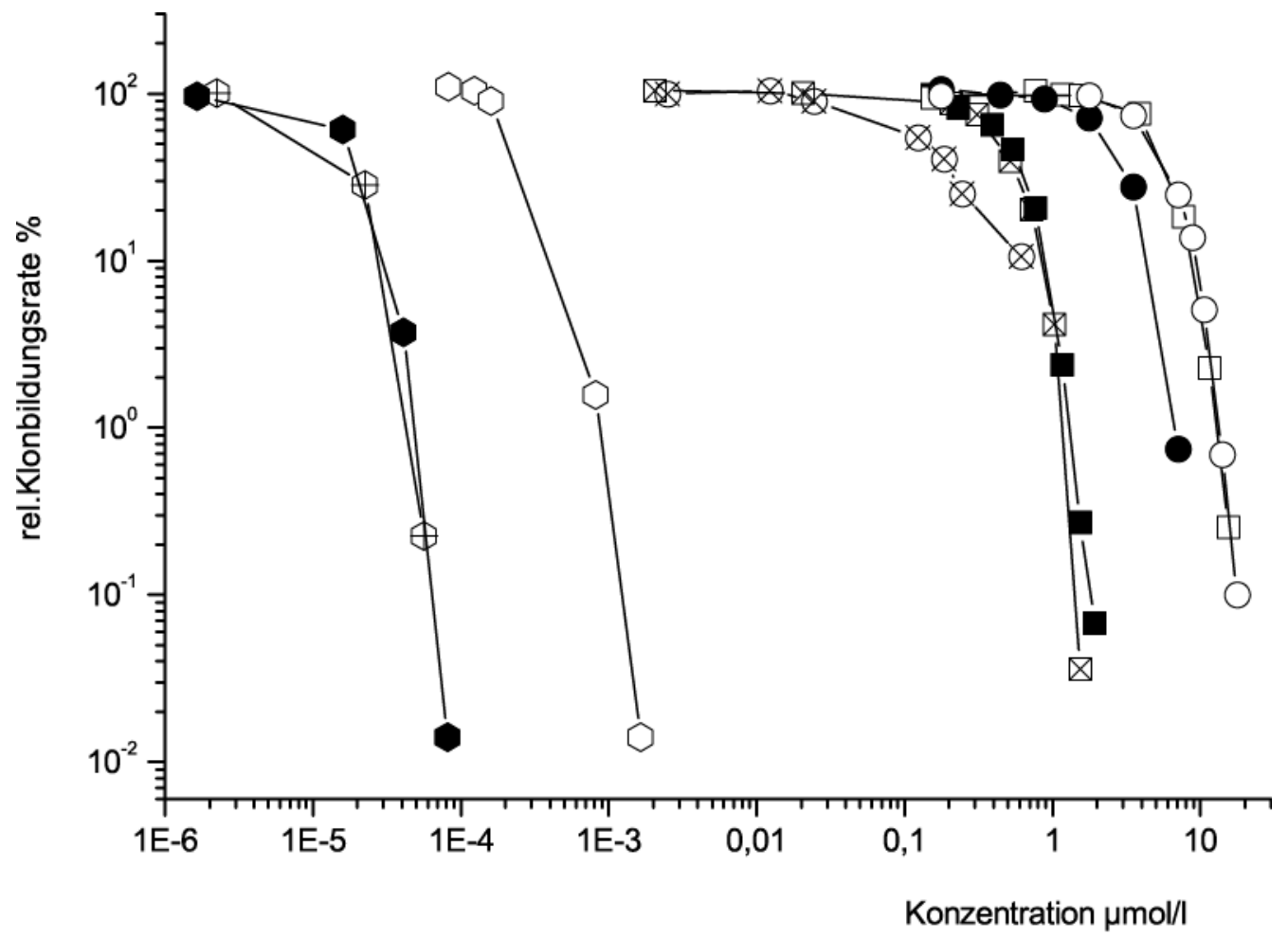

\begin{tabular}{l|c|c} 
& $\begin{array}{c}\mathbf{I C}_{\mathbf{5 0}} \\
(\boldsymbol{\mu} \mathbf{m o l} / \mathbf{l})\end{array}$ & $\mathbf{Q}_{\mathbf{5 0}}$ \\
\hline$-(1 S)-137$ & 0.38 & \\
$-(1 S)-139$ mit Enzym & 0.43 & 12 \\
\hline$(1 S)-139$ ohne Enzym & 5.0 & \\
$-(1 S)-92$ & 0.11 & \\
$-(1 S)-69$ mit Enzym & 2.4 & 2 \\
$-(1 S)-69$ ohne Enzym & 4.8 & \\
$-(1 S)-138$ & 0.000014 & \\
$-(1 S)-140$ mit Enzym & 0.000018 & 14
\end{tabular}

Abbildung 78. Darstellung der Ergebnisse der In vitro-Zytotoxizitätsuntersuchungen für die (1S)-Cumarin-Derivate (1S)-137 und (1S)-139, (1S)-92 und (1S)-69 sowie (1S)-138 und (1S)-140. 


\section{Charakterisierung und Untersuchung der zellulären Aufnahme von fluoreszenzmarkierten (1S)-CBI-Cumarin-Derivaten}

\subsection{Fluorimetrische Bestimmung der Absorptions- und Emissionsmaxima}

Zur Untersuchung der zellulären Aufnahme der in dieser Arbeit synthetisierten CBICumarin-Derivate (1S)-92, (1S)-69, (1S)-137, (1S)-139, (1S)-138 und (1S)-140 sowie 62, 130 und 131 mussten zunächst die Absorptions- und Emissionsspektren bestimmt werden. Hierzu wurden entsprechende Lösung der Substanzen in Methanol und wässrigem Bildgebungspuffer am Fluorimeter untersucht, um die benötigten Maxima zu ermitteln.

\subsubsection{Bestimmung der Fluoreszenz- und Emissionsmaxima für die dargestellten Cumarin-3-Carbonsäure-Derivate (1S)-92, (1S)-69 sowie 131}<smiles>O=C(c1cc2ccccc2oc1=O)N1C[C@H](CCl)c2c1cc(O)c1ccccc21</smiles><smiles>O=C(O)c1cc2ccccc2oc1=O</smiles>

131

$$
\begin{array}{ll}
R=H & (1 S)-92 \\
R=\beta-D-G a l(O H)_{4} & (1 S)-69
\end{array}
$$

Abbildung 79. Untersuchte Verbindungen mit Cumarin-3-Carbonsäure (131) als DNA-Binder.

Wie in den abgebildeten Spektren zu sehen ist, liegt das Absorptionsmaximum, wie es für Cumarine charakteristisch ist, sowohl in Methanol als auch in wässrigem Puffer im Bereich von $\lambda_{\max , A b s}=314-352 \mathrm{~nm}$. Ebenso kann der für Cumarine typische geringe Stokes-Shift beobachtet werden, der sich im Fall der hier dargestellten Verbindungen 
im Bereich von 30-79 $\mathrm{nm}$ bewegt und damit zu einem Emissionsmaximum im Bereich von $\lambda_{\text {max }, \mathrm{Em}}=380-414 \mathrm{~nm}$ führt (Abbildungen $80-82$ ).
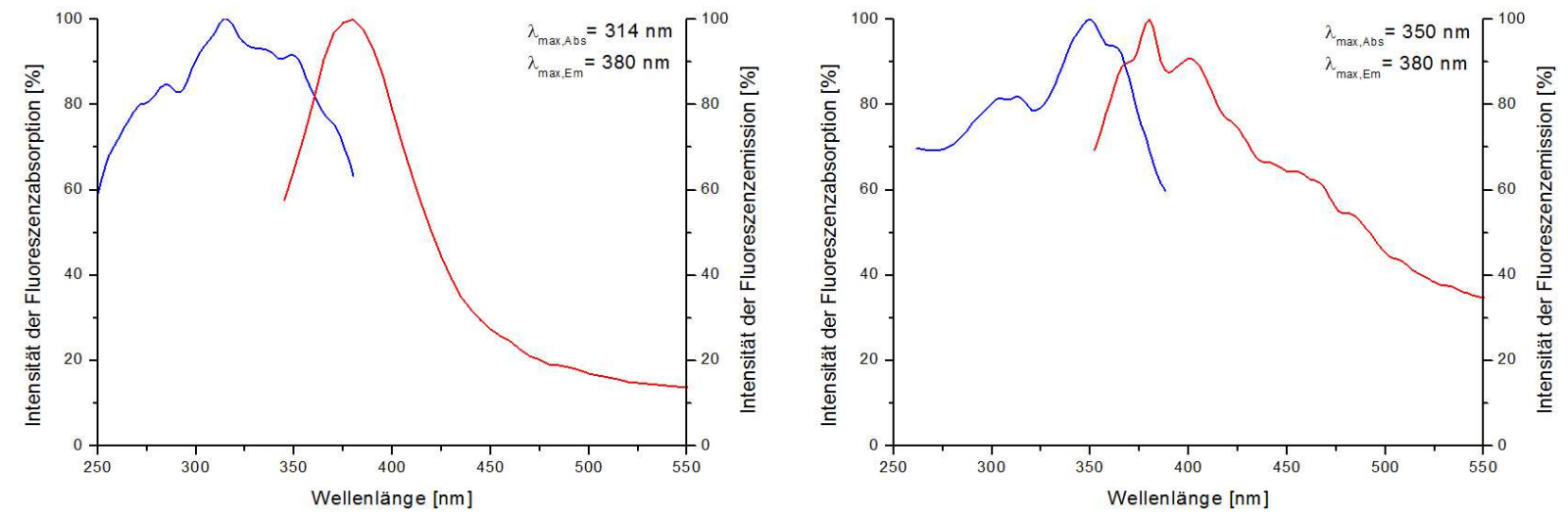

Abbildung 80. Absorptions- und Emissionsspektren von (1S)-92 in Methanol (links) und wässrigem Bildgebungspuffer (rechts).
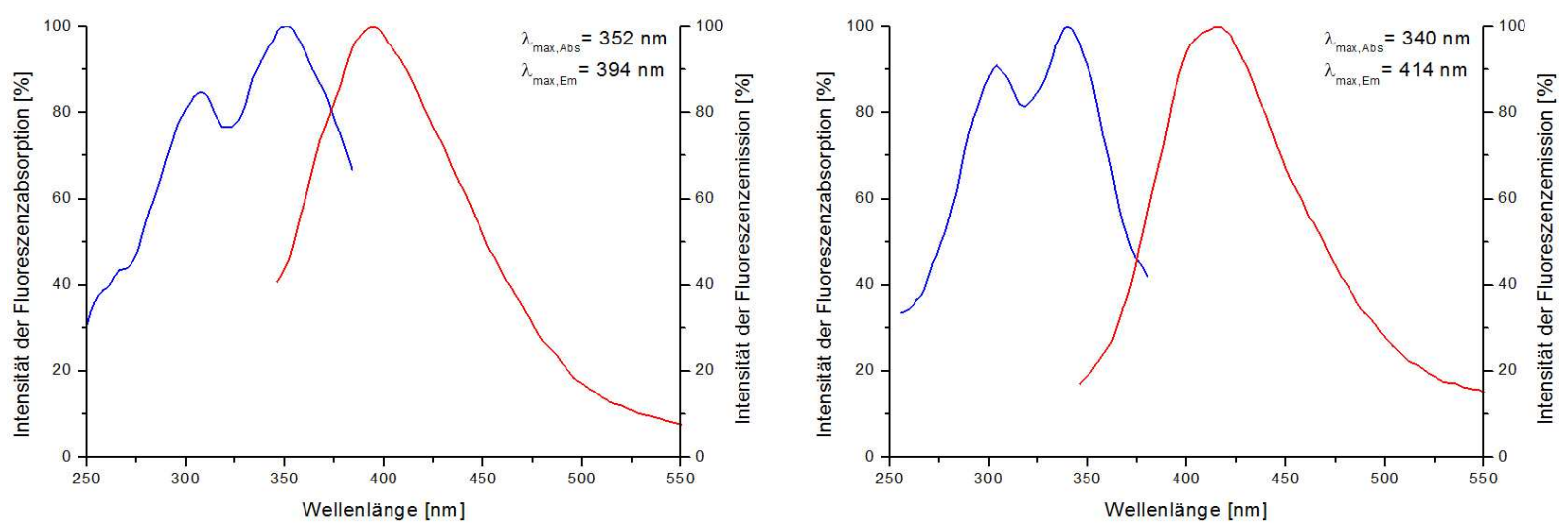

Abbildung 81. Absorptions- und Emissionsspektren von (1S)-69 in Methanol (links) und wässrigem Bildgebungspuffer (rechts).
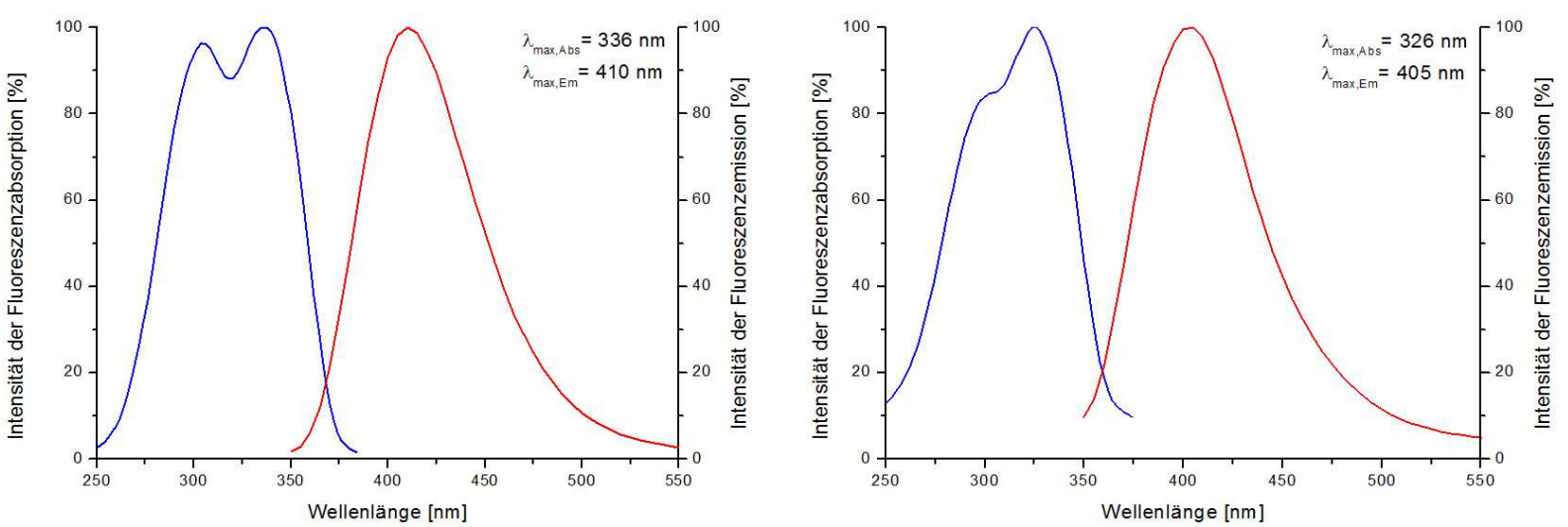

Abbildung 82. Absorptions- und Emissionsspektren von 131 in Methanol (links) und wässrigem Bildgebungspuffer (rechts). 


\subsubsection{Bestimmung der Fluoreszenz- und Emissionsmaxima für die dargestellten DMACA-Derivate (1S)-137, (1S)-139 sowie 62}<smiles>[R20]c1cc2c(c3ccccc13)[C@@H](CCl)CN2C(=O)Cc1cc(=O)oc2cc(N(C)C)ccc12</smiles>

$$
\begin{array}{ll}
\mathrm{R}=\mathrm{H} & (1 S)-137 \\
\mathrm{R}=\beta-\mathrm{D}-\mathrm{Gal}(\mathrm{OH})_{4} & (1 S)-139
\end{array}
$$<smiles>CN(C)c1ccc2c(CC(=O)O)cc(=O)oc2c1</smiles>

62

Abbildung 83. Untersuchte Verbindungen mit DMACA (62) als DNA-Binder.

Bei den DMACA-Derivaten (Abbildung 83) liegt das Absorptionsmaximum sowohl in Methanol als auch in wässrigem Puffer im Bereich von $\lambda_{\max , \mathrm{Abs}}=370-390 \mathrm{~nm}$ und damit im Vergleich zu den Cumarin-3-Carbonsäure-Derivaten etwas rotverschoben. Auch hier kann der für Cumarine typische kleine Stokes-Shift beobachtet werden, der sich über einen Bereich von $80-87 \mathrm{~nm}$ erstreckt und damit zu einem Emissionsmaximum von $\lambda_{\text {max,Em }}=450-478 \mathrm{~nm}$ führt(Abbildungen 84-86).

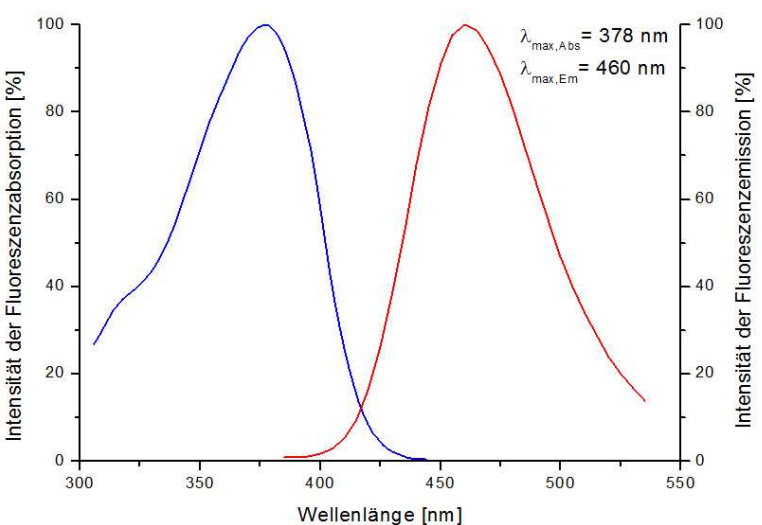

Abbildung 84. Absorptions- und Emissionsspektren von (1S)-137 in Methanol (links) und wässrigem Bildgebungspuffer (rechts).

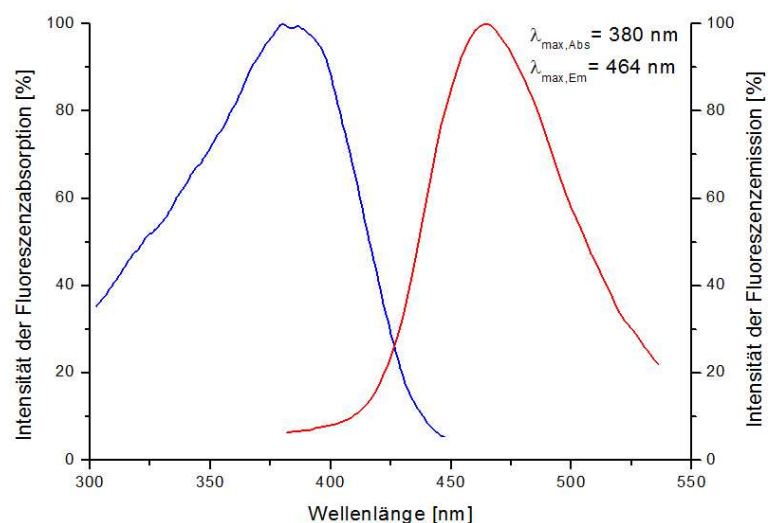



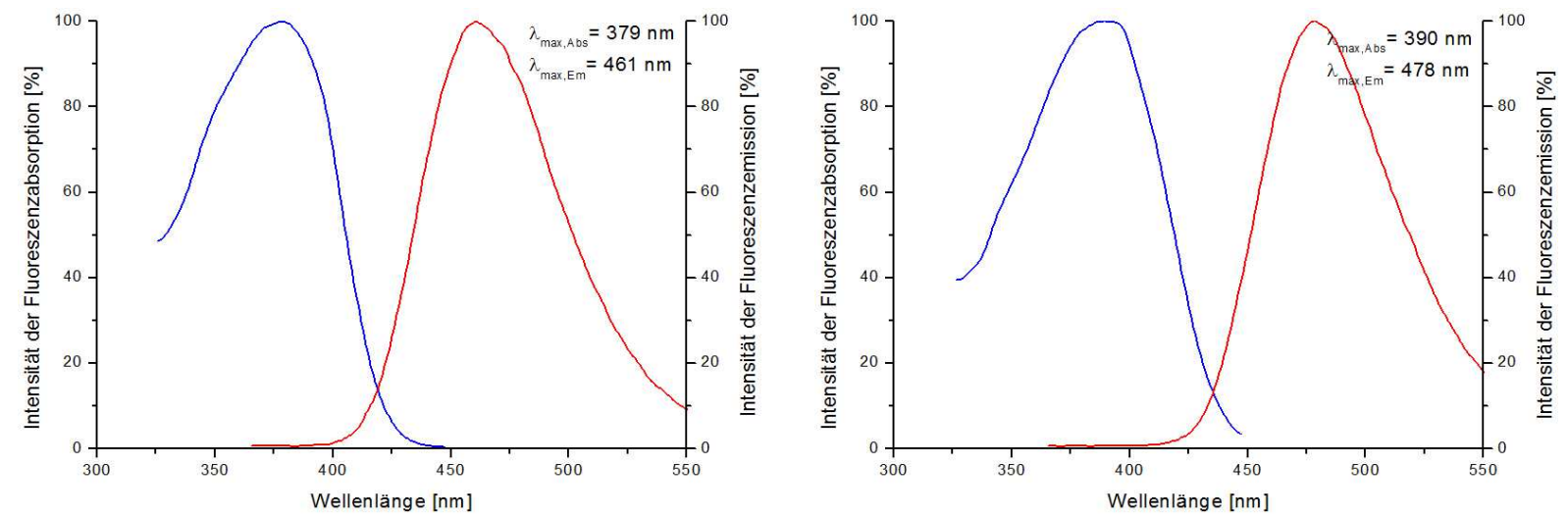

Abbildung 85. Absorptions- und Emissionsspektren von (1S)-139 in Methanol (links) und wässrigem Bildgebungspuffer (rechts).
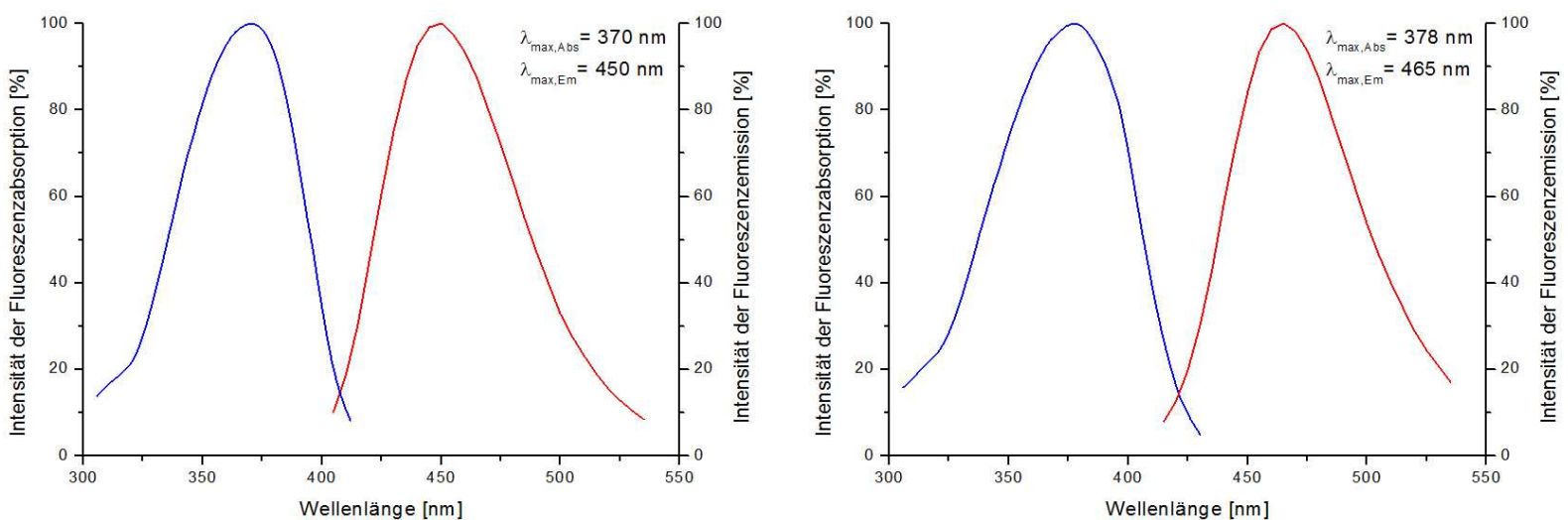

Abbildung 86. Absorptions- und Emissionsspektren von 62 in Methanol (links) und wässrigem Bildgebungspuffer (rechts).

\subsubsection{Bestimmung der Fluoreszenz- und Emissionsmaxima für die} dargestellten Indol-Cumarin-Derivate (1S)-138, (1S)-140 sowie 130<smiles>[R7]Oc1cc2c(c3ccccc13)[C@@H](CCl)CN2C(=O)c1cc2c(ccc3oc(=O)ccc32)[nH]1</smiles><smiles>O=C(O)c1cc2c(ccc3oc(=O)ccc32)[nH]1</smiles>

$$
\begin{array}{ll}
\mathrm{R}=\mathrm{H} & (1 S)-138 \\
\mathrm{R}=\beta-\mathrm{D}-\mathrm{Gal}(\mathrm{OH})_{4} & (1 S)-140
\end{array}
$$

130

Abbildung 87. Untersuchte Verbindungen mit Cumarin-Indol-2-Carbonsäure 130 als DNA-Binder. 
Bei den Cumarinindol-Derivaten (Abbildung 87) liegt das Absorptionsmaximum sowohl in Methanol als auch in wässrigem Puffer im Bereich von $\lambda_{\text {max,Abs }}=346-$ $370 \mathrm{~nm}$ und damit im Vergleich zu den Cumarin-3-Carbonsäure-Derivaten nur leicht rotverschoben. Der im Gegensatz zu den vorherigen Cumarin-Derivaten große StokesShift beträgt in den unten abgebildeten Spektren 106-146 nm und führt zu einem Emissionsmaximum von $\lambda_{\text {max }, \mathrm{Em}}=452-498 \mathrm{~nm}$. Auffälig ist hierbei vor allem der deutlich größere Stokes-Shift in wässrigem Puffer im Vergleich zu Methanol(Abbildungen 88-90).
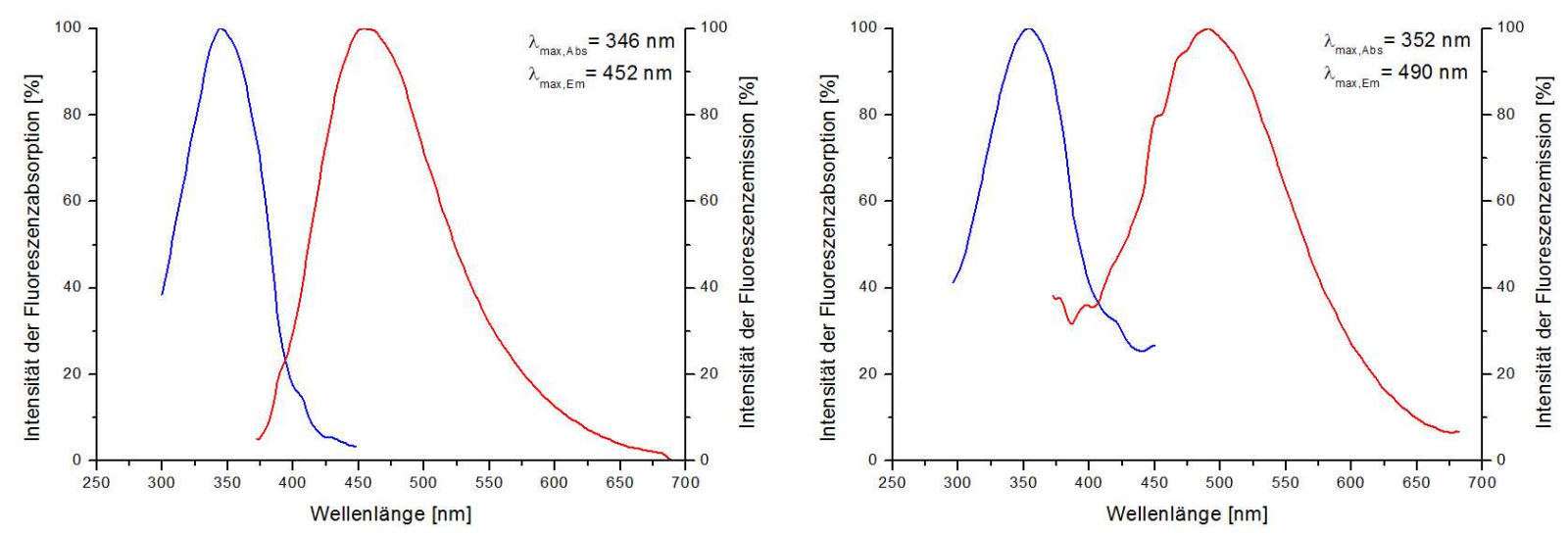

Abbildung 88. Absorptions- und Emissionsspektren von (1S)-138 in Methanol (links) und wässrigem Bildgebungspuffer (rechts).
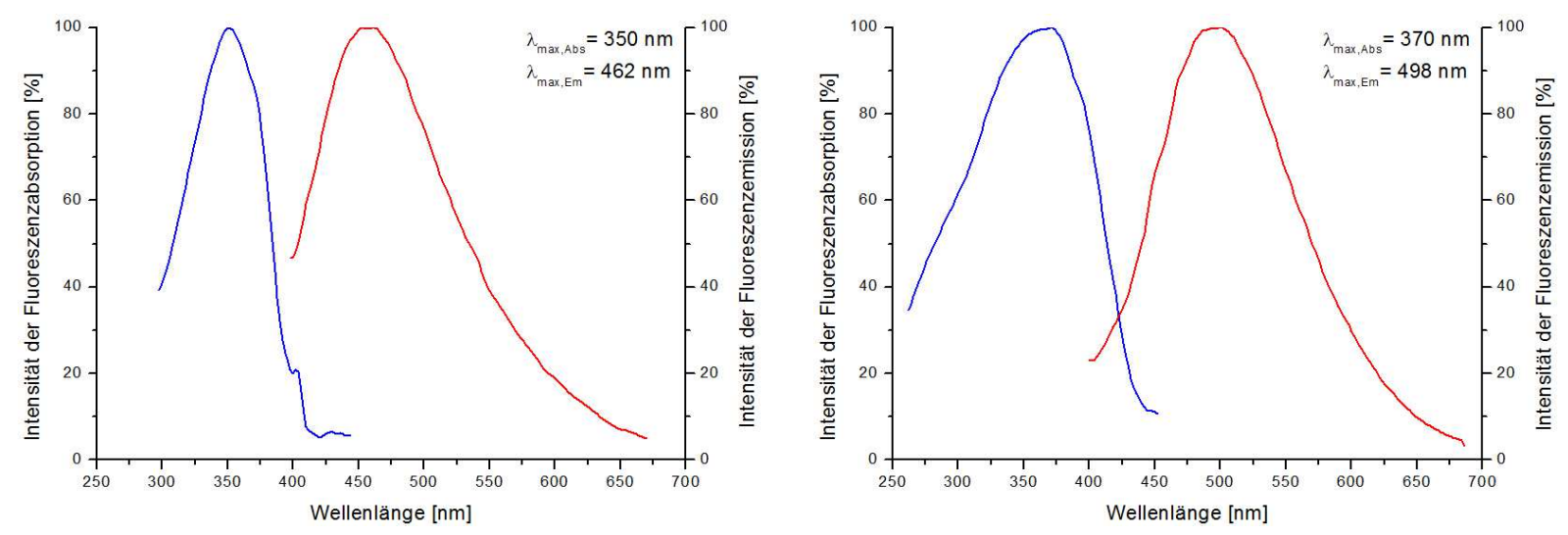

Abbildung 89. Absorptions- und Emissionsspektren von (1S)-140 in Methanol (links) und wässrigem Bildgebungspuffer (rechts). 

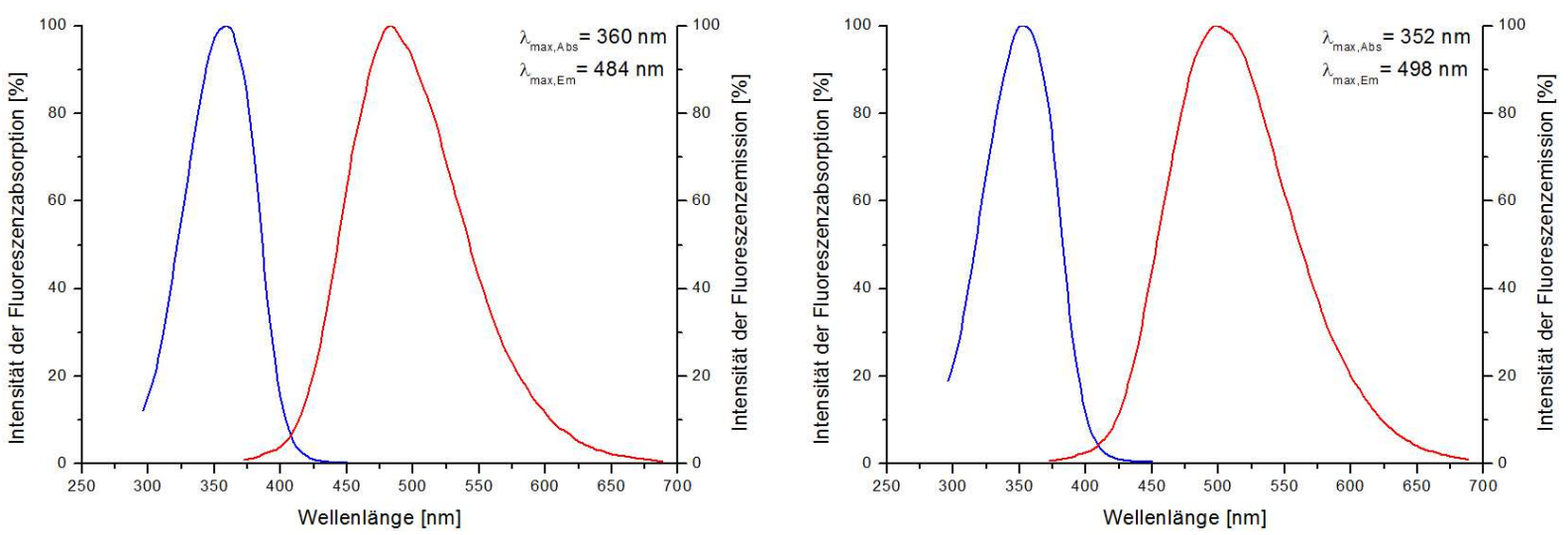

Abbildung 90. Absorptions- und Emissionsspektren von 130 in Methanol (links) und wässrigem Bildgebungspuffer (rechts).

\subsection{Untersuchung der zellulären Aufnahme fluroreszenzmarkierter (1S)-CBI-Cumarin-Derivate in lebende Zellen durch CLSM}

Zu einem besseren Verständnis des Wirkmechanismus der CBI-Derivate wurden die in dieser Arbeit synthetisierten fluoreszenzmarkierten Analoga in Live Cell Imaging Experimenten untersucht. Hierzu wurden adhärent wachsende Zelllinien vom Typ A549 sowie HeLa SS6 für kurze Zeit mit den zu untersuchenden Substanzen inkubiert (5-15 min) und die Verteilung der Substanz mit Hilfe eines Konfokalen Laser Scanning Mikroskops beobachtet und einer CCD-Kamera aufgenommen. Zur Identifikation der angefärbten Zellregion wurde auf das Prinzip der Kolokalisation zurückgegriffen, um eine Unterscheidung zwischen Organellen, wie dem Zellkern (durch Hoechst 33342 (64)) oder mitochondrialen Strukturen (durch MitoTracker Deep Red 633 FM (65)) zu treffen (Abbildung 91). 
<smiles>CCOc1ccc(-c2nc3cc(-c4nc5cc(N6CCN(C)CC6)ccc5[nH]4)ccc3[nH]2)cc1</smiles>

Hoechst 33342 (64)<smiles>C[N+]1=C(C=CC=C2N(Cc3ccc(CCl)cc3)c3ccccc3C2(C)C)C(C)(C)c2ccccc21</smiles>

MitoTracker Deep Red 633 FM (65)

Abbildung 91. Strukturen der für die Kolokalisationsexperimente eingesetzten Anfärbereagenzien: 64: Anfärbung des Zelkerns sowie 65: Anfärbung der Mitochondrien.

\subsubsection{Untersuchung der zellulären Aufnahme fluroreszenzmarkierter (1S)- CBI-Cumarin-3-Carbonsäure-Derivate (1S)-92, (1S)-69 sowie 131 in} lebende Zellen<smiles>[R2]c1cc2c(c3ccccc13)[C@@H](CCl)CN2C(=O)c1cc2ccccc2oc1=O</smiles><smiles>O=C(O)c1cc2ccccc2oc1=O</smiles>

131

$$
\begin{array}{ll}
R=H & (1 S)-92 \\
R=\beta-D-G a l(O H)_{4} & (1 S)-69
\end{array}
$$

Abbildung 92. Untersuchte Verbindungen mit Cumarin-3-Carbonsäure (131) als DNA-Binder.

Abbildung 93 zeigt die Aufnahme des DNA Binders Cumarin-3-Carbonsäure (131) in lebende Zellen. Bereits nach wenigen Minuten penetriert der Fluorochrom die Zellmembran und akkumuliert innerhalb der Zelle (B). Auf Grund der Kolokalisationsexperimente mit Hoechst 33342 (64) (A) sowie MitoTracker Deep Red 633 FM (65) (C) kann man eindeutig erkennen, dass es sich bei der von 131 populierten Zellregion um Mitochondrien handelt. Die auftretende Färbung außerhalb der mitochondrialen Strukturen lässt sich durch eine zu hohe Konzentration des Fluorochroms erklären. 

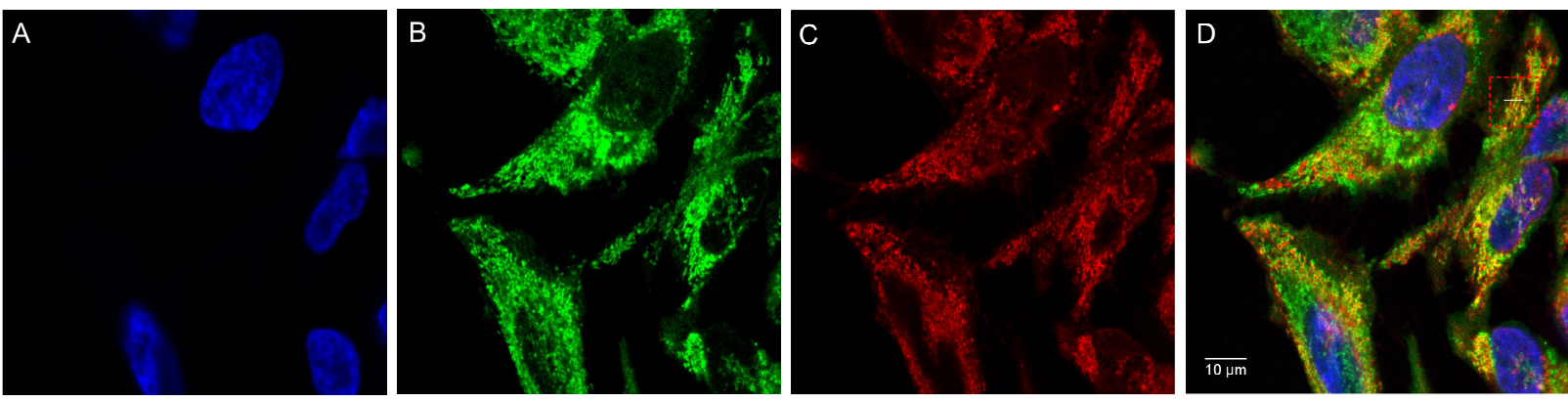

Abbildung 93. Kolokalisationsexperimente zur Aufnahme des Fluorochroms Cumarin-3Carbonsäure (131) in lebende Zellen. A: $\lambda_{\text {exc }}=405 \mathrm{~nm}, \lambda_{\text {em }}=410-429 \mathrm{~nm}$, Anfärbung des Zellkerns durch Hoechst 33342 (64); B: $\lambda_{\text {exc }}=405 \mathrm{~nm}, \lambda_{\text {em }}=415-$ $520 \mathrm{~nm}$, Anfärbung der Mitochondrien durch Cumarin-3-Carbonsäure (131); C: $\lambda_{\text {exc }}=640 \mathrm{~nm}, \lambda_{\text {em }}=650-800 \mathrm{~nm}$, Anfärbung der Mitochondrien durch MitoTracker Deep Red 633 FM (65); D: Überlagerung von A-C.

Das aus Cumarin-3-Carbonsäure (131) dargestellte seco-Drug (1S)-92 zeigt dieselben Eigenschaften wie 131 bei der Untersuchung zur Aufnahme des seco-Drugs (1S)-92 in lebende Zellen. (1S)-92 akkumuliert in Organellen, die durch MitoTracker Deep Red 633 FM (65) als Mitochondrien identifiziert wurden und nicht, wie es von einem secoDrug erwartet würde, im Zellkern (Abbildung 94). Die Toxizität des seco-Drugs (1S)-92 mit $I C_{50}=0.14 \mu \mathrm{M}$ resultiert daher nicht aus einer Alkylierung der Zellkern-DNA sondern höchstwahrscheinlich aus einer Alkylierung der doppelsträngigen mitochondrialen DNA (mtDNA).
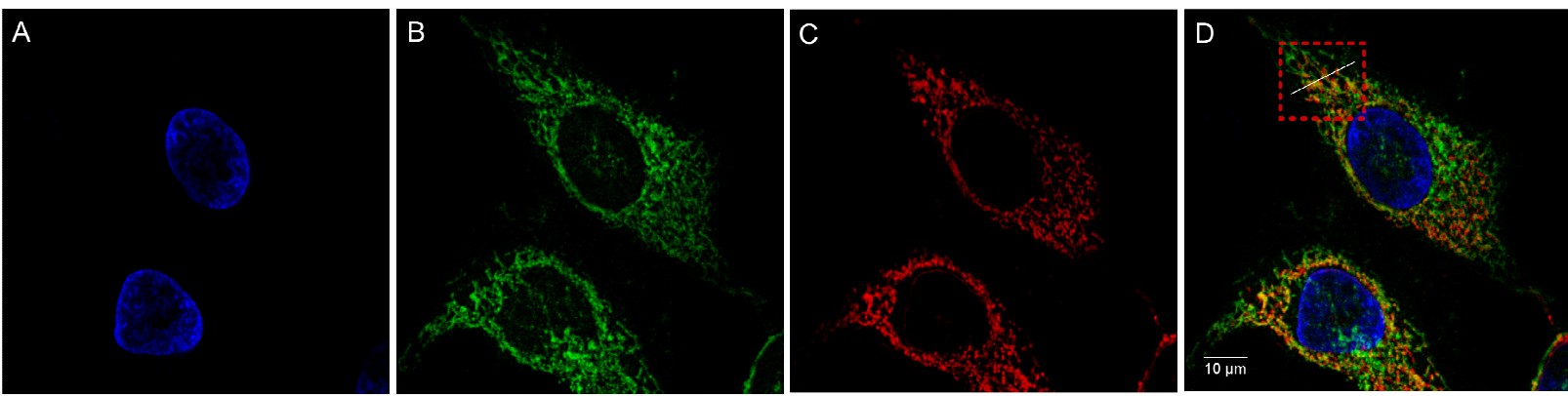

Abbildung 94. Kolokalisationsexperimente zur Aufnahme des seco-Drugs (1S)-92 in lebende Zellen. A: $\lambda_{\text {exc }}=405 \mathrm{~nm}, \lambda_{\text {em }}=415-520 \mathrm{~nm}$, Anfärbung des Zellkerns durch Hoechst 33342 (64); $B: \lambda_{\text {exc }}=405 \mathrm{~nm}, \lambda_{\mathrm{em}}=415-520 \mathrm{~nm}$, Anfärbung der Mitochondrien durch secoDrug (1S)-92; C: $\lambda_{\text {exc }}=640 \mathrm{~nm}, \lambda_{\mathrm{em}}=650-800 \mathrm{~nm}$, Anfärbung der Mitochondrien durch MitoTracker Deep Red 633 FM (65); D: Überlagerung von A-C.

Anders als man es für Verbindungen erwartet, die im Rahmen von ADEPT entwickelt wurden, akkumuliert auch das aus 131 resultierende Galaktosid (1S)-69 bereits nach 
wenigen Minuten innerhalb der Zelle und verweilt nicht, wie angenommen, bis zur enzymatischen Spaltung durch $\beta$-D-Galaktosidase zum seco-Drug (1S)-92 außerhalb der Zelle. Wie bereits bei der Untersuchung des Fluorochroms 131 und des secoDrugs (1S)-92 zeigt sich auch hier eine Akkumulation in mitochondrialen Organellen (Abbildung 95).
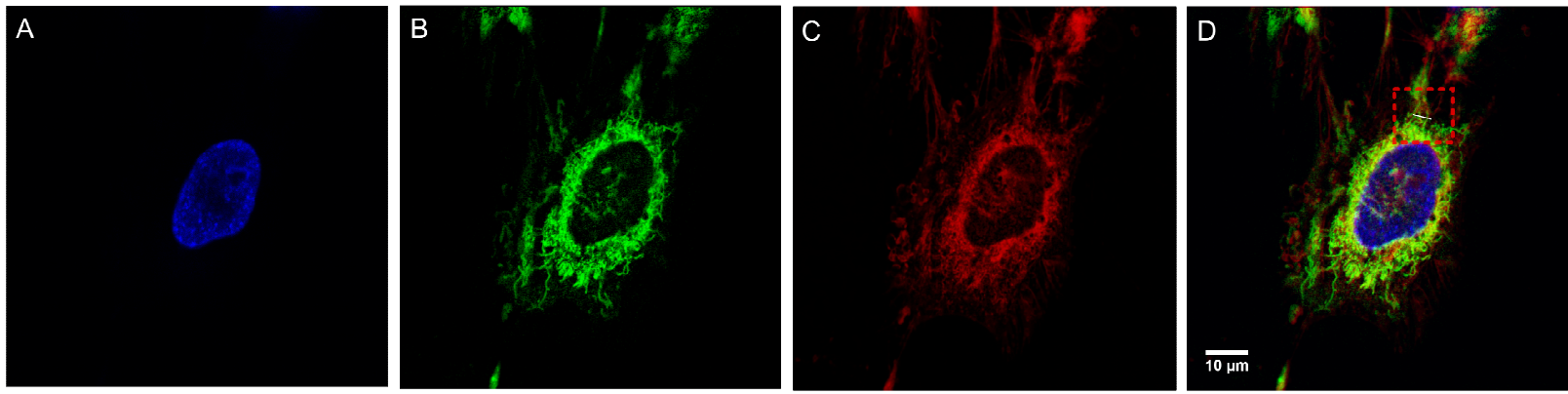

Abbildung 95. Kolokalisationsexperimente zur Aufnahme des Galaktosides (1S)-69 in lebende Zellen. A: $\lambda_{\text {exc }}=405 \mathrm{~nm}, \lambda_{\mathrm{em}}=410-429 \mathrm{~nm}$, Anfärbung des Zellkerns durch Hoechst 33342 (64); B: $\lambda_{\text {exc }}=405 \mathrm{~nm}, \lambda_{\mathrm{em}}=415-520 \mathrm{~nm}$ Anfärbung der Mitochondrien durch Galaktosid (1S)-69; C: $\lambda_{\mathrm{exc}}=640 \mathrm{~nm}, \lambda_{\mathrm{em}}=650-800 \mathrm{~nm}$, Anfärbung der Mitochondrien durch MitoTracker Deep Red 633 FM (65); D: Überlagerung von A-C.

\subsubsection{Untersuchung der zellulären Aufnahme fluroreszenzmarkierter (1S)- CBI-DMACA-Derivate (1S)-137, (1S)-139 sowie 62}<smiles>[R20]c1cc2c(c3ccccc13)[C@@H](CCl)CN2C(=O)Cc1cc(=O)oc2cc(N(C)C)ccc12</smiles>

$$
\begin{array}{ll}
R=H & (1 S)-137 \\
R=\beta-D-G a l(O H)_{4} & (1 S)-139
\end{array}
$$<smiles>CN(C)c1ccc2c(CC(=O)O)cc(=O)oc2c1</smiles>

62

Abbildung 96. Untersuchte Verbindungen mit DMACA (62) als DNA-Binder.

Abbildung 97 zeigt die Aufnahme des DNA Binders DMACA (62) in lebende Zellen. Wie schon im Fall der Cumarin-3-Carbonsäure (131) penetriert 62 bereits nach wenigen Minuten die Zellmembran und akkumuliert innerhalb der Zelle (B). Durch 
Kolokalisationsexperimente mit Hoechst 33342 (64) (A) sowie MitoTracker Deep Red 633 FM (65) (C) kann darauf geschlossen werden, dass es sich bei den von 62 populierten Organellen zum einen um Mitochondrien handelt, zum anderen aber auch eine diffuse Verteilung des Fluorochroms auftritt.
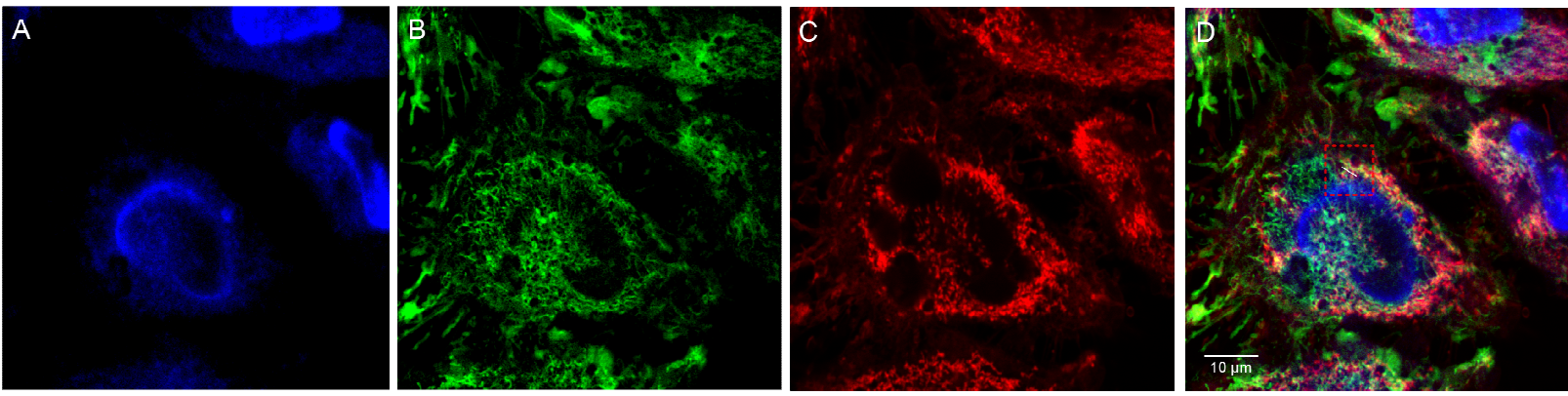

Abbildung 97. Kolokalisationsexperimente zur Aufnahme des Fluorochroms DMACA (62) in lebende Zellen. A: $\lambda_{\text {exc }}=405 \mathrm{~nm}, \lambda_{\mathrm{em}}=410-429 \mathrm{~nm}$, Anfärbung des Zellkerns durch Hoechst 33342 (64); B: $\lambda_{\text {exc }}=405 \mathrm{~nm}, \lambda_{\text {em }}=415-520 \mathrm{~nm}$, Anfärbung der Mitochondrien sowie diffuse Verteilung des Fluorochroms DMACA (62); C: $\lambda_{\text {exc }}=640 \mathrm{~nm}, \lambda_{\text {em }}=650-800 \mathrm{~nm}$, Anfärbung der Mitochondrien durch MitoTracker Deep Red 633 FM (65); D: Überlagerung von A-C.

Das seco-Drug (1S)-137 zeigt bereits nach wenigen Minuten eine Akkumulation innerhalb des Zellkerns sowie der Mitochondrien (Abbildung 98). Möglicherweise führt eine zu hohe Konzenztration des Fluorochroms zu einer Überpopulation des Zellkerns, so dass auch Mitochondrien, populiert werden, die bei geringeren Konzentrationen nicht vom seco-Drug (1S)-137 markiert würden.
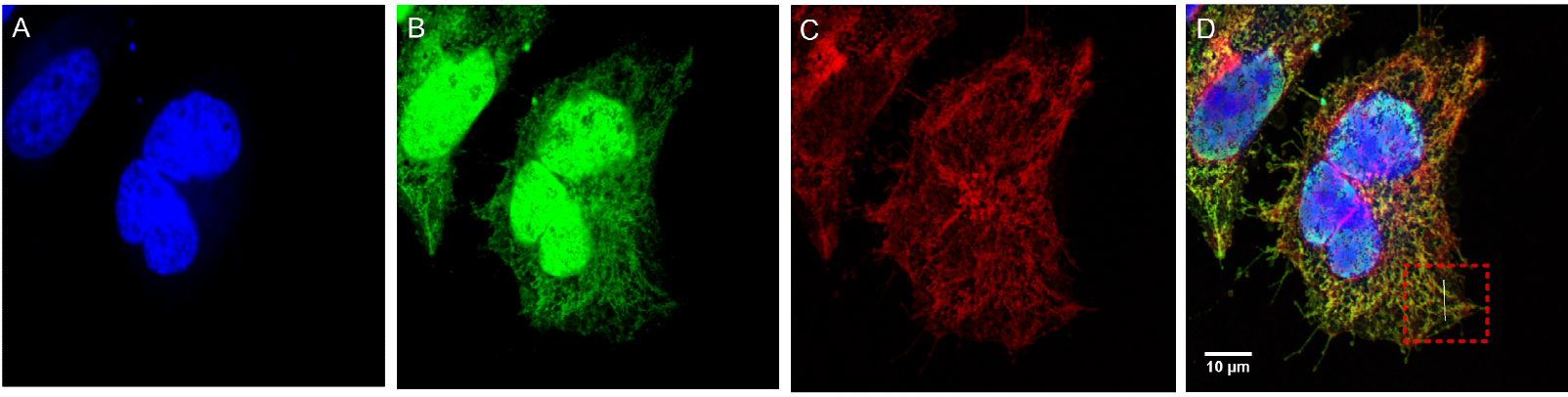

Abbildung 98. Kolokalisationsexperimente zur Aufnahme des seco-Drugs (1S)-137 in lebende Zellen. A: $\lambda_{\text {exc }}=405 \mathrm{~nm}, \lambda_{\text {em }}=410-429 \mathrm{~nm}$, Anfärbung des Zellkerns durch Hoechst 33342 (64); B: $\lambda_{\text {exc }}=405 \mathrm{~nm}, \lambda_{\text {em }}=415-520 \mathrm{~nm}$, Anfärbung der Mitochondrien durch seco-Drug (1S)-137; C: $\lambda_{\text {exc }}=640 \mathrm{~nm}, \lambda_{\text {em }}=650-800 \mathrm{~nm}$, Anfärbung der Mitochondrien durch MitoTracker Deep Red 633 FM (65); D: Überlagerung von A-C. 
Wie schon im Falle des CBI-Cumarin-3-Carbonsäure-Galaktosides (1S)-131 akkumuliert auch das aus DMACA (62) resultierende Galaktosid (1S)-139 bereits nach wenigen Minuten innerhalb der Zelle (Abbildung 99 (B)). Im Gegensatz zum seco-Drug (1S)-137 akkumuliert (1S)-139 jedoch nicht im Zellkern, sondern wie auch das Galaktosid (1S)-69 in den Mitochondrien. So zeigt auch (1S)-139 kein Verweilen außerhalb der Zelle, wie es für das ADEPT-Konzept erforderlich wäre.
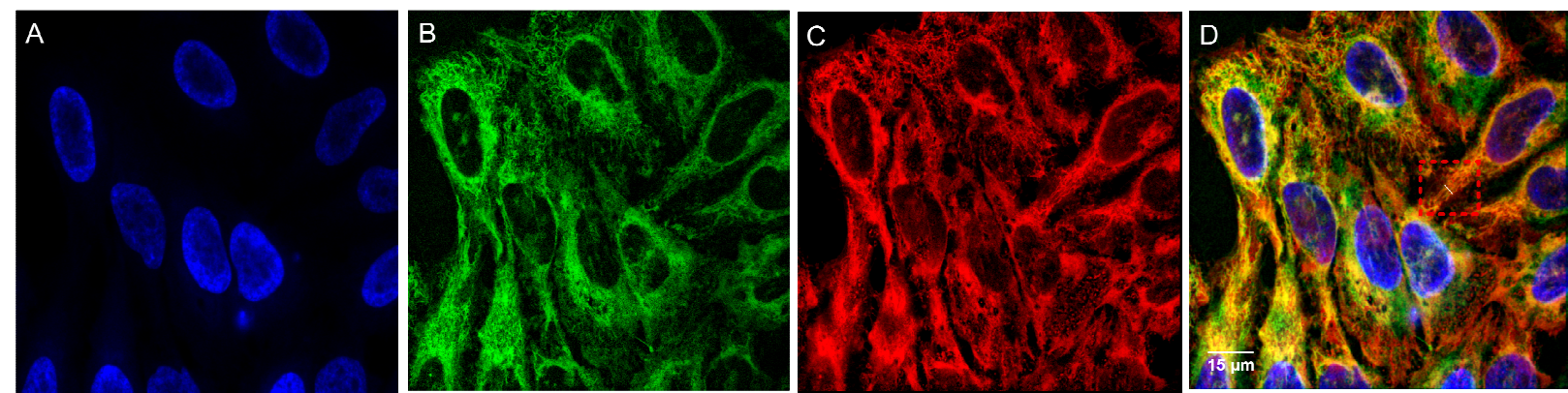

Abbildung 99. Kolokalisationsexperimente zur Aufnahme des Galaktosides (1S)-139 in lebende Zellen. A: $\lambda_{\text {exc }}=405 \mathrm{~nm}, \lambda_{\mathrm{em}}=410-429 \mathrm{~nm}$, Anfärbung des Zellkerns durch Hoechst 33342 (64); B: $\lambda_{\text {exc }}=405 \mathrm{~nm}, \lambda_{\mathrm{em}}=415-520 \mathrm{~nm}$, Anfärbung der Mitochondrien durch Galaktosid (1S)-139; C: $\lambda_{\text {exc }}=640 \mathrm{~nm}, \lambda_{\text {em }}=650-800 \mathrm{~nm}$, Anfärbung der Mitochondrien durch MitoTracker Deep Red 633 FM (65); D: Überlagerung von A-C.

\subsubsection{Untersuchung der zellulären Aufnahme fluroreszenzmarkierter (1S)- CBI-Indol-Cumarin-Derivate (1S)-138, (1S)-140 sowie 130}<smiles>[R20]c1cc2c(c3ccccc13)[C@@H](CCl)CN2C(=O)c1cc2c(ccc3oc(=O)ccc32)[nH]1</smiles><smiles>O=C(O)c1cc2c(ccc3oc(=O)ccc32)[nH]1</smiles>

130

$$
\begin{array}{ll}
\mathrm{R}=\mathrm{H} & (1 S)-\mathbf{1 3 8} \\
\mathrm{R}=\boldsymbol{\beta}-\mathrm{D}-\mathrm{Gal}(\mathrm{OH})_{4} & (1 S)-140
\end{array}
$$

Abbildung 100. Untersuchte Verbindungen mit Cumarin-Indol-2-Carbonsäure (130) als DNA-Binder. 
Auf Grund seiner starken chemischen Ähnlichkeit zu dem im Arbeitskreis Tietze verwendeten DNA-Binder DMAI (104) sollte Cumarin-Indol-2-Carbonsäure (130) gute Aufschlüsse über die Aufnahme der seco-CBI-DMAI-Analoga wie (1S)-48 liefern.

Wie auch die beiden vorherigen Cumarine 62 und 131 akkumuliert 130 bereits nach wenigen Minuten innerhalb der Zelle (Abbildung 101(B)). Auf Grund der Kolokalisationsexperimente mit Hoechst 33342 (64) (A) sowie MitoTracker Deep Red 633 FM (65) (C) konnte eindeutig gezeigt werden, dass es sich bei den von 130 populierten Organellen um Miochondrien handelt
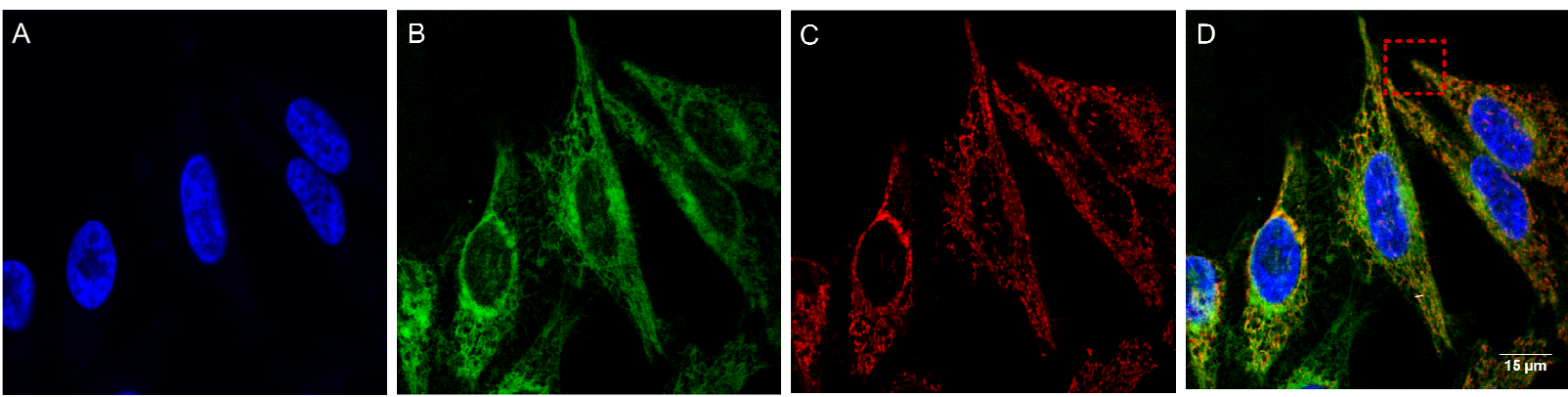

Abbildung 101. Kolokalisationsexperimente zur Aufnahme des Fluorochroms Cumarin-Indol-2Carbonsäure (130) in lebende Zellen. A: $\lambda_{\text {exc }}=405 \mathrm{~nm}, \lambda_{\text {em }}=410-429 \mathrm{~nm}$, Anfärbung des Zellkerns durch Hoechst 33342 (64); B: $\lambda_{\text {exc }}=405 \mathrm{~nm}, \lambda_{\text {em }}=415-$ $520 \mathrm{~nm}$, Anfärbung der Mitochondrien durch Cumarin-Indol-2-Carbonsäure (130); C: $\lambda_{\text {exc }}=640 \mathrm{~nm}, \lambda_{\text {em }}=650-800 \mathrm{~nm}$, Anfärbung der Mitochondrien durch MitoTracker Deep Red 633 FM (65); D: Überlagerung von A-C.

Abbildung 102 zeigt die Verteilung des aus Cumarin-Indol-2-Carbonsäure (130) resultierenden seco-Drugs (1S)-138. Wie das seco-CBI-Cumarin-3-Carbonsäure-Drug (1S)-92 akkumuliert (1S)-138 innerhalb der Mitochondrien und damit nicht wie das seco-CBI-DMACA-Drug (1S)-137 innerhalb des Zellkerns.
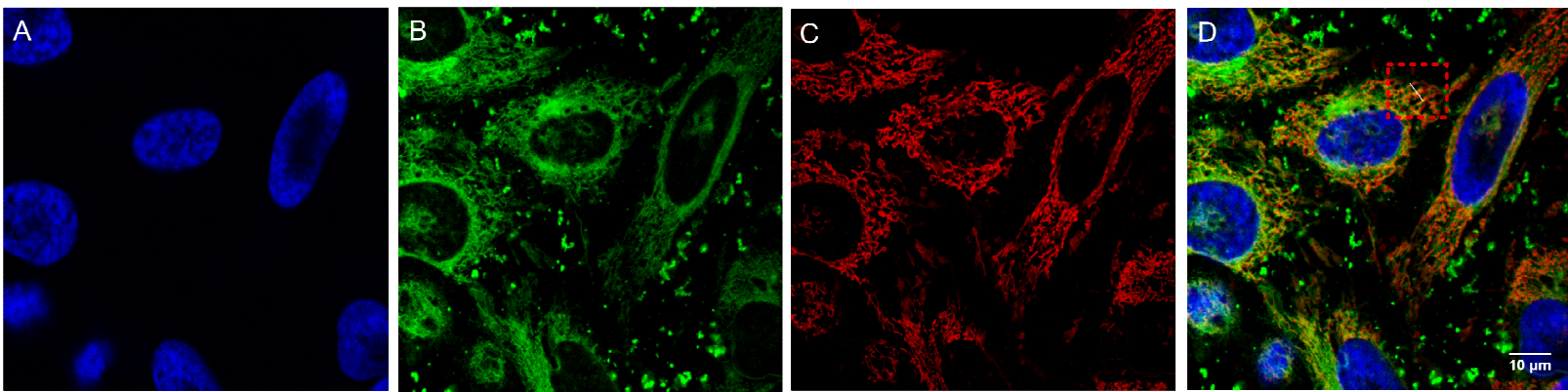

Abbildung 102. Kolokalisationsexperimente zur Aufnahme des seco-Drugs (1S)-138 in lebende Zellen. A: $\lambda_{\text {exc }}=405 \mathrm{~nm}, \lambda_{\text {em }}=410-429 \mathrm{~nm}$, Anfärbung des Zellkerns durch Hoechst 33342 (64); B: $\lambda_{\text {exc }}=405 \mathrm{~nm}, \lambda_{\mathrm{em}}=415-520 \mathrm{~nm}$, Anfärbung der Mitochondrien 
durch seco-Drug (1S)-138; C: $\lambda_{\text {exc }}=640 \mathrm{~nm}, \lambda_{\text {em }}=650-800 \mathrm{~nm}$, Anfärbung der Mitochondrien durch MitoTracker Deep Red 633 FM (65); D: Überlagerung von A-C.

Eine abschließende Betrachtung des Cumarin-Indol-2-Carbonsäure-Galaktosides (1S)140 zeigt auch hier, anders als im Rahmen von ADEPT erwartet, eine Aufnahme des Galaktosides in lebende Zellen bereits nach wenigen Minuten. Es konnte gezeigt werden, dass (1S)-140 wie auch die bisher untersuchten Galaktoside (1S)-131 und (1S)-62 in den Mitochondrien akkumuliert. Zusätzlich kommt es im Fall von (1S)-140 zu einer diffusen Verteilung innerhalb der Zelle (Abbildung 103 (B)).
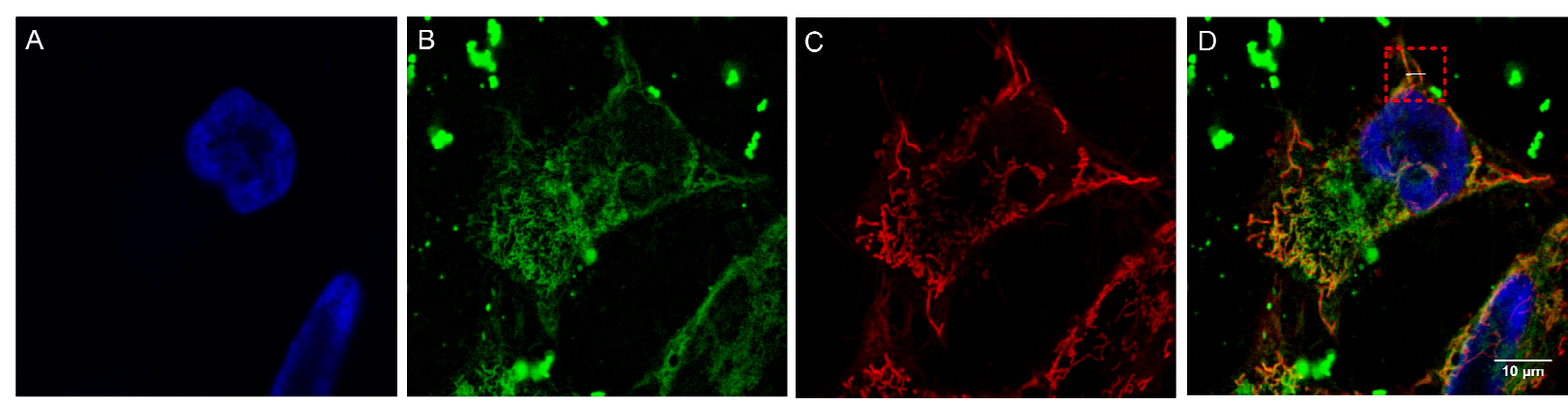

Abbildung 103. Kolokalisationsexperimente zur Aufnahme des Galaktosides (1S)-140 in lebende Zellen. A: $\lambda_{\text {exc }}=405 \mathrm{~nm}, \lambda_{\mathrm{em}}=410-429 \mathrm{~nm}$, Anfärbung des Zellkerns durch Hoechst 33342 (64); B: $\lambda_{\text {exc }}=405 \mathrm{~nm}, \lambda_{\mathrm{em}}=415-520 \mathrm{~nm}$, Anfärbung der Mitochondrien durch Galaktosid (1S)-140; C: $\lambda_{\text {exc }}=640 \mathrm{~nm}, \lambda_{\mathrm{em}}=650-800 \mathrm{~nm}$, Anfärbung der Mitochondrien durch MitoTracker Deep Red 633 FM (65); D: Überlagerung von A-C.

Abschließend kann festgestellt werden, dass keine der dargestellten CBI-CumarinDerivate die von ADEPT postulierten Bedingungen erfüllt.

So konnte für die Galaktoside (1S)-69, (1S)-139 und (1S)-140 keine Akkumulation außerhalb der Zelle beobachtet werden. Stattdessen wurden die untersuchten Galaktoside bereits nach wenigen Minuten in die Zellen aufgenommen und wurden in allen Fällen in den Mitochondrien sichtbar.

Etwas anders verhält es sich bei der Betrachtung der seco-Drugs (1S)-92, (1S)-137 und (1S)-138. Zunächst erfüllen alle untersuchten seco-Drugs die Bedingungen rasch in die Zellen zu gelangen, jedoch unterscheidet sich auch hier die anschließende Akkumulierung in verschiedenen Organellen. Lediglich das aus DMACA resultierende seco-Drug (1S)-137 akkumuliert innerhalb des Zellkerns, während die seco-Drugs (1S)-92 und (1S)-138 in den Mitochondrien zu finden sind. 


\section{Synthese neuartiger fluoreszenzmarkierter (1S)-CBI- Benzothiazol-Duocarmycin-Analoga}

Benzothiazole sind als fluoreszente DNA-Binder mit einer Neigung zur Bindung in ATreichen Regionen der kleinen Furche doppelsträngiger DNA bekannt und eignen sich daher hervorragend für den Einsatz als Fluorochrom. ${ }^{162}$ Aus diesem Grund wurden verschiedene Benzothiazole 63 und 142 genutzt, um neuartige seco-Drugs wie (1S)-141 und den entsprechenden Prodrugs als fluoreszenzmarkierte Analoga von (1S)-48 darzustellen (Abbildung 104). Desweiteren wurden die Methylester der Benzothiazole 144 und 143 dargestellt (Abbildung 105), um die Aufnahme der Fluorochrom in Zellen zu beobachten, da bekannt war, dass die Carbonsäuren 63 und 142 nicht in lebende Zelle aufgenommen werden. ${ }^{162}$

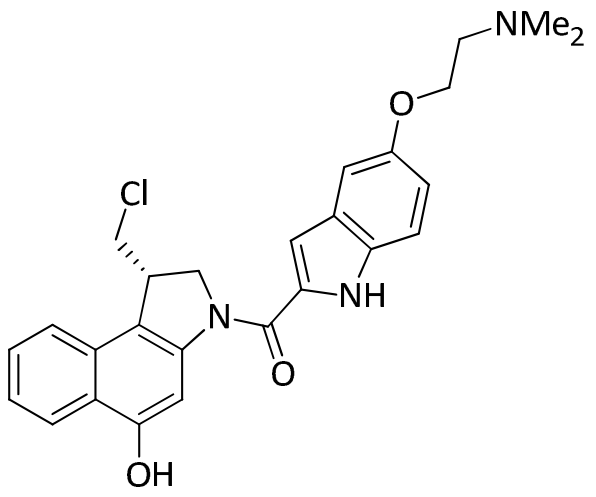

(1S)-48

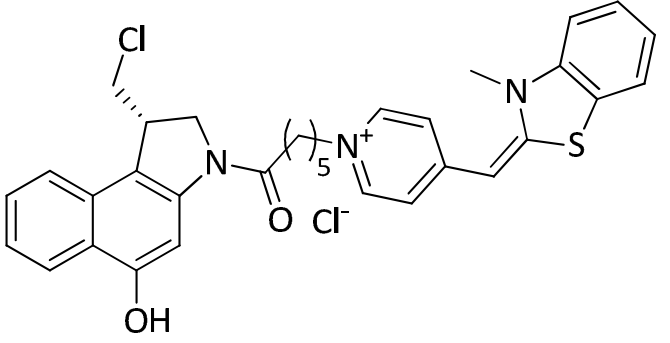

(1S)-141

Abbildung 104. Strukturvergleich der seco-CBI-Analoga mit DMAI (1S)-48 oder Benzothiazol (1S)-141 als DNA-bindende Einheit.

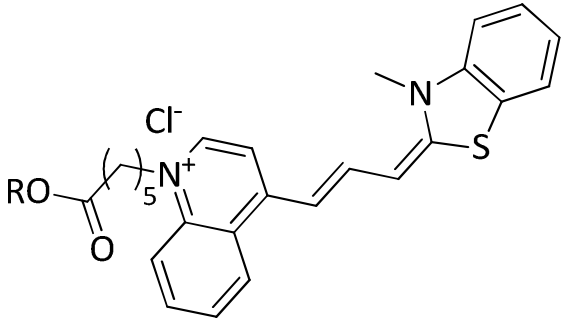

$\mathrm{R}=\mathrm{H} \quad 142$

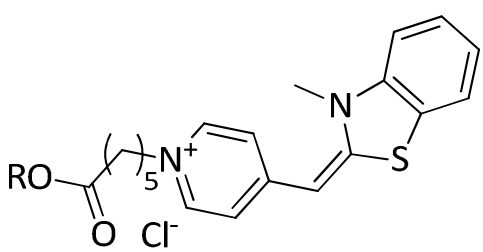

$\mathrm{R}=\mathrm{H} \quad 63$

$\mathrm{R}=\mathrm{Me} 144$

Abbildung 105. Verwendete Benzothiazole TO3 (Thiazol Orange 3) 142 und 143 sowie BO (Benzothiazole Orange) 63 und 144. 


\subsection{Synthese der seco-CBI-Benzothiazol-Drugs (1S)-141 und (1S)-145}

Ausgehend vom Phenol (1S)-128 konnten die seco-CBI-Benzothiazol-Drugs (1S)-141 und (1S)-145 über 2 Stufen hergestellt werden. So wurde zunächst die N-Boc-Schutzgruppe durch Suspendieren des Phenols (1S)-128 in $4 \mathrm{M} \mathrm{HCl/Essigester-Lösung}$ entfernt, um das freie sekundäre Amin im Anschluss unter Verwendung von EDAC $\cdot \mathrm{HCl}$ als Kupplungsreagenz mit der Carbonsäure der Benzothiazole 63 oder 142 zu kuppeln. Abschließend wurden die erhaltenen seco-CBI-Drugs (1S)-141 und (1S)-145 durch präparative HPLC (Kromasil 100 C-18 ) gereinigt (Abbildung 106).

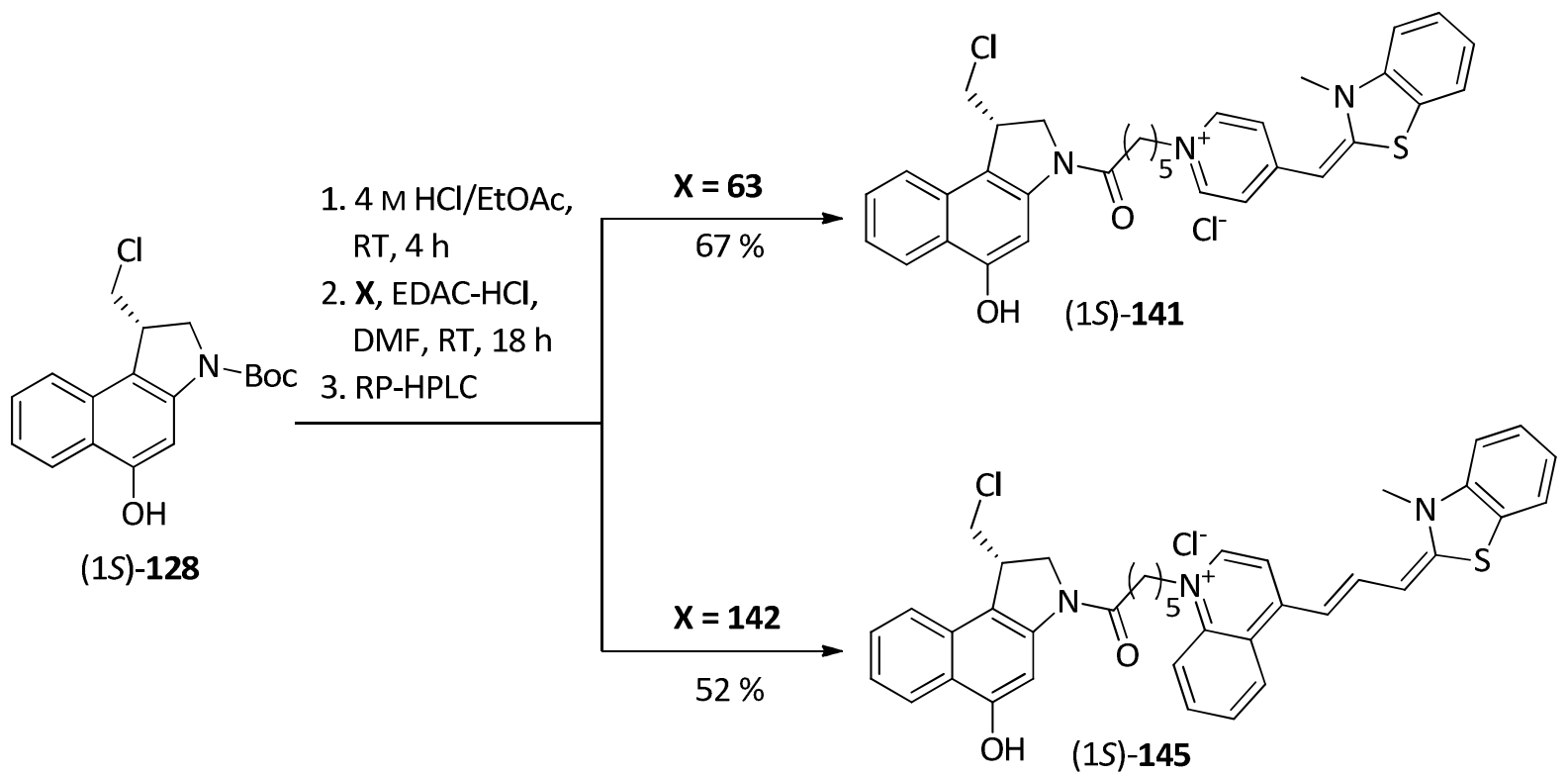

Abbildung 106. Syntheseschema zur Darstellung neuartiger fluoreszenzmarkierter seco-CBIBenzothiazol-Drugs (1S)-141 und (1S)-145.

\subsubsection{Diskussion ausgewählter spektroskopischer Daten des seco-CBI-BO- Drugs (1S)-141}

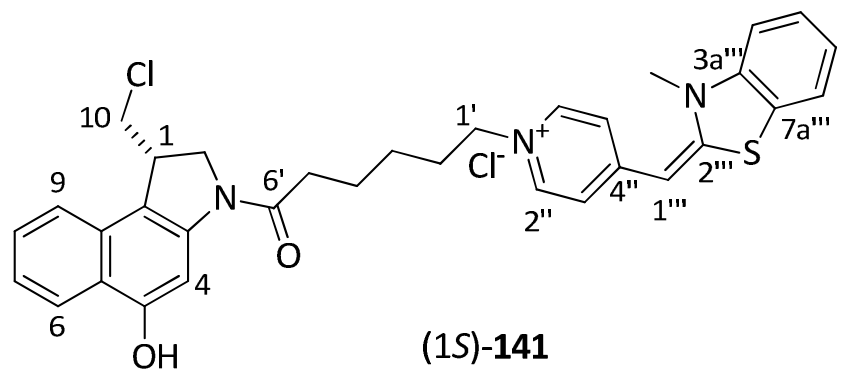

Abbildung 107. Struktur des seco-CBI-BO-Drugs (1S)-141. 


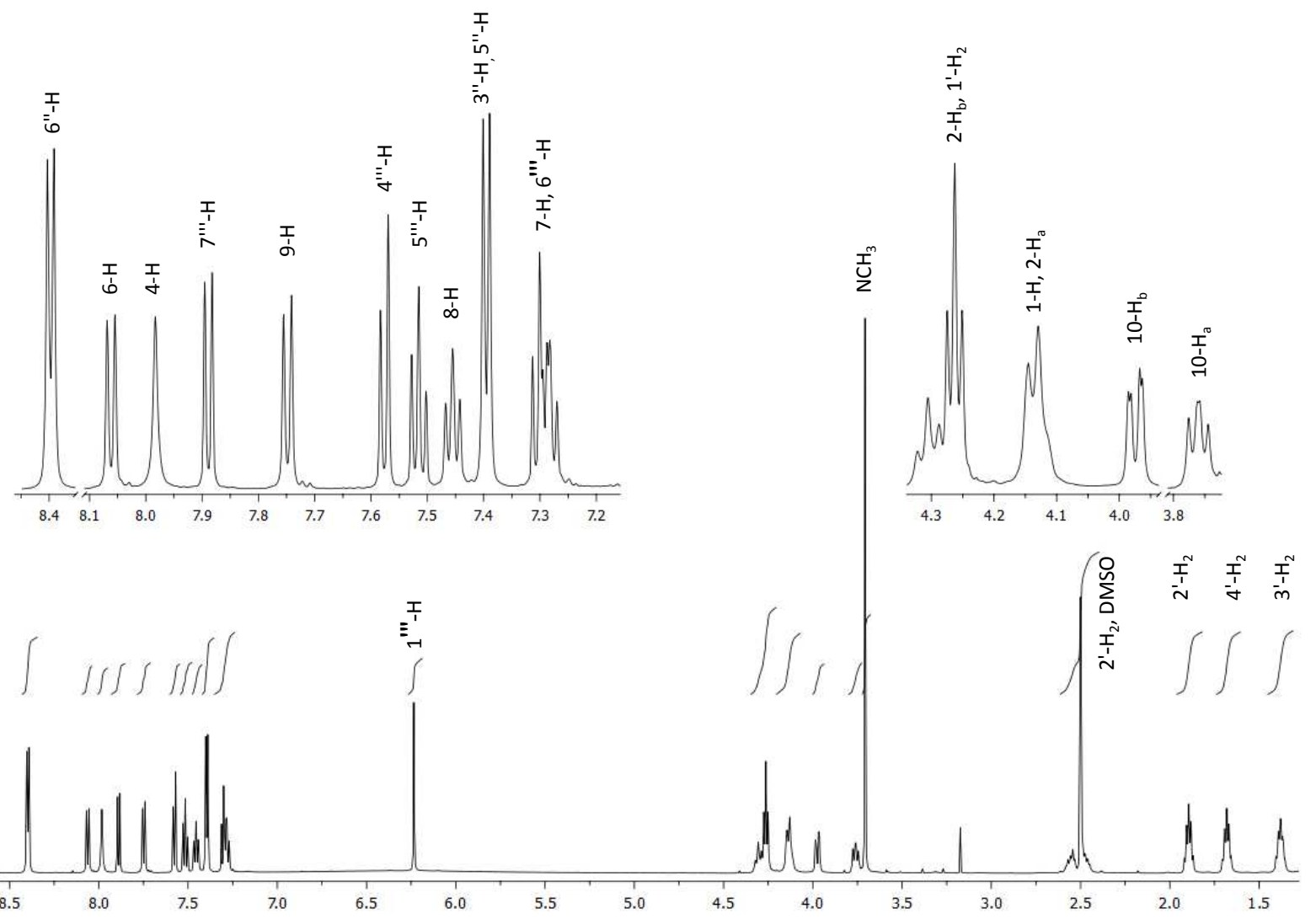

Abbildung 108. ${ }^{1} \mathrm{H}-\mathrm{NMR}$ Spektrum (600 Mhz, DMSO-d $\mathrm{d}_{6}$ ) des seco-CBI-BO-Drugs (1S)-141.

Abbildung 108 zeigt das ${ }^{1} \mathrm{H}-\mathrm{NMR}$ Spektrum sowie die Zuordnung der Signale des secoCBI-BO-Drugs (1S)-141, das in DMSO- $\mathrm{d}_{6}$ bei $600 \mathrm{MHz}$ aufgenommen wurde.

Bei $\delta=1.33-1.44$ ppm resoniert die Mehtylengruppe der Spacereinheit als Multiplett für die Protonen an C-3'. Gefolgt von den benachbarten Protonen an C-4' bei $\delta=1.64-1.73$ ppm und an C-2' bei $\delta=1.85-1.94$ ppm. Das Resonanzsignal für die 5'Protonen findet man bei $\delta=2.43-2.62 \mathrm{ppm}$, die sich in $\alpha$-Position zur Carbonylfunktion befinden. Die Protonen der Methylaminofunktion findet man bei $\delta=3.71 \mathrm{ppm}$ als Singulett. Es folgen die Resonanzsignale für das CBI-Grundgerüst. Bei $\delta=3.76$ ppm ein Dublett vom Dublett mit $J=10.4$ und $8.3 \mathrm{~Hz}$, zunächst das Signal für das Proton $10-\mathrm{H}_{\mathrm{a}}$ sowie bei $\delta=3.97 \mathrm{ppm}$ dessen geminaler Resonanzpartner $10-\mathrm{H}_{\mathrm{b}}$ ebenfalls als Dublett vom Dublett mit $J=10.9$ und $2.6 \mathrm{~Hz}$. Ein Multiplett bei $\delta=4.10-$ 4.17 ppm zeigt die Resonanzen für ein Proton an C-2 sowie 1-H. Ein Multiplett bei $\delta=4.23-4.35$ ppm zeigt die Resonanzen für das zweite Proton an C-2, sowie die Methylenprotonen an C-1', die auf Grund ihrer Nähe zum Pyridiniumion, die am 
weitesten ins Tieffeld verschobene Gruppe der Spacereinheit darstellt. Bei $\delta=6.24 \mathrm{ppm}$ resoniert das Doppelbindungsproton an C-1"' als Singulett. Der aromatische Bereich des Spektrums beginnt mit dem Resonanzsignal für die Protonen an C-6"' sowie an C-7 bei $\delta=7.26-7.32$ ppm. Bei $\delta=7.40$ ppm findet man das Signal für die chemisch äquivalenten Protonen an C-3" sowie an C-5" als Dublett mit einer typischen ortho-Kopplungskonstante von $J=7.0 \mathrm{~Hz}$. Das Signal für das Proton an C-8 beobachtet man bei $\delta=7.45 \mathrm{ppm}$ als Triplett mit $J=7.5 \mathrm{~Hz}$, das durch zwei identische Kopplungen mit $\mathrm{H}-9$ und $\mathrm{H}-7$ entsteht. Bei $\delta=7.52 \mathrm{ppm}$ und $\delta=7.58 \mathrm{ppm}$ folgen die Signale für die aromatischen Protonen an C-4"' und C-5"' als Triplett bzw. als Dublett. Es folgen die Signale für $9-\mathrm{H}$ bei $\delta=7.75 \mathrm{ppm}$ als Dublett durch Kopplung mit $8-\mathrm{H}$ sowie bei $\delta=7.89$ ppm das Resonanzsignal für $7^{\prime \prime \prime}-\mathrm{H}$ als Dublett mit $J=7.8 \mathrm{~Hz}$, das durch ortho-Kopplung mit 6"'-H erklärt werden kann. Bei $\delta=7.98$ ppm folgt ein Singulett für $4-\mathrm{H}$ sowie bei $\delta=8.06 \mathrm{ppm}$ ein Dublett für das Proton an C-6 der CBIEinheit als Dublett mit $J=8.4 \mathrm{~Hz}$. Am weitesten ins Tieffeld verschoben sind die chemisch äquivalenten Protonen an C-2" und C- 6 " bei $\delta=8.40$ ppm als Dublett sowie die phenolische Hydroxyfunktion als breites Singulett bei $\delta=10.34$ ppm. 


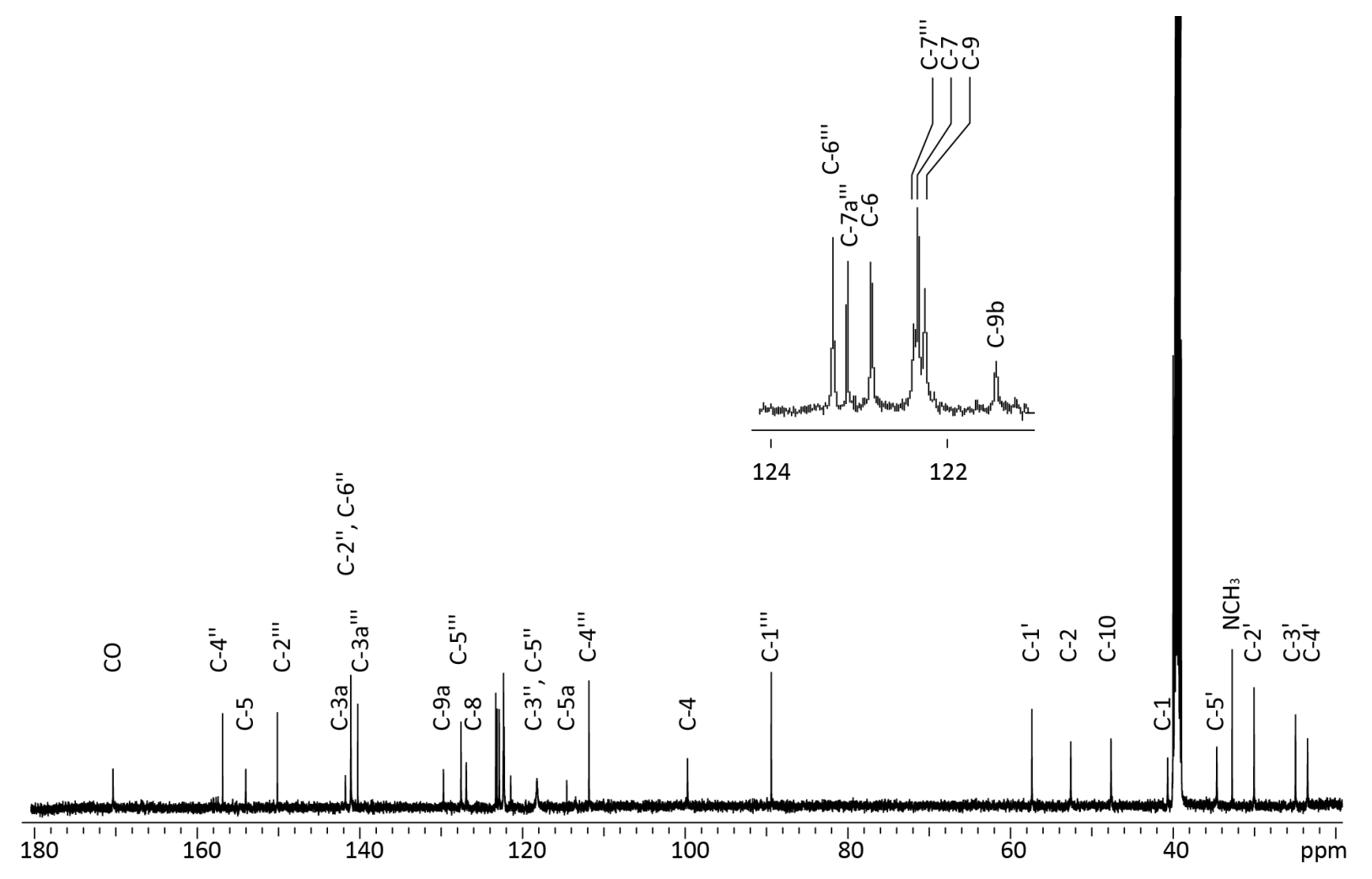

Abbildung 109. ${ }^{13} \mathrm{C}-\mathrm{NMR}$ Spektrum (125 Mhz, DMSO-d $\mathrm{d}_{6}$ ) des seco-CBI-BO-Drugs (1S)-141.

Abbildung 109 zeigt das ${ }^{13} \mathrm{C}$-Spektum (125 Mhz, DMSO-d $\mathrm{d}_{6}$ ) des seco-Drugs (1S)-141. Im Hochfeld beginnt das Spektrum mit den Resonanzsignalen für die Kohlenstoffatome der Spacereinheit mit C-4' bei $\delta=23.3 \mathrm{ppm}$, gefolgt von C-3' $(\delta=24.9)$, C-2' $(\delta=30.0)$ und $\mathrm{C}-5^{\prime}(\delta=34.6)$ sowie bei $\delta=57.4$ ppm für $\mathrm{C}-1^{\prime}$. Ebenso sind auch die Signale für die Kohlenstoffatome der Mehtylaminofunktion (bei $\delta=32.7 \mathrm{ppm}$ ) sowie der aliphatischen Kohlenstoffatome des CBI-Grundgerüstes weit ins Hochfeld verschoben. Man findet die Resonanzsignale für C-1 bei $\delta=40.7 \mathrm{ppm}$, für C-10 bei $\delta=47.6$ ppm und für C-2 bei $\delta=52.6$ ppm. Das Signal für das C-1"'Kohlenstoffatom beobachtet man bei $\delta=89.4 \mathrm{ppm}$. Es folgen die Signale für die aromatischen Kohlenstoffatome beginnend mit C-4 bei $\delta=99.7 \mathrm{ppm}$. Es folgen die Resonanzsignale für die Kohlenstoffatome des Benzothiazolgerüstes bei $\delta=111.8$ (C-4"'), 122.4 (C-7"'), 123.1 (C-7a'"'), 123.3 (C-6"'), 127.5 (C-5"'), 140.2 (C-3a'"') und 150.1 (C-2"') sowie die Resonanzsignale für die pharmakophore CBI-Einheit bei $\delta=114.5$ (C-5a), 121.4 (C-9b), 122.2 (C-9), 122.3 (C-7), 122.8 (C-6), 126.9 (C-8), 129.7 (C-9a), 141.8 (C-3a), 154.0 (C-5) und die Signale für den Pyridiniumteil bei $\delta=118.2$ 
(C-3", C-5"), 141.1 (C-2", C-6"), 156.9 (C-4"). Das Spektrum wird im Tieffeld durch das Resonanzsignal für das Carbonylkohlenstoffatom bei $\delta=170.3$ ppm abgeschlossen.

\subsection{Synthese der (1S)-CBI-Benzothiazol-Galaktoside (1S)-70 und (1S)-147}

Ausgehend vom Phenol (1S)-128 konnten die CBI-Benzothiazol-Galaktoside (1S)-70 und (1S)-145 über 4 Stufen hergestellt werden. Zunächst wurde das freie Phenol (1S)128 nach der Trichloracetimidatmethode nach Schmidt ${ }^{157}$ unter $\mathrm{BF}_{3} \cdot \mathrm{OEt}_{2}$-Katalyse mit tetraacetyliertem D-Galaktose-Trichloracetimidat 116 gekuppelt und anschließend die säurelabile $\mathrm{N}$-Boc-Schutzgruppe $\mathrm{BF}_{3} \cdot \mathrm{OEt}_{2}$-vermittelt abgespalten. Das freie sekundäre Amin wurde dann mit EDAC $\cdot \mathrm{HCl}$ als Kupplungsreagenz in DMF mit der Carbonsäure des entsprechenden Benzothiazols 63 oder 142 gekuppelt um die tetraacetylierten CBI-Benzothiazol-Galaktoside zu erhalten.

Auf Grund der geringen Ausbeuten bei der Entschützung der tetraacetylierten Galaktoside nach Zémplen wurde versucht, die Bildung des unerwünschten Nebenproduktes 143 bzw. 144 zu verringern (Abbildung 110 und Tabelle 4). Es wurde angenommen, dass das Pyridin- bzw. Chinolinisalz des Fluorochroms als Lewissäure fungiert und das Carbonyl gegenüber einem Angriff durch Methanolat aktiviert.

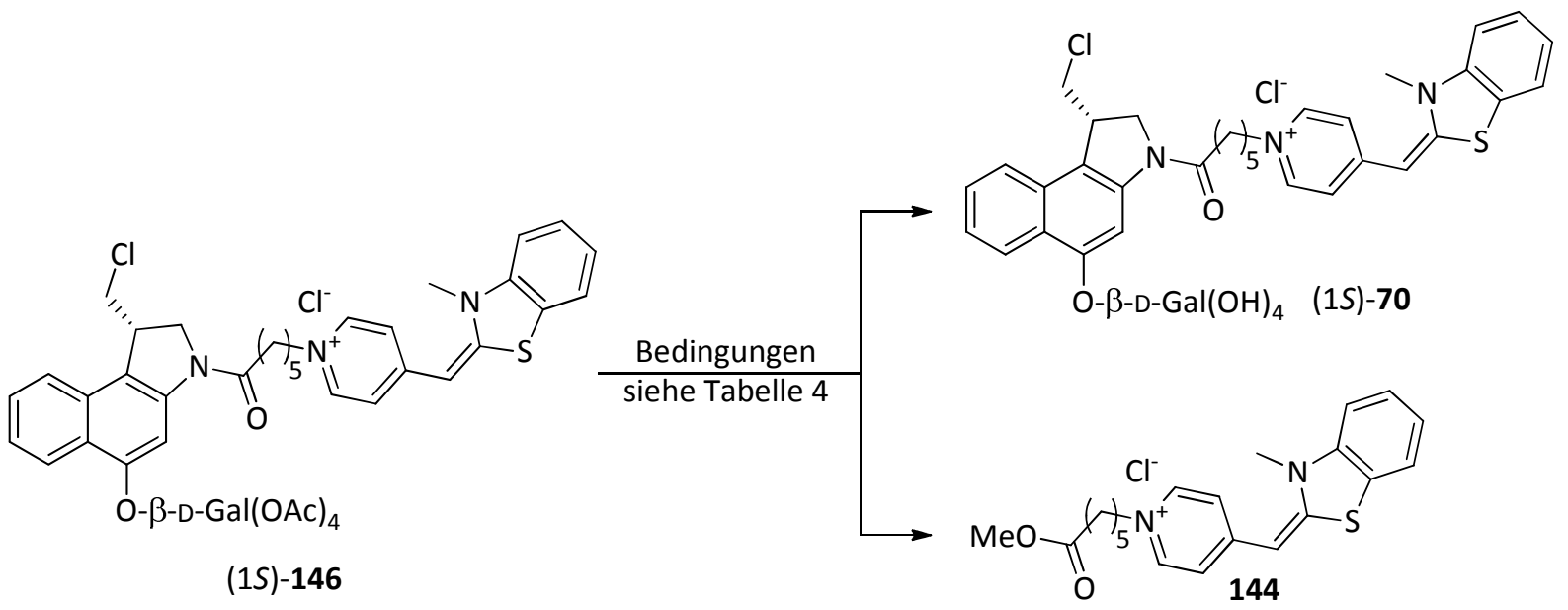

Abbildung 110. Versuche zur Darstellung des CBI-Galaktosides (1S)-70 bei gleichzeitiger Bildung des Mehtylesters 144 als unerwünschtes Nebenprodukt. 


\begin{tabular}{|c|c|c|c|c|c|}
\hline Bedingungen & & Zeit & $(1 S)-146[\%]$ & $(1 S)-70[\%]$ & $144[\%]$ \\
\hline $\mathrm{NaOMe}$ (1.0 eq.) in $\mathrm{MeOH}$ & $-20^{\circ} \mathrm{C}$ & $24 \mathrm{~h}$ & 31 & 18 & 51 \\
\hline $\mathrm{NaOMe}$ (1.0 eq.) in $\mathrm{MeOH}$ & $0^{\circ} \mathrm{C}$ & $60 \mathrm{~min}$ & 14 & 14 & 72 \\
\hline $\mathrm{NaOMe}(0.5$ eq.) in $\mathrm{MeOH}$ & RT & $60 \mathrm{~min}$ & 73 & 9 & 18 \\
\hline $\mathrm{NaOMe}$ (2.0 eq.) in $\mathrm{MeOH}$ & $-20^{\circ} \mathrm{C}$ & $24 \mathrm{~h}$ & 0 & 46 & 54 \\
\hline $\mathrm{NaOMe}$ (2.0 eq.) in $\mathrm{MeOH}$ & $0^{\circ} \mathrm{C}$ & $60 \mathrm{~min}$ & 0 & 17 & 83 \\
\hline $\mathrm{NaOMe}$ (2.0 eq.) in $\mathrm{MeOH}$ & RT & $60 \mathrm{~min}$ & 0 & 29 & 71 \\
\hline $\mathrm{NaOMe}$ (4.0 eq.) in $\mathrm{MeOH}$ & $-20^{\circ} \mathrm{C}$ & $24 \mathrm{~h}$ & 0 & 47 & 53 \\
\hline $\mathrm{NaOMe}$ (4.0 eq.) in $\mathrm{MeOH}$ & $0^{\circ} \mathrm{C}$ & $90 \mathrm{~min}$ & 0 & 63 & 37 \\
\hline $\mathrm{NaOMe}$ (4.0 eq.) in $\mathrm{MeOH}$ & RT & $120 \mathrm{~min}$ & 0 & 17 & 83 \\
\hline $\mathrm{K}_{2} \mathrm{CO}_{3}$ (0.5 eq.) in $\mathrm{MeOH}$ & RT & $160 \mathrm{~min}$ & 5 & 73 & 22 \\
\hline $\mathrm{NaOMe}(1.0$ eq.) in $\mathrm{PrOH}$ & RT & $26 \mathrm{~h}$ & 34 & 38 & 28 \\
\hline$p$ - $\mathrm{TsOH}$ (4.0 eq.) in $\mathrm{MeOH}$ & RT & $48 \mathrm{~h}$ & 32 & 11 & 57 \\
\hline Candida antarctica lipase & RT & $24 \mathrm{~h}$ & 100 & 0 & 0 \\
\hline
\end{tabular}

Tabelle 4. Versuche zur Darstellung von (1S)-70.

Anschließende Deacetylierung durch Kaliumcarbonat in Methanol bei Raumtemperatur lieferte die entsprechenden (1S)-CBI-Cumarin-Galaktoside (1S)-70 und (1S)-147 in guten bis mäßigen Ausbeuten. Abschließend wurden die erhaltenen Prodrugs (1S)-70 und (1S)-147 durch präparative HPLC (Kromasil 100 C-18 ) gereinigt.

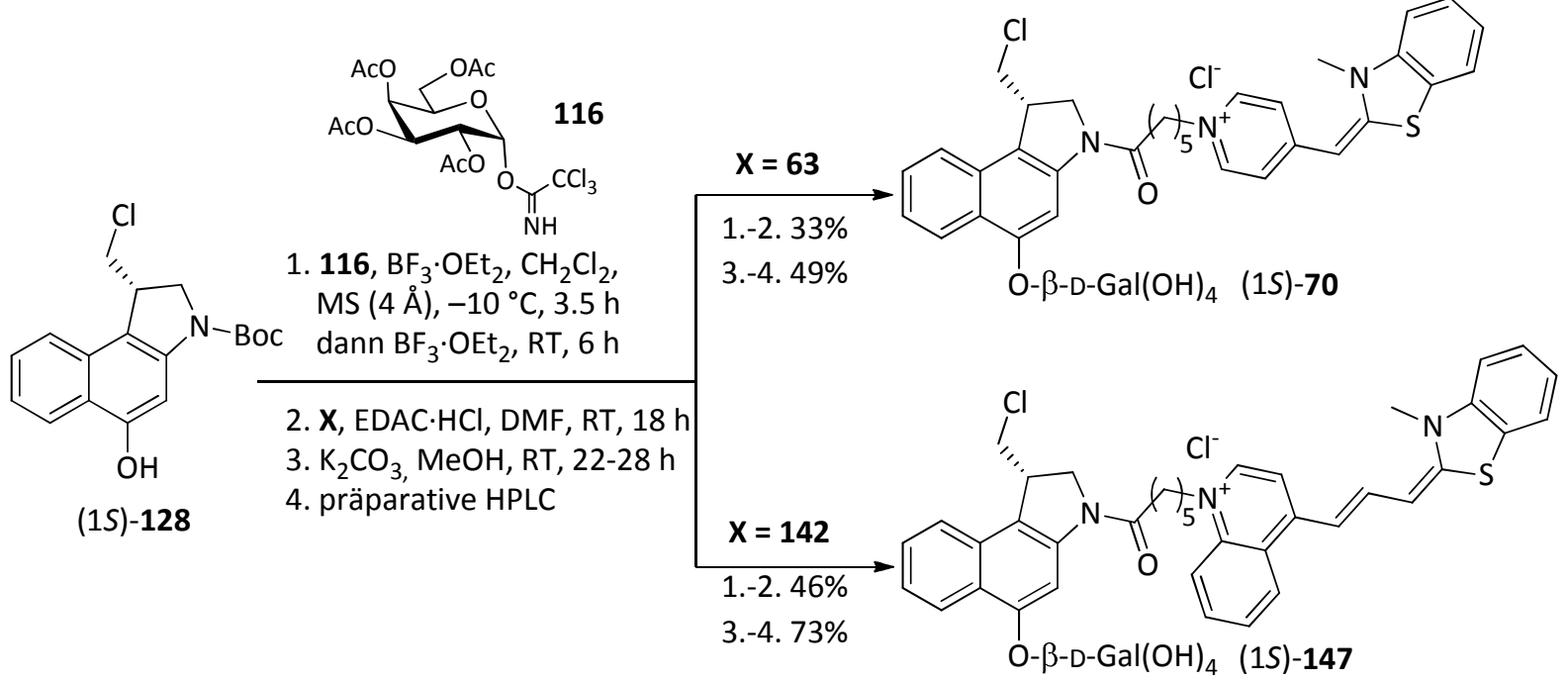

Abbildung 111. Synthese der (1S)-CBI-Benzothiazol-Galaktoside (1S)-70 und (1S)-147 ausgehend von Phenol (1S)-128. 
7.2.1 Ergebnisse zur Untersuchung der In-vitro-Zytotoxizität an humanen Bronchialkarzinomzellen (A549) für die seco-CBI-BenzothiazolDerivate (1S)-141 und (1S)-145 sowie den Galaktosiden (1S)-147 und (1S)-70

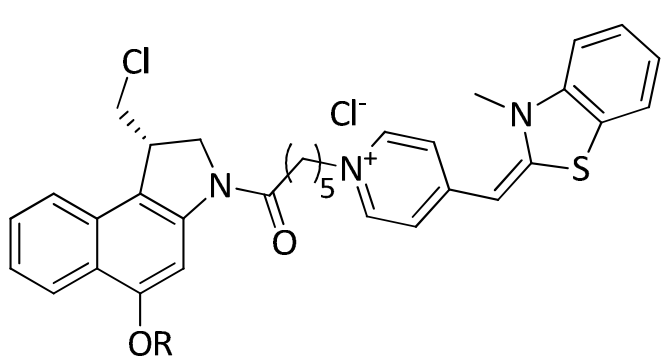

$\begin{array}{ll}\mathrm{R}=\mathrm{H} & (1 S)-\mathbf{1 4 1} \\ \mathrm{R}=\beta-\mathrm{D}-\mathrm{Gal}(\mathrm{OH})_{4} & (1 S)-70\end{array}$

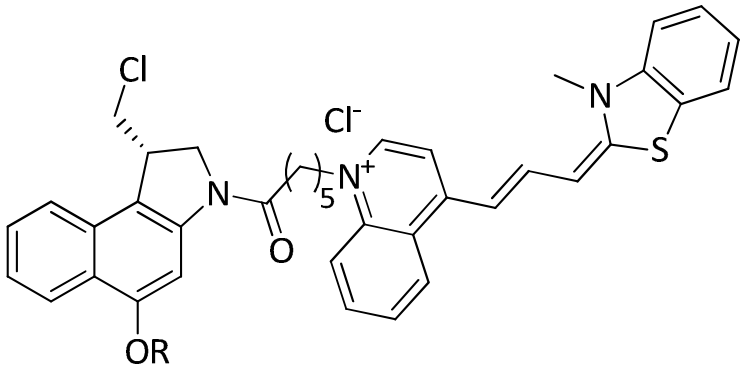

$\begin{array}{ll}\mathrm{R}=\mathrm{H} & (1 \mathrm{~S})-145 \\ \mathrm{R}=\beta-\mathrm{D}-\mathrm{Gal}(\mathrm{OH})_{4} & (1 S)-147\end{array}$

Abbildung 112. Untersuchte (1S)-Benzothiazol Galaktoside (1S)-70 und (1S)-147 sowie secoBenzothiazol-Drugs (1S)-141 und (1S)-145.

Abbildung 112 zeigt die Ergebnisse der Untersuchungen zur In vitro-Zytotoxizität der in dieser Arbeit synthetisierten (1S)-Benzothiazol-Derivate. Die (1S)-Galaktoside (1S)70 und (1S)-147 zeigen mit $\mathrm{IC}_{50}=106 \mathrm{nM}$ und $\mathrm{IC}_{50}=2.3 \mathrm{nM}$ in Abwesenheit des Enzyms große Unterschiede in ihrer Toxizität, während die seco-Drugs (1S)-141 und (1S)-145 nur geringe Unterschiede in ihrer Toxizität aufweisen (IC $C_{50}=18.1 \mathrm{nM}$ für (1S)141 und $I C_{50}=7.2 \mathrm{nM}$ für (1S)-145). Mit QIC $\mathrm{C}_{50}=5.6$ kann für das Galaktosid (1S)-141 nur eine geringe therapeutische Breite gefunden werden. Auch das Galaktosid (1S)147 zeigt in Anwesenheit des Enzyms keine signifikante Toxizitätssteigerung $\left(Q_{I C} C_{50}=1.4\right)$. Vergleicht man die ermittelten Werte mit den Kriterien des ADEPTKonzeptes kann festgestellt werden, dass sich die synthetisierten BenzothiazolDerivate trotz ihrer hervorragenden optischen Eigenschaften, besonders im Fall des Galaktosides (1S)-147 nicht für einen Einsatz im Rahmen von ADEPT eignen. 


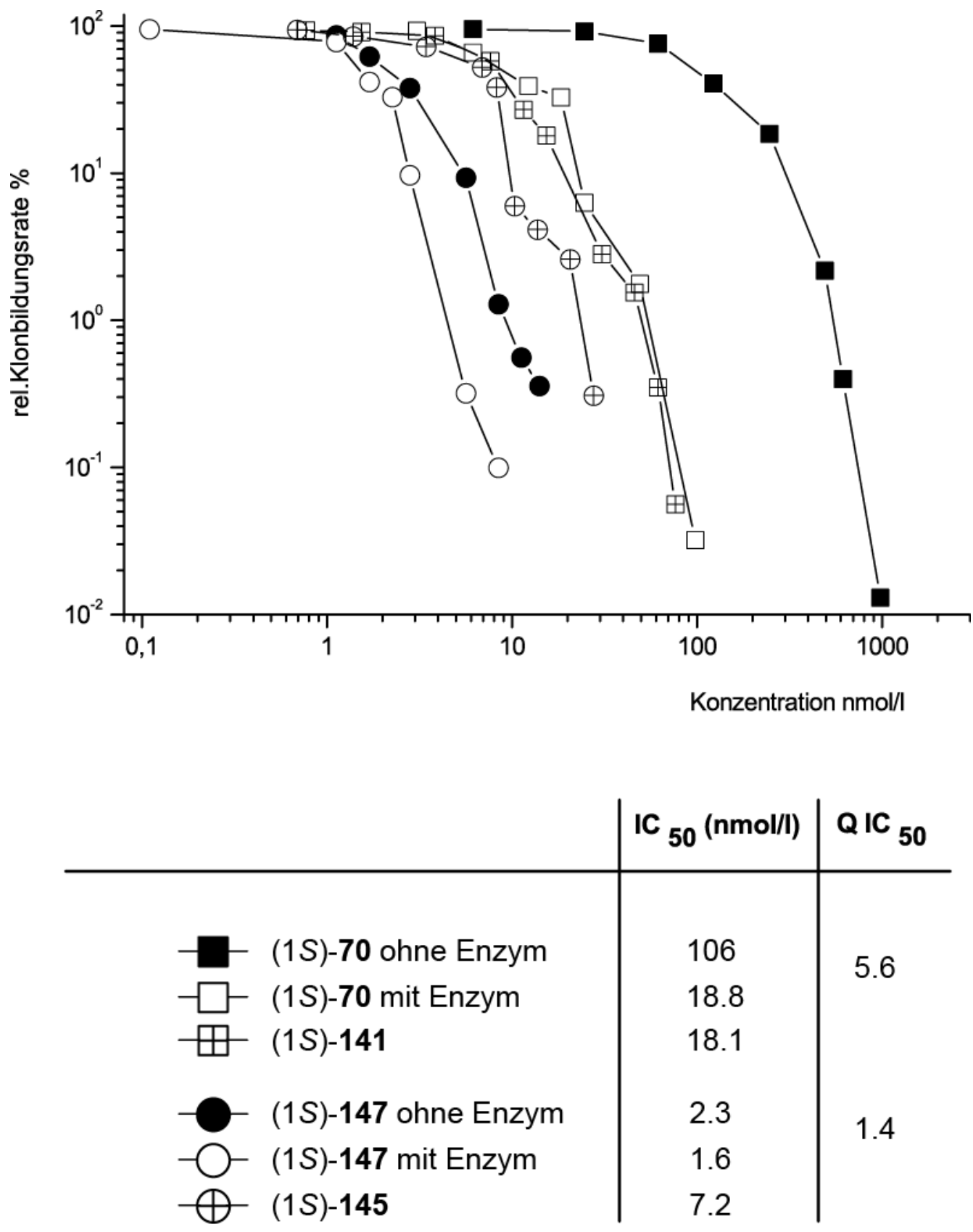

Abbildung 113. Darstellung der Ergebnisse der In vitro-Zytotoxizitätsuntersuchungen für die (1S)-Benzothiazol-Derivate (1S)-70 und (1S)-141 sowie (1S)-147 und (1S)-145.

\subsection{Synthese der Benzothiazol-Methylester 143 und 144}

Ausgehend von den Benzothiazol-Carbonsäuren 63 und 142, die dafür bekannt waren, nicht in lebende Zelle aufgenommen zu werden, wurden auch deren Methylester 143 und 144 dargestellt, um die Aufnahme in lebende Zellen zu untersuchen. Hierzu wurde die Carbonsäure in Methanol gelöst und bei $0^{\circ} \mathrm{C}$ mit Sulfonylchlorid umgesetzt. Das entstandene Säurechlorid konnte dann durch 
Nucleophile Substitution mit Methanol in den entsprechenden Ester überführt werden. Eine Aufreinigung durch präparative HPLC war nicht möglich, da bei Zugabe von Säure unter wässrigen Reverse Phase Bedingungen, die Carbonsäure gebildet wurde. 144 konnte durch Säulenchromatogrpahie an Kieselgel dargestellt werden, während 143 durch präparative Dünnschichtchromatographie aufgereinigt wurde.<smiles>CC(C(=O)O)[n+]1ccc(/C=C2/Sc3ccccc3N2C)cc1</smiles>

63<smiles>CC(=O)CS[n+]1ccc(/C=C/C=C2/Sc3ccccc3N2C)c2ccccc21</smiles>

1. $\mathrm{SOCl}_{2}, \mathrm{MeOH}, 0^{\circ} \mathrm{C}, 3 \mathrm{~h}$

2. Säulenchromatographie an $\mathrm{SiO}_{2}$ $93 \%$

1. $\mathrm{SOCl}_{2}, \mathrm{MeOH}, 0^{\circ} \mathrm{C}, 3 \mathrm{~h}$ 2. Präparative $D C$ $33 \%$<smiles>CC(=O)[S-](Cl)(Cl)(Cl)c1ccc(/C=C2/Sc3ccccc3N2C)cc1</smiles>

144

Abbildung 114. Darstellung der Methylester 144 und 143 ausgehend von den entsprechenden Carbonsäuren 63 und 142. 


\section{Charakterisierung und Untersuchung der zellulären Aufnahme von fluoreszenzmarkierten (1S)-CBI-Benzothiazol-Derivaten}

\subsection{Fluorimetrische Bestimmung der Absorptions- und Emissionsmaxima}

Zur Untersuchung der zellulären Aufnahme der in dieser Arbeit synthetisierten CBIBenzothiazol-Derivate (1S)-141, (1S)-70, (1S)-145 und (1S)-147 sowie 143 und 144 mussten zunächst die Absorptions- und Emissionsspektren bestimmt werden. Hierzu wurden entsprechende Lösung der Substanzen in Methanol und wässrigem Bildgebungspuffer am Fluorimeter untersucht, um die benötigten Maxima zu ermitteln.

\subsubsection{Bestimmung der Fluoreszenz- und Emissionsmaxima für die dargestellten BO-Derivate (1S)-141, (1S)-70 sowie 144}<smiles>[R2]c1cc2c(c3ccccc13)[C@@H](CCl)CN2C(=O)C(C)=[SH][n+]1ccc(/C=C2/Sc3ccccc3N2C)cc1</smiles><smiles>COC(=O)CC[n+]1ccc(C=C2Sc3ccccc3N2C)cc1</smiles>

$$
\begin{array}{ll}
R=H & (1 S)-141 \\
R=\beta-D-G a l(O H)_{4} & (1 S)-70
\end{array}
$$

Abbildung 115. Untersuchte Verbindungen mit BO (144) als DNA-Binder.

Anders als die in Kapitel 6.1.1-6.1.3 beschriebenen Cumarine unterscheiden sich die verwendeten Benzothiazole aus Kapitel 8.1.1 und 8.1.2 in ihren spektralen Eigenschaften deutlich von einander. So zeigen das in dieser Arbeit synthetisierte seco-CBI-BO-Drug (1S)-141, das Galaktosid (1S)-70 sowie der Methylester 144 annähernd identische Eigenschaften. 
Die Verbindungen (1S)-141, (1S)-70 und 144 zeigen, wie in den abgebildeten Spektren zu sehen ist ein Absorptionsmaximum von $\lambda_{\max , A b s}=452-462 \mathrm{~nm}$, während das Emissionsmaximum bei $\lambda_{\max , \mathrm{Em}}=466-482 \mathrm{~nm}$ liegt. Der Stokes-Shift ist in den beschriebenen Spektren mit 14-26 nm sehr gering. Auffällig ist des Weiteren ein zusätzliches Absorptionsmaixmum im Bereich von $\lambda_{\max , A b s}=290 \mathrm{~nm}$, dass mit den zur Verfügung stehenden Mitteln nicht weiter untersucht werden konnte, da es keinen Laser mit einer Anregungswellenlänge in diesem spektralen Bereich gibt (Abbildungen 116-118).
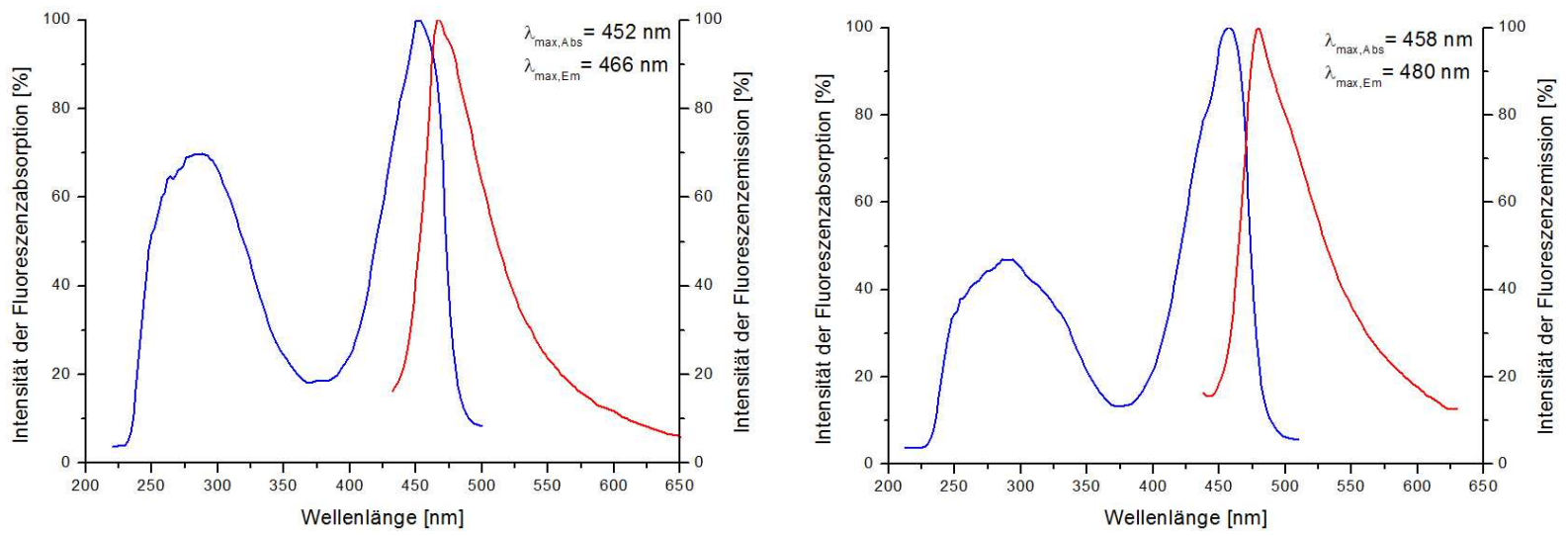

Abbildung 116. Absorptions- und Emissionsspektren von (1S)-141 in Methanol (links) und wässrigem Bildgebungspuffer (rechts).
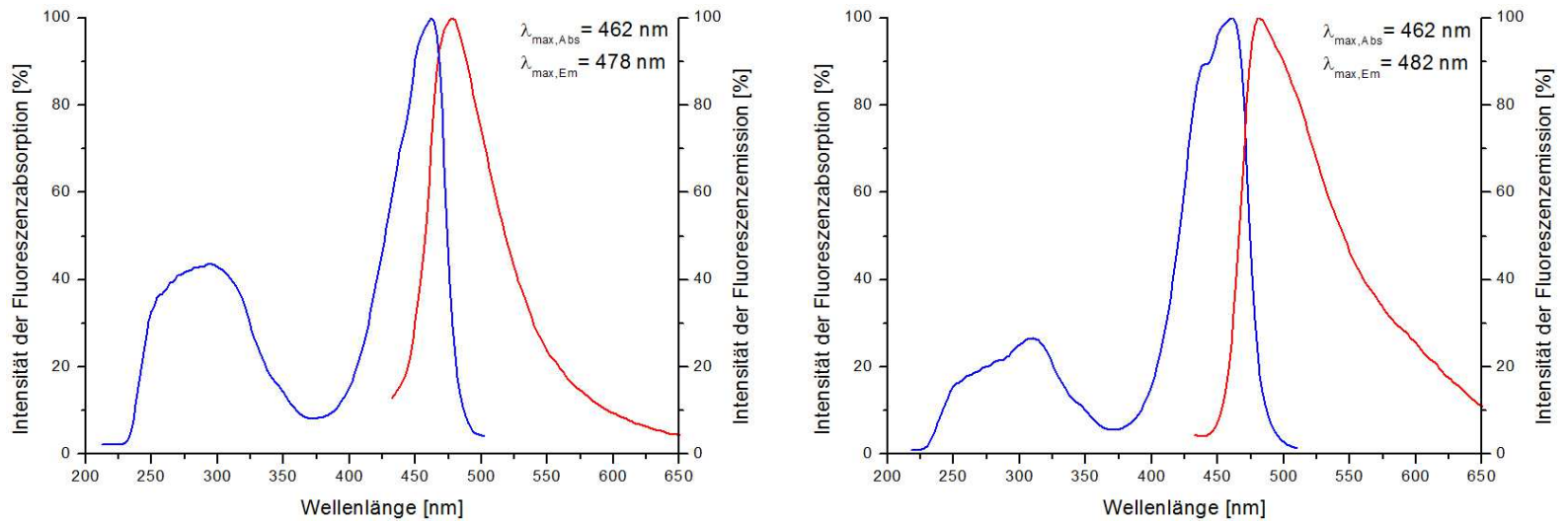

Abbildung 117. Absorptions- und Emissionsspektren von (1S)-70 in Methanol (links) und wässrigem Bildgebungspuffer (rechts). 

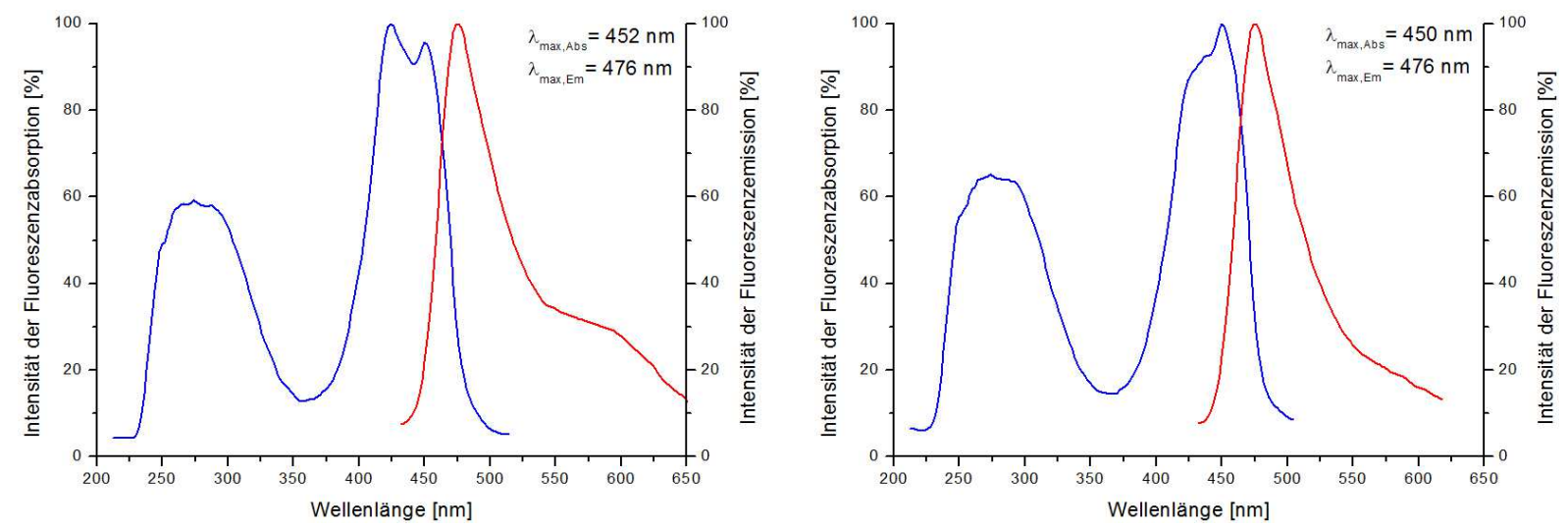

Abbildung 118. Absorptions- und Emissionsspektren von 144 in Methanol (links) und wässrigem Bildgebungspuffer (rechts).

\subsubsection{Bestimmung der Fluoreszenz- und Emissionsmaxima für die dargestellten TO3-Derivate (1S)-145, (1S)-147 sowie 143}

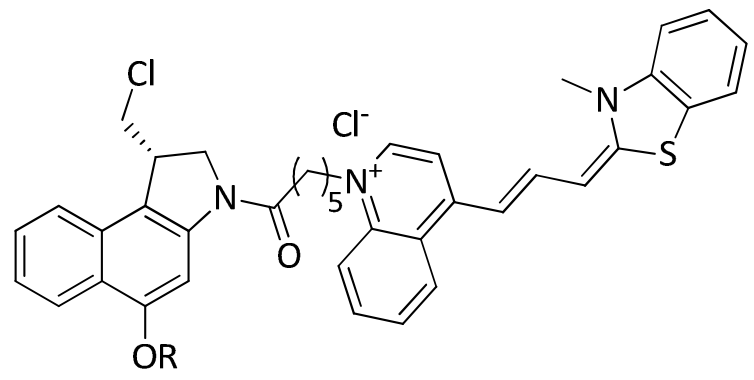

$\mathrm{R}=\mathrm{H}$

$\mathrm{R}=\beta-\mathrm{D}-\mathrm{Gal}(\mathrm{OH})_{4}$

(1S)-145

(1S)-147

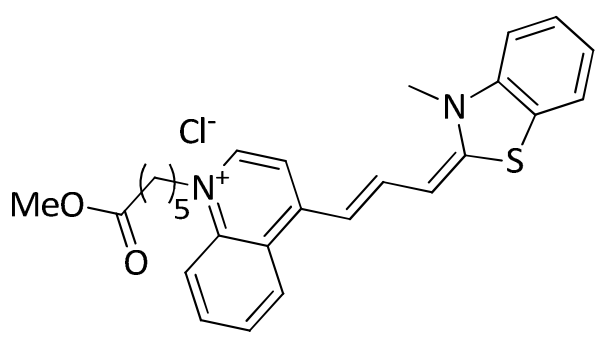

143

Abbildung 119. Untersuchte Verbindungen mit TO3 (143) als DNA-Binder.

Bei den TO3-Derivaten (1S)-145, (1S)-147 sowie 143 ist das Absorptionsspektrum mit $\lambda_{\text {max,Abs }}=628-646 \mathrm{~nm}$ von allen in dieser Arbeit synthetisierten Verbindungen am weitesten rotverschoben. Mit $\lambda_{\text {max,Em }}=647-651 \mathrm{~nm}$ zeigen die TO3-Derivate einen nur sehr geringen Stokes-Shift von 7-19 nm auf (Abbildungen 120-122). Auf Grund Ihrer spektralen Eigenschaften, wie ihrer enorm hohen molaren Extinktionskoeffizienten $\left(\varepsilon=10^{5} \mathrm{M}^{-1} \mathrm{~cm}^{-1}\right)$, den guten Quantenausbeuten $(\Phi=0.024-0.206)^{163}$ sowie den sehr großen Absorptionswellenlängen ${ }^{162}$ eignen sich diese Verbindungen hervorragend für Versuche im Rahmen von Optical Imaging. 

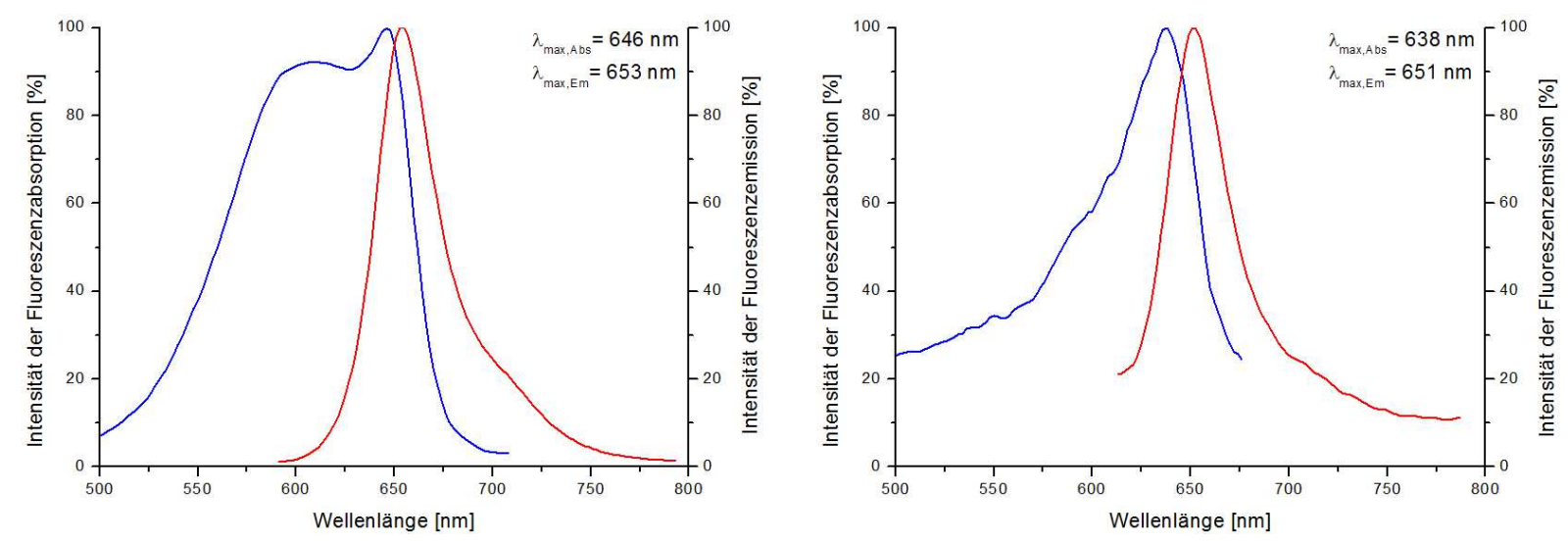

Abbildung 120. Absorptions- und Emissionsspektren von (1S)-145 in Methanol (links) und wässrigem Bildgebungspuffer (rechts).
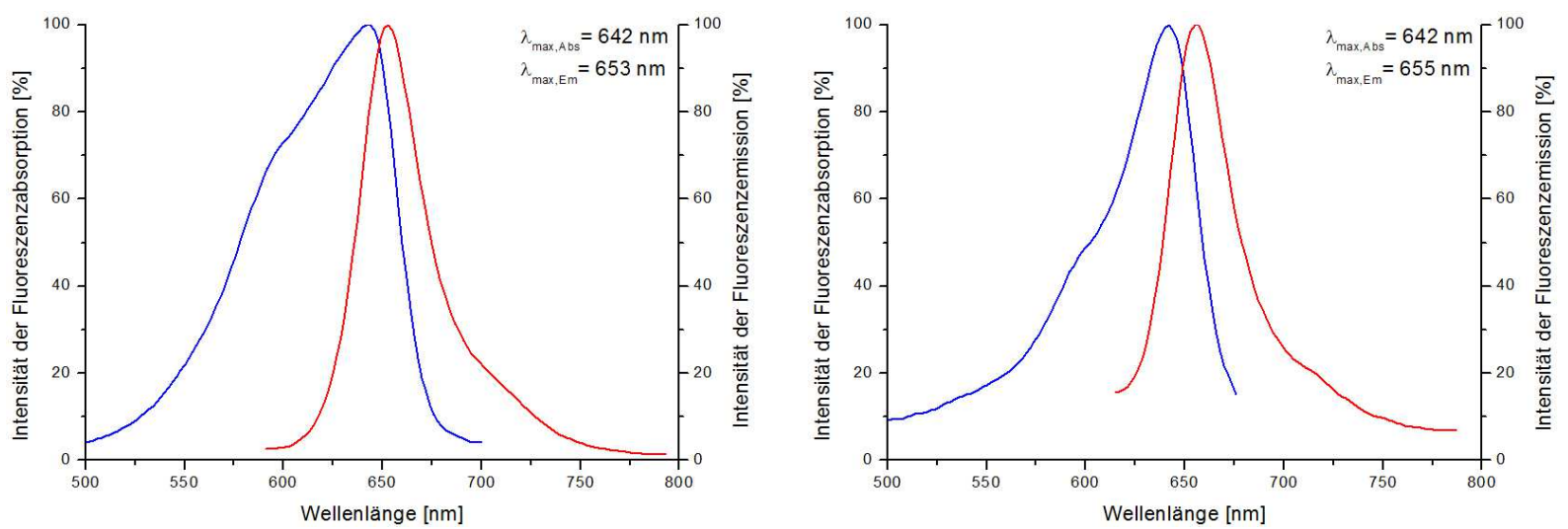

Abbildung 121. Absorptions- und Emissionsspektren von (1S)-147 in Methanol (links) und wässrigem Bildgebungspuffer (rechts).
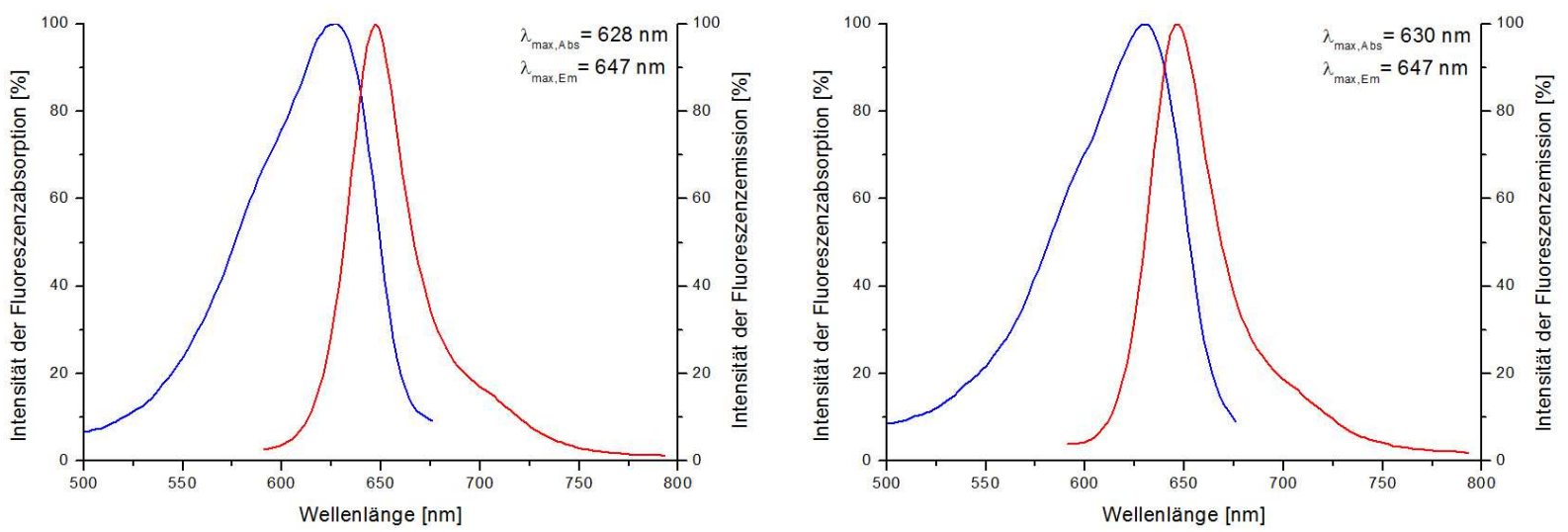

Abbildung 122. Absorptions- und Emissionsspektren von 143 in Methanol (links) und wässrigem Bildgebungspuffer (rechts). 


\subsection{Untersuchung der zellulären Aufnahme fluroreszenzmarkierter (1S)- CBI-Benzothiazol-Derivate in lebende Zellen durch CLSM}

Zu einem besseren Verständnis des Wirkmechanismus der CBI-Derivate wurden die in dieser Arbeit synthetisierten fluoreszenzmarkierten Analoga in Live Cell ImagingExperimenten untersucht. Hierzu wurden adhärent wachsende Zelllinien vom Typ HeLa SS6 für kurze Zeit mit den zu untersuchenden Substanzen inkubiert (5-15 min) und die Verteilung der Substanz mit Hilfe eines Konfokalen Laser Scanning Mikroskops beobachtet und einer CCD-Kamera aufgenommen.

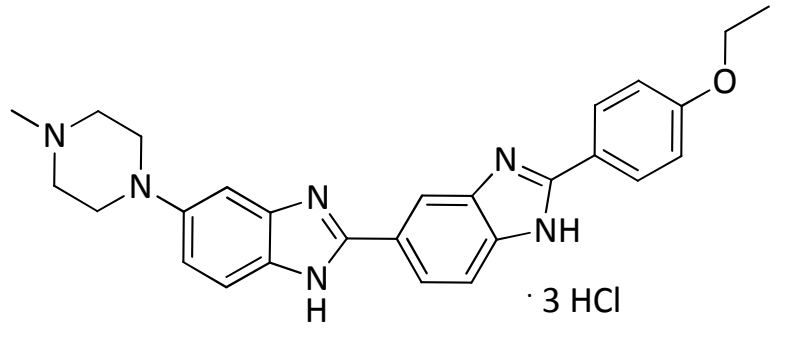

Hoechst 33342 (64) $\lambda_{\text {max }, \text { Abs. }}=346 \mathrm{~nm}$ $\lambda_{\max , \mathrm{Em} .}=460 \mathrm{~nm}$<smiles>C[N+]1=C(C=CC=C2N(Cc3ccc(CCl)cc3)c3ccccc3C2(C)C)C(C)(C)c2ccccc21</smiles>

MitoTracker Deep Red 633 FM (65)

$$
\begin{aligned}
& \lambda_{\text {max, Abs. }}=644 \mathrm{~nm} \\
& \lambda_{\text {max, Em. }}=665 \mathrm{~nm}
\end{aligned}
$$<smiles>CN1C(=CC=Cc2n(-c3ccc(CCl)cc3)c3cc(Cl)c(Cl)cc3[n+]2-c2ccc(CCl)cc2)Oc2ccccc21</smiles>

MitoTracker Green FM (148)

$$
\begin{aligned}
& \lambda_{\text {max }, \text { Abs. }}=490 \mathrm{~nm} \\
& \lambda_{\text {max }, \text { Em. }}=516 \mathrm{~nm}
\end{aligned}
$$

Abbildung 123. Strukturen, der für die Kolokalisationsexperimente eingesetzten Anfärbreagenzien: 64 zur Anfärbung des Zelkerns sowie 65 bzw. 148 zur Anfärbung der Mitochondrien.

Zur Identifikation der angefärbten Zellregion wurde auf das Prinzip der Kolokalisation zurückgegriffen, um eine Unterscheidung zwischen Organellen, wie dem Zellkern 
oder mitochondrialen Strukturen zu treffen. Hierzu eignen sich im besonderen der Mitochondrienfarbstoff MitoTracker Deep Red 633 FM (65), der auf Grund seiner optischen Eigenschaften im Fall der BO-Derivate eingesetzt werden konnte, der Mitochondrienfarbstoff MitoTracker Green FM (148), der auf Grund seiner optischen Eigenschaften im Fall der TO3-Derivate eingesetzt werden konnte, sowie der Zellkernfarbstoff Hoechst 33342 (64) (Abbildung 123).

\subsubsection{Untersuchung der zellulären Aufnahme fluroreszenzmarkierter (1S)- CBI-BO-Derivate (1S)-141, (1S)-70 sowie 144 in lebende Zellen}<smiles>CCC[n+]1ccc(/C=C2\Sc3ccccc3N2C)cc1</smiles><smiles>COC(=O)C(C)(Cl)[n+]1ccc(/C=C2\Sc3ccccc3N2C)cc1</smiles>

$$
\begin{array}{ll}
\mathrm{R}=\mathrm{H} & (1 S)-141 \\
\mathrm{R}=\beta-\mathrm{D}-\mathrm{Gal}(\mathrm{OH})_{4} & (1 S)-70
\end{array}
$$

Abbildung 124. Untersuchte Verbindungen mit BO (144) als DNA-Binder.

Bei der Untersuchung des seco-Drugs (1S)-141 fällt auf, dass eine sehr selektive Akkumulation des Wirkstoffs innerhalb des Zellkerns auftritt (Abbildung 125). Dieses konnte durch Kolokalisationsexperimente mit Hoechst 33342 (64) (A) sowie MitoTracker Green FM (148) (C) bestätigt werden. Ein erstes Kriterium im Rahmen der Anwendung des ADEPT-Konzeptes ist hiermit gegeben, da das seco-Drug (1S)-141 die Zellemembran innerhalb von wenigen Minuten penetriert und sich innerhalb der Zelle, genauer dem Zellkern, aufhält. 

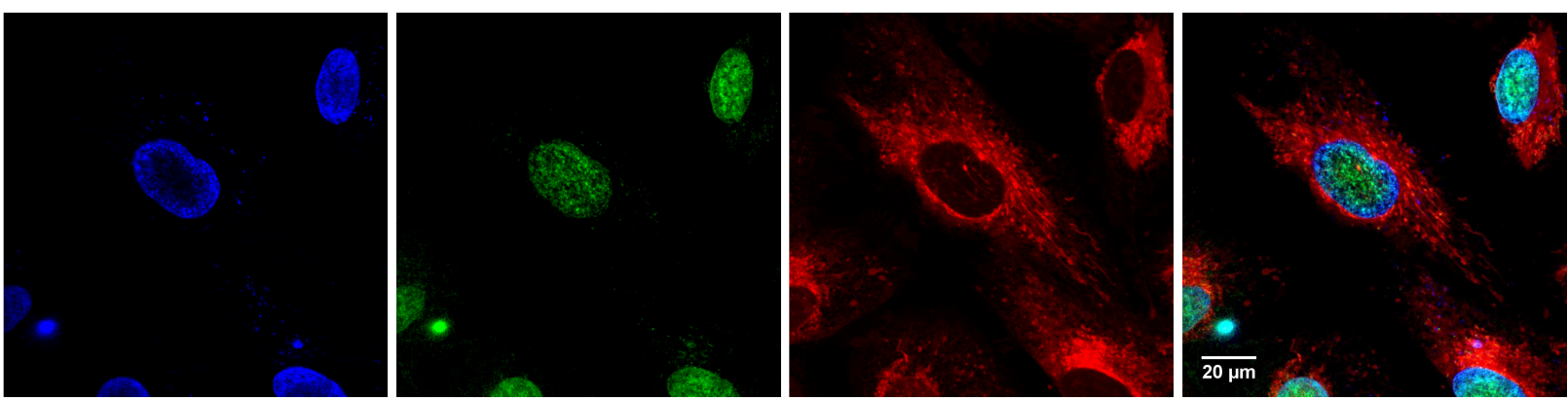

Abbildung 125. Kolokalisationsexperimente zur Aufnahme des seco-Drugs (1S)-141 in lebende Zellen. 1. von links: $\lambda_{\text {exc }}=405 \mathrm{~nm}, \lambda_{\text {em }}=415-450 \mathrm{~nm}$, Anfärbung des Zellkerns durch Hoechst 33342 (64); 2 . von links: $: \lambda_{\text {exc }}=458 \mathrm{~nm}, \lambda_{\text {em }}=463-471 \mathrm{~nm}$, Anfärbung des Zellkerns durch (1S)-141; 3. von links: $\lambda_{\text {exc }}=633 \mathrm{~nm}, \lambda_{\text {em }}=644-703 \mathrm{~nm}$, Anfärbung der Mitochondrien durch MitoTracker Deep Red 633 FM (65); 4. von links: Überlagerung von 1.-3.

Ebenso penetriert das aus 144 synthetisierte Galaktosid (1S)-70 innerhalb weniger Minuten die Zellmembran und verweilt nicht, wie es das ADEPT-Konzept erfordert, bis zur enzymatischen Spaltung und Freisetzung des seco-Drugs (1S)-141 außerhalb der Zelle. Wie in Abbildung 126 zu sehen ist, akkumuliert das Galaktosid (1S)-70 in den Mitochondrien lebender Zellen. Dieses Ergebniss lässt sich durch Kolokalisationsexperimente durch MitoTracker Green FM (148) eindeutig bestätigen.
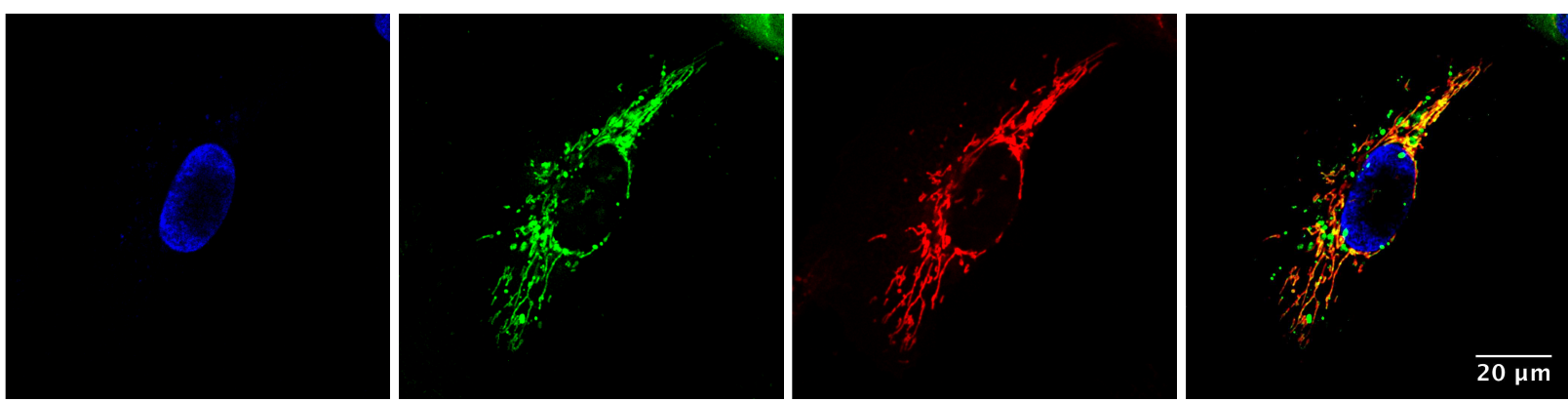

Abbildung 126. Kolokalisationsexperimente zur Aufnahme des Galaktosides (1S)-70 in lebende Zellen. 1. von links: $\lambda_{\mathrm{exc}}=405 \mathrm{~nm}, \lambda_{\mathrm{em}}=415-450 \mathrm{~nm}$,Anfärbung des Zellkerns durch Hoechst 33342 (64); 2 . von links: : $\lambda_{\text {exc }}=458 \mathrm{~nm}, \lambda_{\text {em }}=475-520 \mathrm{~nm}$, Anfärbung der Mitochondrien durch Galaktosid (1S)-70; 3. von links: $\lambda_{\text {exc }}=633 \mathrm{~nm}, \lambda_{\text {em }}=644-$ 703 nm, Anfärbung der Mitochondrien durch MitoTracker Deep Red 633 FM (65); 4. von links: Überlagerung von 1.-3. 
Wie in Abbildung 127 zu sehen ist, penetiert der Methylester 144 die Zellmembran und akkumuliert in den Nukleoli sowie an der Zellkernmembran und zeigt damit ein anderes Verhalten als das seco-Drug (1S)-141 oder das Galaktosid (1S)-70.

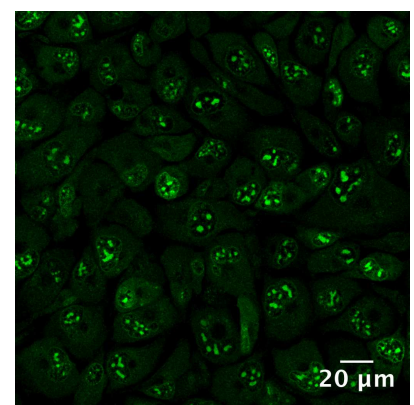

Abbildung 127. Kolokalisationsexperimente zur Aufnahme des Fluorochroms $\mathbf{1 4 4}$ in lebende Zellen ( $\lambda_{\text {exc }}=458 \mathrm{~nm}, \lambda_{\text {em }}=475-520 \mathrm{~nm}$, Nukleoli und Zellkernmembran).

\subsubsection{Untersuchung der zellulären Aufnahme fluroreszenzmarkierter (1S)- CBI-TO3-Derivate (1S)-145, (1S)-147 sowie 143}

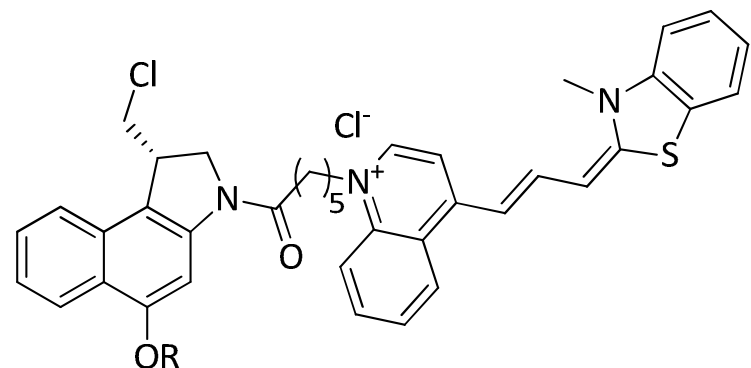

$\mathrm{R}=\mathrm{H}$

$\mathrm{R}=\beta-\mathrm{D}-\mathrm{Gal}(\mathrm{OH})_{4}$

(1S)-145

(1S)-147

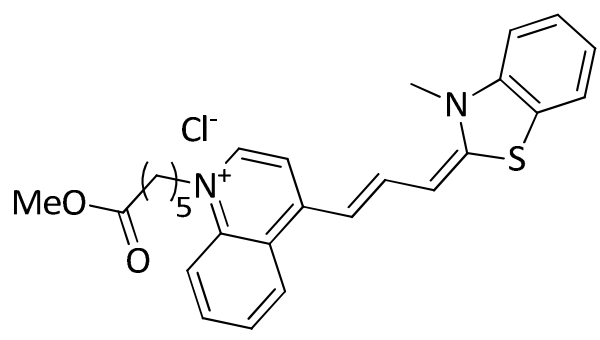

143

Abbildung 128. Untersuchte Verbindungen mit TO3 (143) als DNA-Binder.

Das seco-Drug (1S)-145 zeigt im Live Cell Imaging ein zum seco-Drug (1S)-141 sehr ähnliches Verhalten. (1S)-145 akkumuliert bereits nach wenigen Minuten innerhalb des Zellkerns, jedoch vorwiegend in Nukleoli-artigen Strukturen (Abbildung 129). Die nicht vollständige Färbung des Zellerns durch Kolokalisationsexperimente im Fall von Hoechst 33342 (64) ist durch die zu kurze Wartezeit zu erklären. Dennoch ist klar ersichtlich, dass es sich bei der von (1S)-145 markierten Zellregion um den Zellkern und nicht um mitochondriale Strukturen handelt. 

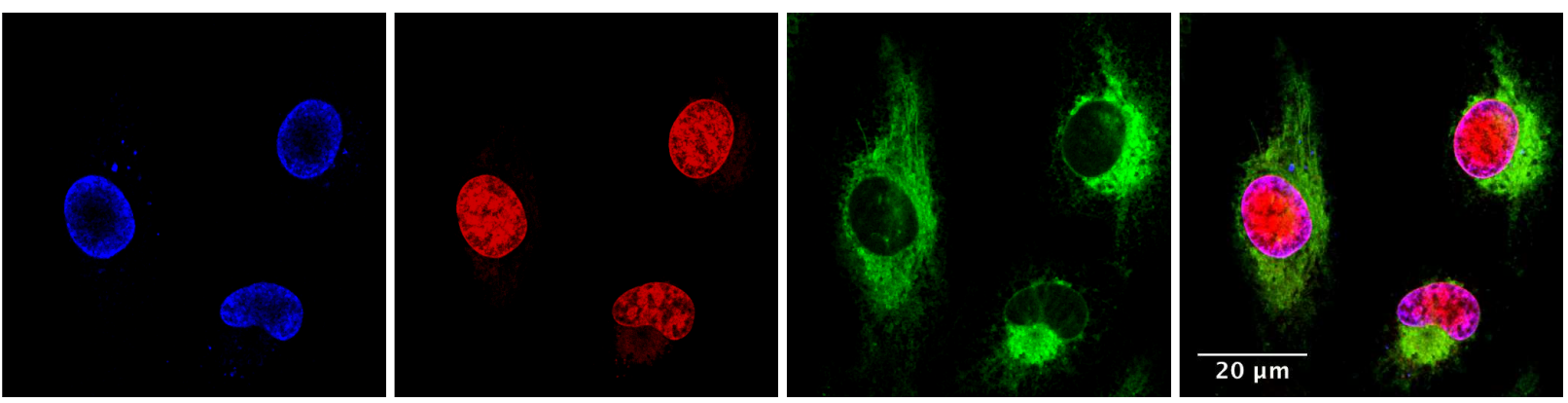

Abbildung 129. Kolokalisationsexperimente zur Aufnahme des seco-Drugs (1S)-145 in lebende Zellen. 1. von links: $\lambda_{\text {exc }}=405 \mathrm{~nm}, \lambda_{\text {em }}=415-485 \mathrm{~nm}$, Anfärbung des Zellkerns durch Hoechst 33342 (64); 2 . von links: $\lambda_{\mathrm{exc}}=633 \mathrm{~nm}, \lambda_{\mathrm{em}}=644-703 \mathrm{~nm}$, Anfärbung des Zellkerns durch seco-Drug (1S)-145; 3. von links: $\lambda_{\text {exc }}=488 \mathrm{~nm}, \lambda_{\mathrm{em}}=497-538 \mathrm{~nm}$, Anfärbung der Mitochondrien durch MitoTracker Green (148); 4. von links: Überlagerung von 1.-3.

Eine weitere Analogie lässt sich durch Live Cell Imaging-Experimente mit dem aus 143 dargestellten Galaktosid (1S)-147 finden. So akkumuliert das Galaktosid (1S)-147 wie auch das Galaktosid (1S)-70 zuvor innerhalb der Mitochondrien. Eine Akkumulation des Zellkerns kann hier nicht beobachtet werden.
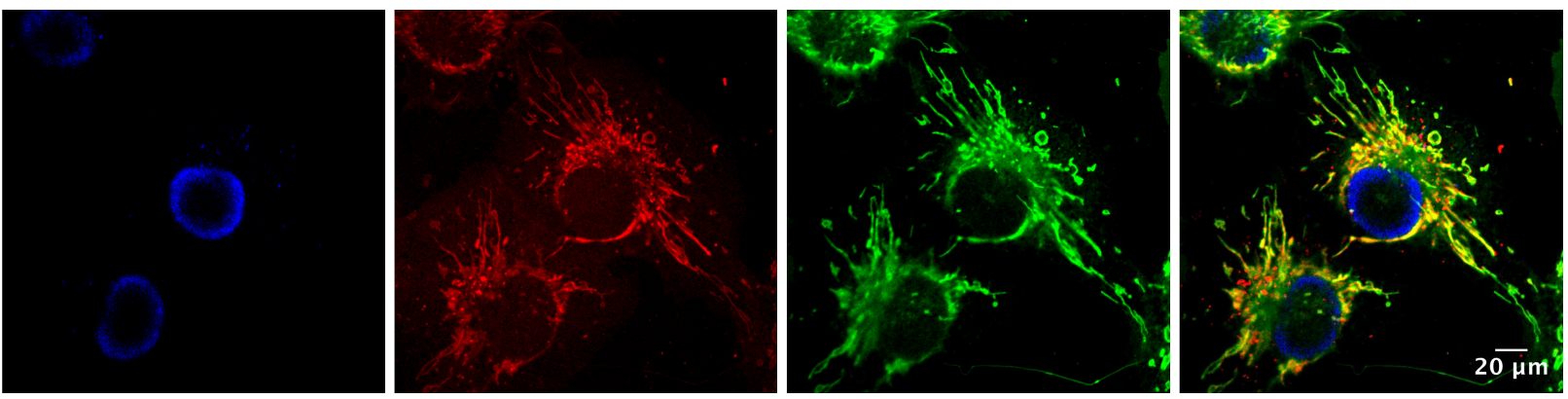

Abbildung 130. Kolokalisationsexperimente zur Aufnahme des Galaktosids (1S)-147 in lebende Zellen. 1. von links: $\lambda_{\text {exc }}=405 \mathrm{~nm}, \lambda_{\text {em }}=415-485 \mathrm{~nm}$, Anfärbung des Zellkerns durch Hoechst 33342 (64); 2. von links: $\lambda_{\text {exc }}=633 \mathrm{~nm}, \lambda_{\mathrm{em}}=655-703 \mathrm{~nm}$, Anfärbung der Mitochondrien durch Galaktosid (1S)-147; 3. von links: $\lambda_{\text {exc }}=488 \mathrm{~nm}, \lambda_{\text {em }}=497-538$ $\mathrm{nm}$, Anfärbung der Mitochondrien durch MitoTracker Green (148); 4. von links: Überlagerung 1.-3.

Eine weitere Analogie der verwendeten Benzothiazole lässt sich in der Auswertung der Erbgenisse für den Metyhlester 143 finden. 143 penetriert, wie auch 144 innerhalb von wenigen Minuten die Zellmembran und akkumuliert in Nukleoli sowie in der Zellkernmembran. 


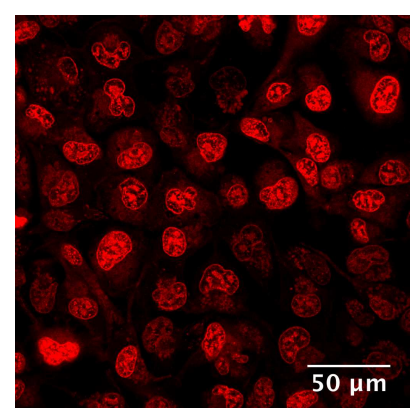

Abbildung 131. Kolokalisationsexperimente zur Aufnahme des Fluorochrmos $\mathbf{1 4 3}$ in lebende Zellen ( $\lambda_{\text {exc }}=633 \mathrm{~nm}, \lambda_{\text {em }}=644-703 \mathrm{~nm}$; Nukleoli und Zellkernmembran).

Zusammenfassend kann man feststellen, dass sich die dargestellten BenzothiazolDerivate in ihren Eigenschaften zur Zellaufnahme sehr stark ähneln. So penetrieren alle untersuchten Verbindungen sehr schnell die Zellmembran. Während die secoDrugs (1S)-141 und (1S)-145 im Zellkern bzw. Nukleoli akkumulieren, populieren die Galaktoside (1S)-70 und (1S)-147 die Mitochondrien. Genauso verhält es sich bei den Methylestern der Fluorochrome. So akkumuliern 143 und 144 in Nukleoli und der Zellkernmembran. Da die Zuckerderivate der seco-Drugs wie auch die seco-Drugs selbst sehr schnell die Zellmembranen penetrieren sind sie nicht für das ADEPTKonzept geeignet, das eine enzymatische Spaltung an der äußeren Zellmembran durch Konjugate aus monoklonalen Antikörpern und Glucohydrolasen vorsieht. 


\section{ZUSAMMENFASSUNG}

Noch immer stellt Krebs einen der größten Teile der tödlichen Erkrankungen in Deutschland dar. So kann jeder vierte Todesfall auf Krebs zurückgeführt werden. Aus diesem Grund und dem in Deutschland herrschenden demographischen Wandel ist eine weitere Entwicklung und eine damit einhergehende Senkung der Mortalitätszahlen, die durch Krebserkrankungen verursacht werden, ein wichtiges Ziel der Krebsforschung.

Die klassische antineoplastische Chemotherapie, die durch starke Nebenwirkungen wie Erbrechen, Immunschwäche, Infertilität oder Haarausfall den Behandlungserfolg stark einschränkt, ist trotz der angesprochenen Nebenwirkungen neben der chirurgischen Entfernung des Tumors und der Strahlentherapie eine der wichtigsten Behandlungsmethoden. Die klassischen Zytostatika beruhen im Allgemeinen auf der erhöhten Proliferationsrate von Tumorzellen. Allerdings gehören auch gesunden Zellen, wie die der Haarfolikel oder des Knochenmarks zu den schnell proliferierenden Zellen und werden somit durch Zytostatika gleichermaßen angegriffen. Eine weitere Untersuchung der Unterschiede zwischen gesunden Zellen und Krebszellen ist daher unabdingbar und moderne Therapieansätze greifen auf phänotypische und genotypische Eigenschaften von Krebszellen zurück um damit eine Selektierung der Krebszellen zu ermöglichen.

Auf der Grundlage dieser Überlegungen konnten im Arbeitskreis Tietze neue Konzepte erarbeitet werden. Hierzu zählt im Besonderen das Konzept der ProdrugTherapie. Hierdurch wird eine hohe Selektivität und Effizienz bei der Behandlung von Krebs erreicht, da weitesgehend untoxische Prodrugs gezielt am Wirkort in die entsprechenden zytotoxischen Drugs durch Nutzung der phänotypischen Eigenschaften der Krebszelle umgewandelt werden.

In besonderem Maß haben sich hierbei die Konzepte der Antibody-Directed Enzyme Prodrug Therapy (ADEPT) sowie der Prodrug-Mono-Therapie (PMT) hervorgetan. So wird bei Anwendung des ADEPT-Konzeptes eine enzymatische Umwandlung des Prodrugs in das Drug durch Antikörper-Enzym-Konjugate erreicht, die selektiv an 
tumorassoziierte Antigene binden. Neben diesem binären Ansatz, stellt die PMT einen ähnlichen Ansatz, bei dem allerdings kein Antikörper-Konjugat genutzt wird. Stattdessen werden in Krebszellen überexprimierte Enzyme genutzt, um das Prodrug selektiv am Tumor in das entsprechende Drug umzuwandeln. So stellt zum Beispiel das tumorassoziierte Enzym $\beta$-D-Glucuronidase ein Ziel im Rahmen der Prodrug Monotherypie dar.

Entsprechende Prodrugs im Rahmen von ADEPT und PMT konnten im Arbeitskreis Tietze synthetisiert werden. Als Leitstruktur für die dargestellten Verbindungen dienten die zytotoxischen Antibiotika (+)-CC-1065 (31) und (+)-Duocarmycin SA (32) Bei der Betrachtung der Antibiotika fällt auf, das beide über eine elektrophile Spirocyclopropylcyclohexadienon-Struktur verfügen, die einen Angriffspunkt für die DNA darstellt sowie eine Indolstruktur, die als DNA-bindende Struktureinheit dient. Des Weiteren weisen die gezeigten Antibiotika eine hohe Affinität zu doppelsträngiger DNA auf.

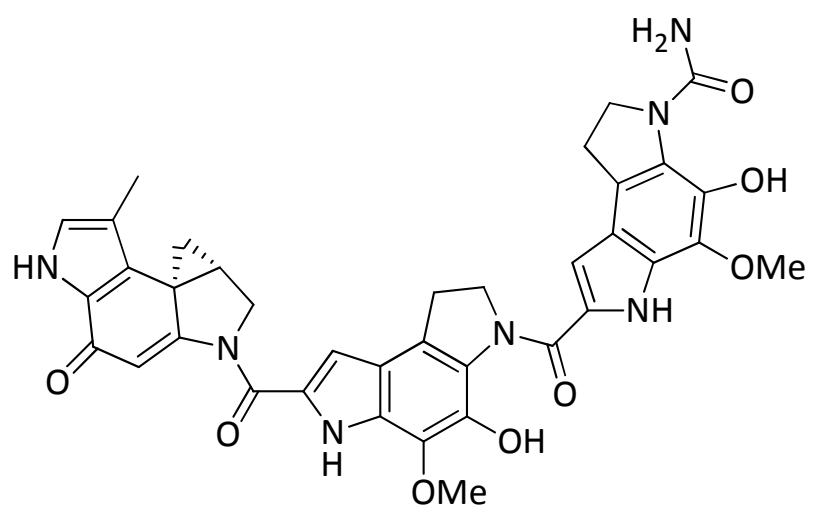

(+)-CC-1065 ((+)-31)

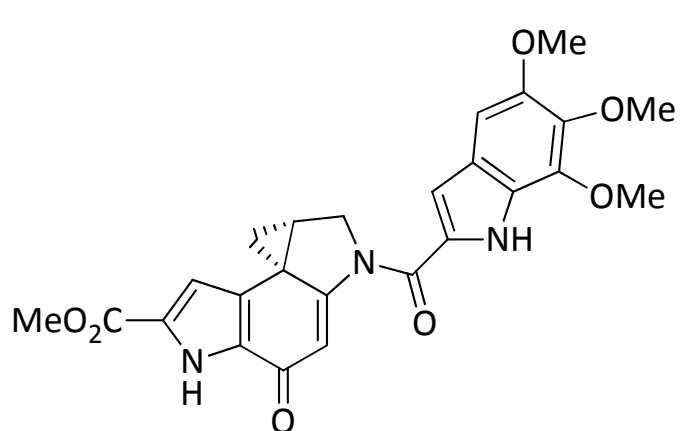

(+)-Duocarmycin SA ((+)-32)

Abbildung 132. Die natürlichen zytotoxischen Antibiotika (+)-CC-1065 (31) und Duocarmycin SA (32).

Die im Arbeitskreis Tietze entwickelten Analoga weisen keine Cyclopropylcyclohexadienon-Struktur auf, da die Cyclopropyl-Einheit in situ aus seco-Drugs durch eine Winstein-3'-Arylspirocyclisierung gebildet wird. Auf diese Weise wurden im Hinblick auf eine Anwendung im Rahmen von ADEPT oder PMT Analoga des Duocarmycins (32) dargestellt. Hierzu wurden Prodrugs synthetisiert, bei denen die Hydroxyfunktion 
durch eine Zuckereinheit als detoxifizierende Einheit genutzt wird. So können untoxische Prodrugs dargestellt werden, die erst am Wirkort, zum Beispiel durch das Enzym $\beta$-D-Galaktosidase, in das enstsprechende seco-Drug enzymatisch überführt werden

Zur weiteren Aufklärung des Wirkortes sowie des Wirkmechanismus der im Arbeitskreis Tietze synthetisierten Prodrugs und seco-Drugs wurden neuartige fluoreszenzmarkierte Analoga dargestellt und in Live Cell Imaging-Versuchen analysiert. Hierbei wurde zum ersten Mal auf eine Fluoreszenzmarkierung durch käuflich erwerbliche Marker verzichtet und stattdessen synthetisch zugängige, von sich aus DNA-bindende Fluorochrome eingesetzt. Zur Evaluierung eines Patentes, wurde ein von B.Krewer ${ }^{4 a}$ synthetisiertes fluoreszenzmarkiertes seco-Drug in einer deutlich verkürtzten und vielfach günstigeren Synthese dargestellt. Eine Untersuchung der fünf neuartigen fluoreszenzmarkierten Prodrugs, der fünf entsprechenden seco-Drugs sowie des fluoreszenzmarkierten seco-Drugs, das über eine neue Syntheseroute dargestellt wurde, konnte in Zusammenarbeit mit Herrn Dr.Miso Mitovski vom MPI für experimentelle Medizin Göttingen im Rahmen von Live Cell Imaging-Experimente am Konfokalen Laser Scanning Mikroskop durchgeführt werden. Darüber hinaus wurde ein neues fluoreszenzmarkiertes FITC-markiertes Prodrug sowie ein Prodrug mit einer Aminofunktion dargestellt.

Zur Synthese des FITC-markierten Prodrugs (1S)-72 wurde zunächst das seco-CCBIGrundgerüst (1S)-115 aus 112 dargestellt.

Hierzu wurde 112 selektiv an C-1 iodiert und im Anschluss das Boc-geschützte Amin mit (E/Z)-1,3-Dichlopropen (113) zu 114 alykliert. Darauffolgend wurde durch radikalische Zyklisierung durch TTMSS und AIBN das racemische CBI-Analoga rac-(1S)74 dargestellt, welches an chiraler startionärer Phase getrennt werden konnte, um nach Debenzylierung das gewünschte seco-CCBI-Grundgerüst (-)-(1S)-115 in enantiome-renreiner Form zu erhalten. 


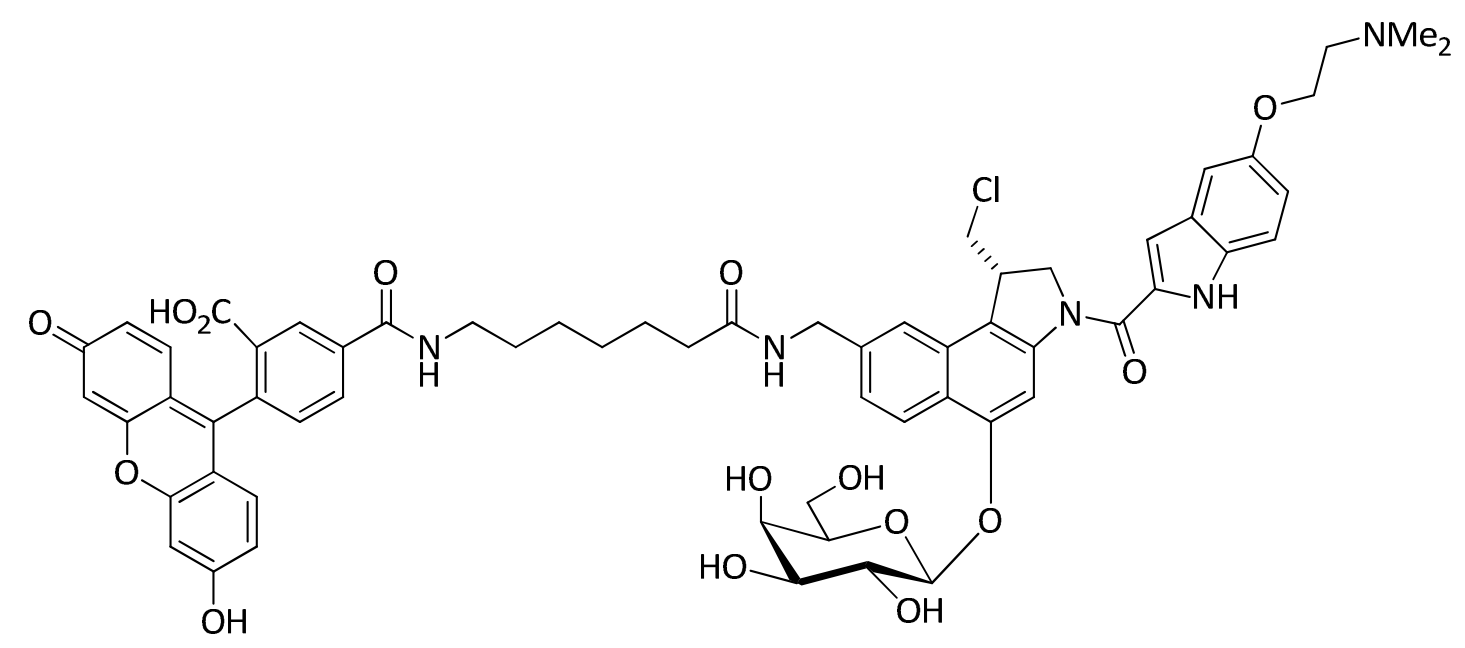

(1S)-72

Abbildung 133. Struktur des fluoreszenzmarkierten Galaktosides (1S)-72.<smiles>CC(C)(C)OCc1cccc(C(=O)Nc2cc(OCc3ccccc3)c3ccc(C#N)cc3c2)c1</smiles>

112

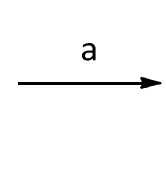<smiles>CC(C)(C)OC(=O)Nc1cc(OCc2ccccc2)c2ccc(C#N)cc2c1I</smiles>

75

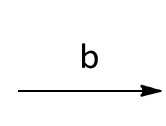

114<smiles>N#Cc1ccc2c(O)cc3c(c2c1)[C@H](CCl)CN3C(=O)OCc1ccccc1</smiles>

(1S)-115<smiles>CC(C)(O)N(C/C=C/Cl)c1cc(Cc2ccccc2)c2ccc(C#N)cc2c1I</smiles><smiles>N#Cc1ccc2c(OCc3ccccc3)cc3c(c2c1)C(CCl)CN3C(=O)OCc1ccccc1</smiles>

74

Abbildung 134. Darstellung des seco-CCBI-Grundkörpers (1S)-115 ausgehend von $\mathbf{1 1 2}$ für die Synthese von (1S)-72. a) NIS, TsOH, MeOH/THF 1:1, $50{ }^{\circ} \mathrm{C}, 1.5 \mathrm{~h}$; b) NaH, DMF, RT, $1.5 \mathrm{~h}$, dann (E/Z)-1,3-Dichlor-propen (113), RT, $14 \mathrm{~h}, 77 \%$; c) TTMSS, AIBN, Toluol, $80{ }^{\circ} \mathrm{C}, 4.5 \mathrm{~h}, 85 \%$; d) Trennung der Enantiomere von rac-74 an Chiralcel IA; e) Pd/C, $\mathrm{H}_{2}$, EtOAc, RT, 5 h, $90 \%$.

Die Boc-Schutzgruppe in (1S)-115 wurde durch $\mathrm{BF}_{3} \cdot \mathrm{OEt}_{2}$ enfernt und im Anschluss mit DMAI- $\mathrm{CO}_{2} \mathrm{H}(\mathbf{1 0 4})$ und EDAC· $\mathrm{HCl}$ alkyliert. Glykosidierung nach der Trichloracetimidat- 
methode nach Schmidt ${ }^{157}$ mit 116 lieferte das tetraacetylierte Galaktosid (1S)-73 in einer Ausbeute von 53\%.<smiles>N#Cc1ccc2c(O)cc3c(c2c1)[C@H](CCl)CN3C(=O)OCc1ccccc1</smiles>

(1S)-115

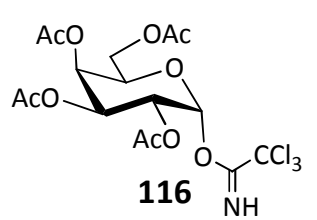

1. 116, $\mathrm{BF}_{3} \cdot \mathrm{OEt}_{2}, \mathrm{CH}_{2} \mathrm{Cl}_{2}$, $\operatorname{MS}(4 \AA),-10{ }^{\circ} \mathrm{C}, 3.5 \mathrm{~h}$ dann $\mathrm{BF}_{3} \cdot \mathrm{OEt}_{2}, \mathrm{RT}, 6 \mathrm{~h}$

2. 104, EDAC $\cdot \mathrm{HCl}, \mathrm{DMF}, \mathrm{RT}$, $18 \mathrm{~h}$

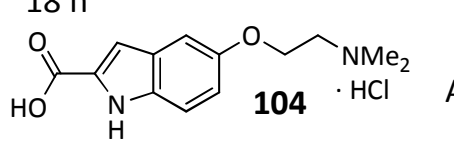

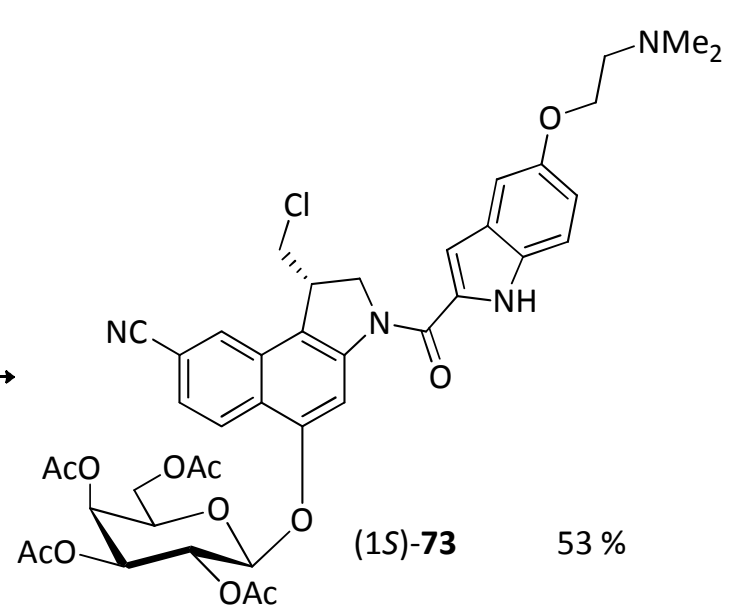

Abbildung 135. Syntheseschema zur Darstellung des tetraacetylierten Galaktosids (1S)-73 ausgehend vom Phenol (1S)-115.

Anschließende Reduktion des Nitrils in (1S)-73 unter Verwendung von $\mathrm{PtO}_{2} \cdot \mathrm{H}_{2} \mathrm{O}$ und $\mathrm{H}_{2}$ in Ethanol für $48 \mathrm{~h}$ bei Raumtemperatur sowie basische Spaltung der Acetylschutzgruppen in (1S)-117 durch Natrimmethanolat in Methanol lieferte das Galaktosid (1S)-118 mit einer Ausbeute von 84\% (Abbildung 136).

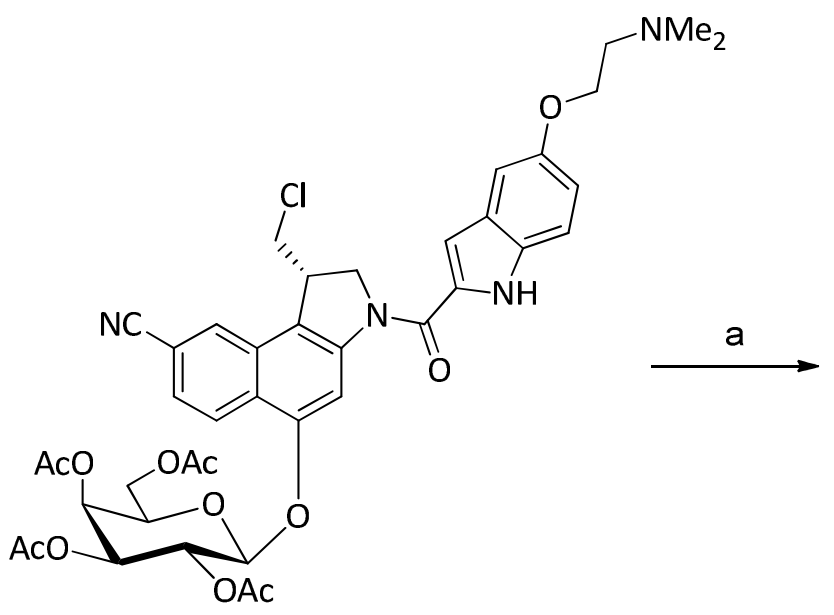

(1S)-73

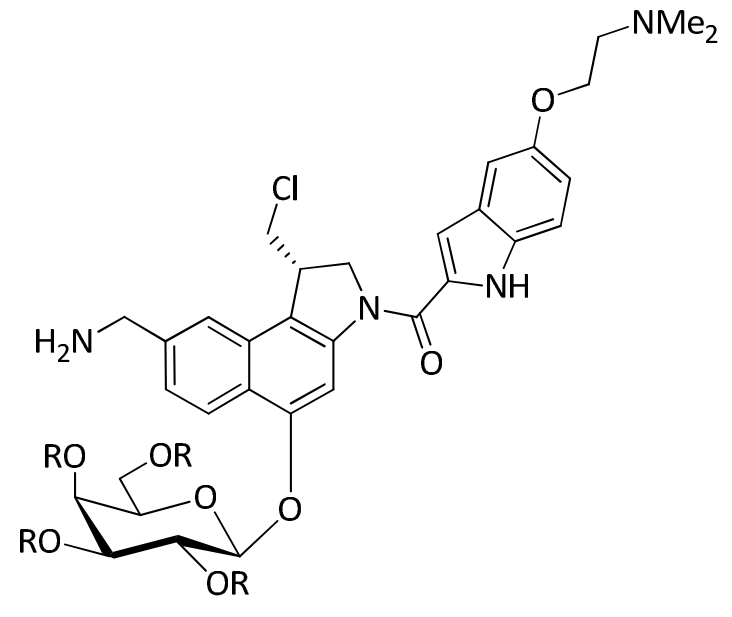

$\mathrm{R}=\mathrm{Ac}(1 \mathrm{~S})-117 \stackrel{\mathrm{b}}{\longrightarrow} \mathrm{R}=\mathrm{H}(1 \mathrm{~S})-118$

Abbildung 136. Syntheseschema zur Darstellung des Galaktosides (1S)-118. a) $\mathrm{PtO}_{2} \cdot \mathrm{H}_{2} \mathrm{O}, \mathrm{H}_{2}, 1.25 \mathrm{M}$ $\mathrm{HCl} / \mathrm{EtOH}, \mathrm{EtOH} / \mathrm{H}_{2} \mathrm{O}$ (96:4), RT, 48 h, 66\%; b) NaOMe, $\mathrm{MeOH}, \mathrm{Rt}, 30 \mathrm{~min}, 84 \%$. 
Das Prodrug (1S)-118 wurde durch Hünig-Base deprotoniert und mit einer Lösung des $\mathrm{N}$-Hydroxy-Succinimid-Esters 119 in DMF versetzt, um nach präparativer HPLC das fluoreszenzmarkierte Galaktosid (1S)-72 mit einer Ausbeute von 46\% zu erhalten. Leider stellen die dargestellten Prodrugs (1S)-118 und (1S)-72 auf Grund ihrer nur geringen $\mathrm{QIC}_{50}$-Werte von $\mathrm{QIC}_{50}=124$ für das Amino-Galaktosid 118 sowie $\mathrm{QIC}_{50}=74$ für das fluoreszenzmarkierte Galaktosid 72 keine geeigneten Verbindungen im Rahmen des ADEPT-Konzeptes dar.

Neben der Darstellung des fluoreszenzmarkierten Prodrugs (1S)-72 konnte ein fluoreszenzmarkiertes seco-Drug in Anlehnung an Arbeiten von B. Krewer in einem um fünf Stufen verkürzten Verfahren dargestellt werden. Außerdem wurde eine zweite neuartige Syntheseroute konzipiert, die eine enorme Kostenersparnis mit sich bringt, da auf den Einsatz des teuren Dapoxyl-Succinimid-Esters verzichtet werden konnte.

(1S)-118

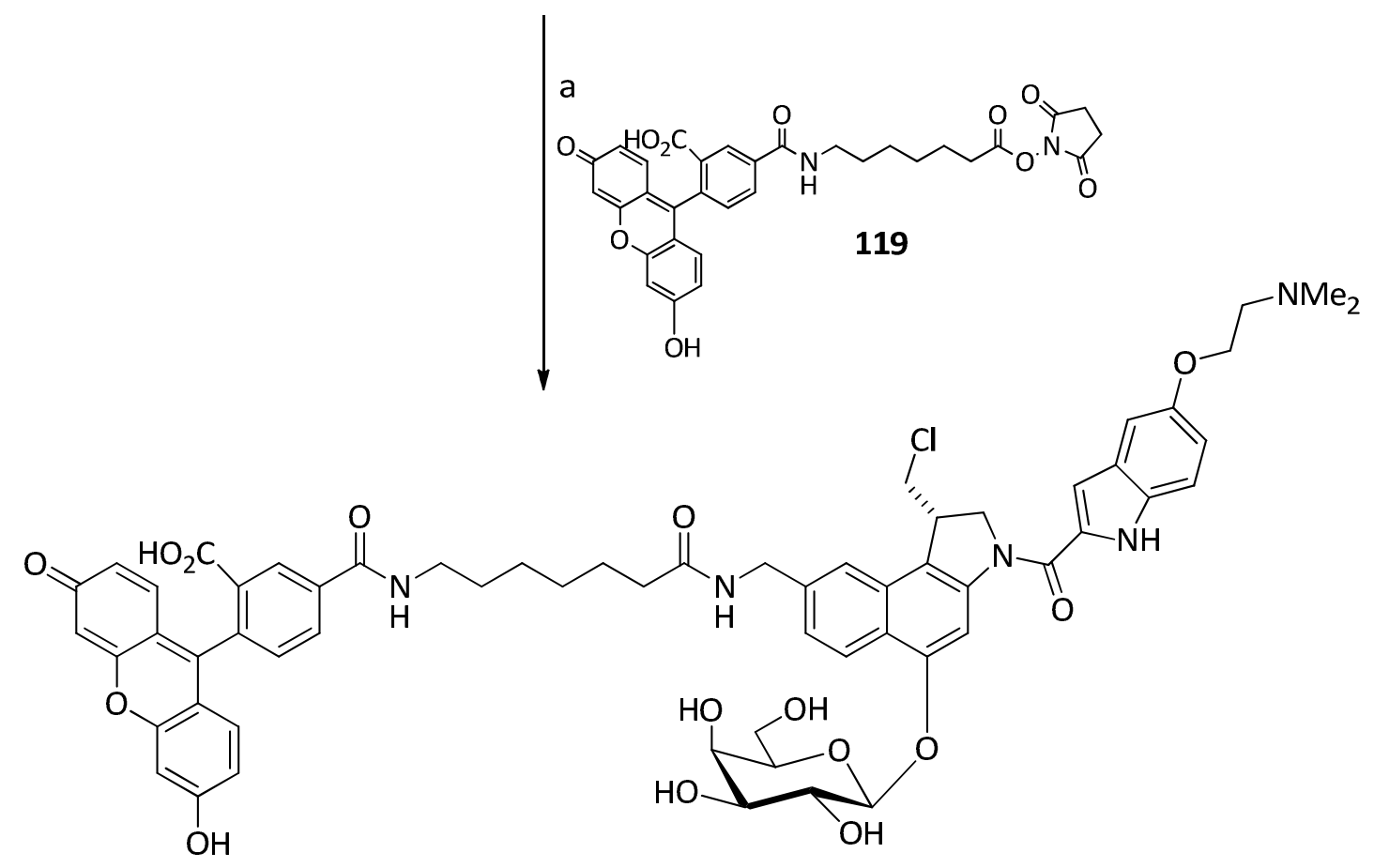

(1S)-72

Abbildung 137. Synthese des fluoreszenzmarkierten Galaktosides (1S)-72. a) DIPEA, 119, DMF, RT, $48 \mathrm{~h}, 46 \%$. 
Hierzu wurde das seco-CCBI-Grundgerüst $(1 S, 10 R)-82$ in 3 Stufen ausgehend von der Verbindung 101 hergestellt, das aus der Carbonsäure 85 über 5 Stufen zugänglich ist.<smiles>O=C(O)c1cc(OCc2ccccc2)c2cc(Br)ccc2c1</smiles>

85<smiles>CC(C)(C)OC(=O)Nc1cc(OCc2ccccc2)c2cc(C#N)ccc2c1Br</smiles>

101<smiles>CCOC(=O)c1cc(OCc2ccccc2)c2cc(Br)ccc2c1</smiles>

98<smiles>CCOC(=O)c1cc(OCc2ccccc2)c2cc(C#N)ccc2c1</smiles>

99<smiles>N#Cc1ccc2cc(NC(=O)OCc3ccccc3)cc(OCc3ccccc3)c2c1</smiles>

79

Abbildung 138. Darstellung des Bromnaphtalins 85. a) $\mathrm{SOCl}_{2}$, EtOH, Rückfluss, 19 h; b) CuCN, DMF, Rückfluss, 20 h; c) LiOH, THF/MeOH/ $\mathrm{H}_{2} \mathrm{O}$ (3:1:1), RT, 20 h.

Für die Umsetzung von 101 zu (1S,10R)-82 wurde eine radikalische Syntheseroute zum racemischen Produkt sowie eine enantioselektive Syntheseroute verwendet (Abbildung 139). Im ersten Ansatz wurde 101 mit 102 alkyliert um im Anschluss eine Zinkat-vermittelte enantioselektive Zyklsierung einzugehen. Nachfolgende Appelreaktion lieferte das seco-CCBI-Grundgerüst (1S,10R)-82. Zum Anderen wurde 101 mit (E/Z)-1,3-Dichlor-2-buten (103) alykliert und anschließend in einer radikalischen Zyklisierung durch TTMSS und AIBN zu den Diastereomeren rac$(1 S, 10 R)-82$ und rac-(1S,10S)-82 umgesetzt, die an Kieselgel getrennt werden konnte. Anschließende Trennung der Enantiomere durch HPLC an chiraler stationärer Phase lieferte das gewünschte Enantiomer (1S,10R)-82. Der Vorteil der racemischen Synthese liegt in der einfacheren Syntheseführung. Nachteilig ist allerdings, dass auch die ungewünschten Verbindungen $(1 R, 10 S)-82$ und das racemische syn-Diastereomer rac-(1S,10S)-82 gebildet werden. Diese konnten jedoch zu Testzwecken genutzt werden. 


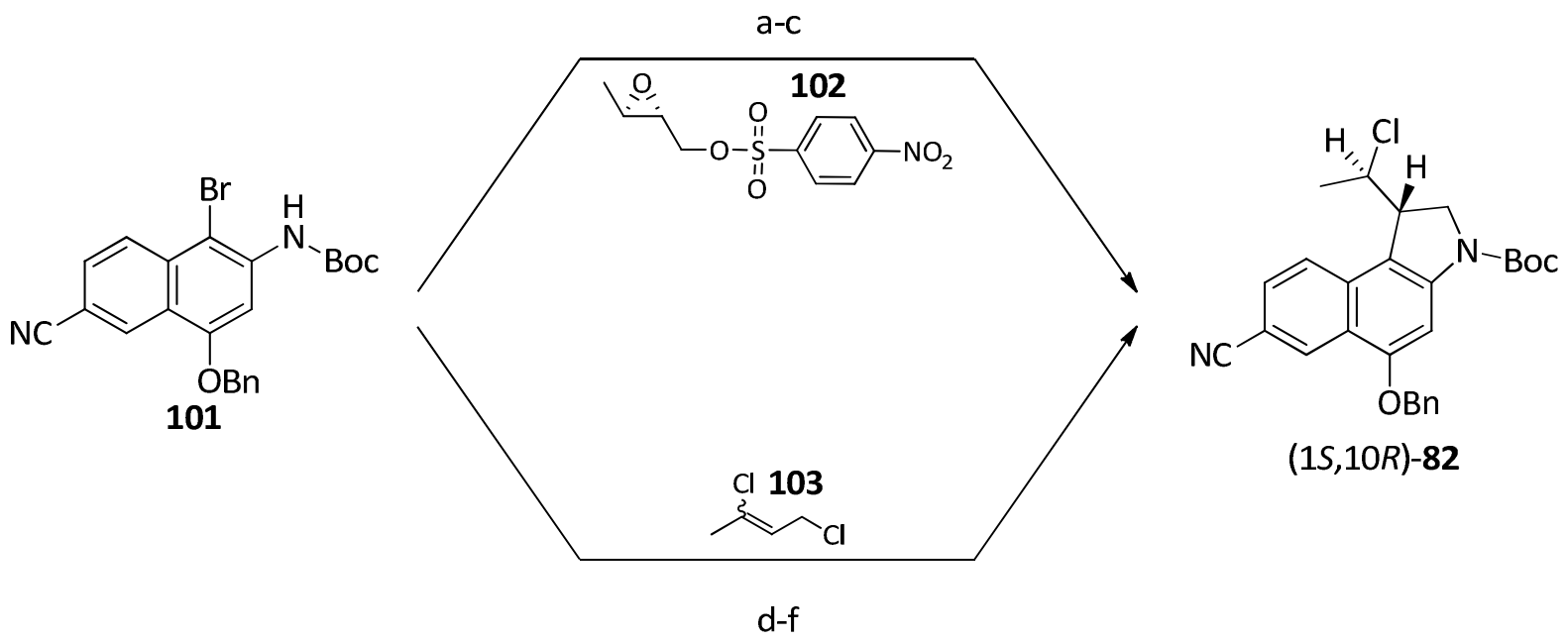

Abbildung 139. Synthese des seco-CCBI-Grundgerüstes (1S,10R)-82. Enantioselektive Synthese: a) $\mathrm{NaH}, \mathrm{DMF}, 40^{\circ} \mathrm{C}, 60 \mathrm{~min}$, dann 102, RT, $24 \mathrm{~h}, 95 \%$; b) $\mathrm{Li}_{2} \mathrm{Zn}(\mathrm{SCN}) \mathrm{Me}_{3}, \mathrm{THF}$, $-78{ }^{\circ} \mathrm{C} \rightarrow \mathrm{RT}, 3.5 \mathrm{~h}, 69 \%$; c) $\mathrm{SOCl}_{2}$, Pyridin, $\mathrm{CH}_{2} \mathrm{Cl}_{2}, \mathrm{RT}, 4 \mathrm{~h}, 34 \%$; Radikalische Synthese: d) NaH, DMF, RT, 4.5 h, dann 103, RT, 4 h, 66\%; e) TTMSS, AIBN, Toluol, $80{ }^{\circ} \mathrm{C}, 4 \mathrm{~h}, 90 \%$; f) Trennung der Enantiomere von rac- $(1 S, 10 R)-82$ an chiraler stationärer Phase Chiralcal IA.

Nachfolgend wurde 82 mit DMAl- $\mathrm{CO}_{2} \mathrm{H}$ (104) nach Boc-Abspaltung zu 88 alkyliert. Anschließende Reduktion der Nitrilfunktion unter Verwendung von $\mathrm{PtO}_{2} \cdot \mathrm{H}_{2} \mathrm{O}$ und $\mathrm{H}_{2}$ in einem Lösungsmittelgemisch aus THF/EtOH/ $\mathrm{H}_{2} \mathrm{O}(2: 4: 1)$ lieferte das gewünschte Amin 81 in guten Ausbeuten von 53-58\% (Abbildung 140).<smiles>CC(=O)OCc1ccc2c(c1)C1=C(c3ccc(C#N)cc31)C(C(C)Cl)CN2C(C)(C)Cl</smiles>

82

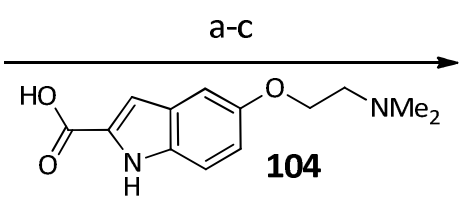

H 104

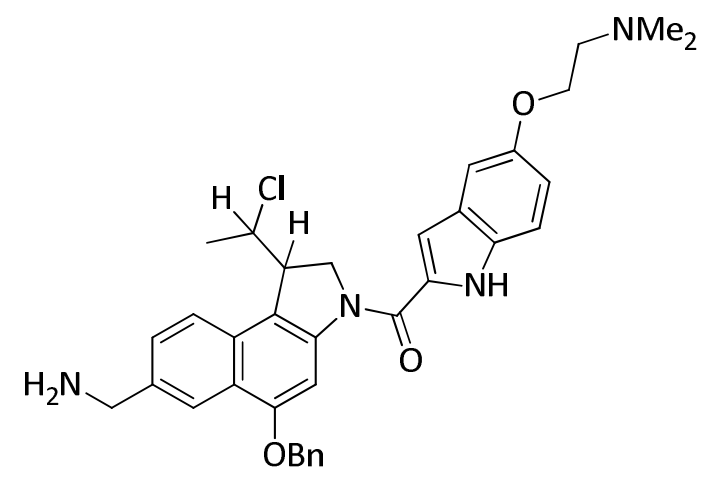

81

Abbildung 140. Synthese des benzylgeschützten Aminomethyl-CBI-DMAl 81. a) $4 \mathrm{M} \mathrm{HCl} / E \mathrm{E}, \mathrm{RT}, 4 \mathrm{~h}$; b) 104, EDAC. $\mathrm{HCl}, \mathrm{RT}, 18 \mathrm{~h}, 47-66 \%$; c) $\mathrm{PtO}_{2} \cdot \mathrm{H}_{2} \mathrm{O}, \mathrm{H}_{2}(1 \mathrm{~atm}), \mathrm{THF} / \mathrm{EtOH} / \mathrm{H}_{2} \mathrm{O}(2: 4: 1)$, $1.25 \mathrm{M} \mathrm{HCl} / \mathrm{EtOH}$ (2 Äq.), RT, 8 h, 53-58\%.

Um das weitaus günstigere Dapoxylderivat 89 zu nutzen, wurde eine Spacer-Einheit eingeführt. Hierzu eignete sich $N$-Cbz geschütztes $\beta$-Alanin (105). Anschließende 
simultane Entfernung der Cbz- und der Benzylschutzgruppe durch hydrogenolytische Spaltung durch $\mathrm{Pd} / \mathrm{C}$ unter $\mathrm{H}_{2}$-Atmosphäre lieferte das Amin (1S,10R)-90 (Abbildung 141), dass im Anschluss mit Dapoxyl-Derivat 89 zum gewünschten fluoreszenzmarkierten seco-Drug $(1 S, 10 R)$-71 gekuppelt werden konnte.<smiles>CON1C[C@H](C(C)Cl)Cc2c1cc(OCc1ccccc1)c1cc(CN)ccc21</smiles>

$(1 S, 10 R)-81$

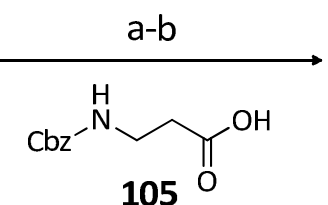

105<smiles>CON1C[C@H](C(C)Cl)c2c1cc(O)c1cc(CNC(=O)CCN)ccc21</smiles>

$(1 S, 10 R)-90$

Abbildung 141. Synthese des Amins (1S,10R)-90. a) 105, PyBOP, DIPEA, DMF, RT, 4 h, 82\%; b) Pd/C, $\mathrm{H}_{2}, \mathrm{MeOH} / \mathrm{AcOH} / \mathrm{H}_{2} \mathrm{O}(3: 1: 1), \mathrm{RT}, 8 \mathrm{~h}, 57 \%$.

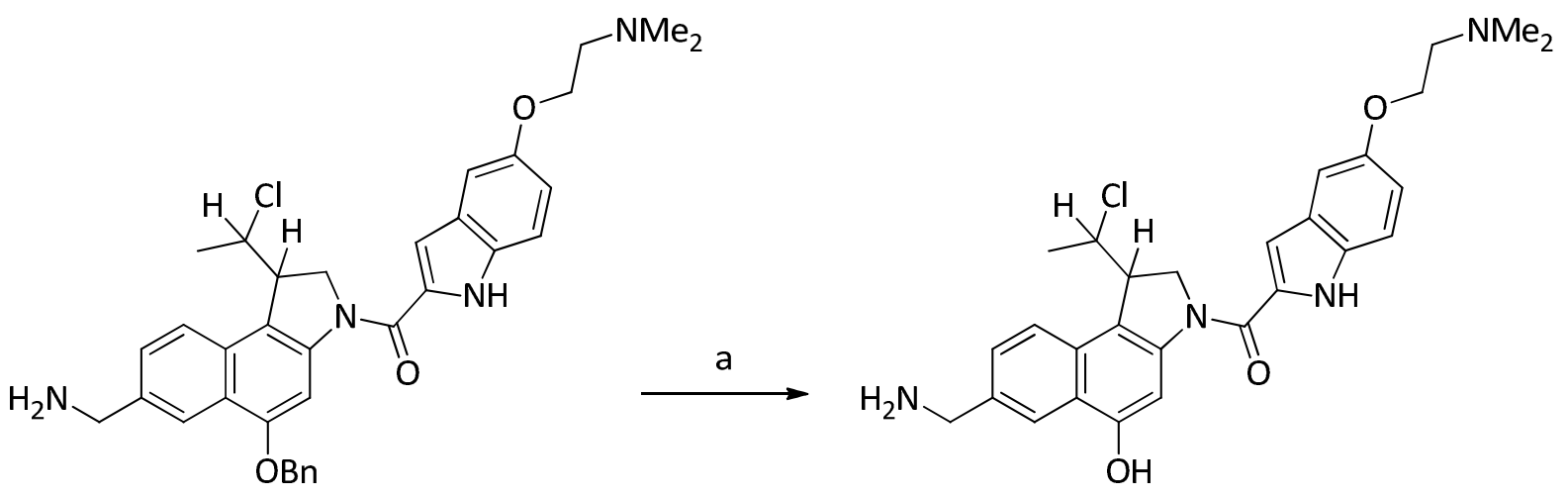

81
87

Abbildung 142. Synthese des Amins 87; a) Pd/C, $\mathrm{H}_{2}, \mathrm{MeOH} / \mathrm{AcOH} / \mathrm{H}_{2} \mathrm{O}(3: 1: 1), \mathrm{RT}, 8 \mathrm{~h}, 51-67 \%$.

Für die Synthese von (1S,10R)-71 unter Verwendung des Farbstoffs 61 wurde die Benzylgruppe in $\mathbf{8 1}$ unter Bildung von $\mathbf{8 7}$ entfernt. Umsetzung von $\mathbf{8 7}$ mit DIPEA in DMF und anschließende Zugabe von 61 lieferte die Zielverbindung (1S,10R)-71. Zusätzlich wurden hierbei $(1 S, 10 R)-\mathbf{1 1 0}$ und $(1 S, 10 R)-\mathbf{1 1 1}$ erhalten, deren Bildung vermutlich über eine Spirocyclopropylzwischenstufe erfolgt. 


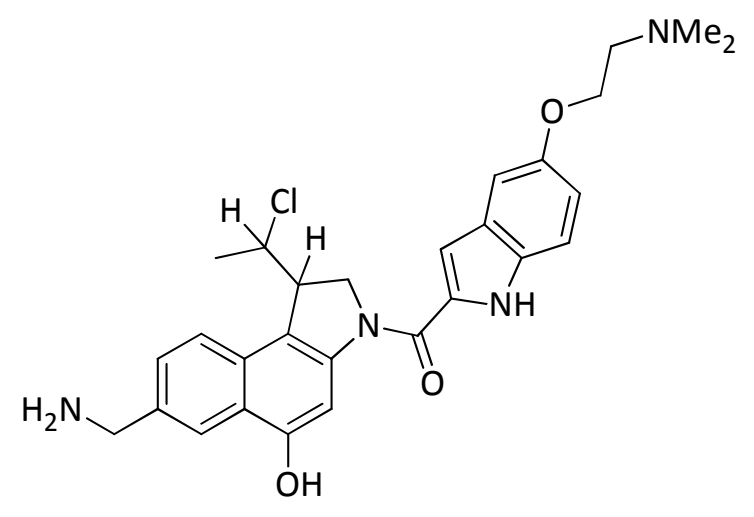

87

für $(1 S, 10 R)-87$ und Verwendung von $i \mathrm{Pr}_{2} \mathrm{NEt}$

$29 \%(1 S, 10 R)-71,13 \%(1 S, 10 R)-110,26 \%(1 S, 10 R)-111$

für (1R,10S)-87 und Verwendung von Pyridin

$47 \%(1 R, 10 S)-71$

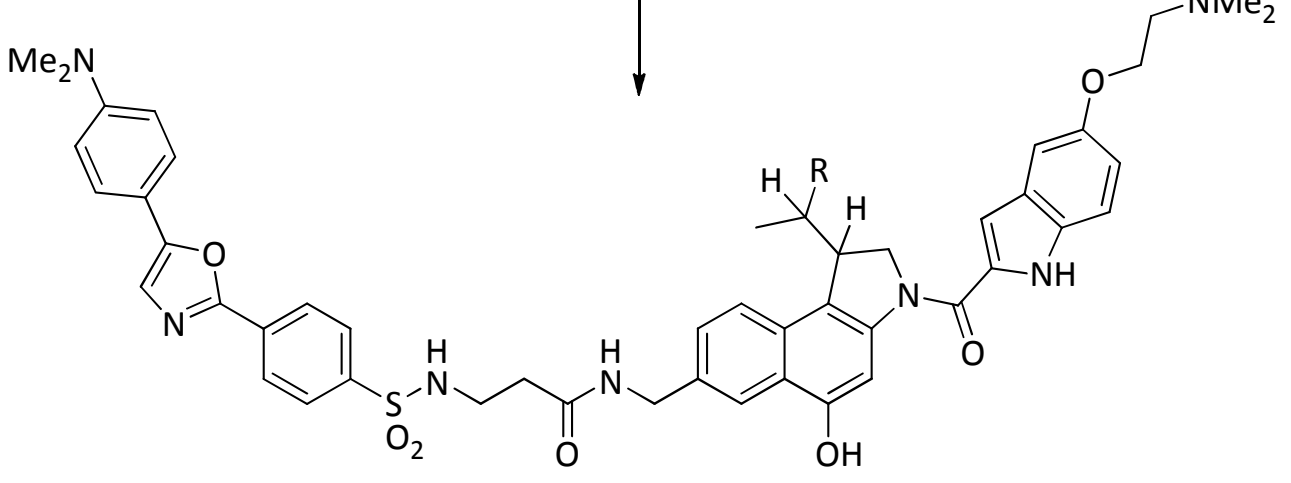

$$
\begin{array}{ll}
R=C l & 71 \\
R=O M e & 110 \\
R=O H & 111
\end{array}
$$

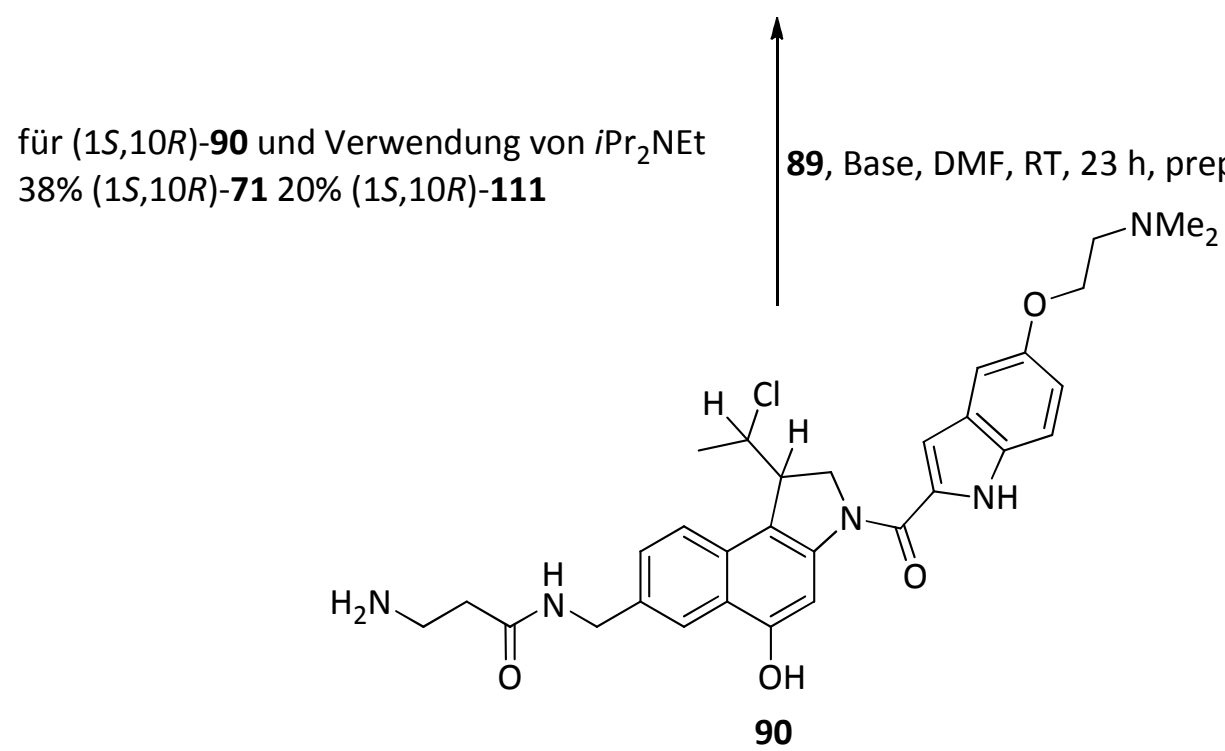

Abbildung 143. Synthese von $\mathbf{7 1}$ durch Verwendung von $\mathbf{8 7}$ und Dapoxylderivat $\mathbf{6 1}$ sowie $\mathbf{1 0 6}$ und Dapoxylderivat 89. 
In Zusammenarbeit mit Dr. Mišo Mitkovski vom MPI für experimentelle Medizin in Göttingen wurden die synthetisierten Verbindung durch CLSM untersucht. Die synthetisierten Verbindungen $(1 S, 10 R)-\mathbf{7 1},(1 S, 10 R)-110$ und $(1 S, 10 R)-111$ verfügen jedoch nicht über eine zweite Absorptionsbande um $\lambda_{\max , A b s}=525 \mathrm{~nm}$. Es muss daher davon ausgegangen werden, dass in der Synthese von $(1 S, 10 R)-71$ durch $B$. Krewer in geringen Mengen ein Nebenprodukt entstanden sein muss, das für die zweite Absorptionsbande verantwortlich ist (Abbildung 144 und 145). Leider war es nicht möglich, die Struktur dieses Nebenproduktes aufzuklären.

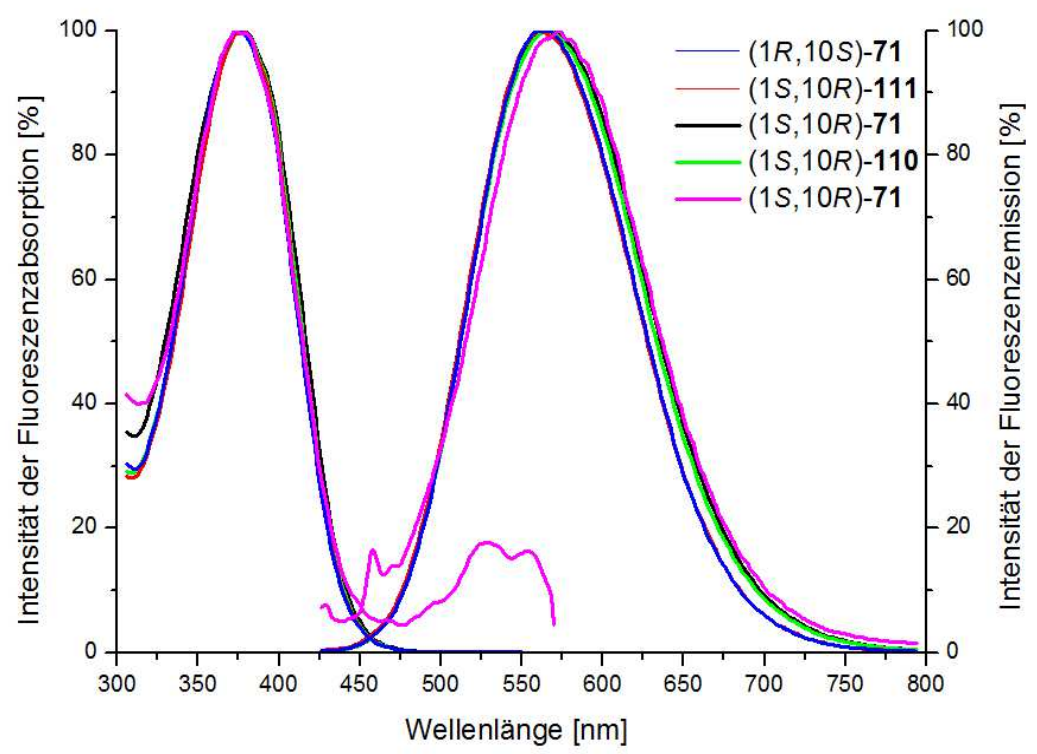

Abbildung 144. Flurorimetrische Bestimmung der Absorptions- und Emissionsspektren in $\mathrm{MeOH}$ für die in dieser Arbeit synthetisierten Verbindungen (blaue, rote, schwarze und grüne Linie) sowie der von B. Krewer synthetisierten Verbindung (magentafarbene Linie). 

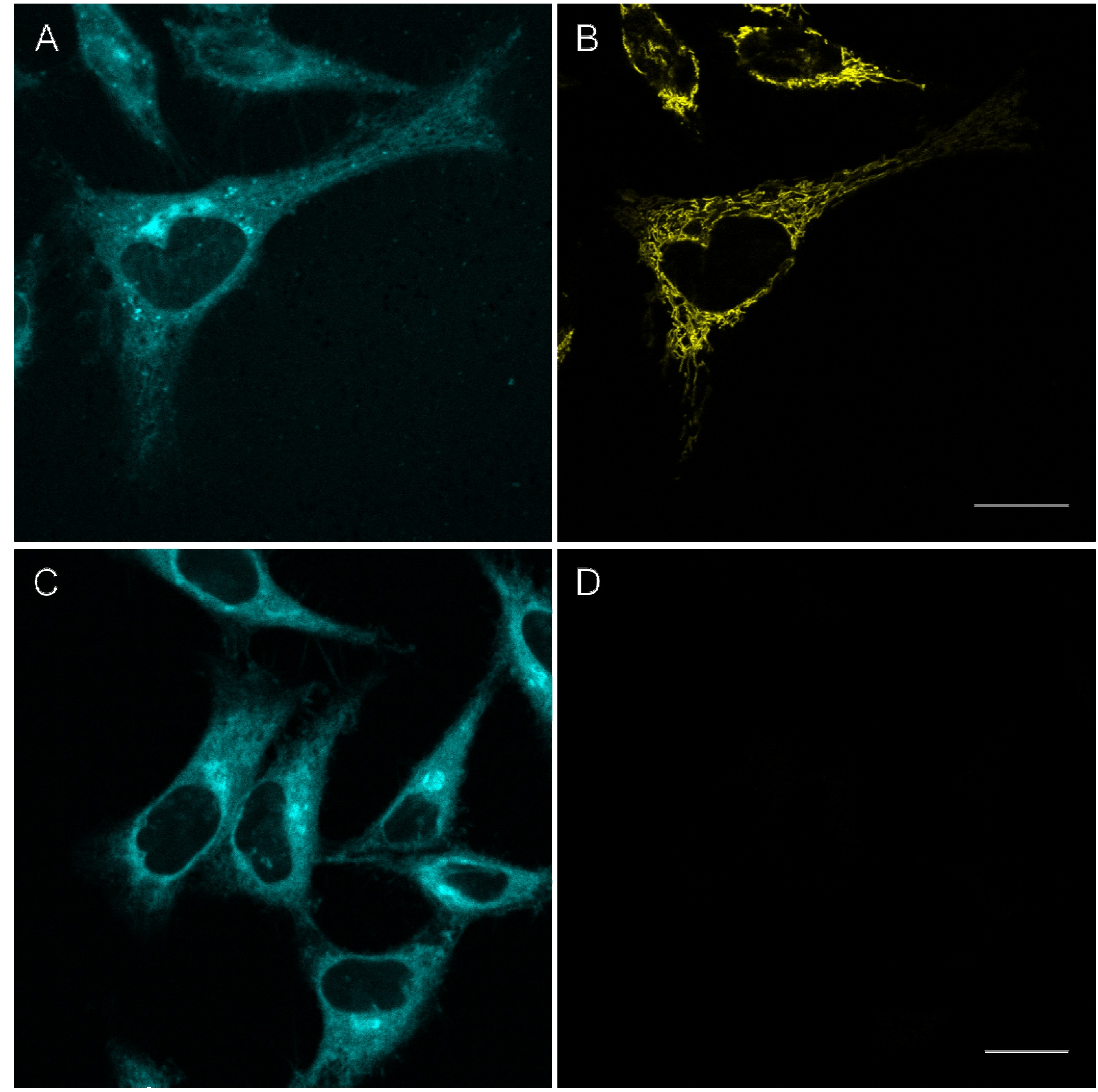

Abbildung 145. Untersuchung der zellulären Aufnahme von einem Gemisch aus (1S,10R)-71 und nicht identifizierbarem Nebenprodukt ( $A$ und $B$ ) sowie von (1S,10R)-71 (C und D). Maßstab $20 \mu \mathrm{m}$.

Neben den dargestellten Ergebnissen zur Synthese fluoreszenzmarkierter Prodrugs und seco-Drugs, die über das CBI-DMAI-Gerüst verfügen wurden in dieser Arbeit erstmals fluoreszenze CBI-Analoga dargestellt, die über einen bifunktionellen DNABinder verfügen, da dieser neben seiner Affinität zu doppelsträngiger DNA auch als Fluorochrom dient. Somit kann die Größe des Wirkstoffs möglichst klein gehalten werden und eine bessere Deutung der erzielten Ergebnisse in Hinblick auf dessen CBIDMAI-Analoga dargestellt werden.

Hierzu wurde zunächst das seco-CBI-Grundgerüst (1S)-128 ausgehend von Benzaldehyd über 12 Stufen dargestellt. Auf Grund der großen Ansatzmenge wurde auf eine Aufreinigung der ersten Stufen durch Säulenchromatographie verzichtet und die Zwischenprodukte wenn möglich durch Umkristallisation gesäubert.

Benzaldehyd (97) wird mit Phosphonat 120 in einer Horner-Wadsworth-EmmonsReaktion und anschließender Hydrolyse zu 96 umgesetzt. Es folgte eine intra- 
molekulare Friedel-Crafts-Acyclierung, Acetatentschützung zu 123, Benzyl-schützung und Verseifung zu 125 sowie Curtius-Umlagerung und abschließende lodierung nach Königstein zum lodnaphthalin 95.<smiles>O=Cc1ccccc1</smiles>

97

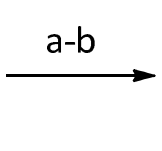<smiles>CCOC(=O)/C(=C/c1ccccc1)CC(=O)O</smiles>

96

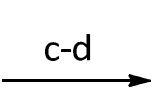<smiles>CCOC(=O)c1cc(O)c2ccccc2c1</smiles>

123<smiles>CC(C)(C)OC(=O)Nc1cc(OCc2ccccc2)c2ccccc2c1I</smiles>

95 e-f<smiles>[3H][13CH3]</smiles><smiles>C1CCCCC1</smiles><smiles>O=C(O)c1cc(Br)c2ccccc2c1</smiles>

125

Abbildung 146. Synthese des lodnaphthalins 95 ausgehend von Benzaldehyd (97) über 8 Stufen. a) 120, $\mathrm{NaH}, \mathrm{THF}, 0^{\circ} \mathrm{C}$ auf RT, $17 \mathrm{~h}$; b) TFA $/ \mathrm{H}_{2} \mathrm{O}, \mathrm{RT}, 20 \mathrm{~h}$; c) $\mathrm{KOAc}, \mathrm{Ac}_{2} \mathrm{O}$, Rückfluss, $2.5 \mathrm{~h}$, d) $\mathrm{K}_{2} \mathrm{CO}_{3}$, EtOH, Rückfluss, $1 \mathrm{~h}$; e) $\mathrm{BnBr}$, TBAl, $\mathrm{K}_{2} \mathrm{CO}_{3}$, DMF, RT, $24 \mathrm{~h}$; f) LiOH, $\mathrm{THF} / \mathrm{MeOH} / \mathrm{H}_{2} \mathrm{O},(3: 1: 1), \mathrm{RT}, 2 \mathrm{~d}$, umkristallisation, 53\% über 6 Stufen; g) DPPA, $\mathrm{NEt}_{3}, \mathrm{MS}(4 \AA ̊)$, ${ }^{\mathrm{t}} \mathrm{BuOH}$, Rückfluss, 2 d, quant.; h) $\mathrm{HIO}_{3}, \mathrm{I}_{2}, \mathrm{MeOH} / \mathrm{H}_{2} \mathrm{O}$ (4:1), Rückfluss, $2 \mathrm{~h}, 83 \%$.

Zur Darstellung des seco-CBI-Grundgerüstes (1S)-128 wurde der lodaromat 95 mit enantiomerenreinem Epoxid 126 alkyliert und einer Zyklisierung durch das Zinkat $\mathrm{Li}_{2} \mathrm{Zn}(\mathrm{SCN}) \mathrm{Me}_{3}$ unterzogen. Anschließend erfolgte eine Appel-Reaktion zu (1S)-93, Debenzylierung zu (1S)-128 und eine Reinigung durch Chromatographie an chiraler stationärer Phase.

Es folgte die Synthese der seco-Drugs und Prodrugs ausgehend vom seco-CBIGrundgerüst (1S)-128 unter Verwendung der in dieser Arbeit synthetisierten Carbonsäuren wie den Cumarinen 62 und 130, sowie der kommerziell erwerblichen 131 bzw. den synthetisierten Benzothiazolen 63 und 142. 
<smiles>CC(C)(C)OC(=O)Nc1cc(OCc2ccccc2)c2ccccc2c1I</smiles>

95
Ö

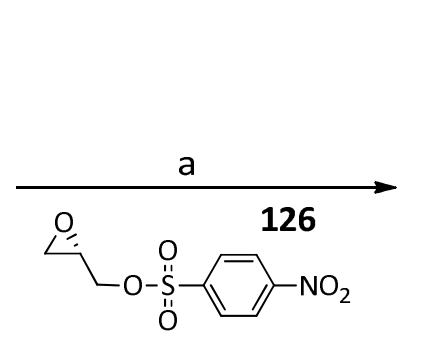

(1)<smiles>CC(C)(C)OC(=O)N1CC2OC2c2c1cc(OCc1ccccc1)c1ccccc21</smiles>

94<smiles>CC(C)(C)OC(=O)N1C[C@H](CCl)c2c1cc(O)c1ccccc21</smiles>

(1S)-128

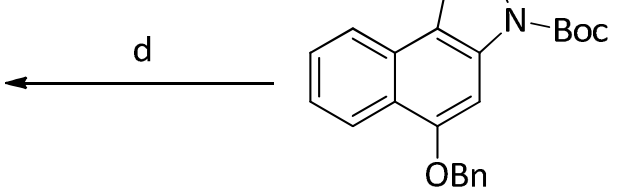

(1S)-93

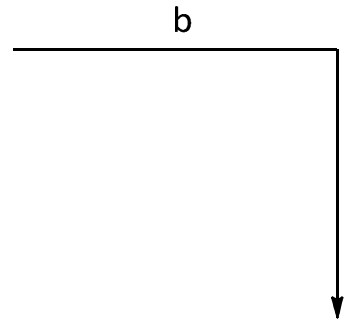

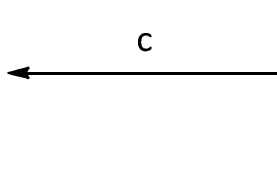

1<smiles>C1=CCCCCCCCCC1</smiles>

(1S)-127

Abbildung 147. Synthese des seco-CBI-Grundgerüstes (1S)-128. a) NaH, DMF, RT, 30 min., dann 126, $\mathrm{RT}, 18 \mathrm{~h}, 87 \%$; b) $\mathrm{Li}_{2} \mathrm{Zn}(\mathrm{SCN}) \mathrm{Me}_{3}, \mathrm{THF},-78^{\circ} \mathrm{C} \rightarrow \mathrm{RT}, 12 \mathrm{~h}, 47 \%$; c) $\mathrm{CCl}_{4}, \mathrm{PPh}_{3}, \mathrm{CH}_{2} \mathrm{Cl}_{2}$, RT, 3 h, 90\%; d) Pd/C, $\mathrm{H}_{2}$ (1atm), $50{ }^{\circ} \mathrm{C}, 6$ h, $89 \%$.

Hierzu wurde die N-Boc-Schutzgruppe durch Suspendieren des Phenols (1S)-128 in $4 \mathrm{M} \mathrm{HCl}$ /Essigester-Lösung entfernt, um das freie sekundäre Amin im Anschluss unter Verwendung von EDAC. $\mathrm{HCl}$ als Kupplungsreagenz mit der entsprechenden Carbonsäure zu kuppeln. Abschließend wurden die erhaltenen seco-CBI-Drugs (1S)92, (1S)-137, (1S)-138, (1S)-141 und (1S)-145 durch präparative HPLC (Kromasil 100 C-18) aufgereinigt (Abbildung 148).

Die Galaktoside konnten über 4 Stufen aus (1S)-128 hergestellt werden. Zunächst wurde (1S)-128 nach der Trichloracetimidatmethode nach Schmidt ${ }^{157}$ unter $\mathrm{BF}_{3} \cdot \mathrm{OEt}_{2}$ Katalyse mit tetraacetyliertem D-Galaktose-Trichloracetimidat 116 gekuppelt und anschließend die säurelabile $\mathrm{N}$-Boc-Schutzgruppe durch Umsetzung mit $\mathrm{BF}_{3} \cdot \mathrm{OEt}_{2}$ abgespalten, um das Amin mit EDAC. $\mathrm{HCl}$ in DMF mit der entsprechenden Carbonsäure zu kuppeln. Entschützung der tetraacetylierten Galaktoside durch $\mathrm{NaOMe}$ in $\mathrm{MeOH}$ bei 62, 130 und 131 bzw. durch $\mathrm{K}_{2} \mathrm{CO}_{3}$ in $\mathrm{MeOH}$ lieferte die gewünschten Galaktoside (1S)-69, (1S)-70,(1S)-139, (1S)-140, (1S)-147 (Abbildung 149). 


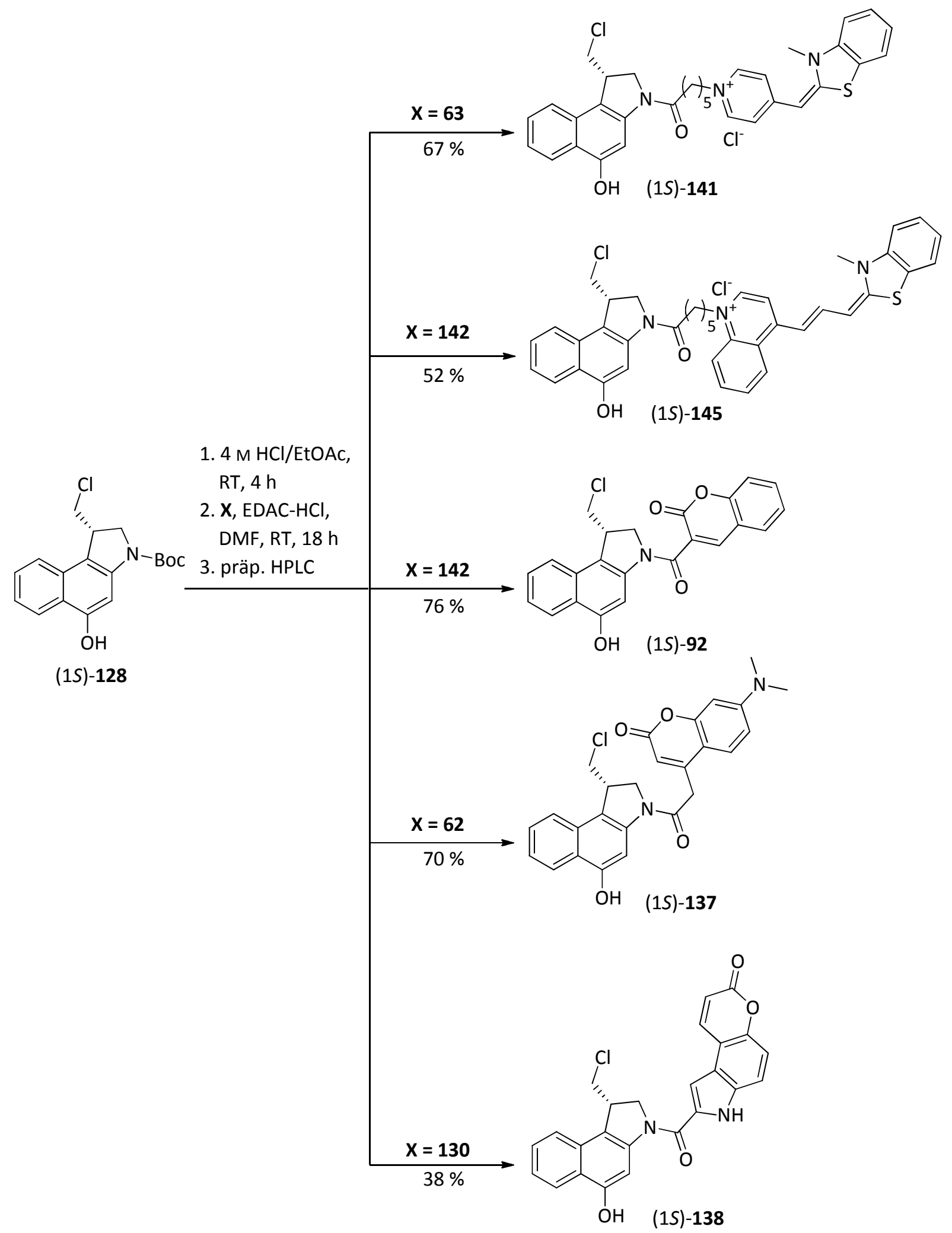

Abbildung 148. Darstellung der seco-CBI-Drugs (1S)-92, (1S)-137, (1S)-138, (1S)-141 und (1S)-145. 


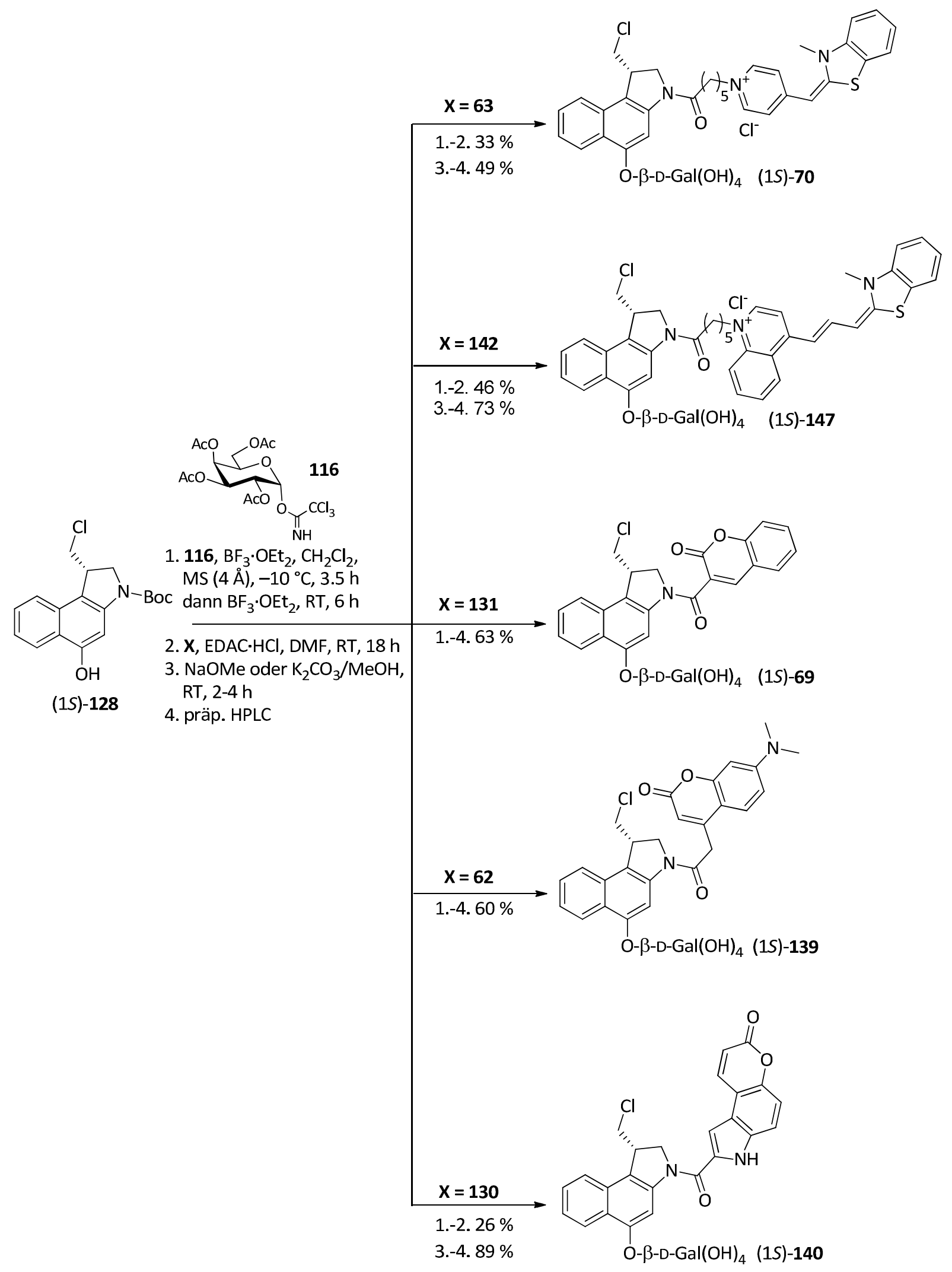

Abbildung 149. Darstellung der Galaktoside (1S)-69, (1S)-70,(1S)-139, (1S)-140, (1S)-147. 
Abschließend wurden die neuartigen (1S)-CBI-Derivate zunächst am Fluorimeter auf ihre Fluoreszenzeigenschaften (Absorptions- und Emissionsmaxima in $\mathrm{MeOH}$ und wässrigem Puffer) sowie in Live Cell Imaging Experimenten auf ihre zelluläre Aufnahme untersucht. So zeigen die in Tabelle 5 gezeigten Verbindungen folgende Gemeinsamkeiten: Im Fall der Benzothiazole (Spalte A und B) akkumulieren die secoDrugs (1S)-141 und (1S)-145 im Zellkern (A1 und B1), während die Galaktoside (1S)-70 und (1S)-147 die Mitochondrien (A2 und B2) und die Fluorochrom 144 und 143 die Nukleoli (A3 und B3) populieren. Im Fall der Cumarine populieren mit Ausnahme von seco-Drug 137 (D1), das auch im Zellkern zu finden ist, alle Verbindungen ausschließlich die Mitochondrien lebender Zellen.
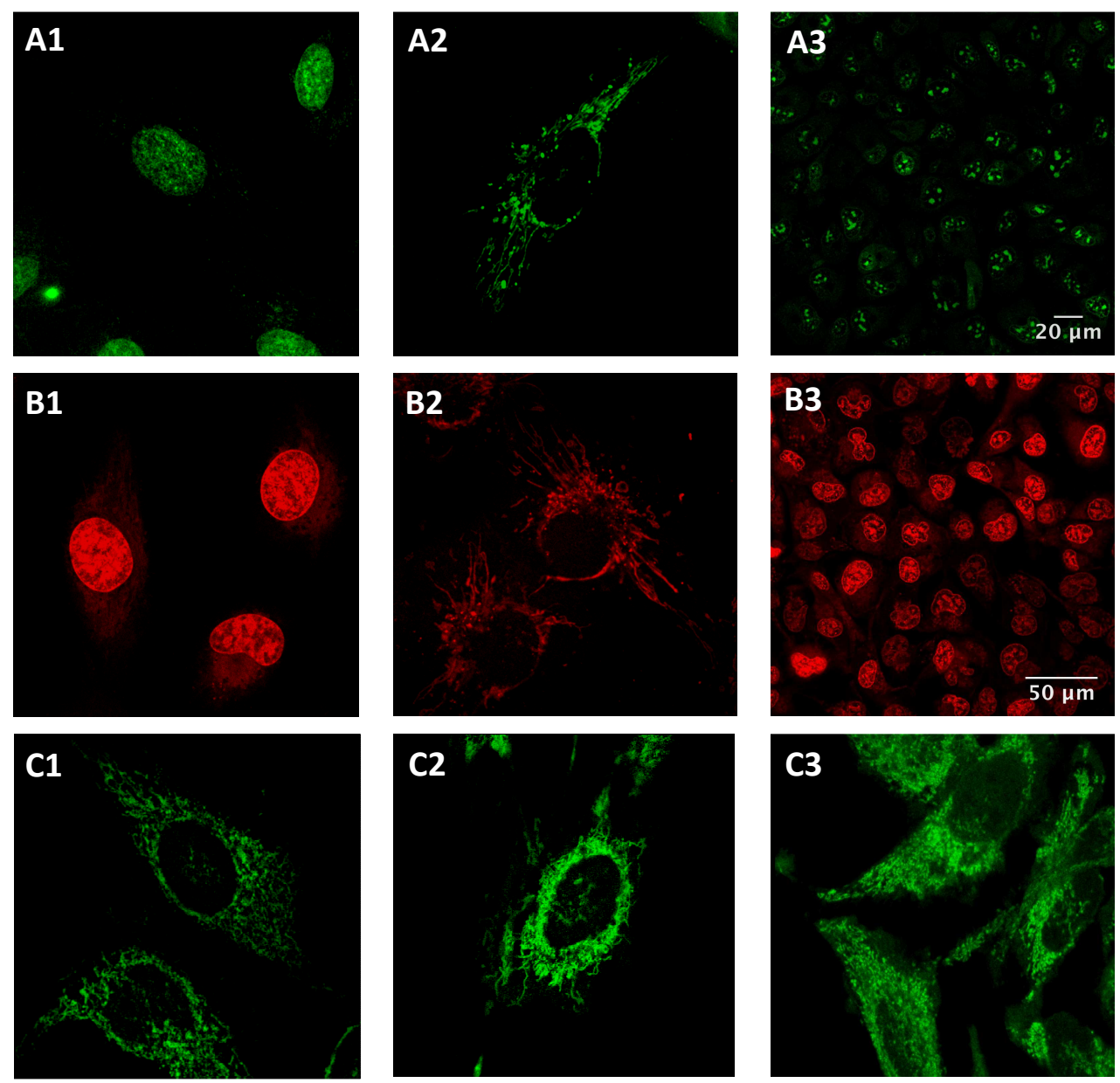

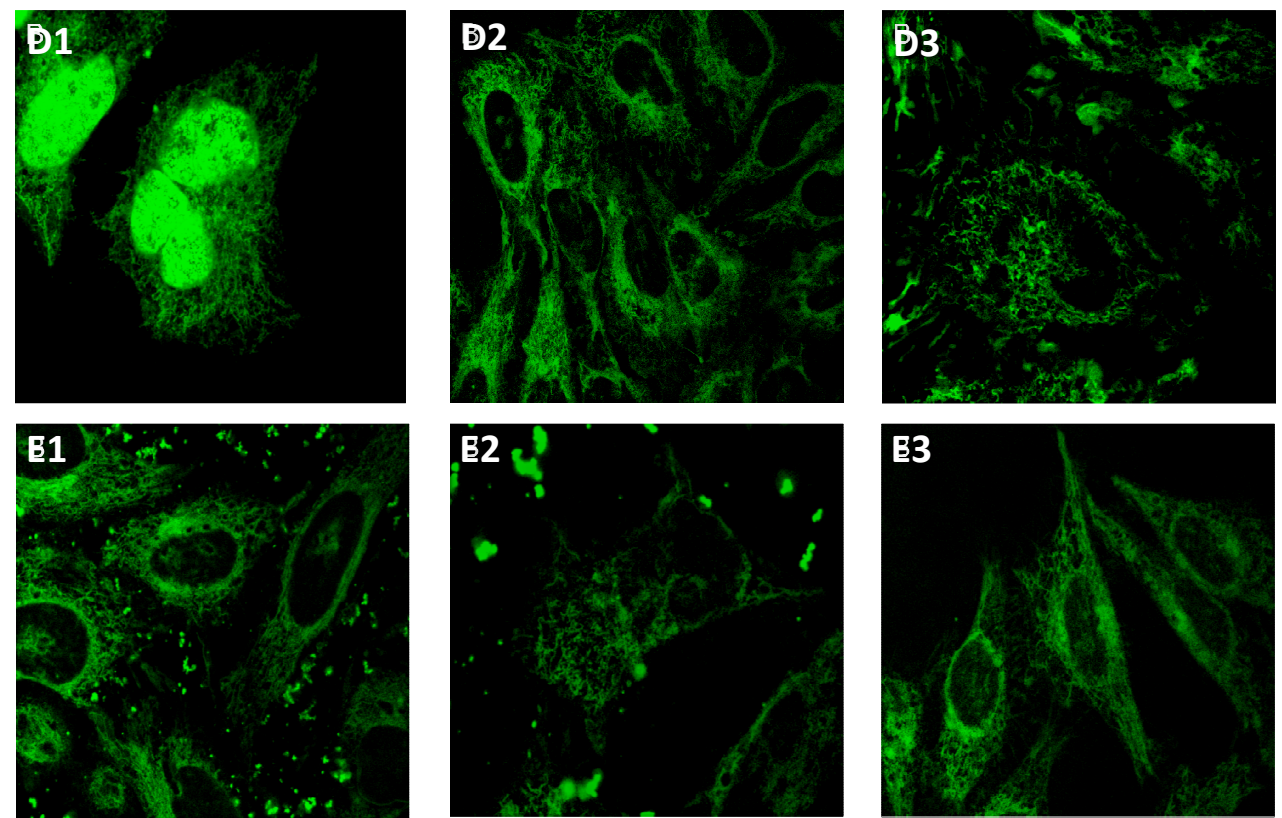

Tabelle 5. Darstellung der Live Cell Imaging Experimente zur Untersuchung der zellulären Aufnahme der seco-Drugs (1S)-141 (A1), (1S)-145 (B1), (1S)-92 (C1), (1S)-137 (D1) und (1S)-138 (E1), der Galaktoside (1S)-70 (A2), (1S)-147 (B2), (1S)-69 (C2), (1S)-139 (D2) und (1S)-140 (E2) sowie der Fluorochrom 144 (A3), 143 (B3), 131 (C3), 62 (D3) und $130(E 3)$.

Zusammenfassend kann festgehalten werden, dass die, in der Zielsetzung der Arbeit, ausgegebenen Punkte erreicht werden konnten. Im ersten Abschnitt dieser Arbeit konnte eine verkürzte Synthese zur Darstellung des fluoreszenzmarkierten antiMethyl-seco-Drugs (1S,10R)-71 dargestellt werden. Zusätzlich wurde eine weitere Syntheseroute, die den Einsatz des kostengünstigeren Fluorochroms 89 zulässt, eingeführt. Außerdem wurde das gewünschte Galaktosid (1S)-72, welches mit Fluorescein markiert wurde erfolgreich synthetisiert. Des weiteren konnten fünf neuartige seco-Drugs sowie fünf Galaktoside einer innovativen Struktur, in der der DNA-Binder DMAI durch einen fluoreszenzaktiven DNA-Binder aus der Klasse der Benzothiazole oder der Cumarine ersetzt wurde, synthetisiert und in Live Cell Imaging Experimenten auf ihre zelluläre Aufnahme untersucht werden. 


\section{EXPERIMENTELLER TEIL}

\section{Allgemeine Arbeitsvorschriften}

Die Umsetzungen wurden, soweit nötig, in ausgeheizten Glasapparaturen unter einem leichten Argon-Überdruck durchgeführt. Die Lösungsmittel wurden entsprechend den üblichen Laboratoriumsmethoden getrocknet und destilliert ${ }^{164}$ bzw. als p.a.-Ware über Molsieb gelagert. Soweit nicht anders vermerkt, wurden kommerziell erhältliche Produkte ohne weitere Reinigung eingesetzt. Das Entgasen von Lösungsmitteln geschah durch Einleiten eines Argon-Gasstroms über einen längeren Zeitraum. Konzentrationsangaben beziehen sich, sofern nicht anders erwähnt, auf wäßrige Lösungen.

\subsection{Verwendete Geräte}

Drehwerte: Drehwerte wurden mit einem Polarimeter Modell 241 der Firma PerkinElmer sowie P-2000 der Firma Jasco gemessen.

Infrarotspektren: Sofern „(KBr)“ oder „(Film)“ hinter der Bezeichnung „IR“ vermerkt ist, wurden die Infrarotspektren mit dem Modell Vector 22 der Firma Bruker aufgenommen, wobei Feststoffe als $\mathrm{KBr}$-Preßlinge und Flüssigkeiten als Film zwischen $\mathrm{KBr}$ Platten gemessen wurden. Findet sich kein Hinweis hinter der Bezeichnung "IR“, wurden die Infrarotspektren mit dem Modell FT/IR-4100 der Firma Jasco ohne $\mathrm{KBr}$ Optik (neat) aufgenommen.

UV/VIS-Spektren: Die Aufnahme der UV/VIS-Spektren erfolgte mit Modell Lambda 2 der Firma Perkin-Elmer und V-630 der Firma Jasco.

${ }^{1}$ H-NMR-Spektren: Die ${ }^{1}$ H-NMR-Spektren wurden mit den Modellen Mercury 300, Mercury-vx 300, Unity 300, Vnmrs 300 (jeweils 300 MHz) sowie Inova 600 (600 MHz) der Firma Varian von in deuterierten Solventien gelösten Proben aufgenommen. Die 
chemischen Verschiebungen sind in Einheiten der $\delta$-Skala angegeben und auf das Signal des angegebenen Lösungsmittels referenziert. Zur Kennzeichnung der Multiplizitäten der Signale werden folgende Abkürzungen verwendet: s (Singulett), d (Dublett), t (Triplett), q (Quartett), dd (Dublett von Dubletts), dt (Dublett von Tripletts) usw. Signale, die durch Überlagerung oder Anteile höherer Ordnung nicht interpretierbar waren, wurden mit $m$ (Multiplett) bzw. $\mathrm{m}_{\mathrm{c}}$ (symmetrisches, zentriertes Multiplett) bezeichnet und verbreiterte Signale durch den Zusatz br indiziert. Zur Bezeichnung aromatischer Protonen oder Kohlenstoffatome werden folgende Abkürzungen verwendet: $i$ (ipso), o (ortho), $m$ (meta), $p$ (para). Sofern nicht anders angegeben, beziehen sich alle Kopplungskonstanten auf ${ }^{1} \mathrm{H}-{ }^{1} \mathrm{H}$-Kopplungen.

${ }^{13}$ C-NMR-Spektren: Die, soweit nicht anders vermerkt, ${ }^{1} \mathrm{H}$-breitbandentkoppelten ${ }^{13} \mathrm{C}$-NMR-Spektren wurden mit den Geräten Unity $300(75 \mathrm{MHz})$, Inova $500(125 \mathrm{MHz})$ und Inova 600 (150 MHz) der Firma Varian aufgenommen. Die chemischen Verschiebungen sind in Einheiten der $\delta$-Skala angegeben. Als interner Standard diente das angegebene Lösungsmittel. Generell erfolgte die Zuordnung der Signale durch die 2DNMR-Experimente COSY, HSQC sowie HMBC.

Massenspektren: Zur Aufnahme der EI- und EI-HRMS-Spektren diente ein Time-ofFlight Massenspektrometer AccuTOF der Firma Jeol. ESI-Spektren wurden mit einem Ion-Trap-Massenspektrometer LCQ der Firma Finnigan sowie einem Time-of-Flight Massenspektrometer micrOTOF der Firma Bruker aufgenommen. Die Messung der ESI-HRMS-Spektren erfolgte an einem 7-Tesla-Fourier-Transform-Ion-CyclotronResonance (FTICR)-Massenspektrometer APEX IV der Firma Bruker und am micrOTOFGerät. Angegeben werden die Quotienten aus Masse zu Ladung sowie in Klammern die relativen Intensitäten bezogen auf den Basispeak ( $I=100)$.

Kältetechnik: Die Reaktionsführung bei konstant tiefen Temperaturen über einen längeren Zeitraum erfolgte unter Einsatz von Kryostaten. Benutzt wurde das Modell EK 90 der Firma Haake. Darüber hinaus wurden übliche Kältemischungen wie Eis/Wasser- und Aceton/ Trockeneis-Gemische zur temporären Kühlung verwendet. 


\subsection{Chromatographische Methoden}

Dünnschichtchromatographie (DC): Es wurden Aluminium-Fertigfolien Si60 $\mathrm{F}_{254}$ der Firma Merck verwendet. Angegeben sind $\mathrm{R}_{f}$-Werte (Laufhöhe relativ zur Laufmittelfront). Neben der UV-Detektion dienten eine Vanillin-Schwefelsäure-Lösung (0.5 g Vanillin, $3 \mathrm{~mL}$ konz. $\mathrm{H}_{2} \mathrm{SO}_{4}, 85 \mathrm{~mL} \mathrm{MeOH}$ und $10 \mathrm{~mL} \mathrm{HOAc}$ ) sowie eine Ninhydrin-Lösung (0.2\% in Ethanol) als Anfärbereagenzien.

Säulenchromatographie: Alle säulenchromatographischen Trennungen wurden mit Kieselgel 60 (Korngröße: 0.032-0.063 mm) der Firma Merck durchgeführt.

\section{Hochdruckflüssigkeitschromatographie (HPLC):}

Analytische HPLC: Analytische Trennungen wurden auf einer HPLC-Anlage der Firma Jasco, ausgestattet mit einer Lösungsmittelpumpe PU-2080, einer Mischkammer LG1590-04, einem Multiwellenlängendetektor MD-2010 Plus und der Steuerung LC-Net II/ADC, vorgenommen. Für die Injektion wurde ein automatischer Probenwechsler (Autosampler AS-2055) derselben Firma verwendet. Zur Bedienung, Datenerfassung und Datenauswertung wurden die Computerprogramme Borwin PDA, HSS 2000 und Borwin Chromatography der Firma Jasco eingesetzt. Für die analytischen Messungen wurden die Säulen Chiralcel ${ }^{\oplus}$ OD $(250 \times 4.6 \mathrm{~mm}, 5 \mu \mathrm{m}$, Daicel Chemical Industries Ltd.), Chiralpak ${ }^{\oplus}$ IA (250 $\times 4.6$ mm, $5 \mu \mathrm{m}$, Daicel Chemical Industries Ltd.) mit chiraler stationärer Phase sowie Aqua ${ }^{\oplus}$ C18 200A $(250 \times 4.6 \mathrm{~mm}, 5 \mu \mathrm{m}$, Phenomenex $)$ mit stationärer RP-Phase verwendet. Angegebene Lösungsmittel waren von HPLC-Qualität und das Wasser bidestilliert. Alle Proben wurden membranfiltriert mit Polytetrafluorethylen-(PTFE)-Filtern der Firma Roth $(\varnothing 25 \mathrm{~mm}, 0.2 \mu \mathrm{m})$ bzw. VWR $(\varnothing 13 \mathrm{~mm}, 0.2 \mu \mathrm{m})$

Präparative HPLC: Präparative Trennungen wurden auf einem HPLC-System der Firma Jasco, ausgestattet mit zwei Lösungsmittelpumpen Modell PU-2087 PLUS und einem UV-Detektor Modell UV-2075 PLUS, vorgenommen. Verwendet wurden die Säulen Chiralpak IA $(250 \times 20 \mathrm{~mm}, 5 \mu \mathrm{m}$, Daicel Chemical Industries Ltd. $)$ mit chiraler stationärer Phase und Kromasil ${ }^{\oplus} 100 \mathrm{C} 18(7 \mu \mathrm{m}, 250 \times 20$ mm, Jasco und Dr. Maisch 
$\mathrm{GmbH})$ sowie Aqua ${ }^{\oplus} \mathrm{C} 18200 \mathrm{~A}(250 \times 21.2 \mathrm{~mm}, 5 \mu \mathrm{m}$, Phenomenex $)$ mit stationärer RP-Phase. Angegebene Lösungsmittel waren von HPLC-Qualität und das Wasser bidestilliert. Alle Proben wurden vor der Trennung membranfiltriert (siehe analytische HPLC).

HPLC-MS: Die analytischen Trennungen wurden auf einer HPLC-Anlage bestehend aus einer Lösungsmittelpumpe Rheos 4000, einem Entgaser ERC-3415 $\alpha$ der Firma Flux Instruments, Autosamplern 851-AS und AS-1555 der Firma Jasco und einem Diodenarray-Detektor der Firma Thermo vorgenommen. Eingesetzt wurde die Säule Synergi Max-RP C12 (150 × 2 mm, 4 mm) der Firma Phenomenex. Zur Bedienung, Datenerfassung und Datenauswertung wurden die Computerprogramme Janeiro und Xcalibur verwendet. Als Laufmittelkomponenten dienten Wasser und Methanol der Firma VWR. Zur Verbesserung der Peakschärfe enthielten beide Laufmittel $0.05 \%$ Ameisensäure der Firma Roth. Die Lösungsmittelgemische wurden auf der Niederdruckseite erzeugt. Der Fluß betrug $300 \mu \mathrm{L} \mathrm{min}^{-1}$ bei folgendem Gradienten:

\begin{tabular}{ll}
\hline Zeit [min] & $\mathrm{H}_{2} \mathrm{O} / \mathrm{MeOH}$ (je 0.05\% Ameisensäure) \\
\hline 0 & $70 / 30$ \\
$0-15$ & $70 / 30 \rightarrow 0 / 100$ \\
$15-22$ & $0 / 100$ \\
$22-23$ & $0 / 100 \rightarrow 70 / 30$ \\
$23-29$ & $70 / 30$ \\
\hline
\end{tabular}

Die der analytischen Trennung online folgende Aufnahme von ESI-Massenspektren wurde an einem Ion-Trap-Massenspektrometer LCQ der Firma Finnigan durchgeführt. Die UV-Detektion erfolgte im Bereich von 200-800 nm und die Massen-Detektion im Bereich von $\mathrm{m} / \mathrm{z}$ 100-2000. Die Kapillartemperatur betrug 220 bzw. $240{ }^{\circ} \mathrm{C}$, die Sprayspannung 4.5 kV und der Sheath-Gas-Fluß 80 (beliebige Einheit). 


\subsection{Materialien für die In-vitro-Zytotoxizitätsuntersuchungen}

Zellinie: A549, ATCC-CCL 185 (American Type Culture Collection Certified Cell Lines), eine 1972 aus einem kanzerogenen Tumorexplanat der Lunge eines 58-jährigen männlichen Patienten etablierte, epithelähnliche, adhärent in Monolayern wachsende Zellinie. Herkunft: Institut für Zellbiologie der Universität Essen.

Medien: Alle Pulvermedien wurden in bidestilliertem Wasser angesetzt, mit $\mathrm{HCl}$ auf pH 7.4 titriert und sterilfiltriert.

Kulturmedium für A549: DMEM (Dulbecco`s Modified Eagles Medium) mit $4.5 \mathrm{~g} \mathrm{~L}^{-1}$ Glucose (Biochrom, T043-10). Das Medium wurde mit $4 \mathrm{mM} \mathrm{L-Glutamin}$ und $3.7 \mathrm{~g} \mathrm{~L}^{-1}$ Natriumhydrogencarbonat supplementiert.

Medium-Zusätze: 10\% FKS (Fötales Kälberserum) der Firma Biochrom, 30 min inaktiviert bei $56^{\circ} \mathrm{C}$.

Enzyme: $4.0 \mathrm{U} \mathrm{mL}^{-1} \quad \beta$-D-Galaktosidase (EC 3.2.1.23) aus Eschericha coli G 5635 (Sigma), Aktivität: 250-600 Units (U) pro mg Protein bei pH7.3 und $37^{\circ} \mathrm{C}$, $1 \mathrm{U}=1 \mu \mathrm{mol}$ Substratumsatz pro Minute.

Zellzählkammer: Netzeinteilung nach Bürker der Firma Assistent. 


\section{Synthese der fluoreszenzaktiven DNA-Binder}

\subsection{1-(5-Methoxycarbonyl-pentyl)-4-(3-Methyl-3H-benzothiazol-2- ylidenmethyl)-pyridinium-chlorid (144)}

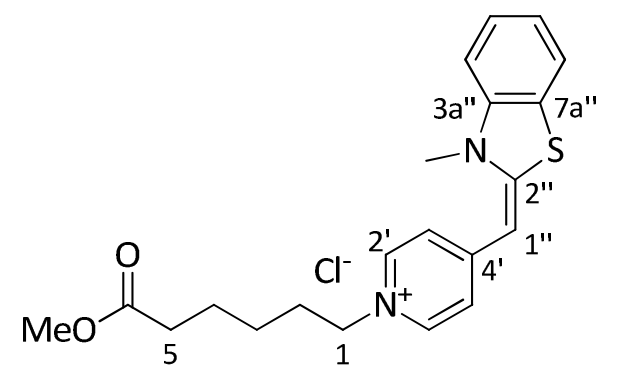

Zu einer Lösung der Carbonsäure 63 (50.0 mg, $111 \mu \mathrm{mol}, 1.0$ Äq.) in absolutem MeOH $(10 \mathrm{~mL})$ wird bei $0{ }^{\circ} \mathrm{C} \mathrm{SOCl}_{2}\left(21.9 \mathrm{mg}, 13.3 \mu \mathrm{L}, 184 \mu \mathrm{mol}, 1.6\right.$ Äq.) gegeben, auf $50^{\circ} \mathrm{C}$ erwärmt, für $3 \mathrm{~h}$ gerührt und das Lösungsmittel im Vakuum entfernt. Nach säulenchromatographischer Reinigung an Kieselgel $\left(\mathrm{CH}_{2} \mathrm{Cl}_{2} / \mathrm{MeOH}=5: 1\right)$ erhielt man die Zielverbindung 144 als gelben Feststoff (46.5 mg, $104 \mu \mathrm{mol}, 93 \%$ ).

UV (MeOH): $\lambda_{\max }(\lg \varepsilon)=204.0 \mathrm{~nm}$ (4.336), 218.0 (4.340), 255.0 (3.891), 341.0 (2.847), 447.0 (4.757).

${ }^{1} \mathrm{H}-\mathrm{NMR}\left(300 \mathrm{MHz}\right.$, DMSO- $\left.\mathrm{d}_{6}\right): \delta=1.16-1.35\left(\mathrm{~m}, 2 \mathrm{H}, 3-\mathrm{H}_{2}\right), 1.51-1.63\left(\mathrm{~m}, 2 \mathrm{H}, 4-\mathrm{H}_{2}\right)$, 1.77-1.89 (m, $\left.2 \mathrm{H}, 2-\mathrm{H}_{2}\right), 2.32\left(\mathrm{t}, \mathrm{J}=7.3 \mathrm{~Hz}, 2 \mathrm{H}, 5-\mathrm{H}_{2}\right), 3.58\left(\mathrm{~s}, 3 \mathrm{H}, \mathrm{CO}_{2} \mathrm{CH}_{3}\right), 3.73(\mathrm{~s}$, $\left.3 \mathrm{H}, \mathrm{NCH}_{3}\right), 4.23\left(\mathrm{t}, \mathrm{J}=7.3 \mathrm{~Hz}, 2 \mathrm{H}, 1-\mathrm{H}_{2}\right), 6.28\left(\mathrm{~s}, 1 \mathrm{H}, 1^{\prime \prime}-\mathrm{H}\right), 7.30(\mathrm{dt}, J=7.7,0.9 \mathrm{~Hz}$, $\left.1 \mathrm{H}, 6^{\prime \prime}-\mathrm{H}\right), 7.41$ (d, J = 7.2 Hz, $\left.2 \mathrm{H}, 3^{\prime}-\mathrm{H}, 5^{\prime}-\mathrm{H}\right), 7.52$ (dt, J = 7.3, $\left.0.9 \mathrm{~Hz}, 1 \mathrm{H}, 5^{\prime \prime}-\mathrm{H}\right), 7.60$ (d, J = 7.9 Hz, $1 \mathrm{H}, 4^{\prime \prime}-\mathrm{H}$ ), 7.92 (d, J = 7.7 Hz, $1 \mathrm{H}, 7^{\prime \prime}-\mathrm{H}$ ), 8.39 (d, J = 7.2 Hz, $2 \mathrm{H}, 2^{\prime}-\mathrm{H}$, 6'-H) ppm.

${ }^{13} \mathrm{C}-\mathrm{NMR}\left(125 \mathrm{MHz}, \mathrm{DMSO}-\mathrm{d}_{6}\right): \delta=23.7$ (C-4), 24.8 (C-3), 29.9 (C-2), $32.8\left(\mathrm{NCH}_{3}\right), 32.9$ (C-5), $51.1\left(\mathrm{CO}_{2} \mathrm{CH}_{3}\right), 57.2$ (C-1), 89.4 (C-1"), 111.8 (C-4"), 118.2 (C-3', C-5'), 122.4 (C-7"), 123.1 (C-7a"), 123.3 (C-6"), 127.6 (C-5"), 140.3 (C-3a"), 141.1 (C-2',C-6'), 150.2 (C-2"), 156.9 (C-4'), $172.9\left(\underline{\mathrm{CO}}_{2} \mathrm{CH}_{3}\right) \mathrm{ppm}$. 
$\mathrm{C}_{21} \mathrm{H}_{25} \mathrm{ClN}_{2} \mathrm{O}_{2} \mathrm{~S}(404.95)$

ber.: $369.1631\left[{\left.\mathrm{M}-\mathrm{Cl}^{-}\right]^{+}}^{+}\right.$

gef.: 369.1630 (ESI-HRMS).

\subsection{1-(5-Methoxycarbonyl-pentyl)-4-[3-(3-Methyl-3H-benzothiazol-2- yliden)-propenyl]-quinolinium-chlorid (143)}

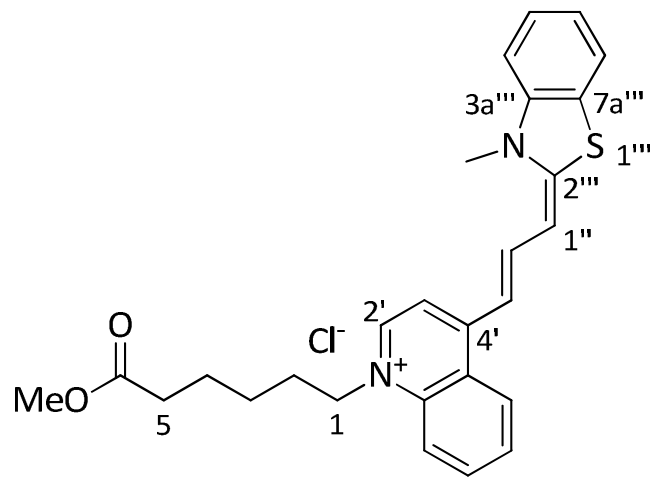

Zu einer Lösung der Carbonsäure 142 (20.0 mg, 39 mol, 1.0 Äq.) in absolutem MeOH $(5 \mathrm{~mL})$ wird bei $0{ }^{\circ} \mathrm{C} \mathrm{SOCl}\left(7.5 \mathrm{mg}, 4.5 \mu \mathrm{L}, 63 \mu \mathrm{mol}, 1.6 \mathrm{Äq}\right.$.) gegeben, auf $50{ }^{\circ} \mathrm{C}$ erwärmt, für $3 \mathrm{~h}$ gerührt und das Lösungsmittel im Vakuum entfernt. Nach Reinigung durch präparative Dünnschichtchromatographie an Kieselgel $\left(\mathrm{CH}_{2} \mathrm{Cl}_{2} / \mathrm{MeOH}=10: 1\right)$ erhielt man die Zielverbindung 143 als blauen Feststoff ( 6.9 mg, 13 rmol, 33\%).

UV (MeOH): $\lambda_{\max }(\lg \varepsilon)=240.0 \mathrm{~nm}$ (4.660), 283.0 (4.535), 304.0 (4.501), 629.0 (4.793). ${ }^{1} \mathrm{H}-\mathrm{NMR}\left(600 \mathrm{MHz}\right.$, DMSO-d $\left.\mathrm{d}_{6}\right): \delta=1.32-1.42\left(\mathrm{~m}, 2 \mathrm{H}, 3-\mathrm{H}_{2}\right), 1.52-1.64\left(\mathrm{~m}, 2 \mathrm{H}, 4-\mathrm{H}_{2}\right)$, 1.77-1.87 (m, $\left.2 \mathrm{H}, 2-\mathrm{H}_{2}\right), 2.32\left(\mathrm{t}, \mathrm{J}=7.3 \mathrm{~Hz}, 2 \mathrm{H}, 5-\mathrm{H}_{2}\right), 3.57\left(\mathrm{~s}, 3 \mathrm{H}, \mathrm{CO}_{2} \mathrm{CH}_{3}\right), 3.74(\mathrm{~s}$, $\left.3 \mathrm{H}, \mathrm{NCH}_{3}\right), 4.55\left(\mathrm{t}, \mathrm{J}=7.3 \mathrm{~Hz}, 2 \mathrm{H}, 1-\mathrm{H}_{2}\right), 6.51(\mathrm{~d}, J=12.4 \mathrm{~Hz}, 1 \mathrm{H}, 1 "-\mathrm{H}), 7.12$ (d, $\left.J=12.4 \mathrm{~Hz}, 1 \mathrm{H}, 3^{\prime \prime}-\mathrm{H}\right), 7.32\left(\mathrm{dt}, J=7.7,0.9 \mathrm{~Hz}, 1 \mathrm{H}, 6^{\prime \prime \prime}-\mathrm{H}\right), 7.50(\mathrm{dt}, J=7.3,0.9 \mathrm{~Hz}, 1 \mathrm{H}$, 5'"'-H), 7.60 (d, J= $\left.7.9 \mathrm{~Hz}, 1 \mathrm{H}, 4^{\prime \prime \prime}-\mathrm{H}\right), 7.71$ (t, J= 7.6 Hz, $\left.1 \mathrm{H}, 7^{\prime}-\mathrm{H}\right), 7.85$ (d, J= $7.6 \mathrm{~Hz}$, $\left.1 \mathrm{H}, 3^{\prime \prime}-\mathrm{H}\right), 7.89\left(\mathrm{~d}, J=8.6 \mathrm{~Hz}, 1 \mathrm{H}, 7^{\prime \prime \prime}-\mathrm{H}\right), 7.95\left(\mathrm{~d}, J=7.7 \mathrm{~Hz}, 1 \mathrm{H}, 66^{\prime}-\mathrm{H}\right), 8.08$ (d, $\left.J=8.6 \mathrm{~Hz}, 1 \mathrm{H}, 5^{\prime}-\mathrm{H}\right), 8.16\left(\mathrm{t}, J=10.6 \mathrm{~Hz}, 1 \mathrm{H}, 2^{\prime \prime}-\mathrm{H}\right), 8.43\left(\mathrm{~d}, J=6.7 \mathrm{~Hz}, 1 \mathrm{H}, 2^{\prime}-\mathrm{H}\right), 8.47$ (d, J=8.6 Hz, $\left.1 \mathrm{H}, 8^{\prime}-\mathrm{H}\right)$ ppm.

${ }^{13} \mathrm{C}-\mathrm{NMR}\left(125 \mathrm{MHz}, \mathrm{DMSO}-\mathrm{d}_{6}\right): \delta=23.9(\mathrm{C}-4), 25.3(\mathrm{C}-3), 28.4(\mathrm{C}-2), 32.8\left(\mathrm{NCH}_{3}\right), 33.0$ (C-5), $51.1\left(\mathrm{CO}_{2} \mathrm{CH}_{3}\right), 53.7$ (C-1), 98.7 (C-1"), 109.1 (C-3"'), 109.5 (C-3'), 112.3 (C-4'"'), 
117.7 (C-5'), 122.3 (C-7'"'), 124.0 (C-4a', C-7a'"'), 124.4 (C-6'"'), 125.0 (C-8'), 126.5 (C-5'"', C-7'), 133.1 (C-6'), 137.6 (C-3a"), 141.8 (C-2'), 142.3 (C-8a"), 150.1 (C-2", C-2'"'), $161.4\left(\mathrm{C}-4{ }^{\prime}\right), 172.9\left(\mathrm{CO}_{2} \mathrm{CH}_{3}\right)$ ppm.

$\mathrm{C}_{27} \mathrm{H}_{29} \mathrm{ClN}_{2} \mathrm{O}_{2} \mathrm{~S}(481.04)$

ber.: $445.1944\left[{\left.\mathrm{M}-\mathrm{Cl}^{-}\right]^{+}}^{+}\right.$

gef.: 445.1941 (ESI-HRMS).

\section{3 (7-Dimethylamino-2-oxo-2H-chromn-4-yl)-essigsäure (62)}

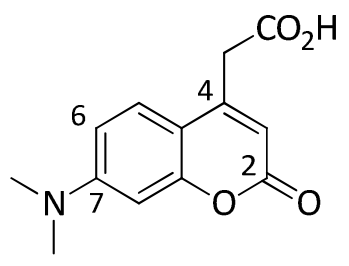

m-Dimethylaminophenol (132) (5.00 g, 36.4 mmol, 1.0 Äq.), Carboxylate 133 (8.11 g, $40.0 \mathrm{mmol}, 1.1 \mathrm{Äq}$.) und $\mathrm{ZnCl}_{2}(5.96 \mathrm{~g}, 43.7 \mathrm{mmol}, 1.2$ Äq.) wurden in absolutem EtOH $(20 \mathrm{~mL})$ gelöst und für $18 \mathrm{~h}$ unter Rückfluss gerührt. Es wurde auf Raumtemperatur abgekühlt und der entstandene Feststoff abfiltriert. Das Filtrat wurde auf Eiswasser $(500 \mathrm{~mL})$ gegeben und erneut abfiltiert. Das so erhaltene grüne Rohprodukt wurde in einem 3:1 Gemisch aus THF / $\mathrm{H}_{2} \mathrm{O}(40 \mathrm{~mL})$ gelöst, auf $0{ }^{\circ} \mathrm{C}$ gekühlt und mit einer $2 \mathrm{M}$ LiOH-Lösung (36.5 mL, $72.9 \mathrm{mmol}, 2.0$ Äq.) versetzt. Es wurde $2.5 \mathrm{~h}$ bei Raumtemperatur gerührt, mit $\mathrm{H}_{2} \mathrm{O}(40 \mathrm{~mL})$ versetzt und mit $\mathrm{Et}_{2} \mathrm{O}(3 \times 30 \mathrm{~mL})$ extrahiert. Die wässrige Phase wurde mit $2 \mathrm{~N} \mathrm{HCl}$ auf $\mathrm{pH} \approx 1$ eingestellt und der entstandene Feststoff abfiltriert. Nach säulenchromatographischer Reinigung an Kieselgel $\left(\mathrm{CH}_{2} \mathrm{Cl}_{2} / \mathrm{MeOH}=19: 1,1 \% \mathrm{CH}_{3} \mathrm{CO}_{2} \mathrm{H}\right)$ wurde die Zielverbindung 62 als grüner Feststoff (1.08 g, $4.35 \mathrm{mmol}, 12 \%)$ erhalten.

UV (MeOH): $\lambda_{\max }(\lg \varepsilon)=209.0 \mathrm{~nm}$ (4.550), 244.0 (4.261), 372.0 (4.305).

${ }^{1} \mathrm{H}-\mathrm{NMR}\left(300 \mathrm{MHz}, \mathrm{DMSO}-\mathrm{d}_{6}\right): \delta=3.00\left(\mathrm{~s}, 6 \mathrm{H}, \mathrm{NMe}_{2}\right), 3.76\left(\mathrm{~s}, 2 \mathrm{H}, \mathrm{CH}_{2}\right), 6.03(\mathrm{~s}, 1 \mathrm{H}$, 3-H), $6.53(\mathrm{~d}, J=2.5 \mathrm{~Hz}, 1 \mathrm{H}, 8-\mathrm{H}), 6.71(\mathrm{dd}, J=9.0,2.5 \mathrm{~Hz}, 1 \mathrm{H}, 6-\mathrm{H}), 7.46$ (d, $J=9.0 \mathrm{~Hz}, 1 \mathrm{H}, 5-\mathrm{H}), 12.5\left(\mathrm{~s}_{\mathrm{br}}, 1 \mathrm{H}, \mathrm{CO}_{2} \mathrm{H}\right) \mathrm{ppm}$. 
${ }^{13}$ C-NMR (125 MHz, DMSO-d $\left.{ }_{6}\right): \delta=37.4\left(\mathrm{CH}_{2} \mathrm{CO}_{2} \mathrm{H}\right), 39.5\left(\mathrm{NMe}_{2}\right), 97.3$ (C-8), 108.8 (C-6), 109.3 (C-3), 109.5 (C-4a), 125.9 (C-5), 150.2 (C-4), 152.7 (C-8a), 155.1 (C-7), $160.6(\mathrm{C}=\mathrm{O}), 170.7\left(\mathrm{CO}_{2} \mathrm{H}\right) \mathrm{ppm}$.

$\mathrm{C}_{13} \mathrm{H}_{13} \mathrm{NO}_{4}(247.24)$

ber.: $248.0917[\mathrm{M}+\mathrm{H}]^{+}$

gef.: 248.0923 (ESI-HRMS).

\subsection{7-0xo-3,7-dihydro-pyrano[3,2-e]indol-2-carbonsäure (130)}

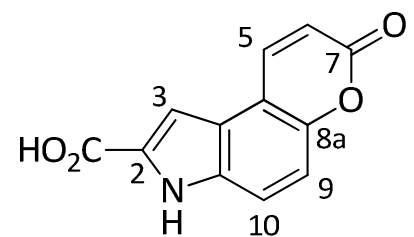

Eine Lösung aus Äpfelsäure (136) (0.89 g, 6.67 mmol, 1.0 Äq.) und 5-Hydroxy-indol-2carbonsäure (135) (1.18 g, $6.67 \mathrm{mmol}, 1.0$ Äq.) in $\mathrm{H}_{2} \mathrm{SO}_{4}(2.0 \mathrm{~mL})$ wurde für $2 \mathrm{~min}$ bei $130{ }^{\circ} \mathrm{C}$ in einer Mikrowellenapparatur gerührt. Es wurde auf Raumtemperatur abgekühlt, auf Eis $(20 \mathrm{~mL})$ gegeben und der entstandene Feststoff abfiltriert. Nach säulenchromatographischer Reinigung an Kieselgel $\left(\mathrm{CH}_{2} \mathrm{Cl}_{2} / \mathrm{MeOH}=5: 1\right)$ wurde die Zielverbindung 130 als brauner Feststoff ( $281 \mathrm{mg}, 1.23$ mmol, 18\%) erhalten.

UV (MeOH): $\lambda_{\max }(\lg \varepsilon)=225.0 \mathrm{~nm}$ (4.391), 259.0 (4.108), 280.0 (4.107), 352.0 (3.871). ${ }^{1} \mathrm{H}-\mathrm{NMR}\left(600 \mathrm{MHz}, \mathrm{DMSO}-\mathrm{d}_{6}\right): \delta=6.45(\mathrm{~d}, J=9.7 \mathrm{~Hz}, 1 \mathrm{H}, 6-\mathrm{H}), 7.18(\mathrm{~d}, J=8.5 \mathrm{~Hz}, 1 \mathrm{H}$, 9-H), $7.30(\mathrm{~s}, 1 \mathrm{H}, 3-\mathrm{H}), 7.69(\mathrm{~d}, J=9.0 \mathrm{~Hz}, 1 \mathrm{H}, 10-\mathrm{H}), 8.47$ (d, J=9.7 Hz, $1 \mathrm{H}, 5-\mathrm{H})$, $11.8\left(s_{b r}, 1 \mathrm{H}, \mathrm{NH}\right) \mathrm{ppm}$.

${ }^{13} \mathrm{C}-\mathrm{NMR}\left(125 \mathrm{MHz}, \mathrm{DMSO}-\mathrm{d}_{6}\right.$ ): $\delta=102.2$ (C-3), 110.7 (C-4), 111.6 (C-9), 114.2 (C-6), 116.7 (C-10), 123.7 (C-3a), 132.3 (C-10a), 136.9 (C-2), 141.9 (C-5), 149.3 (C-8a), 160.7 $(\mathrm{C}=\mathrm{O}), 164.2\left(\mathrm{CO}_{2} \mathrm{H}\right) \mathrm{ppm}$.

$\mathrm{C}_{12} \mathrm{H}_{7} \mathrm{NO}_{4}(229.19)$

ber.: $228.0302[\mathrm{M}-\mathrm{H}]^{-}$

gef.: 228.0309 (ESI-HRMS). 


\section{Synthese des seco-CCBI-Grundgerüstes (1S)-115}

\subsection{2-Amino-4-benzyloxy-1-iod-N-(tert-butyloxycarbonyl)-7-cyano- naphthalin (75)}<smiles>CC(=O)OCc1cc(OCc2ccccc2)c2ccc(C#N)cc2c1I</smiles>

Zu einer Lösung von Naphthalinderivat 112 (7.78 g, 20.8 mmol, 1.0 Äq.) in einem 1:1 Gemisch aus absolutem THF (125 mL) und absolutem MeOH $(125 \mathrm{~mL})$ gab man bei Raumtemperatur eine Lösung von $\mathrm{TsOH} \cdot \mathrm{H}_{2} \mathrm{O}$ (395 mg, $20.8 \mathrm{mmol}, 1.0$ Äq.) in absolutem THF (15 mL). Anschließend wurde $N$-lod-Succinimid $(9.35 \mathrm{~g}, 41.6 \mathrm{mmol}$, 2.0 Äq.) zugegeben und das Reaktionsgemisch für $1.5 \mathrm{~h}$ bei $50^{\circ} \mathrm{C}$ gerührt. Es wurde auf Raumtemperatur abgekühlt, gesättigte $\mathrm{NaHCO}_{3}$-Lösung. (150 mL) und $\mathrm{H}_{2} \mathrm{O}$ $(150 \mathrm{~mL})$ zugegeben und mit EtOAc $(300 \mathrm{~mL})$ extrahiert. Die vereinigten organischen Phasen wurden mit $1 \mathrm{M} \mathrm{Na}_{2} \mathrm{SO}_{3}$-Lösung $(300 \mathrm{~mL}$ ) gewaschen und die wässrige Phase mit EtOAc $(2 \times 200 \mathrm{~mL})$ extrahiert. Die vereinigte organische Phase wurde mit gesättigter $\mathrm{NaCl}$-Lösung $(800 \mathrm{~mL})$ gewaschen und über $\mathrm{MgSO}_{4}$ getrocknet. Das Lösungsmittel wurde im Vakuum entfernt und die Zielverbindung 75 als Rohprodukt $(10.0 \mathrm{~g})$ erhalten, das ohne weitere Aufreinigung später eingesetzt wurde.

$\mathbf{R}_{\mathbf{f}}=0.46(n-$ Pentan $/$ EtOAc $=10: 1)$.

UV $\left(\mathrm{CH}_{3} \mathrm{CN}\right): \lambda_{\max }(\lg \varepsilon)=227.0 \mathrm{~nm}$ (4.401), 265.0 (4.616), 309.0 (3.652), 320.0 (3.666), 359.5 (3.390).

IR (KBr-Pressling): $\tilde{v}\left(\mathrm{~cm}^{-1}\right)=3373,2977,2228,2170,1733,1600,1489,1395,1358$, 1332, 1155.

${ }^{1} \mathrm{H}-\mathrm{NMR}\left(300 \mathrm{MHz}, \mathrm{CDCl}_{3}\right): \delta=1.59\left(\mathrm{~s}, 9 \mathrm{H}, \mathrm{C}\left(\mathrm{CH}_{3}\right)_{3}\right), 5.27\left(\mathrm{~s}, 2 \mathrm{H}, \mathrm{OCH}_{2} \mathrm{Ph}\right), 7.22-7.55$ (m, $7 \mathrm{H}, 5 \times \mathrm{Ph}-\mathrm{H}, \mathrm{NH}, 6-\mathrm{H}), 8.21\left(\mathrm{~s}_{\mathrm{br}}, 1 \mathrm{H}, 3-\mathrm{H}\right), 8.27(\mathrm{dd}, \mathrm{J}=0.5,8.6 \mathrm{~Hz}, 1 \mathrm{H}, 5-\mathrm{H}), 8.44$ (dd, $J=0.5,1.5 \mathrm{~Hz}, 1 \mathrm{H}, 8-\mathrm{H}$ ) ppm. 
${ }^{13} \mathrm{C}-\mathrm{NMR}\left(125 \mathrm{MHz}, \mathrm{CDCl}_{3}\right): \delta=28.28\left(\mathrm{C}\left(\mathrm{CH}_{3}\right)_{3}\right), 70.72\left(\mathrm{OCh}_{2} \mathrm{Ph}\right), 78.81(\mathrm{C}-1), 81.80$ $\left(\underline{\mathrm{C}}\left(\mathrm{CH}_{3}\right)_{3}\right), 102.2(\mathrm{C}-3), 112.2$ (C-7), $119.0(\mathrm{CN}), 124.2$ (C-5), 125.0, 125.1, 127.9 (2 Signale), 128.4, 128.7 (2 Signale), 134.2, 140.2 (C-4a, C-8a, C-6, C-2, $2 \times$ Ph-Co,

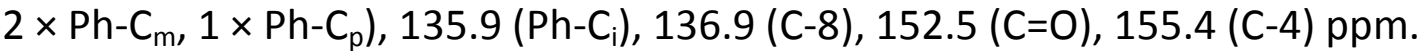

MS (ESI): $m / z(\%)=523.1(15)[\mathrm{M}+\mathrm{Na}]^{+}$.

$\mathrm{C}_{23} \mathrm{H}_{21} \mathrm{~N}_{2} \mathrm{O}_{3} \mathrm{I}(500.33)$

ber.: $523.04891[\mathrm{M}+\mathrm{Na}]^{+}$

gef.: $\quad 523.04891$ (ESI-HRMS).

\section{2 (E/Z)-2-Amino-4-benzyloxy-N-(tert-butyloxycarbonyl)-N-(3-chlor- prop-2-enyl)-7-cyano-1-iod-naphthalin (104)}<smiles>Cc1c(N([C]C=CCl)C(=O)OC(C)(C)C)cc(O)c2ccc(C#N)cc12</smiles>

Zu einer Lösung des geschützten Amins 75 (2.00 g, 4.00 mmol, 1.0 Äq.) in absolutem DMF (50 mL) wurde bei Raumtemperatur NaH (399 mg einer 60\%-igen Suspension in Mineralöl, $10.0 \mathrm{mmol}, 2.5$ Äq.) gegeben und $1.5 \mathrm{~h}$ gerührt. Anschließend wurde (E/Z)1,3-Dichlorpropen (113, $986 \mathrm{mg}, 823 \mu \mathrm{L}, 8.00 \mathrm{mmol}, 2.0$ Äq.) zugetropft und $14 \mathrm{~h}$ bei Raumtemperatur gerührt. Es wurde mit gesättigter $\mathrm{NH}_{4} \mathrm{Cl}$-Lösung auf $\mathrm{pH} \approx 7$ eingestellt und mit EtOAc $(3 \times 100 \mathrm{~mL})$ extrahiert. Die vereinigten organischen Phasen wurden mit $\mathrm{H}_{2} \mathrm{O}(3 \times 100 \mathrm{~mL})$ und gesättigter $\mathrm{NaCl}$-Lösung $(3 \times 100 \mathrm{~mL})$ gewaschen, über $\mathrm{MgSO}_{4}$ getrocknet und das Lösungsmittel im Vakuum entfernt. Nach säulenchromatographischer Reinigung an Kieselgel ( $n$-Pentan / EtOAc $=8: 1)$ erhielt man die Zielverbindung 104 als gelben Feststoff ( $1.76 \mathrm{~g}, 3.06 \mathrm{mmol}, 77 \%)$.

$\mathbf{R}_{\mathbf{f}}=0.27(n-$ Pentan $/$ EtOAc $=10: 1)$.

UV $\left(\mathrm{CH}_{3} \mathrm{CN}\right): \lambda_{\max }(\lg \varepsilon)=221.0 \mathrm{~nm}(4.645), 256.0$ (4.509), 320.5 (3.828), 354.4 (3.638). 
IR (KBr-Pressling): $\tilde{v}\left(\mathrm{~cm}^{-1}\right)=2976,2228,1703,1593,1503,1410,1368,1329,1257$, 1163.

${ }^{1} \mathrm{H}-\mathrm{NMR}\left(300 \mathrm{MHz}, \mathrm{CDCl}_{3}\right): \delta=1.22-1.35,1.49-1.60\left(2 \times \mathrm{m}\right.$, zus. $\left.9 \mathrm{H}, \mathrm{C}\left(\mathrm{CH}_{3}\right)_{3}\right), 3.69-$ 3.83, 4.17-4.33 $\left(2 \times \mathrm{m}\right.$, zus. $\left.1 \mathrm{H}, 1^{\prime}-\mathrm{H}_{\mathrm{a}}\right), 4.42-4.61\left(\mathrm{~m}, 1 \mathrm{H}, 1^{\prime}-\mathrm{H}_{\mathrm{b}}\right), 5.10-5.34(\mathrm{~m}, 2 \mathrm{H}$, OC$\left.{ }_{2} \mathrm{Ph}\right), 5.90-6.12\left(\mathrm{~m}, 1 \mathrm{H}, 2^{\prime}-\mathrm{H}\right), 6.74-6.92(\mathrm{~m}, 1 \mathrm{H}, 3-\mathrm{H}), 7.35-7.69(\mathrm{~m}, 5 \mathrm{H}$, $5 \times \mathrm{Ph}-\mathrm{H}), 7.58-7.69(\mathrm{~m}, 1 \mathrm{H}, 6-\mathrm{H}), 8.32-8.44(\mathrm{~m}, 1 \mathrm{H}, 5-\mathrm{H}), 8.57-8.64(\mathrm{~m}, 1 \mathrm{H}, 8-\mathrm{H})$ ppm.

${ }^{13} \mathrm{C}-\mathrm{NMR}\left(150 \mathrm{MHz}, \mathrm{CDCl}_{3}\right): \delta=28.12,28.14\left(\mathrm{C}\left(\mathrm{CH}_{3}\right)_{3}\right), 46.11,49.19\left(\mathrm{C}-1^{\prime}\right), 71.01$, $71.10\left(\mathrm{OCL}_{2} \mathrm{Ph}\right), 81.01,81.07\left(\underline{\mathrm{C}}\left(\mathrm{CH}_{3}\right)_{3}\right), 94.57,94.66$ (C-1), 110.41, 110.82 (C-3), $112.19,112.26$ (C-7), 118.31, 118.35 (CN), 120.57, 121.72 (C-3'), 124.03, 124.07 (C-5), 126.7, 126.8, 127.0, 127.1, 127.2, 128.2, 128.5, 128.6, 129.5, 134.8, 134.9, 135.6, 135.7, 144.8, 145.1 (C-4a, C-8a, C-2, C-2', $6 \times$ Ph-C), 138.3, 138.4 (C-8), 152.9, 153.1, $155.2(\mathrm{C}-4, \mathrm{C}=\mathrm{O}) \mathrm{ppm}$.

MS (ESI): $m / z(\%)=597.0(22)[\mathrm{M}+\mathrm{Na}]^{+}, 520.0(100)\left[\mathrm{M}-\mathrm{C}_{3} \mathrm{H}_{4} \mathrm{Cl}+\mathrm{Na}\right]^{+}$.

$\mathrm{C}_{26} \mathrm{H}_{24} \mathrm{~N}_{2} \mathrm{O}_{3} \mathrm{ICl}(574.84)$

ber.: $597.04187[\mathrm{M}+\mathrm{Na}]^{+}$

gef.: 597.04123 (ESI-HRMS).

\section{3 rac-\{(1S)-5-Benzyloxy-3-(tert-butyloxycarbonyl)-1-chlormethyl-8- cyano-1,2-dihydro-3H-benz[e]indol (rac-(1S)-74)}<smiles>CC(C)(C)OC(=O)n1cc(CCl)c2c3cc(C#N)ccc3c(OCc3ccccc3)cc21</smiles>

Der lodaromat 104 (1.42 g, $2.47 \mathrm{mmol}, 1.00 \mathrm{Äq})$ wurde in absolutem Toluol (60 mL) gelöst und die Lösung durch Einleiten eines Argonstroms 10 min entgast. Anschließend versetzte man mit Tris(trimethylsilyl)silan (836 $\mu \mathrm{L}, 2.72 \mathrm{mmol}, 1.10$ Äq.) sowie AIBN (102 mg, $618 \mu \mathrm{mol}, 0.25$ Äq.), erwärmte das Reaktionsgemisch mit Hilfe 
eines vorgeheizten Ölbades auf $80{ }^{\circ} \mathrm{C}$ und rührte für $4.5 \mathrm{~h}$. Man gab $\mathrm{SiO}_{2}(3 \mathrm{~g})$ hinzu und entfernte das Lösungsmittel im Vakuum. Nach säulenchromatographischer Reinigung an Kieselgel (Hexan / DCM = 9:1) erhielt man die Zielverbindung rac-(1S)-74 als weißen Feststoff (940 mg, $2.10 \mathrm{mmol}, 85 \%)$.

$\mathbf{R}_{\mathbf{f}}=0.30(n-$ Pentan $/$ EtOAc $=10: 1)$.

UV $\left(\mathrm{CH}_{3} \mathrm{CN}\right): \lambda_{\max }(\lg \varepsilon)=210.0 \mathrm{~nm}$ (4.391), 230.0 (4.469), 268.0 (4.786), 311.5 (3.906), 322.5 (3.977), 381.0 (3.475).

IR (KBr-Pressling): $\tilde{v}\left(\mathrm{~cm}^{-1}\right)=2977,2230,1693,1593,1455,1409,1374,1336,1257$, 1146.

${ }^{1} \mathrm{H}-\mathrm{NMR}\left(300 \mathrm{MHz}, \mathrm{C}_{2} \mathrm{D}_{2} \mathrm{Cl}_{4}\right): \delta=1.64\left(\mathrm{~s}, 9 \mathrm{H}, \mathrm{C}\left(\mathrm{CH}_{3}\right)_{3}\right), 3.59(\mathrm{dd}, J=9.1,11.2 \mathrm{~Hz}, 1 \mathrm{H}$, 2- $\mathrm{H}_{\mathrm{a}}$ ), 3.89 (dd, J = 3.51, $\left.11.2 \mathrm{~Hz}, 1 \mathrm{H}, 2-\mathrm{H}_{\mathrm{b}}\right), 4.00(\mathrm{tt}, J=3.2,8.8 \mathrm{~Hz}, 1 \mathrm{H}, 1-\mathrm{H}), 4.21$ (dd, $\left.J=8.8,11.7 \mathrm{~Hz}, 1 \mathrm{H}, 10-\mathrm{H}_{\mathrm{a}}\right), 4.30\left(\mathrm{dd}, J=3.2,11.8 \mathrm{~Hz}, 1 \mathrm{H}, 10-\mathrm{H}_{\mathrm{b}}\right), 5.32(\mathrm{~s}, 2 \mathrm{H}$, $\left.\mathrm{OC} \underline{\mathrm{H}}_{2} \mathrm{Ph}\right), 7.36-7.57$ (m, $\left.6 \mathrm{H}, 7-\mathrm{H}, 5 \times \mathrm{Ph}-\mathrm{H}\right), 7.93(\mathrm{~s}, 1 \mathrm{H}, 4-\mathrm{H}), 8.02$ (dd, J = 0.7, $1.5 \mathrm{~Hz}$, $1 \mathrm{H}, 9-\mathrm{H}), 8.38(\mathrm{dd}, J=0.5,8.7 \mathrm{~Hz}, 1 \mathrm{H}, 6-\mathrm{H}) \mathrm{ppm}$.

${ }^{13} \mathrm{C}-\mathrm{NMR}\left(100 \mathrm{MHz}, \mathrm{C}_{2} \mathrm{D}_{2} \mathrm{Cl}_{4}\right): \delta=28.26\left(\mathrm{C}\left(\mathrm{CH}_{3}\right)_{3}\right), 41.01$ (C-1), 46.37 (C-2), 53.15 (C-10), $70.84\left(\mathrm{O}_{\underline{C}} \mathrm{H}_{2} \mathrm{Ph}\right), 81.57\left(\underline{\mathrm{C}}\left(\mathrm{CH}_{3}\right)_{3}\right), 99.71$ (C-4), 111.1 (C-8), 114.9 (C-9b), 118.9 (CN), 123.2 (C-7), 123.6 (C-5a), 124.9 (C-6), 127.4 (C-9), 127.4 (2× Ph-Co), 128.0 $\left(\mathrm{Ph}-\mathrm{C}_{\mathrm{p}}\right), 128.4\left(2 \times \mathrm{Ph}^{-C_{m}}\right), 129.4$ (C-9a), $136.1\left(\mathrm{Ph}-\mathrm{C}_{\mathrm{i}}\right), 143.4$ (C-3a), 151.9 (C=O), 155.9 (C-5) ppm.

MS (El, $70 \mathrm{eV}): m / z(\%)=448(5)[\mathrm{M}]^{+}, 391(22)\left[\mathrm{M}-\mathrm{C}_{4} \mathrm{H}_{8}\right]^{+}, 91.0(100)\left[\mathrm{C}_{7} \mathrm{H}_{7}\right]^{+}$.

$\mathrm{C}_{26} \mathrm{H}_{25} \mathrm{ClN}_{2} \mathrm{O}_{3}(448.94)$ ber.: $471.14459[\mathrm{M}+\mathrm{Na}]^{+}$ gef.: 471.14459 (ESI-HRMS). 


\subsection{Trennung der Enantiomere von rac-\{(1S)-5-Benzyloxy-3-(tert- butyloxy-carbonyl)-1-chlormethyl-8-cyano-1,2-dihydro-3H- benz[e]indol (rac-(1S)-74)}

rac-(1S)-74 (500 mg, $1.10 \mathrm{mmol}$ ) wurde in einem Gemisch aus $n$-Hexan $/ \mathrm{CH}_{2} \mathrm{Cl}_{2}=1: 1$ $(10 \mathrm{~mL}$ ) gelöst. Je $0.15 \mathrm{~mL}$ dieser Probenlösung (entsprechend $7.5 \mathrm{mg}$ bzw. $167 \mu \mathrm{mol}$ des racemischen Gemisches) wurden in das präparative HPLC-System (Säule: Chiralpak IA, $250 \times 20 \mathrm{~mm}$, Partikelgröße: $5 \mu \mathrm{m}$, mobile Phase: $n$-Hexan / $\mathrm{CH}_{2} \mathrm{Cl}_{2}=85: 15$, Fluss: $18 \mathrm{~mL} / \min$, Druck: $5.9 \mathrm{MPa}$ ) injiziert. Fraktioniertes Auffangen des Eluats (UV-Detektor: $269 \mathrm{~nm}$ ) lieferte die Enantiomere (+)-(1S)-74 und $(-)-(1 S)-74$

Analytische Daten für (+)-(1S)-74:

$[\alpha]_{D}^{20}=+9.5^{\circ}\left(\mathrm{c}=0.5, \mathrm{CHCl}_{3}\right)$.

HPLC (präparativ): $t_{R}$ :

$12.54 \mathrm{~min}$

Fraktion: $\quad$ 12.2-15.9 $\mathrm{min}$

HPLC (analytisch): Säule: Chiralpak OD

Eluens: $\quad n$-Hexan / 2-Propanol $=93: 7$

Fluss: $\quad 0.8 \mathrm{~mL} / \mathrm{min}$

$t_{R}: \quad 8.35 \min , 99.7 \%$ ee.

Analytische Daten für (-)-(1S)-74:

$[\alpha]_{D}^{20}=-11.0^{\circ}\left(\mathrm{c}=0.5, \mathrm{CHCl}_{3}\right)$.

HPLC (präparativ): $t_{R}$ : 10.87 min

Fraktion: $\quad$ 10.4-13.8 $\mathrm{min}$

HPLC (analytisch): Säule: Chiralpak OD

Eluens: $\quad n$-Hexan / 2-Propanol $=93: 7$

Fluss: $\quad 0.8 \mathrm{~mL} / \mathrm{min}$

$t_{R}: \quad 6.42 \min , 99.2 \%$ ee. 


\section{5 (-)-\{(1S)-5-Benzyloxy-3-(tert-butyloxycarbonyl)-1-chlormethyl-8-} cyano-1,2-dihydro-3H-benz[e]indol ((-)-(1S)-115)<smiles></smiles>

Der Benzylether (-)-(1S)-74(250 mg, $557 \mu \mathrm{mol}, 1.0$ Äq.) wurde in absolutem EtOAc (17 mL) gelöst und die Lösung auf $40{ }^{\circ} \mathrm{C}$ erwärmt. Anschließend wurde Palladium auf Aktivkohle (10\%-ig, $140 \mathrm{mg}, 129 \mu \mathrm{mol}, 0.23$ Äq. bezogen auf Pd) zugegeben, die Reaktionslösung kurz entgast, $\mathrm{H}_{2}$ durch die Lösung geleitet und unter $\mathrm{H}_{2}$-Atmosphäre für $5 \mathrm{~h}$ bei Raumtemperatur gerührt. Anschließend wurde über eine Celite-Schicht abfiltriert und mit Aceton $(800 \mathrm{~mL})$ gespült. Das Lösungsmittel wurde im Vakuum entfernt und nach säulenchromatographischer Reinigung an Kieselgel ( $n$-Pentan/EtOAc 5:1) erhielt man die Zielverbindung (-)-(1S)-115 als weißen Feststoff (179 mg, $499 \mu \mathrm{mol}, 90 \%)$.

$\mathbf{R}_{\mathbf{f}}=0.50(n-$ Pentan $/$ EtOAc $=2: 1)$

UV $\left(\mathrm{CH}_{3} \mathrm{CN}\right): \lambda_{\max }(\lg \varepsilon)=210.5 \mathrm{~nm}$ (4.221), 229.5 (4.369), 269.0 (4.787), 312.5 (3.826), 323.5 (3.861), 381.0 (3.428).

IR (KBr-Pressling): $\tilde{v}\left(\mathrm{~cm}^{-1}\right)=3350,2230,1676,1626,1595,1513,1422,1369,1223$, 1144.

${ }^{1} \mathrm{H}-\mathrm{NMR}\left(300 \mathrm{MHz}, \mathrm{DMSO}-\mathrm{d}_{6}\right): \delta=1.54\left(\mathrm{~s}, 9 \mathrm{H}, \mathrm{C}\left(\mathrm{CH}_{3}\right)_{3}\right), 3.77-4.31\left(\mathrm{~m}, 5 \mathrm{H}, 1-\mathrm{H}, 2-\mathrm{H}_{2}\right.$, $\left.10-\mathrm{H}_{2}\right), 7.50(\mathrm{dd}, J=1.5,8.7 \mathrm{~Hz}, 1 \mathrm{H}, 7-\mathrm{H}), 7.72\left(\mathrm{~s}_{\mathrm{br}}, 1 \mathrm{H}, 4-\mathrm{H}\right), 8.18(\mathrm{~d}, J=8.7 \mathrm{~Hz}, 1 \mathrm{H}$, 6-H), 8.41 (dd, J = 0.5, $1.5 \mathrm{~Hz}, 1 \mathrm{H}, 9-\mathrm{H}), 10.88\left(\mathrm{~s}_{\mathrm{br}}, 1 \mathrm{H}, \mathrm{OH}\right) \mathrm{ppm}$.

${ }^{13} \mathrm{C}-\mathrm{NMR}\left(75 \mathrm{MHz}\right.$, DMSO-d $\left.\mathrm{d}_{6}\right): \delta=27.98\left(\mathrm{C}\left(\mathrm{CH}_{3}\right)_{3}\right), 47.86$ (C-1), 52.50 (C-2), 61.92 (C-10), $80.8\left(\underline{\mathrm{C}}\left(\mathrm{CH}_{3}\right)_{3}\right), 101.0$ (C-4), 109.7 (C-8), 114.1 (C-9b), 119.2 (CN), 122.0 (C-5a), 122.5 (C-7), 124.5 (C-6), 128.8 (C-9), 129.2 (C-9a), 143.2 (C-3a), 151.5 (C=O), 154.6 (C-5) ppm. 
MS $(E l, 70 \mathrm{eV}): m / z(\%)=358(5)[\mathrm{M}]^{+}, 57(100)\left[\mathrm{C}_{4} \mathrm{H}_{9}\right]^{+}$.

$\mathrm{C}_{19} \mathrm{H}_{19} \mathrm{~N}_{2} \mathrm{O}_{3} \mathrm{Cl}$ (358.82)

ber.: $381.09764[\mathrm{M}+\mathrm{Na}]^{+}$

gef.: 381.09764 (ESI-HRMS). 


\section{Synthese des fluoreszenzmarkierten Prodrugs (1S)-72}

\section{$4.1(+)-\left\{\left[(1 S)-1-c h l o r m e t h y l-8-c y a n o-3-\left[\left(5-\left(2-\left(N, N^{\prime}\right.\right.\right.\right.\right.\right.$-dimethyl-amino)- ethoxy)-indol-2-yl)carbonyl]-1,2-dihydro-3H-benz[e]indol-5-yl]- 2,3,4,6-tetra-O-acetyl- $\beta$-D-Galaktopyranosid\} ((+)-(1S)-73)}

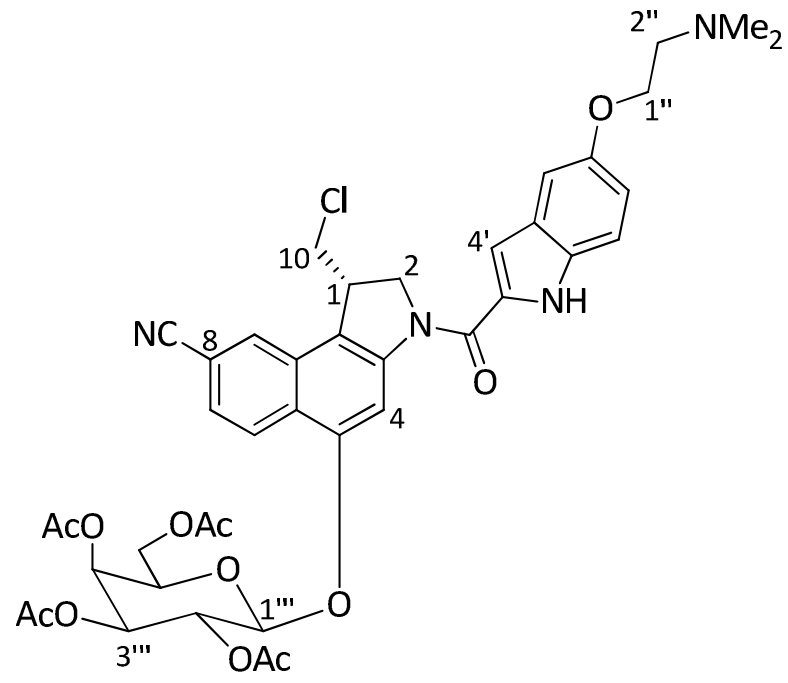

Zu einer Lösung des Phenols (-)-115 (283 mg, $663 \mu \mathrm{mol}, 1.0$ Äq.) in absolutem $\mathrm{CH}_{2} \mathrm{Cl}_{2}$ $(20 \mathrm{~mL})$ gab man aktiviertes Molsieb $4 \AA$ ( $2.00 \mathrm{~g})$ und rührte $30 \mathrm{~min}$ bei Raumtemperatur. Nach Zugabe des $O-(2,3,4,6$-Tetra-O-acetyl- $\alpha$-D-Galaktopyranosyl)-trichloracetimidats (116) (427 mg, $870 \mu \mathrm{mol}, 1.3$ Äq.) kühlte man die Lösung auf $-10{ }^{\circ} \mathrm{C}$ und tropfte langsam eine Lösung von $\mathrm{BF}_{3} \cdot \mathrm{OEt}_{2}(50 \mu \mathrm{L}, 395 \mu \mathrm{mol}, 0.5 \mathrm{Äq}$.) in absolutem $\mathrm{CH}_{2} \mathrm{Cl}_{2}(4.0 \mathrm{~mL})$ zu. Nach $3.5 \mathrm{~h}$ Rühren bei $-10^{\circ} \mathrm{C}$ tropfte man weiteres $\mathrm{BF}_{3} \cdot \mathrm{OEt}_{2}\left(250 \mu \mathrm{L}, 1.97 \mathrm{mmol}, 3.0 \mathrm{Äq}\right.$.) in absolutem $\mathrm{CH}_{2} \mathrm{Cl}_{2}(4.0 \mathrm{~mL})$ hinzu und ließ auf Raumtemperatur erwärmen. Nach $6 \mathrm{~h}$ wurde das Reaktionsgemisch via Transferkanüle in einen zweiten Kolben überführt und so vom Molsieb abgetrennt. Man wusch dieses gründlich mit $\mathrm{CH}_{2} \mathrm{Cl}_{2}(30 \mathrm{~mL})$ und entfernte anschließend das Lösungsmittel im Vakuum. Der erhaltene Rückstand wurde $1.5 \mathrm{~h}$ im Vakuum getrocknet und in absolutem DMF (38 mL) aufgenommen. Man kühlte die Lösung auf $0^{\circ} \mathrm{C}$ und gab EDAC. $\mathrm{HCl}$ (450 mg, $1.35 \mathrm{mmol}, 3.0$ Äq.) sowie DMAI-CO ${ }_{2} \mathrm{H} \cdot \mathrm{HCl}$ (104) (336 mg, 1.35 mmol, 1.5 Äq.) hinzu. Nach $18 \mathrm{~h}$ Rühren bei Raumtemperatur wurde die Reaktionslösung mit EtOAc $(70 \mathrm{~mL}), \mathrm{H}_{2} \mathrm{O}(70 \mathrm{~mL})$ sowie gesättigter $\mathrm{NaHCO}_{3}$-Lösung $(70 \mathrm{~mL})$ versetzt und anschließend mit EtOAc $(4 \times 150 \mathrm{~mL})$ extrahiert. Die vereinigten 
organischen Phasen wurden mit gesättigter NaCl-Lösung $(4 \times 200 \mathrm{~mL})$ gewaschen, über $\mathrm{MgSO}_{4}$ getrocknet und das Lösungsmittel wurde im Vakuum entfernt. Nach säulenchromatographischer Reinigung an Kieselgel $\left(\mathrm{CH}_{2} \mathrm{Cl}_{2} / \mathrm{MeOH} 6: 1\right)$ erhielt man die Zielverbindung (+)-(1S)-73 als weißen Feststoff (340 mg, $416 \mu \mathrm{mol}, 53 \%$ ).

$\mathbf{R}_{\mathbf{f}}=0.44\left(\mathrm{CH}_{2} \mathrm{Cl}_{2} / \mathrm{MeOH}=6: 1\right)$.

$[\alpha]_{D}^{20}=+6.0^{\circ}\left(\mathrm{c}=0.3, \mathrm{CHCl}_{3}\right)$.

UV $\left(\mathrm{CH}_{3} \mathrm{CN}\right): \lambda_{\max }(\lg \varepsilon)=212.5$ (4.712), 261.5 (4.431), 302.0 (4.546), 399.0 (4.519).

IR (KBr): $\tilde{v}\left(\mathrm{~cm}^{-1}\right)=3406(\mathrm{NH}), 2942,2229(\mathrm{CN}), 1754$ (C=O), 1626, 1457, 1396, 1226.

$\left.{ }^{1} \mathrm{H}-\mathrm{NMR}\left(600 \mathrm{MHz}^{\mathrm{CDCl}}\right)_{3}\right): \delta=2.01\left(\mathrm{~s}, 6 \mathrm{H}, 2 \times \mathrm{C}(\mathrm{O}) \mathrm{CH}_{3}\right), 2.03\left(\mathrm{~s}, 3 \mathrm{H}, \mathrm{C}(\mathrm{O}) \mathrm{CH}_{3}\right), 2.16$ $\left(\mathrm{s}, 3 \mathrm{H}, \mathrm{C}(\mathrm{O}) \mathrm{CH}_{3}\right), 2.37\left(\mathrm{~s}, 6 \mathrm{H}, \mathrm{NMe}_{2}\right), 2.79\left(\mathrm{t}, J=5.7 \mathrm{~Hz}, 2 \mathrm{H}, 2^{\prime \prime}-\mathrm{H}_{2}\right), 3.55(\mathrm{dd}, J=11.5$, 10.0 Hz, $\left.1 \mathrm{H}, 10-\mathrm{H}_{\mathrm{a}}\right), 3.78-3.85(\mathrm{~m}, 1 \mathrm{H}, 1-\mathrm{H}), 3.90\left(\mathrm{dd}, J=11.5,3.2 \mathrm{~Hz}, 1 \mathrm{H}, 10-\mathrm{H}_{\mathrm{b}}\right.$ ), 4.05-4.23 (m, 5 H, 1"'- $\mathrm{H}_{2}, 5$ '"'-H, 6'"'- $\left.\mathrm{H}_{2}\right), 4.69-4.75\left(\mathrm{~m}, 1 \mathrm{H}, 2-\mathrm{H}_{\mathrm{a}}\right), 4.84(\mathrm{dd}, J=10.6$, $\left.2.0 \mathrm{~Hz}, 1 \mathrm{H}, 2-\mathrm{H}_{\mathrm{b}}\right), 4.99$ (dd, J = 10.5, 3.2 Hz, $\left.1 \mathrm{H}, 3^{\prime \prime \prime}-\mathrm{H}\right), 5.27$ (d, J = 7.9 Hz, $1 \mathrm{H}, 1$ '"'-H), $5.38(\mathrm{~d}, J=3.3 \mathrm{~Hz}, 1 \mathrm{H}, 4$ '"'-H), $5.63(\mathrm{dd}, J=10.5,7.9 \mathrm{~Hz}, 1 \mathrm{H}, 2$ '"'-H), 7.03-7.06 (m, $2 \mathrm{H}$, 3'-H, 6'-H), 7.14 (d, J = 2.2 Hz, 1 H, 4'-H), 7.37 (d, J = 8.9 Hz, 1 H, 7'-H), 7.53 (dd, J = 8.7, $1.5 \mathrm{~Hz}, 1 \mathrm{H}, 7-\mathrm{H}), 8.08(\mathrm{~d}, J=0.8 \mathrm{~Hz}, 1 \mathrm{H}, 9-\mathrm{H}), 8.21(\mathrm{~d}, J=8.7 \mathrm{~Hz}, 1 \mathrm{H}, 6-\mathrm{H}), 8.51(\mathrm{~s}$, $1 \mathrm{H}, 4-\mathrm{H}), 10.05$ (s, $1 \mathrm{H}, \mathrm{NH}) \mathrm{ppm}$.

${ }^{13} \mathrm{C}-\mathrm{NMR}\left(125 \mathrm{MHz}, \mathrm{CDCl}_{3}\right): \delta=20.63,20.69,20.72,20.84\left(4 \times \mathrm{C}(\mathrm{O}) \underline{\mathrm{CH}}_{3}\right), 45.84$ $\left(\mathrm{NMe}_{2}\right), 45.96$ (C-10), 54.99 (C-2), 58.35 (C-2"), 61.16 (C-6"'), 66.33 (C-1"), 66.78 (C-4'"'), 68.43 (C-2'"'), 70.51 (C-3'"'), 71.20 (C-1), 99.02 (C-1'"'), 103.52 (C-4'), 103.9 (C-4), 106.4 (C-6'*), 111.6 (C-5a), 112.7 (C-7'), 117.8 (C-3'*), 118.6 (C-8), 118.8, 124.4 (C-5a, C-9b), 124.8 (C-6), 125.0 (C-7), 128.0 (C-9), 128.2, 128.7, 130.0, 131.5 (CN, C-2', C-3a', C-7a'), 143.7 (C-3a), 153.3 (C-5), 153.9 (C-5'), 160.8 (C=0), 169.5, 169.7, 170.0, $170.3\left(4 \times \underline{\mathrm{C}}(\mathrm{O}) \mathrm{CH}_{3}\right) \mathrm{ppm}$.

MS (ESI): $m / z(\%)=833.3(100)[\mathrm{M}+\mathrm{H}]^{+}$.

$\mathrm{C}_{41} \mathrm{H}_{43} \mathrm{ClN}_{4} \mathrm{O}_{12}$ (819.25).

ber.: $819.2639[\mathrm{M}+\mathrm{H}]^{+}$

gef.: 819.2651 (ESI-HRMS). 
$4.2(+)-\left\{\left[(1 S)-8-A m i n o m e t h y l-1-c h l o r m e t h y l-3-\left[\left(5-\left(2-\left(N, N^{\prime}-\right.\right.\right.\right.\right.\right.$ dimethylamino)-ethoxy)-indol-2-yl)carbonyl]-1,2-dihydro-3Hbenz[e]indol-5-yl]-2,3,4,6-tetra-O-acetyl- $\beta$-D-Galaktopyranosid\} ((+)-(1S)-117)

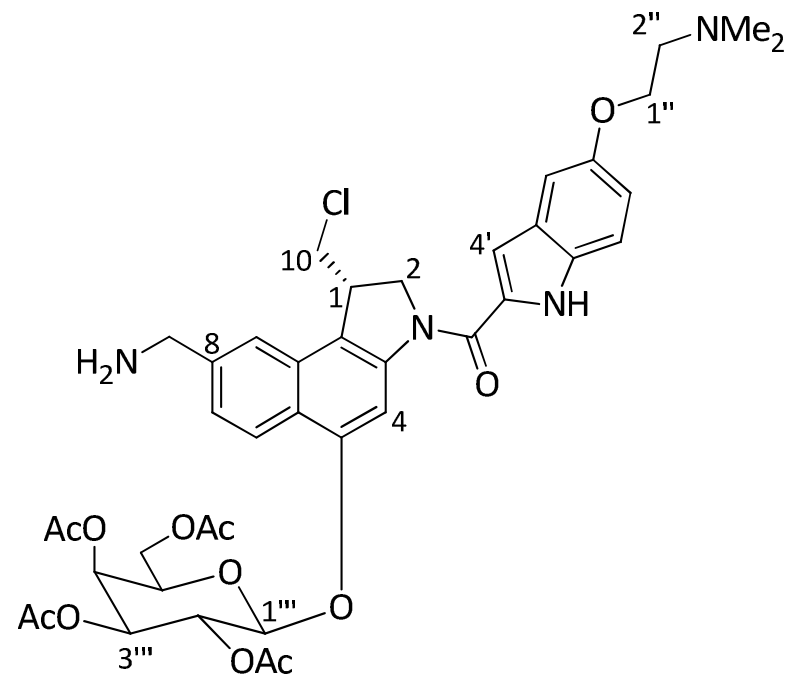

Das acetylgeschützte Galaktosid (+)-(1S)-73(50 mg, $61 \mu \mathrm{mol}, 1.0$ Äq.) wurde in absolutem Ethanol $(3.0 \mathrm{~mL})$ gelöst und die Lösung unter Rühren mittels eines Argongasstroms kurz entgast. Anschließend wurden eine $1.25 \mathrm{M}$ Lösung von $\mathrm{HCl}$ in Ethanol ${ }^{165}\left(98 \mu \mathrm{L}, 122 \mu \mathrm{mol}, 2.0\right.$ Äq.) sowie $\mathrm{H}_{2} \mathrm{O}(0.15 \mathrm{~mL})$ zugetropft und $\mathrm{PtO}_{2} \cdot \mathrm{H}_{2} \mathrm{O}$ (3.0 mg, $12.2 \mu \mathrm{mol}, 0.20$ Äq.) zugegeben. Man leitete kurz einen $\mathrm{H}_{2}$-Gasstrom durch die Reaktionsmischung und rührte dann für $48 \mathrm{~h}$ bei Raumtemperatur unter einer $\mathrm{H}_{2}$ Atmosphäre. Danach filtrierte man über eine Celite-Schicht vom Feststoff ab, spülte gründlich mit $\mathrm{MeOH}(200 \mathrm{~mL})$ nach und entfernte das Lösungsmittel im Vakuum. Nach Säulenchromatographie an Kieselgel $\left(\mathrm{CH}_{2} \mathrm{Cl}_{2} / \mathrm{MeOH}=5: 1\right)$ erhielt man die Zielverbindung (+)-(1S)-117 als weißen Feststoff (33 mg, $40 \mu \mathrm{mol}, 66 \%$ ).

$\mathbf{R}_{\mathrm{f}}=0.17\left(\mathrm{CH}_{2} \mathrm{Cl}_{2} / \mathrm{MeOH}=5: 1\right)$.

$[\alpha]_{\mathrm{D}}^{20}=+2.0^{\circ}(\mathrm{c}=0.3, \mathrm{DMSO})$.

UV $\left(\mathrm{CH}_{3} \mathrm{CN}\right): \lambda_{\max }(\lg \varepsilon)=206.0$ (4.461), 300.0 (4.312), $336.0 \mathrm{~nm}$ (4.249).

IR $(\mathrm{KBr}): \tilde{v}\left(\mathrm{~cm}^{-1}\right)=3421(\mathrm{NH}), 1749(\mathrm{C}=0), 1628,1519,1404$.

${ }^{1} \mathrm{H}-\mathrm{NMR}\left(600 \mathrm{MHz}, \mathrm{DMSO}-\mathrm{d}_{6}\right): \delta=1.98\left(\mathrm{~s}, 3 \mathrm{H}, \mathrm{C}(\mathrm{O}) \mathrm{CH}_{3}\right), 2.01(\mathrm{~s}, 3 \mathrm{H}, \mathrm{C}(\mathrm{O}) \mathrm{CH} 3), 2.04$ $\left(\mathrm{s}, 3 \mathrm{H}, \mathrm{C}(\mathrm{O}) \mathrm{CH}_{3}\right), 2.18\left(\mathrm{~s}, 3 \mathrm{H}, \mathrm{C}(\mathrm{O}) \mathrm{CH}_{3}\right), 2.24\left(\mathrm{~s}, 6 \mathrm{H}, \mathrm{NMe}_{2}\right), 2.65(\mathrm{t}, J=5.8 \mathrm{~Hz}, 2 \mathrm{H}$, 
2"- $\left.\mathrm{H}_{2}\right), 3.89-3.97\left(\mathrm{~m}, 3 \mathrm{H}, \mathrm{C}_{2} \mathrm{NH}_{2}, 10-\mathrm{H}_{\mathrm{a}}\right), 4.07\left(\mathrm{t}, J=5.9 \mathrm{~Hz}, 2 \mathrm{H}, 1^{\prime \prime}-\mathrm{H}_{2}\right), 4.10-4.18$ (m, $3 \mathrm{H}, 6$ 6"'- $\left.\mathrm{H}_{2}, 10-\mathrm{H}_{\mathrm{b}}\right), 4.22-4.28(\mathrm{~m}, 1 \mathrm{H}, 1-\mathrm{H}), 4.47-4.52\left(\mathrm{~m}, 1 \mathrm{H}, 5^{\prime \prime \prime}-\mathrm{H}\right), 4.57-4.62$ $\left(\mathrm{m}, 1 \mathrm{H}, 2-\mathrm{H}_{\mathrm{a}}\right), 4.81-4.86\left(\mathrm{~m}, 1 \mathrm{H}, 2-\mathrm{H}_{\mathrm{b}}\right), 5.38-5.46\left(\mathrm{~m}, 3 \mathrm{H}, 2^{\prime \prime \prime}-\mathrm{H}, 3\right.$ 3"'-H, 4"'-H), 5.54$5.59\left(\mathrm{~m}, 1 \mathrm{H}, 1^{\prime \prime \prime}-\mathrm{H}\right), 6.92$ (dd, J = 8.9, $2.3 \mathrm{~Hz}, 1 \mathrm{H}, 6$ 6'-H), 7.10 (s, $1 \mathrm{H}, 3$ 3'-H), 7.17 (d, $\left.J=2.1 \mathrm{~Hz}, 1 \mathrm{H}, 4^{\prime}-\mathrm{H}\right), 7.41\left(\mathrm{~d}, J=8.9 \mathrm{~Hz}, 1 \mathrm{H}, 7^{\prime}-\mathrm{H}\right), 7.44(\mathrm{~d}, J=8.7 \mathrm{~Hz}, 1 \mathrm{H}, 7-\mathrm{H}), 7.86$ (s, $1 \mathrm{H}, 9-\mathrm{H}), 7.90$ (d, J = 8.7 Hz, $1 \mathrm{H}, 6-\mathrm{H}), 8.18(\mathrm{~s}, 1 \mathrm{H}, 4-\mathrm{H}), 11.55$ (s, $1 \mathrm{H}, \mathrm{NH}) \mathrm{ppm}$.

${ }^{13}$ C-NMR (125 MHz): $\delta=20.29,20.34,20.50\left(4 \times \mathrm{C}(\mathrm{O}) \underline{\mathrm{CH}}_{3}\right), 40.09$ (C-1), 41.14 (C-10), $45.51\left(\mathrm{C}_{2} \mathrm{NH}_{2}\right), 47.42\left(\mathrm{NMe}_{2}\right), 55.01$ (C-2), 57.76 (C-2"), 60.94 (C-1"), 66.22 (C-6"'), 67.03, 68.49, 69.66, 70.29 (C-2'"', C-3'"', C-4'", C-5'"'), 98.90 (C-1'"'), 101.6 (C-4), 103.1 (C-4'), 105.2 (C-3'), 113.1 (C-7'), 115.7 (C-6'), 118.8, 121.2, 127.3, 129.5, 130.7, 131.5 (C-5a, C-9a, C-9b, C-8, C-2', C-3a', C-7a'), 121.6 (C-6), 124.5 (C-7), 141.8 (C-3a), 152.6, $152.8\left(\mathrm{C}-5, \mathrm{C}-5^{\prime}\right), 160.1$ (C=O), 169.2, 169.3, 169.7, $169.8\left(4 \times \underline{\mathrm{C}}(\mathrm{O}) \mathrm{CH}_{3}\right) \mathrm{ppm}$.

$\mathrm{C}_{41} \mathrm{H}_{47} \mathrm{ClN}_{4} \mathrm{O}_{12}$ (823.28).

ber.: $823.2952[\mathrm{M}+\mathrm{H}]^{+}$

gef.: 823.2941 (ESI-HRMS).

4.3 [(1S)-8-Aminomethyl-1-chlormethyl-3-[(5-(2-(N,N'-dimethylamino)ethoxy)-indol-2-yl)carbonyl]-1,2-dihydro-3H-benz[e]indol-5-yl]-O- $\beta$-DGalaktopyranosid-ditrifluoracetat \} ((1S)-118)

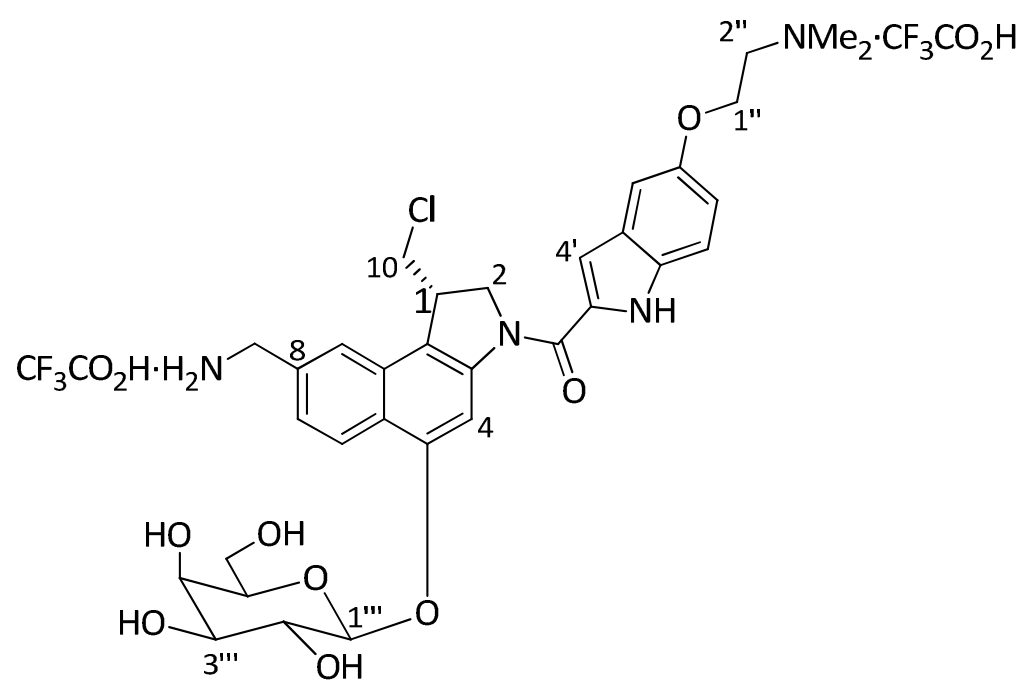

Das acetylgeschützte Galaktosid (+)-(1S)-117 (19 mg, $23 \mu \mathrm{mol}, 1.0$ Äq.) wurde in absolutem $\mathrm{MeOH}(3 \mathrm{~mL})$ gelöst und bei $0^{\circ} \mathrm{C}$ mit einer Lösung von $\mathrm{NaOMe}$ in $\mathrm{MeOH}$ 
(8.5 $\mu \mathrm{L}$ einer $5.4 \mathrm{M}$ Lösung, $46 \mu \mathrm{mol}, 2.0$ Äq.) versetzt. Man rührte $30 \mathrm{~min}$ bei Raumtemperatur, verdünnte die Reaktionslösung anschließend mit $\mathrm{MeOH}(4 \mathrm{~mL})$ sowie $\mathrm{H}_{2} \mathrm{O}$ (4 mL) und gab solange sauren lonentauscher (Amberlite-IR 120) hinzu, bis die Lösung neutral war $(\mathrm{pH} \approx 7)$. Es wurde vom lonentauscher abgetrennt und dieser gründlich mit $\mathrm{MeOH}(25 \mathrm{~mL})$ gewaschen. Das Lösungsmittel wurde im Vakuum entfernt und das Rohprodukt in einem Gemisch aus bidestilliertem $\mathrm{H}_{2} \mathrm{O} \quad(0.1 \%$ TFA) $/ \mathrm{CH}_{3} \mathrm{CN}=4: 1$ (4 mL) aufgenommen. Je $2 \mathrm{~mL}$ dieser Lösung wurden in das präparative HPLC-System (s. u.) injiziert. Fraktioniertes Auffangen des Eluats, Entfernung von $\mathrm{CH}_{3} \mathrm{CN}$ und TFA unter vermindertem Druck sowie Entfernung des restlichen Lösungsmittels mittels Gefriertrocknung lieferte das Galaktosid (1S)-118 als weißen Feststoff (12.7 mg, $19.4 \mu \mathrm{mol}, 84 \%)$.

HPLC (analytisch):

Säule: $\quad$ Kromasil 100 C18

\begin{tabular}{ccc}
\cline { 2 - 3 } Gradient: & Zeit $[\mathrm{min}]$ & $\mathrm{H}_{2} \mathrm{O}(0.1 \% \mathrm{TFA}) / \mathrm{MeOH}$ \\
\cline { 2 - 3 } & 0 & $90 / 10$ \\
& $15-15$ & $0 / 100$ \\
& $22-30$ & $0 / 100 \rightarrow 90 / 10$ \\
& $30-35$ & $90 / 10$ \\
$t_{\mathrm{R}}:$ & $0.8 \mathrm{~mL} \mathrm{~min}^{-1}$ & \\
Fluss: & $14.1 \mathrm{~min}^{22}$ &
\end{tabular}


HPLC (präparativ):

Säule: Kromasil $100 \mathrm{C} 18$

\begin{tabular}{ccc}
\cline { 2 - 3 } Gradient: & Zeit [min] & $\mathrm{H}_{2} \mathrm{O}(0.1 \% \mathrm{TFA}) / \mathrm{MeOH}$ \\
\cline { 2 - 3 } & 0 & $90 / 10$ \\
& $0-15$ & $90 / 10 \rightarrow 0 / 100$ \\
& $15-22$ & $0 / 100$ \\
& $22-30$ & $0 / 100 \rightarrow 90 / 10$ \\
& $30-35$ & $90 / 10$ \\
Fluss: & $12 \mathrm{~mL} \mathrm{~min}^{-1}$ & \\
$t_{\mathrm{R}}:$ & $6.89 \mathrm{~min}$ &
\end{tabular}

${ }^{1} \mathrm{H}-\mathrm{NMR}\left(600 \mathrm{MHz}, \mathrm{DMSO}-\mathrm{d}_{6}\right) \delta=2.90\left(\mathrm{~s}, 6 \mathrm{H}, \mathrm{NMe}_{2}\right), 3.44-4.21\left(\mathrm{~m}, 7 \mathrm{H}, 1^{2}-\mathrm{H}, 2\right.$ '"'-H, 3'"-H, 4'"-H, 5'"-H, 6'"-H), 4.20-4.31 (m, 3 H, $\left.\mathrm{CH}_{2} \mathrm{NH}_{2}, 1-\mathrm{H}\right), 4.31-4.40\left(\mathrm{~m}, 2 \mathrm{H}, 2^{\prime \prime}-\mathrm{H}_{2}\right)$, 4.57-4.66 (m, $\left.2 \mathrm{H}, 10-\mathrm{H}_{2}\right), 4.82-4.93\left(\mathrm{~m}, 3 \mathrm{H}, 2-\mathrm{H}_{2}, 1{ }^{\prime \prime}-\mathrm{H}\right), 7.01$ (dd, J= 8.9, $2.4 \mathrm{~Hz}$, $1 \mathrm{H}, 6^{\prime}-\mathrm{H}$ ), 7.14 (d, J=1.6 Hz, $\left.1 \mathrm{H}, 3^{\prime}-\mathrm{H}\right), 7.26\left(\mathrm{~d}, J=2.3 \mathrm{~Hz}, 1 \mathrm{H}, 4^{\prime}-\mathrm{H}\right), 7.45$ (d, $\left.J=8.9 \mathrm{~Hz}, 1 \mathrm{H}, 7^{\prime}-\mathrm{H}\right), 7.49(\mathrm{dd}, J=8.8,1.3 \mathrm{~Hz}, 1 \mathrm{H}, 7-\mathrm{H}), 8.01(\mathrm{~s}, 1 \mathrm{H}, 9-\mathrm{H}), 8.37$ (d, $J=8.7 \mathrm{~Hz}, 1 \mathrm{H}, 6-\mathrm{H}), 8.42(\mathrm{~s}, 1 \mathrm{H}, 4-\mathrm{H}), 11.74(\mathrm{~s}, 1 \mathrm{H}, \mathrm{NH}) \mathrm{ppm}$.

${ }^{13} \mathrm{C}-\mathrm{NMR}\left(125 \mathrm{MHz}\right.$, DMSO-d $\left.\mathrm{d}_{6}\right): \delta=41.29(\mathrm{C}-1), 42.30\left(\mathrm{CH}_{2} \mathrm{NH}_{2}\right), 42.80\left(\mathrm{NMe}_{2}\right), 47.27$ (C-10), 54.89 (C-2), 55.55 (C-1"), 59.45 (C-6'"'), 62.72 (C-2"), 67.40, 70.38, 73.02, 75.05 (C-2'", C-3"', C-4'"', C-5'"'), 102.1 (C-1'"'), 103.9 (C-4'), 105.2 (C-3'), 113.2 (C-7'), 115.6 (C-6'), 117.8 (C-4*), 122.3 (C-9b), 122.5 (C-9), 123.7 (C-6), 124.1 (C-7), 127.2, 129.1, 131.0, 131.9, 133.1 (C-5a, C-9a, C-2', C-3a', C-7a', C-8), 142.5 (C-3a), 151.9, 153.6 (C-5, C-5'), $160.1(\mathrm{C}=0) \mathrm{ppm}$.

$\mathrm{C}_{37} \mathrm{H}_{41} \mathrm{ClF}_{6} \mathrm{~N}_{4} \mathrm{O}_{12}$ (883.18).

ber.: $655.2529\left[\mathrm{M}-2 \mathrm{CF}_{3} \mathrm{CO}_{2}-\mathrm{H}\right]^{+}$

gef.: $655.2526,($ ESI-HRMS). 
4.4 6-(Fluorescein-5-carboxamido)-Hexansäure-[(1S)-1-chlormethyl-3-[(5(2-( $N, N^{\prime}$-dimethylamino)-ethoxy)-indol-2-yl)carbonyl]-5-O- $\beta$-D-

Galaktopyranosyl-1,2-dihydro-3H-benz[e]indol-8-ylmethyl]-amidtrifluoracetat ((1S)-72)

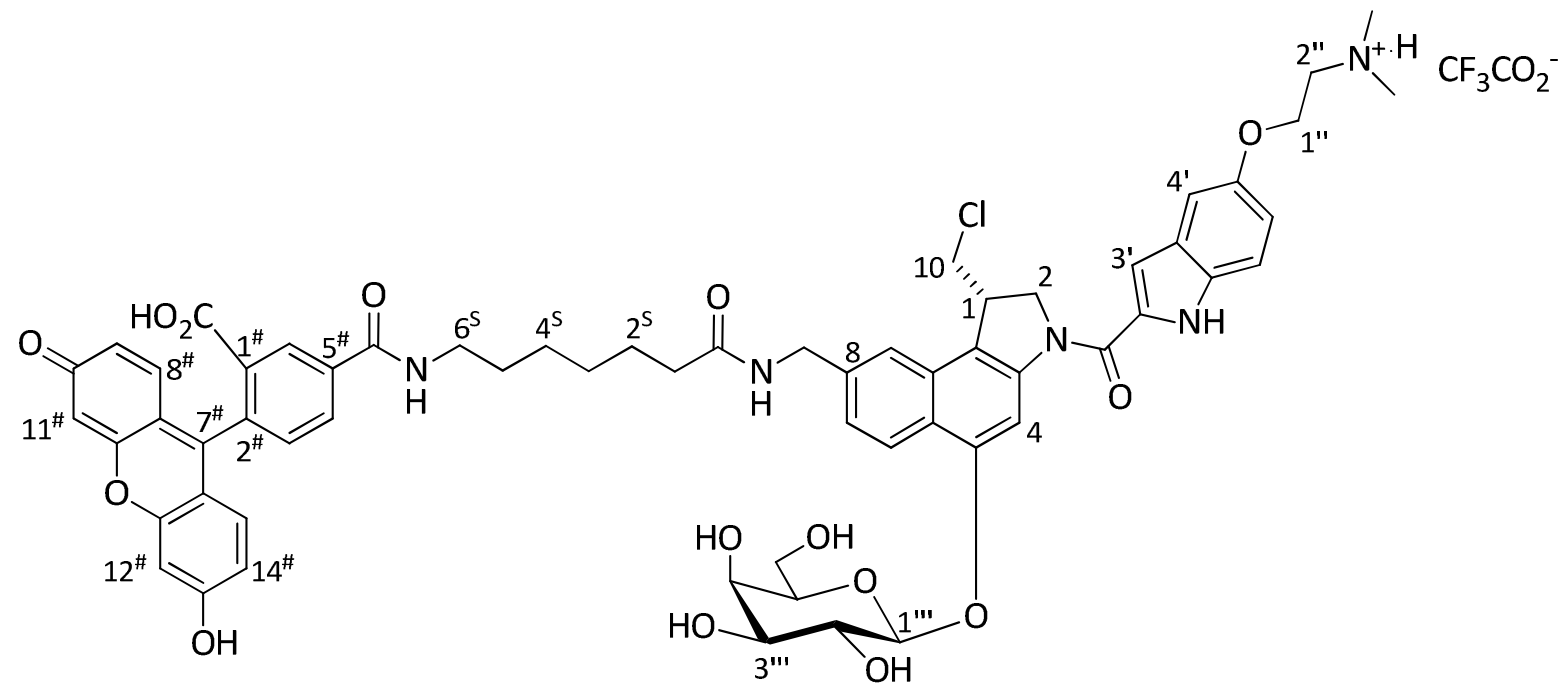

Zu einer Lösung des NHS-Esters (119) (8.90 mg, $15.2 \mu \mathrm{mol}, 1.0$ Äq.) in absolutem DMF $(150 \mu \mathrm{L})$ wurde eine Lösung von Galaktosid (+)-(1S)-118 (11.9 mg, $18.2 \mu \mathrm{mol}, 1.3$ Äq.) und $i$ - $\operatorname{Pr}_{2}$ NEt (11 $\mu \mathrm{L}, 66.6 \mu \mathrm{mol}, 4.5$ Äq.) in absolutem DMF (150 $\left.\mu \mathrm{L}\right)$ getropft. Nach $18 \mathrm{~h}$ Rühren bei Raumtemperatur wurde die Reaktionslösung mit bidestilliertem $\mathrm{H}_{2} \mathrm{O}$ (0.1\% TFA; $3.0 \mathrm{~mL}$ ) und $\mathrm{CH}_{3} \mathrm{CN}(2.5 \mathrm{~mL})$ verdünnt. Je $2 \mathrm{~mL}$ dieser Lösung wurden in das präparative HPLC-System (s. u.) injiziert. Fraktioniertes Auffangen des Eluats, Entfernung von $\mathrm{CH}_{3} \mathrm{CN}$ und TFA unter vermindertem Druck sowie Entfernung des restlichen Lösungsmittels mittels Gefriertrocknung lieferte das fluoreszenzmarkierte glykosidische Prodrug (1S)-72 als gelben Feststoff (7.8 mg, $6.9 \mu \mathrm{mol}, 46 \%$ ).

HPLC (analytisch):

Säule: Kromasil $100 \mathrm{C} 18$

\begin{tabular}{|c|c|c|}
\hline \multirow[t]{3}{*}{ Gradient: } & Zeit [min] & $\mathrm{H}_{2} \mathrm{O}(0.1 \%$ TFA $) / \mathrm{CH}_{3} \mathrm{CN}$ \\
\hline & 0 & $83 / 17$ \\
\hline & $0-60$ & $83 / 17 \rightarrow 50 / 50$ \\
\hline
\end{tabular}


$60-80$

$50 / 50$

80-90 50/50 $\rightarrow 83 / 17$

Fluss: $\quad 0.8 \mathrm{~mL} \mathrm{~min}^{-1}$

$t_{\mathrm{R}}: \quad 30.9 \min$

HPLC (präparativ):

Säule: Kromasil $100 \mathrm{C} 18$

\begin{tabular}{ccc}
\cline { 2 - 3 } Gradient: & Zeit [min] & $\mathrm{H}_{2} \mathrm{O}(0.1 \%$ TFA $) / \mathrm{CH}_{3} \mathrm{CN}$ \\
\cline { 2 - 3 } & 0 & $83 / 17$ \\
& $0-60$ & $83 / 17 \rightarrow 50 / 50$ \\
& $60-70$ & $50 / 50 \rightarrow 83 / 17$ \\
\cline { 2 - 3 } Fluss: & $12 \mathrm{~mL} \mathrm{~min}^{-1}$ & \\
$t_{\mathrm{R}}:$ & $36.6 \mathrm{~min}$ &
\end{tabular}

Fraktion: $\quad 36.0-38.0 \mathrm{~min}$

${ }^{1} \mathrm{H}-\mathrm{NMR}\left(600 \mathrm{MHz}\right.$, DMSO-d $\left.\mathrm{d}_{6}\right) \delta=1.08-1.70\left(\mathrm{~m}, 6 \mathrm{H}, 3^{\mathrm{S}}-\mathrm{H}_{2}, 4^{\mathrm{S}}-\mathrm{H}_{2}, 5^{\mathrm{S}}-\mathrm{H}_{2}\right), 2.18-2.22$ $\left(\mathrm{m}, 2 \mathrm{H}, 2^{\mathrm{S}}-\mathrm{H}\right), 2.87\left(\mathrm{~s}, 6 \mathrm{H}, \mathrm{NMe}_{2}\right), 3.18-4.26\left(\mathrm{~m}, 13 \mathrm{H}, 2^{\prime \prime \prime}-\mathrm{H}, 3^{\prime \prime \prime}-\mathrm{H}, 44^{\prime \prime \prime}-\mathrm{H}, 5\right.$ '"-H, 6"'- $\left.\mathrm{H}_{2}, 10-\mathrm{H}_{2}, 1-\mathrm{H}, 6^{\mathrm{S}}-\mathrm{H}_{2}, 1^{\prime \prime}-\mathrm{H}_{2}\right), 4.35-4.39\left(\mathrm{~m}, 2 \mathrm{H}, 2^{2 "}-\mathrm{H}\right), 4.43-4.48\left(\mathrm{~m}, 2 \mathrm{H}, \mathrm{Ar}-\mathrm{C}_{2}\right)$, 4.57-4.53 (m, $\left.1 \mathrm{H}, 2-\mathrm{H}_{\mathrm{a}}\right), 4.82-4.88\left(\mathrm{~m}, 1 \mathrm{H}, 2-\mathrm{H}_{\mathrm{b}}\right), 4.89-4.93\left(\mathrm{~m}, 1 \mathrm{H}, 1^{\prime \prime}-\mathrm{H}\right), 6.53-$ $6.60(\mathrm{~m}, 4 \mathrm{H}, \mathrm{Ar}-\mathrm{H}), 6.69$ (d, J = 2.1 Hz, 1-H, Ar-H), 7.01 (dd, J = 8.9, $\left.2.4 \mathrm{~Hz}, 1 \mathrm{H}, 6^{\prime}-\mathrm{H}\right)$, 7.15-7.21 (m, $\left.1 \mathrm{H}, 3^{\prime}-\mathrm{H}\right), 7.27$ (d, J = 2.1 Hz, $\left.1 \mathrm{H}, 4^{\prime}-\mathrm{H}\right)$, 7.30-7.38 (m, $2 \mathrm{H}, 7^{\prime}-\mathrm{H}$, Ar-H), $7.45(\mathrm{~d}, J=8.9 \mathrm{~Hz}, 1 \mathrm{H}, 7-\mathrm{H}), 7.69-7.73(\mathrm{~m}, 1 \mathrm{H}, \mathrm{Ar}-\mathrm{H}), 8.22-8.25(\mathrm{~m}, 1 \mathrm{H}, \mathrm{Ar}-\mathrm{H}), 8.30$ (d, J=8.8 Hz, $1 \mathrm{H}, 6-\mathrm{H}), 8.41(\mathrm{t}, J=6.0 \mathrm{~Hz}, 1 \mathrm{H}, \mathrm{Ar}-\mathrm{H}), 8.45(\mathrm{~d}, J=9.6 \mathrm{~Hz}, 1 \mathrm{H}, \operatorname{Ar}-\mathrm{H})$, 
$8.79(\mathrm{t}, J=5.4 \mathrm{~Hz}, 1 \mathrm{H}, \mathrm{Ar}-\mathrm{H}), 9.75,10.05\left(\mathrm{~s}, 2 \mathrm{H}, \mathrm{OH}, \mathrm{CO}_{2} \mathrm{H}\right), 11.70(\mathrm{~s}, 1 \mathrm{H}$, Indol-NH) ppm.

$\mathrm{C}_{63} \mathrm{H}_{63} \mathrm{ClF}_{3} \mathrm{~N}_{5} \mathrm{O}_{17}(1254.65)$.

ber.: $1126.3847\left[\mathrm{M}-\mathrm{CF}_{3} \mathrm{CO}_{2}\right]^{+}$

gef.: 1126.3832 , (ESI-HRMS). 


\section{Synthese des anti-Methyl-seco-CCBI-Grundgerüstes (1S,10R)-82}

\subsection{Ethyl-1-benzyloxy-7-brom-3-naphthalincarboxylat (98)}<smiles>CCCOC(=O)c1cc(Cc2ccccc2)c2cc(Br)ccc2c1</smiles>

Die Carbonsäure $85(10.1 \mathrm{~g}, 28.3 \mathrm{mmol}, 1.0$ Äq.), wurde in absolutem Ethanol $(350 \mathrm{~mL})$ suspendiert und Thionylchlorid $(4.1 \mathrm{~mL}, 6.66 \mathrm{~g}, 56.0 \mathrm{mmol}, 2.0$ Äq.) zugetropft. Das Gemisch wurde für $3 \mathrm{~h}$ unter Rückfluß gerührt, nochmals mit Thionylchlorid $(1.0 \mathrm{~mL}, 1.67 \mathrm{~g}, 14.0 \mathrm{mmol}, 0.5$ Äq.) versetzt und für weitere $16 \mathrm{~h}$ unter Rückfluß gerührt. Nach dem Abkühlen auf Raumtemperatur wurde das Lösungsmittel im Vakuum entfernt und die Zielverbindung 98 als Rohprodukt weiter eingesetzt.

$\mathbf{R}_{\mathbf{f}}=0.59(n-P e n t a n /$ EtOAc $=4: 1)$.

${ }^{1} \mathrm{H}-\mathrm{NMR}\left(300 \mathrm{MHz}, \mathrm{CDCl}_{3}\right): \delta=1.44\left(\mathrm{t}, J=7.1 \mathrm{~Hz}, 3 \mathrm{H}, \mathrm{OCH}_{2} \mathrm{C}_{3}\right), 4.43(\mathrm{q}, J=7.1 \mathrm{~Hz}$, $\left.2 \mathrm{H}, \mathrm{OCH}_{2} \mathrm{CH}_{3}\right), 5.28\left(\mathrm{~s}, 2 \mathrm{H}, \mathrm{OC}_{2} \mathrm{Ph}\right), 7.34-7.48,7.51-7.55(2 \times \mathrm{m}, 2 \times 3 \mathrm{H}, 2-\mathrm{H}$, $5 \times \mathrm{Ph}-\mathrm{H}), 7.61(\mathrm{dd}, J=8.7 \mathrm{~Hz}, 1 \mathrm{H}, 6-\mathrm{H}), 7.76(\mathrm{~d}, J=8.8 \mathrm{~Hz}, 1 \mathrm{H}, 5-\mathrm{H}), 8.17(\mathrm{~s}, 1 \mathrm{H}$, 4-H), $8.47(\mathrm{~d}, J=2.0 \mathrm{~Hz}, 1 \mathrm{H}, 8-\mathrm{H}) \mathrm{ppm}$.

${ }^{13}$ C-NMR $\left(75 \mathrm{MHz}, \mathrm{CDCl}_{3}\right): \delta=14.4\left(\mathrm{OCH}_{2} \underline{C H}_{3}\right), 61.3\left(\mathrm{OCH}_{2} \mathrm{CH}_{3}\right), 70.5\left(\mathrm{OCH}_{2} \mathrm{Ph}\right), 105.1$ (C-2), 122.1, 128.3, 128.8 (C-3, C-4a, C-8a), 123.3, 124.9, 128.2, 130.5130 .6 (C-4, C-5,

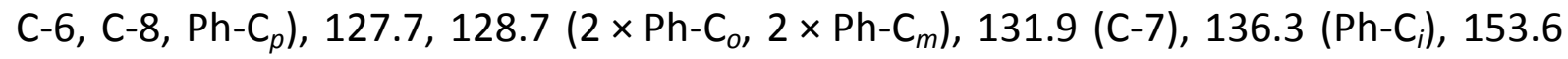
(C-1), $166.5(\mathrm{C}=0)$ ppm.

$\mathrm{C}_{20} \mathrm{H}_{17} \mathrm{BrO}_{3}$ (385.25). 


\subsection{Ethyl-1-benzyloxy-7-cyano-3-naphthalincarboxylat (99)}<smiles>CCOC(=O)c1cc(Br)c2ccc(C#N)cc2c1</smiles>

Der Benzylether 98 (10.7 g, $27.7 \mathrm{mmol}, 1.0$ Äq.) wurde in absolutem DMF (15 mL) gelöst und CuCN (2.98 g, $33.2 \mathrm{mmol}, 1.2 \mathrm{Äq}$.) zugegeben und $20 \mathrm{~h}$ bei Rückfluss gerührt. Nach Abkühlen auf Raumtemperatur wurde das Reaktionsgemisch in eine Suspension von $\mathrm{H}_{2} \mathrm{O}(250 \mathrm{~mL})$ und $\mathrm{FeCl}_{3}(5.79 \mathrm{~g}, 33.2 \mathrm{mmol}, 1.2 \mathrm{Äq}$.) gegossen und für 30 min gerührt. Das Reaktionsgemisch wurde mit EtOAc $(3 \times 200 \mathrm{~mL})$ extrahiert und die vereinigten organischen Phasen mit gesättigter $\mathrm{NaCl}$-Lösung $(2 \times 300 \mathrm{~mL})$ gewaschen und über $\mathrm{MgSO}_{4}$ getrocknet. Man erhielt die Zielverbindung 99 als Rohprodukt, das weiter eingesetzt werden konnte.

$\mathbf{R}_{\mathbf{f}}=0.27(n-$ Pentan / EtOAc $=10: 1)$.

${ }^{1} \mathrm{H}-\mathrm{NMR}\left(300 \mathrm{MHz}, \mathrm{CDCl}_{3}\right): \delta=1.46\left(\mathrm{t}, J=7.2 \mathrm{~Hz}, 3 \mathrm{H}, \mathrm{OCH}_{2} \underline{\mathrm{C}}_{3}\right), 4.46(\mathrm{q}, J=7.2 \mathrm{~Hz}$, $\left.2 \mathrm{H}, \mathrm{OC}_{2} \mathrm{CH}_{3}\right), 5.32\left(\mathrm{~s}, 2 \mathrm{H}, \mathrm{OC}_{2} \mathrm{Ph}\right), 7.37-7.57(\mathrm{~m}, 5 \mathrm{H}, 5 \times \mathrm{Ph}-\mathrm{H}), 7.59(\mathrm{~d}, J=1.2 \mathrm{~Hz}$, $1 \mathrm{H}, 2-\mathrm{H}), 7.67(\mathrm{dd}, J=8.5,1.6 \mathrm{~Hz}, 1 \mathrm{H}, 6-\mathrm{H}), 7.98(\mathrm{~d}, J=8.5 \mathrm{~Hz}, 1 \mathrm{H}, 5-\mathrm{H}), 8.22\left(\mathrm{~s}_{\mathrm{br}}, 1 \mathrm{H}\right.$, 4-H), $8.71\left(m_{c}, 1 \mathrm{H}, 8-\mathrm{H}\right) \mathrm{ppm}$.

$\left.{ }^{13} \mathrm{C}-\mathrm{NMR}(75 \mathrm{MHz}, \mathrm{CDCl})_{3}\right): \delta=14.3\left(\mathrm{OCH}_{2} \mathrm{CH}_{3}\right), 61.6\left(\mathrm{OCH}_{2} \mathrm{CH}_{3}\right), 70.6\left(\mathrm{OCH}_{2} \mathrm{Ph}\right), 105.7$ (C-2), 110.6 (C-7), 119.0 (CN), 122.8 (C-4), 126.8 (C-3), 127.6, 128.4, 128.8 (C-6,

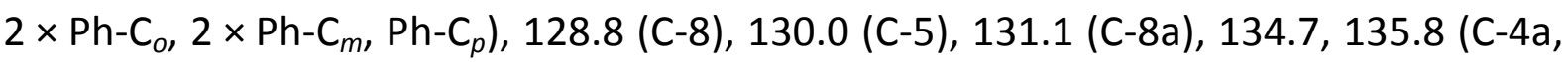
$\left.\mathrm{Ph}-\mathrm{C}_{i}\right), 154.6(\mathrm{C}-1), 166.1$ (C=0) ppm.

$\mathrm{C}_{21} \mathrm{H}_{17} \mathrm{NO}_{3}$ (331.36). 


\subsection{1-Benzyloxy-7-cyano-3-naphthalincarbonsäure (100)}<smiles>N#Cc1ccc2cc(C(=O)O)cc(Br)c2c1</smiles>

Der Ethylester 99 (8.40 g, 25.4 mmol, 1.0 Äq.) wurde in einem Lösungsmittelgemisch aus THF / MeOH / $\mathrm{H}_{2} \mathrm{O}=3: 1: 1\left(250 \mathrm{~mL}\right.$ ) gelöst und nach Zugabe von $\mathrm{LiOH} \cdot \mathrm{H}_{2} \mathrm{O}$ (5.32 g, $127 \mathrm{~mol}$, 5.0 Äq.) $20 \mathrm{~h}$ bei Raumtemperatur gerührt. Anschließend wurde die Reaktionsmischung auf $0{ }^{\circ} \mathrm{C}$ gekühlt und unter Rühren mit $10 \%$-iger $\mathrm{HCl}$ angesäuert $(\mathrm{pH} \approx 1)$, wobei sich ein weißer Niederschlag bildete. Dieser wurde durch Filtration abgetrennt und im Vakuum getrocknet. Man erhielt die Carbonsäure $\mathbf{1 0 0}$ als weißen Feststoff (6.12 g, $20.2 \mathrm{mmol}, 80 \%)$.

$\mathbf{R}_{\mathbf{f}}=0.26(\mathrm{PE} / \mathrm{EtOAc}=1: 1,1 \% \mathrm{HOAc})$.

${ }^{1} \mathrm{H}-\mathrm{NMR}\left(300 \mathrm{MHz}, \mathrm{DMSO}-\mathrm{d}_{6}\right): \delta=5.43\left(\mathrm{~s}, 2 \mathrm{H}, \mathrm{OCH}_{2} \mathrm{Ph}\right), 7.32-7.50(\mathrm{~m}, 3 \mathrm{H}, 2-\mathrm{H}$, $2 \times \mathrm{Ph}-\mathrm{H}), 7.91(\mathrm{dd}, J=8.5,1.6 \mathrm{~Hz}, 1 \mathrm{H}, 6-\mathrm{H}), 8.28(\mathrm{~s}, 1 \mathrm{H}, 5-\mathrm{H}), 8.31(\mathrm{~s}, 1 \mathrm{H}, 4-\mathrm{H}), 8.65$ (d, J = 0.8 Hz, $1 \mathrm{H}, 8-\mathrm{H}), 13.32\left(\mathrm{~s}_{\mathrm{br}}, 1 \mathrm{H}, \mathrm{CO}_{2} \mathrm{H}\right) \mathrm{ppm}$.

$\mathrm{C}_{19} \mathrm{H}_{13} \mathrm{NO}_{3}$ (303.31).

\subsection{3-Amino-1-benzyloxy- $N$-(tert-butyloxycarbonyl)-7-cyano-naphthalin} (79)<smiles>CC(C)(C)OC(=O)Nc1cc(OCc2ccccc2)c2cc(C#N)ccc2c1</smiles>

Zu einer Suspension der Carbonsäure 100 (6.12 g, 20.2 mmol, 1.0 Äq.) in absolutem tert-Butylalkohol (1000 mL) gab man aktiviertes Molsieb $4 \AA$ (60.0 g), Triethylamin (3.37 mL, 2.46 g, 24.2 mmol, 1.2 Äq.) und DPPA (5.35 mL, 6.80 g, 24.2 mmol, 1.2 Äq.). Das Reaktionsgemisch wurde $22 \mathrm{~h}$ ohne Rühren zum Sieden erhitzt und nach Abkühlen auf Raumtemperatur vom Molsieb abfiltriert, welches gründlich mit EtOAc 
$(500 \mathrm{~mL})$ gewaschen wurde. Nach Entfernen des Lösungsmittels im Vakuum wurde der Rückstand in EtOAc $(300 \mathrm{~mL})$ aufgenommen und die Lösung mit $2 \mathrm{~N} \mathrm{HCl}$ $(2 \times 200 \mathrm{~mL})$, gesättigter $\mathrm{NaHCO}_{3}$-Lösung $(200 \mathrm{~mL})$ sowie gesättigter $\mathrm{NaCl}$-Lösung $\left(200 \mathrm{~mL}\right.$ ) gewaschen. Man trocknete die organische Phase über $\mathrm{MgSO}_{4}$ und entfernte das Lösungsmittel im Vakuum. Nach säulenchromatographischer Reinigung an Kieselgel $(\mathrm{PE} / \mathrm{EtOAC}=7: 1 \rightarrow 5: 1 \rightarrow 4: 1)$ erhielt man die Zielverbindung 79 als weißen Feststoff (6.06 g, $16.2 \mathrm{mmol}, 80 \%)$.

$\mathbf{R}_{\mathrm{f}}=0.51(\mathrm{PE} / \mathrm{EtOAC}=4: 1)$.

${ }^{1} \mathrm{H}-\mathrm{NMR}\left(300 \mathrm{MHz}, \mathrm{CDCl}_{3}\right) \delta=1.54\left(\mathrm{~s}, 9 \mathrm{H}, \mathrm{C}\left(\mathrm{CH}_{3}\right)_{3}\right), 5.20\left(\mathrm{~s}, 1 \mathrm{H}, \mathrm{OCH}_{2} \mathrm{Ph}\right), 6.79\left(\mathrm{~s}_{\mathrm{br}}\right.$, $1 \mathrm{H}, \mathrm{NH}), 7.14(\mathrm{~d}, \mathrm{~J}=1.6 \mathrm{~Hz}, 1 \mathrm{H}, 2-\mathrm{H}), 7.30-7.55(\mathrm{~m}, 7 \mathrm{H}, 4-\mathrm{H}, 6-\mathrm{H}, 5 \times \mathrm{Ph}-\mathrm{H}), 7.67$ (s, $1 \mathrm{H}, 5-\mathrm{H}), 7.68(\mathrm{~d}, \mathrm{~J}=8.5 \mathrm{~Hz}, 1 \mathrm{H}, 5-\mathrm{H}), 8.50-8.58(\mathrm{~m}, 1 \mathrm{H}, 8-\mathrm{H}) \mathrm{ppm}$.

$\mathrm{C}_{23} \mathrm{H}_{22} \mathrm{~N}_{2} \mathrm{O}_{3}(374.43)$.

\subsection{2-Amino-4-benzyloxy-1-brom-N-(tert-butyloxycarbonyl)-6-cyano- naphthalin (101)}<smiles>CC(=O)Nc1cc(OCc2ccccc2)c2cc(C#N)ccc2c1Br</smiles>

Zu einer Lösung von Naphthalinderivat 79 (6.00 g, $16.0 \mathrm{mmol}, 1.0$ Äq.) in absolutem THF (300 mL) wurde bei $-78{ }^{\circ} \mathrm{C}$ eine Lösung von $\mathrm{H}_{2} \mathrm{SO}_{4}$ in absolutem THF (435 $\mu \mathrm{L}$ einer Lösung von $1 \mu \mathrm{L} \mathrm{H}_{2} \mathrm{SO}_{4}$ in $1.00 \mathrm{~mL} \mathrm{THF}, 8.02 \mu \mathrm{mol}, 0.05$ mol-\%) getropft. Nach $20 \mathrm{~min}$ Rühren bei $-78{ }^{\circ} \mathrm{C}$ wurde portionsweise $N$-Bromsuccinimid $(3.42 \mathrm{~g}, 19.3 \mathrm{mmol}$, 1.2 Äq.) zugegeben und für weitere $4 \mathrm{~h}$ bei dieser Temperatur gerührt. Anschließend ließ man innerhalb von $1 \mathrm{~h}$ auf Raumtemperatur erwärmen und beendete die Reaktion durch Zugabe von gesättigter NaCl-Lösung $(110 \mathrm{~mL}), \mathrm{H}_{2} \mathrm{O}(60 \mathrm{~mL})$ sowie gesättigter $\mathrm{NaHCO}_{3}$-Lösung $(60 \mathrm{~mL})$. Man extrahierte mit EtOAc $(3 \times 100 \mathrm{~mL})$, wusch die vereinigten organischen Phasen mit gesättigter $\mathrm{NaCl}$-Lösung $(3 \times 200 \mathrm{~mL})$ und 
trocknete über $\mathrm{MgSO}_{4}$. Man erhielt die Zielverbindung 101 als Rohprodukt (7.56 g), das weiter eingesetzt werden konnte.

$\mathbf{R}_{\mathrm{f}}=0.52(\mathrm{PE} / \mathrm{EtOAC}=10: 1)$.

${ }^{1} \mathrm{H}-\mathrm{NMR}\left(300 \mathrm{MHz}, \mathrm{CDCl}_{3}\right) \delta=1.57\left(\mathrm{~s}, 9 \mathrm{H}, \mathrm{C}\left(\mathrm{CH}_{3}\right)_{3}\right), 5.25\left(\mathrm{~s}, 2 \mathrm{H}, \mathrm{OCH}_{2} \mathrm{Ph}\right), 7.33-7.56$ (m, $6 \mathrm{H}, \mathrm{NH}, 5 \times \mathrm{Ph}-\mathrm{H}), 7.62(\mathrm{dd}, J=8.8,1.7 \mathrm{~Hz}, 1 \mathrm{H}, 7-\mathrm{H}), 8.09$ (dd, $J=8.8,0.5 \mathrm{~Hz}, 1 \mathrm{H}$, 8-H), $8.24(\mathrm{~d}, J=5.3 \mathrm{~Hz}, 1 \mathrm{H}, 3-\mathrm{H}), 8.53-8.59(\mathrm{~m}, 1 \mathrm{H}, 5-\mathrm{H}) \mathrm{ppm}$.

$\mathrm{C}_{23} \mathrm{H}_{21} \mathrm{BrN}_{2} \mathrm{O}_{3}$ (453.33).

\section{$5.6(+)-\left\{\left(2^{\prime} R, 3^{\prime} R\right)\right.$-2-Amino-4-benzyloxy- $N$-(2,3-epoxybutyl)-1-brom- $N$ - (tert-butyloxycarbonyl)-6-cyano-naphthalin\} ((+)-(2'R,3'R)-80)}

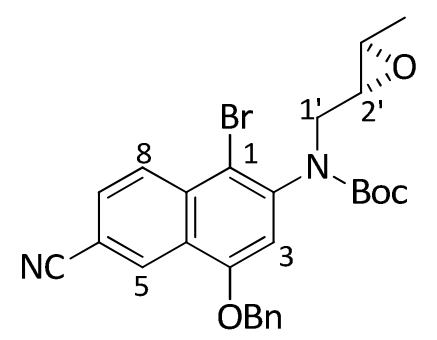

Eine Lösung des Carbamats 101 (4.98 g, 11.0 mmol, 1.0 Äq.) in DMF (100 mL) wurde bei $60{ }^{\circ} \mathrm{C}$ mit NaH (1.05 g, $44.0 \mathrm{mmol}, 4.0$ Äq.; 60\%ige Suspension in Mineralöl) versetzt und $45 \mathrm{~min}$ bei dieser Temperatur gerührt. Nach dem Abkühlen auf Raumtemperatur wurde weitere $30 \mathrm{~min}$ gerührt, anschließend das Nosylat (+)-(2R,3R)-102 (4.50 g, $16.5 \mathrm{mmol}, 1.5$ Äq.) zugegeben und nach weiterem Rühren für $24 \mathrm{~h}$ bei dieser Temperatur wurde die Suspension vorsichtig mit gesättigter $\mathrm{NH}_{4} \mathrm{Cl}$ Lösung $(300 \mathrm{~mL})$ versetzt. Nach Extraktion des Gemisches mit EtOAc $(3 \times 300 \mathrm{~mL})$ wurden die vereinigten organischen Phasen über $\mathrm{Na}_{2} \mathrm{SO}_{4}$ getrocknet und das Lösungsmittel im Vakuum entfernt. Säulenchromatographische Reinigung an Kieselgel (PE/EtOAC $=5: 1)$ lieferte die Zielverbindung $(+)-\left(2^{\prime} R, 3^{\prime} R\right)-80 \quad(2.59 \mathrm{~g}$, $4.09 \mathrm{mmol}, 45 \%)$ als Gemisch von Atropisomeren in Form eines weißen Feststoffes.

$\mathbf{R}_{\mathbf{f}}=0.26(\mathrm{PE} / \mathrm{EtOAC}=6: 1)$ 
${ }^{1} \mathrm{H}-\mathrm{NMR}$ (300 MHz, $\mathrm{CDCl}_{3}$; Gemisch von Rotameren): $\delta=1.21-1.38,1.49-1.63(2 \times \mathrm{m}$, zus. $\left.12 \mathrm{H}, \mathrm{C}\left(\mathrm{CH}_{3}\right)_{3}, 4^{\prime}-\mathrm{H}_{3}\right), 2.56-2.67,2.73-2.89,3.00-3.21,3.23-3.37,3.49-3.60$, 3.68-4.31 (7 × m, zus. $\left.4 \mathrm{H}, 1^{\prime}-\mathrm{H}_{2}, 2^{\prime}-\mathrm{H}, 3^{\prime}-\mathrm{H}\right), 5.13-5.33\left(\mathrm{~m}, 2 \mathrm{H}, \mathrm{OC}_{2} \mathrm{Ph}\right), 6.87-7.10$ (m, $1 \mathrm{H}, 3-\mathrm{H}), 7.33-7.55(\mathrm{~m}, 5 \mathrm{H}, 5 \times \mathrm{Ph}-\mathrm{H}), 7.64-7.76(\mathrm{~m}, 1 \mathrm{H}, 7-\mathrm{H}), 8.28-8.38(\mathrm{~m}, 1 \mathrm{H}$, 8-H), 8.62-8.72 (m, $1 \mathrm{H}, 5-\mathrm{H}) \mathrm{ppm}$.

$\mathrm{C}_{27} \mathrm{H}_{27} \mathrm{BrN}_{2} \mathrm{O}_{4}$ (523.42).

ber.: $523.1227[\mathrm{M}+\mathrm{H}]^{+}$

gef.: 523.1217 (ESI-HRMS).

\section{$5.7(+)-\{(1 S, 10 R)-5-B e n z y l o x y-3-(t e r t-b u t y l o x y c a r b o n y l-1-(10-H y d r o x y-$ ethyl)-7-cyano-1,2-dihydro-3H-benz[e]indol ((+)-(1S,10R)-81)}

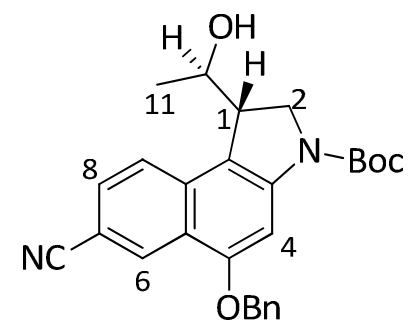

Eine Lösung von frisch sublimiertem $\mathrm{ZnCl}_{2}(1.42 \mathrm{~g}, 10.4 \mathrm{mmol}, 1.5 \mathrm{Äq}$.) in absolutem THF (100 mL) wurde auf $0{ }^{\circ} \mathrm{C}$ gekühlt, langsam mit Methyllithium $(26.1 \mathrm{~mL}$ einer $1.6 \mathrm{M}$ Lösung in $\mathrm{Et}_{2} \mathrm{O}, 41.8 \mathrm{mmol}, 6.0$ Äq.) versetzt und für 30 min bei dieser Temperatur gerührt. Anschließend wurde die Lösung auf $-78^{\circ} \mathrm{C}$ gekühlt, tropfenweise mit TMSNCS (1.48 $\mu \mathrm{L}, 1.37 \mathrm{~g}, 10.4 \mathrm{mmol}, 1.5$ Äq.) versetzt, wieder auf $0{ }^{\circ} \mathrm{C}$ erwärmt und für 30 min bei dieser Temperatur gerührt. Nach dem erneuten Abkühlen auf $-78^{\circ} \mathrm{C}$ wurde zu dem Gemisch über eine Transferkanüle eine Lösung des Epoxids (+)-(2'R,3'R)-80 (2.93 g, $6.94 \mathrm{mmol}, 1.0$ Äq.) in absolutem THF (50 mL) getropft und für $30 \mathrm{~min}$ bei dieser Temperatur gerührt. Nach Erwärmen auf $0^{\circ} \mathrm{C}$ wurde die Reaktionslösung zunächst für $1 \mathrm{~h}$ bei dieser Temperatur gerührt, dann auf Raumtemperatur erwärmt und weitere $2.5 \mathrm{~h}$ gerührt. Anschließend wurde das Gemisch vorsichtig mit gesättigter $\mathrm{NH}_{4} \mathrm{Cl}$-Lösung $(400 \mathrm{~mL})$ versetzt, mit $\mathrm{CH}_{2} \mathrm{Cl}_{2}$ $(4 \times 400 \mathrm{~mL})$ extrahiert, die vereinigten organischen Phasen über $\mathrm{Na}_{2} \mathrm{SO}_{4}$ getrocknet und das Lösungsmittel im Vakuum entfernt. Säulenchromatographische Reinigung an 
Kieselgel (PE / EtOAc $=5: 2) \quad$ lieferte die Zielverbindung $(+)-(1 S, 10 R)-81 \quad(1.73 \mathrm{~g}$, $3.89 \mathrm{mmol}, 69 \%, 98.8 \%$ ee) als weißen Feststoff.

Analytische Daten für (-)-(1R,10S)-81:

HPLC (analytisch): Säule: $\quad$ Chiralpak $^{\oplus} \mathrm{IA}, 250 \times 4.6 \mathrm{~mm}, 5 \mu \mathrm{m}$

Eluens $\quad 5 \%$ iso-Propanol in $n$-Hexan

Fluss: $\quad 0.8 \mathrm{~mL} / \mathrm{min}$

$t_{\mathrm{R}}: \quad 21.2 \mathrm{~min}$

Analytische Daten für $(+)-(1 S, 10 R)-81:$

HPLC (analytisch): Säule: $\quad$ Chiralpak ${ }^{\circledR} \mathrm{IA}, 250 \times 4.6 \mathrm{~mm}, 5 \mu \mathrm{m}$

Eluens $\quad 5 \%$ iso-Propanol in $n$-Hexan

Fluss: $\quad 0.8 \mathrm{~mL} / \mathrm{min}$

$t_{\mathrm{R}}: \quad 27.6 \mathrm{~min}$

$\mathbf{R}_{\mathbf{f}}=0.47(\mathrm{PE} / \mathrm{EtOAC}=2: 1)$

${ }^{1} \mathrm{H}-\mathrm{NMR}\left(300 \mathrm{MHz}, \mathrm{CDCl}_{3}\right): \delta=1.27\left(\mathrm{~d}, J=6.4 \mathrm{~Hz}, 3 \mathrm{H}, 10-\mathrm{CH}_{3}\right), 1.64\left(\mathrm{~s}, 9 \mathrm{H}, \mathrm{C}\left(\mathrm{CH}_{3}\right)_{3}\right.$, $3.70(\mathrm{dt}, J=9.9,3.1 \mathrm{~Hz}, 1 \mathrm{H}, 1-\mathrm{H}), 4.03-4.16\left(\mathrm{~m}, 1 \mathrm{H}, 2-\mathrm{H}_{\mathrm{a}}\right), 4.26(\mathrm{dd}, J=11.6,3.4 \mathrm{~Hz}$, $\left.1 \mathrm{H}, 2-\mathrm{H}_{\mathrm{b}}\right), 4.30-4.39(\mathrm{~m}, 1 \mathrm{H}, 10-\mathrm{H}), 5.32\left(\mathrm{~s}, 2 \mathrm{H}, \mathrm{OC}_{2}{ }_{2} \mathrm{Ph}\right), 7.35-7.61(\mathrm{~m}, 6 \mathrm{H}, 8-\mathrm{H}$, $5 \times \mathrm{Ph}-\mathrm{H}), 7.83(\mathrm{~d}, J=8.7 \mathrm{~Hz}, 1 \mathrm{H}, 9-\mathrm{H}), 7.89(\mathrm{~s}, 1 \mathrm{H}, 4-\mathrm{H}), 8.64(\mathrm{~d}, J=1.4 \mathrm{~Hz}, 1 \mathrm{H}, 6-\mathrm{H})$ ppm.

$\mathrm{C}_{27} \mathrm{H}_{28} \mathrm{~N}_{2} \mathrm{O}_{4}(444.52)$. 
$5.8(+)-\{(1 S, 10 R)-5-B e n z y l o x y-3-(t e r t-b u t y l o x y c a r b o n y l-1-(10-c h l o r e t h y l)-$ 7-cyano-1,2-dihydro-3H-benz[e]indol ((+)-(1S,10R)-82)<smiles>[H][Z]([H])(Cl)[C@H]1CN(C(=O)OC(C)(C)C)c2cc(Cc3ccccc3)c3cc(C#N)ccc3c21</smiles>

Eine Lösung des Alkohols (+)-(1S,10R)-81 (2.00 g, $4.50 \mathrm{mmol}, 1.0$ Äq.) in absolutem $\mathrm{CH}_{2} \mathrm{Cl}_{2}(100 \mathrm{~mL})$ wurde bei $0{ }^{\circ} \mathrm{C}$ mit Thionylchlorid $(3.26 \mathrm{~mL}, 5.35 \mathrm{~g}, 45.0 \mathrm{mmol}$, 10.0 Äq.) sowie Pyridin $(9.08 \mathrm{~mL}, 8.90 \mathrm{~g}, 113 \mathrm{mmol}, 25.0$ Äq.) versetzt und anschließend auf Raumtemperatur erwärmt. Nach $4 \mathrm{~h}$ Rühren bei dieser Temperatur wurden die flüchtigen Bestandteile im Vakuum entfernt und der Rückstand mittels Säulenchromatographie an Kieselgel $(P E / E t O A c=5: 1)$ gereinigt. Das Produkt $(+)$ (1S,10R)-82 (0.70 g, $151 \mu \mathrm{mol}, 34 \%, 99.0 \%$ ee) wurde als weißer Feststoff erhalten.

Analytische Daten für (+)-(1S,10R)-82:

HPLC (analytisch): Säule: Chiralpak OD

Eluens: $\quad n$-Hexan / 2-Propanol $=98: 2$

Fluss: $\quad 0.8 \mathrm{~mL} / \mathrm{min}$

$\mathrm{t}_{\mathrm{R}}: \quad 37.6 \mathrm{~min}$.

Analytische Daten für (-)-(1S,10R)-82:

HPLC (analytisch): Säule: Chiralpak OD

Eluens: $\quad n$-Hexan / 2-Propanol $=98: 2$

Fluss: $\quad 0.8 \mathrm{~mL} / \mathrm{min}$

$t_{R}: \quad 33.9 \mathrm{~min}, 99.2 \%$ ee.

$\mathbf{R}_{\mathrm{f}}=0.31(\mathrm{PE} / \mathrm{EtOAC}=5: 1)$.

$\mathrm{C}_{27} \mathrm{H}_{27} \mathrm{ClN}_{2} \mathrm{O}_{3}$ (462.97). 
5.9 (E/Z)-2-Amino-4-benzyloxy-1-brom-N-(tert-butyloxycarbonyl)-N-(3chlor-2-butenyl)-6-cyano-naphthalin (104)<smiles>CC(=O)[C]N(C(=O)C=C(C)Cl)c1cc(Br)c2cc(C#N)ccc2c1Br</smiles>

Zu einer Lösung des Naphthalinderivats 101 (2.89 g, 6.38 mmol, 1.0 Äq.) in absolutem DMF $(60 \mathrm{~mL})$ wurde bei Raumtemperatur portionsweise $\mathrm{NaH}(383 \mathrm{mg}, 9.58 \mathrm{mmol}$, 1.5 Äq. als 60\%ige Suspension in Mineralöl) gegeben. Man ließ $2.5 \mathrm{~h}$ bei Raumtemperatur rühren, tropfte (E/Z)-1,3-Dichlor-2-buten $(103)(1.40 \mathrm{~mL}, 1.59 \mathrm{~g}, 12.8 \mathrm{mmol}$, 2.0 Äq.) hinzu und rührte für weitere $4 \mathrm{~h}$ bei Raumtemperatur. Die Reaktion wurde durch vorsichtige Zugabe einer gesättigter $\mathrm{NH}_{4} \mathrm{Cl}$-Lösung $(100 \mathrm{~mL})$ sowie $\mathrm{H}_{2} \mathrm{O}(50 \mathrm{~mL})$ beendet und die Reaktionsmischung wurde mit EtOAc $(3 \times 100 \mathrm{~mL})$ extrahiert. Die vereinigten organischen Phasen wurden mit $\mathrm{H}_{2} \mathrm{O}(3 \times 100 \mathrm{~mL})$ sowie gesättigter $\mathrm{NaCl}$ Lösung $(3 \times 100 \mathrm{~mL})$ gewaschen, über $\mathrm{MgSO}_{4}$ getrocknet und das Lösungsmittel wurde im Vakuum entfernt. Säulenchromatographie an Kieselgel (PE / EtOAc = 10:1) lieferte die Zielverbindung 104 als weißen Feststoff (2.29 g, 4.22 mmol, 66\%).

$\mathbf{R}_{\mathbf{f}}=0.46(\mathrm{PE} / \mathrm{EtOAC}=5: 1)$.

$\mathrm{C}_{27} \mathrm{H}_{26} \mathrm{BrClN}_{2} \mathrm{O}_{3}$ (541.86). 
5.10 rac-\{(1S,10R)-5-Benzyloxy-3-(tert-butyloxycarbonyl)-1-(10-chlorethyl)-7-cyano-1,2-dihydro-3H-benz[e]indol\} (rac-(1S,10R)-82) und rac-\{(1S,10S)-5-Benzyloxy-3-(tert-butyloxycarbonyl)-1-(10-chlorethyl)-7-cyano-1,2-dihydro-3H-benz[e]indol\} (rac-(1S,10S)-82)<smiles>[H][Z]([H])(Cl)[C@H]1CN(C(=O)OC(C)(C)C)c2cc(Cc3ccccc3)c3cc(C#N)ccc3c21</smiles><smiles>[H][Z]([H])([H])[C@H]1CN(C(=O)OC(C)(C)C)c2cc(OCc3ccccc3)c3cc(C#N)ccc3c21</smiles>

Der lodaromat 104 (2.29 g, $4.23 \mathrm{mmol}, 1.0$ Äq.) wurde in absolutem Toluol (125 mL) gelöst und die Lösung gründlich durch Einleiten eines lebhaften Argon-Gasstroms entgast. Dann versetzte man mit Tris-(trimethylsilyl)-silan $(1.43 \mathrm{~mL}, 4.63 \mathrm{mmol}$, 1.1 Äq.) sowie AIBN (173 mg, 1.05 mmol, 0.25 Äq.), erwärmte das Gemisch mittels eines vorgeheizten Ölbades auf $80^{\circ} \mathrm{C}$ und rührte bei dieser Temperatur für $4 \mathrm{~h}$. Man ließ auf Raumtemperatur abkühlen und entfernte das Lösungsmittel im Vakuum. Waschen des öligen braunen Rohprodukts mit $n$-Pentan sowie Kristallisation aus EtOAc / $n$-Pentan lieferte einen hellbraunen Feststoff, der durch Säulenchromatographie an Kieselgel (Gradient: PE / EtOAc $=10: 1 \rightarrow 8: 1$ ) gereingt wurde. Man erhielt ein Gemisch aus dem syn-Diastereomer rac-(1S,10S)-82 und dem dehalogenierten Cyclisierungsprodukt als hellgelben Feststoff $(853 \mathrm{mg}, 1.84 \mathrm{mmol}, 44 \%)$ und das gewünschte anti-Diastereomer rac-(1S,10R)-82 als weißen Feststoff (902 mg, $1.95 \mathrm{mmol}, 46 \%)$.

Analytische Daten für rac-(1S,10R)-82:

$\mathbf{R}_{\mathrm{f}}=0.28(\mathrm{PE} / \mathrm{EtOAC}=5: 1)$.

Analytische Daten für rac-(1S,10S)-82:

$\mathbf{R}_{\mathrm{f}}=0.52(\mathrm{PE} / \mathrm{EtOAC}=5: 1)$. 


$$
\begin{aligned}
& \mathrm{C}_{27} \mathrm{H}_{27} \mathrm{ClN}_{2} \mathrm{O}_{3} \text { (462.97). } \quad \text { ber.: } 485.1602[\mathrm{M}+\mathrm{Na}]^{+} \\
& \text {gef.: } 485.1589 \text { (ESI-HRMS). }
\end{aligned}
$$

\subsection{Trennung der Enantiomere von rac-\{(1S,10R)-5-Benzyloxy-3-(tert- butyloxycarbonyl)-1-(10-chlor-ethyl)-7-cyano-1,2-dihydro-3H- benz $[e]$ indol\} $($ rac- $(1 S, 10 R)-82)$}

$\operatorname{rac}-(1 S, 10 R)-82$ (900 mg, $1.95 \mathrm{mmol})$ wurde in einem Gemisch aus $n$-Hexan $/ \mathrm{CH}_{2} \mathrm{Cl}_{2}=$ 1:1 (20 mL) gelöst. Je $0.8 \mathrm{~mL}$ dieser Probelösung (entsprechend $36 \mathrm{mg}$ bzw. $778 \mu \mathrm{mol}$ des racemischen Gemisches) wurden in das präparative HPLC-System (Säule: Chiralpak IA, $250 \times 20$ mm, Partikelgröße: $5 \mu \mathrm{m}$, mobile Phase: $n$-Heptan $/ \mathrm{CH}_{2} \mathrm{Cl}_{2}=$ 4:1, Fluss: $18 \mathrm{~mL} \mathrm{~min}^{-1}$, Druck: 7.4 MPa) injiziert. Fraktioniertes Auffangen des Eluats (UV-Detektor: $\lambda=250 \mathrm{~nm}$ ) lieferte die Enantiomere (+)-82 und (-)-82.

Analytische Daten für $(+)-(1 R, 10 S)-82$ :

$\begin{array}{lll}\text { HPLC (präparativ): } & t_{\mathrm{R}}: & 8.2 \mathrm{~min} \\ & \text { Fraktion: } & 7.7-9.5 \mathrm{~min} \\ \text { HPLC (analytisch): } & \text { Säule: } & \text { Chiralcel OD } \\ & \text { Eluens: } & n \text {-Hexan } / \mathrm{i}-\mathrm{PrOH}=98: 2 \\ & \text { Fluss: } & 0.8 \mathrm{~mL} \mathrm{\textrm {min } ^ { - 1 }} \\ & t_{\mathrm{R}}: & 37.6 \mathrm{~min}, 99.9 \% \text { ee } . \\ {[\alpha]_{\mathrm{D}}^{\mathbf{2 0}}=+21.6^{\circ}\left(\mathrm{c}=0.5, \mathrm{CHCl}_{3}\right) .} & \end{array}$

Analytische Daten für (-)-(1S,10R)-82:

$\begin{array}{lll}\text { HPLC (präparativ): } & t_{\mathrm{R}}: & 10.8 \mathrm{~min} \\ & \text { Fraktion: } & 10.2-14.0 \mathrm{~min} \\ \text { HPLC (analytisch): } & \text { Säule: } & \text { Chiralcel OD } \\ & \text { Eluens: } & n-\text { Hexan } / i-\mathrm{PrOH}=98: 2\end{array}$




$$
\begin{array}{ll}
\text { Fluss: } & 0.8 \mathrm{~mL} \mathrm{~min}^{-1} \\
t_{\mathrm{R}}: & 33.9 \mathrm{~min}, 98.7 \% \text { ee. }
\end{array}
$$

$[\alpha]_{\mathrm{D}}^{20}=-24.0^{\circ}\left(\mathrm{c}=0.5, \mathrm{CHCl}_{3}\right)$.

\section{$5.12(+)-\{(1 S, 10 R)-3-($ tert-Butyloxycarbonyl)-1-(10-chlor-ethyl)-7-cyano-} 5-hydroxy-1,2-dihydro-3H-benz[e]indol\} $((+)-(1 S, 10 R)-149)$

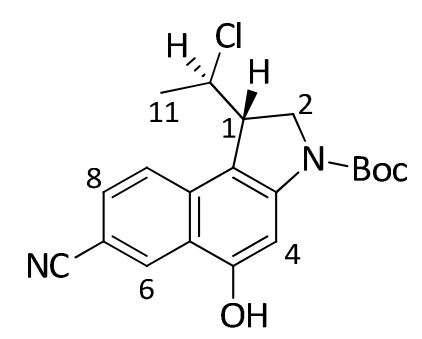

Benzylether (+)-82 (700 mg, $1.51 \mathrm{mmol}, 1.0$ Äq.) wurde in frisch destilliertem THF $(50 \mathrm{~mL})$ gelöst und die Lösung wurde auf $40{ }^{\circ} \mathrm{C}$ erwärmt. Dann wurde Palladium auf Aktivkohle (10\%ig, 162 mg, $151 \mu \mathrm{mol}, 0.1$ Äq. bez. Pd) zugegeben und nach kurzem Rühren wurde eine 25\%ige Lösung von Ammoniumformiat in $\mathrm{H}_{2} \mathrm{O}(1.60 \mathrm{~mL}$ ) langsam zugetropft. Nach 120 min Rühren bei $40{ }^{\circ} \mathrm{C}$ (DC-Kontolle!) wurde über eine CeliteSchicht vom Feststoff abfiltriert, wobei gründlich mit Aceton $(1000 \mathrm{~mL})$ nachgespült wurde. Das Filtrat wurde im Vakuum konzentriert, säulenchromatographisch an Kieselgel gereinigt $(\mathrm{PE} / \mathrm{EtOAC}=5: 1)$ und man erhielt $(+)-\mathbf{1 4 9}$ als weißen Feststoff (477 mg, $1.28 \mathrm{mmol}, 85 \%)$.

$\mathbf{R}_{\mathbf{f}}=0.50(\mathrm{PE} / \mathrm{EtOAC}=2: 1)$.

${ }^{1} \mathrm{H}-\mathrm{NMR}\left(300 \mathrm{MHz}, \mathrm{DMSO}-\mathrm{d}_{6}\right): \delta=1.54\left(\mathrm{~s}, 9 \mathrm{H}, \mathrm{C}\left(\mathrm{CH}_{3}\right)_{3}\right), 1.57(\mathrm{~d}, J=6.7 \mathrm{~Hz}, 3 \mathrm{H}$, $\left.11-\mathrm{H}_{3}\right), 4.00-4.16\left(\mathrm{~m}, 3 \mathrm{H}, 1-\mathrm{H}, 2-\mathrm{H}_{2}\right), 4.66-4.79\left(\mathrm{~m}_{\mathrm{c}}, 1 \mathrm{H}, 10-\mathrm{H}\right), 7.66\left(\mathrm{~m}_{\mathrm{c}}, 2 \mathrm{H}, 4-\mathrm{H}\right.$, 8-H), $7.95(\mathrm{~d}, J=8.8 \mathrm{~Hz}, 1 \mathrm{H}, 9-\mathrm{H}), 8.47(\mathrm{~d}, J=1.7 \mathrm{~Hz}, 1 \mathrm{H}, 6-\mathrm{H}), 10.89\left(\mathrm{~s}_{\mathrm{br}}, 1 \mathrm{H}, \mathrm{OH}\right)$ ppm.

$\mathrm{C}_{20} \mathrm{H}_{21} \mathrm{ClN}_{2} \mathrm{O}_{3}$ (372.85). 


\section{Synthese der anti-Methyl-CBI-Derivate mit DMAI-Seitenkette}

\section{1 rac-\{(1S,10S)-5-Benzyloxy-1-(10-chlor-ethyl)-7-cyano-3-[(5-(2-(N,N'- dimethylamino)-ethoxy)-indol-2-yl)carbonyl]-1,2-dihydro-3H- benz $[e]$ indol\} (rac-(1S,10S)-88)}

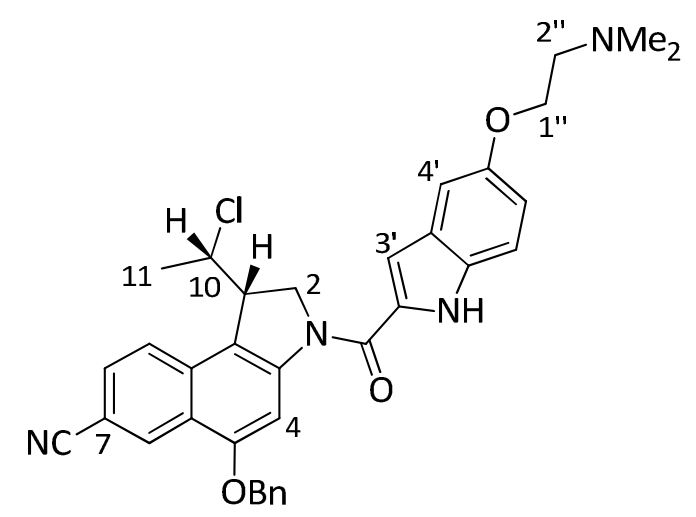

rac-(1S,10S)-82(200 mg, $431 \mu \mathrm{mol}, 1.0$ Äq.) wurde in $4 \mathrm{M} \mathrm{HCl} /$ EtOAc (15 mL) suspendiert und $4 \mathrm{~h}$ bei Raumtemperatur gerührt. Anschließend engte man die Lösung im Vakuum ein und trocknete $1.5 \mathrm{~h}$ im Vakuum. Der grüne Rückstand wurde in DMF $(11 \mathrm{~mL})$ gelöst, auf $0{ }^{\circ} \mathrm{C}$ gekühlt und mit EDAC $\cdot \mathrm{HCl}(248 \mathrm{mg}, 1.30 \mathrm{mmol}, 3.0$ Äq.) sowie DMAl- $\mathrm{CO}_{2} \mathrm{H} \cdot \mathrm{HCl}(104)(160 \mathrm{mg}, 561 \mu \mathrm{mol}, 1.3$ Äq.) versetzt. Nach $18 \mathrm{~h}$ Rühren bei Raumtemperatur wurde die Reaktionslösung mit EtOAc $(70 \mathrm{~mL}), \mathrm{H}_{2} \mathrm{O}(70 \mathrm{~mL})$ sowie gesättigter $\mathrm{NaHCO}_{3}$-Lösung $(70 \mathrm{~mL})$ versetzt und anschließend mit EtOAc $(4 \times 70 \mathrm{~mL})$ extrahiert. Die vereinigten organischen Phasen wurden mit gesättigter $\mathrm{NaCl}$-Lösung $(4 \times 130 \mathrm{~mL})$ gewaschen, über $\mathrm{MgSO}_{4}$ getrocknet und das Lösungsmittel wurde im Vakuum entfernt. Nach Säulenchromatographie an Kieselgel $\left(\mathrm{CH}_{2} \mathrm{Cl}_{2} / \mathrm{MeOH} 10: 1\right)$ erhielt man die Zielverbindung rac- $(1 S, 10 S)-82$ als gelben Feststoff $(169 \mathrm{mg}$, $285 \mu \mathrm{mol}, 66 \%)$.

$\mathbf{R}_{\mathbf{f}}=0.53\left(\mathrm{CH}_{2} \mathrm{Cl}_{2} / \mathrm{MeOH}=9: 1\right)$.

${ }^{1} \mathrm{H}-\mathrm{NMR}\left(300 \mathrm{MHz}, \mathrm{CDCl}_{3}\right): \delta=1.14\left(\mathrm{~d}, J=6.7 \mathrm{~Hz}, 3 \mathrm{H}, 11-\mathrm{H}_{3}\right), 2.36\left(\mathrm{~s}, 6 \mathrm{H}, \mathrm{NMe}_{2}\right), 2.77$ $\left(\mathrm{t}, J=5.7 \mathrm{~Hz}, 2 \mathrm{H}, 2^{\prime \prime}-\mathrm{H}_{2}\right), 4.04-4.16\left(\mathrm{~m}, 3 \mathrm{H}, 1-\mathrm{H}, 2 "-\mathrm{H}_{2}\right), 4.55-4.72\left(\mathrm{~m}, 2 \mathrm{H}, 2-\mathrm{H}_{\mathrm{a}}\right.$ $10-\mathrm{H}), 4.92\left(\mathrm{dd}, J=11.3,1.9 \mathrm{~Hz}, 1 \mathrm{H}, 2-\mathrm{H}_{\mathrm{b}}\right), 5.17-5.37\left(\mathrm{~m}, 2 \mathrm{H}, \mathrm{OCH}_{2} \mathrm{Ph}\right), 6.92-7.18(\mathrm{~m}$, $\left.3 \mathrm{H}, 3^{\prime}-\mathrm{H}, 4^{\prime}-\mathrm{H}, 6^{\prime}-\mathrm{H}\right), 7.27-7.53\left(\mathrm{~m}, 6 \mathrm{H}, 7^{\prime}-\mathrm{H}, 5 \times \mathrm{Ph}-\mathrm{H}\right), 7.61$ (dd, J = 8.6, $1.7 \mathrm{~Hz}, 1 \mathrm{H}$, 
8-H), 7.75 (d, J = 8.6 Hz, $1 \mathrm{H}, 9-\mathrm{H}), 8.29(\mathrm{~s}, 1 \mathrm{H}, 4-\mathrm{H}), 8.66(\mathrm{~d}, J=1.5 \mathrm{~Hz}, 1 \mathrm{H}, 6-\mathrm{H}), 9.50$ $\left(\mathrm{s}_{\mathrm{br}}, 1 \mathrm{H}, \mathrm{NH}\right) \mathrm{ppm}$.

${ }^{13} \mathrm{C}-\mathrm{NMR}\left(125 \mathrm{MHz}, \mathrm{CDCl}_{3}\right): \delta=18.0$ (C-11), $45.9\left(\mathrm{NMe}_{2}\right), 47.1$ (C-1), 52.4 (C-2), 57.5 (C-2"), 58.4 (C-10), 66.6 (C-1"), $70.8\left(\mathrm{OCH}_{2} \mathrm{Ph}\right), 99.7$ (C-4), 103.6 (C-4'), 106.7, 106.9 (C-7, C-3'), 112.7 (C-7'), 116.3, 118.0 (C-5a, C-6'), 119.4, 122.5 (C-9b, CN), 123.4 (C-9),

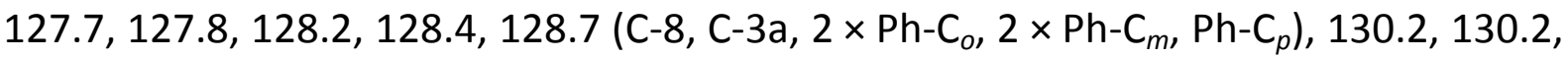
131.2, 131.5 (C-6, C-9a, C-2', C-7a'), $135.8\left(\mathrm{Ph}^{\left.-C_{i}\right)}, 145.7\right.$ (C-3a), 154.1 (C-5'), 156.1 (C-5), $160.6(\mathrm{C}=0)$ ppm.

$\mathrm{C}_{35} \mathrm{H}_{33} \mathrm{ClN}_{4} \mathrm{O}_{3}(593.11)$.

ber.: $\quad 593.2314[\mathrm{M}+\mathrm{H}]^{+}$

gef.: $\quad 593.2317$ (ESI-HRMS).

\section{$6.2(+)-\left\{(1 S, 10 R)-5-B e n z y l o x y-1-(10-c h l o r-e t h y l)-7-c y a n o-3-\left[\left(5-\left(2-\left(N, N^{\prime}-\right.\right.\right.\right.\right.$ dimethylamino)-ethoxy)-indol-2-yl)carbonyl]-1,2-dihydro-3H- benz $[e]$ indol\} $((+)-(1 S, 10 R)-88)$}

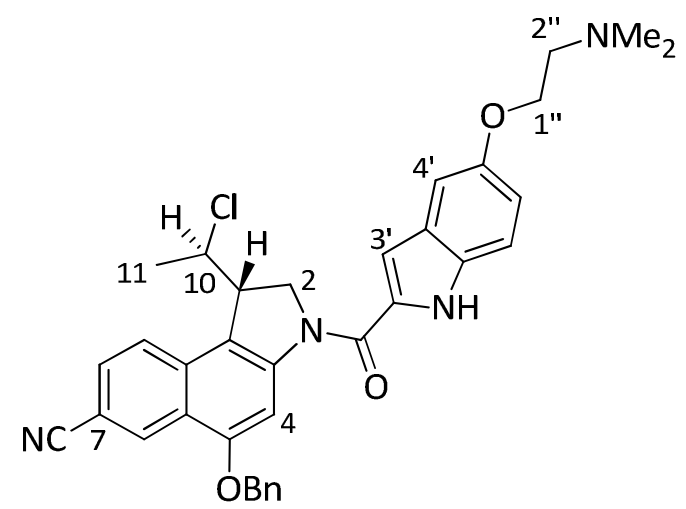

(+)-(1S,10R)-82 (400 mg, $862 \mu \mathrm{mol}, 1.0$ Äq.) wurde in $4 \mathrm{M} \mathrm{HCl} /$ EtOAc (30 mL) suspendiert und $4 \mathrm{~h}$ bei Raumtemperatur gerührt. Anschließend engte man die Lösung im Vakuum ein und trocknete $1.5 \mathrm{~h}$ im Vakuum. Der grüne Rückstand wurde in DMF (25 mL) gelöst, auf $0^{\circ} \mathrm{C}$ gekühlt und mit EDAC. $\mathrm{HCl}(496 \mathrm{mg}, 2.60 \mathrm{mmol}, 3.0$ Äq.) sowie DMAI- $\mathrm{CO}_{2} \mathrm{H} \cdot \mathrm{HCl}(104)(320 \mathrm{mg}, 1.02 \mathrm{mmol}, 1.3$ Äq.) versetzt. Nach $18 \mathrm{~h}$ Rühren bei Raumtemperatur wurde die Reaktionslösung mit EtOAc (140 mL), $\mathrm{H}_{2} \mathrm{O}(140 \mathrm{~mL})$ sowie gesättigter $\mathrm{NaHCO}_{3}$-Lösung $(140 \mathrm{~mL})$ versetzt und anschließend mit EtOAc $(4 \times 140 \mathrm{~mL})$ extrahiert. Die vereinigten organischen Phasen wurden mit gesättigter 
$\mathrm{NaCl}$-Lösung $(4 \times 250 \mathrm{~mL})$ gewaschen, über $\mathrm{MgSO}_{4}$ getrocknet und das Lösungsmittel wurde im Vakuum entfernt. Nach Säulenchromatographie an Kieselgel $\left(\mathrm{CH}_{2} \mathrm{Cl}_{2} / \mathrm{MeOH} 10: 1\right)$ erhielt man die Zielverbindung $(+)-(1 S, 10 R)-88$ als gelben Feststoff (317 mg, $534 \mu \mathrm{mol}, 62 \%$ ).

$\mathbf{R}_{\mathbf{f}}=0.63\left(\mathrm{CH}_{2} \mathrm{Cl}_{2} / \mathrm{MeOH}=5: 1\right)$.

${ }^{1} \mathrm{H}-\mathrm{NMR}\left(300 \mathrm{MHz}, \mathrm{CDCl}_{3}\right): \delta=1.63\left(\mathrm{~d}, J=6.8 \mathrm{~Hz}, 3 \mathrm{H}, 11-\mathrm{H}_{3}\right), 2.37\left(\mathrm{~s}, 6 \mathrm{H}, \mathrm{NMe}_{2}\right), 2.77$ $\left(\mathrm{t}, J=5.8 \mathrm{~Hz}, 2 \mathrm{H}, 2^{\prime \prime}-\mathrm{H}_{2}\right), 3.98(\mathrm{dd}, J=6.9,3.8 \mathrm{~Hz}, 1 \mathrm{H}, 1-\mathrm{H}), 4.11(\mathrm{t}, J=5.8 \mathrm{~Hz}, 2 \mathrm{H}$, 1"-H), 4.45-4.67 (m, $\left.2 \mathrm{H}, 2-\mathrm{H}_{\mathrm{a}}, 10-\mathrm{H}\right), 4.85\left(\mathrm{dd}, J=10.8,2.1 \mathrm{~Hz}, 1 \mathrm{H}, 2-\mathrm{H}_{\mathrm{b}}\right), 5.27$ (q, $\left.J=11.3 \mathrm{~Hz}, 2 \mathrm{H}, \mathrm{OCH}_{2} \mathrm{Ph}\right), 6.99-7.08\left(\mathrm{~m}, 2 \mathrm{H}, 3^{\prime}-\mathrm{H}, 6^{\prime}-\mathrm{H}\right), 7.12\left(\mathrm{~d}, J=2.3 \mathrm{~Hz}, 1 \mathrm{H}, 4^{\prime}-\mathrm{H}\right)$, 7.29-7.53 (m, 6 H, 7'-H, $5 \times \mathrm{Ph}-\mathrm{H}), 7.61(\mathrm{dd}, J=8.7,1.7 \mathrm{~Hz}, 1 \mathrm{H}, 8-\mathrm{H}), 7.72$ (d, $J=8.7 \mathrm{~Hz}, 1 \mathrm{H}, 9-\mathrm{H}), 8.24(\mathrm{~s}, 1 \mathrm{H}, 4-\mathrm{H}), 8.67(\mathrm{~d}, J=1.6 \mathrm{~Hz}, 1 \mathrm{H}, 6-\mathrm{H}), 9.47\left(\mathrm{~s}_{\mathrm{br}}, 1 \mathrm{H}, \mathrm{NH}\right)$ ppm.

${ }^{13} \mathrm{C}-\mathrm{NMR}\left(125 \mathrm{MHz}, \mathrm{CDCl}_{3}\right.$ ): $\delta=23.8$ (C-11), $45.9\left(\mathrm{NMe}_{2}\right), 47.2$ (C-1), 53.4 (C-2), 58.4 (C-2"), 59.5 (C-10), 66.6 (C-1"), $70.8\left(\mathrm{OCH}_{2} \mathrm{Ph}\right), 99.6$ (C-4), 103.6 (C-4'), 106.3, 106.6 (C-7, C-3'), 112.7 (C-7'), 117.0, 117.7 (C-5a, C-6'), 119.6, 122.6 (C-9b, CN), 123.5 (C-9), 127.7, 128.1, 128.2, 128.4, 128.7 (C-8, C-3a, $\left.2 \times \mathrm{Ph}_{-} \mathrm{C}_{o}, 2 \times \mathrm{Ph}-\mathrm{C}_{m}, \mathrm{Ph}-\mathrm{C}_{p}\right), 130.2,130.3$, 131.4, 131.5 (C-6, C-9a, C-2', C-7a'), 135.9 (Ph-C) 145.7 (C-3a), 154.0 (C-5'), 155.9 (C-5), 160.7 (C=0) ppm.

$\mathrm{C}_{35} \mathrm{H}_{33} \mathrm{CIN}_{4} \mathrm{O}_{3}$ (593.11).

ber.: $593.2314[\mathrm{M}+\mathrm{H}]^{+}$

gef.: 593.2320 (ESI-HRMS). 
$6.3(-)-\left\{(1 R, 10 S)-5-B e n z y l o x y-1-(10-c h l o r-e t h y l)-7-c y a n o-3-\left[\left(5-\left(2-\left(N, N^{\prime}-\right.\right.\right.\right.\right.$ dimethylamino)-ethoxy)-indol-2-yl)carbonyl]-1,2-dihydro-3Hbenz $[e]$ indol\} $((-)-(1 R, 10 S)-88)$

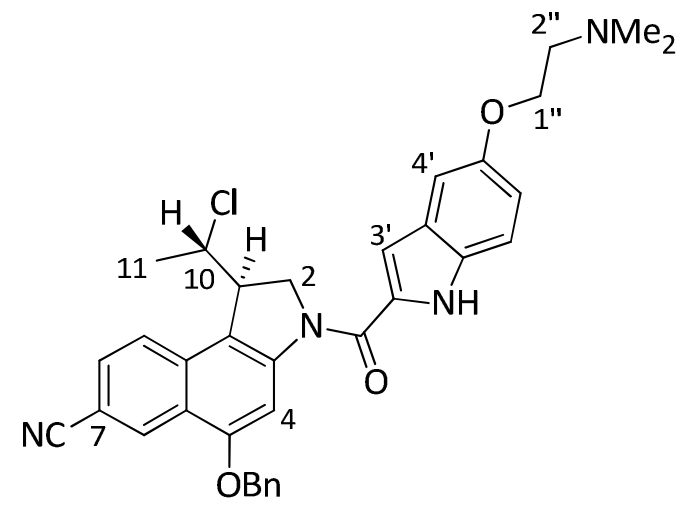

(-)-(1R,10S)-82 (200 mg, $431 \mu \mathrm{mol}, 1.0$ Äq.) wurde in $4 \mathrm{M} \mathrm{HCl} /$ EtOAc (15 mL) suspendiert und $4 \mathrm{~h}$ bei Raumtemperatur gerührt. Anschließend engte man die Lösung im Vakuum ein und trocknete $1.5 \mathrm{~h}$ im Vakuum. Der grüne Rückstand wurde in DMF $(11 \mathrm{~mL})$ gelöst, auf $0{ }^{\circ} \mathrm{C}$ gekühlt und mit EDAC $\cdot \mathrm{HCl}(248 \mathrm{mg}, 1.30 \mathrm{mmol}, 3.0$ Äq.) sowie DMAl- $\mathrm{CO}_{2} \mathrm{H} \cdot \mathrm{HCl}$ (104) (160 mg, $561 \mu \mathrm{mol}, 1.3$ Äq.) versetzt. Nach $18 \mathrm{~h}$ Rühren bei Raumtemperatur wurde die Reaktionslösung mit EtOAc $(70 \mathrm{~mL}), \mathrm{H}_{2} \mathrm{O}(70 \mathrm{~mL})$ sowie gesättigter $\mathrm{NaHCO}_{3}$-Lösung $(70 \mathrm{~mL})$ versetzt und anschließend mit EtOAc $(4 \times 70 \mathrm{~mL})$ extrahiert. Die vereinigten organischen Phasen wurden mit gesättigter $\mathrm{NaCl}$-Lösung $(4 \times 130 \mathrm{~mL})$ gewaschen, über $\mathrm{MgSO}_{4}$ getrocknet und das Lösungsmittel wurde im Vakuum entfernt. Nach Säulenchromatographie an Kieselgel $\left(\mathrm{CH}_{2} \mathrm{Cl}_{2} / \mathrm{MeOH} 10: 1\right)$ erhielt man die Zielverbindung (-)-(1R,10S)-88 als gelben Feststoff $(120 \mathrm{mg}$, $202 \mu \mathrm{mol}, 47 \%)$.

$\mathbf{R}_{\mathbf{f}}=0.67\left(\mathrm{CH}_{2} \mathrm{Cl}_{2} / \mathrm{MeOH}=5: 1\right)$.

${ }^{1} \mathrm{H}-\mathrm{NMR}\left(300 \mathrm{MHz}, \mathrm{CDCl}_{3}\right): \delta=1.64\left(\mathrm{~d}, J=6.8 \mathrm{~Hz}, 3 \mathrm{H}, 11-\mathrm{H}_{3}\right), 2.37\left(\mathrm{~s}, 6 \mathrm{H}, \mathrm{NMe}_{2}\right), 2.78$ $\left(\mathrm{t}, J=5.8 \mathrm{~Hz}, 2 \mathrm{H}, 2^{\prime \prime}-\mathrm{H}_{2}\right), 3.95-4.04(\mathrm{~m}, 1 \mathrm{H}, 1-\mathrm{H}), 4.12(\mathrm{t}, J=5.8 \mathrm{~Hz}, 2 \mathrm{H}, 1$ 1"-H), 4.43$4.72\left(\mathrm{~m}, 2 \mathrm{H}, 2-\mathrm{H}_{\mathrm{a}}, 10-\mathrm{H}\right), 4.86\left(\mathrm{~d}, J=10.7 \mathrm{~Hz}, 1 \mathrm{H}, 2-\mathrm{H}_{\mathrm{b}}\right), 5.27(\mathrm{q}, J=11.3 \mathrm{~Hz}, 2 \mathrm{H}$, $\left.\mathrm{OCH}_{2} \mathrm{Ph}\right), 6.99-7.10\left(\mathrm{~m}, 2 \mathrm{H}, 3^{\prime}-\mathrm{H}, 6^{\prime}-\mathrm{H}\right), 7.14\left(\mathrm{~d}, J=2.0 \mathrm{~Hz}, 1 \mathrm{H}, 4^{\prime}-\mathrm{H}\right), 7.31-7.56(\mathrm{~m}$, $\left.6 \mathrm{H}, 7^{\prime}-\mathrm{H}, 5 \times \mathrm{Ph}-\mathrm{H}\right), 7.62(\mathrm{dd}, J=8.7,1.7 \mathrm{~Hz}, 1 \mathrm{H}, 8-\mathrm{H}), 7.73(\mathrm{~d}, J=8.7 \mathrm{~Hz}, 1 \mathrm{H}, 9-\mathrm{H})$, $8.27(\mathrm{~s}, 1 \mathrm{H}, 4-\mathrm{H}), 8.69(\mathrm{~d}, J=1.6 \mathrm{~Hz}, 1 \mathrm{H}, 6-\mathrm{H}), 9.61\left(\mathrm{~s}_{\mathrm{br}}, 1 \mathrm{H}, \mathrm{NH}\right) \mathrm{ppm}$. 
${ }^{13} \mathrm{C}-\mathrm{NMR}\left(125 \mathrm{MHz}, \mathrm{CDCl}_{3}\right): \delta=23.8$ (C-11), $45.9\left(\mathrm{NMe}_{2}\right), 47.2$ (C-1), 53.4 (C-2), 58.4 (C-2"), 59.5 (C-10), 66.6 (C-1"), $70.8\left(\mathrm{OCH}_{2} \mathrm{Ph}\right), 99.6$ (C-4), 103.6 (C-4'), 106.3, 106.6 (C-7, C-3'), 112.7 (C-7'), 117.0, 117.7 (C-5a, C-6'), 119.6, 122.6 (C-9b, CN), 123.5 (C-9),

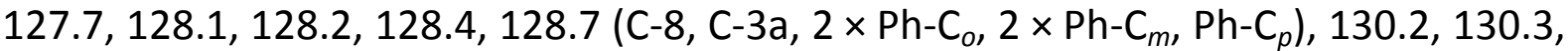
131.4, 131.5 (C-6, C-9a, C-2', C-7a'), $135.9\left(\mathrm{Ph}-\mathrm{C}_{i}\right), 145.7$ (C-3a), 154.0 (C-5'), 155.9 (C-5), 160.7 (C=O) ppm.

$\mathrm{C}_{35} \mathrm{H}_{33} \mathrm{ClN}_{4} \mathrm{O}_{3}$ (593.11).

ber.: $\quad 593.2314[\mathrm{M}+\mathrm{H}]^{+}$

gef.: 593.2310 (ESI-HRMS).

\section{4 rac-\{(1S,10S)-7-Aminomethyl-5-benzyloxy-1-(10-chlor-ethyl)-3-[(5-(2-} ( $N, N^{\prime}$-dimethylamino)-ethoxy)-indol-2-yl)carbonyl]-1,2-dihydro-3Hbenz $[e]$ indol\} $($ rac- $(1 S, 10 S)-81)$

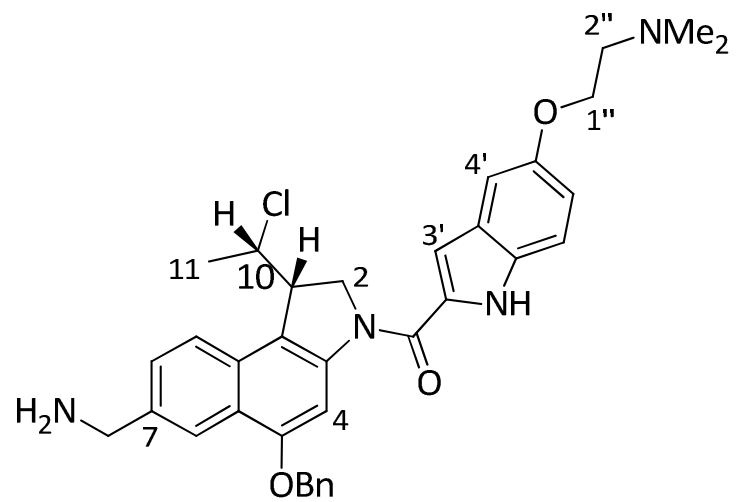

Eine Lösung des Nitrils rac-(1S,10S)-88 (140 mg, $236 \mu \mathrm{mol}, 1.0$ Äq.) in einem Lösungsmittelgemisch aus absolutem THF $(5 \mathrm{~mL})$ und absolutem Ethanol $(10 \mathrm{~mL})$ wurde entgast und $1.25 \mathrm{M}$ Lösung von $\mathrm{HCl}$ in Ethanol ${ }^{165}(378 \mu \mathrm{L}, 472 \mu \mathrm{mol}, 2.0 \mathrm{Äq}$.) sowie $\mathrm{H}_{2} \mathrm{O}$ (2.5 mL) zugetropft und $\mathrm{PtO}_{2} \cdot \mathrm{H}_{2} \mathrm{O}$ (11.8 mg, $\left.47.2 \mu \mathrm{mol}, 0.20 \mathrm{Äq}.\right)$ zugegeben. Man leitete kurz einen $\mathrm{H}_{2}$-Gasstrom durch die Reaktionsmischung und rührte dann für $8 \mathrm{~h}$ bei Raumtemperatur unter einer $\mathrm{H}_{2}$-Atmosphäre. Danach filtrierte man über eine Celite-Schicht vom Feststoff $a b$, spülte gründlich mit $\mathrm{MeOH}(200 \mathrm{~mL})$ nach und entfernte das Lösungsmittel im Vakuum. Nach Säulenchromatographie an Kieselgel $\left(\mathrm{CH}_{2} \mathrm{Cl}_{2} / \mathrm{MeOH}=5: 1\right)$ erhielt man die Zielverbindung rac-(1S,10S)-81 als hell gelben Feststoff (76 mg, $127 \mu \mathrm{mol}, 54 \%)$. 
$\mathbf{R}_{\mathbf{f}}=0.21\left(\mathrm{CH}_{2} \mathrm{Cl}_{2} / \mathrm{MeOH}=5: 1\right)$.

${ }^{1} \mathrm{H}-\mathrm{NMR}\left(600 \mathrm{MHz}, \mathrm{DMSO}-\mathrm{d}_{6}\right): \delta=1.08\left(\mathrm{~d}, J=6.7 \mathrm{~Hz}, 3 \mathrm{H}, 11-\mathrm{H}_{3}\right), 2.24\left(\mathrm{~s}, 6 \mathrm{H}, \mathrm{NMe}_{2}\right)$, $2.77\left(t, J=5.8 \mathrm{~Hz}, 2 \mathrm{H}, 2^{\prime \prime}-\mathrm{H}_{2}\right), 3.95\left(\mathrm{~s}, 2 \mathrm{H}, 12-\mathrm{H}_{2}\right), 4.04-4.09,4.29-4.35,4.63-4.68$, 4.75-4.85 (4 × m, $\left.6 \mathrm{H}, 1-\mathrm{H}, 2-\mathrm{H}_{2}, 10-\mathrm{H}, 1^{\prime \prime}-\mathrm{H}_{2}\right), 5.32$ (q, J = 11.8 Hz, $\left.2 \mathrm{H}, \mathrm{OCH}_{2} \mathrm{Ph}\right), 6.93$ (dd, J = 8.8, 2.4 Hz, $\left.1 \mathrm{H}, 6^{\prime}-\mathrm{H}\right), 7.10\left(\mathrm{~d}, J=4.4 \mathrm{~Hz}, 1 \mathrm{H}, 3^{\prime}-\mathrm{H}\right), 7.20(\mathrm{~d}, J=2.4 \mathrm{~Hz}, 1 \mathrm{H}$, 4'-H), 7.32-7.46 (m, $\left.6 \mathrm{H}, 7^{\prime}-\mathrm{H}, 5 \times \mathrm{Ph}-\mathrm{H}\right), 7.57$ (d, J=7.4, Hz, $\left.1 \mathrm{H}, 8-\mathrm{H}\right), 7.63$ (dd, $J=8.6,1.6 \mathrm{~Hz}, 1 \mathrm{H}, 6-\mathrm{H}), 7.90(\mathrm{~d}, J=8.6 \mathrm{~Hz}, 1 \mathrm{H}, 9-\mathrm{H}), 8.18(\mathrm{~s}, 1 \mathrm{H}, 4-\mathrm{H}), 11.65(\mathrm{~s}, 1 \mathrm{H}$, $\mathrm{NH}) \mathrm{ppm}$.

${ }^{13}$ C-NMR (125 MHz, DMSO-d 6 ): $\delta=18.3$ (C-11), 45.5 (C-12), $45.5\left(\mathrm{NMe}_{2}\right), 46.1$ (C-1), 52.6 (C-2), 57.8 (C-2"), 59.0 (C-10), 66.2 (C-1"), $69.7\left(\mathrm{OCH}_{2} \mathrm{Ph}\right), 98.5$ (C-4), 103.2 (C-4'), 105.3 (C-3'), 113.1 (C-7'), 115.9, 116.5 (C-5a, C-6'), 120.7, 122.5, 123.3, 123.4 (C-7, C-8, C-9, C-9b), 127.8, 128.1, 128.4, 130.7, 136.7 (C-6, C-9a, C-2', C-3a', C-7a'), 141.9 (C-3a), 153.0 (C-5'), 154.4 (C-5), 160.1 (C=O) ppm.

$\mathrm{C}_{35} \mathrm{H}_{37} \mathrm{ClN}_{4} \mathrm{O}_{3}$ (598.15).

ber.: $\quad 597.2627[\mathrm{M}+\mathrm{H}]^{+}$

gef.: 597.2627 (ESI-HRMS).

$6.5\{(1 S, 10 R)-7-A m i n o m e t h y l-5-b e n z y l o x y-1-(10-c h l o r-e t h y l)-3-[(5-(2-$ (N,N'-dimethylamino)-ethoxy)-indol-2-yl)carbonyl]-1,2-dihydro-3Hbenz $[e]$ indol\} $((1 S, 10 R)-81)$

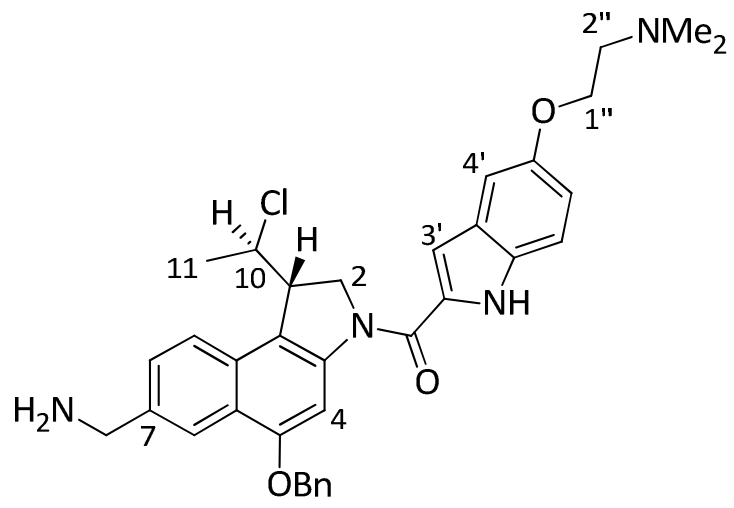

Eine Lösung des Nitrils (+)-(1S,10R)-88 (175 mg, $295 \mu \mathrm{mol}, 1.0$ Äq.) in einem Lösungsmittelgemisch aus absolutem THF $(6 \mathrm{~mL})$ und absolutem Ethanol $(12 \mathrm{~mL})$ wurde entgast und $1.25 \mathrm{M}$ Lösung von $\mathrm{HCl}$ in Ethanol ${ }^{165}(476 \mu \mathrm{L}, 594 \mu \mathrm{mol}, 2.0$ Äq.) 
sowie $\mathrm{H}_{2} \mathrm{O} \quad(3.0 \mathrm{~mL})$ zugetropft und $\mathrm{PtO}_{2} \cdot \mathrm{H}_{2} \mathrm{O} \quad(14.9 \mathrm{mg}, 59.4 \mu \mathrm{mol}, \quad 0.20$ Äq.) zugegeben. Man leitete kurz einen $\mathrm{H}_{2}$-Gasstrom durch die Reaktionsmischung und rührte dann für $8 \mathrm{~h}$ bei Raumtemperatur unter einer $\mathrm{H}_{2}$-Atmosphäre. Danach filtrierte man über eine Celite-Schicht vom Feststoff ab, spülte gründlich mit $\mathrm{MeOH}(300 \mathrm{~mL})$ nach und entfernte das Lösungsmittel im Vakuum. Nach Säulenchromatographie an Kieselgel $\left(\mathrm{CH}_{2} \mathrm{Cl}_{2} / \mathrm{MeOH}=5: 1\right)$ erhielt man die Zielverbindung $(15,10 R)-81$ als hell gelben Feststoff ( $87.2 \mathrm{mg}, 146 \mu \mathrm{mol}, 50 \%)$.

$\mathbf{R}_{\mathrm{f}}=0.14\left(\mathrm{CH}_{2} \mathrm{Cl}_{2} / \mathrm{MeOH}=5: 1\right)$.

${ }^{1} \mathrm{H}-\mathrm{NMR}\left(600 \mathrm{MHz}, \mathrm{DMSO}-\mathrm{d}_{6}\right): \delta=1.65\left(\mathrm{~d}, J=6.7 \mathrm{~Hz}, 3 \mathrm{H}, 11-\mathrm{H}_{3}\right), 2.24\left(\mathrm{~s}, 6 \mathrm{H}, \mathrm{NMe}_{2}\right)$, $2.66\left(t, J=5.8 \mathrm{~Hz}, 2 \mathrm{H}, 2^{\prime \prime}-\mathrm{H}_{2}\right), 4.01\left(\mathrm{~s}, 2 \mathrm{H}, 12-\mathrm{H}_{2}\right), 4.04-4.09,4.20-4.30,4.55-4.84$, $\left(3 \times \mathrm{m}, 6 \mathrm{H}, 1-\mathrm{H}, 2-\mathrm{H}_{2}, 10-\mathrm{H}, 1 "-\mathrm{H}_{2}\right), 5.32$ (q, J = 12.0 Hz, $2 \mathrm{H}, \mathrm{OCH}_{2} \mathrm{Ph}$ ), 6.92 (dd, $\left.J=8.9,2.4 \mathrm{~Hz}, 1 \mathrm{H}, 66^{\prime}-\mathrm{H}\right), 7.12-7.19\left(\mathrm{~m}, 2 \mathrm{H}, 3^{\prime}-\mathrm{H}, 4^{\prime}-\mathrm{H}\right), 7.30-7.47\left(\mathrm{~m}, 6 \mathrm{H}, 7^{\prime}-\mathrm{H}\right.$, $5 \times \mathrm{Ph}-\mathrm{H}), 7.58(\mathrm{~d}, J=7.4, \mathrm{~Hz}, 1 \mathrm{H}, 8-\mathrm{H}), 7.65$ (dd, J=8.6, $1.6 \mathrm{~Hz}, 1 \mathrm{H}, 6-\mathrm{H}), 7.97$ (d, $J=8.6 \mathrm{~Hz}, 1 \mathrm{H}, 9-\mathrm{H}), 8.21(\mathrm{~s}, 1 \mathrm{H}, 4-\mathrm{H}), 11.61(\mathrm{~s}, 1 \mathrm{H}, \mathrm{NH}) \mathrm{ppm}$.

${ }^{13} \mathrm{C}-\mathrm{NMR}\left(125 \mathrm{MHz}, \mathrm{DMSO}-\mathrm{d}_{6}\right): \delta=23.3$ (C-11), 45.5 (C-12), $45.9\left(\mathrm{NMe}_{2}\right), 46.1$ (C-1), 51.9 (C-2), 57.8 (C-2"), 61.4 (C-10), 66.2 (C-1"), $69.6\left(\mathrm{OCH}_{2} \mathrm{Ph}\right.$ ), 98.6 (C-4), 103.2 (C-4'), 105.4 (C-3'), 113.1 (C-7'), 115.8, 117.6 (C-5a, C-6'), 121.4, 122.4, 123.2, 127.3 (C-7, C-8, C-9, C-9b), 127.9, 128.4, 128.4, 131.6, 136.7 (C-6, C-9a, C-2', C-3a', C-7a'), 142.0 (C-3a), 152.9 (C-5'), 154.1 (C-5), 160.0 (C=0) ppm.

$\mathrm{C}_{35} \mathrm{H}_{37} \mathrm{ClN}_{4} \mathrm{O}_{3}$ (598.15).

ber.: $597.2625[\mathrm{M}+\mathrm{H}]^{+}$

gef.: 597.2627 (ESI-HRMS). 
6.6 \{(1R,10S)-7-Aminomethyl-5-benzyloxy-1-(10-chlor-ethyl)-3-[(5-(2( $N, N^{\prime}$-dimethylamino)-ethoxy)-indol-2-yl)carbonyl]-1,2-dihydro-3Hbenz[e]indol\} $((1 R, 10 S)-81)$

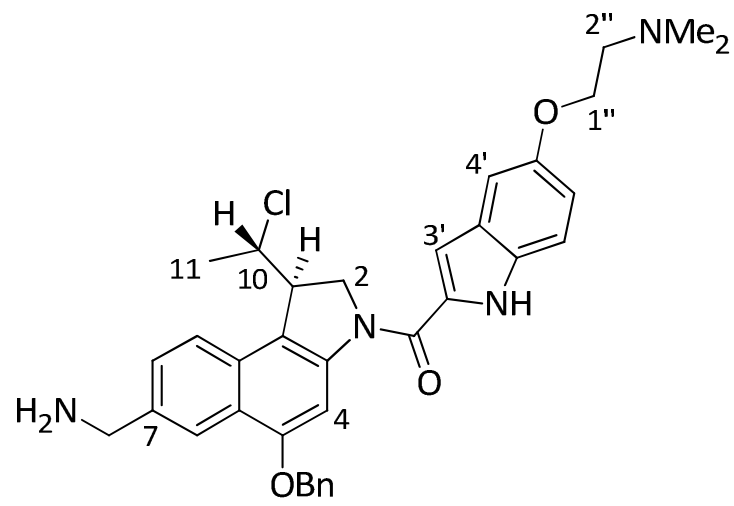

Eine Lösung des Nitrils (-)-(1S,10R)-88 (110 mg, $185 \mu \mathrm{mol}, 1.0$ Äq.) in einem Lösungsmittelgemisch aus absolutem THF $(4 \mathrm{~mL})$ und absolutem Ethanol $(8 \mathrm{~mL})$ wurde entgast und $1.25 \mathrm{M}$ Lösung von $\mathrm{HCl}$ in Ethanol ${ }^{165}(299 \mu \mathrm{L}, 371 \mu \mathrm{mol}, 2.0 \mathrm{Äq}$.) sowie $\mathrm{H}_{2} \mathrm{O}(1.9 \mathrm{~mL})$ zugetropft und $\mathrm{PtO}_{2} \cdot \mathrm{H}_{2} \mathrm{O}(9.3 \mathrm{mg}, 37.1 \mu \mathrm{mol}, 0.20$ Äq.) zugegeben. Man leitete kurz einen $\mathrm{H}_{2}$-Gasstrom durch die Reaktionsmischung und rührte dann für $8 \mathrm{~h}$ bei Raumtemperatur unter einer $\mathrm{H}_{2}$-Atmosphäre. Danach filtrierte man über eine Celite-Schicht vom Feststoff ab, spülte gründlich mit $\mathrm{MeOH}(250 \mathrm{~mL})$ nach und entfernte das Lösungsmittel im Vakuum. Nach Säulenchromatographie an Kieselgel $\left(\mathrm{CH}_{2} \mathrm{Cl}_{2} / \mathrm{MeOH}=5: 1\right)$ erhielt man die Zielverbindung $(1 S, 10 R)-81$ als hell gelben Feststoff (58 mg, $97 \mu \mathrm{mol}, 52 \%)$.

$\mathbf{R}_{\mathbf{f}}=0.14\left(\mathrm{CH}_{2} \mathrm{Cl}_{2} / \mathrm{MeOH}=5: 1\right)$.

${ }^{1} \mathrm{H}-\mathrm{NMR}\left(600 \mathrm{MHz}, \mathrm{DMSO}-\mathrm{d}_{6}\right): \delta=1.65\left(\mathrm{~d}, J=6.6 \mathrm{~Hz}, 3 \mathrm{H}, 11-\mathrm{H}_{3}\right), 2.25\left(\mathrm{~s}, 6 \mathrm{H}, \mathrm{NMe}_{2}\right)$, $2.67\left(\mathrm{t}, \mathrm{J}=5.8 \mathrm{~Hz}, 2 \mathrm{H}, 2^{\prime \prime}-\mathrm{H}_{2}\right), 4.02-4.12,4.20-4.32,4.56-4.85$, (3 × m, $8 \mathrm{H}, 12-\mathrm{H}_{2}$, 1-H, 2- $\left.\mathrm{H}_{2}, 10-\mathrm{H}, 1 "-\mathrm{H}_{2}\right), 5.27-5.38\left(\mathrm{~m}, 2 \mathrm{H}, \mathrm{OCH}_{2} \mathrm{Ph}\right), 6.93(\mathrm{dd}, J=8.8,2.4 \mathrm{~Hz}, 1 \mathrm{H}$, 6'-H), 7.13-7.19 (m, $\left.2 \mathrm{H}, 3^{\prime}-\mathrm{H}, 4^{\prime}-\mathrm{H}\right), 7.34-7.48\left(\mathrm{~m}, 6 \mathrm{H}, 7^{\prime}-\mathrm{H}, 5 \times \mathrm{Ph}-\mathrm{H}\right), 7.59(\mathrm{~d}$, $J=7.5, \mathrm{~Hz}, 1 \mathrm{H}, 8-\mathrm{H}), 7.65(\mathrm{~d}, J=8.8 \mathrm{~Hz}, 1 \mathrm{H}, 6-\mathrm{H}), 8.01(\mathrm{~d}, J=8.5 \mathrm{~Hz}, 1 \mathrm{H}, 9-\mathrm{H}), 8.25$ (s, $1 \mathrm{H}, 4-\mathrm{H}), 11.59$ (s, $1 \mathrm{H}, \mathrm{NH}) \mathrm{ppm}$.

${ }^{13} \mathrm{C}-\mathrm{NMR}\left(125 \mathrm{MHz}, \mathrm{DMSO}-\mathrm{d}_{6}\right): \delta=23.2$ (C-11), 45.5 (C-12), $45.9\left(\mathrm{NMe}_{2}\right), 46.1$ (C-1), 52.0 (C-2), 57.8 (C-2"), 61.4 (C-10), 66.2 (C-1"), $69.7\left(\mathrm{OCH}_{2} \mathrm{Ph}\right), 98.9$ (C-4), 103.4 (C-4'), 
105.4 (C-3'), 113.2 (C-7'), 115.9, 117.7 (C-5a, C-6'), 121.4, 122.4, 123.2, 127.3 (C-7, C-8, C-9, C-9b), 127.6, 128.4, 128.6, 131.7, 136.7 (C-6, C-9a, C-2', C-3a', C-7a'), 142.0 (C-3a), 153.0 (C-5'), 154.4 (C-5), 160.2 (C=O) ppm.

$\mathrm{C}_{35} \mathrm{H}_{37} \mathrm{ClN}_{4} \mathrm{O}_{3}$ (598.15).

ber.: $\quad 597.2606[\mathrm{M}+\mathrm{H}]^{+}$

gef.: $\quad 597.2627$ (ESI-HRMS).

6.7 $\left\{(1 S, 10 R)-2-\left(\left\{5-B e n z y l o x y-1-(10-c h l o r-e t h y l)-3-\left[\left(5-\left(2-N, N^{\prime}-\right.\right.\right.\right.\right.\right.$ dimethylamino)-ethoxy)-1H-indol-2-yl)carbonyl]-1,2-dihydro-3Hbenz[e]indol-7-ylmethyl\}-carbamoyl)-ethyl]-carbaminsäurebenzylester ((1S,10R)-90)

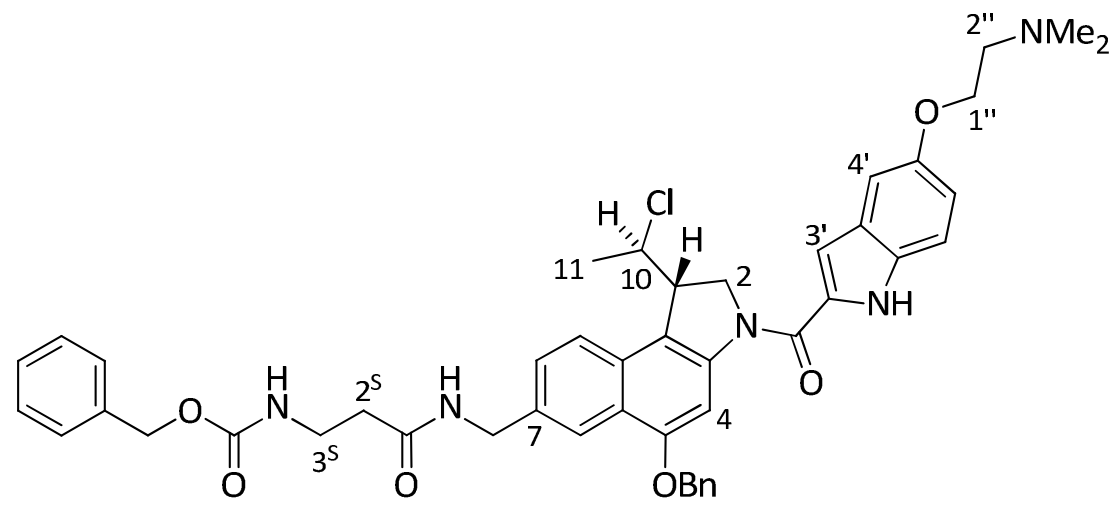

Zu einer auf $0{ }^{\circ} \mathrm{C}$ gekühlten Lösung des Amins (1S,10R)-81 (20.0 mg, $33.5 \mathrm{mmol}$, 1.0 Äq.) in absolutem DMF (4.0 mL) wurde $i \operatorname{Pr}_{2} \operatorname{Net}(17 \mu \mathrm{L}, 101 \mu \mathrm{mol}, 3.0$ Äq.), PyBOP (19.2 mg, $37 \mu \mathrm{mol}, 1.1$ Äq.) und Cbz-geschütztes $\beta$-Alanin (105) (8.2 mg, $37 \mu \mathrm{mol}$, 1.1 Äq.) gegeben, $30 \mathrm{~min}$ bei $0^{\circ} \mathrm{C}$ und $4 \mathrm{~h}$ bei Raumtemperatur gerührt. Das Lösungsmittel wurde im Vakuum entfernt und nach Säulenchromatographie an Kieselgel $\left(\mathrm{CH}_{2} \mathrm{Cl}_{2} / \mathrm{MeOH}=7: 1\right)$ erhielt man die Zielverbindung $(1 S, 10 R)-90$ als hell gelben Feststoff (22.1 mg, $27.6 \mu \mathrm{mol}, 82 \%)$.

$\mathbf{R}_{\mathbf{f}}=0.60\left(\mathrm{CH}_{2} \mathrm{Cl}_{2} / \mathrm{MeOH}=5: 1\right)$.

${ }^{1} \mathrm{H}-\mathrm{NMR}\left(600 \mathrm{MHz}, \mathrm{DMSO}-\mathrm{d}_{6}\right): \delta=1.64\left(\mathrm{~d}, J=6.6 \mathrm{~Hz}, 3 \mathrm{H}, 11-\mathrm{H}_{3}\right), 2.25\left(\mathrm{~s}, 6 \mathrm{H}, \mathrm{NMe}_{2}\right)$, $2.37\left(t, J=7.3 \mathrm{~Hz}, 2 \mathrm{H}, 2 \mathrm{~S}-\mathrm{H}_{2}\right), 2.65-2.70\left(\mathrm{~m}, 2 \mathrm{H}, 2^{\prime \prime}-\mathrm{H}_{2}\right), 3.22-3.35\left(\mathrm{~m}, 2 \mathrm{H}, 3 \mathrm{~S}-\mathrm{H}_{2}\right)$, $4.07\left(\mathrm{t}, J=5.9 \mathrm{~Hz}, 2 \mathrm{H}, 1^{\prime \prime}-\mathrm{H}_{2}\right), 4.22(\mathrm{~d}, J=9.4 \mathrm{~Hz}, 1 \mathrm{H}, 1-\mathrm{H}), 4.37-4.46\left(\mathrm{~m}, 2 \mathrm{H}, 12-\mathrm{H}_{2}\right)$, 
$4.61(\mathrm{dd}, J=11.0,2.1 \mathrm{~Hz}, 1 \mathrm{H}, 2-\mathrm{Ha}), 4.69-4.83\left(\mathrm{~m}, 3 \mathrm{H}, 2-\mathrm{H}_{\mathrm{b}}, 10-\mathrm{H}\right), 5.02(\mathrm{~s}, 2 \mathrm{H}$, $\mathrm{OCH}_{2} \mathrm{Ph}$ ), 5.31 (q, J = 11.9 Hz, $2 \mathrm{H}, \mathrm{OCH}_{2} \mathrm{Ph}$ ), 6.93 (dd, J=8.9, 2.4 Hz, $1 \mathrm{H}, 66^{\prime}-\mathrm{H}$ ), 7.13$7.58\left(\mathrm{~m}, 13 \mathrm{H}, 10 \times \mathrm{Ph}-\mathrm{H}, 3^{\prime}-\mathrm{H}, 4^{\prime}-\mathrm{H}, 7^{\prime}-\mathrm{H}, 8-\mathrm{H}\right), 7.92$ (d, J= $\left.8.6 \mathrm{~Hz}, 1 \mathrm{H}, 9-\mathrm{H}\right), 8.08$ (s, $1 \mathrm{H}, 4-\mathrm{H}), 8.47(\mathrm{t}, J=5.9 \mathrm{~Hz}, 1 \mathrm{H}, 6-\mathrm{H}), 11.55-11.61(\mathrm{~m}, 1 \mathrm{H}, \mathrm{NH}) \mathrm{ppm}$.

${ }^{13} \mathrm{C}-\mathrm{NMR}\left(125 \mathrm{MHz}, \mathrm{DMSO}-\mathrm{d}_{6}\right): \delta=23.3(\mathrm{C}-11), 25.8\left(\mathrm{C}-2^{\mathrm{S}}\right), 30.4\left(\mathrm{C}-3^{\mathrm{S}}\right), 42.3(\mathrm{C}-12)$, $45.5\left(\mathrm{NMe}_{2}\right), 45.9$ (C-1), 51.9 (C-2), 57.7 (C-2"), 61.3 (C-10), 65.1 (C-1"), 98.5 (C-4), 103.2 (C-4'), 105.3 (C-3'), 113.1 (C-7'), 115.8 (C-6'), 117.5, 120.5 (C-7, C-9b), 122.4, $123.3,127.3,127.4,127.5,127.6,127.6,127.8,128.2,128.4,130.8,131.6,134.9$, 136.8, 137.1, (12 × Ph-C, C-5a, C-8, C-9a, C-2', C-3a', C-7a'), 141.8 (C-3a), 152.9, 154.0 (C-5, C-5'), 155.9 (NC=0), 160.0 ( $\underline{\mathrm{COOCH}} 2 \mathrm{Ph}),(170.1$ (NHC=O) ppm.

$\mathrm{C}_{46} \mathrm{H}_{48} \mathrm{ClN}_{5} \mathrm{O}_{6}$ (802.34).

ber.: $802.3375[\mathrm{M}+\mathrm{H}]^{+}$

gef.: 802.3366 (ESI-HRMS).

$6.8\left\{(1 S, 10 R)-3-A m i n o-N-2-\left(\left\{1-(10-c h l o r-e t h y l)-3-\left[\left(5-\left(2-N, N^{\prime}-\right.\right.\right.\right.\right.\right.$ dimethylamino)-ethoxy)-1H-indol-2-yl)carbonyl]-5-hydroxy-1,2dihydro-3H-benz[e]indol-7-ylmethyl\}-propionamid $((1 S, 10 R)-106)$

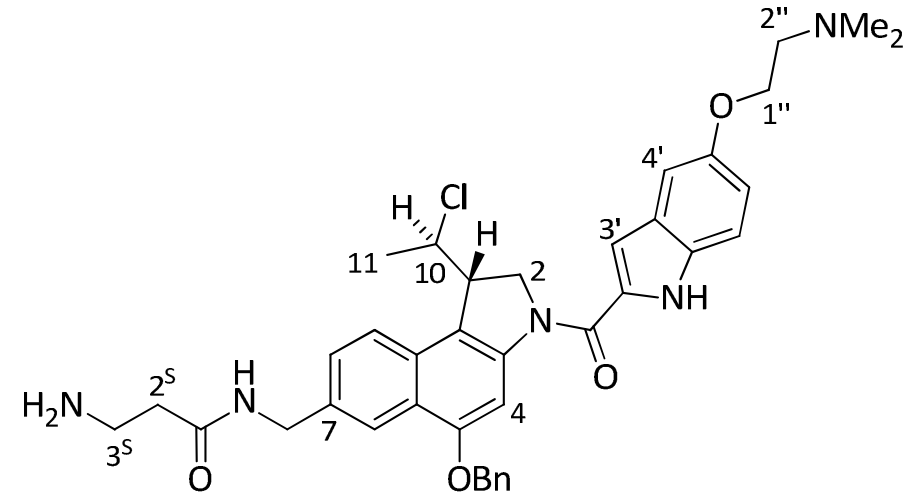

Zu einer Lösung des CBZ-geschützten Amins (1S,10R)-90 (20.0 mg, $25.0 \mu \mathrm{mol}, 1.0$ Äq.) in $\mathrm{MeOH} / \mathrm{AcOH} / \mathrm{H}_{2} \mathrm{O}(3: 1: 1,10 \mathrm{~mL}$ ) wird bei Raumtemperatur Pd/C (10\%-ig, $5.3 \mathrm{mg}$, $4.99 \mu \mathrm{mol}, 0.2$ Äq.) zugegeben und für $8 \mathrm{~h}$ gerührt. Es wurde mittels Spritzenfilter vom Pd abgetrennt und mit $\mathrm{MeOH}(2 \times 5 \mathrm{~mL})$ gewaschen. Das Lösungsmittel wurde entfernt und der Rückstand in einem 1:1 Gemisch aus $\mathrm{H}_{2} \mathrm{O} / \mathrm{MeOH}$ (4 mL) aufgenommen. Je $2 \mathrm{~mL}$ dieser Lösung wurden in das präparative HPLC-System (s. u.) 
injiziert. Fraktioniertes Auffangen des Eluats, Entfernung der Lösungsmittel unter vermindertem Druck sowie Entfernung des restlichen Lösungsmittels mittels Gefriertrocknung lieferte die Zielverbindung $(1 S, 10 R)$-106 als blassgelben Feststoff $(8.2 \mathrm{mg}$, $14.2 \mu \mathrm{mol}, 57 \%)$.

HPLC (analytisch):

Säule: $\quad$ Kromasil 100 C18

\begin{tabular}{|c|c|c|}
\hline \multirow[t]{6}{*}{ Gradient: } & Zeit [min] & $\mathrm{H}_{2} \mathrm{O}(0.06 \% \mathrm{HCl}) / \mathrm{MeOH}$ \\
\hline & 0 & $70 / 30$ \\
\hline & $0-20$ & $70 / 30 \rightarrow 0 / 100$ \\
\hline & $20-25$ & $0 / 100$ \\
\hline & $25-26$ & $0 / 100 \rightarrow 70 / 30$ \\
\hline & $26-30$ & $70 / 30$ \\
\hline
\end{tabular}

Fluss: $\quad 0.8 \mathrm{~mL} \mathrm{~min}^{-1}$

$t_{\mathrm{R}}: \quad 10.9 \mathrm{~min}$

HPLC (präparativ):

Säule: Kromasil 100 C18

\begin{tabular}{|c|c|c|}
\hline \multirow[t]{6}{*}{ Gradient: } & Zeit [min] & $\mathrm{H}_{2} \mathrm{O}(0.06 \% \mathrm{HCl}) / \mathrm{MeOH}$ \\
\hline & $0-1$ & $70 / 30$ \\
\hline & $1-20$ & $70 / 30 \rightarrow 0 / 100$ \\
\hline & $20-25$ & $0 / 100$ \\
\hline & $25-26$ & $0 / 100 \rightarrow 70 / 30$ \\
\hline & $26-30$ & $70 / 30$ \\
\hline
\end{tabular}


Fluss: $\quad 18 \mathrm{~mL} \mathrm{~min}^{-1}$

$t_{\mathrm{R}}: \quad 8.0 \mathrm{~min}$

${ }^{1} \mathrm{H}-\mathrm{NMR}\left(600 \mathrm{MHz}, \mathrm{DMSO}-\mathrm{d}_{6}\right): \delta=1.62\left(\mathrm{~d}, J=6.6 \mathrm{~Hz}, 3 \mathrm{H}, 11-\mathrm{H}_{3}\right), 2.57(\mathrm{t}, J=6.8 \mathrm{~Hz}$, $\left.2 \mathrm{H}, 2 \mathrm{~S}-\mathrm{H}_{2}\right), 2.83\left(\mathrm{~s}, 6 \mathrm{H}, \mathrm{NMe}_{2}\right), 3.04\left(\mathrm{t}, J=6.8 \mathrm{~Hz}, 2 \mathrm{H}, 3 \mathrm{~S}-\mathrm{H}_{2}\right), 3.41-3.52(\mathrm{~m}, 2 \mathrm{H}$, 2"- $\left.\mathrm{H}_{2}\right), 4.17(\mathrm{~d}, J=9.3 \mathrm{~Hz}, 1 \mathrm{H}, 1-\mathrm{H}), 4.34\left(\mathrm{t}, J=4.9 \mathrm{~Hz}, 2 \mathrm{H}, 1 "-\mathrm{H}_{2}\right), 4.39-4.50(\mathrm{~m}, 2 \mathrm{H}$, 12- $\left.\mathrm{H}_{2}\right), 4.57\left(\mathrm{dd}, J=8.9,2.4 \mathrm{~Hz}, 1 \mathrm{H}, 2-\mathrm{H}_{\mathrm{a}}\right), 4.70-4.77\left(\mathrm{~m}, 2 \mathrm{H}, 2-\mathrm{H}_{\mathrm{b}}, 10-\mathrm{H}\right), 7.00$ (dd, $\left.J=8.9,2.4 \mathrm{~Hz}, 1 \mathrm{H}, 6^{\prime}-\mathrm{H}\right), 7.17$ (s, $\left.1 \mathrm{H}, 3^{\prime}-\mathrm{H}\right), 7.25$ (d, J = 2.2 Hz, $\left.1 \mathrm{H}, 4^{\prime}-\mathrm{H}\right), 7.40-7.47$ (m, $\left.2 \mathrm{H}, 8-\mathrm{H}, 7^{\prime}-\mathrm{H}\right), 7.82\left(\mathrm{~s}_{\mathrm{br}}, 2 \mathrm{H}, 3 \mathrm{~S}-\mathrm{NH}_{2}\right), 7.87(\mathrm{dd}, J=8.6,1.7 \mathrm{~Hz}, 1 \mathrm{H}, 9-\mathrm{H}), 7.97-8.03$ (m, $2 \mathrm{H}, 4-\mathrm{H}, 6-\mathrm{H}), 8.69$ (s, $1 \mathrm{H}, 12-\mathrm{NH}), 10.37$ (s, $1 \mathrm{H}, \mathrm{OH}), 11.62$ (s, $1 \mathrm{H}, \mathrm{NH}) \mathrm{ppm}$.

${ }^{13}$ C-NMR (125 MHz, DMSO-d 6 ): $\delta=23.3$ (C-11), 32.0 (C-2S), 35.3 (C-3S), 42.5 (C-12), $43.0\left(\mathrm{NMe}_{2}\right), 45.9$ (C-1), 52.1 (C-2), 55.7 (C-2"), 61.5 (C-10), 63.0 (C-1"), 100.5 (C-4), 104.0 (C-4'), 105.2 (C-3'), 113.2 (C-7'), 115.5 (C-6'), 115.8, 121.3 (C-7, C-9b), 123.1 (C-9), 123.7 (C-6), 127.2, 127.4, 128.9, 131.3, 131.8, 133.6 (C-5a, C-8, C-9a, C-2', C-3a', C-7a'), 141.9 (C-3a), 151.9, 153.6 (C-5, C-5'), 159.8 (NC=O), 169.2 (NHC=O) ppm.

$\mathrm{C}_{31} \mathrm{H}_{36} \mathrm{ClN}_{5} \mathrm{O}_{4}(578.10)$.

ber.: $578.2529[\mathrm{M}+\mathrm{H}]^{+}$

gef.: 578.2532 (ESI-HRMS).

$6.9\{(1 R, 10 S)$-[7-Aminomethyl-1-(1-chlor-ethyl)-5-hydroxy-1,2-dihydrobenz[e]indol-3-yl]-[5-(2-dimethylamino-ethoxy)-1H-indol-2-yl]methanon $((1 R, 10 S)-87)$

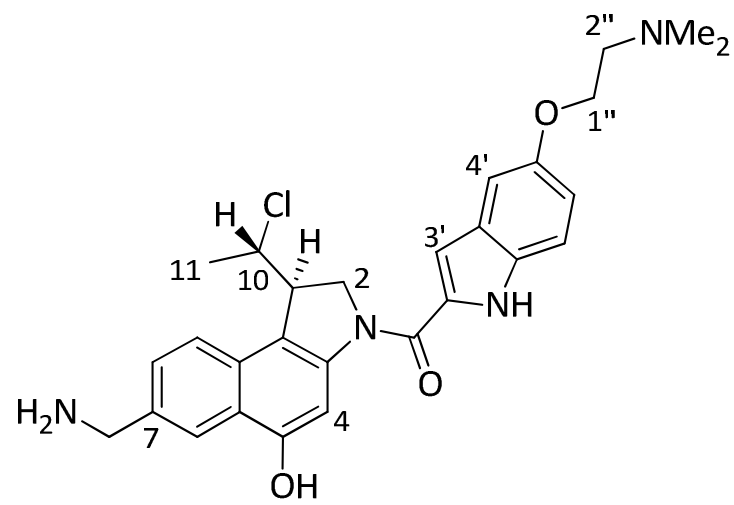


Zu einer Lösung des Benzyl-geschützten Naphtols (1R,10S)-91 (58.0 mg, $97.1 \mu \mathrm{mol}$, 1.0 Äq.) in $\mathrm{MeOH} / \mathrm{AcOH} / \mathrm{H}_{2} \mathrm{O}(3: 1: 1,40 \mathrm{~mL})$ wird bei Raumtemperatur Pd/C (10\%-ig, $20.6 \mathrm{mg}, 19.4 \mu \mathrm{mol}, 0.2 \mathrm{Äq}$.) zugegeben und für $9 \mathrm{~h}$ unter $\mathrm{H}_{2}$-atm gerührt. Es wurde mittels Spritzenfilter vom Pd abgetrennt und mit $\mathrm{MeOH}(2 \times 5 \mathrm{~mL})$ gewaschen. Das Lösungsmittel wurde entfernt und der Rückstand in einem 1:2 Gemisch aus $\mathrm{H}_{2} \mathrm{O} / \mathrm{MeOH}(6 \mathrm{~mL})$ aufgenommen und Je $1.0 \mathrm{~mL}$ dieser Lösung wurden in das präparative HPLC-System (s. u.) injiziert. Fraktioniertes Auffangen des Eluats, Entfernung der Lösungsmittel unter vermindertem Druck sowie Entfernung des restlichen Lösungsmittels mittels Gefriertrocknung lieferte die Zielverbindung $(1 R, 10 S)-87$ als blassgelben Feststoff ( $25.1 \mathrm{mg}, 49.5 \mu \mathrm{mol}, 51 \%)$.

HPLC (analytisch):

Säule: Kromasil 100 C18

\begin{tabular}{|c|c|c|}
\hline \multirow[t]{6}{*}{ Gradient: } & Zeit [min] & $\mathrm{H}_{2} \mathrm{O}(0.06 \% \mathrm{HCl}) / \mathrm{MeOH}$ \\
\hline & 0 & $70 / 30$ \\
\hline & $0-20$ & $70 / 30 \rightarrow 0 / 100$ \\
\hline & $20-25$ & $0 / 100$ \\
\hline & $25-26$ & $0 / 100 \rightarrow 70 / 30$ \\
\hline & $26-30$ & $70 / 30$ \\
\hline
\end{tabular}

Fluss: $\quad 0.8 \mathrm{~mL} \mathrm{~min}^{-1}$
$t_{\mathrm{R}}$
$9.72 \mathrm{~min}$ 
HPLC (präparativ):

Säule: $\quad$ Kromasil 100 C18

\begin{tabular}{ccc} 
Gradient: & Zeit [min] & $\mathrm{H}_{2} \mathrm{O}(0.06 \% \mathrm{HCl}) / \mathrm{MeOH}$ \\
\cline { 2 - 3 } & $0-1$ & $70 / 30$ \\
$20-25$ & $0 / 100$ \\
& $25-26$ & $0 / 100 \rightarrow 70 / 30$ \\
& $26-30$ & $70 / 30$ \\
Fluss: & $18 \mathrm{~mL} \mathrm{~min}^{-1}$ & \\
$t_{\mathrm{R}}:$ & $7.00 \mathrm{~min}^{2}$ &
\end{tabular}

${ }^{1} \mathrm{H}-\mathrm{NMR}\left(600 \mathrm{MHz}, \mathrm{DMSO}-\mathrm{d}_{6}\right): \delta=1.63\left(\mathrm{~d}, J=6.6 \mathrm{~Hz}, 3 \mathrm{H}, 11-\mathrm{H}_{3}\right), 2.86\left(\mathrm{~s}, 6 \mathrm{H}, \mathrm{NMe}_{2}\right)$, $3.52\left(t, J=5.0 \mathrm{~Hz}, 2 \mathrm{H}, 2^{\prime \prime}-\mathrm{H}_{2}\right), 4.13-4.23\left(\mathrm{~m}, 3 \mathrm{H}, 1-\mathrm{H}, 12-\mathrm{H}_{2}\right), 4.38(\mathrm{t}, J=5.0 \mathrm{~Hz}, 1 \mathrm{H}$, $\left.1^{\prime \prime}-\mathrm{H}_{2}\right), 4.58\left(\mathrm{dd}, J=10.9,2.2 \mathrm{~Hz}, 1 \mathrm{H}, 2-\mathrm{H}_{\mathrm{a}}\right), 4.71-4.79\left(\mathrm{~m}, 2 \mathrm{H}, 2-\mathrm{H}_{\mathrm{b}}, 10-\mathrm{H}\right), 7.01$ (dd, $\left.J=8.9,2.4 \mathrm{~Hz}, 1 \mathrm{H}, 6^{\prime}-\mathrm{H}\right), 7.19\left(\mathrm{~s}, 1 \mathrm{H}, 3^{\prime}-\mathrm{H}\right), 7.26\left(\mathrm{~d}, J=2.3 \mathrm{~Hz}, 1 \mathrm{H}, 4^{\prime}-\mathrm{H}\right), 7.45$ (d, $\left.J=8.9 \mathrm{~Hz}, 1 \mathrm{H}, 7^{\prime}-\mathrm{H}\right), 7.63(\mathrm{dd}, J=8.6,1.7 \mathrm{~Hz}, 1 \mathrm{H}, 8-\mathrm{H}), 7.96(\mathrm{~d}, J=8.6 \mathrm{~Hz}, 1 \mathrm{H}, 9-\mathrm{H})$, $8.01(\mathrm{~s}, 1 \mathrm{H}, 4-\mathrm{H}), 8.23(\mathrm{~s}, 1 \mathrm{H}, 6-\mathrm{H}), 10.55$ (s, $1 \mathrm{H}, \mathrm{OH}), 11.65$ (s, $1 \mathrm{H}, \mathrm{NH}) \mathrm{ppm}$.

${ }^{13}$ C-NMR (125 MHz, DMSO-d 6 ): $\delta=23.3$ (C-11), 42.5 (C-12), $42.8\left(\mathrm{NMe}_{2}\right), 46.0$ (C-1), 52.0 (C-2), 55.4 (C-2"), 61.5 (C-10), 62.9 (C-1"), 100.7 (C-4), 104.0 (C-4'), 105.3 (C-3'), 113.2 (C-7'), 115.6 (C-6'), 115.9, 121.7 (C-7, C-9b), 123.4 (C-9), 123.7 (C-6), 127.4, 127.7, 128.3, 129.5, 131.2, 132.0 (C-5a, C-8, C-9a, C-2', C-3a', C-7a'), 142.7 (C-3a), 152.0, $154.0\left(\mathrm{C}-5, \mathrm{C}-5^{\prime}\right), 159.9$ (C=O) ppm.

$\mathrm{C}_{28} \mathrm{H}_{31} \mathrm{ClN}_{4} \mathrm{O}_{3}$ (507.02).

ber.: $\quad 507.2157[\mathrm{M}+\mathrm{H}]^{+}$

gef.: 507.2153 (ESI-HRMS). 
$6.10\{(1 S, 10 R)-[7-A m i n o m e t h y l-1-(1-c h l o r-e t h y l)-5-h y d r o x y-1,2-d i h y d r o-$ benz[e]indol-3-yl]-[5-(2-dimethylamino-ethoxy)-1H-indol-2-yl]methanon $(1 S, 10 R)-87$

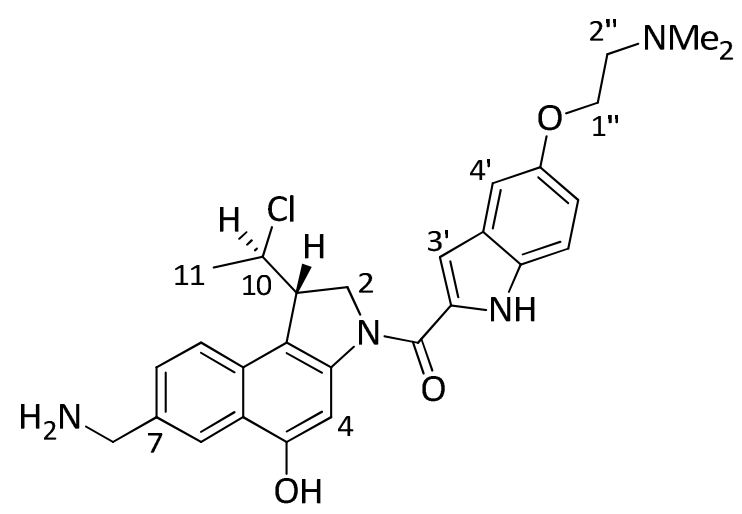

Zu einer Lösung des Benzyl-geschützten Naphtols (1S,10R)-91 (20.0 mg, $33.5 \mu \mathrm{mol}$, 1.0 Äq.) in $\mathrm{MeOH} / \mathrm{AcOH} / \mathrm{H}_{2} \mathrm{O}(3: 1: 1,15 \mathrm{~mL})$ wird bei Raumtemperatur $\mathrm{Pd} / \mathrm{C}(10 \%$-ig, $7.1 \mathrm{mg}, 6.7 \mu \mathrm{mol}, 0.2 \mathrm{Äq}$.) zugegeben und für $6 \mathrm{~h}$ unter $\mathrm{H}_{2}$-atm gerührt. Es wurde mittels Spritzenfilter vom Pd abgetrennt und mit $\mathrm{MeOH}(2 \times 5 \mathrm{~mL})$ gewaschen. Das Lösungsmittel wurde entfernt und der Rückstand in einem 1:2 Gemisch aus $\mathrm{H}_{2} \mathrm{O} / \mathrm{MeOH}(7.5 \mathrm{~mL})$ aufgenommen und je $1.5 \mathrm{~mL}$ dieser Lösung wurden in das präparative HPLC-System (s. u.) injiziert. Fraktioniertes Auffangen des Eluats, Entfernung der Lösungsmittel unter vermindertem Druck sowie Entfernung des restlichen Lösungsmittels mittels Gefriertrocknung lieferte die Zielverbindung (1S,10R)-87 als blassgelben Feststoff (11.4 mg, $22.5 \mu \mathrm{mol}, 67 \%)$.

HPLC (analytisch):

Säule: Kromasil $100 \mathrm{C} 18$

\begin{tabular}{ccc}
\cline { 2 - 3 } Gradient: & Zeit [min] & $\mathrm{H}_{2} \mathrm{O}(0.06 \% \mathrm{HCl}) / \mathrm{MeOH}$ \\
\cline { 2 - 3 } & 0 & $70 / 30$ \\
$0-20$ & $70 / 30 \rightarrow 0 / 100$ \\
$20-25$ & $0 / 100$ \\
$25-26$ & $0 / 100 \rightarrow 70 / 30$
\end{tabular}


$26-30$

$70 / 30$

Fluss: $\quad 0.8 \mathrm{~mL} \mathrm{~min}^{-1}$

$t_{\mathrm{R}}: \quad 9.40 \mathrm{~min}$

HPLC (präparativ):

Säule: $\quad$ Kromasil 100 C18

\begin{tabular}{ccc}
\cline { 2 - 3 } Gradient: & Zeit [min] & $\mathrm{H}_{2} \mathrm{O}(0.06 \% \mathrm{HCl}) / \mathrm{MeOH}$ \\
\cline { 2 - 3 } & $0-1$ & $70 / 30$ \\
$20-25$ & $0 / 100$ \\
& $25-26$ & $0 / 100 \rightarrow 70 / 30$ \\
& $26-30$ & $70 / 30$ \\
Fluss: & $18 \mathrm{~mL} \mathrm{~min}^{-1}$ & \\
$t_{\mathrm{R}}:$ & $7.10 \mathrm{~min}^{20}$ &
\end{tabular}

${ }^{1} \mathrm{H}-\mathrm{NMR}\left(600 \mathrm{MHz}, \mathrm{DMSO}-\mathrm{d}_{6}\right): \delta=1.63\left(\mathrm{~d}, J=6.6 \mathrm{~Hz}, 3 \mathrm{H}, 11-\mathrm{H}_{3}\right), 2.84\left(\mathrm{~s}, 6 \mathrm{H}, \mathrm{NMe}_{2}\right)$, 3.47-3.51 (m, $\left.2 \mathrm{H}, 2^{\prime \prime}-\mathrm{H}_{2}\right), 4.14-4.23\left(\mathrm{~m}, 3 \mathrm{H}, 1-\mathrm{H}, 12-\mathrm{H}_{2}\right), 4.35-4.40\left(\mathrm{~m}, 1 \mathrm{H}, 1 "-\mathrm{H}_{2}\right)$, $4.58\left(\mathrm{dd}, J=10.9,2.0 \mathrm{~Hz}, 1 \mathrm{H}, 2-\mathrm{H}_{\mathrm{a}}\right), 4.71-4.79\left(\mathrm{~m}, 2 \mathrm{H}, 2-\mathrm{H}_{\mathrm{b}}, 10-\mathrm{H}\right), 7.01(\mathrm{dd}, J=8.9$, $\left.2.3 \mathrm{~Hz}, 1 \mathrm{H}, 6^{\prime}-\mathrm{H}\right), 7.18\left(\mathrm{~s}, 1 \mathrm{H}, 3^{\prime}-\mathrm{H}\right), 7.26\left(\mathrm{~d}, J=2.1 \mathrm{~Hz}, 1 \mathrm{H}, 4^{\prime}-\mathrm{H}\right), 7.45(\mathrm{~d}, J=8.9 \mathrm{~Hz}$, $\left.1 \mathrm{H}, 7^{\prime}-\mathrm{H}\right), 7.63(\mathrm{~d}, J=8.7 \mathrm{~Hz}, 1 \mathrm{H}, 8-\mathrm{H}), 7.96(\mathrm{~d}, J=8.6 \mathrm{~Hz}, 1 \mathrm{H}, 9-\mathrm{H}), 8.01(\mathrm{~s}, 1 \mathrm{H}, 4-\mathrm{H})$, $8.23(\mathrm{~s}, 1 \mathrm{H}, 6-\mathrm{H}), 10.55$ (s, $1 \mathrm{H}, \mathrm{OH}), 11.64(\mathrm{~s}, 1 \mathrm{H}, \mathrm{NH}) \mathrm{ppm}$.

${ }^{13} \mathrm{C}-\mathrm{NMR}\left(125 \mathrm{MHz}, \mathrm{DMSO}-\mathrm{d}_{6}\right): \delta=23.3$ (C-11), 42.5 (C-12), $42.8\left(\mathrm{NMe}_{2}\right), 45.8(\mathrm{C}-1)$, 52.1 (C-2), 55.5 (C-2"), 61.5 (C-10), 63.0 (C-1"), 100.7 (C-4), 104.0 (C-4'), 105.4 (C-3'), 113.2 (C-7'), 115.6 (C-6'), 115.9, 121.7 (C-7, C-9b), 123.4 (C-9), 123.7 (C-6), 127.4, 
127.7, 128.3, 129.5, 131.2, 132.0 (C-5a, C-8, C-9a, C-2', C-3a', C-7a'), 142.7 (C-3a), 152.0, $154.0\left(\mathrm{C}-5, \mathrm{C}-5^{\prime}\right), 159.9$ (C=0) ppm.

$\mathrm{C}_{28} \mathrm{H}_{31} \mathrm{CIN}_{4} \mathrm{O}_{3}$ (507.02).

ber.: $\quad 507.2157[\mathrm{M}+\mathrm{H}]^{+}$

gef.: 507.2152 (ESI-HRMS). 
7 Synthese der fluoreszenzmarkierten anti-Methyl-seco-CBIDMAI-Derivate $(1 S, 10 R)-71$ und $(1 R, 10 S)-71$

\subsection{3-[4-(5-(4-Dimethylaminophenyl)oxazol-2-yl)benzolsulfonylamino]- propionsäure-[(1S,10R)-1-(10-chlor-ethyl)-3-[(5-(2-(N,N'-dimethyl- amino)ethoxy)indol-2-yl)carbonyl]-5-hydroxy-1,2-dihydro-3H- benz[e]indol-7-ylmethyl]amid ((1S,10R)-71)}

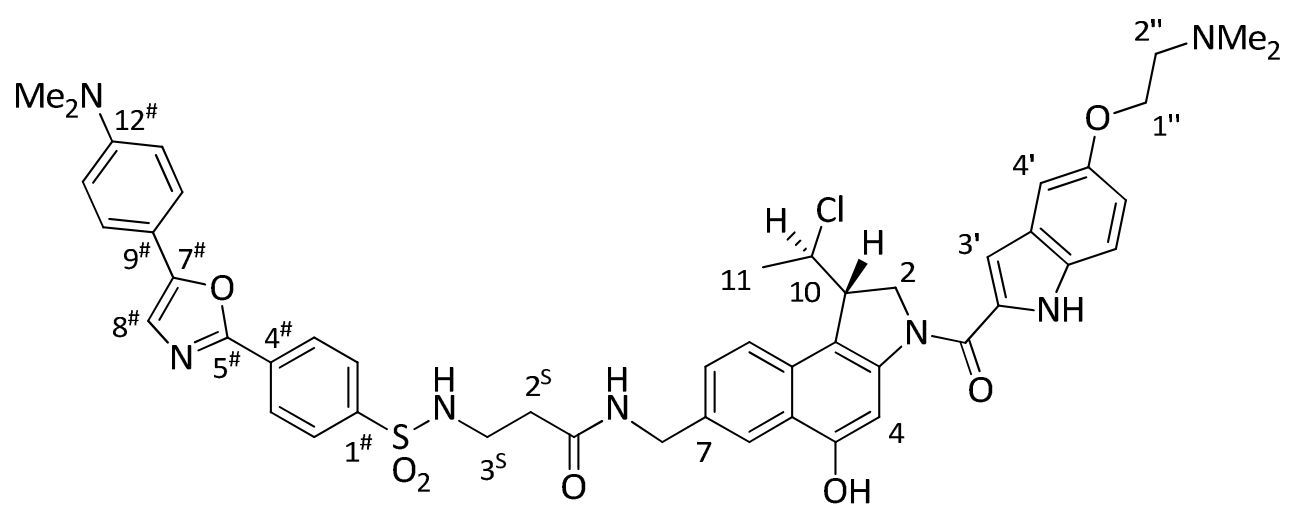

Zu einer Lösung des NHS-Esters 61 (7.5 mg, $14.6 \mu \mathrm{mol}, 1.0$ Äq.) in absolutem DMF $(200 \mu \mathrm{L})$ wurde eine Lösung des Phenols (1S,10R)-87 (9.5 mg, $17.5 \mu \mathrm{mol}, 1.2$ Äq.) und $i$ - $\operatorname{Pr}_{2}$ NEt (14.0 $\mu \mathrm{L}, 98 \mu \mathrm{mol}, 5.8$ Äq.) in absolutem DMF (150 $\left.\mu \mathrm{L}\right)$ getropft. Nach $23 \mathrm{~h}$ Rühren bei Raumtemperatur wurde die Reaktionslösung mit bidestilliertem $\mathrm{H}_{2} \mathrm{O}$ $(0.06 \% \mathrm{HCl}, 3.0 \mathrm{~mL})$ und $\mathrm{MeOH}(6.0 \mathrm{~mL})$ verdünnt. Je $1.5 \mathrm{~mL}$ dieser Lösung wurden in das präparative HPLC-System (s. u.) injiziert. Fraktioniertes Auffangen des Eluats, Entfernung der Lösungsmittel unter vermindertem Druck sowie Entfernung des restlichen Lösungsmittels mittels Gefriertrocknung lieferte das Hydroxyderivat (1S,10R)-111 (3.6 mg, $4.07 \mu \mathrm{mol}, 28 \%)$, sowie eine Mischung der Zielverbindung $(1 S, 10 R)-71$ und des Cl-substituierten Methoxyderivates (1S,10R)-110. Das Gemisch wurde in $\mathrm{MeOH}(5.0 \mathrm{~mL})$ aufgenommen. Je $1.0 \mathrm{~mL}$ dieser Lösung wurden in das präparative HPLC-System (s. u.) injiziert. Fraktioniertes Auffangen des Eluats, Entfernung der Lösungsmittel unter vermindertem Druck sowie Entfernung des restlichen Lösungsmittels mittels Gefriertrocknung lieferte die Zielverbindung als hellgelben Feststoff (1S,10R)-71 (4.0 mg, $4.42 \mu \mathrm{mol}, 30 \%)$, sowie das Cl-substituierte Methoxyderivat (1S,10R)-110 (1.8 mg, $2.00 \mu \mathrm{mol}, 14 \%)$. 
1. Aufreinigung HPLC (analytisch):

Säule: Kromasil 100 C18

\begin{tabular}{|c|c|c|}
\hline \multirow[t]{5}{*}{ Gradient: } & Zeit [min] & $\mathrm{H}_{2} \mathrm{O}(0.06 \% \mathrm{HCl}) / \mathrm{MeOH}$ \\
\hline & 0 & $50 / 50$ \\
\hline & $0-20$ & $50 / 50 \rightarrow 0 / 100$ \\
\hline & $20-26$ & $0 / 100$ \\
\hline & $26-30$ & $0 / 100 \rightarrow 50 / 50$ \\
\hline
\end{tabular}

Fluss: $\quad 0.8 \mathrm{~mL} \mathrm{~min}^{-1}$

$t_{\mathrm{R}}: \quad 19.1 \mathrm{~min} \mathbf{1 1 1} \quad 21.3 \mathrm{~min}$. Gemisch aus 110 \& 71

1. Aufreinigung HPLC (präparativ):

Säule: Kromasil 100 C18

\begin{tabular}{|c|c|c|}
\hline \multirow[t]{6}{*}{ Gradient: } & Zeit [min] & $\mathrm{H}_{2} \mathrm{O}(0.06 \% \mathrm{HCl}) / \mathrm{MeOH}$ \\
\hline & 0 & $50 / 50$ \\
\hline & $0-20$ & $50 / 50 \rightarrow 25 / 75$ \\
\hline & $20-23$ & $25 / 75 \rightarrow 0 / 100$ \\
\hline & $23-25$ & $0 / 100 \rightarrow 50 / 50$ \\
\hline & $25-30$ & $50 / 50$ \\
\hline
\end{tabular}

Fluss: $\quad 18 \mathrm{~mL} \mathrm{~min}^{-1}$

$t_{\mathrm{R}}: \quad 13.2 \min \mathbf{1 1 1} \quad 15.4 \mathrm{~min}$. Gemisch aus $110 \& 71$ 
2. Aufreinigung HPLC (analytisch):

Säule: Kromasil 100 C18

\begin{tabular}{|c|c|c|}
\hline \multirow[t]{6}{*}{ Gradient: } & Zeit [min] & $\mathrm{H}_{2} \mathrm{O}(0.06 \% \mathrm{HCl}) / \mathrm{CH}_{3} \mathrm{CN}$ \\
\hline & $0-30$ & $70 / 30$ \\
\hline & $30-31$ & $60 / 40 \rightarrow 0 / 100$ \\
\hline & $31-35$ & $0 / 100$ \\
\hline & $35-36$ & $0 / 100 \rightarrow 70 / 30$ \\
\hline & $36-40$ & $70 / 30$ \\
\hline
\end{tabular}

Fluss: $\quad 0.8 \mathrm{~mL} \mathrm{~min}^{-1}$

$t_{\mathrm{R}}: \quad 24.4 \mathrm{~min}$ für $\mathbf{1 1 0} \quad 27.5 \mathrm{~min}$. für $\mathbf{7 1}$

2. Aufreinigung HPLC (präparativ):

Säule: Kromasil 100 C18

\begin{tabular}{|c|c|c|}
\hline \multirow[t]{6}{*}{ Gradient: } & Zeit [min] & $\mathrm{H}_{2} \mathrm{O}(0.06 \% \mathrm{HCl}) / \mathrm{CH}_{3} \mathrm{CN}$ \\
\hline & $0-30$ & $70 / 30$ \\
\hline & $30-31$ & $60 / 40 \rightarrow 0 / 100$ \\
\hline & $31-35$ & $0 / 100$ \\
\hline & $35-36$ & $0 / 100 \rightarrow 70 / 30$ \\
\hline & $36-40$ & $70 / 30$ \\
\hline
\end{tabular}

Fluss: $\quad 18 \mathrm{~mL} \mathrm{~min}^{-1}$

$t_{\mathrm{R}}: \quad 20.1 \mathrm{~min}$ für $\mathbf{1 1 0} \quad 22.7 \mathrm{~min}$. für $\mathbf{7 1}$

${ }^{1} \mathrm{H}-\mathrm{NMR}\left(600 \mathrm{MHz}\right.$, DMSO-d 6 ): $\delta=1.61\left(\mathrm{~d}, J=6.3 \mathrm{~Hz}, 3 \mathrm{H}, 11-\mathrm{H}_{3}\right), 2.37(\mathrm{t}, J=7.3 \mathrm{~Hz}$, $2 \mathrm{H}, 2 \mathrm{~S}-\mathrm{H}_{2}$ ), $2.88\left(\mathrm{~d}, J=4.8 \mathrm{~Hz}, 6 \mathrm{H}, 2^{\prime \prime}-\mathrm{NMe}_{2}\right.$ ), 2.97 (s, $6 \mathrm{H}, 12^{\#}-\mathrm{NMe}_{2}$ ), 3.07 (dd, 
$\left.J=13.5,7.1 \mathrm{~Hz}, 2 \mathrm{H}, 3 \mathrm{~S}-\mathrm{H}_{2}\right), 3.53-3.56\left(\mathrm{~m}, 2 \mathrm{H}, 2^{\prime \prime}-\mathrm{H}_{2}\right), 4.14(\mathrm{~d}, J=8.6 \mathrm{~Hz}, 1 \mathrm{H}, 1-\mathrm{H})$, 4.34-4.40 (m, $\left.4 \mathrm{H}, 1^{\prime \prime}-\mathrm{H}_{2}, 12-\mathrm{H}_{2}\right), 4.56\left(\mathrm{~d}, J=9.2 \mathrm{~Hz}, 1 \mathrm{H}, 2-\mathrm{H}_{\mathrm{a}}\right), 4.67-4.76(\mathrm{~m}, 2 \mathrm{H}$, $\left.2-\mathrm{H}_{\mathrm{b}}, 10-\mathrm{H}\right), 6.82(\mathrm{~d}, J=8.8 \mathrm{~Hz}, 2 \mathrm{H}, 2 \times \mathrm{Ar}-\mathrm{H}), 7.00\left(\mathrm{dd}, J=8.8,2.3 \mathrm{~Hz}, 1 \mathrm{H}, 6{ }^{\prime}-\mathrm{H}\right), 7.16$ (s, $\left.1 \mathrm{H}, 3^{\prime}-\mathrm{H}\right), 7.26\left(\mathrm{~d}, J=2.1 \mathrm{~Hz}, 1 \mathrm{H}, 4^{\prime}-\mathrm{H}\right), 7.39$ (d, J = 8.5 Hz, $\left.1 \mathrm{H}, 8-\mathrm{H}\right), 7.44$ (d, $\left.J=8.9 \mathrm{~Hz}, 1 \mathrm{H}, 7^{\prime}-\mathrm{H}\right), 7.62\left(\mathrm{~s}, 1 \mathrm{H}, 8^{\#}-\mathrm{H}\right), 7.67(\mathrm{~d}, J=8.8 \mathrm{~Hz}, 2 \mathrm{H}, 2 \times \mathrm{Ar}-\mathrm{H}), 7.77-7.86$ (m, 2 H, 3S-NH, 9-H), 7.92-7.99 (m, $4 \mathrm{H}, 4-\mathrm{H}, 6-\mathrm{H}, 2 \times \mathrm{Ar}-\mathrm{H}), 8.23(\mathrm{~d}, J=8.4 \mathrm{~Hz}, 2 \mathrm{H}$, $2 \times \mathrm{Ar}-\mathrm{H}), 8.45(\mathrm{t}, \mathrm{J}=5.9 \mathrm{~Hz}, 1 \mathrm{H}, 12-\mathrm{NH}), 10.15\left(\mathrm{~s}_{\mathrm{br}}, 1 \mathrm{H}, \mathrm{OH}\right), 10.34(\mathrm{~s}, 1 \mathrm{H}, \mathrm{NH}), 11.63$ (s, $\left.1 \mathrm{H}, 1^{\prime}-\mathrm{NH}\right) \mathrm{ppm}$.

$\mathrm{C}_{48} \mathrm{H}_{50} \mathrm{CIN}_{7} \mathrm{O}_{7} \mathrm{~S}_{6}$ (904.47).

ber.: $904.3254[\mathrm{M}+\mathrm{H}]^{+}$

gef.: 904.3258 (ESI-HRMS).

7.2 3-[4-(5-(4-Dimethylaminophenyl)oxazol-2-yl)benzolsulfonylamino]propionsäure-[(1S,10R)-1-(10-chlor-ethyl)-3-[(5-(2-(N,N'-dimethylamino)ethoxy)indol-2-yl)carbonyl]-5-Hydroxy-1,2-dihydro-3Hbenz[e]indol-7-ylmethyl]amid $((1 R, 10 S)-71)$

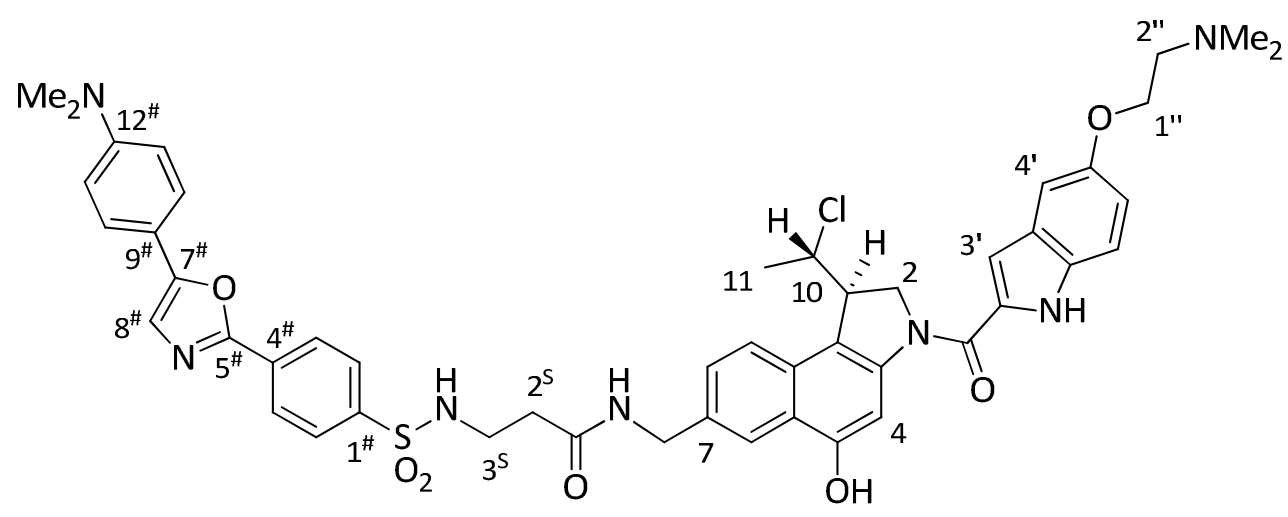

Zu einer Lösung des NHS-Esters $61(8.8 \mathrm{mg}, 17.2 \mu \mathrm{mol}, 1.0$ Äq.) in absolutem DMF $(200 \mu \mathrm{L}$ ) wurde eine Lösung des Phenols 87 (10.0 mg, $17.2 \mu \mathrm{mol}, 1.2$ Äq.) und Pyridin (4.2 $\mu \mathrm{L}, 52 \mu \mathrm{mol}, 3.0$ Äq.) in absolutem DMF (150 $\mu \mathrm{L}$ ) getropft. Nach $23 \mathrm{~h}$ Rühren bei Raumtemperatur wurde die Reaktionslösung mit bidestilliertem $\mathrm{H}_{2} \mathrm{O}(0.06 \% \mathrm{HCl}$, $3.0 \mathrm{~mL}$ ) und $\mathrm{MeOH}(6.0 \mathrm{~mL})$ verdünnt. Je $1.5 \mathrm{~mL}$ dieser Lösung wurden in das präparative HPLC-System (s. u.) injiziert. Fraktioniertes Auffangen des Eluats, 
Entfernung der Lösungsmittel unter vermindertem Druck sowie Entfernung des restlichen Lösungsmittels mittels Gefriertrocknung lieferte die Zielverbindung $(1 S, 10 R)-71$ als hellgelben Feststoff (7.9 mg, $8.75 \mu \mathrm{mol}, 47 \%)$.

${ }^{1} \mathrm{H}-\mathrm{NMR}\left(600 \mathrm{MHz}\right.$, DMSO-d 6 ): $\delta=1.61\left(\mathrm{~d}, J=6.6 \mathrm{~Hz}, 3 \mathrm{H}, 11-\mathrm{H}_{3}\right), 2.37(\mathrm{t}, J=7.4 \mathrm{~Hz}$, $2 \mathrm{H}, 2^{\mathrm{S}}-\mathrm{H}_{2}$ ), $2.88\left(\mathrm{~d}, J=4.8 \mathrm{~Hz}, 6 \mathrm{H}, 2^{\prime \prime}-\mathrm{NMe}_{2}\right.$ ), 2.97 (d, J = $7.5 \mathrm{~Hz}, 6 \mathrm{H}, 12^{\#}-\mathrm{NMe}_{2}$ ), 3.07 (dd, J = 13.4, 7.0 Hz, $\left.2 \mathrm{H}, 3^{\mathrm{S}}-\mathrm{H}_{2}\right), 3.43-3.59\left(\mathrm{~m}, 2 \mathrm{H}, 2^{\prime \prime}-\mathrm{H}_{2}\right), 4.14(\mathrm{~d}, J=9.2 \mathrm{~Hz}, 1 \mathrm{H}$, 1-H), 4.32-4.42 (m, $4 \mathrm{H}, 1$ 1"- $\left.\mathrm{H}_{2}, 12-\mathrm{H}_{2}\right), 4.56\left(\mathrm{~d}, \mathrm{~J}=9.4 \mathrm{~Hz}, 1 \mathrm{H}, 2-\mathrm{H}_{\mathrm{a}}\right), 4.67-4.77(\mathrm{~m}$, $\left.2 \mathrm{H}, 2-\mathrm{H}_{\mathrm{b}}, 10-\mathrm{H}\right), 6.85(\mathrm{~d}, J=8.4 \mathrm{~Hz}, 2 \mathrm{H}, 2 \times \mathrm{Ar}-\mathrm{H}), 7.00\left(\mathrm{dd}, J=8.9,2.3 \mathrm{~Hz}, 1 \mathrm{H}, 6^{\prime}-\mathrm{H}\right)$, 7.16 (s, 1 H, 3'-H), 7.26 (d, J = 2.0 Hz, 1 H, 4'-H), 7.39 (d, J = 8.8 Hz, 1 H, 8-H), 7.44 (d, $\left.J=8.9 \mathrm{~Hz}, 1 \mathrm{H}, 7^{\prime}-\mathrm{H}\right), 7.63\left(\mathrm{~s}, 1 \mathrm{H}, 8^{\#}-\mathrm{H}\right), 7.68(\mathrm{~d}, J=8.7 \mathrm{~Hz}, 2 \mathrm{H}, 2 \times \mathrm{Ar}-\mathrm{H}), 7.77-7.85$ (m, $\left.2 \mathrm{H}, 3^{\mathrm{S}}-\mathrm{NH}, 9-\mathrm{H}\right), 7.93-7.99(\mathrm{~m}, 4 \mathrm{H}, 4-\mathrm{H}, 6-\mathrm{H}, 2 \times \mathrm{Ar}-\mathrm{H}), 8.23(\mathrm{~d}, J=8.4 \mathrm{~Hz}, 2 \mathrm{H}$, $2 \times \operatorname{Ar}-\mathrm{H}), 8.46(\mathrm{t}, J=5.8 \mathrm{~Hz}, 1 \mathrm{H}, 12-\mathrm{NH}), 10.25(\mathrm{~s}, 1 \mathrm{H}, \mathrm{OH}), 10.34(\mathrm{~s}, 1 \mathrm{H}, \mathrm{NH}), 11.63$ (s, $\left.1 \mathrm{H}, 1^{\prime}-\mathrm{NH}\right) \mathrm{ppm}$.

$\mathrm{C}_{48} \mathrm{H}_{50} \mathrm{CIN}_{7} \mathrm{O}_{7} \mathrm{~S}_{6}$ (904.47).

ber.: $904.3254[\mathrm{M}+\mathrm{H}]^{+}$

gef.: 904.3258 (ESI-HRMS).

7.3 3-[4-(5-(4-Dimethylaminophenyl)oxazol-2-yl)benzolsulfonylamino]propionsäure-[(1S,10R)-1-(10-chlor-ethyl)-3-[(5-(2-(N,N'-dimethylamino)ethoxy)indol-2-yl)carbonyl]-5-hydroxy-1,2-dihydro-3Hbenz[e]indol-7-ylmethyl]amid ((1S,10R)-71)

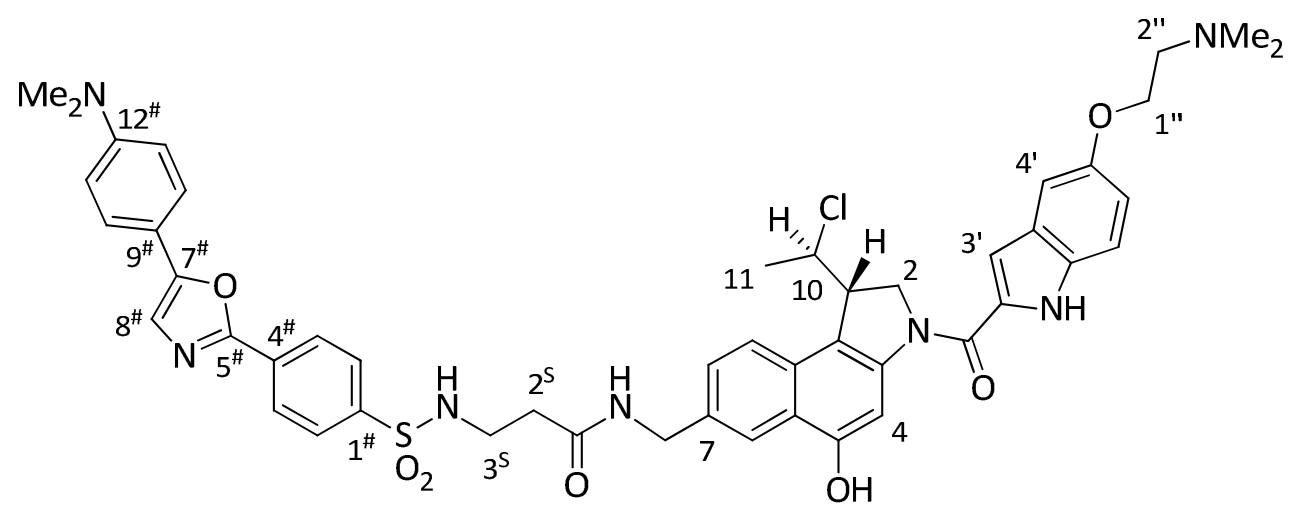


$\mathrm{Zu}$ einer Suspension des Sulfonylchlorids $89(2.73 \mathrm{mg}, 7.52 \mu \mathrm{mol}, 1.0$ Äq.) in absolutem DMF $(200 \mu \mathrm{L})$ wurde eine Lösung des Amins (1S,10R)-106 (4.4 mg, $6.77 \mu \mathrm{mol}, 1.0$ Äq.) und $i \operatorname{Pr}_{2} \mathrm{NEt}(5.8 \mu \mathrm{L}, 33.9 \mu \mathrm{mol}$, 4.5 Äq.) in absolutem DMF $(150 \mu \mathrm{L})$ getropft. Nach $23 \mathrm{~h}$ Rühren bei Raumtemperatur wurde die Reaktionslösung mit bidestilliertem $\mathrm{H}_{2} \mathrm{O}(0.06 \% \mathrm{HCl}, 0.5 \mathrm{~mL})$ und $\mathrm{MeOH}(1.5 \mathrm{~mL})$ verdünnt. Je $0.5 \mathrm{~mL}$ dieser Lösung wurden in das präparative HPLC-System (s. u.) injiziert. Fraktioniertes Auffangen des Eluats, Entfernung der Lösungsmittel unter vermindertem Druck sowie Entfernung des restlichen Lösungsmittels mittels Gefriertrocknung lieferte die Zielverbindung (1S,10R)-71 als hellgelben Feststoff ( $2.3 \mathrm{mg}, 2.6 \mu \mathrm{mol}, 34 \%)$, sowie das Chlorubstituierte Derivat (1S,10R)-111 als hellgelben Feststoff $(1.2 \mathrm{mg}, 1.35 \mu \mathrm{mol}$, $18 \%)$. 


\section{Synthese des seco-CBI-Grundgerüstes (1S)-128}

\section{1 tert-Butyl-(E)-3-(ethoxycarbonyl)-4-phenyl-3-butenoat (121)}<smiles>CCOC(=O)CC(=Cc1ccccc1)C(=O)OCC</smiles>

Zu einer Suspension aus $\mathrm{NaH}(8.0 \mathrm{~g}, 199 \mathrm{mmol}, 1.1 \mathrm{Äq}$. als 60\%ige Suspension in Mineralöl) in absolutem THF $(240 \mathrm{~mL})$ wurde bei $0{ }^{\circ} \mathrm{C}$ das Phosphonat 120 (60.0 g, $177 \mathrm{mmol}, 1.0$ Äq.) langsam zugetropftund für $5 \mathrm{~h}$ bei Raumtemperatur gerührt. Die Reaktionslösung wurde auf $0^{\circ} \mathrm{C}$ gekühlt und via Transferkanüle zu einer auf $0{ }^{\circ} \mathrm{C}$ gekühlten Lösung von frisch destilliertem Benzaldehyd (97) (20.8 g, 177 mmol, 1.1 Äq.) in absolutem THF $(160 \mathrm{~mL})$ gegeben. Das Reaktionsgemisch wurde auf Raumtemperatur erwärmt und $17 \mathrm{~h}$ gerührt. Anschließend wurde das Lösungsmittel im Vakuum entfernt und der orange Rückstand in $\mathrm{CH}_{2} \mathrm{Cl}_{2}(250 \mathrm{~mL})$ aufgenommen. Man wusch mit $\mathrm{H}_{2} \mathrm{O}(4 \times 200 \mathrm{~mL})$, trocknete über $\mathrm{MgSO}_{4}$ und entfernte das Lösungsmittel im Vakuum. Die Zielverbindung 121 wurde als gelbliches Öl (55.2 g, $190 \mathrm{mmol}, 97 \%)$ erhalten, welche ohne weitere Aufreinigung später eingesetzt wurde.

$\mathbf{R}_{\mathbf{f}}=0.57(\mathrm{PE} / \mathrm{EtOAC}=10: 1)$.

${ }^{1} \mathrm{H}-\mathrm{NMR}\left(300 \mathrm{MHz}, \mathrm{CDCl}_{3}\right): \delta=1.32\left(\mathrm{t}, J=7.1 \mathrm{~Hz}, 3 \mathrm{H}, \mathrm{OCH}_{2} \mathrm{CH}_{3}\right), 1.44\left(\mathrm{~s}, 9 \mathrm{H}, \mathrm{C}\left(\mathrm{CH}_{3}\right)_{3}\right)$, $3.43\left(\mathrm{~s}, 2 \mathrm{H}, 2-\mathrm{H}_{2}\right), 4.26\left(\mathrm{q}, J=7.1 \mathrm{~Hz}, 2 \mathrm{H}, \mathrm{OC}_{2} \mathrm{CH}_{3}\right), 7.24-7.40(\mathrm{~m}, 5 \mathrm{H}, 5 \times \mathrm{Ph}-\mathrm{H})$, $7.83(\mathrm{~s}, 1 \mathrm{H}, 4-\mathrm{H}) \mathrm{ppm}$.

${ }^{13} \mathrm{C}-\mathrm{NMR}\left(125 \mathrm{MHz}, \mathrm{CDCl}_{3}\right): \delta=14.23\left(\mathrm{OCH}_{2} \underline{\mathrm{CH}}_{3}\right), 27.98\left(\mathrm{C}\left(\mathrm{CH}_{3}\right)_{3}\right), 34.83(\mathrm{C}-2), 60.99$ $\left(\mathrm{O}_{\mathrm{CH}} \mathrm{CH}_{3}\right), 80.91\left(\underline{\mathrm{C}}\left(\mathrm{CH}_{3}\right)_{3}\right), 126.9$ (C-3), 128.5, 128.5, 128.7, 129.0, 129.0 (C-2', C-3', C-4', C-5', C-6'), 135.2 (C-1'), 141.1 (C-4), 167.5 ( CO $\left._{2} \mathrm{Et}\right), 170.2$ (C-1) ppm.

$\mathrm{C}_{17} \mathrm{H}_{22} \mathrm{O}_{4}$ (290.35).

ber.: $313.1410[\mathrm{M}+\mathrm{Na}]^{+}$ gef.: 313.1412 (ESI-HRMS). 


\section{2 (E)-3-Ethoxycarbonyl-4-phenyl-3-butencarbonsäure (96)}<smiles>CCC(=O)C(=Cc1ccccc1)CC(=O)O</smiles>

Eine Lösung des ${ }^{\mathrm{t}} \mathrm{Bu}$-Esters 121 (62.7 g, $216 \mathrm{mmol}$ ) in TFA / $\mathrm{H}_{2} \mathrm{O}=9: 1$ (300 mL) wurde für $20 \mathrm{~h}$ bei Raumtemperatur gerührt. Das Lösungsmittel wurde im Vakuum entfernt und der Rückstand zur azeotropen Entfernung von TFA zweimal in Toluol (jeweils $100 \mathrm{~mL}$ ) aufgenommen und das Lösungsmittel im Vakuum entfernt. Nach vorsichtiger Zugabe einer gesättigten $\mathrm{NaHCO}_{3}$-Lösung $(200 \mathrm{~mL})$ versetzte man bis $\mathrm{pH} \approx 1$ mit $2 \mathrm{~N}$ $\mathrm{HCl}(150 \mathrm{~mL})$ und extrahierte die Lösung mit EtOAc $(4 \times 125 \mathrm{~mL})$. Die vereinigten organischen Phasen wurden mit gesättigter NaCl-Lösung $(150 \mathrm{~mL})$ gewaschen, über $\mathrm{MgSO}_{4}$ getrocknet und das Lösungsmittel im Vakuum entfernt. Man erhielt die Zielverbindung 96 als gelbes Öl $(55.8 \mathrm{~g}, 238 \mathrm{mmol})$, welches ohne weitere Aufreinigung später eingesetzt wurde.

$\mathbf{R}_{\mathrm{f}}=0.55(\mathrm{PE} / \mathrm{EtOAC}=3: 1)$.

${ }^{1} \mathrm{H}-\mathrm{NMR}\left(300 \mathrm{MHz}, \mathrm{CDCl}_{3}\right): \delta=1.33\left(\mathrm{t}, J=7.2 \mathrm{~Hz}, 3 \mathrm{H}, \mathrm{OCH}_{2} \underline{\mathrm{C}}_{3}\right), 3.57\left(\mathrm{~s}, 2 \mathrm{H}, 2-\mathrm{H}_{2}\right)$, $4.29\left(\mathrm{q}, J=7.2 \mathrm{~Hz}, 2 \mathrm{H}, \mathrm{OCH}_{2} \mathrm{CH}_{3}\right), 7.30-7.45(\mathrm{~m}, 5 \mathrm{H}, 5 \times \mathrm{Ph}-\mathrm{H}), 7.91(\mathrm{~s}, 1 \mathrm{H}, 4-\mathrm{H})$, $10.70\left(\mathrm{~s}_{\mathrm{br}}, 1 \mathrm{H}, \mathrm{CO}_{2} \mathrm{H}\right) \mathrm{ppm}$.

${ }^{13} \mathrm{C}-\mathrm{NMR}\left(125 \mathrm{MHz}, \mathrm{CDCl}_{3}\right): \delta=14.14\left(\mathrm{OCH}_{2} \mathrm{CH}_{3}\right), 33.58(\mathrm{C}-2), 61.35\left(\mathrm{OCH}_{2} \mathrm{CH}_{3}\right), 125.5$ (C-3), 128.7 (C-2', C-6'), 128.8, 128.9 (C-3', C-4', C-5'), 134.8 (C-1'), 142.3 (C-4), 167.4 $\left(\mathrm{CO}_{2} \mathrm{Et}\right), 177.2(\mathrm{C}-1) \mathrm{ppm}$.

$\mathrm{C}_{13} \mathrm{H}_{14} \mathrm{O}_{4}$ (234.25).

ber.: $257.0784[\mathrm{M}+\mathrm{Na}]^{+}$

gef.: 257.0787 (ESI-HRMS). 


\subsection{Ethyl-1-acetoxy-3-naphthalincarboxylat (122)}<smiles>CCOC(=O)c1cc(OC(C)=O)c2ccccc2c1</smiles>

Zu einer Lösung aus der Carbonsäure 96 (76.0 g, $324 \mathrm{mmol}, 1.0$ Äq.) in Essigsäureanhydrid (1.7 L) wurde Kaliumacetat (47.7 g, 486 mmol, 1.5 Äq.) gegeben und für $2.5 \mathrm{~h}$ unter Rückfluss gerührt. Anschließend wurden $1.2 \mathrm{~L}$ des Lösungsmittels im Vakuum entfernt und das Reaktionsgemisch auf Eiswasser (2.0 L) gegeben. Das Lösungsmittel wurde im Vakuum eingeengt und mit $2 \mathrm{~N} \mathrm{NaOH}$ auf $\mathrm{pH} \approx 7$ eingestellt. Es wurde mit EtOAc $(4 \times 300 \mathrm{~mL})$ extrahiert, die vereinigte organische Phase mit gesättigter $\mathrm{NaCl}$-Lösung $(1 \mathrm{~L})$ gewaschen und über $\mathrm{MgSO}_{4}$ getrocknet. Man entfernte das Lösungsmittel im Vakuum und erhielt die Zielverbindung 122 als schwarzes Öl (64.0 g, 248 mmol), das ohne weitere Aufreinigung später eingesetzt wurde.

$\mathbf{R}_{\mathbf{f}}=0.35(\mathrm{PE} / \mathrm{EtOAC}=5: 1)$.

${ }^{1} \mathrm{H}-\mathrm{NMR}\left(300 \mathrm{MHz}, \mathrm{CDCl}_{3}\right): \delta=1.42\left(\mathrm{t}, J=7.1 \mathrm{~Hz}, 3 \mathrm{H}, \mathrm{OCH}_{2} \mathrm{C}_{3}\right), 2.46\left(\mathrm{~s}, 3 \mathrm{H}, \mathrm{COC}_{3}\right)$ ), $4.42\left(\mathrm{q}, J=7.1 \mathrm{~Hz}, 2 \mathrm{H}, \mathrm{OCH}_{2} \mathrm{CH}_{3}\right), 7.57\left(\mathrm{~m}_{\mathrm{c}}, 2 \mathrm{H}, 6-\mathrm{H}, 7-\mathrm{H}\right), 7.82(\mathrm{~d}, J=1.6 \mathrm{~Hz}, 1 \mathrm{H}$, 2-H), 7.84-7.88 (m, $1 \mathrm{H}, 5-\mathrm{H}), 7.94-7.99$ (m, $1 \mathrm{H}, 8-\mathrm{H}), 8.50$ (s, $1 \mathrm{H}, 4-\mathrm{H}) \mathrm{ppm}$.

${ }^{13}$ C-NMR $\left.\left(125 \mathrm{MHz}, \mathrm{CDCl}_{3}\right): \delta=14.31\left(\mathrm{OCH}_{2} \underline{\mathrm{CH}}_{3}\right), 20.87(\mathrm{CO} \underline{\mathrm{CH}})_{3}\right), 61.26\left(\mathrm{OCL}_{2} \mathrm{CH}_{3}\right)$, 117.6 (C-2), 121.2 (C-8), 127.2 (C-6), 127.7 (C-3), 128.7, 128.9, 129.5 (C-4, C-6, C-7), 129.0 (C-8a), 133.7 (C-4a), 146.6 (C-1), $165.9\left(\mathrm{CO}_{2} \mathrm{Et}\right), 169.2\left(\underline{\left.\mathrm{COCH}_{3}\right) \mathrm{ppm} .}\right.$

$\mathrm{C}_{15} \mathrm{H}_{14} \mathrm{O}_{4}$ (258.27).

ber.: $281.0784[\mathrm{M}+\mathrm{Na}]^{+}$

gef.: 281.0782 , (ESI-HRMS). 


\subsection{Ethyl-1-Hydroxy-3-naphthalincarboxylat (123)}

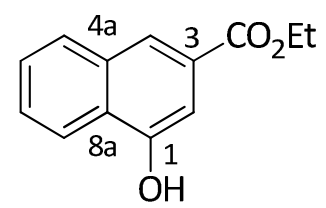

Zu einer Lösung von 122 (64.0 g, 247 mmol, 1.0 Äq.) in Ethanol (1.3 L) gab man $\mathrm{K}_{2} \mathrm{CO}_{3}$ (81.9 g, 592 mmol, 2.4 Äq.) und rührte 1 h unter Rückfluss. Nach Abkühlen auf Raumtemperatur goss man auf Eiswasser $(1.6 \mathrm{~L})$ und brachte mit halbkonzentrierter $\mathrm{HCl}$ auf $\mathrm{pH} \approx 1$. Der ausgefallene Feststoff wurde abfiltriert, mit $\mathrm{H}_{2} \mathrm{O}(300 \mathrm{~mL})$ gewaschen und die wässrige Phase mit EtOAc $(2 \times 300 \mathrm{~mL})$ extrahiert. Man vereinigte den Feststoff mit den Extrakten und wusch die organische Phase mit gesättigter $\mathrm{NaCl}$ Lösung $(2 \times 200 \mathrm{~mL})$, trocknete über $\mathrm{MgSO}_{4}$ und entfernte das Lösungsmittel im Vakuum. Man erhielt man die Zielverbindung 123 als braunen Feststoff (50.0 g, 93\%), der ohne weitere Aufreinigung später eingesetzt wurde.

$\mathbf{R}_{\mathbf{f}}=0.25(\mathrm{PE} / \mathrm{EtOAC}=5: 1)$.

${ }^{1} \mathrm{H}-\mathrm{NMR} \quad\left(300 \mathrm{MHz}, \mathrm{DMSO}-\mathrm{d}_{6}\right): \delta=1.35\left(\mathrm{t}, J=7.1 \mathrm{~Hz}, 3 \mathrm{H}, \mathrm{OCH}_{2} \mathrm{C}_{3}\right), 4.35$ (q, $\left.J=7.1 \mathrm{~Hz}, 2 \mathrm{H}, \mathrm{OC}_{2} \mathrm{CH}_{3}\right), 7.40(\mathrm{~d}, J=1.6 \mathrm{~Hz}, 1 \mathrm{H}, 2-\mathrm{H}), 7.52-7.63(\mathrm{~m}, 2 \mathrm{H}, 6-\mathrm{H}, 7-\mathrm{H})$, 7.97-8.04 (m, $1 \mathrm{H}, 5-\mathrm{H}), 8.07(\mathrm{~s}, 1 \mathrm{H}, 4-\mathrm{H}), 8.15-8.22(\mathrm{~m}, 1 \mathrm{H}, 8-\mathrm{H}), 10.47\left(\mathrm{~s}_{\mathrm{br}}, 1 \mathrm{H}, \mathrm{OH}\right)$ ppm.

${ }^{13} \mathrm{C}-\mathrm{NMR}\left(125 \mathrm{MHz}, \mathrm{DMSO}-\mathrm{d}_{6}\right): \delta=14.13\left(\mathrm{OCH}_{2} \mathrm{CH}_{3}\right), 60.62\left(\mathrm{OCH}_{2} \mathrm{CH}_{3}\right), 106.5(\mathrm{C}-2)$, 121.0 (C-8), 121.9 (C-4), 126.7, 127.0, 127.0, 127.6 (C-3, C-6, C-7, C-8a), 129.0 (C-5), 133.4 (C-4a), 153.5 (C-1), 165.9 (C=O) ppm.

$\mathrm{C}_{13} \mathrm{H}_{12} \mathrm{O}_{3}$ (216.23).

ber.: $\quad 239.0679[\mathrm{M}+\mathrm{Na}]^{+}$

gef.: 239.0685 (ESI-HRMS). 


\subsection{Ethyl-1-benzyloxy-3-naphthalincarboxylat (124)}<smiles>CCOC(=O)c1cc(Cc2ccccc2)c2ccccc2c1</smiles>

Zu einer Lösung des Naphtholderivats 123 (50.0 g, 231 mmol, 1.0 Äq.) in absolutem DMF (800 mL) wurden $\mathrm{K}_{2} \mathrm{CO}_{3}(48.0 \mathrm{~g}, 347 \mathrm{mmol}, 1.5$ Äq.) und TBAI (3.46 g, $9.2 \mathrm{mmol}$, 0.04 Äq.) gegeben. Nach Zutropfen von Benzylbromid (43.5 g, 254 mmol, 1.1 Äq.) wurde $24 \mathrm{~h}$ bei Raumtemperatur gerührt. Anschließend versetzte man das Reaktionsgemisch mit $\mathrm{H}_{2} \mathrm{O}(600 \mathrm{~mL})$, rührte 30 min bei Raumtemperatur und goss das Reaktionsgemisch auf $\mathrm{H}_{2} \mathrm{O}(1.2 \mathrm{~L})$. Der ausgefallene Feststoff wurde abfiltriert, mit $\mathrm{H}_{2} \mathrm{O}(800 \mathrm{~mL})$ gewaschen und im Vakuum getrocknet. Man erhielt die Zielverbindung 124 als braunen Feststoff $(72.5 \mathrm{~g}, 237 \mathrm{mmol})$, der ohne weitere Aufreinigung später eingesetzt wurde.

$\mathbf{R}_{\mathbf{f}}=0.62(\mathrm{PE} / \mathrm{EtOAC}=3: 1)$.

${ }^{1} \mathrm{H}-\mathrm{NMR}\left(300 \mathrm{MHz}, \mathrm{CDCl}_{3}\right): \delta=1.44\left(\mathrm{t}, J=7.1 \mathrm{~Hz}, 3 \mathrm{H}, \mathrm{OCH}_{2} \mathrm{CH}_{3}\right), 4.44(\mathrm{q}, J=7.1 \mathrm{~Hz}$, $\left.2 \mathrm{H}, \mathrm{OCH}_{2} \mathrm{CH}_{3}\right), 5.30\left(\mathrm{~s}, 2 \mathrm{H}, \mathrm{OCH}_{2} \mathrm{Ph}\right), 7.32-7.60(\mathrm{~m}, 8 \mathrm{H}, 2-\mathrm{H}, 6-\mathrm{H}, 7-\mathrm{H}, 5 \times \mathrm{Ph}-\mathrm{H})$, 7.86-7.94 (m, $1 \mathrm{H}, 5-\mathrm{H}), 8.23(\mathrm{~s}, 1 \mathrm{H}, 4 \mathrm{H}), 8.31-8.38(\mathrm{~m}, 1 \mathrm{H}, 8-\mathrm{H}) \mathrm{ppm}$.

${ }^{13} \mathrm{C}-\mathrm{NMR}\left(75 \mathrm{MHz}, \mathrm{CDCl}_{3}\right): \delta=14.41\left(\mathrm{OCH}_{2} \underline{\mathrm{CH}}_{3}\right), 61.15\left(\mathrm{O}_{\mathrm{CH}} \mathrm{CH}_{3}\right), 70.29\left(\mathrm{O}_{\underline{C}} \mathrm{H}_{2} \mathrm{Ph}\right)$, 104.1 (C-2), 122.3 (C-8), 123.7 (C-4), 127.1, 128.1, 129.0 (C-5, C-6, C-7, Ph-C ), 127.6 $\left(2 \times \mathrm{Ph}^{-\mathrm{C}_{o}}\right), 127.9,127.9(\mathrm{C}-3, \mathrm{C}-8 \mathrm{a}), 128.6\left(2 \times \mathrm{Ph}^{-\mathrm{C}_{m}}\right), 133.5(\mathrm{C}-4 \mathrm{a}), 136.8\left(\mathrm{Ph}-\mathrm{C}_{i}\right)$, $154.6(\mathrm{C}-1), 166.9$ (믈 $\left.{ }_{2} \mathrm{Et}\right) \mathrm{ppm}$.

$\mathrm{C}_{20} \mathrm{H}_{18} \mathrm{O}_{3}$ (306.35).

ber.: $329.1148[\mathrm{M}+\mathrm{Na}]^{+}$

gef.: 329.1152 (ESI-HRMS). 


\subsection{1-Benzyloxy-3-naphthalincarbonsäure (125)}

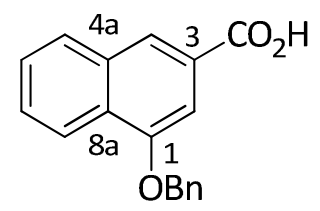

Zu einer Lösung des Ethylesters 124 (72.5 g, 237 mmol, 1.0 Äq.) in einem Gemisch aus THF/MeOH/ $\mathrm{H}_{2} \mathrm{O}=3: 1: 1$ (1.5 L) wurde $\mathrm{LiOH} \cdot \mathrm{H}_{2} \mathrm{O}(39.8 \mathrm{~g}, 951 \mathrm{mmol}, 4.0$ Äq.) und für $2 \mathrm{~d}$ bei Raumtemperatur gerührt. Anschließend wurde die Reaktionsmischung mit $\mathrm{H}_{2} \mathrm{O}(500 \mathrm{~mL})$ versetzt und unter Rühren mit halbkonzentrierter $\mathrm{HCl}$ auf $\mathrm{pH} \approx 1$ eingestellt, wobei sich ein Niederschlag bildete. Dieser wurde abfiltriert, mit $0.2 \mathrm{~N} \mathrm{HCl}$ $(1.0 \mathrm{~L})$ gewaschen und im Vakuum getrocknet. Nach Umkristallisation des Rohprodukts aus EtOAc/n-Hexan erhielt man die Zielverbindung $\mathbf{1 2 5}$ als weißen Feststoff (28.9 g, 104 mmol, 53\% über 6 Stufen).

$\mathbf{R}_{\mathbf{f}}=0.52($ EtOAc $/ \mathrm{MeOH}=10: 1)$.

${ }^{1} \mathrm{H}-\mathrm{NMR}\left(300 \mathrm{MHz}, \mathrm{DMSO}-\mathrm{d}_{6}\right): \delta=5.36\left(\mathrm{~s}, 2 \mathrm{H}, \mathrm{OC}_{2}{ }_{2} \mathrm{Ph}\right), 7.32-7.47(\mathrm{~m}, 3 \mathrm{H}, 1 \times \mathrm{Ph}-\mathrm{H}$, 6-H, 7-H), $7.51(\mathrm{~d}, J=1.1 \mathrm{~Hz}, 1 \mathrm{H}, 2-\mathrm{H}), 7.55-7.67(\mathrm{~m}, 4 \mathrm{H}, 4 \times \mathrm{Ph}-\mathrm{H}), 8.03-8.10(\mathrm{~m}$, $1 \mathrm{H}, 5-\mathrm{H}), 8.22-8.29(\mathrm{~m}, 1 \mathrm{H}, 8-\mathrm{H}), 8.24(\mathrm{~s}, 1 \mathrm{H}, 4-\mathrm{H}) \mathrm{ppm}$.

${ }^{13}$ C-NMR (125 MHz, DMSO-d $\left.\mathrm{d}_{6}\right): \delta=69.59\left(\mathrm{OC}_{2} \mathrm{Ph}\right), 104.4$ (C-2), 121.6 (C-8), 123.2 (C-4), 127.0 (C-8a), 127.2, 127.7, 127.8, 129.0 (C-5, C-6, C-7, Ph-C $), 127.4$ (2 × Ph-Co), $128.4\left(\mathrm{C}-3,2 \times \mathrm{Ph}^{-\mathrm{C}_{m}}\right), 133.1$ (C-4a), $136.8\left(\mathrm{Ph}-\mathrm{C}_{i}\right), 153.9$ (C-1), 167.4 (C=O) ppm.

$\mathrm{C}_{18} \mathrm{H}_{14} \mathrm{O}_{3}$ (278.30).

ber.: $301.0835[\mathrm{M}+\mathrm{Na}]^{+}$ gef.: 301.0835 (ESI-HRMS). 


\subsection{3-Amino-1-benzyloxy-N-(tert-butyloxycarbonyl)-naphthalin (126)}<smiles>CC(=O)OCc1ccccc1</smiles>

Zu einer Suspension der Carbonsäure 125 (21.4 g, $76.8 \mathrm{mmol}, 1.0$ Äq.) in absolutem tert-Butylalkohol (1.5 L) gab man aktiviertes Molsieb $4 \AA$ (150 g), Triethylamin (12.8 mL, $9.3 \mathrm{~g}, 92.2 \mathrm{mmol}, 1.2$ Äq.) und DPPA (23.3 mL, $84.5 \mathrm{mmol}, 1.1$ Äq.). Das Reaktionsgemisch wurde $3 \mathrm{~d}$ unter Rückfluss gerührt und nach Abkühlen auf Raumtemperatur vom Molsieb abgetrennt, welches gründlich mit EtOAc $(1.5 \mathrm{~L})$ gewaschen wurde. Nach Entfernen des Lösungsmittels im Vakuum wurde der Rückstand in EtOAc $(1.0 \mathrm{~L})$ aufgenommen, mit $2 \mathrm{~N} \mathrm{HCl}(2 \times 500 \mathrm{~mL})$, gesättigter $\mathrm{NaHCO}_{3}$-Lösung $(2 \times 300 \mathrm{~mL}), \mathrm{H}_{2} \mathrm{O}(500 \mathrm{~mL})$ und gesättigter $\mathrm{NaCl}$-Lösung $(500 \mathrm{~mL})$ gewaschen, über $\mathrm{MgSO}_{4}$ getrocknet und das Lösungsmittel im Vakuum entfernt. Die Zielverbindung 126 wurde als gelber Feststoff $(28.0 \mathrm{~g}, 80.1 \mathrm{mmol})$ erhalten, der ohne weitere Aufreinigung später eingesetzt wurde.

$\mathbf{R}_{\mathbf{f}}=0.46(\mathrm{PE} / \mathrm{EtOAC}=10: 1)$.

${ }^{1} \mathrm{H}-\mathrm{NMR}\left(300 \mathrm{MHz}, \mathrm{CDCl}_{3}\right): \delta=1.55\left(\mathrm{~s}, 9 \mathrm{H}, \mathrm{C}\left(\mathrm{CH}_{3}\right)_{3}\right), 5.21\left(\mathrm{~s}, 2 \mathrm{H}, \mathrm{OCH}_{2} \mathrm{Ph}\right), 6.64\left(\mathrm{~s}_{\mathrm{br}}\right.$, $1 \mathrm{H}, \mathrm{NH}), 7.06(\mathrm{~d}, J=1.7 \mathrm{~Hz}, 1 \mathrm{H}, 2-\mathrm{H}), 7.29-7.53(\mathrm{~m}, 8 \mathrm{H}, 4-\mathrm{H}, 6-\mathrm{H}, 7-\mathrm{H}, 5 \times \mathrm{Ph}-\mathrm{H})$, $7.68(\mathrm{~d}, J=8.1 \mathrm{~Hz}, 1 \mathrm{H}, 5-\mathrm{H}), 8.22(\mathrm{~d}, J=8.4 \mathrm{~Hz}, 1 \mathrm{H}, 8-\mathrm{H}) \mathrm{ppm}$.

${ }^{13} \mathrm{C}-\mathrm{NMR}\left(125 \mathrm{MHz}, \mathrm{CDCl}_{3}\right): \delta=28.41\left(\mathrm{C}\left(\mathrm{CH}_{3}\right)_{3}\right), 70.19\left(\underline{\mathrm{C}}\left(\mathrm{CH}_{3}\right)_{3}\right), 80.66\left(\mathrm{OCH}_{2} \mathrm{Ph}\right), 99.28$ (C-2), 107.0 (C-4), 122.1, 123.8 (C-7, C-8), 122.6 (C-8a), 126.9, 127.1, 128.0 (C-5, C-6, Ph- $\left.\mathrm{C}_{p}\right), 127.5\left(2 \times \mathrm{Ph}^{-\mathrm{C}_{o}}\right), 128.6\left(2 \times \mathrm{Ph}^{-\mathrm{C}_{m}}\right), 134.8$ (C-4a), 136.2, 136.9 (C-3, Ph- $\left.\mathrm{C}_{i}\right)$, $152.8(\mathrm{C}=0), 155.2$ (C-1) ppm.

$\mathrm{C}_{22} \mathrm{H}_{23} \mathrm{NO}_{3}$ (349.42).

ber.: $372.1570[\mathrm{M}+\mathrm{Na}]^{+}$ gef.: 372.1567 (ESI-HRMS). 


\subsection{2-Amino-4-benzyloxy-N-(tert-butyloxycarbonyl)-1-iod-naphthalin (95)}<smiles>CC(C)(C)OC(=O)Nc1cc(OCc2ccccc2)c2ccccc2c1I</smiles>

Zu einer Lösung des Naphthalinderivats 126 (28.0 g, $80.1 \mathrm{mmol}, 1.0$ Äq.) in einem Lösungsmittelgemisch aus $\mathrm{MeOH} / \mathrm{H}_{2} \mathrm{O}=4: 1 \quad(1500 \mathrm{~mL})$ wurde $\mathrm{HIO}_{3} \quad(2.81 \mathrm{~g}$, $16.0 \mathrm{mmol}, 0.2 \mathrm{Äq}$.) und lod $(8.20 \mathrm{~g}, 32.3 \mathrm{mmol}, 0.4$ Äq.) gegeben und $2 \mathrm{~h}$ unter Rückfluss gerührt. Man ließ auf Raumtemperatur abkühlen, filtrierte den ausgefallenen Feststoff ab und wusch diesen mit $\mathrm{H}_{2} \mathrm{O}(2 \times 500 \mathrm{~mL})$. Die Zielverbindung 95 wurde als hellbrauner Feststoff $(31.5 \mathrm{~g}, 66.3 \mathrm{mmol}$, 83\%) erhalten, der ohne weitere Aufreinigung später eingesetzt wurde.

$\mathbf{R}_{\mathbf{f}}=0.64(\mathrm{PE} / \mathrm{EtOAC}=10: 1)$.

${ }^{1} \mathrm{H}-\mathrm{NMR}\left(300 \mathrm{MHz}, \mathrm{CDCl}_{3}\right): \delta=1.59\left(\mathrm{~s}, 9 \mathrm{H}, \mathrm{C}\left(\mathrm{CH}_{3}\right)_{3}\right), 5.27\left(\mathrm{~s}, 2 \mathrm{H}, \mathrm{OC}_{2} \mathrm{Ph}\right), 7.31\left(\mathrm{~s}_{\mathrm{br}}\right.$, $1 \mathrm{H}, \mathrm{NH}), 7.33-7.58(\mathrm{~m}, 7 \mathrm{H}, 6-\mathrm{H}, 7-\mathrm{H}, 5 \times \mathrm{Ph}-\mathrm{H}), 8.01\left(\mathrm{~d}, J=7.0 \mathrm{~Hz}, 1 \mathrm{H}, 8-\mathrm{H}^{*}\right), 8.03(\mathrm{~s}$, $1 \mathrm{H}, 3-\mathrm{H}), 8.23\left(\mathrm{dd}, J=8.5 \mathrm{~Hz}, 1 \mathrm{H}, 5-\mathrm{H}^{*}\right) \mathrm{ppm}$.

${ }^{13} \mathrm{C}-\mathrm{NMR}\left(125 \mathrm{MHz}, \mathrm{CDCl}_{3}\right): \delta=28.36\left(\mathrm{C}\left(\underline{\mathrm{CH}}_{3}\right)_{3}\right), 70.38\left(\underline{\mathrm{C}}\left(\mathrm{CH}_{3}\right)_{3}\right), 80.26(\mathrm{C}-1), 81.15$

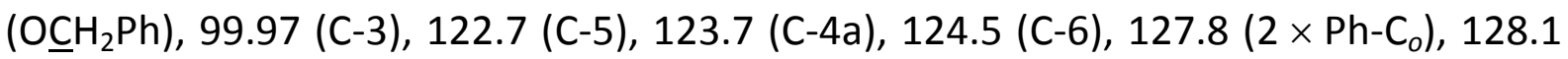
$\left(\mathrm{Ph}-\mathrm{C}_{p}\right), 128.6$ (2 × $\left.\mathrm{Ph}-\mathrm{C}_{m}\right), 128.6$ (C-7), 131.2 (C-8), 134.8 (C-8a), $136.6\left(\mathrm{Ph}-\mathrm{C}_{i}\right), 138.3$ (C-2), $152.8(\mathrm{C}=0), 155.7(\mathrm{C}-4) \mathrm{ppm}$.

$\mathrm{C}_{22} \mathrm{H}_{22} \mathrm{INO}_{3}$ (475.32).

ber.: $498.0537[\mathrm{M}+\mathrm{Na}]^{+}$

gef.: 498.0529 (ESI-HRMS). 


\section{$8.9(+)-\left\{\left(2^{\prime} R\right)-2-\right.$ Amino-4-benzyloxy- $\mathrm{N}$-(tert-butyloxycarbonyl)- $\mathrm{N}-(2,3-$} epoxypropyl)-1-iod-naphthalin\} ((+)-(2'R)-94)

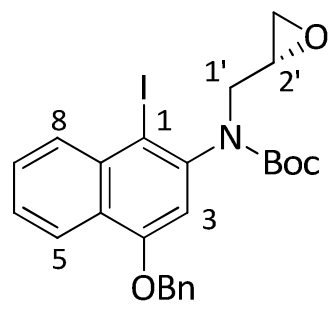

Zu einer Lösung des Amids 95 ( $3.00 \mathrm{~g}, 6.31 \mathrm{mmol}, 1.0$ Äq.) in absolutem DMF (30 mL) wurde bei Raumtemperatur NaH (1.01 g, 25.2 mmol, 4.0 Äq.; 60\%-ige Suspension in Mineralöl) gegeben und 30 min gerührt. Anschließend wurde das Nosylat (+)-(S)-126 ( $2.45 \mathrm{~g}, 9.47 \mathrm{mmol}, 1.5$ Äq.) portionsweise zugegeben und das Gemisch für $18 \mathrm{~h}$ bei Raumtemperatur gerührt. Es wurde Suspension mit gesättigter $\mathrm{NH}_{4} \mathrm{Cl}$-Lösung $(200 \mathrm{~mL})$ versetzt und $\mathrm{mit}^{\mathrm{Et}} \mathrm{t}_{2} \mathrm{O}(3 \times 150 \mathrm{~mL})$ extrahiert. Die vereinigte organische Phase wurde mit gesättigter $\mathrm{NaCl}$-Lösung $(3 \times 150 \mathrm{~mL})$ gewaschen, über $\mathrm{MgSO}_{4}$ getrocknet und das Lösungsmittel im Vakuum entfernt. Nach säulenchromatographischer Reinigung an Kieselgel ( $n$-Pentan/EtOAc $=5: 1$ ) erhielt man die Zielverbindung (+)-(2' $R$ )-94 als hellgelben Feststoff $(2.92 \mathrm{~g}, 5.49 \mathrm{mmol}, 87 \%)$.

$\mathbf{R}_{\mathbf{f}}=0.64$ (n-Pentan / EtOAc $=5: 1$ ).

${ }^{1} \mathrm{H}-\mathrm{NMR}\left(300 \mathrm{MHz}, \mathrm{C}_{2} \mathrm{D}_{2} \mathrm{Cl}_{4}, 100{ }^{\circ} \mathrm{C}\right.$, die Signale der Rotamere sind mit (a) und (b) gekennzeichnet): $\delta=1.46\left(\mathrm{~s}, 18 \mathrm{H}, 2 \times \mathrm{C}\left(\mathrm{CH}_{3}\right)_{3}\right), 2.46\left(\mathrm{~m}_{\mathrm{c}}, 2 \mathrm{H}, 3^{\prime}-\mathrm{H}_{\mathrm{a}}(\mathrm{a}), 3^{\prime}-\mathrm{H}_{\mathrm{a}}(\mathrm{b})\right), 2.73$ $\left(d t, J=20.5,8.9,4.3 \mathrm{~Hz}, 2 \mathrm{H}, 3^{\prime}-\mathrm{H}_{b}(\mathrm{a}), 3^{\prime}-\mathrm{H}_{b}(\mathrm{~b})\right), 3.12-3.19\left(\mathrm{~m}_{\mathrm{c}}, 1 \mathrm{H}, 2^{\prime}-\mathrm{H}(\mathrm{a})\right), 3.29$ (dd, $\left.\mathrm{J}=14.4,6.7 \mathrm{~Hz}, 1 \mathrm{H}, 1^{\prime}-\mathrm{H}_{\mathrm{a}}(\mathrm{a})\right), 3.34-3.42\left(\mathrm{~m}, 1 \mathrm{H}, 2^{\prime}-\mathrm{H}(\mathrm{b})\right), 3.49$ (dd, J = 14.8, $4.9 \mathrm{~Hz}$, $\left.1 \mathrm{H}, 1^{\prime}-\mathrm{H}_{\mathrm{a}}(\mathrm{b})\right), 4.08-4.17\left(\mathrm{~m}, 2 \mathrm{H}, 1^{\prime}-\mathrm{H}_{\mathrm{b}}(\mathrm{a}), 1^{\prime}-\mathrm{H}_{\mathrm{b}}(\mathrm{b}), 5.31,5.33\left(2 \times \mathrm{s}, 4 \mathrm{H}, \mathrm{OC}_{2} \mathrm{Ph}(\mathrm{a})\right.\right.$, $\left.\mathrm{OC}_{2} \mathrm{Ph}(\mathrm{b})\right), 6.89,6.99(2 \times \mathrm{s}, 2 \mathrm{H}, 3-\mathrm{H}(\mathrm{a}), 3-\mathrm{H}(\mathrm{b})), 7.34-7.66(\mathrm{~m}, 14 \mathrm{H}, 6-\mathrm{H}(\mathrm{a}), 6-\mathrm{H}(\mathrm{b})$, 7-H(a), 7-H(b), $5 \times \mathrm{Ph}-\mathrm{H}(\mathrm{a}), 5 \times \mathrm{Ph}-\mathrm{H}(\mathrm{b})), 8.25(2 \times \mathrm{m}, 2 \mathrm{H}, 8-\mathrm{H}(\mathrm{a}), 8-\mathrm{H}(\mathrm{b})), 8.36(2 \times \mathrm{m}$, $2 \mathrm{H}, 5-\mathrm{H}(\mathrm{a}), 5-\mathrm{H}(\mathrm{b})) \mathrm{ppm}$.

${ }^{13} \mathrm{C}-\mathrm{NMR}\left(150 \mathrm{MHz}, \mathrm{C}_{2} \mathrm{D}_{2} \mathrm{Cl}_{4}, 100{ }^{\circ} \mathrm{C}\right.$, die Signale der Rotamere sind mit (a) und (b) gekennzeichnet): $\delta=28.15,28.18$ (2 Signale) $\left(2 \times \mathrm{C}\left(\mathrm{CH}_{3}\right)_{3}\right), 45.71$ (C-3'(a)), 45.87 (C-3'(b)), 49.38 (C-2'(a)), 49.93 (C-2'(b)), 51.33 (C-1'(b)), 52.85 (C-1'(a)), 70.61, 70.68 
$\left(2 \times \mathrm{OCH}_{2} \mathrm{Ph}\right), 80.52$ (2 Signale) $\left(2 \times \underline{\mathrm{C}}\left(\mathrm{CH}_{3}\right)_{3}\right), 94.59$ (C-1(a)), 94.74 (C-1(b)), 108.0 (C-3(a)), 108.1 (C-3(b)), 122.4 (C-5(a), C-5(b)), 125.6 (C-4a(a), C-4a(b)), 126.1 (2 Signale), 127.0, 127.2, 127.9, 128.2, 128.3, 128.4 (2 Signale) (C-6(a), C-6(b), C-7(a),

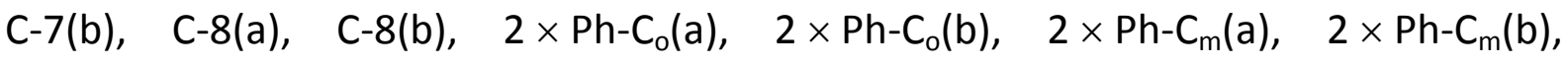
$\left.2 \times \mathrm{Ph}_{-} \mathrm{C}_{\mathrm{p}}(\mathrm{a}), 2 \times \mathrm{Ph}-\mathrm{C}_{\mathrm{p}}(\mathrm{b})\right), 135.3,135.3$ (C-8a(a), C-8a(b)), 136.4, $136.4\left(\mathrm{Ph}-\mathrm{C}_{\mathrm{i}}(\mathrm{a})\right.$, Ph- $\left.C_{i}(b)\right), 143.2,143.7$ (C-2(a), C-2(b)), 153.4, 153.6 ( $\left.C=O(a), C=O(b)\right), 155.4,155.5$ (C-4(a), C-4(b)) ppm.

$\mathrm{C}_{25} \mathrm{H}_{26} \mathrm{INO}_{4}$ (531.38)

ber.: $554.0799[\mathrm{M}+\mathrm{Na}]^{+}$

gef.: 554.0795 (ESI-HRMS).

\section{$8.10(+)-\{(1 S)-5-B e n z y l o x y-3-(t e r t-b u t y l o x y c a r b o n y l)-1-h y d r o x y m e t h y l-1,2-$ dihydro-3H-benz[e]indol\} $((+)-(1 S)-127)$}<smiles>CC(C)(C)OC(=O)N1CC([14CH](O)O)c2c1cc(Cc1ccccc1)c1ccccc21</smiles>

$\mathrm{Zu}$ einer Lösung aus frisch sublimiertem $\mathrm{ZnCl}_{2}$ (2.06 g, $15.1 \mathrm{mmol}, 2.5$ Äq.) in absolutem THF $(160 \mathrm{~mL})$ wurde bei $0{ }^{\circ} \mathrm{C}$, langsam Methyllithium $(37.8 \mathrm{~mL}$ einer $1.6 \mathrm{M}$ Lösung in $\mathrm{Et}_{2} \mathrm{O}, 60.4 \mathrm{mmol}, 10$ Äq.) getropft und für $30 \mathrm{~min}$ bei dieser Temperatur gerührt. Anschließend wurde die Lösung auf $-78{ }^{\circ} \mathrm{C}$ gekühlt und tropfenweise TMSNCS (1.98 g, $2.12 \mathrm{~mL}, 15.1 \mathrm{mmol}, 2.5$ Äq.) zugegeben. Es wurde auf $0{ }^{\circ} \mathrm{C}$ erwärmt und für 30 min bei dieser Temperatur gerührt. Nach erneutem Abkühlen auf $-78{ }^{\circ} \mathrm{C}$ wurde zu dem Reaktionsgemisch eine Lösung des Epoxids ((+)-(2'R)-94 (3.21 g, 6.05 mmol, 1.0 Äq.) in absolutem THF (45 mL) getropft und für $15 \mathrm{~min}$ bei $-78^{\circ} \mathrm{C}$, für $1 \mathrm{~h}$ bei $0{ }^{\circ} \mathrm{C}$ und anschließend für $12 \mathrm{~h}$ bei Raumtemperatur gerührt. Das Gemisch wurde mit gesättigter $\mathrm{NH}_{4} \mathrm{Cl}$-Lösung $(250 \mathrm{~mL})$ versetzt, mit $\mathrm{CH}_{2} \mathrm{Cl}_{2}(4 \times 250 \mathrm{~mL})$ extrahiert, die vereinigten organischen Phasen über $\mathrm{Na}_{2} \mathrm{SO}_{4}$ getrocknet und das Lösungsmittel im Vakuum entfernt. Nach säulenchromatographischer Reinigung an Kieselgel 
$(\mathrm{PE} / \mathrm{EtOAC}=4: 1)$ erhielt man die Zielverbindung $(+)-(1 S)-127$ als weißen Feststoff (2.26 g, $5.57 \mathrm{mmol}, 47 \%)$.

Analytische Daten für (+)-(1S)-127:

HPLC (analytisch):

Säule:

Chiralcel OD

Eluens:

$n$-Hexan $/ i-\mathrm{PrOH}=95: 5$

Fluss:

$0.8 \mathrm{~mL} \mathrm{~min}^{-1}$

$t_{\mathrm{R}}$ :

$14.3 \mathrm{~min}$.

Analytische Daten für (-)-(1R)-127:

$\begin{array}{lll}\text { HPLC (analytisch): } & \text { Säule: } & \text { Chiralcel OD } \\ & \text { Eluens: } & n \text {-Hexan } / i-\operatorname{PrOH}=95: 5 \\ & \text { Fluss: } & 0.8 \mathrm{~mL} \mathrm{~min}^{-1} \\ t_{\mathrm{R}}: & 13.6 \mathrm{~min} .\end{array}$

$\mathbf{R}_{\mathbf{f}}=0.14$ (PE / EtOAC $\left.=3: 1\right)$.

${ }^{1} \mathrm{H}-\mathrm{NMR}\left(300 \mathrm{MHz}, \mathrm{CDCl}_{3}\right): \delta=1.59\left(\mathrm{~s}_{\mathrm{br}}, 9 \mathrm{H}, \mathrm{C}\left(\mathrm{CH}_{3}\right)_{3}, 3.68-3.86\left(\mathrm{~m}_{\mathrm{c}}, 2 \mathrm{H}, 1-\mathrm{H}, 10-\mathrm{H}_{\mathrm{b}}\right)\right.$, 3.89-3.98 $\left(m_{c}, 1 \mathrm{H}, 10-\mathrm{H}_{\mathrm{a}}\right), 4.06-4.15\left(\mathrm{~m}_{\mathrm{c}}, 1 \mathrm{H}, 2-\mathrm{H}_{\mathrm{a}}\right), 4.17-4.27\left(\mathrm{~m}_{\mathrm{c}}, 1 \mathrm{H}, 2-\mathrm{H}_{\mathrm{b}}\right), 5.25$ (s, $\left.2 \mathrm{H}, \mathrm{OC} \underline{\mathrm{H}}_{2} \mathrm{Ph}\right), 7.27-7.56(\mathrm{~m}, 7 \mathrm{H}, 7-\mathrm{H}, 8-\mathrm{H}, 5 \times \mathrm{Ph}-\mathrm{H}), 7.69(\mathrm{~d}, J=8.3 \mathrm{~Hz}, 1 \mathrm{H}, 9-\mathrm{H})$, $7.89\left(\mathrm{~s}_{\mathrm{br}}, 1 \mathrm{H}, 4-\mathrm{H}\right), 8.22(\mathrm{~d}, J=8.3 \mathrm{~Hz}, 1 \mathrm{H}, 6-\mathrm{H}) \mathrm{ppm}$.

$\left.\left.{ }^{13} \mathrm{C}-\mathrm{NMR}(125 \mathrm{MHz}, \mathrm{CDCl})_{3}\right): \delta=28.47\left(\mathrm{C}_{\left(\mathrm{CH}_{3}\right.}\right)_{3}\right), 41.30$ (C-1), 52.42 (C-2), 64.74 (C-10), $70.24\left(\mathrm{O}_{\mathrm{C}} \mathrm{H}_{2} \mathrm{Ph}\right), 80.74\left(\underline{\mathrm{C}}\left(\mathrm{CH}_{3}\right)_{3}\right), 96.51$ (C-4), 114.3 (C-9b), 122.3 (C-5a), 122.4, 122.9, 123.4 (C-6, C-7, C-9), 127.3 (C-8), 127.5, 127.9, 128.5 (5 × Ph- $\underline{\mathrm{CH}}$ ), 130.6 (C-9a), 136.9 $\left(\mathrm{Ph}-\mathrm{C}_{i}\right), 142.0(\mathrm{C}-3 \mathrm{a}), 152.7(\mathrm{C}=0), 155.6(\mathrm{C}-5) \mathrm{ppm}$.

$\mathrm{C}_{25} \mathrm{H}_{27} \mathrm{NO}_{4}$ (405.49).

ber.: $428.1832[\mathrm{M}+\mathrm{Na}]^{+}$

gef.: 428.1832 (ESI-HRMS). 


\section{$8.11(-)-\{(1 S)-5-B e n z y l o x y-3-(t e r t-B u t y l o x y c a r b o n y l)-1-c h l o r m e t h y l-1,2-$ dihydro-3H-benz[e]indol\} ((-)-(1S)-93)}<smiles></smiles>

Zu einer Lösung von (+)-(1S)-127 (2.06 g, $5.08 \mathrm{mmol}, 1.0$ Äq.) in absolutem $\mathrm{CH}_{2} \mathrm{Cl}_{2}$ (50 mL) wurde bei Raumtemperatur Triphenylphosphin (3.99 g, $15.2 \mathrm{mmol}, 3.0$ Äq.) und $\mathrm{CCl}_{4}\left(4.45 \mathrm{~mL}, 45.7 \mathrm{mmol}, 9.0 \mathrm{Äq}\right.$ ) gegeben und für $3 \mathrm{~h}$ gerührt. Es wurde $\mathrm{SiO}_{2}$ (12 g) zugegeben und das Lösungsmittel im Vakuum entfernt. Nach anschließender säulenchromatographischer Reinigung an Kieselgel $(P E /$ EtOAc $=7: 1)$ erhielt man die Zielverbindung (-)-(1S)-93 als weißen Feststoff (1.93 g, $4.55 \mathrm{mmol}, 90 \%)$.

Analytische Daten für $(+)-(1 R)-93:$

$\begin{array}{lll}\text { HPLC (analytisch): } & \text { Säule: } & \text { Chiralcel OD } \\ & \text { Eluens: } & n \text {-Hexan } / i \text {-PrOH }=98: 2 \\ & \text { Fluss: } & 0.8 \mathrm{~mL} \mathrm{~min}^{-1} \\ & t_{\mathrm{R}}: & 9.6 \mathrm{~min} .\end{array}$

Analytische Daten für (-)-(1S)-93:

$\begin{array}{lll}\text { HPLC (analytisch): } & \text { Säule: } & \text { Chiralcel OD } \\ & \text { Eluens: } & n \text {-Hexan } / i-\operatorname{PrOH}=98: 2 \\ & \text { Fluss: } & 0.8 \mathrm{~mL} \mathrm{~min}^{-1} \\ & t_{\mathrm{R}}: & 13.4 \mathrm{~min} .\end{array}$

$\mathbf{R}_{\mathbf{f}}=0.73(\mathrm{PE} / \mathrm{EtOAC}=3: 1)$.

${ }^{1} \mathrm{H}$-NMR $\left(300 \mathrm{MHz}, \mathrm{CDCl}_{3}\right) \delta=1.60\left(\mathrm{~s}, 9 \mathrm{H}, \mathrm{C}\left(\mathrm{CH}_{3}\right)_{3}\right), 3.42\left(\mathrm{t}, J=10.5 \mathrm{~Hz}, 1 \mathrm{H}, 10-\mathrm{H}_{\mathrm{a}}\right)$, 3.86-4.01 (m, $\left.2 \mathrm{H}, 1-\mathrm{H}, 10-\mathrm{H}_{\mathrm{b}}\right), 4.05-4.17\left(\mathrm{~m}, 1 \mathrm{H}, 2-\mathrm{H}_{\mathrm{a}}\right), 4.26(\mathrm{~d}, J=11.7 \mathrm{~Hz}, 1 \mathrm{H}$, 2- $\left.\mathrm{H}_{\mathrm{b}}\right), 5.25\left(\mathrm{~s}, 2 \mathrm{H}, \mathrm{OCH}_{2} \mathrm{Ph}\right), 7.27-7.58(\mathrm{~m}, 7 \mathrm{H}, 7-\mathrm{H}, 8-\mathrm{H}, 5 \times \mathrm{Ph}-\mathrm{H}), 7.63(\mathrm{~d}, J=8.4 \mathrm{~Hz}$, $1 \mathrm{H}, 9-\mathrm{H}), 7.85\left(\mathrm{~s}_{\mathrm{br}}, 1 \mathrm{H}, 4-\mathrm{H}\right), 8.28(\mathrm{~d}, J=8.4 \mathrm{~Hz}, 1 \mathrm{H}, 6-\mathrm{H}) \mathrm{ppm}$. 
${ }^{13} \mathrm{C}-\mathrm{NMR}\left(125 \mathrm{MHz}, \mathrm{CDCl}_{3}\right): \delta=28.6\left(\mathrm{C}\left(\mathrm{CH}_{3}\right)_{3}\right), 41.8$ (C-1), 46.5 (C-10), 53.1 (C-2), 70.3 $\left(\mathrm{OCH}_{2} \mathrm{Ph}\right), 81.2\left(\underline{\mathrm{C}}\left(\mathrm{CH}_{3}\right)_{3}\right), 96.4$ (C-4), 114.3 (C-9b), 122.4 (C-5a), 121.7, 123.0, 123.5 (C-6, C-7, C-9), 127.5 (Ph-Co), 127.6 (C-8), 127.9 (Ph-Cp), 128.5 (Ph-C ( $\left._{m}\right), 130.1$ (C-9a), $136.7\left(\mathrm{Ph}^{-\mathrm{C}_{i}}\right), 141.5$ (C-3a), 152.5 (C=0), 156.0 (C-5) ppm.

$\mathrm{C}_{25} \mathrm{H}_{26} \mathrm{CINO}_{3}$ (423.93).

ber.: $446.1493[\mathrm{M}+\mathrm{Na}]^{+}$

gef.: 449.1489 (ESI-HRMS).

\section{$8.12(-)-\{(1 S)-3-($ tert-Butyloxycarbonyl)-1-chlormethyl-5-hydroxy-1,2- dihydro-3H-benz $[e]$ indol\} $((-)-(1 S)-128)$}<smiles>CC(C)(C)OC(=O)N1C[C@H](Cl)c2c1cc(O)c1ccccc21</smiles>

Zu einer Lösung von (+)-(1S)-93 (250 mg, 59.0 mmol, 1.0 Äq.) in absolutem THF wurde bei Raumtemperatur Pd/C (10\%-ig, 125 mg, $117 \mu \mathrm{mol}, 0.2$ Äq.) gegeben. Es wurde $\mathrm{H}_{2}$ durch die Lösung geleitet und für $6 \mathrm{~h}$ bei $50{ }^{\circ} \mathrm{C}$ unter $\mathrm{H}_{2}$-atm gerührt. Das Reaktionsgemisch wurde über Celite filtiert und mit EtOAc $(500 \mathrm{~mL})$ gewaschen. Anschließend wurde das Lösungsmittel im Vakuum entfernt und nach säulenchromatographischer Reinigung an Kieselgel (PE / EtOAC $=4: 1)$ erhielt man die Zielverbindung (-)-(1S)-128 als weißen Feststoff (176 mg, 52.7 mmol, 89\%).

\subsection{Anreichung von $\{(1 S)-3-($ tert-butyloxycarbonyl)-1-(chlormethyl)-1,2- di-hydro-5-hydroxy-3H-benz[e]indol\} ((1S)-128) durch präparative HPLC}

(-)-(1S)-128 (1.76 g, $5.27 \mathrm{mmol}$ ) wurde in einem Gemisch aus $n$-Hexan / iPrOH= 1:1 $(50 \mathrm{~mL}$ ) gelöst. Je $0.5 \mathrm{~mL}$ dieser Probelösung (entsprechend $17 \mathrm{mg}$ bzw. $527 \mu \mathrm{mol}$ des Gemisches) wurden in das präparative HPLC-System (Säule: Chiralpak IA, $250 \times 20$ mm, Partikelgröße: $5 \mu \mathrm{m}$, mobile Phase: $n$-Hexan $/ \mathrm{PrOH}=97 / 3$, Fluss: 
$18 \mathrm{~mL} \mathrm{~min}^{-1}$, Druck: 7.4 MPa) injiziert. Fraktioniertes Auffangen des Eluats (UVDetektor: $\lambda=250 \mathrm{~nm}$ ) lieferte das reine Enantiomer (-)-(1S)-128.

Analytische Daten für (-)-(1S)-128:

HPLC (analytisch):

$\begin{array}{ll}\text { Säule: } & \text { Chiralcel IA } \\ \text { Eluens: } & n \text {-Hexan / } i \text {-PrOH }=98: 2 \\ \text { Fluss: } & 18 \mathrm{~mL} \mathrm{~min}^{-1} \\ t_{\mathrm{R}}: & 14.0 \mathrm{~min} .\end{array}$

HPLC (analytisch):

Säule: $\quad$ Chiralcel IA

Eluens: $\quad n$-Hexan $/ i-\mathrm{PrOH}=98: 2$

Fluss: $\quad 0.8 \mathrm{~mL} \mathrm{~min}^{-1}$

$t_{\mathrm{R}}: \quad 22.9 \mathrm{~min}$.

$\mathbf{R}_{\mathbf{f}}=0.60(\mathrm{PE} / \mathrm{EtOAC}=6: 1)$.

${ }^{1} \mathbf{H}-N M R\left(300 \mathrm{MHz} \mathrm{CDCl}_{3}\right): \delta=1.62\left(\mathrm{~s}, 9 \mathrm{H}, \mathrm{C}\left(\mathrm{CH}_{3}\right)_{3}\right), 3.41\left(\mathrm{t}, J=11.4 \mathrm{~Hz}, 10-\mathrm{H}_{\mathrm{a}}\right), 3.90-$ $3.98\left(\mathrm{~m}, 2 \mathrm{H}, 1-\mathrm{H}, 10-\mathrm{H}_{\mathrm{b}}\right), 4.12\left(\mathrm{t}, J=10.5 \mathrm{~Hz}, 1 \mathrm{H}, 2-\mathrm{H}_{\mathrm{a}}\right), 4.25\left(\mathrm{~d}, J=11.5 \mathrm{~Hz}, 1 \mathrm{H}, 2-\mathrm{H}_{\mathrm{b}}\right)$, 7.33 (dt, J = 7.6, 1.0 Hz, $1 \mathrm{H}, 7-\mathrm{H}$ ), 7.49 (dt, J = 7.6, 1.0 Hz, $1 \mathrm{H}, 8-\mathrm{H}), 7.62$ (d, J = 8.4 Hz, $1 \mathrm{H}, 9-\mathrm{H}), 7.82\left(\mathrm{~s}_{\mathrm{br}}, 1 \mathrm{H}, 4-\mathrm{H}\right), 8.21(\mathrm{~d}, J=8.4 \mathrm{~Hz}, 1 \mathrm{H}, 6-\mathrm{H}) \mathrm{ppm}$.

${ }^{13} \mathrm{C}-\mathrm{NMR}\left(125 \mathrm{MHz}, \mathrm{CDCl}_{3}\right): \delta=28.5\left(\mathrm{C}\left(\mathrm{CH}_{3}\right)_{3}\right), 41.8$ (C-1), 46.5 (C-10), 53.1 (C-2), 81.8 $\left(\underline{\mathrm{C}}\left(\mathrm{CH}_{3}\right)_{3}\right), 99.1$ (C-4), 114.3 (C-9b*), 121.6 (C-5*), 121.7 (C-9), 122.9 (C-7), 123.6 (C-6), 127.6 (C-8), 130.4 (C-9a), 141.1 (C-3a), 153.3 (C=0), 154.1 (C-5) ppm.

$\mathrm{C}_{18} \mathrm{H}_{20} \mathrm{ClNO}_{3}$ (333.81).

ber.: $356.1024[\mathrm{M}+\mathrm{Na}]^{+}$

gef.: 356.1026 (ESI-HRMS). 


\section{Synthese neuartiger fluoreszenzmarkierter CBI-Prodrugs, sowie der entsprechenden seco-CBI-Derivate}

9.1 (-)-(1S)-1-[6-(1-Chlormethyl-5-hydroxy-1,2-dihydro-benz[e]indol-3-yl)6-oxo-hexyl]-4-(3-Methyl-3H-benzothiazol-2-ylidenmethyl)pyridinium-chlorid ((-)-(1S)-141)

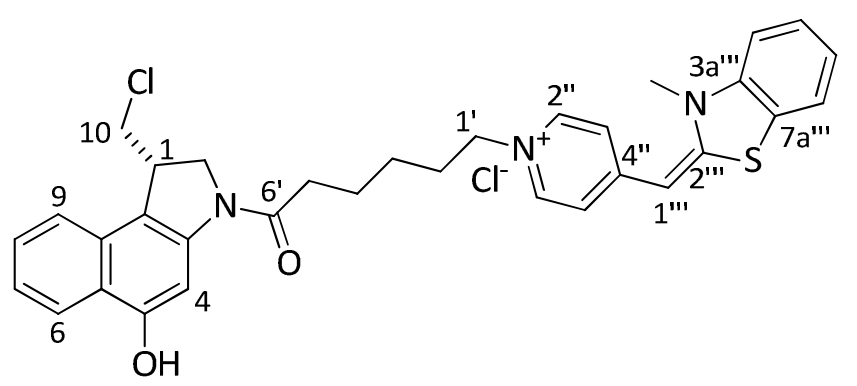

Phenol (1S)-128 (50.0 mg, $150 \mu \mathrm{mol}, 1.0$ Äq.) wurde bei Raumtemperatur in $4 \mathrm{M}$ $\mathrm{HCl} /$ EtOAc $(3.75 \mathrm{~mL})$ suspendiert, für $4 \mathrm{~h}$ gerührt und das Lösungsmittel im Vakuum entfernt. Der Rückstand wird $1 \mathrm{~h}$ im Hochvakuum getrocknet und anschließend in DMF (2.5 mL) gelöst und bei $0{ }^{\circ} \mathrm{C}$ mit EDAC. $\mathrm{HCl}(86.0 \mathrm{mg}, 450 \mu \mathrm{mol}, 3.0$ Äq.) und 63 ( $85 \mathrm{mg}, 195 \mu \mathrm{mol}, 1.3$ Äq.) versetzt und anschließend für $18 \mathrm{~h}$ bei Raumtemperatur gerührt. Das Lösungsmittel wurde entfernt und der Rückstand in einem 5:1 Gemisch aus $\mathrm{MeOH} / \mathrm{CH}_{3} \mathrm{CN}$ (12 mL) aufgenommen. Je $1 \mathrm{~mL}$ dieser Lösung wurden in das präparative HPLC-System (s. u.) injiziert. Fraktioniertes Auffangen des Eluats, Entfernung der Lösungsmittel unter vermindertem Druck sowie Entfernung des restlichen Lösungsmittels mittels Gefriertrocknung lieferte die Zielverbindung (1S)141 als gelben Feststoff (65.1 mg, $100 \mu \mathrm{mol}, 67 \%)$. 
HPLC (analytisch):

Säule: Kromasil $100 \mathrm{C18}$

\begin{tabular}{ccc}
\cline { 2 - 3 } Gradient: & Zeit [min] & $\mathrm{H}_{2} \mathrm{O}(0.06 \% \mathrm{HCl}) / \mathrm{MeOH}$ \\
\cline { 2 - 3 } & 0 & $40 / 60$ \\
$0-20$ & $40 / 60 \rightarrow 0 / 100$ \\
$20-25$ & $0 / 100$ \\
$25-27$ & $0 / 100 \rightarrow 40 / 60$ \\
$27-30$ & $40 / 60$ \\
\hline
\end{tabular}

Fluss: $\quad 0.8 \mathrm{~mL} \mathrm{~min}^{-1}$

$t_{\mathrm{R}}: \quad 18.1 \mathrm{~min}$

HPLC (präparativ):

Säule: Kromasil $100 \mathrm{C} 18$

\begin{tabular}{ccc}
\cline { 2 - 3 } Gradient: & Zeit [min] & $\mathrm{H}_{2} \mathrm{O}(0.06 \% \mathrm{HCl}) / \mathrm{MeOH}$ \\
\cline { 2 - 3 } & $0-2.5$ & $40 / 60$ \\
$2.5-20$ & $40 / 60 \rightarrow 0 / 100$ \\
$20-25$ & $0 / 100$ \\
$25-27$ & $0 / 100 \rightarrow 40 / 60$ \\
$27-30$ & $40 / 60$ \\
\hline
\end{tabular}

Fluss: $\quad 18 \mathrm{~mL} \mathrm{~min}^{-1}$

$t_{\mathrm{R}}: \quad 12.9 \mathrm{~min}$

$[\alpha]_{\mathrm{D}}^{20}=-13.3^{\circ}(\mathrm{c}=0.15, \mathrm{MeOH})$. 
UV (MeOH): $\lambda_{\max }(\lg \varepsilon)=228.0$ (4.490), 257.0 (4.738), 319.0 (4.073), $449.0 \mathrm{~nm}$ (4.865).

${ }^{1} \mathrm{H}-N M R\left(600 \mathrm{MHz}, \mathrm{DMSO}-\mathrm{d}_{6}\right): \delta=1.33-1.44\left(\mathrm{~m}, 2 \mathrm{H}, 3^{\prime}-\mathrm{H}_{2}\right), 1.64-1.73\left(\mathrm{~m}, 2 \mathrm{H}, 4^{\prime}-\mathrm{H}_{2}\right)$, 1.85-1.94 (m, $\left.2 \mathrm{H}, 2^{\prime}-\mathrm{H}_{2}\right), 2.43-2.62\left(\mathrm{~m}, 2 \mathrm{H}, 5{ }^{\prime}-\mathrm{H}_{2}\right), 3.71\left(\mathrm{~s}, 3 \mathrm{H}, \mathrm{NCH}_{3}\right), 3.76$ (dd, $\left.J=10.4,8.3 \mathrm{~Hz}, 1 \mathrm{H}, 10-\mathrm{H}_{\mathrm{a}}\right), 3.97\left(\mathrm{dd}, J=10.9,2.6 \mathrm{~Hz}, 1 \mathrm{H}, 10-\mathrm{H}_{\mathrm{b}}\right), 4.07-4.21(\mathrm{~m}, 2 \mathrm{H}$, 1-H, 2- $\left.\mathrm{H}_{\mathrm{a}}\right), 4.23-4.35\left(\mathrm{~m}, 3 \mathrm{H}, 2-\mathrm{H}_{\mathrm{b}}, 1^{\prime}-\mathrm{H}_{2}\right), 6.24\left(\mathrm{~s}, 1 \mathrm{H}, 1^{\prime \prime \prime}-\mathrm{H}\right), 7.25-7.32(\mathrm{~m}, 2 \mathrm{H}, 7-\mathrm{H}$, 6"'-H), $7.40\left(\mathrm{~d}, J=7.0 \mathrm{~Hz}, 2 \mathrm{H}, 3^{\prime \prime}-\mathrm{H}, 5 "-\mathrm{H}\right), 7.45(\mathrm{t}, J=7.5 \mathrm{~Hz}, 1 \mathrm{H}, 8-\mathrm{H}), 7.52$ (t, $J=7.8 \mathrm{~Hz}, 1 \mathrm{H}, 5$ '"'-H), 7.58 (d, J = 8.2 Hz, 1 H, 4'"-H), 7.75 (d, J = 8.4 Hz, 1 H, 9-H), 7.89 (d, J= 7.8 Hz, $\left.1 \mathrm{H}, 7^{\prime \prime \prime}-\mathrm{H}\right), 7.98\left(\mathrm{~s}_{\mathrm{br}}, 1 \mathrm{H}, 4-\mathrm{H}\right), 8.06(\mathrm{~d}, J=8.4 \mathrm{~Hz}, 1 \mathrm{H}, 6-\mathrm{H}), 8.40$ (d, $J=7.0 \mathrm{~Hz}, 2 \mathrm{H}, 2 "-\mathrm{H}, 6$ 6"-H), $10.34\left(\mathrm{~s}_{\mathrm{br}}, 1 \mathrm{H}, \mathrm{OH}\right) \mathrm{ppm}$.

${ }^{13} \mathrm{C}-\mathrm{NMR}\left(125 \mathrm{MHz}, \mathrm{DMSO}-\mathrm{d}_{6}\right): \delta=23.3$ (C-4'), 24.9 (C-3'), 30.0 (C-2'), $32.7\left(\mathrm{NCH}_{3}\right)$, 34.6 (C-5'), 40.7 (C-1), 47.6 (C-10), 52.6 (C-2), 57.4 (C-1'), 89.4 (C-1'"'), 99.7 (C-4), 111.8 (C-4"'), 113.5 (C-5a), 118.2 (C-3", C-5"), 121.4 (C-9b), 122.2 (C-9), 122.3 (C-7), 122.4 (C-7"'), 122.8 (C-6), 123.1 (C-7a'"'), 123.3 (C-6"'), 126.9 (C-8), 127.5 (C-5'"'), 129.7 (C-9a), 140.2 (C-3a'"'), 141.1 (C-2", C-6"), 141.8 (C-3a), 150.1 (C-2"'), 154.0 (C-5), 156.9 $(\mathrm{C}-4 "), 170.3(\mathrm{C}=0)$ ppm.

$\mathrm{C}_{33} \mathrm{H}_{33} \mathrm{Cl}_{2} \mathrm{~N}_{3} \mathrm{O}_{2} \mathrm{~S}$ (606.61).

ber.: $570.1977[\mathrm{M}-\mathrm{Cl}]^{+}$

gef.: 570.1971 (ESI-HRMS).

9.2 (-)-\{(1S)-[1-[6-(1-Chlormethyl-1,2-dihydro-benz[e]indol-3-yl)-6oxo-hexyl]-4-(3-Methyl-3H-benzothiazol-2-ylidenmethyl)-pyridiniumchlorid-5-yl]-2,3,4,6-tetra-O-acetyl- $\beta$-D-Galaktopyranosid\} ((-)-(1S)146)

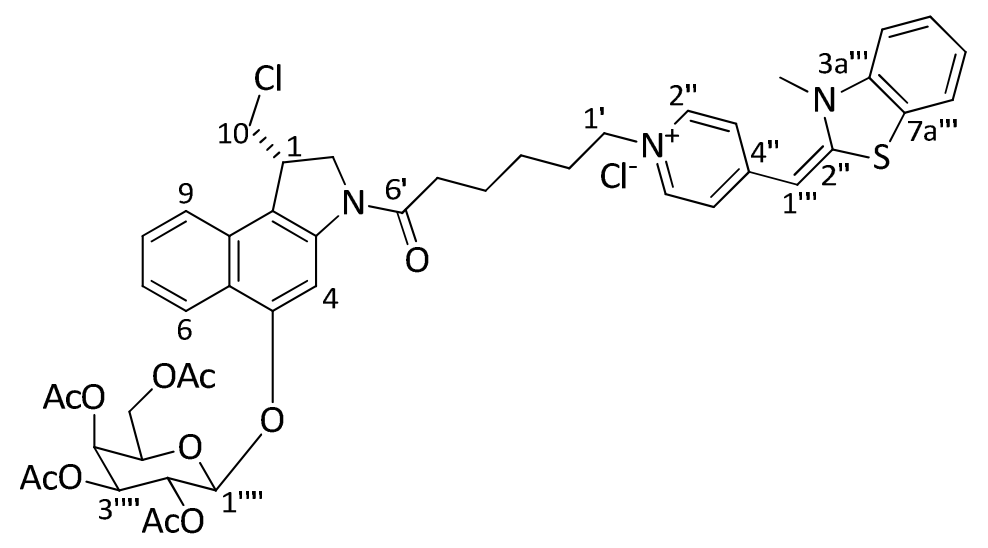


Zu einer Lösung des Phenols (1S)-128 (100 mg, $300 \mu \mathrm{mol}, 1.0$ Äq.) in absolutem $\mathrm{CH}_{2} \mathrm{Cl}_{2}(15 \mathrm{~mL})$ gab man aktiviertes Molsieb $4 \AA$ ( $\left.0.80 \mathrm{~g}\right)$ und rührte 30 min bei Raumtemperatur. Nach Zugabe des $O-(2,3,4,6$-Tetra-O-acetyl- $\alpha$-D-Galaktopyranosyl)-trichloracetimidats (116) (162 mg, $330 \mu \mathrm{mol}, 1.10$ Äq.) kühlte man die Lösung auf $10{ }^{\circ} \mathrm{C}$ und tropfte langsam eine Lösung von $\mathrm{BF}_{3} \cdot \mathrm{OEt}_{2}(19.0 \mu \mathrm{L}, 150 \mu \mathrm{mol}, 0.5 \mathrm{Äq}$.) in absolutem $\mathrm{CH}_{2} \mathrm{Cl}_{2}(1.5 \mathrm{~mL})$ zu. Nach $3.5 \mathrm{~h}$ Rühren bei $-10{ }^{\circ} \mathrm{C}$ tropfte man weiteres $\mathrm{BF}_{3} \cdot \mathrm{OEt}_{2}(113 \mu \mathrm{L}, 900 \mu \mathrm{mol}, 3.0 \mathrm{Aq}$. $)$ in absolutem $\mathrm{CH}_{2} \mathrm{Cl}_{2}(1.5 \mathrm{~mL})$ hinzu und ließ auf Raumtemperatur erwärmen. Nach $6 \mathrm{~h}$ wurde das Reaktionsgemisch via Transferkanüle in einen zweiten Kolben überführt und so vom Molsieb abgetrennt. Dieses wurde gründlich mit $\mathrm{CH}_{2} \mathrm{Cl}_{2}(2 \times 10 \mathrm{~mL})$ gewaschen und das Lösungsmittel im Vakuum entfernt. Der Rückstand wurde $1 \mathrm{~h}$ im Hochvakuum getrocknet und in absolutem DMF (15 mL) gelöst. Man kühlte die Lösung auf $0^{\circ} \mathrm{C}$ und gab EDAC $\cdot \mathrm{HCl}$ (126 mg, $660 \mu \mathrm{mol}, 2.2$ Äq.) sowie 63 (143 mg, $330 \mu \mathrm{mol}, 1.1$ Äq.) hinzu. Nach $17 \mathrm{~h}$ Rühren bei Raumtemperatur wurde das Lösungsmittel entfernt und der Rückstand in einem 2:1 Gemisch aus $\mathrm{MeOH} / \mathrm{CH}_{3} \mathrm{CN}(15 \mathrm{~mL}$ ) aufgenommen. Je $0.5 \mathrm{~mL}$ dieser Lösung wurden in das präparative HPLC-System (s. u.) injiziert. Fraktioniertes Auffangen des Eluats, Entfernung der Lösungsmittel unter vermindertem Druck sowie Entfernung des restlichen Lösungsmittels mittels Gefriertrocknung lieferte die Zielverbindung (-)(1S)-146 als gelben Feststoff (97.3 mg, $99.1 \mu \mathrm{mol}, 33 \%)$.

HPLC (analytisch):

Säule: $\quad$ Kromasil 100 C18

\begin{tabular}{ccc}
\cline { 2 - 2 } Gradient: & Zeit $[\mathrm{min}]$ & $\mathrm{H}_{2} \mathrm{O}(0.06 \% \mathrm{HCl}) / \mathrm{MeOH}$ \\
\hline 0 & $40 / 60$ \\
$0-20$ & $40 / 60 \rightarrow 0 / 100$ \\
$20-25$ & $0 / 100$ \\
$25-27$ & $0 / 100 \rightarrow 40 / 60$
\end{tabular}


27-30

$40 / 60$

Fluss: $\quad 0.8 \mathrm{~mL} \mathrm{~min}^{-1}$

$t_{\mathrm{R}}: \quad 16.5 \mathrm{~min}$

HPLC (präparativ):

Säule: $\quad$ Kromasil 100 C18

\begin{tabular}{|c|c|c|}
\hline \multirow[t]{6}{*}{ Gradient: } & Zeit [min] & $\mathrm{H}_{2} \mathrm{O}(0.06 \% \mathrm{HCl}) / \mathrm{MeOH}$ \\
\hline & 0 & $40 / 60$ \\
\hline & $0-20$ & $40 / 60 \rightarrow 0 / 100$ \\
\hline & $20-25$ & $0 / 100$ \\
\hline & $25-27$ & $0 / 100 \rightarrow 40 / 60$ \\
\hline & $27-30$ & $40 / 60$ \\
\hline
\end{tabular}

Fluss: $\quad 18 \mathrm{~mL} \mathrm{~min}^{-1}$

$t_{\mathrm{R}}: \quad 20.1 \mathrm{~min}$

$[\alpha]_{D}^{20}=-49.7^{\circ}(\mathrm{c}=0.15, \mathrm{MeOH})$.

UV (MeOH): $\lambda_{\max }(\lg \varepsilon)=254.5$ (4.634), 288.0 (3.923), 313.0 (4.026), $449.0 \mathrm{~nm}$ (4.734).

${ }^{1} \mathrm{H}-N M R\left(600 \mathrm{MHz}, \mathrm{DMSO}-\mathrm{d}_{6}\right): \delta=1.33-1.44\left(\mathrm{~m}, 2 \mathrm{H}, 3^{\prime}-\mathrm{H}_{2}\right), 1.62-1.72\left(\mathrm{~m}, 2 \mathrm{H}, 4^{\prime}-\mathrm{H}_{2}\right)$, 1.85-1.92 (m, $\left.2 \mathrm{H}, 2^{\prime}-\mathrm{H}_{2}\right), 1.97,2.02,2.06,2.17\left(4 \times \mathrm{s}, 4 \times 3 \mathrm{H}, 4 \times \mathrm{C}(\mathrm{O}) \mathrm{CH}_{3}\right), 2.54-2.64$ $\left(\mathrm{m}, 2 \mathrm{H}, 5^{\prime}-\mathrm{H}_{2}\right), 3.71\left(\mathrm{~s}, 3 \mathrm{H}, \mathrm{NCH}_{3}\right), 3.74-3.78\left(\mathrm{~m}, 1 \mathrm{H}, 10-\mathrm{H}_{\mathrm{a}}\right), 3.83-3.88(\mathrm{~m}, 1 \mathrm{H}$, $\left.10-H_{b}\right), 3.95-4.51\left(m, 8 H, 1-H, 2-H_{2}, 1^{\prime}-H_{2}, 5\right.$ '"'-H, 6'"'- $\left.H_{2}\right), 5.36-5.46(m, 3 ~ H, ~ 2 ' " '-H$, 3'"'-H, 4'"'-H), 5.54 (d, J = 6.4 Hz, 1 H, 1'"'-H ), 6.11-6.32 (m, 1 H, 1"'-H), 7.25-7.34 (m, $2 \mathrm{H}, 7-\mathrm{H}, 6$ 6"'-H), 7.40-7.42 (m, $2 \mathrm{H}, 3^{\prime}-\mathrm{H}, 5$ 5'-H), 7.44-7.48 (m, 1 H, 8-H), 7.54 (dd, $\left.J=7.8,6.6 \mathrm{~Hz}, 1 \mathrm{H}, 5^{\prime \prime \prime}-\mathrm{H}\right), 7.59(\mathrm{~d}, J=8.1 \mathrm{~Hz}, 1 \mathrm{H}, 4$ '"'-H), $7.75(\mathrm{~d}, J=8.3 \mathrm{~Hz}, 1 \mathrm{H}, 9-\mathrm{H})$, 
$7.87\left(\mathrm{~d}, J=8.4 \mathrm{~Hz}, 1 \mathrm{H}, 7^{\prime \prime \prime}-\mathrm{H}\right), 7.97\left(\mathrm{~s}_{\mathrm{br}}, 1 \mathrm{H}, 4-\mathrm{H}\right), 8.06(\mathrm{~d}, J=8.4 \mathrm{~Hz}, 1 \mathrm{H}, 6-\mathrm{H}), 8.39$ (d, $\left.J=7.2 \mathrm{~Hz}, 2 \mathrm{H}, 2^{\prime}-\mathrm{H}, 6^{\prime}-\mathrm{H}\right) \mathrm{ppm}$.

${ }^{13} \mathrm{C}-\mathrm{NMR}\left(125 \mathrm{MHz}, \mathrm{DMSO}-\mathrm{d}_{6}\right): \delta=20.3\left(\mathrm{C}(\mathrm{O}) \underline{\mathrm{C}}_{3}\right), 20.3\left(\mathrm{C}(\mathrm{O}) \underline{\mathrm{C}}_{3}\right), 20.5\left(\mathrm{C}(\mathrm{O}) \underline{\mathrm{CH}}_{3}\right)$, $20.5\left(\mathrm{C}(\mathrm{O}) \mathrm{CH}_{3}\right), 23.4$ (C-4'), 25.0 (C-3'), 30.1 (C-2'), $32.7\left(\mathrm{NCH}_{3}\right), 34.7$ (C-5'), 40.6 (C-1), 47.5 (C-10), 52.6 (C-2), 57.4 (C-1'), 61.5 (C-6'"'), 67.3, 68.5, 69.7, 70.7 (C-2'"', C-3'"', C-4'"', C-5'"'), 89.4 (C-1'"'), 98.8 (C-1'"'), 101.5 (C-4), 111.8 (C-4'"'), 115.3 (C-5a), 118.2 (C-3", C-5"), 121.7 (C-9b), 121.9 (C-9), 122.2 (C-7), 122.3 (C-7"'), 122.7 (C-6), 123.1 (C-7a'"'), 123.3 (C-6"'), 127.4 (C-8), 127.6 (C-5'"'), 129.3 (C-9a), 140.3 (C-3a'"'), 141.1 (C-2", C-6"), 141.5 (C-3a), 150.1 (C-2'"'), 152.7 (C-5), 156.9 (C-4"), 169.2, 169.3, 169.7, $169.9\left(4 \times \underline{\mathrm{C}}(\mathrm{O}) \mathrm{CH}_{3}\right), 170.5(\mathrm{C}=\mathrm{O}) \mathrm{ppm}$.

$\mathrm{C}_{47} \mathrm{H}_{51} \mathrm{Cl}_{2} \mathrm{~N}_{3} \mathrm{O}_{11} \mathrm{~S}$ (936.89).

ber.: $900.2927[\mathrm{M}-\mathrm{Cl}]^{+}$

gef.: $\quad 900.2932^{+}($ESI-HRMS).

\section{3 (-)-\{(1S)-[1-[6-(1-Chlormethyl-1,2-dihydro-benz[e]indol-3-yl)-6- oxo-hexyl]-4-(3-Methyl-3H-benzothiazol-2-ylidenmethyl)-pyridinium- chlorid-5-yl]-O- $\beta$-D-Galaktopyranosid\} ((-)-(1S)-70)}

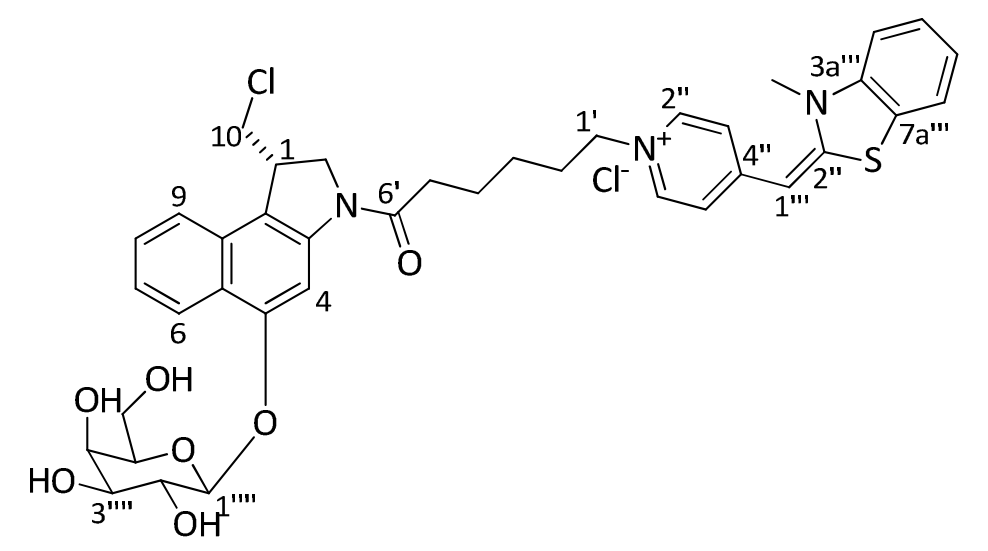

Zu einer Lösung des AC-geschützten Zuckers (-)-(1S)-146 (40 mg, $40.8 \mu \mathrm{mol}, 1.0$ Äq.) in absolutem MeOH (4 mL) gab man $\mathrm{K}_{2} \mathrm{CO}_{3}(2.8 \mathrm{mg}, 20 \mu \mathrm{mol}, 0.5 \mathrm{Äq}$.) und rührte $28 \mathrm{~h}$ bei Raumtemperatur. Nach Zugabe von $1 \mathrm{M} \mathrm{HCl}(22 \mu \mathrm{L}, 22 \mu \mathrm{mol}, 0.55$ Äq.) wurde je $0.7 \mathrm{~mL}$ dieser Lösung in das präparative HPLC-System (s. u.) injiziert. Fraktioniertes Auffangen des Eluats, Entfernung der Lösungsmittel unter vermindertem Druck sowie 
Entfernung des restlichen Lösungsmittels mittels Gefriertrocknung lieferte die Zielverbindung (-)-(1S)-70 als gelben Feststoff (16.2 mg, $19.9 \mu \mathrm{mol}, 49 \%$ ).

HPLC (analytisch):

Säule: $\quad$ Kromasil 100 C18

Isokratisch: $\mathrm{H}_{2} \mathrm{O}(0.06 \% \mathrm{HCl}) / \mathrm{CH}_{3} \mathrm{CN} \quad 60 / 40$

Fluss: $\quad 0.8 \mathrm{~mL} \mathrm{~min}^{-1}$

$t_{\mathrm{R}}: \quad 13.2 \mathrm{~min}$

HPLC (präparativ):

Säule: $\quad$ Kromasil 100 C18

Isokratisch: $\mathrm{H}_{2} \mathrm{O}(0.06 \% \mathrm{HCl}) / \mathrm{CH}_{3} \mathrm{CN} \quad 60 / 40$

Fluss: $\quad 18 \mathrm{~mL} \mathrm{~min}^{-1}$

$t_{\mathrm{R}}: \quad 16.8 \mathrm{~min}$

UV (MeOH): $\lambda_{\max }(\lg \varepsilon)=205.0 \mathrm{~nm}$ (4.613), 256.0 (4.693), 288.0 (4.054), 314.0 (4.122), 450.0 (4.755).

$[\alpha]_{D}^{20}=-66.7^{\circ}(\mathrm{c}=0.15, \mathrm{MeOH})$.

${ }^{1} \mathrm{H}-\mathrm{NMR}\left(600 \mathrm{MHz}, \mathrm{DMSO}-\mathrm{d}_{6}\right): \delta=1.29-1.46\left(\mathrm{~m}, 2 \mathrm{H}, 3^{\prime}-\mathrm{H}_{2}\right), 1.62-1.72\left(\mathrm{~m}, 2 \mathrm{H}, 4^{\prime}-\mathrm{H}_{2}\right)$, 1.84-1.94 (m, $\left.2 \mathrm{H}, 2^{\prime}-\mathrm{H}_{2}\right), 2.42-2.64\left(\mathrm{~m}, 2 \mathrm{H}, 5^{\prime}-\mathrm{H}\right), 3.14-4.40\left(\mathrm{~m}, 20 \mathrm{H}, \mathrm{NCH}_{3}, 4 \times \mathrm{OH}\right.$,

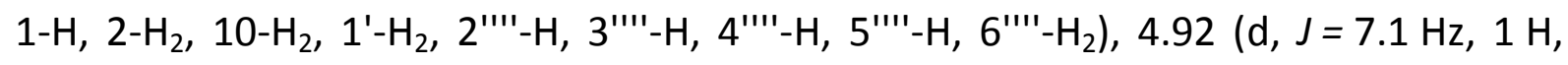
1'"'-H), 6.21 (s, $\left.1 \mathrm{H}, 1^{\prime \prime \prime}-\mathrm{H}\right), 7.29-7.32\left(\mathrm{~m}, 2 \mathrm{H}, 7-\mathrm{H}, 6\right.$ 6"'-H), 7.32-7.42 (m, $2 \mathrm{H}, 3^{\prime}-\mathrm{H}$, 5'-H), 7.48-7.53 (m, $\left.2 \mathrm{H}, 8-\mathrm{H}, 5^{\prime \prime \prime}-\mathrm{H}\right), 7.57$ (d, J = 8.2 Hz, $\left.1 \mathrm{H}, 4^{\prime \prime \prime}-\mathrm{H}\right), 7.82(\mathrm{~d}, J=8.4 \mathrm{~Hz}$, 
$1 \mathrm{H}, 9-\mathrm{H}), 7.90\left(\mathrm{dd}, J=7.8,0.6 \mathrm{~Hz}, 1 \mathrm{H}, 7^{\prime \prime \prime}-\mathrm{H}\right), 8.24-8.30(\mathrm{~d}, J=8.7 \mathrm{~Hz}, 2 \mathrm{H}, 4-\mathrm{H}, 6-\mathrm{H})$, 8.36-8.40 (m, 2 H, 2'-H, 6'-H) ppm.

${ }^{13} \mathrm{C}-\mathrm{NMR}\left(125 \mathrm{MHz}, \mathrm{DMSO}-\mathrm{d}_{6}\right): \delta=23.3$ (C-4'), 24.9 (C-3'), 30.0 (C-2'), $32.7\left(\mathrm{NCH}_{3}\right)$, 34.7 (C-5'), 40.6 (C-1), 47.5 (C-10), 52.6 (C-2), 57.4 (C-1'), 59.7 (C-6'"'), 67.6 (C-4'"'), 70.4 (C-2'"'), 73.1 (C-3'"'), 75.2 (C-5'"'), 89.4 (C-1'"'), 101.2 (C-1'"'), 101.9 (C-4), 111.8 (C-4"'), 116.5 (C-5a), 118.2 (C-3", C-5"), 122.3, 122.3, 123.1, 123.1, 123.3 (C-7, C-7"', C-9b, C-9, C-6, C-7a'"', C-6"'), 127.1 (C-8), 127.6 (C-5'"'), 129.4 (C-9a), 140.2 (C-3a'"'), 141.1 (C-2", C-6"), 141.6 (C-3a), 150.1 (C-2'"'), 153.6 (C-5), 156.9 (C-4"), 170.6 (C=O) ppm.

$\mathrm{C}_{39} \mathrm{H}_{43} \mathrm{Cl}_{2} \mathrm{~N}_{3} \mathrm{O}_{7} \mathrm{~S}$ (768.75).

ber.: $732.2505[\mathrm{M}-\mathrm{Cl}]^{+}$

gef.: $732.2508(E S I-H R M S)$.

\section{4 (1S)-1-[6-(1-Chlormethyl-5-hydroxy-1,2-dihydro-benz[e]indol-3-yl)-6- oxo-hexyl]-4-[3-(3-Methyl-3H-benzothiazol-2-yliden)-propenyl]- quinolinium-chlorid ((1S)-145)}

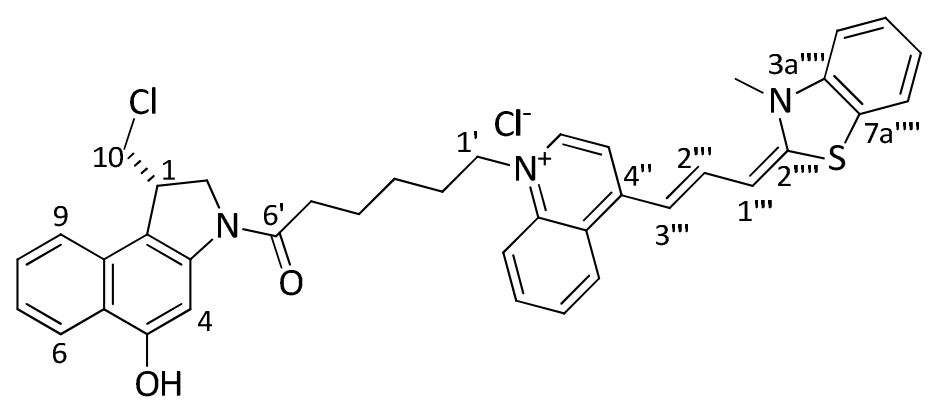

Phenol (1S)-128 (40.0 mg, $120 \mu \mathrm{mol}, 1.0$ Äq.) wurde bei Raumtemperatur in $4 \mathrm{M}$ $\mathrm{HCl} /$ EtOAc $(3.0 \mathrm{~mL})$ suspendiert, für $3 \mathrm{~h}$ gerührt und das Lösungsmittel im Vakuum entfernt. Der Rückstand wurde 1h im Hochvakuum getrocknet und anschließend in DMF (2.0 mL) gelöst und bei $0{ }^{\circ} \mathrm{C}$ mit EDAC. HCl $(68.8 \mathrm{mg}, 360 \mu \mathrm{mol}, 3.0 \mathrm{Äq}$.) und 142 (80.0 mg, $156 \mu \mathrm{mol}, 1.3$ Äq.) versetzt und anschließend für $18 \mathrm{~h}$ bei Raumtemperatur gerührt. Das Lösungsmittel wurde entfernt und der Rückstand in einem 5:1 Gemisch aus $\mathrm{MeOH} / \mathrm{CH}_{3} \mathrm{CN}(9.6 \mathrm{~mL})$ aufgenommen. Je $1 \mathrm{~mL}$ dieser Lösung wurden in das präparative HPLC-System (s. u.) injiziert. Fraktioniertes Auffangen des Eluats, Entfernung der Lösungsmittel unter vermindertem Druck sowie Entfernung des 
restlichen Lösungsmittels mittels Gefriertrocknung lieferte die Zielverbindung (1S)145 als blauen Feststoff (45.3 mg, $62.3 \mu \mathrm{mol}, 52 \%)$.

HPLC (analytisch):

Säule: Kromasil 100 C18

\begin{tabular}{|c|c|c|}
\hline \multirow[t]{6}{*}{ Gradient: } & Zeit [min] & $\mathrm{H}_{2} \mathrm{O}(0.06 \% \mathrm{HCl}) / \mathrm{MeOH}$ \\
\hline & 0 & $40 / 60$ \\
\hline & $0-20$ & $40 / 60 \rightarrow 0 / 100$ \\
\hline & $20-25$ & $0 / 100$ \\
\hline & $25-27$ & $0 / 100 \rightarrow 40 / 60$ \\
\hline & $27-30$ & $40 / 60$ \\
\hline
\end{tabular}

Fluss: $\quad 0.8 \mathrm{~mL} \mathrm{~min}^{-1}$

$t_{\mathrm{R}}: \quad 19.0 \mathrm{~min}$

HPLC (präparativ):

Säule: Kromasil 100 C18

\begin{tabular}{|c|c|c|}
\hline \multirow[t]{6}{*}{ Gradient: } & Zeit [min] & $\mathrm{H}_{2} \mathrm{O}(0.06 \% \mathrm{HCl}) / \mathrm{MeOH}$ \\
\hline & $0-2.5$ & $40 / 60$ \\
\hline & $2.5-20$ & $40 / 60 \rightarrow 0 / 100$ \\
\hline & $20-25$ & $0 / 100$ \\
\hline & $25-27$ & $0 / 100 \rightarrow 40 / 60$ \\
\hline & $27-30$ & $40 / 60$ \\
\hline
\end{tabular}

Fluss: $\quad 18 \mathrm{~mL} \mathrm{~min}^{-1}$

$t_{\mathrm{R}}: \quad 15.9 \mathrm{~min}$ 
UV (MeOH): $\lambda_{\max }(\lg \varepsilon)=256.0$ (4.563), 307.0 (4.216), $631.0 \mathrm{~nm}$ (4.951).

${ }^{1} \mathrm{H}-\mathrm{NMR}\left(600 \mathrm{MHz}, \mathrm{DMSO}-\mathrm{d}_{6}\right): \delta=1.41-1.53\left(\mathrm{~m}, 2 \mathrm{H}, 3^{\prime}-\mathrm{H}_{2}\right), 1.61-1.76\left(\mathrm{~m}, 2 \mathrm{H}, 4^{\prime}-\mathrm{H}_{2}\right)$, 1.82-1.97 (m, $\left.2 \mathrm{H}, 2^{\prime}-\mathrm{H}_{2}\right), 2.42-2.60\left(\mathrm{~m}, 2 \mathrm{H}, 5^{\prime}-\mathrm{H}_{2}\right), 3.72\left(\mathrm{~s}, 3 \mathrm{H}, \mathrm{NCH}_{3}\right), 3.75-3.87(\mathrm{~m}$, $\left.1 \mathrm{H}, 10-\mathrm{H}_{\mathrm{a}}\right), 3.99\left(\mathrm{dt}, J=15.9,8.0 \mathrm{~Hz}, 1 \mathrm{H}, 10-\mathrm{H}_{\mathrm{b}}\right), 4.13-4.24\left(\mathrm{~m}, 2 \mathrm{H}, 1-\mathrm{H}, 2-\mathrm{H}_{\mathrm{a}}\right), 4.32$ $\left(\mathrm{d}, J=8.8 \mathrm{~Hz}, 1 \mathrm{H}, 2-\mathrm{H}_{\mathrm{b}}\right), 4.57\left(\mathrm{t}, J=7.2 \mathrm{~Hz}, 2 \mathrm{H}, 1^{\prime}-\mathrm{H}_{2}\right), 6.46\left(\mathrm{~d}, J=12.3 \mathrm{~Hz}, 1 \mathrm{H}, 1^{\prime \prime}-\mathrm{H}\right)$, 7.07 (d, J = 13.3 Hz, $\left.1 \mathrm{H}, 3^{\prime \prime \prime}-\mathrm{H}\right), 7.28-7.32(\mathrm{~m}, 1 \mathrm{H}, 6$ '"'-H), 7.34-7.41 (m, $1 \mathrm{H}, 7-\mathrm{H})$, 7.44-7.54 (m, $\left.2 \mathrm{H}, 5^{\prime \prime \prime '}-\mathrm{H}, 8-\mathrm{H}\right), 7.56\left(\mathrm{~d}, J=8.2 \mathrm{~Hz}, 1 \mathrm{H}, 44^{\prime \prime \prime}-\mathrm{H}\right), 7.65-7.72(\mathrm{~m}, 1 \mathrm{H}$, 7"-H), 7.76-7.89 (m, 3 H, 9-H, 3"-H, 7"'-H), 7.94 (t, J = 7.4 Hz, 1 H, 6"-H), 8.03-8.14 (m, $2 \mathrm{H}, 5$ "'-H, 2"'-H), 8.25-8.31 (m, $2 \mathrm{H}, 4-\mathrm{H}, 6-\mathrm{H}), 8.38-8.47$ (m, $2 \mathrm{H}, 2^{\prime \prime}-\mathrm{H}, 8$ "'-H) ppm.

${ }^{13} \mathrm{C}-\mathrm{NMR}\left(125 \mathrm{MHz}, \mathrm{DMSO}-\mathrm{d}_{6}\right): \delta=23.5\left(\mathrm{C}-4^{\prime}\right), 25.5\left(\mathrm{C}-3^{\prime}\right), 28.6\left(\mathrm{C}-2^{\prime}\right), 32.8\left(\mathrm{NCH}_{3}\right)$, 34.8 (C-5'), 40.7 (C-1), 47.5 (C-10), 52.6 (C-2), 53.8 (C-1'), 98.6 (C-1'"'), 101.1 (C-4), 109.0 (C-3"'), 109.4 (C-3"), 112.3 (C-4'"'), 116.5 (C-5a), 117.7 (C-5"), 122.3 (C-7"'"), 122.3 (C-9), 122.4 (C-7), 123.1 (C-7a"'"'), 123.2 (C-6), 123.9 (C-6"'"), 124.0 (C-9b), 124.4 (C-4a"), 125.0 (C-8"), 126.4 (C-5"'"), 127.2 (C-8), 127.4 (C-7"), 129.4 (C-9a), 133.1 (C-6"), 137.6 (C-3a"'"'), 141.7 (C-3a), 142.2 (C-2"), 143.5 (C-8a"), 143.8 (C-2"'), 150.1 (C-2'"'), 153.6 (C-5), 161.4 (C-4"), 170.6 (C=0) ppm.

$\mathrm{C}_{39} \mathrm{H}_{37} \mathrm{Cl}_{2} \mathrm{~N}_{3} \mathrm{O}_{2} \mathrm{~S}(682.70)$.

ber.: $646.2290[\mathrm{M}-\mathrm{Cl}]^{+}$ gef.: 646.2292 (ESI-HRMS). 
$9.5\{(1 S)$-[1-[6-(1-Chlormethyl-5-hydroxy-1,2-dihydro-benz[e]indol-3-yl)6-oxo-hexyl]-4-[3-(3-Methyl-3H-benzothiazol-2-yliden)-propenyl]quinolinium-chlorid-5-yl]-2,3,4,6-tetra- $O$-acetyl- $\beta$-D-galatopyranosid\} ((1S)-150)

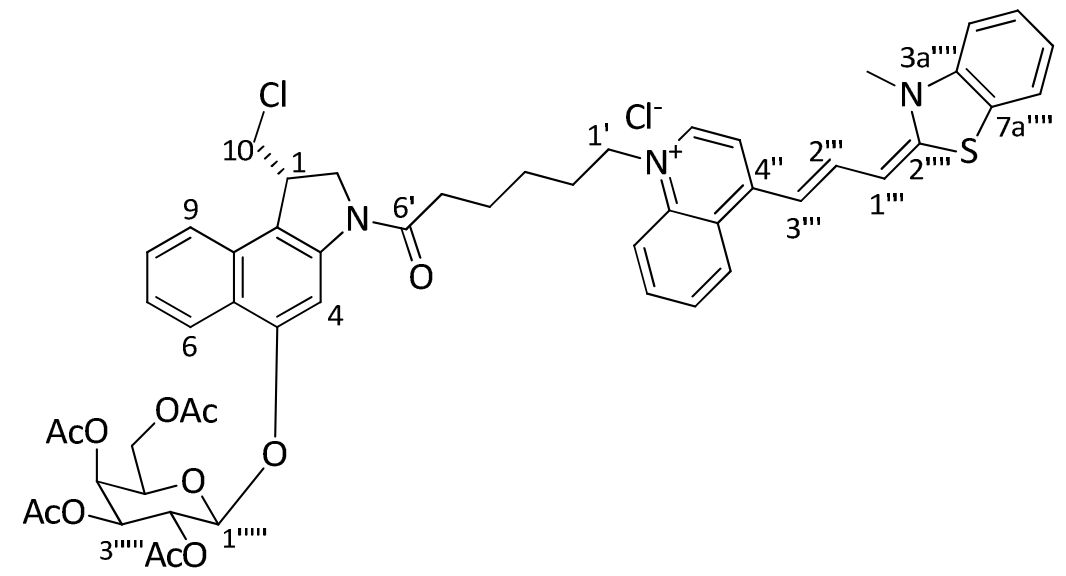

Zu einer Lösung des Phenols (1S)-128 (100 mg, $300 \mu \mathrm{mol}, 1.0$ Äq.) in absolutem $\mathrm{CH}_{2} \mathrm{Cl}_{2}(15 \mathrm{~mL})$ gab man aktiviertes Molsieb $4 \AA$ ( $\left.0.80 \mathrm{~g}\right)$ und rührte $30 \mathrm{~min}$ bei Raumtemperatur. Nach Zugabe des O-(2,3,4,6-Tetra-O-acetyl- $\alpha$-D-Galaktopyranosyl)-trichloracetimidats (116) (162 mg, $330 \mu \mathrm{mol}, 1.10$ Äq.) kühlte man die Lösung auf $10{ }^{\circ} \mathrm{C}$ und tropfte langsam eine Lösung von $\mathrm{BF}_{3} \cdot \mathrm{OEt}_{2}(19.0 \mu \mathrm{L}, 150 \mu \mathrm{mol}, 0.5 \mathrm{Äq}$.) in absolutem $\mathrm{CH}_{2} \mathrm{Cl}_{2}(1.5 \mathrm{~mL})$ zu. Nach $3.5 \mathrm{~h}$ Rühren bei $-10^{\circ} \mathrm{C}$ tropfte man weiteres $\mathrm{BF}_{3} \cdot \mathrm{OEt}_{2}\left(113 \mu \mathrm{L}, 900 \mu \mathrm{mol}, 3.0\right.$ Äq.) in absolutem $\mathrm{CH}_{2} \mathrm{Cl}_{2}(1.5 \mathrm{~mL})$ hinzu und ließ auf Raumtemperatur erwärmen. Nach $6 \mathrm{~h}$ wurde das Reaktionsgemisch via Transferkanüle in einen zweiten Kolben überführt und so vom Molsieb abgetrennt. Dieses wurde gründlich mit $\mathrm{CH}_{2} \mathrm{Cl}_{2}(2 \times 10 \mathrm{~mL})$ gewaschen und das Lösungsmittel im Vakuum entfernt. Der Rückstand wurde $1 \mathrm{~h}$ im Hochvakuum getrocknet und in absolutem DMF (15 mL) gelöst. Man kühlte die Lösung auf $0{ }^{\circ} \mathrm{C}$ und gab EDAC.HCl (172 mg, $900 \mu \mathrm{mol}, 3.0$ Äq.) sowie 142 (230 mg, 450 mol, 1.5 Äq.) hinzu. Nach 18 h Rühren bei Raumtemperatur wurde das Lösungsmittel entfernt und der Rückstand in einem 2:1 Gemisch aus $\mathrm{MeOH} / \mathrm{CH}_{3} \mathrm{CN}(15 \mathrm{~mL})$ aufgenommen. Je $0.5 \mathrm{~mL}$ dieser Lösung wurden in das präparative HPLC-System (s. u.) injiziert. Fraktioniertes Auffangen des Eluats, Entfernung der Lösungsmittel unter vermindertem Druck sowie Entfernung 
des restlichen Lösungsmittels mittels Gefriertrocknung lieferte die Zielverbindung (1S)-150 als blauen Feststoff (148 mg, $138 \mu \mathrm{mol}, 46 \%$ ).

HPLC (analytisch):

Säule: Kromasil 100 C18

\begin{tabular}{ccc} 
Gradient: & Zeit [min] & $\mathrm{H}_{2} \mathrm{O}(0.06 \% \mathrm{HCl}) / \mathrm{CH}_{3} \mathrm{CN}$ \\
\cline { 2 - 3 } 0 & $40 / 60$ \\
$25-27$ & $40 / 60 \rightarrow 0 / 100$ \\
$27-30$ & $0 / 100$ \\
$30-40$ & $0 / 100 \rightarrow 40 / 60$ \\
\end{tabular}

Fluss: $\quad 0.8 \mathrm{~mL} \mathrm{~min}^{-1}$
$t_{\mathrm{R}}$ :
$22.2 \mathrm{~min}$

HPLC (präparativ):

Säule: Kromasil 100 C18

\begin{tabular}{ccc} 
Gradient: & Zeit [min] & $\mathrm{H}_{2} \mathrm{O}(0.06 \% \mathrm{HCl}) / \mathrm{CH}_{3} \mathrm{CN}$ \\
\cline { 2 - 3 } & $0-2.5$ & $40 / 60$ \\
$2.5-25$ & $40 / 60 \rightarrow 0 / 100$ \\
$25-27$ & $0 / 100$ \\
$27-30$ & $0 / 100 \rightarrow 40 / 60$ \\
& $30-40$ & $40 / 60$ \\
Fluss: & $18 \mathrm{~mL} \mathrm{~min}{ }^{-1}$ & \\
$t_{\mathrm{R}}:$ & $15.7 \mathrm{~min}$ &
\end{tabular}


${ }^{1} \mathbf{H}-\mathbf{N M R}\left(600 \mathrm{MHz}, \mathrm{DMSO}-\mathrm{d}_{6}\right): \delta=1.43-1.53\left(\mathrm{~m}, 2 \mathrm{H}, 3^{\prime}-\mathrm{H}_{2}\right), 1.61-1.75\left(\mathrm{~m}, 2 \mathrm{H}, 4^{\prime}-\mathrm{H}_{2}\right)$, 1.85-1.94 (m, $\left.2 \mathrm{H}, 2^{\prime}-\mathrm{H}_{2}\right), 1.97,2.01,2.03,2.17\left(4 \times \mathrm{s}, 4 \times 3 \mathrm{H}, 4 \times \mathrm{C}(\mathrm{O}) \mathrm{CH}_{3}\right), 2.43-2.64$ $\left(\mathrm{m}, 2 \mathrm{H}, 5^{\prime}-\mathrm{H}_{2}\right), 3.73\left(\mathrm{~s}, 3 \mathrm{H}, \mathrm{NCH}_{3}\right), 3.86\left(\mathrm{dd}, J=10.8,7.3 \mathrm{~Hz}, 1 \mathrm{H}, 10-\mathrm{H}_{\mathrm{a}}\right), 3.93-4.16$

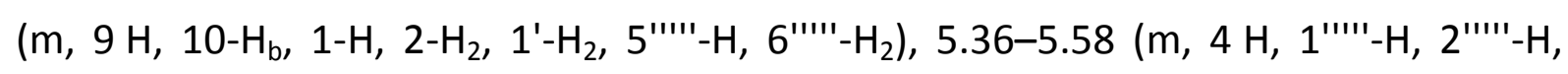
3"'"'-H, 4"'"'-H), 6.48 (d, J = 12.4 Hz, 1 H, 1"'-H), 7.09 (d, J= 13.1 Hz, 1 H, 3'"'-H), 7.28$7.32(\mathrm{~m}, 1 \mathrm{H}, 6$ 6"'-H), 7.37-7.44 (m, $1 \mathrm{H}, 7-\mathrm{H}), 7.44-7.61(\mathrm{~m}, 3 \mathrm{H}, 4$ "'"-H, 5"'"-H, 8-H), 7.65-7.72 (m, 1 H, 7"'-H), 7.76-7.94 (m, 4 H, 9-H, 3"'-H, 7"'"-H, 6"-H), 8.05-8.19 (m, $2 \mathrm{H}, 5^{\prime \prime}-\mathrm{H}, 2$ '"'-H), 8.25-8.31 (m, 2 H, 4-H, 6-H), 8.37-8.49 (m, 2 H, 2"-H, 8"-H) ppm.

${ }^{13} \mathrm{C}-\mathrm{NMR}\left(125 \mathrm{MHz}, \mathrm{DMSO}-\mathrm{d}_{6}\right): \delta=20.3\left(\mathrm{C}(\mathrm{O}) \underline{\mathrm{CH}}_{3}\right), 20.3\left(\mathrm{C}(\mathrm{O}) \underline{\mathrm{CH}}_{3}\right), 20.5\left(\mathrm{C}(\mathrm{O}) \underline{\mathrm{CH}}_{3}\right)$, $20.5\left(\mathrm{C}(\mathrm{O}) \underline{\mathrm{CH}}_{3}\right), 23.5\left(\mathrm{C}-4^{\prime}\right), 25.5\left(\mathrm{C}-3^{\prime}\right), 28.7\left(\mathrm{C}-2^{\prime}\right), 32.8\left(\mathrm{NCH}_{3}\right), 34.8\left(\mathrm{C}-5^{\prime}\right), 40.7(\mathrm{C}-1)$, 47.6 (C-10), 52.6 (C-2), 53.8 (C-1'), 61.5 (C-6"'"'), 67.3, 68.4, 69.7, 70.7 (C-2'"'", C-3'"'", C-4"'"', C-5'"'"), 98.7 (C-1'"'), 98.8 (C-1'"'), 101.5 (C-4), 109.1 (C-3'"'), 109.4 (C-3"), 112.3 (C-4'"'), 117.7 (C-5a), 117.8 (C-5"), 121.7 (C-7'"'), 121.9 (C-9), 122.3 (C-7), 122.7 (C-7a'"'), 123.8 (C-6), 123.9 (C-6"'"), 124.1 (C-9b), 124.4 (C-4a"), 125.0 (C-8"), 126.4 (C-5"'"), 127.4 (C-8), 127.4 (C-7"), 129.4 (C-9a), 133.1 (C-6"), 137.6 (C-3a'"'), 141.5 (C-3a), 142.2 (C-2"), 143.6 (C-8a"), 143.7 (C-2'"'), 150.1 (C-2'"'), 152.7 (C-5), 161.4 (C-4"), 169.2, 169.3, 169.7, $169.9\left(4 \times \underline{\mathrm{C}}(\mathrm{O}) \mathrm{CH}_{3}\right), 170.5$ (C=O) ppm.

$\mathrm{C}_{53} \mathrm{H}_{55} \mathrm{Cl}_{2} \mathrm{~N}_{3} \mathrm{O}_{11} \mathrm{~S}$ (1012.99).

ber.: $976.3240[\mathrm{M}-\mathrm{Cl}]^{+}$ gef.: 976.3242 (ESI-HRMS). 
9.6 \{(1S)-[1-[6-(1-Chlormethyl-5-hydroxy-1,2-dihydro-benz[e]indol-3-yl)6-oxo-hexyl]-4-[3-(3-Methyl-3H-benzothiazol-2-yliden)-propenyl]quinolinium-chlorid-5-yl]-O- $\beta$-D-galatopyranosid\} ((1S)-147)

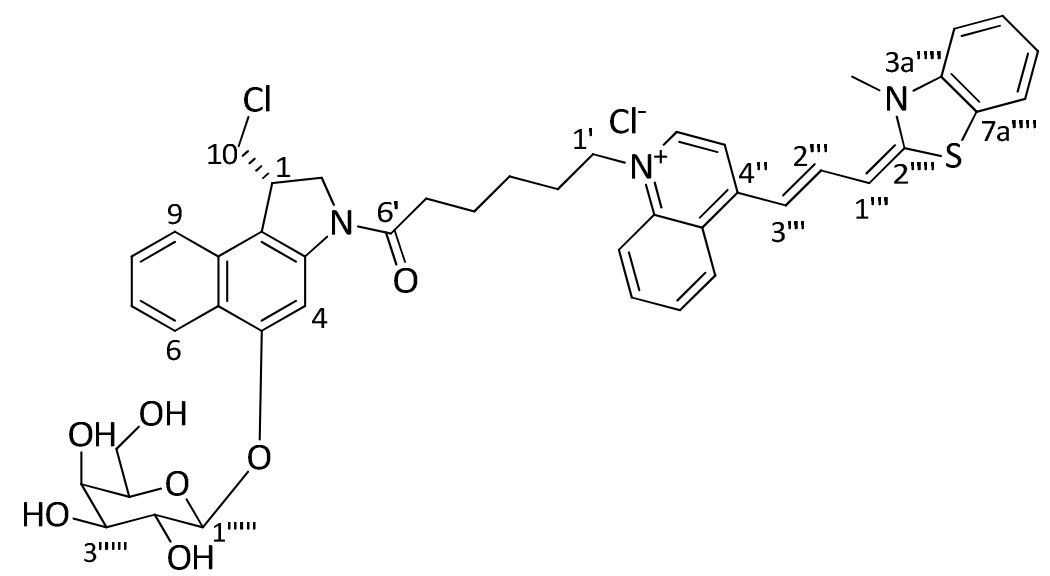

Zu einer Lösung des AC-geschützten Zuckers (1S)-150 (50 mg, $46.7 \mu \mathrm{mol}, 1.0$ Äq.) in absolutem $\mathrm{MeOH}(5 \mathrm{~mL})$ gab man $\mathrm{K}_{2} \mathrm{CO}_{3}(12.8 \mathrm{mg}, 94 \mu \mathrm{mol}, 2.0$ Äq.) und rührte $22 \mathrm{~h}$ bei Raumtemperatur. Nach Zugabe von $1 \mathrm{M} \mathrm{HCl}(100 \mu \mathrm{L}, 100 \mu \mathrm{mol}, 2.0$ Äq.) wurde je $0.7 \mathrm{~mL}$ dieser Lösung in das präparative HPLC-System (s. u.) injiziert. Fraktioniertes Auffangen des Eluats, Entfernung der Lösungsmittel unter vermindertem Druck sowie Entfernung des restlichen Lösungsmittels mittels Gefriertrocknung lieferte die Zielverbindung (1S)-147 als gelben Feststoff (30.3 mg, $34.1 \mu \mathrm{mol}, 73 \%$ ).

HPLC (analytisch):

Säule: $\quad$ Kromasil $100 \mathrm{C} 18$

Isokratisch: $\quad \mathrm{H}_{2} \mathrm{O}(0.06 \% \mathrm{HCl}) / \mathrm{CH}_{3} \mathrm{CN} \quad 50 / 50$

Fluss: $\quad 0.8 \mathrm{~mL} \mathrm{~min}^{-1}$

$t_{\mathrm{R}}: \quad 12.5 \min$ 
HPLC (präparativ):

Säule: Kromasil 100 C18

Isokratisch: $\mathrm{H}_{2} \mathrm{O}(0.06 \% \mathrm{HCl}) / \mathrm{CH}_{3} \mathrm{CN} \quad 40 / 60$

Fluss: $\quad 18 \mathrm{~mL} \mathrm{~min}^{-1}$

$t_{\mathrm{R}}: \quad 12.0 \mathrm{~min}$

UV (MeOH): $\lambda_{\max }(\lg \varepsilon)=254.0 \mathrm{~nm}$ (4.882), 289.0 (4.656), 302.0 (4.656), 632.0 (4.970).

${ }^{1} \mathrm{H}-N M R\left(600 \mathrm{MHz}, \mathrm{DMSO}-\mathrm{d}_{6}\right): \delta=1.42-1.52\left(\mathrm{~m}, 2 \mathrm{H}, 3^{\prime}-\mathrm{H}_{2}\right), 1.62-1.74\left(\mathrm{~m}, 2 \mathrm{H}, 4^{\prime}-\mathrm{H}_{2}\right)$, 1.84-1.96 (m, 2 H, 2'- $\left.\mathrm{H}_{2}\right), 2.41-2.61\left(\mathrm{~m}, 2 \mathrm{H}, 5^{\prime}-\mathrm{H}_{2}\right), 3.46-4.62\left(\mathrm{~m}, 20 \mathrm{H}, \mathrm{NCH}_{3}, 4 \times \mathrm{OH}\right.$,

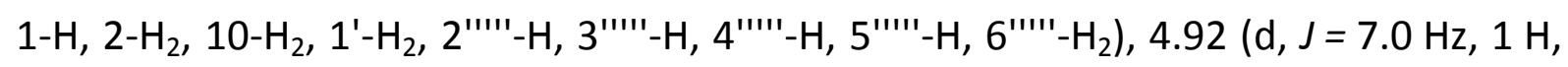
1"'"'-H), 6.46 (d, J = 12.3 Hz, $\left.1 \mathrm{H}, 1^{\prime \prime \prime}-\mathrm{H}\right), 7.07$ (d, J = $\left.13.3 \mathrm{~Hz}, 1 \mathrm{H}, 3^{\prime \prime \prime}-\mathrm{H}\right), 7.28-7.32$ (m, 1 H, 6"'"-H), 7.34-7.39 (m, 1 H, 7-H), 7.46-7.58 (m, 3 H, 4'"'-H, 5'"'-H, 8-H), 7.66-7.70 (m, 1 H, 7"-H), 7.77-7.97 (m, 4 H, 9-H, 3"-H, 7"'"-H, 6"-H), 8.04-8.15 (m, 2 H, 5"'-H, 2"'-H), 8.23-8.32 (m, 2 H, 4-H, 6-H), 8.39-8.47 (m, 2 H, 2"'-H, 8"'-H) ppm.

${ }^{13}$ C-NMR (125 MHz, DMSO-d $\left.{ }_{6}\right): \delta=23.5\left(\mathrm{C}-4^{\prime}\right), 25.5\left(\mathrm{C}-3^{\prime}\right), 28.6\left(\mathrm{C}-2^{\prime}\right), 32.8\left(\mathrm{NCH}_{3}\right)$, 34.8 (C-5'), 40.7 (C-1), 47.5 (C-10), 52.6 (C-2), 53.8 (C-1'), 59.7 (C-6'"'"), 67.6, 70.4, 73.1, 75.1 (C-2'"'", C-3"'"', C-4'"'", C-5'"'"), 98.6 (C-1'"'), 101.1 (C-4), 101.8 (C-1'"'), 109.0 (C-3"'), 109.4 (C-3"), 112.3 (C-4"'"), 117.7 (C-5a), 117.8 (C-5"), 121.7 (C-7"'"), 122.3 (C-9), 122.3 (C-7), 123.1 (C-7a"'"), 123.9 (C-6), 124.0 (C-6"'"'), 124.4 (C-9b), 125.0 (C-4a"), 125.0 (C-8"'), 126.4 (C-5"'"), 127.2 (C-8), 127.4 (C-7"), 129.4 (C-9a), 133.1 (C-6"), 137.6 (C-3a"'"), 141.6 (C-3a), 142.2 (C-2"), 143.5 (C-8a"), 143.8 (C-2'"'), 150.1 (C-2"'"), 153.6 (C-5), 161.4 (C-4"), 170.6 (C=0) ppm.

$\mathrm{C}_{45} \mathrm{H}_{47} \mathrm{Cl}_{2} \mathrm{~N}_{3} \mathrm{O}_{7} \mathrm{~S}$ (844.84).

ber.: $808.2818[\mathrm{M}-\mathrm{Cl}]^{+}$

gef.: $\quad 808.2819$ (ESI-HRMS). 
9.7 (-)-(1S)-3-(1-Chlormethyl-5-hydroxy-1,2-dihydro-benz[e]indol-3carbonyl)-chromn-2-on ((-)-(1S)-92)<smiles>O=C(c1cc2ccccc2oc1=O)N1C[C@@H](Cl)c2c1cc(O)c1ccccc21</smiles>

Phenol (1S)-92 (100.0 mg, $300 \mu \mathrm{mol}, 1.0$ Äq.) wurde bei Raumtemperatur in $4 \mathrm{M}$ $\mathrm{HCl} /$ EtOAc $(7.5 \mathrm{~mL})$ suspendiert, für $4 \mathrm{~h}$ gerührt und das Lösungsmittel im Vakuum entfernt. Der Rückstand wurde $1 \mathrm{~h}$ im Hochvakuum getrocknet und anschließend in DMF (10 mL) gelöst und bei $0^{\circ} \mathrm{C}$ mit EDAC.HCl (173 mg, $900 \mu \mathrm{mol}, 3.0$ Äq.) und 131 (74.1 mg, $390 \mu \mathrm{mol}, 1.3$ Äq.) versetzt und anschließend für $18 \mathrm{~h}$ bei Raumtemperatur gerührt. Das Lösungsmittel wurde entfernt und der Rückstand in DMF $(3.0 \mathrm{~mL})$ aufgenommen. Je $0.5 \mathrm{~mL}$ dieser Lösung wurden in das präparative HPLC-System (s. u.) injiziert. Fraktioniertes Auffangen des Eluats, Entfernung der Lösungsmittel unter vermindertem Druck sowie Entfernung des restlichen Lösungsmittels mittels Gefriertrocknung lieferte die Zielverbindung (1S)-92 als gelben Feststoff (92.7 mg, $228 \mu \mathrm{mol}, 76 \%)$.

HPLC (analytisch):

Säule: Kromasil 100 C18

\begin{tabular}{ccc}
\cline { 2 - 3 } Gradient: & Zeit [min] & $\mathrm{H}_{2} \mathrm{O}(0.06 \% \mathrm{HCl}) / \mathrm{CH}_{3} \mathrm{CN}$ \\
\cline { 2 - 3 } & 0 & $60 / 40$ \\
$0-40$ & $60 / 40 \rightarrow 30 / 70$ \\
$40-41$ & $30 / 70 \rightarrow 0 / 100$ \\
$41-45$ & $0 / 100$ \\
$45-46$ & $0 / 100 \rightarrow 60 / 40$
\end{tabular}


$46-60$

$60 / 40$

Fluss: $\quad 0.8 \mathrm{~mL} \mathrm{~min}^{-1}$

$t_{\mathrm{R}}: \quad 22.9 \mathrm{~min}$

HPLC (präparativ):

Säule: Kromasil 100 C18

\begin{tabular}{|c|c|c|}
\hline \multirow[t]{2}{*}{ Gradient: } & Zeit [min] & $\mathrm{H}_{2} \mathrm{O}(0.06 \% \mathrm{HCl}) / \mathrm{CH}_{3} \mathrm{CN}$ \\
\hline & $0-1$ & $60 / 40$ \\
\hline & $1-40$ & $60 / 40 \rightarrow 30 / 70$ \\
\hline & $40-41$ & $30 / 70 \rightarrow 0 / 100$ \\
\hline & $41-45$ & $0 / 100$ \\
\hline & $45-46$ & $0 / 100 \rightarrow 60 / 40$ \\
\hline & $46-50$ & $60 / 40$ \\
\hline
\end{tabular}

Fluss: $\quad 18 \mathrm{~mL} \mathrm{~min}^{-1}$

$t_{\mathrm{R}}: \quad 26.8 \mathrm{~min}$

UV (MeOH): $\lambda_{\max }(\lg \varepsilon)=258.0 \mathrm{~nm}$ (4.514), 297.0 (4.318), 315.0 (4.299)..

$[\alpha]_{\mathrm{D}}^{20}=-4.25^{\circ}(\mathrm{c}=0.15, \mathrm{DMSO})$.

${ }^{1} \mathrm{H}-\mathrm{NMR}\left(600 \mathrm{MHz}, \mathrm{DMSO}-\mathrm{d}_{6}\right): \delta=3.76-3.85\left(\mathrm{~m}, 1 \mathrm{H}, 10-\mathrm{H}_{\mathrm{a}}\right), 3.94(\mathrm{~d}, J=10.1 \mathrm{~Hz}, 1 \mathrm{H}$, 10- $\left.\mathrm{H}_{\mathrm{b}}\right), 4.05-4.19\left(\mathrm{~m}, 2 \mathrm{H}, 1-\mathrm{H}, 2-\mathrm{H}_{\mathrm{a}}\right), 4.32\left(\mathrm{t}, J=9.9 \mathrm{~Hz}, 1 \mathrm{H}, 2-\mathrm{H}_{\mathrm{b}}\right), 7.38(\mathrm{t}, J=7.3 \mathrm{~Hz}$, $1 \mathrm{H}, 7-\mathrm{H}), 7.45$ (t, J = $\left.7.5 \mathrm{~Hz}, 1 \mathrm{H}, 66^{\prime}-\mathrm{H}\right), 7.49-7.57\left(\mathrm{~m}, 2 \mathrm{H}, 8^{\prime}-\mathrm{H}, 8-\mathrm{H}\right), 7.74(\mathrm{t}, J=7.8 \mathrm{~Hz}$, $\left.1 \mathrm{H}, 7^{\prime}-\mathrm{H}\right), 7.80-7.88$ (m, $\left.2 \mathrm{H}, 5^{\prime}-\mathrm{H}, 9-\mathrm{H}\right), 7.97$ (s, $\left.1 \mathrm{H}, 4-\mathrm{H}\right), 8.14$ (d, J = 8.1 Hz, $\left.1 \mathrm{H}, 6-\mathrm{H}\right)$, $8.44\left(\mathrm{~s}, 1 \mathrm{H}, 4^{\prime}-\mathrm{H}\right), 10.48$ (s, $\left.1 \mathrm{H}, \mathrm{OH}\right) \mathrm{ppm}$.

${ }^{13}$ C-NMR (125 MHz, DMSO-d 6 ): $\delta=40.7$ (C-1), 47.3 (C-10), 53.4 (C-2), 99.5 (C-4), 115.3 (C-5a), 116.3 (C-8'), 118.1 (C-3'), 122.3 (C-9b), 122.7 (C-9), 123.1 (C-6), 123.2 (C-7), 
124.9 (C-6'), 125.1 (C-4a'), 127.3 (C-8), 129.3 (C-5'), 129.8 (C-9a), 133.1 (C-7'), 140.9 (C-3a), 143.1 (C-4'), 153.6 (C-5), 154.3 (C-8a'), 157.3 (COO), 162.0 (NCO) ppm.

$\mathrm{C}_{23} \mathrm{H}_{16} \mathrm{ClNO}_{4}$ (405.83).

ber.: $428.0657[\mathrm{M}+\mathrm{Na}]^{+}$

gef.: 428.0660 (ESI-HRMS).

\section{$9.8\{(1 S)-[3-(1-C h l o r m e t h y l-5-h y d r o x y-1,2-d i h y d r o-b e n z[e] i n d o l-3-$} carbonyl)-chromn-2-on]-2,3,4,6-tetra-O-acetyl- $\beta$-D-Galaktopyranosid\} ((1S)-151)

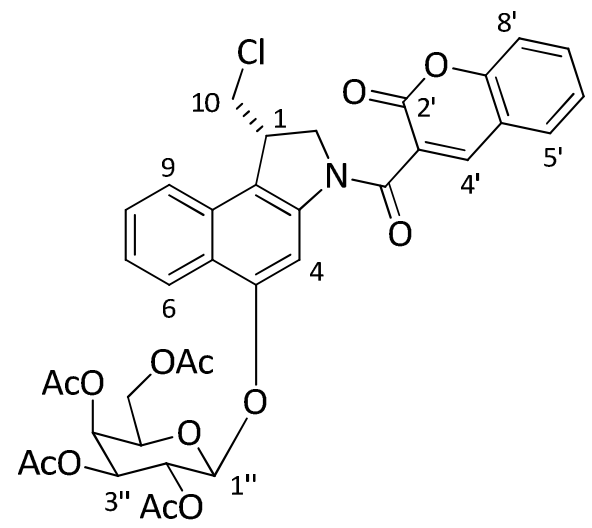

Zu einer Lösung des Phenols (1S)-128 (100 mg, $300 \mu \mathrm{mol}, 1.0$ Äq.) in absolutem $\mathrm{CH}_{2} \mathrm{Cl}_{2}(15 \mathrm{~mL})$ gab man aktiviertes Molsieb $4 \AA$ ( $\left.0.80 \mathrm{~g}\right)$ und rührte 30 min bei Raumtemperatur. Nach Zugabe des $O-(2,3,4,6$-Tetra-O-acetyl- $\alpha$-D-Galaktopyranosyl)-trichloracetimidats (116) (162 mg, $330 \mu \mathrm{mol}, 1.10$ Äq.) kühlte man die Lösung auf $10{ }^{\circ} \mathrm{C}$ und tropfte langsam eine Lösung von $\mathrm{BF}_{3} \cdot \mathrm{OEt}_{2}(19.0 \mu \mathrm{L}, 150 \mu \mathrm{mol}, 0.5$ Äq.) in absolutem $\mathrm{CH}_{2} \mathrm{Cl}_{2}(1.5 \mathrm{~mL})$ zu. Nach $3.5 \mathrm{~h}$ Rühren bei $-10^{\circ} \mathrm{C}$ tropfte man weiteres $\mathrm{BF}_{3} \cdot \mathrm{OEt}_{2}\left(113 \mu \mathrm{L}, 900 \mu \mathrm{mol}, 3.0\right.$ Äq.) in absolutem $\mathrm{CH}_{2} \mathrm{Cl}_{2}(1.5 \mathrm{~mL})$ hinzu und ließ auf Raumtemperatur erwärmen. Nach $6 \mathrm{~h}$ wurde das Reaktionsgemisch via Transferkanüle in einen zweiten Kolben überführt und so vom Molsieb abgetrennt. Dieses wurde gründlich mit $\mathrm{CH}_{2} \mathrm{Cl}_{2}(2 \times 10 \mathrm{~mL})$ gewaschen und das Lösungsmittel im Vakuum entfernt. Der Rückstand wurde $1 \mathrm{~h}$ im Hochvakuum getrocknet und in absolutem DMF $(15 \mathrm{~mL})$ gelöst. Man kühlte die Lösung auf $0^{\circ} \mathrm{C}$ und gab $\mathrm{EDAC} \cdot \mathrm{HCl}$ (172 mg, $900 \mu \mathrm{mol}, 3.0$ Äq.) sowie 131 (86 mg, $450 \mu \mathrm{mol}, 1.5$ Äq.) hinzu. Nach 18 h 
Rühren bei Raumtemperatur wurde das Lösungsmittel entfernt. Nach säulenchromatographischer Filtration an Kieselgel $\left(\mathrm{CH}_{2} \mathrm{Cl}_{2} / \mathrm{MeOH} 50: 1\right)$ erhielt man die leicht verunreinigte Zielverbindung (1S)-151 als braunen Feststoff (249 mg).

$\mathrm{C}_{37} \mathrm{H}_{34} \mathrm{CINO}_{13}(736.12)$.

$9.9\{(1 S)$-[3-(1-Chlormethyl-5-hydroxy-1,2-dihydro-benz[e]indol-3carbonyl)-chromn-2-on]-O- $\beta$-D-Galaktopyranosid\} ((1S)-69)

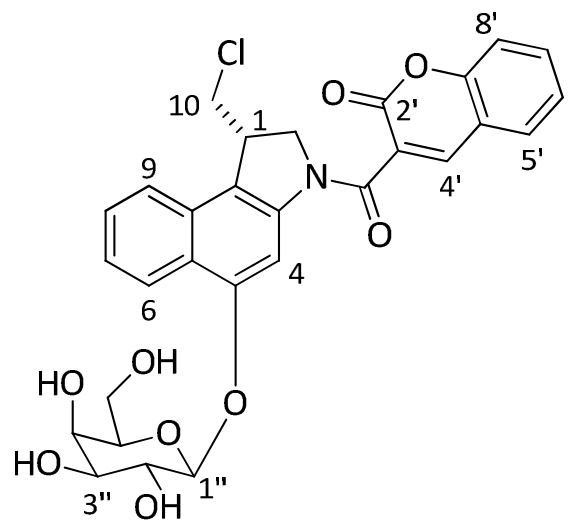

Zu einer Suspension des AC-geschützten Zuckers (1S)-151 (249 mg) in absolutem $\mathrm{MeOH}(50 \mathrm{~mL})$ wurde bei $0^{\circ} \mathrm{C}$ eine Lösung von NaOMe in $\mathrm{MeOH}(111 \mu \mathrm{L}$ einer $5.4 \mathrm{M}$ Lösung) zugegeben und für $4 \mathrm{~h}$ bei Raumtemperatur gerührt. Die Reaktionslösung wurde mit $\mathrm{H}_{2} \mathrm{O}(5 \mathrm{~mL}$ ) verdünnt und mit saurem lonentauscher (Amberlite-IR 120) auf $\mathrm{pH} \approx 7$ eingestellt. Es wurde vom lonentauscher abfiltiert, mit $\mathrm{MeOH}(20 \mathrm{~mL})$ gewaschen und das Lösungsmittel im Vakuum entfernt. Das Rohprodukt wurde in DMF $(6 \mathrm{~mL})$ gelöst und es wurde je $0.5 \mathrm{~mL}$ dieser Lösung in das präparative HPLC-System (s. u.) injiziert. Fraktioniertes Auffangen des Eluats, Entfernung der Lösungsmittel unter vermindertem Druck sowie Entfernung des restlichen Lösungsmittels mittels Gefriertrocknung lieferte die Zielverbindung (1S)-69 als gelben Feststoff (108 mg, $190 \mu \mathrm{mol}, 63 \%$ ausgehend von (1S)-128). 
HPLC (analytisch):

Säule: Kromasil 100 C18

\begin{tabular}{|c|c|c|}
\hline \multirow[t]{6}{*}{ Gradient: } & Zeit [min] & $\mathrm{H}_{2} \mathrm{O}(0.06 \% \mathrm{HCl}) / \mathrm{CH}_{3} \mathrm{CN}$ \\
\hline & 0 & $70 / 30$ \\
\hline & $0-20$ & $70 / 30 \rightarrow 0 / 100$ \\
\hline & $20-25$ & $0 / 100$ \\
\hline & $25-26$ & $0 / 100 \rightarrow 70 / 30$ \\
\hline & $26-30$ & $70 / 30$ \\
\hline
\end{tabular}

Fluss: $\quad 0.8 \mathrm{~mL} \mathrm{~min}^{-1}$
$t_{\mathrm{R}}:$
$9.7 \mathrm{~min}$

HPLC (präparativ):

Säule: Kromasil 100 C18

\begin{tabular}{ccc}
\cline { 2 - 3 } Gradient: & Zeit $[\mathrm{min}]$ & $\mathrm{H}_{2} \mathrm{O}(0.06 \% \mathrm{HCl}) / \mathrm{CH}_{3} \mathrm{CN}$ \\
\cline { 2 - 3 } & $0-1$ & $70 / 30$ \\
& $20-25$ & $0 / 100$ \\
& $25-26$ & $0 / 100 \rightarrow 70 / 30$ \\
& $26-30$ & $70 / 30$ \\
Fluss: & $18 \mathrm{~mL} \mathrm{~min}{ }^{-1}$ & \\
$t_{\mathrm{R}}:$ & $9.5 \mathrm{~min}$ &
\end{tabular}

UV (MeOH): $\lambda_{\max }(\lg \varepsilon)=204.0 \mathrm{~nm}(4.542), 256.0$ (4.506), 298.0 (4.344). 
${ }^{1} \mathrm{H}-\mathrm{NMR}\left(600 \mathrm{MHz}, \mathrm{DMSO}-\mathrm{d}_{6}\right): \delta=3.44-3.69,3.74-3.93\left(2 \times \mathrm{m}, 7 \mathrm{H}, 2^{2}-\mathrm{H}, 3\right.$ "'-H, 4"-H, 5"-H, 6"- $\left.\mathrm{H}_{2}, 10-\mathrm{H}_{\mathrm{a}}\right), 3.97\left(\mathrm{~d}, J=10.1 \mathrm{~Hz}, 1 \mathrm{H}, 10-\mathrm{H}_{\mathrm{b}}\right), 4.13\left(\mathrm{~d}, J=10.5 \mathrm{~Hz}, 1 \mathrm{H}, 2-\mathrm{H}_{\mathrm{a}}\right)$, 4.18-4.27 (m, $1 \mathrm{H}, 1-\mathrm{H}), 4.36\left(\mathrm{t}, J=9.9 \mathrm{~Hz}, 1 \mathrm{H}, 2-\mathrm{H}_{\mathrm{b}}\right), 4.49-4.59(\mathrm{~m}, 2 \mathrm{H}, \mathrm{OH}), 4.84(\mathrm{~d}$, $J=3.9 \mathrm{~Hz}, 1 \mathrm{H}, \mathrm{OH}), 4.96\left(\mathrm{~d}, J=7.1 \mathrm{~Hz}, 1 \mathrm{H}, 1^{\prime \prime}-\mathrm{H}\right), 5.31(\mathrm{~d}, J=5.4 \mathrm{~Hz}, 1 \mathrm{H}, \mathrm{OH}), 7.43-$ $7.49(\mathrm{~m}, 2 \mathrm{H}, 6$ '-H, 7-H), 7.52 (d, J = $8.3 \mathrm{~Hz}, 1 \mathrm{H}, 8$ '-H), 7.56-7.61 (m, $1 \mathrm{H}, 8-\mathrm{H}), 7.72-$ $7.77\left(\mathrm{~m}, 1 \mathrm{H}, 7^{\prime}-\mathrm{H}\right), 7.84-7.88\left(\mathrm{~m}, 1 \mathrm{H}, 5^{\prime}-\mathrm{H}\right), 7.90$ (d, J = 8.3 Hz, $\left.1 \mathrm{H}, 9-\mathrm{H}\right), 8.27$ (s, $1 \mathrm{H}$, 4-H), 8.37 (d, J = 8.0 Hz, $1 \mathrm{H}, 6-\mathrm{H}), 8.46$ (s, $\left.1 \mathrm{H}, 4^{\prime}-\mathrm{H}\right) \mathrm{ppm}$.

${ }^{13}$ C-NMR (125 MHz, DMSO-d 6 ): $\delta=40.7$ (C-1), 47.2 (C-10), 53.4 (C-2), 59.5 (C-6"), 67.5, 70.4, 73.1, 75.2 (C-2", C-3", C-4", C-5"), 101.3 (C-4), 102.3 (C-1"), 116.3 (C-8'), 118.2 (C-3'), 118.4 (C-5a), 122.8 (C-9b), 123.2 (C-9), 123.3 (C-6), 124.0 (C-7), 124.8 (C-6'), 125.0 (C-4a'), 127.5 (C-8), 129.4 (C-5'), 129.4 (C-9a), 133.1 (C-7'), 140.8 (C-3a), 143.4 (C-4'), 153.6 (C-5), 153.9 (C-8a'), 157.3 (COO), 162.2 (NCO) ppm.

$\mathrm{C}_{29} \mathrm{H}_{26} \mathrm{CINO}_{9}$ (567.97).

ber.: $590.1187[\mathrm{M}+\mathrm{Na}]^{+}$

gef.: 590.1188 (ESI-HRMS).

\section{$9.10\{(-)-(1 S)-[4-[2-(1-C h l o r m e t h y l-5-h y d r o x y-1,2-d i h y d r o-b e n z[e]$ indol-3-} yl)-2-oxo-ethyl]-7-dimethylamino-chromn-2-on]\} ((-)-(1S)-137)

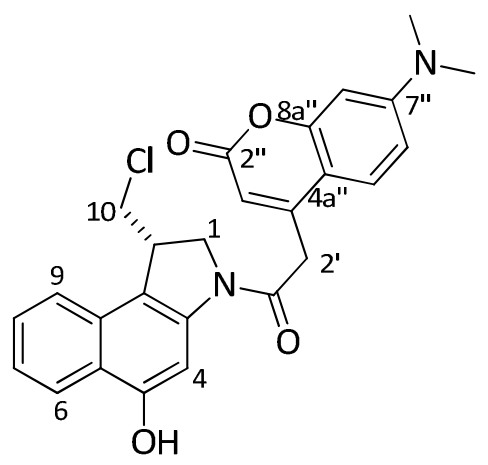

Phenol (1S)-128 (100.0 mg, $300 \mu \mathrm{mol}, 1.0$ Äq.) wurde bei Raumtemperatur in $4 \mathrm{M}$ $\mathrm{HCl} /$ EtOAc $(7.5 \mathrm{~mL})$ suspendiert, für $4 \mathrm{~h}$ gerührt und das Lösungsmittel im Vakuum entfernt. Der Rückstand wurde $1 \mathrm{~h}$ im Hochvakuum getrocknet und anschließend in DMF (10 mL) gelöst und bei $0{ }^{\circ} \mathrm{C}$ mit EDAC. $\mathrm{HCl}(173 \mathrm{mg}, 900 \mu \mathrm{mol}, 3.0$ Äq.) und 62 (96.4 mg, $390 \mu \mathrm{mol}, 1.3$ Äq.) versetzt und anschließend für $18 \mathrm{~h}$ bei Raumtemperatur 
gerührt. Das Lösungsmittel wurde entfernt und der Rückstand in DMF $(3.0 \mathrm{~mL})$ aufgenommen. Je $0.5 \mathrm{~mL}$ dieser Lösung wurden in das präparative HPLC-System (s. u.) injiziert. Fraktioniertes Auffangen des Eluats, Entfernung der Lösungsmittel unter vermindertem Druck sowie Entfernung des restlichen Lösungsmittels mittels Gefriertrocknung lieferte die Zielverbindung (1S)-137 als gelben Feststoff $(96.7 \mathrm{mg}$, $209 \mu \mathrm{mol}, 70 \%)$.

HPLC (analytisch):

Säule: Kromasil 100 C18

\begin{tabular}{ccc} 
Gradient: & Zeit [min] & $\mathrm{H}_{2} \mathrm{O}(0.06 \% \mathrm{HCl}) / \mathrm{CH}_{3} \mathrm{CN}$ \\
\cline { 2 - 3 } 0 & $60 / 40$ \\
$40-41$ & $30 / 70 \rightarrow 0 / 100$ \\
$41-45$ & $0 / 100$ \\
$45-46$ & $0 / 100 \rightarrow 60 / 40$ \\
$46-60$ & $60 / 40$ \\
\hline
\end{tabular}

Fluss: $\quad 0.8 \mathrm{~mL} \mathrm{~min}^{-1}$

$t_{\mathrm{R}}: \quad 25.2 \mathrm{~min}$

HPLC (präparativ):

Säule: Kromasil 100 C18

\begin{tabular}{ccc} 
Gradient: & Zeit [min] & $\mathrm{H}_{2} \mathrm{O}(0.06 \% \mathrm{HCl}) / \mathrm{CH}_{3} \mathrm{CN}$ \\
\hline $0-1$ & $60 / 40$ \\
$1-40$ & $60 / 40 \rightarrow 30 / 70$
\end{tabular}




\begin{tabular}{|c|c|c|}
\hline & $40-41$ & $30 / 70 \rightarrow 0 / 100$ \\
\hline & $41-45$ & $0 / 100$ \\
\hline & $45-46$ & $0 / 100 \rightarrow 60 / 40$ \\
\hline & $46-50$ & $60 / 40$ \\
\hline Fluss: & $18 \mathrm{~mL} \mathrm{~min}^{-1}$ & \\
\hline$t_{\mathrm{R}}:$ & $28.7 \mathrm{~min}$ & \\
\hline
\end{tabular}

UV (MeOH): $\lambda_{\max }(\lg \varepsilon)=209.0 \mathrm{~nm}$ (4.665), 259.0 (4.758), 319.0 (4.161), 375.0 (4.297). $[\alpha]_{D}^{20}=-55.06^{\circ}(c=0.15, \mathrm{DMSO})$.

${ }^{1} \mathrm{H}-\mathrm{NMR}\left(600 \mathrm{MHz}, \mathrm{DMSO}-\mathrm{d}_{6}\right): \delta=3.02\left(\mathrm{~s}, 6 \mathrm{H}, \mathrm{NMe}_{2}\right), 3.76-3.88\left(\mathrm{~m}, 1 \mathrm{H}, 10-\mathrm{H}_{\mathrm{a}}\right)$, 3.97-4.00 (m, $\left.2 \mathrm{H}, 10-\mathrm{H}_{\mathrm{b}}, 2^{\prime}-\mathrm{H}_{\mathrm{a}}\right), 4.12-4.27\left(\mathrm{~m}, 2 \mathrm{H}, 1-\mathrm{H}, 2^{\prime}-\mathrm{H}_{\mathrm{b}}\right), 4.34(\mathrm{dd}, J=10.9$, $\left.2.4 \mathrm{~Hz}, 1 \mathrm{H}, 2-\mathrm{H}_{\mathrm{a}}\right), 4.50\left(\mathrm{t}, J=10.1 \mathrm{~Hz}, 1 \mathrm{H}, 2-\mathrm{H}_{\mathrm{b}}\right), 6.05\left(\mathrm{~s}, 1 \mathrm{H}, 3^{\prime}-\mathrm{H}\right), 6.58(\mathrm{~d}, J=2.5 \mathrm{~Hz}$, $\left.1 \mathrm{H}, 8^{\prime}-\mathrm{H}\right), 6.71$ (dd, J=9.1, 2.5 Hz, $\left.1 \mathrm{H}, 6^{\prime}-\mathrm{H}\right), 7.34$ (t, J= $\left.7.3 \mathrm{~Hz}, 1 \mathrm{H}, 7-\mathrm{H}\right), 7.51$ (t, $J=7.6 \mathrm{~Hz}, 1 \mathrm{H}, 8-\mathrm{H}), 7.56\left(\mathrm{~d}, J=9.0 \mathrm{~Hz}, 1 \mathrm{H}, 5^{\prime}-\mathrm{H}\right), 7.81(\mathrm{~d}, J=8.4 \mathrm{~Hz}, 1 \mathrm{H}, 9-\mathrm{H}), 7.88$ (s, $1 \mathrm{H}, 4-\mathrm{H}), 8.10(\mathrm{~d}, J=8.3 \mathrm{~Hz}, 1 \mathrm{H}, 6-\mathrm{H}), 10.32(\mathrm{~s}, 1 \mathrm{H}, \mathrm{OH}) \mathrm{ppm}$.

${ }^{13} \mathrm{C}-\mathrm{NMR}\left(125 \mathrm{MHz}, \mathrm{DMSO}-\mathrm{d}_{6}\right): \delta=38.7$ (C-2'), $39.6\left(\mathrm{NMe}_{2}\right), 40.8$ (C-1), 47.6 (C-10), 52.9 (C-2), 97.3 (C-8"), 99.6 (C-4), 108.5 (C-4a"), 108.9 (C-6"), 109.5 (C-3"), 114.1 (C-5a), 121.8 (C-9b), 122.5 (C-9), 122.8 (C-7), 123.0 (C-6), 126.4 (C-5"), 127.2 (C-8), 129.8 (C-9a), 141.7 (C-3a), 151.0 (C-4"), 152.7 (C-8a"), 154.2 (C-5), 155.3 (C-7"), 160.6 (COO), 166.8 (NCO) ppm.

$\mathrm{C}_{26} \mathrm{H}_{23} \mathrm{ClN} \mathrm{O}_{4}$ (462.92).

ber.: $461.1265[\mathrm{M}-\mathrm{H}]^{-}$

gef.: 461.1274 (ESI-HRMS). 


\section{$9.11\{[(1 S)$-[4-[2-(1-Chlormethyl-5-hydroxy-1,2-dihydro-benz[e]indol-3-yl)-} 2-oxo-ethyl]-7-dimethylamino-chromn-2-on]-5-yl]-2,3,4,6-tetra-Oacetyl- $\beta$-D-Galaktopyranosid\} ((1S)-151)

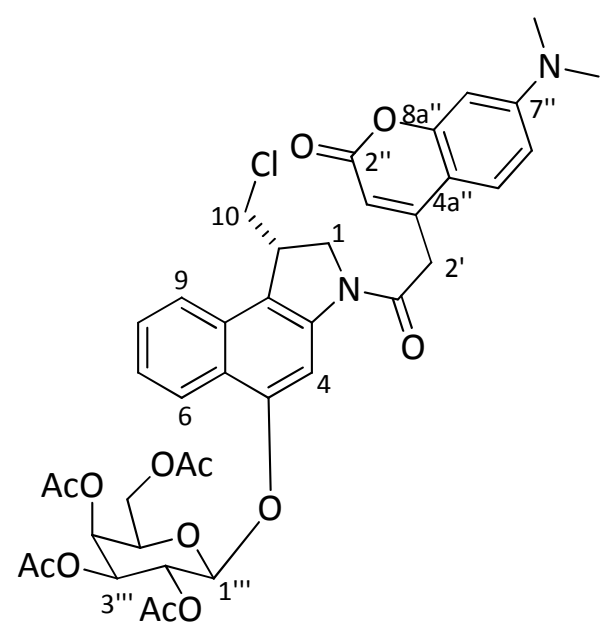

Zu einer Lösung des Phenols (1S)-128 (100 mg, $300 \mu \mathrm{mol}, 1.0$ Äq.) in absolutem $\mathrm{CH}_{2} \mathrm{Cl}_{2}(15 \mathrm{~mL})$ gab man aktiviertes Molsieb $4 \AA$ ( $\left.0.80 \mathrm{~g}\right)$ und rührte $30 \mathrm{~min}$ bei Raumtemperatur. Nach Zugabe des O-(2,3,4,6-Tetra-O-acetyl- $\alpha$-D-Galaktopyranosyl)-trichloracetimidats (116) (162 mg, $330 \mu \mathrm{mol}, 1.10$ Äq.) kühlte man die Lösung auf $10{ }^{\circ} \mathrm{C}$ und tropfte langsam eine Lösung von $\mathrm{BF}_{3} \cdot \mathrm{OEt}_{2}(19.0 \mu \mathrm{L}, 150 \mu \mathrm{mol}, 0.5 \mathrm{Äq}$.) in absolutem $\mathrm{CH}_{2} \mathrm{Cl}_{2}(1.5 \mathrm{~mL})$ zu. Nach $3.5 \mathrm{~h}$ Rühren bei $-10^{\circ} \mathrm{C}$ tropfte man weiteres $\mathrm{BF}_{3} \cdot \mathrm{OEt}_{2}\left(113 \mu \mathrm{L}, 900 \mu \mathrm{mol}, 3.0\right.$ Äq.) in absolutem $\mathrm{CH}_{2} \mathrm{Cl}_{2}(1.5 \mathrm{~mL})$ hinzu und ließ auf Raumtemperatur erwärmen. Nach $6 \mathrm{~h}$ wurde das Reaktionsgemisch via Transferkanüle in einen zweiten Kolben überführt und so vom Molsieb abgetrennt. Dieses wurde gründlich mit $\mathrm{CH}_{2} \mathrm{Cl}_{2}(2 \times 10 \mathrm{~mL})$ gewaschen und das Lösungsmittel im Vakuum entfernt. Der Rückstand wurde $1 \mathrm{~h}$ im Hochvakuum getrocknet und in absolutem DMF $(15 \mathrm{~mL})$ gelöst. Man kühlte die Lösung auf $0{ }^{\circ} \mathrm{C}$ und gab EDAC $\cdot \mathrm{HCl}$ (172 mg, $900 \mu \mathrm{mol}, 3.0$ Äq.) sowie 62 (111 mg, $450 \mu \mathrm{mol}, 1.5$ Äq.) hinzu. Nach $18 \mathrm{~h}$ Rühren bei Raumtemperatur wurde das Lösungsmittel entfernt. Nach säulenchromatographischer Filtration an Kieselgel $\left(\mathrm{CH}_{2} \mathrm{Cl}_{2} / \mathrm{MeOH}\right.$ 50:1) erhielt man die leicht verunreinigte Zielverbindung (1S)-151 als braunen Feststoff (226 mg).

$\mathrm{C}_{40} \mathrm{H}_{41} \mathrm{ClN}_{2} \mathrm{O}_{13}$ (793.21). 


\section{$9.12\{[(-)-(1 S)$-[4-[2-(1-Chlormethyl-5-hydroxy-1,2-dihydro-benz[e]indol-3-}

yl)-2-oxo-ethyl]-7-dimethylamino-chromn-2-on]-5-yl]-O- $\beta$-D-

\section{Galaktopyranosid\} ((-)-(1S)-139)}

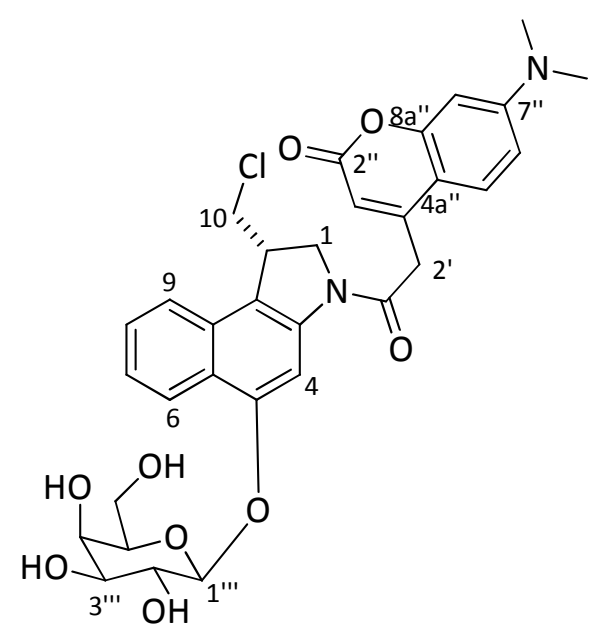

Zu einer Suspension des AC-geschützten Zuckers (1S)-151 (226 mg) in absolutem $\mathrm{MeOH}(50 \mathrm{~mL})$ wurde bei $0^{\circ} \mathrm{C}$ eine Lösung von $\mathrm{NaOMe}$ in $\mathrm{MeOH}(111 \mu \mathrm{L}$ einer $5.4 \mathrm{M}$ Lösung) zugegeben und für $4 \mathrm{~h}$ bei Raumtemperatur gerührt. Die Reaktionslösung wurde mit $\mathrm{H}_{2} \mathrm{O}(5 \mathrm{~mL}$ ) verdünnt und mit saurem lonentauscher (Amberlite-IR 120) auf $\mathrm{pH} \approx 7$ eingestellt. Es wurde vom lonentauscher abfiltiert, mit $\mathrm{MeOH}(20 \mathrm{~mL})$ gewaschen und das Lösungsmittel im Vakuum entfernt. Das Rohprodukt wurde in DMF $(6 \mathrm{~mL})$ gelöst und es wurde je $0.5 \mathrm{~mL}$ dieser Lösung in das präparative HPLC-System (s. u.) injiziert. Fraktioniertes Auffangen des Eluats, Entfernung der Lösungsmittel unter vermindertem Druck sowie Entfernung des restlichen Lösungsmittels mittels Gefriertrocknung lieferte die Zielverbindung (1S)-139 als gelben Feststoff (113 mg, $181 \mu \mathrm{mol}, 60 \%$ ausgehend von (1S)-128).

HPLC (analytisch):

Säule: Kromasil 100 C18

\begin{tabular}{|c|c|c|}
\hline \multirow[t]{2}{*}{ Gradient: } & Zeit [min] & $\mathrm{H}_{2} \mathrm{O}(0.06 \% \mathrm{HCl}) / \mathrm{CH}_{3} \mathrm{CN}$ \\
\hline & 0 & $70 / 30$ \\
\hline & $0-20$ & $70 / 30 \rightarrow 0 / 100$ \\
\hline
\end{tabular}




$\begin{array}{cc}20-25 & 0 / 100 \\ 25-26 & 0 / 100 \rightarrow 70 / 30 \\ 26-30 & 70 / 30\end{array}$

Fluss: $\quad 0.8 \mathrm{~mL} \mathrm{~min}^{-1}$

$t_{\mathrm{R}}: \quad 10.2 \mathrm{~min}$

HPLC (präparativ):

Säule: Kromasil $100 \mathrm{C} 18$

\begin{tabular}{ccc}
\cline { 2 - 3 } Gradient: & Zeit [min] & $\mathrm{H}_{2} \mathrm{O}(0.06 \% \mathrm{HCl}) / \mathrm{CH}_{3} \mathrm{CN}$ \\
\cline { 2 - 3 } & $0-1$ & $70 / 30$ \\
& $1-20$ & $70 / 30 \rightarrow 0 / 100$ \\
& $20-25$ & $0 / 100$ \\
& $25-26$ & $0 / 100 \rightarrow 70 / 30$ \\
& $26-30$ & $70 / 30$ \\
Fluss: & $18 \mathrm{~mL} \mathrm{~min}^{-1}$ & \\
$t_{\mathrm{R}}:$ & $10.1 \mathrm{~min}$ &
\end{tabular}

UV (MeOH): $\lambda_{\max }(\lg \varepsilon)=256.0 \mathrm{~nm}$ (4.898), 280.0 (4.470), 310.0 (4.504), 375.0 (4.232). $[\alpha]_{\mathrm{D}}^{20}=-52.98^{\circ}(\mathrm{c}=0.15, \mathrm{DMSO})$.

${ }^{1} \mathrm{H}-\mathrm{NMR}\left(600 \mathrm{MHz}, \mathrm{DMSO}-\mathrm{d}_{6}\right) \delta=3.02\left(\mathrm{~s}, 6 \mathrm{H}, \mathrm{NMe}_{2}\right), 3.38-3.58(\mathrm{~m}, 4 \mathrm{H}, 3$ '"'-H, 5"'-H, 6'"'- $\left.\mathrm{H}_{2}\right), 3.72-3.76\left(\mathrm{~m}, 2 \mathrm{H}, 2\right.$ '"-H, 4'"-H), $3.90\left(\mathrm{dd}, J=11.1,7.8 \mathrm{~Hz}, 1 \mathrm{H}, 10-\mathrm{H}_{\mathrm{a}}\right), 4.04-$ $4.11\left(\mathrm{~m}, 2 \mathrm{H}, 10-\mathrm{H}_{\mathrm{b}}, 2^{\prime}-\mathrm{H}_{\mathrm{a}}\right), 4.18\left(\mathrm{~d}, J=16.5 \mathrm{~Hz}, 1 \mathrm{H}, 2^{\prime}-\mathrm{H}_{\mathrm{b}}\right), 4.28-4.33(\mathrm{~m}, 1 \mathrm{H}, 1-\mathrm{H})$, $4.38\left(\mathrm{~d}, J=10.7 \mathrm{~Hz}, 1 \mathrm{H}, 2-\mathrm{H}_{\mathrm{a}}\right), 4.56\left(\mathrm{t}, J=10.0 \mathrm{~Hz}, 1 \mathrm{H}, 2-\mathrm{H}_{\mathrm{b}}\right), 4.94(\mathrm{t}, J=11.1 \mathrm{~Hz}, 1 \mathrm{H}$, 1''-H), $6.06\left(\mathrm{~s}, 1 \mathrm{H}, 3^{\prime}-\mathrm{H}\right), 6.58\left(\mathrm{~d}, J=2.1 \mathrm{~Hz}, 1 \mathrm{H}, 8^{\prime}-\mathrm{H}\right), 6.72\left(\mathrm{~d}, J=8.9 \mathrm{~Hz}, 1 \mathrm{H}, 6^{\prime}-\mathrm{H}\right)$, 
7.38-7.44 (m, $1 \mathrm{H}, 7-\mathrm{H}), 7.54-7.59(\mathrm{~m}, 2 \mathrm{H}, 8-\mathrm{H}, 5$ '-H), $7.88(\mathrm{~d}, J=8.3 \mathrm{~Hz}, 1 \mathrm{H}, 9-\mathrm{H})$, $8.16(\mathrm{~s}, 1 \mathrm{H}, 4-\mathrm{H}), 8.29$ (d, J = 8.4 Hz, $1 \mathrm{H}, 6-\mathrm{H}) \mathrm{ppm}$.

${ }^{13} \mathrm{C}-\mathrm{NMR}\left(125 \mathrm{MHz}, \mathrm{DMSO}-\mathrm{d}_{6}\right) \delta=38.6$ (C-2'), $40.1\left(\mathrm{NMe}_{2}\right), 40.9$ (C-1), 47.6 (C-10), 52.9 (C-2), 59.5 (C-6'"'), 67.5, 70.4, 73.0, 75.1 (C-2'", C-3'"', C-4'", C-5'"'), 97.3 (C-8'"), 100.6 (C-4), 101.6 (C-1"'), 108.6 (C-4a"), 109.0 (C-6"), 109.7 (C-3"'), 117.0 (C-5a), 122.6 (C-9b), 122.7 (C-9), 123.3 (C-7), 123.5 (C-6), 126.4 (C-5"), 127.5 (C-8), 129.5 (C-9a), 141.5 (C-3a), 150.9 (C-4"), 152.7 (C-8a"), 153.8 (C-5), 155.3 (C-7"), 160.6 (COO), 167.0 (NCO) ppm.

$\mathrm{C}_{32} \mathrm{H}_{33} \mathrm{ClN}_{2} \mathrm{O}_{9}$ (625.07).

ber.: $647.1766[\mathrm{M}+\mathrm{Na}]^{+}$

gef.: 647.1767 (ESI-HRMS).

\subsection{3 (1S)-2-(1-Chlormethyl-5-hydroxy-1,2-dihydro-benz[e]indol-3-} carbonyl)-3H-pyrano[3,2-e]indol-7-on ((1S)-138)

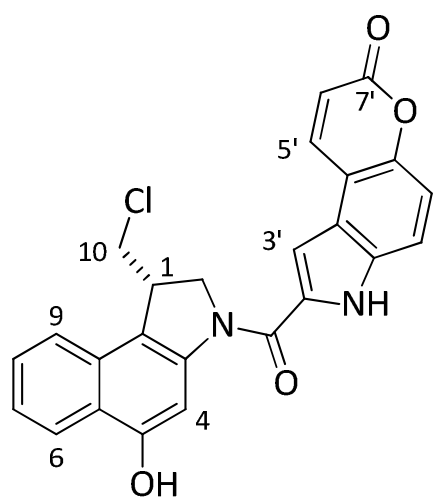

Phenol (1S)-128 (20.0 mg, $60.0 \mu \mathrm{mol}, 1.0$ Äq.) wurde bei Raumtemperatur in $4 \mathrm{M}$ $\mathrm{HCl} /$ EtOAc $(3.0 \mathrm{~mL})$ suspendiert, für $4 \mathrm{~h}$ gerührt und das Lösungsmittel im Vakuum entfernt. Der Rückstand wurde $1 \mathrm{~h}$ im Hochvakuum getrocknet und anschließend in DMF (3 mL) gelöst und bei $0{ }^{\circ} \mathrm{C}$ mit EDAC. $\mathrm{HCl}(35 \mathrm{mg}, 180 \mu \mathrm{mol}, 3.0$ Äq.) und 130 (17.9 mg, $78 \mu \mathrm{mol}, 1.3$ Äq.) versetzt und anschließend für $18 \mathrm{~h}$ bei Raumtemperatur gerührt. Das Lösungsmittel wurde entfernt und der Rückstand in DMF $(2.0 \mathrm{~mL})$ aufgenommen. Je $0.5 \mathrm{~mL}$ dieser Lösung wurden in das präparative HPLC-System (s. u.) injiziert. Fraktioniertes Auffangen des Eluats, Entfernung der Lösungsmittel unter vermindertem Druck sowie Entfernung des restlichen Lösungsmittels mittels 
Gefriertrocknung lieferte die Zielverbindung (1S)-138 als gelben Feststoff $(10.1 \mathrm{mg}$, $22.7 \mu \mathrm{mol}, 38 \%)$.

HPLC (analytisch):

Säule: Kromasil 100 C18

\begin{tabular}{ccc} 
Gradient: & Zeit $[\mathrm{min}]$ & $\mathrm{H}_{2} \mathrm{O}(0.06 \% \mathrm{HCl}) / \mathrm{CH}_{3} \mathrm{CN}$ \\
\hline 0 & $60 / 40$ \\
$0-40$ & $30 / 70 \rightarrow 0 / 100$ \\
$40-41$ & $0 / 100$ \\
$41-45$ & $0 / 100 \rightarrow 60 / 40$ \\
$45-46$ & $60 / 40$ \\
$46-50$ & \\
\hline
\end{tabular}

Fluss: $\quad 0.8 \mathrm{~mL} \mathrm{~min}^{-1}$

$t_{\mathrm{R}}: \quad 20.5 \mathrm{~min}$

HPLC (präparativ):

Säule: Kromasil 100 C18

\begin{tabular}{ccc} 
Gradient: & Zeit $[\mathrm{min}]$ & $\mathrm{H}_{2} \mathrm{O}(0.06 \% \mathrm{HCl}) / \mathrm{CH}_{3} \mathrm{CN}$ \\
\hline $1-40$ & $60 / 40$ \\
$40-41$ & $30 / 70 \rightarrow 0 / 100$ \\
$41-45$ & $0 / 100$ \\
$45-46$ & $0 / 100 \rightarrow 60 / 40$
\end{tabular}


$46-50$

$60 / 40$

Fluss: $\quad 18 \mathrm{~mL} \mathrm{~min}^{-1}$

$t_{\mathrm{R}}: \quad 25.6 \mathrm{~min}$

UV (MeOH): $\lambda_{\max }(\lg \varepsilon)=228.0 \mathrm{~nm}$ (4.663), 245.0 (4.641), 283.0 (4.520), 325.0 (4.423), 357.0 (4.418).

${ }^{1} \mathrm{H}-\mathrm{NMR}\left(600 \mathrm{MHz}, \mathrm{DMSO}-\mathrm{d}_{6}\right): \delta=3.90\left(\mathrm{dd}, J=11.1,7.2 \mathrm{~Hz}, 1 \mathrm{H}, 10-\mathrm{H}_{\mathrm{a}}\right), 4.01(\mathrm{dd}, J=$ 11.1, 2.7 Hz, $\left.1 \mathrm{H}, 10-\mathrm{H}_{\mathrm{b}}\right), 4.25-4.32(\mathrm{~m}, 1 \mathrm{H}, 1-\mathrm{H}), 4.60\left(\mathrm{~d}, J=10.7 \mathrm{~Hz}, 1 \mathrm{H}, 2-\mathrm{H}_{\mathrm{a}}\right), 4.83$ $\left(\mathrm{t}, J=9.9 \mathrm{~Hz}, 1 \mathrm{H}, 2-\mathrm{H}_{\mathrm{b}}\right), 6.57\left(\mathrm{~d}, J=9.5 \mathrm{~Hz}, 1 \mathrm{H}, 6^{\prime}-\mathrm{H}\right), 7.32\left(\mathrm{~d}, J=9.0 \mathrm{~Hz}, 1 \mathrm{H}, 9^{\prime}-\mathrm{H}\right)$, $7.37(\mathrm{~d}, J=7.6 \mathrm{~Hz}, 1 \mathrm{H}, 7-\mathrm{H}), 7.54(\mathrm{t}, J=7.5 \mathrm{~Hz}, 1 \mathrm{H}, 8-\mathrm{H}), 7.73-7.78\left(\mathrm{~m}, 2 \mathrm{H}, 3^{\prime}-\mathrm{H}\right.$, $\left.10^{\prime}-\mathrm{H}\right), 7.87(\mathrm{~d}, J=8.3 \mathrm{~Hz}, 1 \mathrm{H}, 9-\mathrm{H}), 7.97(\mathrm{~s}, 1 \mathrm{H}, 4-\mathrm{H}), 8.14(\mathrm{~d}, J=8.4 \mathrm{~Hz}, 1 \mathrm{H}, 6-\mathrm{H})$, $8.63\left(\mathrm{dd}, J=9.5 \mathrm{~Hz}, 1 \mathrm{H}, 5^{\prime}-\mathrm{H}\right), 10.41(\mathrm{~s}, 1 \mathrm{H}, \mathrm{OH}), 12.16(\mathrm{~s}, 1 \mathrm{H}, \mathrm{NH}) \mathrm{ppm}$.

${ }^{13} \mathrm{C}-\mathrm{NMR}\left(125 \mathrm{MHz}, \mathrm{DMSO}-\mathrm{d}_{6}\right): \delta=41.1$ (C-1), 47.5 (C-10), 55.0 (C-2), 100.2 (C-4), 103.8 (C-3'), 111.1 (C-4'), 113.5 (C-9'), 114.9 (C-6'), 115.1 (C-5a), 116.7 (C-10'), 122.2 (C-9b), 122.7 (C-9), 123.1 (C-6), 123.1 (C-7), 123.4 (C-3a'), 127.2 (C-8), 129.8 (C-9a), 132.4 (C-10a'), 132.7 (C-2'), 141.6 (C-5'), 142.0 (C-3a), 149.6 (C-8'), 154.1 (C-5), 159.6 (COO), 160.5 (NCO) ppm.

$\mathrm{C}_{25} \mathrm{H}_{17} \mathrm{ClN}_{2} \mathrm{O}_{4}$ (444.87).

ber.: $467.0769[\mathrm{M}+\mathrm{Na}]^{+}$

gef.: 467.0769 (ESI-HRMS). 


\section{$9.14\{(1 S)$-[2-(1-Chlormethyl-5-hydroxy-1,2-dihydro-benz[e]indol-3-} carbonyl)-3H-pyrano[3,2-e]indol-7-on-5-yl]-2,3,4,6-tetra-O-acetyl- $\beta$-DGalaktopyranosid\} ((1S)-152)

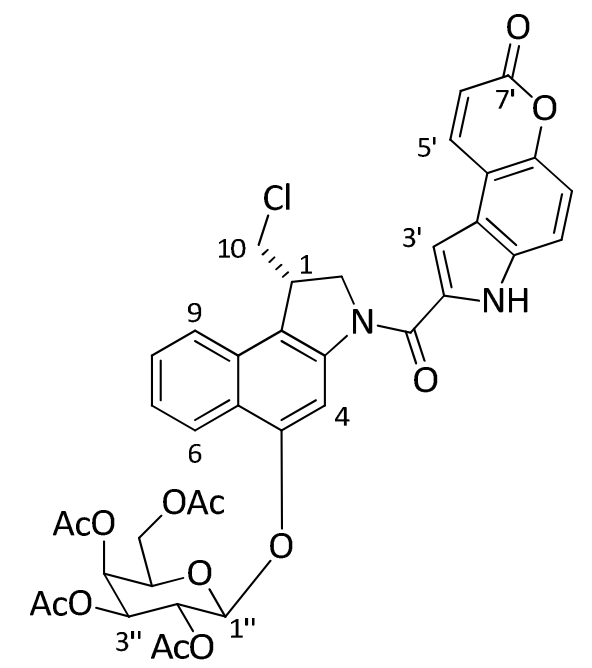

Zu einer Lösung des Phenols (1S)-128 (20.0 mg, $60.0 \mu \mathrm{mol}, 1.0$ Äq.) in absolutem $\mathrm{CH}_{2} \mathrm{Cl}_{2}(3.0 \mathrm{~mL})$ gab man aktiviertes Molsieb $4 \AA$ ( $\left.0.15 \mathrm{~g}\right)$ und rührte 30 min bei Raumtemperatur. Nach Zugabe des $O$-(2,3,4,6-Tetra-O-acetyl- $\alpha$-D-Galaktopyranosyl)-trichloracetimidats (116) (31.2 mg, $63 \mu \mathrm{mol}, 1.05$ Äq.) kühlte man die Lösung auf $-10{ }^{\circ} \mathrm{C}$ und tropfte langsam eine Lösung von $\mathrm{BF}_{3} \cdot \mathrm{OEt}_{2}(3.8 \mu \mathrm{L}, 30.0 \mu \mathrm{mol}, 0.5 \mathrm{Äq}$ ) in absolutem $\mathrm{CH}_{2} \mathrm{Cl}_{2}(1.5 \mathrm{~mL}) \mathrm{zu}$. Nach $3.5 \mathrm{~h}$ Rühren bei $-10{ }^{\circ} \mathrm{C}$ tropfte man weiteres $\mathrm{BF}_{3} \cdot \mathrm{OEt}_{2}\left(23 \mu \mathrm{L}, 180 \mu \mathrm{mol}, 3.0 \mathrm{Äq}\right.$.) in absolutem $\mathrm{CH}_{2} \mathrm{Cl}_{2}(1.5 \mathrm{~mL})$ hinzu und ließ auf Raumtemperatur erwärmen. Nach $6 \mathrm{~h}$ wurde das Reaktionsgemisch via Transferkanüle in einen zweiten Kolben überführt und so vom Molsieb abgetrennt. Dieses wurde gründlich mit $\mathrm{CH}_{2} \mathrm{Cl}_{2}(2 \times 10 \mathrm{~mL})$ gewaschen und das Lösungsmittel im Vakuum entfernt. Der Rückstand wurde $1 \mathrm{~h}$ im Hochvakuum getrocknet und in absolutem DMF $(3.0 \mathrm{~mL})$ gelöst. Man kühlte die Lösung auf $0{ }^{\circ} \mathrm{C}$ und gab EDAC $\cdot \mathrm{HCl}$ (34.6 mg, $180 \mu \mathrm{mol}, 3.0$ Äq.) sowie 130 (18.0 mg, $90 \mu \mathrm{mol}, 1.5$ Äq.) hinzu. Nach $18 \mathrm{~h}$ Rühren bei Raumtemperatur wurde das Lösungsmittel entfernt und der Rückstand in DMF $(2.0 \mathrm{~mL})$ aufgenommen. Je $0.5 \mathrm{~mL}$ dieser Lösung wurden in das präparative HPLC-System (s. u.) injiziert. Fraktioniertes Auffangen des Eluats, Entfernung der Lösungsmittel unter vermindertem Druck sowie Entfernung des restlichen 
Lösungsmittels mittels Gefriertrocknung lieferte die Zielverbindung (1S)-152 als gelben Feststoff (12.0 mg, $15.5 \mu \mathrm{mol}, 26 \%$ ).

HPLC (analytisch):

Säule: Kromasil 100 C18

\begin{tabular}{|c|c|c|}
\hline \multirow[t]{6}{*}{ Gradient: } & Zeit [min] & $\mathrm{H}_{2} \mathrm{O}(0.06 \% \mathrm{HCl}) / \mathrm{CH}_{3} \mathrm{CN}$ \\
\hline & 0 & $70 / 30$ \\
\hline & $0-20$ & $70 / 30 \rightarrow 0 / 100$ \\
\hline & $20-25$ & $0 / 100$ \\
\hline & $25-26$ & $0 / 100 \rightarrow 70 / 30$ \\
\hline & $26-30$ & $70 / 30$ \\
\hline
\end{tabular}

Fluss: $\quad 0.8 \mathrm{~mL} \mathrm{~min}^{-1}$

$t_{\mathrm{R}}: \quad 18.0 \mathrm{~min}$

HPLC (präparativ):

Säule: Kromasil 100 C18

\begin{tabular}{ccc}
\cline { 2 - 3 } Gradient: & Zeit $[\mathrm{min}]$ & $\mathrm{H}_{2} \mathrm{O}(0.06 \% \mathrm{HCl}) / \mathrm{CH}_{3} \mathrm{CN}$ \\
\cline { 2 - 3 } & $0-1$ & $70 / 30$ \\
$20-25$ & $0 / 100$ \\
& $25-26$ & $0 / 100 \rightarrow 70 / 30$ \\
& $26-30$ & $70 / 30$ \\
Fluss: & $18 \mathrm{~mL} \mathrm{~min}^{-1}$ & \\
$t_{\mathrm{R}}:$ & $17.3 \mathrm{~min}^{2}$ &
\end{tabular}


$\mathrm{C}_{39} \mathrm{H}_{35} \mathrm{ClN}_{2} \mathrm{O}_{13}$ (775.15).

\section{$9.15\{(1 S)$-[2-(1-Chlormethyl-5-hydroxy-1,2-dihydro-benz[e]indol-3-} carbonyl)-3H-pyrano[3,2-e]indol-7-on-5-yl]-O- $\beta$-D-Galaktopyranosid\} ((1S)-140)

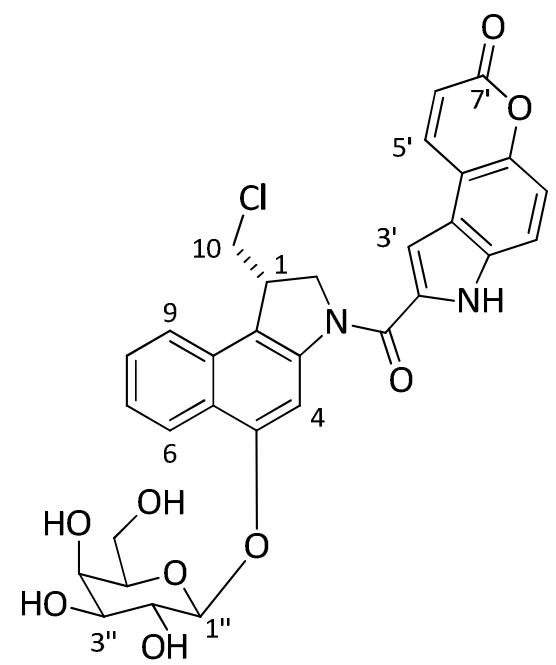

Zu einer Lösung des AC-geschützten Zuckers (1S)-152 (12 mg, $15.5 \mu \mathrm{mol}, 1.0$ Äq.) in absolutem $\mathrm{MeOH}(5.0 \mathrm{~mL})$ wurde bei $0^{\circ} \mathrm{C}$ eine Lösung von $\mathrm{NaOMe}$ in $\mathrm{MeOH}(6 \mu \mathrm{L}$ einer $5.4 \mathrm{M}$ Lösung, $31 \mu \mathrm{mol}, 2.0$ Äq.) zugegeben und für $2 \mathrm{~h}$ bei Raumtemperatur gerührt. Die Reaktionslösung wurde mit $\mathrm{H}_{2} \mathrm{O}(1 \mathrm{~mL})$ und $\mathrm{MeOH}(1 \mathrm{~mL})$ verdünnt und mit saurem lonentauscher (Amberlite-IR 120) auf $\mathrm{pH} \approx 7$ eingestellt. Es wurde vom lonentauscher abfiltiert, mit $\mathrm{MeOH}(20 \mathrm{~mL})$ gewaschen und das Lösungsmittel im Vakuum entfernt. Das Rohprodukt wurde in DMF (2 mL) gelöst und es wurde je $0.5 \mathrm{~mL}$ dieser Lösung in das präparative HPLC-System (s. u.) injiziert. Fraktioniertes Auffangen des Eluats, Entfernung der Lösungsmittel unter vermindertem Druck sowie Entfernung des restlichen Lösungsmittels mittels Gefriertrocknung lieferte die Zielverbindung (1S)-140 als gelben Feststoff (8.4 mg, $13.8 \mu \mathrm{mol}, 89 \%)$. 
HPLC (analytisch):

Säule: Kromasil 100 C18

\begin{tabular}{|c|c|c|}
\hline \multirow[t]{6}{*}{ Gradient: } & Zeit [min] & $\mathrm{H}_{2} \mathrm{O}(0.06 \% \mathrm{HCl}) / \mathrm{CH}_{3} \mathrm{CN}$ \\
\hline & 0 & $70 / 30$ \\
\hline & $0-20$ & $70 / 30 \rightarrow 0 / 100$ \\
\hline & $20-25$ & $0 / 100$ \\
\hline & $25-26$ & $0 / 100 \rightarrow 70 / 30$ \\
\hline & $26-30$ & $70 / 30$ \\
\hline
\end{tabular}

Fluss: $\quad 0.8 \mathrm{~mL} \mathrm{~min}^{-1}$

$t_{\mathrm{R}}: \quad 8.7 \mathrm{~min}$

HPLC (präparativ):

Säule: Kromasil 100 C18

\begin{tabular}{|c|c|c|}
\hline \multirow[t]{6}{*}{ Gradient: } & Zeit [min] & $\mathrm{H}_{2} \mathrm{O}(0.06 \% \mathrm{HCl}) / \mathrm{CH}_{3} \mathrm{CN}$ \\
\hline & $0-1$ & $70 / 30$ \\
\hline & $1-20$ & $70 / 30 \rightarrow 0 / 100$ \\
\hline & $20-25$ & $0 / 100$ \\
\hline & $25-26$ & $0 / 100 \rightarrow 70 / 30$ \\
\hline & $26-30$ & $70 / 30$ \\
\hline
\end{tabular}

Fluss: $\quad 18 \mathrm{~mL} \mathrm{~min}^{-1}$

$t_{\mathrm{R}}: \quad 8.9 \mathrm{~min}$ 
UV (MeOH): $\lambda_{\max }(\lg \varepsilon)=206.0 \mathrm{~nm}$ (4.403), 228.0 (4.364), 288.0 (4.139), 355.0 (4.243). ${ }^{1}$ H-NMR (600 MHz, DMSO-d 6 ) $\delta=3.41-4.10(\mathrm{~m}, 8 \mathrm{H}, 3$ "'-H, 5"-H, 6"--H 2,4 "-H, 2"-H, $\left.10-\mathrm{H}_{2}\right), 4.34-4.38(\mathrm{~m}, 1 \mathrm{H}, 1-\mathrm{H}), 4.50-4.68\left(\mathrm{~m}, 4 \mathrm{H}, \mathrm{OH}, 2-\mathrm{H}_{\mathrm{a}}\right), 4.81-4.98(\mathrm{~m}, 4 \mathrm{H}, \mathrm{OH}$, 2- $\left.\mathrm{H}_{\mathrm{b}}, 1^{\prime \prime}-\mathrm{H}\right), 5.30(\mathrm{~d}, J=5.4 \mathrm{~Hz}, 1 \mathrm{H}, \mathrm{OH}), 6.58\left(\mathrm{~d}, J=9.5 \mathrm{~Hz}, 1 \mathrm{H}, 6^{\prime}-\mathrm{H}\right), 7.32(\mathrm{~d}, J=$ $\left.8.9 \mathrm{~Hz}, 1 \mathrm{H}, 9^{\prime}-\mathrm{H}\right), 7.43-7.48(\mathrm{~m}, 1 \mathrm{H}, 7-\mathrm{H}), 7.59(\mathrm{t}, J=7.5 \mathrm{~Hz}, 1 \mathrm{H}, 8-\mathrm{H}), 7.72-7.80(\mathrm{~m}$, $\left.2 \mathrm{H}, 3^{\prime}-\mathrm{H}, 10^{\prime}-\mathrm{H}\right), 7.95(\mathrm{~d}, J=8.3 \mathrm{~Hz}, 1 \mathrm{H}, 9-\mathrm{H}), 8.25(\mathrm{~s}, 1 \mathrm{H}, 4-\mathrm{H}), 8.36(\mathrm{~d}, J=8.4 \mathrm{~Hz}$, $1 \mathrm{H}, 6-\mathrm{H}), 8.64\left(\mathrm{~d}, J=9.5 \mathrm{~Hz}, 1 \mathrm{H}, 5^{\prime}-\mathrm{H}\right), 12.24(\mathrm{~s}, 1 \mathrm{H}, \mathrm{NH}) \mathrm{ppm}$.

${ }^{13} \mathrm{C}-\mathrm{NMR}$ (125 MHz, DMSO-d 6 ) $\delta=41.2$ (C-1), 47.4 (C-10), 55.0 (C-2), 59.5 (C-6"), 67.5, 70.4, 73.1, 75.1 (C-2", C-3", C-4", C-5"), 101.7 (C-4), 102.1 (C-1"), 103.9 (C-3'), 111.2 (C-4'), 113.6 (C-9'), 114.9 (C-6'), 116.7 (C-5a), 118.2 (C-10'), 122.8 (C-9b), 123.0 (C-9), 123.3 (C-6), 123.4 (C-7), 123.9 (C-3a'), 127.5 (C-8), 129.4 (C-9a), 132.5 (C-2'), 132.5 (C-10a'), 141.5 (C-5'), 141.9 (C-3a), 149.6 (C-8a'), 153.7 (C-5), 159.7 (COO), 160.5 (NCO) ppm.

$\mathrm{C}_{31} \mathrm{H}_{27} \mathrm{ClN}_{2} \mathrm{O}_{9}$ (607.01).

ber.: $\quad 629.1293[\mathrm{M}+\mathrm{Na}]^{+}$

gef.: 629.1297 (ESI-HRMS). 
10 Untersuchung der Fluoreszenzeigenschaften und der zellulären Aufnahme der dargestellten CBI-Derivate

Untersuchungen zur zellulären Aufnahme sowie der Extinktions- und Emissionsspektren am Fluorimeter wurden in Zusammenarbeit mit Herrn Dr. Mišo Mitkovski am Max-Planck-Institutes für experimentelle Medizin in Göttingen untersucht.

10.1 Charakterisierung der Fluoreszenzeigenschaften der synthetisierten CBI-Derivate

10.1.1 AAV 10.1.1. Allgemeine Arbeitsvorschrift zur Bestimmung der Fluoreszenzintensität bei festgelegter Wellenlänge in Abhängigkeit von der Anregungswellenlänge (Absorption-Scan)

1-5 Aliquots der Stammlösung $(c=1 \mathrm{mM})$ der zu untersuchenden Substanz in DMSO (entsprechend 2-10 $\mu \mathrm{L}, 2-10 \mathrm{nmol})$ wurden im gewählten Lösungsmittel $(200 \mu \mathrm{L}$, Lösungsmittel: Methanol oder Bildgebungspuffer) gelöst. Nach anschließender Durchmischung mit Hilfe eines Vortexgerät wurde diese Lösung in eine Quarz-Küvette pipettiert. Mit einem Fluorimeter wurde die Fluoreszenzintensität der Emissionen bei verschiedenen Anregungswellenlängen gemessen.

10.1.2 AAV 10.1.2 Allgemeine Arbeitsvorschrift zur Messung des Fluoreszenzemissionsspektrums bei festgelegter Anregungswellenlänge (Emission-Scan)

1-5 Aliquots der Stammlösung $(c=1 \mathrm{mM})$ der zu untersuchenden Substanz in DMSO (entsprechend 2-10 $\mu \mathrm{L}, 2-10 \mathrm{nmol})$ wurden im gewählten Lösungsmittel $(200 \mu \mathrm{L}$, 
Lösungsmittel: Methanol oder Bildgebungspuffer) gelöst. Nach anschließender Durchmischung mit Hilfe eines Vortexgerät wurde diese Lösung in eine Quarz-Küvette pipettiert. Mit einem Fluorimeter wurde die Fluoreszenzintensität bei verschiedenen Anregungen mit Laserlicht gemessen.

10.2 Fluoreszenzmikroskopische Untersuchungen zur Aufnahme und intrazellulären Verteilung der synthetisierten fluoreszenzmarkierten CBI-Derivate

10.2.1 AAV 10.2.1. Allgemeine Arbeitsvorschrift zur Vorbereitung der A549-Zellen zur Untersuchung der zellulären Aufnahme fluoreszenzmarkierter CBI-Derivate am konfokalen Laserscanning-Mikroskop

Als Zelllinie dienten das adhärent wachsende humane Bronchialkarzinom der Linie A549. Die Aussaat der Tumorzellen erfolgte in D10F (DMEM mit Zusatz von 10\% fötalem Kälberserum, $44 \mathrm{mM} \mathrm{NaHCO}$ und $4 \mathrm{mM}$ Glutamin) in einer Konzentrationen von $25 \times 10^{3}$ Zellen in $500 \mu \mathrm{L}$ Medium pro Kammer des Deckgläschens (Nunc ${ }^{\oplus}$ LabTek $^{\circledR}$ Chamber Slide mit 4 wells auf Permanox). Die Zellen wurden für $24 \mathrm{~h}$ bei $37^{\circ} \mathrm{C}$ und $7.5 \% \mathrm{CO}_{2}$ adheriert. Alternativ wurden die Zellen in einer Konzentration von $5 \times 10^{3}$ Zellen in $500 \mu \mathrm{L}$ Medium pro Kammer des Deckgläschens ausgesät und für 3 Tage bei $37^{\circ} \mathrm{C}$ und $7.5 \% \mathrm{CO}_{2}$ adheriert.

10.2.2 AAV 10.2.2. Allgemeine Arbeitsvorschrift zur Vorbereitung der HeLa SS6-Zellen zur Untersuchung der zellulären Aufnahme fluoreszenzmarkierter CBI-Derivate am konfokalen LaserscanningMikroskop

Als Zelllinie dienten adhärent wachsende Zervixkarzinomzellen der Linie HeLa SS6.

Die Aussaat der Tumorzellen erfolgte in D10F (DMEM mit Zusatz von 10\% fötalem 
Kälberserum, $0.4 \mathrm{mM}$ Glutamin und $0.2 \mathrm{mM}$ Natriumpyruvat) in einer Konzentrationen von $25 \times 10^{3}$ Zellen in $500 \mu \mathrm{L}$ Medium pro Kammer des Deckgläschens (Nunc ${ }^{\oplus}$ Lab-Tek ${ }^{\circledast}$ Chamber Slide mit 4 wells auf Permanox). Die Zellen wurden für $24 \mathrm{~h}$ bei $37^{\circ} \mathrm{C}$ und $5 \% \mathrm{CO}_{2}$ adheriert.

10.2.3 AAV 10.2.2. Allgemeine Arbeitsvorschrift zur Untersuchung der zellulären Aufnahme fluoreszenzmarkierter CBI- Derivate inklusive des Waschens der Zellen nach Inkubation

Die nach AAV 10.2.1. und AAV 10.2.2. vorbereiteten Zellen wurden mit serumfreiem UltraCulture Medium $(2 \times 1 \mathrm{~mL})$ gewaschen und mit einer Lösung der zu untersuchenden Substanz in DMSO und UltraCulture Medium (500 $\mu \mathrm{L}, 1 \% \mathrm{DMSO}$ ) oder mit einer Lösung aus DMSO in UltraCulture Medium (500 $\mu \mathrm{L}$, 1\% DMSO) versetzt. Die Konzentration der Verbindungen in der Inkubationslösung betrug 1-100 $\mu \mathrm{M}$. Nach einer bestimmten Inkubationszeit ( $5 \mathrm{~min}-0.5 \mathrm{~h}$ ) bei $37^{\circ} \mathrm{C}$ und $7.5 \%$ $\mathrm{CO}_{2}$ wurden die Zellen mit UltraCulture Medium $(1 \mathrm{~mL})$ sowie mit dem Bildgebungspuffer $(2 \times 1 \mathrm{~mL})$ gewaschen und im Bildgebungspuffer mittels eines konfokalen Fluoreszenzmikroskopes untersucht.

\subsubsection{AAV 10.2.4. Allgemeine Arbeitsvorschrift zur Anfärbung mit Hoechst 33342 (64), Mitochondrienfarbstoff MitoTracker Deep Red FM (65) oder dem Mitochondrienfarbstoff MitoTracker Grenn FM (148)}

Zur Anfärbung der Zellkerne wurde eine Lösung des Farbstoffs Hoechst 33342 (64) in Puffer (1 $\mu \mathrm{L}$, Konzentration der Stammlösung: 36 mM, 36 nmol) mit dem Bildgebungspuffer in den Kammern vermischt $\left(c_{\text {final }}=72 \mu \mathrm{M}\right)$ und die Zellen vor der Beobachtung einige Minuten bei Raumtemperatur inkubiert. 
Zur Co-Lokalisation der mitochondrialen Strukturen wurde mit den Mitochondrienfarbstoffen 65 oder 148 angefärbt. Hierzu wurde eine Lösung von 65 oder 148 in DMSO (0.5-1.5 $\mu \mathrm{L}$, Konzentration der Stammlösung: $10 \mu \mathrm{M})$ mit dem Bildgebungspuffer in den Kammern vermischt $\left(c_{\text {final }}=10-30 \mathrm{nM}\right)$ und die Zellen vor der Beobachtung bei Raumtemperatur 5 min- 0.5 h inkubiert. 


\section{E. ANHANG}

\section{In-vitro-Zytotoxizitätstests}

Die Zytotoxizität der in dieser Arbeit dargestellten seco-Drugs und Galaktoside wurde mit Hilfe des HTCFA-Assays an humanen Bronchialkarzinomzellen der Linie A549 bestimmt. Angegeben sind die aus mindestens drei voneinander unabhängig durchgeführten Experimenten gemittelten relativen Klonbildungsraten (Kapitel B.3.4).Die Untersuchungen wurden von Dr. Ingrid Schuberth, Angela Rübeling und Anja Herdlitschke in der Abteilung für Zellkultur des Instituts für Organische und Biomolekulare Chemie der Georg-August-Universität Göttingen durchgeführt.

\subsection{Ergebnisse für das (1S)-CBI-DMAI-Galaktosid (1S)-118}

\begin{tabular}{|c|c|c|c|}
\hline \multicolumn{2}{|c|}{ ohne $\beta$-D-Galaktosidase } & \multicolumn{2}{|c|}{ mit $\beta$-D-Galaktosidase $\left(4 \mathrm{U} \mathrm{mL}^{-1}\right)$} \\
\hline Konzentration & $\begin{array}{c}\text { rel. } \\
\text { Klonbildungsrate }\end{array}$ & Konzentration & $\begin{array}{c}\text { rel. } \\
\text { Klonbildungsrate }\end{array}$ \\
\hline$[\mu \mathrm{M}]$ & [\%] & {$[\mu \mathrm{M}]$} & [\%] \\
\hline 0 & 100 & 0 & 100 \\
\hline 7.63 & 91.46 & 0.0153 & 101.67 \\
\hline 15.26 & 88.3 & 0.076 & 101.34 \\
\hline 22.9 & 86.56 & 0.153 & 84.3 \\
\hline 38.16 & 60.4 & 0.229 & 67.77 \\
\hline 76.32 & 6.52 & 0.382 & 42.93 \\
\hline 152.64 & 0.29 & 0.763 & 4.11 \\
\hline 305.28 & 0.12 & 1.144 & 1.96 \\
\hline \multicolumn{2}{|c|}{$\mathrm{IC}_{50}=40.8 \mathrm{nM}$} & \multicolumn{2}{|c|}{$I C_{50}=0.33 \mathrm{nM}$} \\
\hline \multicolumn{4}{|c|}{$\mathrm{QIC}_{50}=124$} \\
\hline
\end{tabular}


1.2 Ergebnisse für das fluoreszenzmarkierte (1S)-CBI-DMAI-Galaktosid (1S)-72

\begin{tabular}{|c|c|c|c|}
\hline \multicolumn{2}{|c|}{ ohne $\beta$-D-Galaktosidase } & \multicolumn{2}{|c|}{ mit $\beta$-D-Galaktosidase $\left(4 \mathrm{U} \mathrm{mL}^{-1}\right)$} \\
\hline Konzentration & $\begin{array}{c}\text { rel. } \\
\text { Klonbildungsrate }\end{array}$ & Konzentration & $\begin{array}{c}\text { rel. } \\
\text { Klonbildungsrate }\end{array}$ \\
\hline$[\mu \mathrm{M}]$ & [\%] & {$[\mu \mathrm{M}]$} & [\%] \\
\hline 0 & 100 & 0 & 100 \\
\hline 8.88 & 101.17 & 0.044 & 94.66 \\
\hline 44.38 & 104.68 & 0.444 & 94.16 \\
\hline 88.76 & 96.47 & 0.888 & 100.31 \\
\hline 221.9 & 71.33 & 2.22 & 75.93 \\
\hline 310.67 & 52.04 & 4.44 & 49.97 \\
\hline 443.81 & 34.34 & 6.66 & 15.78 \\
\hline 665.72 & 5.74 & 8.88 & 4.27 \\
\hline \multicolumn{2}{|c|}{$\mathrm{IC}_{50}=317 \mathrm{nM}$} & \multicolumn{2}{|c|}{$\mathrm{IC}_{50}=4.3 \mathrm{nM}$} \\
\hline \multicolumn{4}{|c|}{$\mathrm{QIC}_{50}=74$} \\
\hline
\end{tabular}

1.3 Ergebnisse für das (1S)-CBI-BO-Galaktosid (1S)-70

\begin{tabular}{|c|c|c|c|}
\hline \multicolumn{2}{|c|}{ ohne $\beta$-D-Galaktosidase } & \multicolumn{2}{|c|}{ mit $\beta$-D-Galaktosidase $\left(4 \mathrm{U} \mathrm{mL}^{-1}\right)$} \\
\hline Konzentration & $\begin{array}{c}\text { rel. } \\
\text { Klonbildungsrate }\end{array}$ & Konzentration & $\begin{array}{c}\text { rel. } \\
\text { Klonbildungsrate }\end{array}$ \\
\hline [nM] & [\%] & [nM] & [\%] \\
\hline 0 & 100 & 0 & 100 \\
\hline 3.07 & 93.01 & 6.15 & 95.65 \\
\hline 6.15 & 66.22 & 24.59 & 92.07 \\
\hline 12.3 & 39.19 & 61.49 & 76.01 \\
\hline
\end{tabular}




\begin{tabular}{cc|cc}
18.45 & 32.8 & 122.97 & 40.59 \\
24.59 & 6.28 & 245.94 & 18.55 \\
49.19 & 1.77 & 491.88 & 2.17 \\
98.38 & 0.032 & 614.85 & 0.4 \\
\hline \multicolumn{2}{c}{$\mathrm{IC}_{50}=106 \mathrm{nM}$} & \multicolumn{2}{|c}{$\mathrm{IC}_{50}=18.8 \mathrm{nM}$} \\
\hline
\end{tabular}

1.4 Ergebnisse für das (1S)-seco-CBI-BO-Drug (1S)-141

\begin{tabular}{cc}
\hline $\begin{array}{c}\text { Konzentration } \\
{[\mathrm{nM}]}\end{array}$ & $\begin{array}{c}\text { rel. } \\
\text { Klonbildungsrate } \\
{[\%]}\end{array}$ \\
\hline 0 & 100 \\
0.77 & 94.19 \\
1.54 & 91.13 \\
3.84 & 85.95 \\
7.68 & 57.61 \\
11.52 & 27.18 \\
15.36 & 18.04 \\
30.72 & 2.81 \\
\hline & $\mathrm{IC}_{50}=18.1 \mathrm{nM}$ \\
\hline
\end{tabular}

1.5 Ergebnisse für das (1S)-CBI-TO3-Galaktosid (1S)-147

\begin{tabular}{cc|cc}
\hline \multicolumn{2}{c|}{ ohne $\beta$-D-Galaktosidase } & \multicolumn{2}{c}{ mit $\beta$-D-Galaktosidase $\left(4 \mathrm{U} \mathrm{mL}^{-1}\right)$} \\
\hline Konzentration & rel. & Konzentration & rel. \\
& Klonbildungsrate & & Klonbildungsrate \\
{$[\mathrm{nM}]$} & {$[\%]$} & {$[\mathrm{nM}]$} & {$[\%]$} \\
\hline
\end{tabular}




\begin{tabular}{cc|cc}
\hline 0 & 100 & 0 & 100 \\
1.12 & 87.09 & 0.11 & 95.82 \\
1.69 & 62.14 & 1.12 & 78.48 \\
2.81 & 38.14 & 1.69 & 41.81 \\
5.62 & 9.4 & 2.25 & 33.1 \\
8.43 & 1.29 & 2.81 & 9.74 \\
11.24 & 0.56 & 5.62 & 0.32 \\
14.06 & 0.36 & 8.43 & 0.1 \\
\hline & $\mathrm{IC}_{50}=2.3 \mathrm{nM}$ & \multicolumn{2}{|c|}{$\mathrm{IC}_{50}=1.6 \mathrm{nM}$} \\
\hline
\end{tabular}

1.6 Ergebnisse für das (1S)-seco-CBI-TO3-Drug (1S)-145

\begin{tabular}{cc}
\hline Konzentration & $\begin{array}{c}\text { rel. } \\
\text { Klonbildungsrate } \\
{[\mathrm{nM}]}\end{array}$ \\
\hline 0 & 100 \\
0.69 & 94.66 \\
1.38 & 85.58 \\
3.44 & 72.59 \\
6.88 & 52.8 \\
8.25 & 38.33 \\
10.31 & 6.03 \\
13.75 & 4.13 \\
\hline & $\mathrm{IC} \mathrm{C}_{50}=7.2 \mathrm{nM}$ \\
\hline
\end{tabular}


1.7 Ergebnisse für das (1S)-CBI-Galaktosid (1S)-69

\begin{tabular}{|c|c|c|c|}
\hline \multicolumn{2}{|c|}{ ohne $\beta$-D-Galaktosidase } & \multicolumn{2}{|c|}{ mit $\beta$-D-Galaktosidase $\left(4 \mathrm{U} \mathrm{mL}^{-1}\right)$} \\
\hline Konzentration & $\begin{array}{c}\text { rel. } \\
\text { Klonbildungsrate }\end{array}$ & Konzentration & $\begin{array}{c}\text { rel. } \\
\text { Klonbildungsrate }\end{array}$ \\
\hline$[\mu \mathrm{M}]$ & [\%] & {$[\mu \mathrm{M}]$} & [\%] \\
\hline 0 & 100 & 0 & 100 \\
\hline 0.176 & 97.21 & 0.176 & 105.8 \\
\hline 1.76 & 97.27 & 0.44 & 98.22 \\
\hline 3.52 & 73.83 & 0.88 & 93.39 \\
\hline 7.04 & 25 & 1.76 & 71.9 \\
\hline 8.8 & 13.8 & 3.52 & 27.79 \\
\hline 10.56 & 5.11 & 7.04 & 0.75 \\
\hline 14.09 & 0.69 & & \\
\hline \multicolumn{2}{|c|}{$\mathrm{IC}_{50}=4.8 \mu \mathrm{M}$} & \multicolumn{2}{|c|}{$\mathrm{IC}_{50}=2.4 \mu \mathrm{M}$} \\
\hline \multicolumn{4}{|c|}{$\mathrm{QIC}_{50}=2$} \\
\hline
\end{tabular}

1.8 Ergebnisse für das (1S)-seco-CBI-Drug (1S)-92

\begin{tabular}{cc}
\hline Konzentration & $\begin{array}{c}\text { rel. } \\
\text { Klonbildungsrate } \\
{[\mu \mathrm{M}]}\end{array}$ \\
\hline 0 & 100 \\
0.0025 & 99.56 \\
0.0123 & 103.52 \\
0.0246 & 90.61 \\
0.123 & 54.7 \\
0.185 & 40.78
\end{tabular}



0.246
25.24
0.616
10.6

$\mathrm{IC}_{50}=0.11 \mu \mathrm{M}$

1.9 Ergebnisse für das (1S)-CBI-DMACA-Galaktosid (1S)-139

\begin{tabular}{|c|c|c|c|}
\hline \multicolumn{2}{|c|}{ ohne $\beta$-D-Galaktosidase } & \multicolumn{2}{|c|}{ mit $\beta$-D-Galaktosidase $\left(4 \mathrm{U} \mathrm{mL}^{-1}\right)$} \\
\hline Konzentration & $\begin{array}{c}\text { rel. } \\
\text { Klonbildungsrate }\end{array}$ & Konzentration & $\begin{array}{c}\text { rel. } \\
\text { Klonbildungsrate }\end{array}$ \\
\hline$[\mu \mathrm{M}]$ & [\%] & {$[\mu \mathrm{M}]$} & {$[\%]$} \\
\hline 0 & 100 & 0 & 100 \\
\hline 0.154 & 94.32 & 0.154 & 97.98 \\
\hline 0.77 & 104.43 & 0.23 & 81.85 \\
\hline 1.16 & 98.17 & 0.39 & 65.26 \\
\hline 1.54 & 97.24 & 0.54 & 46.19 \\
\hline 3.86 & 75.82 & 0.77 & 20.63 \\
\hline 7.72 & 18.4 & 1.16 & 2.38 \\
\hline 11.58 & 2.28 & 1.54 & 0.27 \\
\hline \multicolumn{2}{|c|}{$\mathrm{IC}_{50}=5.0 \mu \mathrm{M}$} & \multicolumn{2}{|c|}{$\mathrm{IC}_{50}=0.43 \mu \mathrm{M}$} \\
\hline \multicolumn{4}{|c|}{$\mathrm{QIC}_{50}=12$} \\
\hline
\end{tabular}

1.10 Ergebnisse für das (1S)-seco-CBI-DMACA-Drug (1S)-137

Konzentration rel. Klonbildungsrate

\begin{tabular}{cl}
{$[\mu \mathrm{M}]$} & {$[\%]$} \\
\hline 0 & 100
\end{tabular}

$0.00206 \quad 104.16$ 


\begin{tabular}{ll}
0.0206 & 100.68 \\
0.206 & 87.53 \\
0.309 & 75.15 \\
0.515 & 39.17 \\
0.721 & 20.19 \\
1.03 & 4.17 \\
\hline \multicolumn{2}{c}{$\mathrm{IC}_{50}=0.38 \mu \mathrm{M}$}
\end{tabular}

1.11 Ergebnisse für das (1S)-CBI-Cumarinindol-Galaktosid (1S)-140

\begin{tabular}{|c|c|c|c|}
\hline \multicolumn{2}{|c|}{ ohne $\beta$-D-Galaktosidase } & \multicolumn{2}{|c|}{ mit $\beta$-D-Galaktosidase $\left(4 \mathrm{U} \mathrm{mL}^{-1}\right)$} \\
\hline Konzentration & $\begin{array}{c}\text { rel. } \\
\text { Klonbildungsrate }\end{array}$ & Konzentration & $\begin{array}{c}\text { rel. } \\
\text { Klonbildungsrate }\end{array}$ \\
\hline [nM] & [\%] & [nM] & [\%] \\
\hline 0 & 100 & 0 & 100 \\
\hline 0.0824 & 109.8 & 0.00165 & 96.3 \\
\hline 0.124 & 104.2 & 0.016 & 61.01 \\
\hline 0.16 & 90.19 & 0.041 & 3.74 \\
\hline 0.82 & 1.57 & 0.082 & 0.014 \\
\hline 1.65 & 0.014 & & \\
\hline \multicolumn{2}{|c|}{$\mathrm{IC}_{50}=0.26 \mathrm{nM}$} & \multicolumn{2}{|c|}{$\mathrm{IC}_{50}=0.018 \mathrm{nM}$} \\
\hline \multicolumn{4}{|c|}{$\mathrm{QIC}_{50}=14$} \\
\hline
\end{tabular}

1.12 Ergebnisse für das (1S)-seco-CBI-Cumarinindol-Drug (1S)-138

\begin{tabular}{cc}
\hline Konzentration & rel. \\
& $\begin{array}{c}\text { Klonbildungsrate } \\
\text { [nM] }\end{array}$ \\
\hline
\end{tabular}




\begin{tabular}{cc}
\hline 0 & 100 \\
0.00225 & 100.9 \\
0.0225 & 28.5 \\
0.0562 & 0.225 \\
\hline $\mathrm{IC}_{50}=0.014 \mathrm{nM}$ \\
\hline
\end{tabular}




\section{Abkürzungen und Akronyme}

A

ABPP

Ac

ADEPT

Äq.

APT

aq.

atm

ber.

$\mathrm{Bn}$

Boc

$\mathrm{Bu}$

BuLi

CBI

CBIQ

$\mathrm{Cl}$

COSY

CPI

CPyl

CT

Cys

DA

DC

DMAI

DMBI

DMEM

DMF

DMMI

DMSO
Adenin

activity-based protein profiling

Acetyl

antibody-directed enzyme prodrug therapy

Äquivalente

attached proton test

wäßrig

Atmosphäre

berechnet

Benzyl

tert-Butyloxycarbonyl

Butyl

Butyllithium

Cyclopropabenzoindol

Cyclopropabenzochinolin

Cyclopropaindol

correlated spectroscopy

Cyclopropapyrroloindol

7-Methoxycarbonyl-cyclopropapyridoindol

Computertomographie

Cystein

Duocarmycin A

Dünnschichtchromatographie

5-[2-(N,N-Dimethylamino)-ethoxy]-1H-indol

5-[2-(N,N-Dimethylamino)-ethoxy]-6-benzyloxy- $1 H$-indol

Dulbeccos Modified Eagles Medium

$N, N$-Dimethylformamid

5-(2-N,N-Dimethylaminoethoxy)-6-methoxy-1H-indol

Dimethylsulfoxid 


\begin{tabular}{|c|c|}
\hline DNA & Desoxyribonucleinsäure \\
\hline DPPA & Diphenylphosphorylazid \\
\hline DSA & Duocarmycin SA \\
\hline $\mathrm{EDAC} \cdot \mathrm{HCl}$ & $N$-(3-Dimethylaminopropyl)-N'-ethylcarbodiimid-Hydrochlorid \\
\hline EDTA & Ethylendiamintetraessigsäure \\
\hline$e e$ & Enantiomerenüberschuß \\
\hline ESI & Elektrospray-Ionisation \\
\hline Et & Ethyl \\
\hline EtOAc & Essigsäureethylester \\
\hline Gal & Galaktosyl \\
\hline GDEPT & gene-directed enzyme prodrug therapy \\
\hline gef. & gefunden \\
\hline ges. & gesättigt \\
\hline GlcA & Glucuronyl \\
\hline HER2 & human epidermal growth factor receptor 2 \\
\hline HMBC & heteronuclear multiple-bond correlation \\
\hline HOAC & Eisessig \\
\hline HPLC & high performance (pressure) liquid chromatography \\
\hline HRMS & high resolution mass spectrometry \\
\hline HSQC & heteronuclear single-quantum correlation \\
\hline HTCFA & human tumor colony forming ability \\
\hline $\mathrm{Hz}$ & Hertz \\
\hline$i$ & ipso \\
\hline $\mathrm{IC}_{50}$ & $\begin{array}{l}\text { inhibitory concentration; Toxinkonzentration, bei der das } \\
\text { Zellwachstum um 50\% unterbunden wird }\end{array}$ \\
\hline $\operatorname{lnd}_{2}$ & Bisindol- \\
\hline Inj. & Injektion \\
\hline i.p. & intraperitoneal \\
\hline i.v. & intravenös \\
\hline in vitro & $\begin{array}{l}\text { außerhalb lebender Organismen; hier: Versuche an lebenden } \\
\text { Zellen in Zellkultur }\end{array}$ \\
\hline in vivo & im lebenden Organismus \\
\hline IR & Infrarot-Spektroskopie \\
\hline
\end{tabular}




\begin{tabular}{|c|c|}
\hline$m$ & meta \\
\hline kat. & katalytische Mengen \\
\hline konz. & konzentriert \\
\hline Man & Mannosyl \\
\hline MCBI & 7-Methoxy-CBI \\
\hline MCCPI & Methoxycarbonyl-CPI \\
\hline $\mathrm{Me}$ & Methyl \\
\hline $\mathrm{MeOH}$ & Methanol \\
\hline MMI & 5-Methoxy-1H-indol \\
\hline MPMI & $\begin{array}{l}\text { 5-[2-(N-Methyl- } N \text {-propargylamino)ethoxy]-6-methoxy-1H- } \\
\text { indol }\end{array}$ \\
\hline MS & Molsieb \\
\hline MS & Massenspektrometrie \\
\hline MTBE & Methyl-tert-butylether \\
\hline NMR & nuclear magnetic resonance \\
\hline Ns & Nosyl (Nitrophenylsulfonyl) \\
\hline 0 & ortho \\
\hline$p$ & para \\
\hline PBS & phosphate buffered saline, phosphatgepufferte $\mathrm{NaCl}$-Lösung \\
\hline PDE & Pyrrolodihydroindol \\
\hline PET & Positronenemissionstomographie \\
\hline $\mathrm{Ph}$ & Phenyl \\
\hline $\mathrm{QIC}_{50}$ & $\begin{array}{l}I_{50} \text { (Prodrug) / IC } 50 \text { (Prodrug in Anwesenheit des aktivieren- } \\
\text { den Enzyms) }\end{array}$ \\
\hline RAS & rat sarcoma \\
\hline $\mathrm{R}_{f}$ & Retentionswert für DC \\
\hline$t_{\mathrm{R}}$ & Retentionszeit für HPLC \\
\hline RNA & Ribonucleinsäure \\
\hline $\mathrm{RP}$ & reversed-phase (Umkehrphase) \\
\hline RT & Raumtemperatur \\
\hline SDS-PAGE & sodium dodecyl sulfate polyacrylamide gel electrophoresis \\
\hline seco & $\begin{array}{l}\text { Präfix zur Bezeichnung von Vorstufen zyklischer Verbindungen } \\
\text { (Ringöffnung) }\end{array}$ \\
\hline
\end{tabular}


SiRNA

Std.

T

TBAF

TBAI

TBS

tert

TFA

THF

THP

TMI

TMS

TMS-NCS

TOCSY

$\operatorname{Tr}$

Ts

UV small interfering ribonucleic acid

Standard

Thymin

Tetra- $n$-butylammoniumfluorid

Tetra- $n$-butylammoniumiodid

tert-butyldimethylsilyl

tertiär

Trifluoressigsäure

Tetrahydrofuran

Tetrahydro-2-pyranyl

5,6,7-Trimethoxy- $1 H$-indol

Trimethylsilyl

Trimethylsilylisothiocyanat

total correlation spectroscopy

Triphenylmethyl (trityl)

Tosyl (Toluolsulfonyl)

Ultraviolett-Spektroskopie 


\section{Literatur}

http://www.destatis.de/jetspeed/portal/cms/Sites/destatis/Internet/DE/Conte nt/Statistiken/Zeitreihen/LangeReihen/Bevoelkerung/Content100/Irbev01ga,t emplateld=renderPrint.psml, 29.08.2011.

http://www.isf-muenchen.de/pdf/Zukunft-dt.pdf, 29.08.2001.

http://www.rki.de/cln_160/nn_205770/DE/Content/GBE/Gesundheitsberichter stattung/GBEDownloadsB/KID2010,templateld=raw, property=publicationFile.p df/KID2010.pdf, 29.08.2011.

a) L. F. Tietze, K. Schmuck, Curr. Pharm. Des. 2011, zur Publikation angenommen; b) L. F. Tietze, B. Krewer, Anti-Cancer Agents Med. Chem. 2009, 9, 304325; c) L. F. Tietze, B. Krewer, Chem. Biol. Drug Des. 2009, 74, 205-211; d) L. F. Tietze, T. Feuerstein Curr. Pharm. Des. 2003, 9, 2155-2175; e) L. F. Tietze, T. Feuerstein, Aust. J. Chem. 2003, 56, 841-854.

Der Allgemeine Teil wurde in weiten Teilen übernommen aus: a) B. Krewer, Dissertation, Göttingen 2008; b) J. M. von Hof, Dissertation, Göttingen 2009; c) F. Major, Dissertation, Göttingen 2006; d) K. Schmuck. Dissertation, Göttingen 2011, e) F. Behrendt. Diplomarbeit, Göttingen 2008.

a) S. Grimm, Chem. Unserer Zeit 2003, 37, 172-178; b) A. Lawen, Bioassays 2003, 25, 888-896; c) A. Gewies, ApoReview 2003, 1-26.

W. Pschyrembel, Pschyrembel, de Gruyter, 261. Auflage, Berlin 2007, S. 714, 1315.

E. Mutschler, G. Geisslinger, H. K. Kroemer, P. Ruth, M. Schäfer-Korting, Mutschler Arzneimittelwirkungen, Wissenschaftliche Verlagsgesellschaft mbH, 9. Auflage, Stuttgart 2008, S. 907, 919, 923.

C. Lengauer, K. W. Kinzler, B. Vogelstein, Nature 1998, 396, 643-649.

H.-G. Neumann, Chemie in unserer Zeit 1991, 25, 102-107. 
D. M. Prescott, A. S. Flexer, Krebs, Spektrum der Wissenschaft Verlagsgesellschaft, Heidelberg, 1990.

W. Burkart, Spektrum der Wissenschaft 1994, 12, 112-120.

G. Löffler, Basiswissen Biochemie mit Pathobiochemie, Springer Verlag, Berlin, 2000.

P. Tiollais, M. Buendia in Krebs - Forschung, Diagnostik, Therapie, Spektrum Akademischer Verlag, Heidelberg, 1992.

L. M. Coussens, Z. Werb, Nature 2002, 420, 860-867.

a) R. A. Weinberg in Spektrum der Wissenschaft Spezial: Krebsmedizin, Spektrum der Wissenschaft Verlagsgesellschaft, Heidelberg, 1996; b) W. W. Gibbs in Spektrum der Wissenschaft Spezial: Krebsmedizin II, Spektrum der Wissenschaft Verlagsgesellschaft, Heidelberg, 2003.

H. Varmus, R. A. Weinberg, Gene und Krebs - Biologische Wurzeln der Tumorentstehung, Spektrum Akademischer Verlag, Heidelberg, 1996.

a) R. A. Weinberg, Science 1985, 230, 770-776; b) L. Römer, C. Klein, A. Dehner, H. Kessler, J. Buchner, Angew. Chem. 2006, 118, 6590-6611.

O. D. Schärer, Angew. Chem. 2003, 115, 3052-3082.

K. E. Hellström, I. Hellström, Tumor Antigens, in J. R. Bertino, Encyclopedia of Cancer, 1. Auflage, Academic Press, San Diego 1997.

L. J. van't Veer, R. Bernards, Nature 2008, 452, 564-570.

a) C. Nieder, N. Wieden Andratschke, M. Molls, Cancer Treatment Rev. 2003, 32, 348-364; b) C. Verhoef, J. H. W. de Wilt, H. M. W. Verheul, Curr. Pharm. Des. 2006, 12, 2623-2630; c) F. Hillen, A. W. Griffioen, Cancer Metast. Rev. 2007, 26, 489-502.

a) S. P. Davies, H. Reddy, M. Caivano, P. Cohen, Biochem. J. 2000, 351, 95-105;

b) M. Deininger, E. Buchdunger, B. J. Druker, Blood 2005, 105, 2640-2653; c) V. Gandhi, J. M. Kilpatrick, W. Plunkett, M. Ayres, L. Harman, M. Du, S. Bantia, J. 
Davisson, W. G. Wierda, S. Faderl, H. Kantarjian, D. Thomas, Blood 2005, 106, 4253-4260.

a) Spektrum der Wissenschaft Spezial 5: Krebsmedizin, Spektrum Akademischer Verlag, Heidelberg, 1996; b) E. Jäger, D. Jäger, A. Knuth, Int. J. Cancer 2003, 106, 817-820; c) L. Gattinoni, D. J. Powell Jr.; S. A. Rosenberg, N. P. Restifo, Nat. Rev. Immunol. 2006, 6, 383-393.

a) S. Kumar, M. Shelley, C. Harrison, B. Coles, T. J. Wilt, M. D. Mason, Cochrane database Syst. Rev. 2006, 4, CD006019; b) C.-H. Lai, H.-J- Huang, Curr. Opin. Obstet. Gynecol. 2006, 18, 29-34; c) S. B. Decruze, J. A. Green, Int. J. Gynecol. Canc. 2007, 17, 964-978; d) R. E. Greene, J. Pharmacy Practice 2008, 21, 3645.

K. Aktories, U. Förstermann, F. Hofmann, K. Starke, Allgemeine und spezielle Pharmakologie und Toxikologie, Urban \& Fischer, 10. Auflage, München 2009, S. 233 f., 948,970 .

a) O. M. Friedman, A. M. Seligman, J. Am. Chem. Soc. 1954, 76, 655-658; b) H. Arnold, F. Bourseaux, Angew. Chem. 1958, 70, 539-544; c) H. Arnold, F. Bourseaux, N. Brock, Nature 1958, 181, 931.

a) A. I. Pinto, S. J. Lippard, Biochim. Biophys. Acta 1985, 780, 167-180; b) S. E. Sherman, S. J. Lippard, Chem. Rev. 1987, 87, 1153-1181; c) J. P. Caradonna, S. J. Lippard, Inorg. Chem. 1988, 27, 1454-1466.

C. Bystroff, S. J. Oatley, J. Kraut, Biochemistry 1990, 29, 3263-3277.

E. K. Rowinsky, R. C. Donehower, Pharmac. Ther. 1991, 52, 35-84.

a) J. Rohr, Angew. Chem. 1997, 109, 2284-2289; b) K. C. Nicolaou, R. K. Guy, P. Potier, Sci. Am. 1996, 272, 84-88; c) M. E. Wall, M. C. Wani, Cancer Res. 1995, 55, 753-760; d) K. C. Nicolaou, W.-M. Dai, R. K. Guy, Angew. Chem. 1994, 106, 38-69; e) A. G. Chaudhary, M. M. Gharpure, J. M. Rimoldi, M. D. Chordia, A. A. L. Gunatilaka, D. G. I. Kingston, S. Grover, C. M. Lin, E. Hamel, J. Am. Chem. Soc. 1994, 116, 4097-4098; f) K. C. Nicolaou, W.-M. Dai, R. K. Guy, Angew. Chem. 
1994, 106, 38-69; g) D. G. I. Kingston, Pharmac. Ther. 1991, 52, 1-34; h) S. B. Horwitz, I. Ringel, J. Nat. Cancer Inst. 1991, 83, 288-291; i) M. C. Wani, H. L. Taylor, M. E. Wall, P. Coggon, A. T. McPhail, J. Am. Chem. Soc. 1971, 93, 23252327.

R. C. Young, R. F. Ozols, C. E. Myers, New Eng. J. Med. 1981, 305, 139-153.

H. ZurHausen, Krebs - Forschung, Diagnostik, Therapie, Spektrum Akad, Vlg., Heidelberg 1992.

a) P. C. Cander, Nutr. Rev. 1998, 56, 70-83; b) T. Boon, P. G. Coulie, B. Van den Eynde, Immunology Today 1997, 18, 267-268.

a) M. Frank, A. Ihan, Radiol. Oncol. 2006, 40, 219-229; b) J. Stanley, Essentials of Immunology \& Serology, Delmar, 1. Auflage, Albany 2002, S. 440.

J. S. Ross, K. Gray, D. Schenkein, B. Greene, Gary S. Gray, J. Shulok, P. J. Worland, A. Celniker, M. Rolfe, Expert Rev. Anticancer Ther. 2003, 3, 107-121.

G. Köhler, C. Milstein, Nature 1975, 256, 495-497.

A. Brufsky, Am. J. Clin. Oncol. 2010, 33, 186-195; b) D. J. Slamon, G. M. Clark, S.

G. Wong, W. J. Levin, A. Ulrich, W. L. McGuire, Science 1987, 235, 177-182.

D. J. Slamon, G. M. Clark, S. G. Wong, W. J. Levin, A. Ulrich, W. L. McGuire, Science 1987, 235, 177-182.

H. Cortes-Funes, Clin. Transl. Oncol. 2009, 11, 349-355.

H. Kirchner, A. Kruse, P. Neustock, L. Rink, Cytokine und Interferone, Spektrum Akademischer Verlag, Heidelberg, 1994.

D. Ockert, M. Schmitz, M. Hampl, E. P. Rieber, Immunology Today 1999, 20, 63-65.

R. T. Costello, L.-A. Gastaut, D. Olive, Immunology Today 1999, 20, 488-493.

P. Sanderson, G. G. MacPherson, C. H. Jenkins, P. C. Calder, J. Leuk. Biol. 1997, $62,771-777$.

A. Kugler, G. Stuhler, Nature Medicine 2000, 6, 332-336. 
Abbildung in Anlehnung an: a) Literaturstelle 42; b) P. Carter, Nature Rev. Cancer 2001, 1, 118-129.

a) L. Ducry, B. Stump, Bioconjugate Chem. 2010, 21, 5-13; b) J. M. Lambert, Curr. Opin. Pharmacol. 2005, 5, 543-549; c) B. Hughes, Nat. Rev. Drug Discovery 2010, 9, 665-667; d) P. R. Hamann, Expert Opin. Ther. Patents 2005, 15, 1087-1103.

J. K. McGavin, C. M. Spencer, Drugs 2001, 61, 1317-1322.

I. E. Krop, M. Beeram, S. Modi, S. F. Jones, S. N. Holden, W. Yu, S. Girish, J. Tibbitts, J.-H. Yi, M. X. Sliwkowski, F. Jacobson, S. G. Lutzker, H. A. Burris, J. Clin. Oncol. 2010, 28, 2698-2704.

R. A. Herbertson, N. C. Tebbutt, F.-T. Lee, D. J. MacFarlane, B. Chappell, N. Micallef, S.-T. Lee, T. Saunder, W. Hopkins, F. E. Smyth, D. K. Wyld, J. Bellen, D. S. Sonnichsen, M. W. Brechbiel, C. Murone, A. M. Scott, Clin. Cancer Res. 2009, 15, 6709-6715.

A. Albert, Nature 1958, 182, 421-423.

a) K. D. Bagshawe, Br. J. Cancer 1987, 56, 531-532; b) K. D. Bagshawe, Expert Rev. Anticancer Ther. 2006, 6, 1421-1431; c) K. D. Bagshawe, Curr. Drug Targets 2009, 10, 152-157; d) K. D. Bagshawe, S. K. Sharma, R. H. J. Begent, Expert Opin. Biol. Ther. 2004, 4, 1777-1789; e) T. W. Doyle, Pharmacology \& Therapeutics 1999, 83, 67-123; f) P. D. Senter, C. J. Springer, Adv. Drug Delivery Rev. 2001, 53, 247-264.

a) J. C. Reubi, H. R. Mäcke, E. P. Krenning, J. Nucl. Med. 2005, 46, 67S-75S; b) M. Dyba, N. I. Tarasova, C. J. Michejda, Curr. Pharm. Des. 2004, 10, 2311-2334; c) J. C. Reubi, Endocrine Reviews 2003, 24, 389-427; d) J. C. Reubi, B. Waser, Eur. J. Nucl. Med. 2003, 30, 781-793; e) M. Langer, A. G. Beck-Sickinger, Curr. Med. Chem. 2001, 1, 71-93; f) W. A. P. Breeman, M. de Jong, D. J. Kwekkeboom, R. Valkema, W. H. Bakker, P. P. M. Kooij, T. J. Visser, E. P. Krenning, Eur. J. Nucl. Med. 2001, 28, 1421-1429; g) A. Heppeler, S. 
Froidevaux, A. N. Eberle, H. R. Maecke, Curr. Med. Chem. 2000, 7, 971-994; h) A. V. Schally, A. Nagy, Eur. J. Endocrin. 1999, 141, 1-14; h) G. S. Baldwin, A. Shulkes, Gut 1998, 42, 581-584.

a) F. Kratz, I. A. Müller, C. Ryppa, A. Warnecke, ChemMedChem 2008, 3, 20-53;

b) Y. Singh, M. Palombo, P. J. Sinko, Curr. Med. Chem. 2008, 15, 1802-1826; c)

G. S. Shukla, D. N. Krag, Expert Opin. Biol. Ther. 2006, 6, 39-54; d) R. V. J. Chari, Adv. Drug Deliv. Rev. 1998, 31, 89-104; e) A. T. de Albuquerque Silva, M. C. Chung, L. F. Castro, R. V. Carvalho Güido, E. I. Ferriera, Mini-Rev. Med. Chem. 2005, 5, 893-914; f) H. Fuchs, C. Bachran, Curr. Drug Targets 2009, 10, 89-93. O. Warburg, The Metabolism of Tumors, Constable, London, 1930.

a) X. Zhang, Y. Lin, R. J. Gillies, J. Nucl. Med. 2010, 51, 1167-1170; b) R. J. Gillies, I. Robey, R. A. Gatenby, J. Nucl. Med. 2008, 49, 24S-42S.

a) R. K. Jain, Spektrum der Wissenschaft 1994, 9, 48-55; b) P. Vaupel, F. Kallinowski, P. Okunieff, Cancer Res. 1989, 49, 6449-6465. M. von Ardenne, P. G. Reitnauer, Acta Biol. Med. Germ. 1970, 25, 483-493. a) S. P. Osinsky, L. N. Bubnovskaja, T. Sergienko, Anticancer Res. 1987, 7, 199201; b) S. P. Osinsky, L. N. Bubnovskaja, Arch. Geschwulstforschung 1984, 54, 463-469; c) E. Jähde, M. F. Rajewsky, Cancer Res. 1982, 42, 1505-1512; d) M. von Ardenne, P. G. Reitnauer, Arch. Geschwulstforschung 1975, 45, 135-145. L. F. Tietze, Nachr. Chem. Tech. Lab. 1988, 36, 728-737.

a) L. F. Tietze, M. Beller, R. Fischer, M. Lögers, E. Jähde, K. H. Glüsenkamp, M. F. Rajewsky, Angew. Chem. 1990, 102, 812-813; b) L. F. Tietze, R. Fischer, M. Beller, R. Seele, Liebigs Ann. Chem. 1990, 151-157; c) L. F. Tietze, R. Fischer, H. J. Guder, M. Neumann, Liebigs Ann. Chem. 1987, 847-856; d) L. F. Tietze, R. Fischer, H. J. Guder, A. Goerlach, M. Neumann, T. Krach, Carbohydr. Res. 1987, 164, 177-194; e) L. F. Tietze, R. Fischer, Angew. Chem. 1981, 93, 1002. a) M. Jung, Mini-Rev. Med. Chem. 2001, 1, 399-407; b) K. N. Syrigos, A. A. Epenetos, Anticancer Res. 1999, 19, 605-613; c) G. M. Dubowchik, M. A. 
Walker, Pharmacol. Ther. 1999, 83, 67-123; d) L. N. Jungheim, T. A. Shepherd, Chem. Rev. 1994, 94, 1553-1566.

M. P. Napier, S. K. Sharma, C. J. Springer, K. D. Bagshawe, A. J. Green, J. Martin, S. M. Stribbling, N. Cushen, D. O’Malley, R. H. J. Begent, Clin. Cancer. Res. 2000, $6,765-772$.

S. K. Sharma, R. B. Pedley, J. Bhatia, G. M. Boxer, E. El-Emir, U. Qureshi, B. Tolner, H. Lowe, N. P. Michael, N. Minton, R. H. J. Begent, K. A. Chester, Clin. Cancer. Res. 2005, 11, 814-825.

T. L. Cheng, S. L. Wei, B. M. Chen, J. W. Chern, M. F. Wu, P. W. Liu, S. R. Roffler, Br. J. Cancer 1999, 79, 1378-1385.

a) G. Xu, H. L. McLeod, Clin. Cancer Res. 2001, 7, 3314-3324; b) I. NiculescuDuvaz, C. J. Springer, Adv. Drug Delivery Rev. 1997, 26, 151-172.

L. F. Tietze, T. Herzig, T. Feuerstein, I. Schuberth, Eur. J. Org. Chem. 2002, 2002, 1634-1645.

a) P. Wentworth, A. Datta, D. Blakey, T. Boyle, L. J. Partridge, G. M. Blackburn, Proc. Natl. Acad. Sci. U.S.A. 1996, 93, 799-803; b) D. Shabat, C. Rader, B. List, R. A. Lerner, C. F. Barbas III, Proc. Natl. Acad. Sci. U.S.A. 1999, 96, 6925-6930; c) T. A. Houston, Curr. Drug Delivery 2007, 4, 264-268.

a) P. M. Wallace, P. D. Senter, Bioconjugate Chem. 1991, 2, 349-352; b) H. P. Svensson, J. F. Kadow, V. M. Vrudhula, P. M. Wallace, P. D. Senter, Bioconjugate Chem. 1992, 3, 176-181.

a) M. A. Smal, Z. Dong, H. T. Cheung, Y. Asano, L. Escoffier, M. Costello, M. H. N. Tattersall, Biochem. Pharmacol. 1995, 49, 567-574; b) P. M. Deckert, W. G. Bornmann, G. Ritter, C. Williams Jr, J. Franke, U. Keilholz, E. Thiel, L. J. Old, J. R. Bertino, S. Welt, Int. J. Oncol. 2004, 24, 1289-1295.

a) J. C. Florent, C. Monneret, Top. Curr. Chem. 2008, 283, 99-140; b) J. C. Florent, X. Dong, G. Gaudel, S. Mitaku, C. Monneret, J. P. Gesson, J.-C. Jacquesy, M. Mondon, B. Renoux, S. Andrianomenjanahary, S. Michel, M. 
Koch, F. Tillequin, M. Gerken, J. Czech, R. Straub, K. Bosslet J. Med. Chem. $1998,41,3572-3581$.

P. D. Senter, P. C. D. Su, T. Katsuragi, T. Sakai, W. L. Cosand, I. Hellström, K. E. Hellström, Bioconjugate Chem. 1991, 2, 447-451.

P. D. Senter, M. G. Saulnier, G. J. Schreiber, D. L. Hirschberg, J. P. Brown, I. Hellström, K. E. Hellström, Proc. Natl. Acad. Sci. U.S.A. 1988, 85, 4842-4846.

L. N. Jungheim, T. A. Shepherd, D. L. Meyer, J. Org. Chem. 1992, 57, 23342240.

M. L. Rodrigues, P. Carter, C. Wirth, S. Mullins, A. Lee, B. K. Blackburn, Chem. Biol. 1995, 2, 223-227.

N. Pessah, M. Reznik, M. Shamis, F. Yantiri, H. Xin, K. Bowdish, N. Shomron, G. Ast, D. Shabat, Bioorg. Med. Chem. 2004, 12, 1859-1866.

a) C. J. Springer, P. Antoniw, K. D. Bagshawe, F. Searle, G. M. F. Bisset, M. Jarman, J. Med. Chem. 1990, 33, 677-681; b) D. C. Blakey, B. E. Valcaccia, S. East, A. F. Wright, F. T. Boyle, C. J. Springer, P. J. Burke, R. G. Melton, K. D. Bagshawe, Cell Biophys. 1993, 22, 1-8; c) K. D. Bagshawe, Adv. Pharmacol. 1993, 24, 99-121; d) I. Niculescu-Duvaz, F. Friedlos, D. Niculescu-Duvaz, L. Davies, C. J. Springer, Anticancer Drug Des. 1999, 19, 517-538; e) L. A. Wolfe, R. J. Mullin, R. Laethem, T. A. Blumenkopf, M. Cory, J. F. Miller, B. R. Keith, J. Humphreys, G. K. Smith, Bioconj. Chem. 1999, 10, 38-48.

a) M. L. Rodrigues, P. Carter, C. Wirth, S. Mullins, A. Lee, B. K. Blackburn, Chem. Biol. 1995, 2, 223-227; b) U. M. Vrudhula, H. P. Svensson, K. A. Kennedy, P. M. Senter, P. M. Wallace, Bioconj. Chem. 1993, 4, 334-340. W. A. Denny, Eur. J. Med. Chem. 2001, 36, 577-595.

P. M. Deckert, C. Renner, L. S. Cohen, A. Jungbluth, G. Ritter, J. R. Bertino, L. J. Old, S. Welt, British Journal of Cancer 2003, 88, 937-939.

a) H. B. Bosmann, T. C. Hall, Proc. Natl. Acad. Sci. U.S.A. 1974, 71, 1833-1837;

b) M. Y. Torgov, S. C. Alley, C. G. Cerveny, D. Farquhar, P. D. Senter, 
Bioconjugate Chem. 2005, 16, 717-721; c) L. Fang, R. F. Battisti, H. Cheng, P. Reigan, Y. Xin, J. Shen, D. Ross, K. K. Chan, E. W. Martin Jr., P. G. Wang, D. Sun, J. Med. Chem. 2006, 49, 6290-6297; d) H. Devalapally, R. S. Navath, V. Yenamandra, R. R. Akkinepally, R. K. Devarakonda, Ach. Pharm. Res. 2007, 6, 723-732.

N. R. Monks, D. C. Blakey, S. J. East, R. I. Dowell, J. A. Calvete, N. J. Curtin, Eur. J. Cancer 2002, 38, 1543-1552.

N. R. Monks, D. C. Blakey, S. J. East, R. I. Dowell, J. A. Calvete, N. J. Curtin, Eur. J. Cancer 2002, 38, 1543-1552.

A. Mayer, R. J. Francis, S. K. Sharma, B. Tolner, C. J. Springer, J. Martin, G. M. Boxer, J. Bell, A. J. Green, J. A. Hartley, C. Cruickshank, J. Wren, K. A. Chester, R. H. J. Begent, Clin. Cancer Res. 2006, 12, 6509-6516.

a) L. F. Tietze, F. Major, I. Schuberth, D. A. Spiegl, B. Krewer, K. Maksimenka, G. Bringmann, J. Magull, Chem. Eur. J. 2007, 13, 4396-4409; b) L. F. Tietze, F. Major, I. Schuberth, Angew. Chem. 2006, 118, 6724-6727; c) L. F. Tietze, B. Krewer, H. Frauendorf, F. Major, I. Schuberth, Angew. Chem. 2006, 118, 67206724. M. Zientkowska, Dissertation, Göttingen, 2007.

a) S. Schepelmann, I. Niculescu-Duvaz, C. J. Springer, Suicide Gene Therapy. In: M. H. Bronchud, M. A. Foote, G. Giaccone, O. Olopade, P. Workman, Principles of Molecular Oncology, Humana Press Inc., Totowa 2008, 367-382; b) C. Altaner, Cancer Lett. 2008, 270, 191-201; c) G. U. Dachs, J. Tupper, G. M. Tozer, Anti-Cancer Drugs 2005, 16, 349-359; d) I. Niculescu-Duvaz, C. J. Springer, Mol. Biotechnol. 2005, 30, 71-88; e) D. Niculescu-Duvaz, I. Niculescu-Duvaz, C. J. Springer, Methods Mol. Med. 2004, 90, 161-202; f) D. S. Riddick, C. Lee, S. Ramji, E. C. Chinje, R. L. Cowen, K. J. Williams, A. V. Patterson, I. J. Stratford, C. S. Morrow, A. J. Townsend, Y. Jounaidi, C.-S. Chen, T. Su, H. Lu, P. S. Schwartz, D. J. Waxman, Drug Metab. Dispos. 2005, 33, 1083- 
1096; g) D. Portsmouth, J. Hlavaty, M. Renner, Mol. Aspects Med. 2007, 28, 441.

R. Duncan, S. Gac-Breton, R. Keane, R. Musila, Y. N. Sat, R. Satchi, F. Searle, J. Control. Release 2001, 74, 135-146. N. P. Minton, Nat. Rev. Microbiol. 2003, 1, 237-42.

S. Patyar, R. Joshi, D. S. Prasad Byrav, A. Prakash, B. Medhi, B. K. Das, J. Biomed. Sci. 2010, 17, 21.

Q. Zhang, G. Xiang, Y. Zhang, K. Yang, W. Fan, J. Lin, F. Zeng, J. Wu, J. Pharm. Sci. 2006, 95, 2266-2275.

M. A. Robinson, S. T. Charlton, P. Garnier, X. Wang, S. S. Davis, A. C. Perkins, M. Frier, R. Duncan, T. J. Savage, D. A. Wyatt, S. A. Watson, B. G. Davis, Proc. Natl. Acad. Sci. U.S.A. 2004, 101, 14527-14532.

D. Shabat, J. Polym. Sci., Part A: Polym. Chem. 2006, 44, 1569-1578.

K. Bosslet, J. Czech, D. Hoffmann, Tumor Target. 1995, 1, 45- 50.

a) F. M. H. De Groot, E. W. P. Damen, H. W. Scheeren, Curr. Med. Chem. 2001, 8, 1093-1122; b) W. A. Denny, Cancer Invest. 2004, 22, 604-619.

a) T. A. Connors, M. E. Whisson, Nature 1966, 210, 866-867; b) K. Bosslet, R. Straub, M. Blumrich, J. Czech, M. Gerken, B. Sperker, H. K. Kroemer, J.-P. Gesson, M. Koch, C. Monneret, Cancer Res. 1998, 58, 1195-1201; c) A. Sinhababu, D. Thakker, Adv. Drug Deliv. Rev. 1996, 19, 241-273.

T. E. Mürdter, G. Friedel, J. T. Backmann, M. McClellan, M. Schick, M. Gerken, K. Bosslet, P. Fritz, H. Toomes, H. K. Kroemer, B. Sperker, J. Pharmacol. Exp. Ther. 2002, 301, 223-228. L. F. Tietze, R. Seele, B. Leiting, T. Krach, Carbohydr. Res. 1988, 180, 253-262. a) M. de Graaf, E. Boven, H. W. Scheeren, H. J. Haisma, H. M. Pinedo, Curr. Pharm. Des. 2002, 8, 1391-1403; b) X. Chen, B. Wu, P. G. Wang, Curr. Med. Chem. Anti Canc. Agents 2003, 3, 139-150. 
a) S. Brüsselbach, Methods Mol. Med. 2004, 90, 303-330; b) D. Weyel, H.-H. Sedlacek, R. Müller, S. Brüsselbach, Gene Ther. 2000, 7, 224-231.

P. H. J. Houba, E. Boven, I. H. van der Meulen-Muileman, R. G. G. Leenders, J. W. Scheeren, H. M. Pinedo, H. J. Haisma, Int. J. Cancer 2001, 91, 550-554. F. Schmidt, I. Ungureanu, R. Duval, A. Pompon, C. Monneret, Eur. J. Org. Chem. 2001, 2001, 2129-2134.

a) E. Bouvier, S. Thirot, F. Schmidt, C. Monneret, Org. Biomol. Chem. 2003, 1, 3343-3352; b) A. El Alaoui, N. Saha, F. Schmidt, C. Monneret, J. C. Florent, Bioorg. Med. Chem. 2006, 14, 5012-2019.

E. Bouvier, S. Thirot, F. Schmidt, C. Monneret, Bioorg. Med. Chem. 2004, 12, 969-977.

a) L. Devy, F. M. H. de Groot, S. Blacher, A. Hajitou, P. H. Beusker, H. W. Scheeren, J.-M. Foidart, A. Noël, FASEB J. 2004, 18, 565-567; b) F. M. H. de Groot, A. C. W. de Bart, J. H. Verheijen, H. W. Scheeren, J. Med. Chem. 1999, 42, 5277-5283; c) K. Bielawski, A. Bielawska, ChemMedChem 2008, 3, 536542; d) M. Rooseboom, J. N. M. Commandeur, N. P. E. Vermeulen, Pharmacol. Rev. 2004, 56, 53-102.

a) J. M. Brown, W. R. Wilson, Nat. Rev. Cancer 2004, 4, 437-447; b) Y. Chen, L. Hu, Med. Res. Rev. 2009, 29, 29-64; c) K. Binley, Z. Askham, L. Martin, H. Spearman, D. Day, S. Kingsman, S. Naylor, Gene Ther. 2003, 10, 540-549.

a) L. J. Hanka, A. Dietz, S. A. Gerpheide, S. L. Kuentzel, D. G. Martin, J. Antibiot. 1978, 31, 1211-1217; b) D. G. Martin, C. Biles, S. A. Gerpheide, L. J. Hanka, W. C. Krueger, J. P. McGovren, S. A. Mizsak, G. L. Neil, J. C. Stewart, J. Visser, J. Antibiot. 1981, 34, 1119-1125.

J. P. McGroven, G. L. Clarke, E. A. Pratt, T. F. DeKoning, J. Antibiot. 1984, 37, 63-70.

M. Ichimura, T. Ogawa, K. Takahashi, E. Kobayashi, I. Kawamoto, T. Yasuzawa, I. Takahashi, H. Nakano, J. Antibiot. 1990, 43, 1037-1038. 
a) Y. Igarashi, K. Futamata, T. Fujita, A. Sekine, H. Senda, H. Naoki, T. Furumai, J. Antibiot. 2003, 56, 107; b) M. S. Tichenor, D. B. Kastrinsky, D. L. Boger, J. Am. Chem. Soc. 2004, 126, 8396-8398.

a) L. H. Hurley, C.-S. Lee, J. P. McGovren, M. A. Warpehoski, M. A. Mitchell, R. C. Kelly, P. A. Aristoff, Biochemistry 1988, 27, 3886-3892; b) L. H. Hurley, M. A. Warpehoski, C.-S. Lee, J. P. McGovren, T. A. Scahill, R. C. Kelly, M. A. Mitchell, N. A. Wicnienski, I. Gebhard, P. D. Joohnson, V. S. Bradford, J. Am. Chem. Soc. 1990, 112, 4633-4649.

a) Y. Ambroise, D. L. Boger, Bioorg. Med. Chem. 2002, 12, 303-306; b) K. Spiegel, Dissertaion, Triest 2004; c) K. Spiegel, U. Rothlisberger, P. Carloni, J. Phys. Chem. B 2006, 110, 3647-3660.

a) W. C. Tse, D. L. Boger, Chem. Biol. 2004, 11, 1607-1617; b) D. L. Boger, D. L. Hertzog, B. Bollinger, D. S. Johnson, H. Cai, J. Goldberg, P. Turnbull, J. Am. Chem. Soc. 1997, 119, 4977-4986; c) D. L. Boger, B. Bollinger, D. L. Hertzog, D. S. Johnson, H. Cai, P. Mésini, R. M. Garbaccio, Q. Jin, P. A. Kitos, J. Am. Chem. Soc. 1997, 119, 4987-4998; d) D. L. Boger, R. M. Garbaccio, Acc. Chem. Res. 1999, 32, 1043-1052. K. S. MacMillan, D. L. Boger, J. Med. Chem. 2009, 52, 5771-5780.

a) K. N. Kirschner, M. Lee, R. C. Stanley, J. P. Bowen, Bioorg. Med. Chem. 2000, 8, 329-335; b) P. Cimino, G. Bifulco, R. Riccio, L. Gomez-Paloma, V. Barone, Org. Biomol. Chem. 2006, 4, 1242-1251.

A. V. Vargiu, P. Ruggerone, A. Magistrato, P. Carloni, Biophys. J. 2008, 94, 550561.

J. P. McGovren, G. L. Clarke, E. A. Pratt, T. F. DeKoning, J. Antibiot. 1984, 37, 63-70.

a) J. P. McGovern, G. L. Clarke, E. A. Pratte, T. F. deKoning, J. Antibiot. 1983, 37, 63-70; b) S. Nagamura, A. Asai, E. Kobayashi, K. Gomi, H. Saito, Bioorg. Med. Chem. 1997, 5, 623-630; c) B. Cacciari, R. Romagnoli, P. G. Baraldi, T. Da Ros, 
G. Spalluto, Exp. Opin. Ther. Pat. 2000, 10, 1853-1871; d) A. Sato, L. McNulty, K. Cox, S. Kim, A. Scott, K. Daniell, K. Summerville, C. Price, S. Hudson, K. Kiakos, L. A. Hartley, T. Asao, M. Lee, J. Med. Chem. 2005, 48, 3903-3918; e) P. G. Baraldi, A. Bovero, F. Fruttarolo, D. Preti, M. A. Tabrizi, M. G. Pavani, R. Romagnoli, Med. Res. Rev. 2004, 24, 475-528; f) W. A. Denny, Curr. Med. Chem. 2001, 8, 533-544; g) B. S. P. Reddy, S. K. Sharma, J. P. W. Lown, Curr. Med. Chem. 2001, 8, 475-508.

a) D. L. Boger, C. W. Boyce, R. M. Garbaccio, J. A. Goldberg, Chem. Rev. 1997, 97, 787-828; b) D. L. Boger, D. S. Johnson, Angew. Chem. 1996, 108, 15421580; c) S. E. Wolkenberg, D. L. Boger, Chem. Rev. 2002, 102, 2477-2496.

N. Ghosh, H. M. Sheldrake, M. Searcey, K. Pors, Curr. Top. Med. Chem. 2009, 9, 1494-1524.

a) J. P. Parrish, T. V. Hughes, I. Hwang, D. L. Boger, J. Am. Chem. Soc. 2004, 126, 80-81; b) M. S. Tichenor, K. S. MacMillen, J. S. Stover, S. E. Wolkenberg, M. G. Pavani, L. Zanella, A. N. Zaid, G. Spalluto, T. J. Rayl, I. Hwang, P. G. Baraldi, D. L. Boger, J. Am Chem. Soc. 2007, 129, 14092-14099. a) P. G. Baraldi, G. Balboni, M. G. Pavani, G. Spalluto, M. A. Tabrizi, E. De Clercq, J. Balzarini, T. Bando, H. S., R. Romagnoli, J. Med. Chem. 2001, 44, 2536-2543; b) J. P. Parrish, D. B. Kastrinsky, F. Stauffer, M. P. Hedrick, I. Hwang, D. L. Boger, Bioorg. Med. Chem. 2003, 11, 3815-3838; c) Y. Wang, L. Li, W. Ye, Z. Tian, W. Jiang, H. Wang, S. C. Wright, J. W. Larrick, J. Med. Chem. 2003, 46, 634-637.

a) D. L. Boger, T. Ishizaki, Tetrahedron Lett. 1990, 31, 793-796; b) D. B. Kastrinsky, D. L. Boger, J. Org. Chem. 2004, 69, 2284-2289. a) R. I. Pinto, S. J. Lippard, Biochem. Biophys. Acta 1985, 780, 167-180; b) S. E. Sherman, S. J. Lippard, Chem. Rev. 1987, 87, 1153-1181; c) J. P. Caradonna, S. J. Lippard, Inorg. Chem. 1988, 27, 1454, -1466. 
a) R. D. Hightower, B. U. Sevin, J. P. Pevras, M. Untch, R. Angioli, H. Averette, Gynecol. Oncol. 1992, 42, 186-190; b) B. J. Foster, P. M. Larusso, E. Poplin, M. Zalupski, M. Valdivieso, A. Wozniak, L. Flaherty, D. A. Kasunic, R. H. Earhart, L. H. Baker, Invest. New Drugs 1996, 13, 321-326; c) H. A. Burris, V. C. Dleras, M. Tunca, R. H. Earhart, J. R. Echardt, G. I. Rodriguez, D. S. Shaffer, S. M. Fields, E. Campbell, L. Scaaf, D. Kasunic, D. D. Von Hoff, Anticancer Drugs 1997, 8, 588596.

a) L. H. Li, T. F. deKoning, R. C. Kelly, W. C. Krueger, J. P. McGovren, G. E. Padbury, G. L. Petzold, T. L. Wallace, R. J. Ouding, M. D. Prairie, I. Gebhard, Canc. Res. 1992, 52, 4904-4913; b) I. Wolff, K. Bench, J. S. Beijnen, U. Bruntsch, F. Cavalli, J. de Jong, Y. Groot, O. van Tellingen, J. Wanders, C. Sessa, Clin. Canc. Res. 1996, 2, 1717-1723; c) O. van Telligen, C. J. A. Punt, A. Awada, D. J. T. Wagener, M. J. Piccart, Y. Groot, L. J. Scaaf, R. E. C. Henrar, W. J. Nooijen, J. H. Beijnen, Cancer Chemother. Pharmacol. 1998, 41, 377-384.

a) H. C. Pitot, C. Erlichman, J. M. Reid, J. A. Sloan, M. M. Ames, P. G. Bagniewski, P. Atherton-Skaff, A. A. Adjei, J. Rubin, D. Rayson, R. M. Goldberg, Proc. Am. Assoc. Cancer Res. 1999, 40, 91; b) H. C. Pitot, J. M. Reid, J. A. Sloan, M. M. Ames, A. A. Adjei, J. Rubin, P. G. Bagniewski, P. Atherton, D. Rayson, R. M. Goldberg, C. Ehrlichman, Clin. Cancer Res. 2002, 8, 712-717; c) G. H. Schwartz, A. Patnaik, L. A. Hammond, J. Rizzo, K. Berg, D. D. Von Hoff, E. K. Rowinsky, Ann. Oncol. 2003, 14, 775-782.

a) S. R. Alberts, C. Ehrlichman, J. M. Reid, J. A. Sloan, M. M. Ames, R. L. Richardson, R. M. Goldberg, Clin. Canc. Res. 1998, 4, 2111-2117; b) A. Asai, S. Nagamura, E. Kobayashi, K. Gomi, H. Saito, Bioorg. Med. Chem. Lett. 1999, 9, 2995-2998; c) J. Rubin, S. Alberts, V. Suman, H. Pitot, M. Greene, J. Camoriano, Proc. Am. Soc. Clin. Oncol. 2000, 19, abstr. 1198; d) S. N. Markovic, V. J. Suman, A. M. Vokov, T. R. Fitch, D. W. Hillman, A. A. Adjei, S. R. Alberts, J. S. Kaur, T. A. Braich, J. M. Leitch, E. T. Creagan, Am. J. Clin. Oncol. 2002, 25, 308-312. 
R. Baird, S. Winstein, J. Am. Chem. Soc. 1963, 85, 567-578.

a) L. F. Tietze, M. Lieb, T. Herzig, F. Haunert, I. Schuberth, Bioorg. Med. Chem. 2001, 9, 1929-1939; b) M. P. Hay, R. F. Anderson, D. M. Ferry, W. R. Wilson, W. A. Denny, J. Med. Chem. 2003, 46, 5533-5545; c) S. C. Jeffrey, M. Y. Torgov, J. B. Andreyka, L. Boddington, C. G. Cerveny, W. A. Denny, K. A. Gordon, D. Gustin, J. Haugen, T. Kline, M. T. Nguyen, P. D. Senter, J. Med. Chem. 2005, 48, 1344-1358.

a) M. P. Hay, B. M. Sykes, W. A. Denny, W. R. Wilson, Bioorg. Med. Chem. Lett. 1999, 9, 2237-2242; b) W. A. Denny, J. Biomed. Biotech. 2003, 1, 48-70.

M. P. Hay, G. J. Atwell, W. R. Wilson, S. M. Pullen, W. A. Denny, J. Med. Chem. 2003, 46, 2456-2466.

a) W. Jin, J. D. Trzupek, T. J. Rayl, M. A. Broward, G. A. Vielhauer, S. J. Weir, I. Hwang, D. L. Boger, J. Am. Chem. Soc. 2007, 129, 15391-15397; b) J. P. Lajiness, W. R. Robertson, I. Dunwiddie, M. A. Broward, G. A. Vielhauer, S. J. Weir, D. L. Boger, J. Med. Chem. 2010, 53, 7731-7738.

a) J. M. von Hof, Dissertation, Göttingen 2009; b) B. Krewer, Dissertation, Göttingen 2008; c) H. Schuster, Dissertation, Göttingen, 2008; d) F. Major, Dissertation, Göttingen, 2006; e) O. Panknin, Dissertation, Göttingen 2004; f) T. Feuerstein, Dissertation, Göttingen 2003; g) F. Colunga, Dissertation, Göttingen 2007; h) A. Fecher, Dissertation, Göttingen 2000; i) T. Herzig, Dissertation, Göttingen 2000.

a) L. F. Tietze, T. Herzig, A. Fecher, F. Haunert, I. Schuberth, ChemBioChem 2001, 2, 758-765; b) L. F. Tietze, T. Feuerstein, A. Fecher, F. Haunert, O. Panknin, U. Borchers, I. Schuberth, F. Alves, Angew. Chem. 2002, 114, 785787; c) L. F. Tietze, T. Herzig, T. Feuerstein, I. Schuberth, Eur. J. Org. Chem. 2002, 1634-1645.

L. F. Tietze, J. M. von Hof, B. Krewer, M. Müller, F. Major, H. J. Schuster, I. Schuberth, ChemMedChem 2008, 3, 1946-1955. 
L. F. Tietze, H. J. Schuster, K. Schmuck, I. Schuberth, F. Alves, Bioorg. Med. Chem. 2008, 16, 6312-6318.

a) L. F. Tietze, B. Krewer, H. Frauendorf, F. Major, I. Schuberth, Angew. Chem. 2006, 118, 6720-6724; b) L. F. Tietze, B. Krewer, H. Frauendorf, Anal. Bioanal. Chem. 2009, 437-448; c) L. F. Tietze, B. Krewer, F. Major, I. Schuberth, J. Am. Chem. Soc. 2009, 131, 13031-13036; d) L. F. Tietze, B. Krewer, H. Frauendorf, Eur. J. Mass Spectrom. 2009, 15, 661-672; e) L. F. Tietze, B. Krewer, J. M. von Hof, H. Frauendorf, I. Schuberth, Toxins 2009, 1, 134-150.

L. F. Tietze, J. M. von Hof, M. Müller, B. Krewer, I. Schuberth, Angew. Chem. 2010, 122, 7494-7497.

a) S. K. Sharma, G. Jia, J. W. Lown, Curr. Med. Chem. Anti Canc. Agents 2001, 1, 27-45; b) K. M. Rahman, A. S. Thompson, C. H. James, M. Narayanaswarmy, D. E. Thurston, J. Am. Chem. Soc. 2009, 131, 13756-13766. Informationen zur Fluoreszenzmikroskopie: http://de.wikipedia.org/wiki/ Fluoreszenzmikroskopie.

a) O. Shimomura, F. H. Johnson und Y. Saiga. Journal of Cellular and Comparative Physiology 1962, 59, 223-239; b) O. Shimomura. Journal of Microscopy 2005, 217, 1-15.

B. G. Giepmans, S. R. Adams, M. H. Ellisman, R. Y. Tsien, Science 2006, 312, 217-224.

a) http://probes.invitrogen.com/handbook/sections/0806.html; b) S. A. Latt, G. Stetten, J. Histochem. and Cytochem. 1976, 24, 24-33; c) G. A. Daxhelet, M. M. Coene, P.P. Hoet, G. C. Cocito, Anal Biochem. 1989, 179, 401-403; c) F. G. Loontiens, P. Regenfuss, A. Zechel, L. Dumortier, R. M. Clegg, Biochemistry 1990, 29, 9029-9039.
a) http://probes.invitrogen.com/handbook/sections/1202.html;
b) http://probes.invitrogen.com/media/pis/mp07510.pdf. 
a) A. Macho, D. Decaudin, M. Castedo, T. Hirsch, S. A. Susin, N. Zamzami, G. Kroemer, Cytometry 1996, 25, 333-340; b) M. Castedo, T. Hirsch, S. A. Susin, N. Zamzami, P. Marchetti, A. Macho, G. Kroemer. J. of Immunol. 1996, 512-521.

http://products.invitrogen.com/ivgn/en/US/adirect/invitrogen?cmd=IVGNcat DisplayCategory\&catKey $=72201$.

F. Alves, M. Zientkowska, unveröffentlichte Ergebnisse. http://www.mpin-koeln.mpg.de/index.php?id=77.

a) J. Krneta, J. Kroll, F. Alves, C. Prahst, F. Sananbenesi, C. Dullin, S. Kimmina, D. J. Phillips, H. G. Augustin, Cancer Res. 2006, 66, 5686-5694; b) N. V. Evgenov, Z. Medarova, G. Dai, S. Bonner-Weir, A. Moore, Nat. Med. 2006, 12, 144-148; c) S. H. Thorne, R. S. Negrin, C. H. Contag, Science 2006, 311, 1780-1784; d) L. A. Pardo, C. Contreras-Jurado, M. Zientkowska, F. Alves, W. Stühmer, J. Membrane Biol. 2005, 205, 115-124; e) X. Chen, P. S. Conti, R. A. Moats, Cancer Res. 2004, 64, 8009-8014; f) C.-H. Tung, Q. Zeng, K. Shah, D.-E. Kim, D. Schellingerhout, R. Weissleder, Cancer Res. 2004, 64, 1579-1583.

a) R. M. Hoffman, Nature 2005, 5, 796-806; b) G. Seitz, S. W. Warmann, J. Fuchs, H. Heitmann, J. Mahrt, A.-C. Busse, P. Ruck, R. M. Hoffman, J. T. Wessels. Cell Prolif. 2008, 41, 365-374; c) M. Yang, G. Luiken, E. Baranov, R. M. Hofman, BioTechniques 2005, 39, 170-172.

F. F. Jobsis, Science 1977, 198, 1264-1267.

D. L. Boger, J. A. McKie, J. Org. Chem. 1995, 60, 1271-1275.

a) L. F. Tietze, H. J. Schuster, S. M. Hampel, S. Rühl, R. Pfohl, Chem. Eur. J. 2008, 14, 895-901.

H. Tecle, Chem. Biol. Drug Des. 2009, 74, 547-559.

a) J. Scherkenbeck, Eur. J. Org. Chem. 2004, 38-47; b) O. Buchardt et al., J. Org. Chem. 1994, 19, 5767-5773. 
a) R. R. Schmidt, Angew. Chem. 1986, 98, 213-236; Angew. Chem. Int. Ed. Engl. 1986, 25, 212-235; b) W. Dullenkopf, J. C. Castro-Palomino, L. Manzoni, R. R. Schmidt, Carbohydr. Res. 1996, 296, 135-147. H. O. Wirth, O. Königstein, W. Kern, Ann. Chem. 1960, 643, 84-104.

a) P. Portonovo, M. Joullie, Tetrahedron 2000, 56, 3687-3690; b) G. Wells et al., Bioorg. Med. Chem. Lett. 2008, 18, 2147-2151; c) M. D. Burkart, J. Am. Chem. Soc. 2008, 130, 5443-5445; d) L. Desaubry, J. Med. Chem. 2009, 52, 5176-5187; e) E. A. Theodorakis, Org. Biomol. Chem. 2009, 7, 4886-4894. K. MacMillan , J. Am. Chem. Soc. 2011, 133, 1428-1437. T. Symeonidis et al., Bioorg. Med. Chem. Lett. 2009, 19, 1139-1142.

S. O. Kelley, Bioorg. \& Med. Chem. Letters 2007, 17, 5182-5185.

S. M. Yarmoluk et al., Bioorg. Med. Chem. Letters 2000, 10, 2201-2204.

D. D. Perrin, W. L. F. Arnarego, Purification of Laboratory Chemicals, $3^{\text {rd }}$ Ed., Pergamon Press, Oxford, 1988. CAS: 123864-74-4) verwendet. 


\section{$4 \quad$ Danksagung}

Die wichtigste und schönste Unterstützung habe ich während der letzten drei Jahre von meiner Familie erhalten, für die ich ihr unendlich dankbar bin. Hierzu zählen neben meinen Eltern, Großeltern und Geschwistern die gesamte Familie Jakobi. Ein besonderer Dank gilt hierbei Dir, liebe Vera. Du hast es geschafft, mich stets zu motivieren, mir auch in schwierigen Situationen bei zu stehen aber natürlich auch die schönen Stunden des Lebens miteinander zu verbringen.

Meinen „Göttinger Jungs“, Arne, Timo, Julian, Johannes, Jan und Miggen danke ich für die entspannten Fussballabende, das ein oder andere Weizen im Thanners und am Willi sowie dem guten Zusammenhalt und der gegenseitigen Unterstützung während unserer Promotionszeit.

Desweiteren möchte ich mich bei allen Mitarbeitern der analytischen Abteilungen bedanken. Denn ohne ihre tatkräftige Unterstützung wäre die Anfertigung dieser Dissertations nicht möglich gewesen.

Im Besonderen möchte ich mich bei Herrn Dr. Holm Frauendorf für die schnelle und unkomplizierte Hilfe bei der Bearbeitung von Sonderwünschen und dem Messen der zahlreichen HPLC-MS-Spektren danken. Gleiches gilt natürlich für Herrn Frank Hambloch, Frau Györgyi Sommer-Udvarnoki und Frau Gabriele Krökel, die mir bei der Messung der ESI-Spektren stets mit Rat und Tat zur Seite standen.

Ebenso wichtig war für mich der Kontakt mit der NMR-Abteilung, die während der letzten drei Jahre immer wieder hilfreich waren und mir die benötigten Kernresonanzspektren schnellst möglich gemessen und für diese Arbeit bestmöglich dargestellt haben. Hierfür bedanke ich mich bei Herrn R. Machinek, Frau C. Siebert, Herrn M. Weitemeyer, Frau C. Zolke und Frau E. Pfeil.

Für die Auswertung und Durchführung der In-vitro-Zytotoxizitätsuntersuchungen danke ich Frau Dr. Ingrid Schuberth, Frau Anja Herdlitschke und Frau Angela Rübeling. 
So konnte ich jederzeit vorbeikommen, wenn einmal das Wasser knapp war oder die neuesten Toxizitätswerte vorlagen. Vielen Dank für die spannenden und angenehmen Gespräche.

Für das schnelle und sorgfältige Korrekturlesen dieser Arbeit danke ich Michael Müller, Christoph Eichhorst, Stefan Jackenkroll, Galina Pestel und Julian Strohmeier. Herrn Dr. Miso Mitkovski danke ich für die zahlreichen Abende und Nächte gemeinsamer Messzeit am Fluoreszenzmikroskop sowie der Hilfe bei der Auswertung der Ergebnisse der Fluorimeterexperimente.

Desweiteren möchte ich mich bei Merck KGaA für die Unterstüzung während meiner Dissertation bedanken. Ein besonderer Dank gilt hierbei meinem ehemaligen Praktikumstutor Herrn Dr. Achim Schwämmle, der mir spannende Einblicke in die Arbeitswelt eines Chemikers verschafft hat.

Bei allen Mitstreitern und Weggefährten aus dem Arbeitskreis Tietze sowie Frau Sabine Schacht und Frau Martian Pretor danke ich für die nette Arbeitsatmosphäre und die zahlreichen Diskussionen und Ratschläge.

Meine akademischen Lehrer waren u.a. die folgenden Professoren und Dozenten:

G. Beuermann, P. Botschwina, A. de Meijere, U. Diederichsen, C. Ducho, L. Fitjer, H. Frauendorf, C. Griesinger, S. Grond, W. Hack, U. Klingebiel, H. Laatsch, T. Lenzer, R. Machinek, J. Magull, F. Meyer, C. Mösch-Zanetti, H. W. Roesky, J. Schroeder, C. Schulzke, G. M. Sheldrick, D. Stalke, C. Steinem, J. Stülke, M. Suhm, L. F. Tietze, J. Troe, S. Tsogoeva, D. B. Werz, A. Zeeck und P. von Zezschwitz. 


\section{Lebenslauf}

\begin{tabular}{|c|c|}
\hline \multicolumn{2}{|l|}{ Persönliche Daten } \\
\hline Name: & Frank Behrendt \\
\hline Geburtsdatum: & 29.11 .1982 \\
\hline Geburtsort: & Paderborn \\
\hline Eltern: & Heinz-Otto und Albertine Behrendt \\
\hline Staatsangehörigkeit: & deutsch \\
\hline Familienstand: & ledig \\
\hline \multicolumn{2}{|c|}{ Schulausbildung und Zivildienst: } \\
\hline $1989-1993$ & Grundschule Elisabethschule, Paderborn \\
\hline $1993-2002$ & Gymnasium Theodorianum, Paderborn \\
\hline 29.06 .2002 & Allgemeine Hochschulreife \\
\hline \multicolumn{2}{|l|}{ Hochschulausbildung } \\
\hline $10 / 2003-07 / 2008$ & Chemiestudium an der Universität Göttingen \\
\hline $09 / 2007-04 / 2008$ & $\begin{array}{l}\text { Diplomarbeit am Institut für Organische und Biomolekulare } \\
\text { Chemie Göttingen unter der Leitung von Prof. Dr. Dr. h.c. } \\
\text { Lutz F. Tietze über das Thema: "Synthese von seco- } \\
\text { CBI-Derivaten als Analoga des zytotoxischen Antibiotikums } \\
\text { CC-1065" }\end{array}$ \\
\hline 17.07.2008 & Diplomprüfung an der Georg-August Universität Göttingen \\
\hline $09 / 2008-09 / 2011$ & $\begin{array}{l}\text { Dissertation am Institut für Organische und Biomolekulare } \\
\text { Chemie der Georg-August-Universität Göttingen unter der } \\
\text { Leitung von Prof. Dr. Dr. h.c. Lutz F. Tietze über das Thema } \\
\text { "Synthese und biologische Evaluierung von } \\
\text { fluoreszenzmarkierten Duocarmycin-Analoga". }\end{array}$ \\
\hline
\end{tabular}

